\title{
Abstracts of the ECTS Congress 2018
}

\section{ECTS 2018}

\section{6-29 May 2018, Valencia, Spain}

\section{5th European Calcified Tissue Society Congress}

Published online: 4 May 2018

(C) Springer Science+Business Media, LLC, part of Springer Nature 2018

\section{Scientific Programme Committee}

Chair:

Anna Teti (L’Aquila, Italy)

\section{Co-Chairs:}

Claus-Christian Glüer (Kiel, Germany)

Clinical Co-Chair:

Juliet Compston (Cambridge, UK)

Basic and Translational Co-Chair:

André Uitterlinden (Rotterdam, Netherlands)

\section{Members:}

Bo Abrahamsen (Copenhagen, Denmark)

Nuria Guañabens (Barcelona, Spain)

Leonor Cancela (Faro, Portugal)

Roland Chapurlat (Lyon, France)

\section{Local Organising Committee}

\section{Chair:}

Núria Guañabens (Barcelona, Spain)

\section{Members:}

Josep Blanch Rubió (Barcelona, Spain) Cristina Campos (Valencia, Spain)

Antonio Cano (Valencia, Spain)

Placido Llaneza (Oviedo, Spain)

Angels Martinez-Ferrer (Barcelona, Spain)

Xavier Nogués (Barcelona, Spain)

\section{ECTS Academy Members}

Ozge Uluckan (Madrid, Spain)

Carmen Huesa (Paisley, UK) 


\section{Abstract Review Panel}

Each abstract was scored blind.

Abrahamsen, Bo (Denmark)

Agis, Hermann (Austria)

Alonso, Nerea (United Kingdom)

Arnett, Tim (United Kingdom)

Aspden, Richard (United Kingdom)

Athanasio, Anastasilakis (Greece)

Baldini, Nicola (Italy)

Banfi, Giuseppe (Italy)

Baschant, Ulrike (Germany)

Bäuerle, Tobias (Germany)

Berenbaum, Francis (France)

Bergwitz, Clemens,

Bianchi, Gerolamo (Italy)

Bianchi, Maria Luisa (Italy)

Blin-Wakkach, Claudine (France)

Bonnet, Nicolas (Switzerland)

Boot, Annemieke (Netherlands)

Bouillon, Roger (Belgium)

Bouxsein, Mary (United States)

Boyde, Alan (United Kingdom)

Briggs, Michael (United Kingdom)

Briot, Karine (France)

Burden, Andrea (Netherlands)

Busse, Björn (Germany)

Campbell, Graeme (Germany)

Cancela, Leonor (Portugal)

Cappariello, Alfredo (Italy)

Capulli, Mattia (Italy)

Carmeliet, Gertrudis (Belgium)

Cenci, Simone (Italy)

Chapurlat, Roland (France)

Chenu, Chantal (United Kingdom)

Civitelli, Roberto (United States)

Clark, Emma (United Kingdom)

Clezardin, Philippe (France)

Cohen-Solal, Martine (France)

Colaianni, Graziana (Italy)

Compston, Juliet (United Kingdom)

Cooper, Cyrus (United Kingdom)

Cooper, Mark (United Kingdom)

Cormier-Daire, Valerie (France)

Cosman, Felicia

Cummings, Steve (United States)

Dawson-Hughes, Bess (United States) de Vries, Teun (Netherlands)

Diez-Perez, Adolfo (Spain)

Ducy, Patricia (United States)

Eastell, Richard (United Kingdom)

Edwards, Claire (United Kingdom)

Edwards, James (United Kingdom)

Elefteriou, Florent (United States)

Engelke, Klaus (Germany)

Eriksen, Erik (Norway)

Fahrleitner-Pammer, Astrid (Austria)

Farquharson, Colin (United Kingdom)

Ferrari, Serge (Switzerland)

Forlino, Antonella (Italy)

Fraser, William (United Kingdom)

Fukumoto, Seiji (Japan)

Funck-Brentano, Thomas (France)

Galbusera, Fabio (Italy)

Garnero, Patrick (France)

Gautvik, Kaare (Norway)

Gennari, Luigi (Italy)

Geoffroy, Valerie (France)

Gianfrancesco, Fernando (Italy)

Glueer, Claus (Germany)

Goldring, Mary (United States)

Goldring, Steven (United States)

Gregory, Jenny (United Kingdom)

Grigoriadis, Agi (United Kingdom)

Grinberg, Daniel (Spain)

Guanabens, Nuria (Spain)

Guarnieri, Vito (Italy)

Hackl, Matthias (Austria)

Hadji, Peyman (Germany)

Hay, Eric (France)

Hesse, Eric (Germany)

Holen, Ingunn (United Kingdom)

Huesa, Carmen (United Kingdom)

Ignatius, Anita (Germany)

Jafari, Abbas (Denmark)

Jähn, Katharina (Germany)

Jakob, Franz (Germany)

Javaid, Kassim (United Kingdom)

Karperien, Marcel (Netherlands)

Kassem, Moustapha (Denmark)

Kiel, Doug (United States)
Kjaer, Mchael (Denmark)

Klaushofer, Klaus (Austria)

Klein Nulend, Jenneke (Netherlands)

Kornak, Uwe (Germany)

Kraenzlin, Marius (Switzerland)

Krönke, Gerhard (Germany)

Lafage-Proust, Marie-Helene (France)

Langman, Craig (United States)

Lanske, Beate (United States)

Legai-Mallet, Laurence (France)

Lems, Willem (Netherlands)

Lips, Paul (Netherlands)

Machuca, Irma (France)

Marie, Pierre (France)

Matsumoto, Toshio (Japan)

Maurizi, Antonio (Italy)

Mazzaferro, Sandro (Italy)

Medina-Gomez, Carolina (Netherlands)

Merlotti, Daniela (Italy)

Minisola, Salvatore (Italy)

Müller, Ralph (Switzerland)

Napoli, Nicola (Italy)

Nishimura, Riko (Japan)

Noda, Masaki (Japan)

Nuki, George (United Kingdom)

Obermayer-Pietsch, Barbara (Austria)

Orriss, Isabel (United Kingdom)

Orwoll, Eric (United States)

Pacifici, Roberto (United States)

Paschalis, Eleftherios (Austria)

Peris, Pilar (Spain)

Peyruchaud, Olivier (France)

Pietschmann, Peter (Austria)

Pitsillides, Andrew (United Kingdom)

Provot, Sylvain (France)

Ralston, Stuart (United Kingdom)

Ramasamy, Saravana (United Kingdom)

Rauner, Martina (Germany)

Reid, David (United Kingdom)

Rejnmark, Lars (Denmark)

Riminucci, Mara (Italy)

Roato, Ilaria (Italy)

Robey, Pamela Gehron (United States)

Rossi, Antonio (Italy)
Roux, Christian (France)

Rucci, Nadia (Italy)

Santini, Daniele (Italy)

Scheafer, Arne (Germany)

Schinke, Thorsten (Germany)

Schnabel, Dirk (Germany)

Schwartz, Jean-Marc (United Kingdom)

Schwarz, Peter (Denmark)

Seibel, Markus (Austria)

Settembre, Carmine (Italy)

Sobacchi, Cristina (Italy)

Sonntag, Stephan (Switzerland)

Staines, Katherine (United Kingdom)

Stenbeck, Gudrun (United Kingdom)

Szulc, Pawel (France)

Taipaleenmäki, Hanna (Germany)

Teti, Anna (Italy)

Tobias, Jon (United Kingdom)

Tsourdi, Elena (Germany)

Tuckermann, Jan (Germany)

Uluckan, Ozge (Spain)

van de Peppel, Jeroen (Netherlands)

van der Eerden, Bram (Netherlands)

van der Pluijm, Gabri (Netherlands)

van Driel, Marjolein (Netherlands)

van Hul, Wim (Belgium)

van Lenthe, G Harry (Belgium)

van Wijnen, Andre (United States)

Veldhuis-Vlug, Annegreet (Netherlands)

Vestergaard, Peter (Denmark)

Vico, Laurence (France)

Villa, Anna (Italy)

Ward, Kate (United Kingdom)

Williams, Graham (United Kingdom)

Young, Marian (United States)

Zambuzzi, Willian (Brazil)

Zaucke, Frabk (Germany)

Zillikens, Carola (Netherlands)

Zwerina, Jochen (Austria)

Zysset, Philippe (Switzerland) 


\section{Plenary Oral Presentations 1: Bone regulators}

\section{PLO01}

A high resolution Capture-C promoter 'interactome' implicates causal genes at BMD GWAS loci

Alessandra Chesi ${ }^{1}$, Matthew E. Johnson ${ }^{1}$, Yadav Wagley ${ }^{2}$, Sumei Lu $^{1}$, Michelle E. Leonard ${ }^{1}$, Kenyaita M. Hodge ${ }^{1}$, James A. Pippin ${ }^{1}$, Elisabetta Manduchi ${ }^{1,3}$, Andrew D. Wells ${ }^{1,4}$, Kurt D. Hankenson ${ }^{2}$, Struan F.A. Grant ${ }^{1,5,6}$

${ }^{1}$ Center for Spatial and Functional Genomics, Children's Hospital of Philadelphia, Philadelphia, United States, ${ }^{2}$ Department of Orthopaedic Surgery, University of Michigan, Ann Arbor, United States, ${ }^{3}$ Institute for Biomedical Informatics, University of Pennsylvania Perelman School of Medicine, Philadelphia, United States, ${ }^{4}$ Department of Pathology and Laboratory Medicine, University of Pennsylvania Perelman School of Medicine, Philadelphia, United States, ${ }^{5}$ Department of Pediatrics, University of Pennsylvania Perelman School of Medicine, Philadelphia, United States, ${ }^{6}$ Divisions of Genetics and Endocrinology, Children's Hospital of Philadelphia, Philadelphia, United States

There is clear evidence for a genetic component to the pathogenesis of osteoporosis. Intense genome wide association study (GWAS) efforts have been successful in discovering key genetic variants robustly associated with bone mineral density (BMD), both in children and adults. However, it is known that GWAS only reports genomic signals and not necessarily the precise localization of culprit genes. Given the notable paucity of genomic data available on bone in the public domain, and to improve upon the low resolution of typical Hi-C approaches, we developed a massively parallel, high-resolution Capture-C based method to characterize the genome-wide interactions of all human promoters in an osteoblast model setting. We designed a custom Agilent SureSelect RNA library targeting DpnII restriction fragments overlapping 36,691 promoters of protein-coding, noncoding, antisense, snRNA, miRNA, snoRNA and lincRNA genes. We applied our method of SPATIaL-seq (genome-Scale, Promoterfocused Analysis of chromaTIn Looping) to primary human mesenchymal progenitor cell (MSC)-derived osteoblasts. We also generated ATAC-seq open chromatin maps from the same samples to determine informative proxy SNPs for each of 111 adult and pediatric BMD GWAS loci. By intersecting our sub- $1 \mathrm{~kb}$ promoter 'interactome' data with the ATAC-seq data, we observed consistent contacts between "open" promoters and proxy SNPs for at least 31 loci. Some 'nearest' genes to the sentinel SNP were supported e.g. SMAD3, SMG6 and SOST1, while at other loci more distant genes were implicated e.g. EPDR1 at the 'STARD3NL' locus and NAA50 at the 'KIAA2018' locus. In conclusion, we observed consistent contacts to at least $\sim 30 \%$ of BMD GWAS loci using the highest resolution promoter 'interactome' to date in a single, disease-relevant cell type. Only by establishing which specific genes at such BMD loci are regulated in the correct cellular context can one truly translate GWAS findings in to more efficacious treatments for bone-related diseases like osteoporosis.

\section{PLO02}

Genome-wide association of bone mineral density in the UK Biobank full release identifies 301 novel loci and implicates DAAM2 in osteoporosis

\section{John Morris ${ }^{1}$, GEFOS Consortium}

${ }^{1}$ Human Genetics, McGill University, Montreal, Canada

Bone mineral density (BMD) is the most clinically relevant predictor of fracture and osteoporosis, a common, aging-related disease.
Previously, a genome-wide association study (GWAS) of calcaneal ultrasound estimated BMD (eBMD) in 142,487 individuals was performed, identifying 203 loci, explaining $12 \%$ of the trait variance and identifying novel determinants of osteoporosis. Here, we performed a GWAS of eBMD in 426,824 White-British UK Biobank participants (55\% female). Using a bioinformatics workflow that integrated statistical fine-mapping and functional genomics data, we prioritized genes for functional experiments in whole animal models and demonstrated the utility of this workflow by focusing on dishevelled-associated activator of morphogenesis 2 (DAAM2), a novel gene involved in Wnt-signalling.

We identified 1,106 conditionally-independent signals $\left(P<6.6 \times 10^{-9}\right)$ mapping to 518 loci $(301$ novel $)$ and explained $20 \%$ of the trait variance. Our workflow prioritized 538 proteincoding genes (247 from novel loci), which we found were strongly enriched for expression in osteoblasts and osteocytes, and known genes for osteoporosis drug targets and bone disorders. Our workflow identified plausibly causal coding SNPs for DAAM2, therefore we generated homozygous (Daam $2^{-l-}$ ) and heterozygous knockout mice for skeletal phenotyping with X-ray microradiography, microtomography, and biomechanical testing. Daam $2^{-1-}$ mice had a small nonsignificant reduction in femur length, and reduced femur and vertebral bone mineral content. Structural parameters of trabecular and cortical bone did not differ between wild-type and Daam $2^{-1-}$ mice. Strikingly, despite normal bone structural parameters, maximum load was greatly reduced in Daam2 $2^{-1-}$ femurs and vertebrae (2.14 standard deviations below predicted strength), suggesting Daam2 deficiency impaired bone quality.

Here, we increased the number of associated loci 2.5 -fold, the variance explained to $20 \%$, provided an efficient strategy to prioritize genes, and identified a novel determinant of bone quality, DAAM2. These results provide further insight into novel, potentially druggable targets contributing to osteoporosis.

Keywords: Osteoporosis, GWAS, DAAM2, UK Biobank, finemapping

\section{PLO03}

Aging-related bone loss is attenuated through Tgif1-ERK1/2 signaling in osteoclasts

Miki Maeda $^{1}$, Hiroaki Saito ${ }^{1}$, Hanna Taipaleenmäki ${ }^{1}$, Eric Hesse ${ }^{1,2}$

${ }^{1}$ Molecular Skeletal Biology Laboratory, Department of Trauma, Hand and Reconstructive Surgery, University Medical Center Hamburg-Eppendorf, Hamburg, Germany, ${ }^{2}$ Department of Anatomy and Cell Biology, Indiana University School of Medicine, Indianapolis, United States

High resorption activity is a key component of bone loss during aging. We identified an increased expression of the homeodomain protein TG-interacting factor 1 (Tgif1) in osteoclasts after RANKL/ M-CSF stimulation, suggesting a functional role of Tgif1 in osteoclast differentiation. To test this hypothesis, we deleted Tgif1 in the germline $\left(\right.$ Tgifl $\left.^{-l-}\right)$ and in the osteoclast lineage $\left(\right.$ Ctsk-Cre ${ }^{+}$; Tgifl $1^{f l}$ $\left.{ }_{\mathrm{fl}}\right)$. Interestingly, 8-month old $\mathrm{Ctsk}-\mathrm{Cr} \mathrm{e}^{+} ;$Tgifl $^{\text {fllfl }}$ mice were protected from aging-related bone loss due to reduced osteoclast number and bone resorption $(\mathrm{p}<0.05)$. Furthermore, in vitro differentiation of bone marrow macrophages (BMMs) obtained from $\mathrm{Tgif1}^{-1-}$ or Ctsk$\mathrm{Cre}^{+} ;$Tgifl $^{\mathrm{fl} f \mathrm{fl}}$ mice demonstrated decreased number and size of osteoclasts $(p<0.05)$ and reduced expression of osteoclast-related genes such as NFATc1 and Cathepsin K, leading to fewer and shallower resorption pits. These findings indicate that Tgif1 promotes osteoclast differentiation and function in a cell-autonomous manner and establish Tgif 1 as a novel regulator of bone resorption. To elucidate the underlying molecular mechanism, we performed signaling analyses, which revealed reduction of phosphorylated ERK1/2 in Tgif1 $1^{-1-}$ BMMs. While the de novo phosphorylation of ERK1/2 in 
response to RANKL/M-CSF stimulation was comparable between wild-type and Tgif1 ${ }^{-1-}$ BMMs, ERK1/2 was rapidly de-phosphorylated in the absence of Tgif1. This suggests that Tgif1 controls ERK1/ 2 activity by inhibiting the expression of a specific phosphatase. Indeed, expression analysis of several ERK phosphatases demonstrated an increased expression of the Protein Phosphatase 2A catalytic subunit isoform $\beta$ (PP2A-C $\beta$ ) in Tgifl $^{-/-}$BMMs compared to control cells. Mechanistically, inhibition of PP2A using okadaic acid or targeted silencing of PP2A-C $\beta$ using the GapmeR technology normalized the amount of phosphorylated ERK1/2 and restored the reduced osteoclast differentiation in Tgifl ${ }^{-1-}$ BMMs. In summary, Tgif1-deficiency in osteoclasts impairs ERK1/2 signaling and subsequently osteoclast differentiation, function and bone resorption. Thus, Tgif1 is a novel regulator of bone remodeling with an important function in aging-related bone loss.

Keywords: Osteoclast, Tgif1

\section{PLO04}

Androgen receptor in neurons is protective against age-related loss of cortical bone in male mice

Ferran Jardi ${ }^{1}$, Michael Laurent ${ }^{1}$, Nari Kim $^{1}$, Rougin Khalil ${ }^{1}$, Geert Carmeliet $^{1}$, Ludo Deboel ${ }^{1}$, Dieter Schollaert ${ }^{1}$, Brigitte Decallonne ${ }^{1}$, Frank Claessens ${ }^{1}$, Dirk Vanderschueren ${ }^{1}$

\section{${ }^{1}$ KU Leuven, Leuven, Belgium}

Both androgens and normal androgen receptor (AR) function are crucial for male bone health. The stimulatory effects of androgens on the cortical compartment appear to be mediated by AR-signaling outside bone tissue. In females, neuronal estrogen receptor alpha inhibits cortical bone mass. So far, a similar role for central AR regulating male bone is not proved. To this end, we knocked out the AR in the nervous system of AR floxed male mice expressing a tamoxifen-inducible CreER under the control of the neuronal promoter Thy. Tamoxifen induced a substantial loss of AR mRNA levels in Thy1-CreER-positive regions (cerebral cortex: $\sim 80 \%$; brainstem: $\sim 75 \%$ ) but not in the hypothalamus or non-neuronal tissues of male N-ARKO mice. There were no differences between genotypes in body weight, body composition or serum testosterone or LH. In contrast to aged-matched orchiectomised (ORX) mice, deletion of neuronal AR did not alter grip strength or hindlimb muscle mass. In young adults (16 weeks), microct analysis of the femur revealed no changes in the trabecular or cortical compartments. However, at both 36 and 46 weeks, deletion of neuronal AR aggravated the age-related losses of cortical thickness and area. The decline in cortical thickness in N-ARKO mice was comparable to that observed in ORX mice $(-8 \%)$. In addition, N-ARKO mice showed trabecular thinning, which did not translate into a loss of trabecular bone. We corroborated our findings in the tibia and following replacement with testosterone or dihydrotestosterone. The trabecular but not cortical surface covered by osteoclasts was increased in the femur of N-ARKO mice whilst osteoblast counts remained unaltered. Finally, both ORX and N-ARKO mice showed an increased expression of uncoupling protein 1 in brown fat, a widely used readout for sympathetic tone. We conclude that androgen actions on the nervous system play a beneficial role decelerating cortical thinning in male mice.

\section{PLO05}

PGC1 $\alpha$ deficiency negatively regulates bone mass and strength

Graziana Colaianni ${ }^{1}$, Luciana Lippo ${ }^{1}$, Lorenzo Sanesi ${ }^{1}$, Giacomina Brunetti $^{2}$, Monica Celi ${ }^{3}$, Nunzio Cirulli ${ }^{2}$, Giovanni Passeri ${ }^{4}$, Janne Reseland $^{5}$, Umberto Tarantino ${ }^{3}$, Silvia Colucci ${ }^{2}$, Maria Grano ${ }^{1}$
${ }^{I}$ Department of Emergency and Organ Transplantation, University of Bari, Bari, Italy, ${ }^{2}$ Department of Basic Medical Science, Neuroscience and Sense Organs, University of Bari, Bari, Italy, ${ }^{3}$ Department of Orthopedics and Traumatology, Tor Vergata University of Rome, Rome, Italy, ${ }^{4}$ Department of Clinical and Experimental Medicine, University of Parma, Parma, Italy, ${ }^{5}$ Department of Biomaterials, University of Oslo, Oslo, Norway

Peroxisome proliferator-activated receptor- $\gamma$ coactivator $1-\alpha$ $(\mathrm{PGC} 1 \alpha)$ is a transcription factor regulating adaptive thermogenesis, glucose metabolism and skeletal muscle fiber type specialization. Here we show that PGC- $1 \alpha$ has a dominant action on the skeleton since PGC- $1 \alpha$ heterozygous (PGC- $1 \alpha^{+/-}$) and deficient (PGC- $1 \alpha^{-/-}$) mice have impaired bone structure and increased fragility. Micro-CT of the tibial mid-shaft showed a marked decrease of cortical thickness in PGC- $1 \alpha^{+/-}(-8.4 \%, \mathrm{p}<0.05)$ and PGC- $1 \alpha^{-/-}(-11.9 \%$, $\mathrm{p}<0.05)$ mice compared to wild type littermates. For trabecular bone, knock out mice displayed lower trabecular thickness (Tb.Th) $\left(-5.9 \% \mathrm{Vs}\right.$ PGC- $\left.1 \alpha^{+/+}, \mathrm{p}<0.05\right)$, whereas trabecular number (Tb.N) was higher than wild type mice $\left(+72 \%\right.$ Vs PGC- $1 \alpha^{+/+}$, $\mathrm{p}<0.05)$, thus resulting in increased $\left(+31.7 \%\right.$ Vs PGC- $1 \alpha^{+/+}$, $\mathrm{p}<0.05$ ) degree of anisotropy (DA), despite unchanged bone volume fraction (BV/TV). Notably, the impairment of cortical and trabecular bone in PGC- $1 \alpha$ knock out mice led to a dramatic $\sim 48.4 \%$ decrease in bending strength $(\mathrm{p}<0.01)$. Bone defects were paralleled by a significant increase in osteoclast number at the cortical bone surface and in serum levels of the bone resorption marker, namely C-terminal crosslinked telopeptides of type I collagen (CTX-I) (+ 42\% Vs PGC$\left.1 \alpha^{+/+}, \mathrm{p}<0.05\right)$, whereas no difference was observed in serum levels of N-terminal propeptide of type 1 procollagen (P1NP) among the genotypes. In PGC- $1 \alpha^{-1-}$, we also found a lower expression of mRNAs codifying for Osteocalcin $(O c n)$ in cortical bone and for Collagen $I$ in bone marrow stromal cells. Moreover, we found that PGC- $1 \alpha^{-1-}$ mice displayed a lower ratio of inguinal WAT (iWAT)/body weight and histological analysis of iWAT revealed profound $\sim 75 \%$ decrease in adipocyte area and a lower expression of Adiponectin (AdipoQ) mRNA, whereas Leptin mRNA was several folds higher than wild type mice. Overall, results presented herein suggest for the first time that PGC- $1 \alpha$ may play a key role in bone metabolism.

\section{PLO06}

Tram2 is a novel genetic determinant of bone mass and strength

Victoria D. Leitch ${ }^{1}$, Penny Sparkes ${ }^{1}$, John G. Logan ${ }^{1}$, Agnes Swiatkowska $^{2}$, Natalie C. Butterfield ${ }^{1}$, Peter I. Croucher ${ }^{3}$, Christoper J. Lelliott ${ }^{2}$, Graham R. Williams ${ }^{1}$, J. H. Duncan Bassett ${ }^{1}$

${ }^{1}$ Molecular Endocrinology Laboratory, Department of Medicine, Imperial College London, London, United Kingdom, ${ }^{2}$ Mouse Pipelines, Wellcome Trust Sanger Institute, Cambridge, United Kingdom, ${ }^{3}$ Bone Biology Division, Garvan Institute of Medical Research, Sydney, Australia

Osteoporosis is the commonest skeletal disorder, costing billions of euros annually. Bone mineral density is highly heritable, but only $12 \%$ of phenotype variance is currently accounted for. Treatments reduce fracture risk by only $50 \%$ and there is urgent need to define new pathways that regulate bone turnover and strength.

We hypothesised that rapid-throughput phenotyping of knockout mice would identify novel susceptibility alleles for bone and mineral disorders and provide in-vivo models to elucidate their molecular basis.

Translocation associated membrane protein-2 knockout mice $\left(\operatorname{Tram} 2^{-/-}\right)$were identified in this screen with reduced body weight, deafness, and spontaneous fractures, despite normal serum biochemistry. 
Detailed analysis (X-ray microradiography, micro-CT, backscattered-electron scanning-electron microscopy, biomechanical testing, $\mathrm{n}=6$ per gender, per genotype) demonstrated short stature $(\mathrm{P}<0.001$, ANOVA), grossly reduced bone mineral content and mineralisation ( $\mathrm{P}<0.001$, Kolmogorov-Smirnov test), profoundly reduced cortical $(\mathrm{P}<0.001$, ANOVA) and trabecular bone mass $(\mathrm{P}<0.001$, ANOVA), and decreased bone strength and stiffness $\left(\mathrm{P}<0.001\right.$, ANOVA) in Tram $2^{-1-}$ mice. Tram $2^{-1-}$ primary osteoblasts had reduced proliferation $(\mathrm{P}<0.001$, t-test $)$ and mineralisation; while osteoclasts had increased nuclei $(\mathrm{P}<0.001$, t-test $)$ and resorption $(\mathrm{P}<0.01$, t-test) compared to wildtype.

Tram2 lies downstream of BMP/Runx2 in osteoblasts and is associated with fracture in genome-wide association studies. Tram 2 is a component of the translocon responsible for the folding of type-1 collagen, however, we detected no abnormalities of type- 1 collagen structure by electron microscopy or protein amount by western blot in $\operatorname{Tram} 2^{-1-}$ mice.

These data demonstrate that $\operatorname{Tram} 2$ is required for normal bone mineralisation, structure and strength. The abnormal skeletal phenotype in Tram $2^{-1-}$ mice likely results from impaired bone formation during growth, together with uncoupling of adult bone turnover resulting in severe bone loss. Elucidation of the cellular and molecular mechanisms underlying this gross skeletal phenotype may identify novel tractable therapeutic targets for prevention and treatment of osteoporosis.

\section{Plenary Oral Presentations 2: FGF23}

\section{PLO07}

FGFR3-gain-of-function mutation modifies lumbar vertebrae structure and cranial synchondroses in a hypochondroplasia mouse model

$\underline{\text { Léa Loisay }}^{1}$, Nabil Kaci ${ }^{1}$, Maxence Cornille ${ }^{1}$, Valentin Estibals ${ }^{1}$, Morad Bensidhoum $^{2}$, Yann Heuzé ${ }^{3}$, Laurence Legeai-Mallet ${ }^{1}$

${ }^{1}$ Imagine Institute, Paris, France, ${ }^{2}$ Université Paris Diderot, B2OAUMR CNRS 7052, Paris, France, ${ }^{3}$ CNRS, Université de Bordeaux, MCC, PACEA, UMR5199, Pessac, France

Hypochondroplasia $(\mathrm{HCH})$ is a moderate rhizomelic dwarfism with lordosis and kyphosis. This syndrome is caused by a gain-offunction mutation in Fibroblast Growth Factor Receptor 3 (Fgfr3) gene. We generated the first mouse model of HCH $\left(F g f r 3^{\text {Asn } 534 L y s /+}\right)$, expressing the most common missense mutation of this disease (pAsn540Lys). Macroscopic analysis of $F g f r 3^{\text {Asn } 534 L y s /+}$ mice showed a growth defect with reduction of appendicular at 14 days of age (e.g. $-13 \%$ femur length, $-19 \%$ humerus length) and axial skeleton ( $-7 \%$ naso-anal length) compared to controls. $\mu \mathrm{CT}$ scanner analyses of the skull base showed a fusion of intersphenoid and spheno occipital synchondroses with histological anomalies at 14 days of age. Studying L4-L6 lumbar vertebrae, we showed a defective chondrocyte differentiation in cartilage end-plate (CEP) of mutant mice from one week to three months of age. We also observed that the size and the shape of nucleus pulposus (NP) and annulus fibrosus (AF) inside the intervertebral disc (IVD) are modified. The height of NP is enhanced $(+31 \%)$ and the weight is decreased $(-18 \%)$ in Fofr $3^{\text {Asn } 534 \text { Lys/ }+}$ mice compared to control mice at 3 weeks of age. After that, we studied the AF matrix using collagen type I and type II staining and we observed that collagen type II area is increased, while there is no modification of collagen type I expression in $\mathrm{FgFr}^{\mathrm{As}-}$ ${ }^{n 534 L y s} /+$ mice compared to control littermates. In parallel, we observed an overexpression of FGFR3 and Sox9 in CEP and AF of Fgfr $3^{\text {Asn } 534 \text { Lys } /+}$ mice.
In conclusion, we show a fusion of the synchondroses at the skull base and anomalies of IVD in Fgfr $3^{\text {Asn } 534 L y s /+}$ mice. This study highlights an essential role of Fgfr3 in both synchondroses and IVD development.

\section{PLO08}

Dissecting the alterations of bone remodeling activity in Cystinosis Giulia Battafarano ${ }^{1}$, Michela Rossi ${ }^{1}$, Laura Rita Rega ${ }^{2}$, Gianna Di Giovamberardino $^{3}$, Anna Pastore ${ }^{3}$, Matteo D'Agostini ${ }^{4}$, Ottavia Porzio $^{4}$, Francesco Emma ${ }^{2}$, Anna Taranta ${ }^{2}$, Andrea Del Fattore ${ }^{1}$

${ }^{1}$ Bone Physiopathology Group, Multifactorial Disease and Complex Phenotype Research Area, Bambino Gesù Children's Hospital, Rome, Italy, ${ }^{2}$ Department of Nephrology and Urology, Division of Nephrology, Bambino Gesù Children's Hospital, Rome, Italy, ${ }^{3}$ Laboratory of Metabolomics and Proteomics, Bambino Gesù Children's Hospital, Rome, Italy, ${ }^{4}$ Clinical Laboratory, Bambino Gesù Children's Hospital, Rome, Italy

Cystinosis is a rare disease caused by loss-of-function mutation of CTNS gene, encoding for cystinosin lysosomal transporter. It is characterized by cystine accumulation into the lysosomes leading to crystals formation and cellular damage. Cystinotic patients develop nephropathic Fanconi syndrome, bone deformity and fragility, osteomalacia and rickets. Skeletal alterations in cystinotic patients have been considered until now as a consequence of renal impairment. To understand if cystinosin deficiency primarily affects the skeleton, we studied ctns $-/-(\mathrm{KO})$ male mice that develop skeletal alterations without Fanconi syndrome up to 9 months of age.

We analyzed the bone phenotype of 1-month-old KO animals. Initially we excluded the nephropathy by histological evaluation of kidney and biochemical analysis. HPLC analysis revealed intralysosomal cystine accumulation in $\mathrm{KO}$ bones. $\mu \mathrm{CT}$ analysis of femurs isolated from $\mathrm{KO}$ mice displayed a reduction of trabecular bone volume and bone mineral density (BMD $\mathrm{mgHA} / \mathrm{cm}^{3}$, WT: $188.6 \pm 4.13$; KO: $162.8 \pm 4.11 . \mathrm{p}<0.005)$ with a decrease of trabecular number and thickness and increased trabecular separation. Histomorphometric analysis revealed a reduction of osteoclasts (Oc.S/BS \%, WT: $22.45 \pm 6.62$; KO: $12.91 \pm 4.85 . \mathrm{p}<0.05$ ) and osteoblast parameters (Ob.S/BS \%, WT: $20.87 \pm 3.26$; KO: $12.08 \pm 6.69 . \mathrm{p}<0.05)$ in KO animals compared to WT. These alterations were confirmed by reduced TRAcP and P1NP levels in KO sera. In vitro analysis revealed intra-lysosomal cystine accumulation in $\mathrm{KO}$ osteoclasts and osteoblasts with a perinuclear engulfment of lysosomes. Cultures from bone marrow of $c t n s-/-$ mice displayed reduced number of osteoclasts (Osteoclast number/field, WT: $100.70 \pm 4.59$; KO: $75.34 \pm 26.44 . \mathrm{p}<0.05$ ). Moreover a defect of differentiation (ALP positive cells \%, WT: $99.67 \pm 0.58$; KO: $59.63 \pm 20.65 . \mathrm{p}<0.05)$ with reduced alp, colla2, runx 2 and $s p 7$ expression was observed in KO osteoblasts compared to WT cells. All together our data reveal that the absence of cystinosin primarily affects bone cell differentiation leading to osteoporotic phenotype in ctns $-/$ - mice.

Keywords: Cystinosis, Remodeling

\section{PLO09}

Neutralizing FGF-23 restores the altered bone phenotype and delays the onset of anemia in myelodysplastic mice

Heike Weidner $^{1,2}$, Ekaterina Balaian ${ }^{1}$, Ulrike Baschant ${ }^{2,3}$, Bill Richards $^{4}$, Lorenz C. Hofbauer ${ }^{2,3,5}$, Uwe Platzbecker ${ }^{1,3,5}$, Martina Rauner ${ }^{2,3}$ 
${ }^{I}$ Technische Universität, Department of Medicine I, Dresden, Germany, ${ }^{2}$ Technische Universität, Department of Medicine III, Dresden, Germany, ${ }^{3}$ Technische Universität, Center for Healthy Aging, Dresden, Germany, ${ }^{4}$ Amgen Inc., Thousand Oaks, United States, ${ }^{5}$ German Cancer Consortium (DKTK), partner site Dresden and German Cancer Research Center (DKFZ), Heidelberg, Germany

Introduction: Myelodysplastic syndromes (MDS) are hematopoietic disorders characterized by ineffective hematopoiesis and cytopenias. We recently showed that MDS patients develop osteoporosis more frequently than age-matched controls. Moreover, NUP98-HOXD13 (NHD13) mice with MDSlike symptoms revealed structural abnormalities with high osteoblast activity, even before the onset of MDS ( $\sim 4$ months of age). Here, we investigated the underlying cellular and molecular mechanism of the bone abnormalities.

Methods: Using von Kossa/van Gieson staining bone mineralization was assessed in tibiae of 2- and 6-month-old NHD13 and wildtype (WT) mice. FGF-23 serum levels were determined by ELISA. Eight-week-old NHD13 and WT mice were treated with $5 \mathrm{mg} / \mathrm{kg}$ FGF-23 antibody for 8 weeks and blood counts were monitored. Bones were used for histological and structural analyses. FGF-23 serum levels were further measured in 44 treatment naïve MDS patients and in 32 age-matched controls. Osteoid was evaluated in bone biopsies from 14 MDS patients and compared to the reference range of healthy individuals.

Results: At both ages, NHD13 mice showed a higher osteoid volume [4/1.7-fold; $P<0.05]$ but FGF-23 serum levels were increased only in 6-month-old mice [2.2-fold; $P<0.001$ ]. Elevated osteoid volume and FGF-23 serum levels were also found in MDS patients [2/4fold; $P<.05]$. Treatment of 2 -month-old NHD13 mice with FGF-23 antibodies improved erythropoiesis [hemoglobin: $+5 \% ; P<0.05]$ and the trabecular number increased up to WT levels $[+16 \% ; P<0.01]$. In both groups, the antibody treatment reduced the osteoid surface [WT: $-65 \% ; P<0.05$; NHD13: $-54 \% ; P<001$.] and the mineralization lag time [WT: $-80 \%$; $P<0.01$; NHD13: $-73 \% ; P<0.05$ ].

Conclusion: MDS patients and NHD13 mice show impaired bone mineralization and high FGF-23 serum levels. Neutralizing FGF-23 improved bone mineralization and delayed the onset of anemia. Thus, targeting FGF-23 may be a novel strategy to improve the dysfunctional osteo-hematopoietic niche.

Keywords: bone mineralization, FGF-23, myelodysplastic syndromes

\section{PLO10}

Increased cancer mortality in older men with higher serum concentrations of fibroblast growth factor 23-the STRAMBO study Pawel Szulc $^{1}$, Roland Chapurlat ${ }^{1}$, Lorenz Hofbauer ${ }^{2}$

${ }^{1}$ INSERM UMR 1033, Université de Lyon, Lyon, France, ${ }^{2}$ Technische Universität Dresden, Dresden, Germany

Higher levels of fibroblast growth factor 23 (FGF23) are associated with higher mortality in chronic kidney disease. By contrast, data on the link between FGF23 and mortality in general population are limited and discordant. Our aim was to study the association of baseline serum FGF23 levels with mortality in a cohort of 814 men aged 60-87 followed up prospectively for 8 years. FGF23 was measured by ELISA (Biomedica, Vienna, Austria). The study obtained permission from the ethics committee. During the follow-up, 167 men died. Data were analysed using Cox' model adjusted for age, BMI, smoking, physical activity, co-morbidities (ischemic heart disease, hypertension, diabetes mellitus, chronic obstructive pulmonary disease, cancer), treatments and serum levels of testosterone, periostin, osteoprotegerin, and high-sensitivity C-reactive protein. Other factors associated with FGF23 were not significant.
Higher FGF23 levels were associated with increased all-cause mortality (HR 1.25 per SD increase, 95\% CI 1.08-1.45, $\mathrm{p}<0.005$ ). All-cause mortality was higher in the upper FGF23 quartile (Q4) vs. the lowest one (Q1): HR 1.81, 95\% CI 1.08-3.05, $\mathrm{p}<0.05$. Higher FGF23 levels were associated with increased cancer mortality $(\mathrm{n}=69)$ : HR 1.27 per SD, 95\% CI 1.01-1.60, $\mathrm{p}<0.05$. Cancer mortality was higher in the highest vs. the lowest FGF23 quartile: HR $2.44,95 \%$ CI $1.16-5.14, p<0.05$. The association persisted after exclusion of 105 men self-reporting cancer at baseline (Q4 vs. Q1: HR 2.84, 95\% CI 1.18-6.75, p <0.05) and after exclusion of the cancer deaths which occurred during the first two years of the followup (Q4 vs. Q1: HR 4.24, 95\% CI 1.57-11.44, p < 0.005). Cardiovascular mortality $(n=54)$ and mortality for other causes $(n=43)$ were not associated with the FGF23 concentrations.

In the prospective 8-year follow-up of a cohort of older men, high FGF23 levels were associated with increased cancer mortality. This association was independent of the prevalent cancer and other factors supposed to be associated with FGF23 and mortality.

\section{PLO11}

A phase 3 randomized, double-blind, placebo-controlled study investigating the efficacy and safety of burosumab, an anti-FGF23 antibody, in adult X-Linked Hypophosphatemia (XLH)

Robin Lachmann ${ }^{1}$, Peter Kamenický ${ }^{2}$, Thomas O. Carpenter ${ }^{3}$, Martine Cohen-Solal ${ }^{4}$, Richard Eastell ${ }^{5}$, Maria Luisa Brandi ${ }^{6}$, Rachel K. Crowley ${ }^{7}$, Stuart H. Ralston ${ }^{8}$, Muhammad K. Javaid ${ }^{9}$, Richard Keen $^{10}$, Karine Briot ${ }^{11}$, Hae Il Cheong ${ }^{12}$, Yasuo Imanishi ${ }^{13}$, Nobuaki Ito $^{14}$, Hiroyuki Tanaka ${ }^{15}$, Lin Zhang ${ }^{16}$, Christina Theodore-Oklota ${ }^{16}$, Matt Mealiffe ${ }^{16}$, Javier San Martin ${ }^{16}$, Karl L. Insogna ${ }^{3}$

${ }^{1}$ Charles Dent Metabolic Unit, Univerisity College of London Hospital, London, United Kingdom, ${ }^{2}$ Université Paris-Sud, Le Kremlin Bicêtre, France, ${ }^{3}$ Yale School of Medicine, New Haven, United States, ${ }^{4}$ Hôpital Lariboisière, Paris, France, ${ }^{5}$ University of Sheffield, Sheffield, United Kingdom, ${ }^{6}$ Azienda Ospedaliero Universitaria Careggi, Firenze, Italy, ${ }^{7}$ St Vincent's University Hospital and University College, Dublin, Ireland, ${ }^{8}$ University of Edinburgh, Western General Hospital, Edinburgh, United Kingdom, ${ }^{9}$ NDORMS, University of Oxford, Oxford, United Kingdom, ${ }^{10}$ Royal National Orthopaedic Hospital, Stanmore, United Kingdom, ${ }^{11}$ Centre d'Evaluation des Maladies Osseuses, Hôpital Cochin, Paris, France, ${ }^{12}$ Seoul National University Children's Hospital, Seoul, Republic of Korea, ${ }^{13}$ Osaka City University Graduate School of Medicine, Osaka, Japan, ${ }^{14}$ University of Tokyo, Tokyo, Japan, ${ }^{15}$ Okayama Saiseikai General Hospital, Oakayama, Japan, ${ }^{16}$ Ultragenyx Pharmaceutical Inc., Novato, United States

CL303 is a Phase 3, double-blind, multicenter study examining the efficacy and safety of burosumab, a monoclonal antibody against FGF23, in adults with XLH. Subjects were randomized 1:1 to receive burosumab $1 \mathrm{mg} / \mathrm{kg}$ or placebo subcutaneously every 4 weeks. After 24 weeks, subjects in the placebo group crossed-over to burosumab and all subjects continued treatment for an additional 24 weeks.

A significantly greater percentage of subjects in the burosumab group attained the primary endpoint of mean serum phosphorus above the LLN at the midpoint of the dosing intervals through week 24 compared with the placebo group ( 94.1 vs. $7.6 \% ; \mathrm{p}<0.0001)$; increases were maintained between weeks 24-48 (crossover $89.4 \%$, burosumab-only $83.8 \%$ ). At baseline, 91 and 65 active fractures/pseudofractures $(\mathrm{Fx} / \mathrm{PFx})$ were present in 58 and $47 \%$ subjects in the crossover and burosumab-only group, respectively. At week 24, burosumab-treated subjects were more likely to show Fx/PFx healing (odds ratio vs. placebo 16.8, $\mathrm{p}<0.0001$ ). At week 48, burosumab-only subjects demonstrated additional Fx/PFx healing; crossover subjects showed healing similar to that of burosumabonly subjects at week 24 . Pain, stiffness, and physical functioning scores 
decreased with burosumab. Analgesic use declined in both groups. Fifteen subjects had serious AEs, none were drug-related. There were no meaningful changes in calcium, iPTH, or nephrocalcinosis scores.

Burosumab was well tolerated in adults with XLH and associated with improvements in serum phosphorus, pain, stiffness, physical functioning, and Fx/PFx healing.

Summary of burosumab efficacy in CL303 at week 48

\begin{tabular}{lll}
\hline Assessment & $\begin{array}{l}\text { Placebo } \\
\text { burosumab }\end{array}$ & $\rightarrow \begin{array}{l}\text { Burosumab } \\
\text { Only } \\
(\mathrm{n}=68)\end{array}$ \\
$\begin{array}{l}\text { Change reported as least squares } \\
\text { mean }\end{array}$ & $\begin{array}{l}\text { Cross-over } \\
(\mathrm{n}=66)\end{array}$ & \\
\hline
\end{tabular}

Serum phosphorus, $\mathrm{mmol} / \mathrm{L}$

\begin{tabular}{|c|c|c|}
\hline Baseline & $0.62 \pm 0.01$ & $0.65 \pm 0.01$ \\
\hline $\begin{array}{l}\text { Week } 22 \text { (midpoint of the dose } \\
\text { interval) }\end{array}$ & $0.65 \pm 0.01$ & $0.94 \pm 0.02$ \\
\hline Week 24 (trough) & $0.67 \pm 0.01$ & $0.82 \pm 0.02$ \\
\hline $\begin{array}{l}\text { Week } 46 \text { (mid-point of the dose } \\
\text { interval) }\end{array}$ & $0.98 \pm 0.02$ & $0.96 \pm 0.02$ \\
\hline Week 48 (trough) & $0.80 \pm 0.02$ & $0.79 \pm 0.02$ \\
\hline \multicolumn{3}{|l|}{ Percentage of healed Fx/PFx, \% } \\
\hline Week 24 (placebo-controlled) & 8 & 43 \\
\hline Week 48 (all on burosumab) & 35 & 63 \\
\hline \multicolumn{3}{|l|}{ BPI worst pain } \\
\hline Baseline & $6.5 \pm 0.2$ & $6.8 \pm 0.2$ \\
\hline $\begin{array}{l}\text { Week } 24 \text { (placebo-controlled; key } \\
\text { secondary) }\end{array}$ & $6.1 \pm 0.3$ & $5.8 \pm 0.2$ \\
\hline Week 48 (all on burosumab) & $4.9 \pm 0.3$ & $5.6 \pm 0.2$ \\
\hline Change at week 48 & $-1.5 \pm 0.2$ & $-1.1 \pm 0.2$ \\
\hline \multicolumn{3}{|l|}{ WOMAC physical functioning } \\
\hline Baseline & $43.9 \pm 2.5$ & $50.8 \pm 2.4$ \\
\hline $\begin{array}{l}\text { Week } 24 \text { (placebo-controlled; key } \\
\text { secondary) }\end{array}$ & $42.7 \pm 2.8$ & $43.4 \pm 2.4$ \\
\hline Week 48 (all on burosumab) & $34.7 \pm 2.8$ & $38.4 \pm 2.3$ \\
\hline Change at week 48 & $-6.4 \pm 2.9$ & $-7.8 \pm 2.1$ \\
\hline \multicolumn{3}{|l|}{ WOMAC stiffness } \\
\hline Baseline & $61.4 \pm 2.6$ & $64.7 \pm 2.5$ \\
\hline $\begin{array}{l}\text { Week } 24 \text { (placebo-controlled; key } \\
\text { secondary) }\end{array}$ & $60.4 \pm 2.7$ & $53.7 \pm 2.5$ \\
\hline Week 48 (all on burosumab) & $44.7 \pm 2.8$ & $45.3 \pm 2.7$ \\
\hline Change at week 48 & $-15.3 \pm 3.5$ & $-16.0 \pm 3.3$ \\
\hline \multicolumn{3}{|c|}{ Percentage of subjects using pain medication, $\%$ Opioid $/ \%$ NSAID } \\
\hline Baseline & $20 / 65$ & $25 / 69$ \\
\hline Week 24 (placebo-controlled) & $21 / 58$ & $24 / 63$ \\
\hline Week 48 (all on burosumab) & $6 / 17$ & $6 / 19$ \\
\hline
\end{tabular}

\section{PLO12}

Fetal serum phosphorus is set independently of maternal serum phosphorus and phosphorus intake, including the extremes of maternal hyperphosphatemia and hypophosphatemia

K. Berit Sellars ${ }^{1}$, Brittany A. Ryan ${ }^{1}$, Beth J. Kirby ${ }^{1}$, Christopher S. Kovacs $^{1}$

${ }^{1}$ Memorial University of Newfoundland, St. John's, Canada
During fetal development, neither genetic loss of FGF23 action nor excess FGF23 action disturbed any phosphorus-related parameter. This implies that factors independent of FGF23 and PTH regulate fetal serum phosphorus, placental phosphorus transport, and accretion of phosphorus into bone.

We studied whether altering maternal serum phosphorus through dietary phosphorus intake would in turn alter fetal phosphorus parameters, FGF23, or PTH.

Thirty C57BL/6 female mice were separated into three groups to receive low phosphate $(0.3 \%)$, normal phosphate $(0.7 \%)$ or high phosphate $(1.65 \%)$ diets (Envigo/Teklad). One week later, maternal blood was taken and mice were mated. One day before expected birth, maternal and fetal blood were collected, fetuses were ashed, mineral content of ash was determined, and qPCR measured expression of relevant calcium and phosphorus transporting genes. Statistical analysis used ANOVA with Tukey's for multiple comparisons. Animal ethics approved these studies.

Increasing phosphate intake resulted in progressively higher maternal serum phosphorus $(1.61 \pm 0.22$ vs. $2.64 \pm 0.10$ vs. $3.11 \pm 0.14 \mathrm{mM} ; \mathrm{p}<0.05)$ and FGF23 $(62.7 \pm 29.5$ vs. $676 \pm 66.3$ vs. $818 \pm 18.4 \mathrm{pg} / \mathrm{mL} ; \mathrm{p}<0.05)$ during pregnancy, while PTH remained suppressed. Fetal serum phosphorus was maintained at the same high value independent of maternal phosphorus $(3.53 \pm 0.13$ vs. $3.95 \pm 0.19$ vs. $3.73 \pm 0.27 \mathrm{mM}$; $\mathrm{p}=\mathrm{NS})$. Fetal FGF23 and PTH remained low and unresponsive to maternal phosphorus. There were no differences in fetal ash weight, phosphorus, or calcium content. Nor were there changes in placental expression of genes relevant to phosphorus or calcium transport.

In summary, altering dietary phosphorus intake had predictable effects on maternal serum phosphorus, FGF23, and PTH. However, fetal phosphotropic hormones, skeletal phosphorus content, and placental gene expression remained unaffected.

In conclusion, fetal serum phosphorus, and phosphorus delivery and incorporation into bone, are regulated independently of maternal serum phosphorus. A phosphate sensor may set fetal serum phosphorus, while factors other than FGF23 and PTH must regulate fetal phosphorus delivery.

\section{Plenary Oral Presentations 3: Cortical bone and osteocytes}

\section{PLO13}

Effects of romosozumab in postmenopausal women with osteoporosis after 2 and 12 months by micro computed tomography (MicroCT) and histomorphometry on iliac crest bone biopsies

Pascale Chavassieux ${ }^{1}$, Roland Chapurlat ${ }^{1}$, Nathalie Portero-Muzy ${ }^{1}$, Jean-Paul Roux ${ }^{1}$, Pedro Garcia ${ }^{2}$, Jacques P. Brown ${ }^{3}$, Stéphane Horlait ${ }^{4}$, Cesar Libanati ${ }^{5}$, Rogely Boyce ${ }^{6}$, Andrea Wang ${ }^{6}$, Andreas Grauer $^{6}$

${ }^{1}$ INSERM UMR 1033, Université de Lyon, Lyon, France, ${ }^{2}$ Hospital Universitario de Monterrey, Monterrey, Mexico, ${ }^{3} \mathrm{CHU}$ de Quebec Research Centre and Laval University, Quebec, Canada, ${ }^{4}$ Amgen Inc., Boulogne Billancourt, Finland, ${ }^{5}$ UCB Pharma, Brussels, Belgium, ${ }^{6}$ Amgen Inc,, Thousand Oaks, CA, United States

Romosozumab increases bone formation and bone mineral density (BMD), and reduces bone resorption and fracture risk. We evaluated the effects of romosozumab on iliac crest bone biopsies by microCT and histomorphometry in postmenopausal women with osteoporosis participating in the FRAME trial (NCT01575834). ${ }^{1}$

Women received romosozumab $210 \mathrm{mg}$ or placebo monthly for 12 months $(\mathrm{m})$ and underwent transiliac biopsies at 2 and $12 \mathrm{~m}$. For microCT assessment, 28 women (14 placebo/14 romosozumab) were 
evaluable at $2 \mathrm{~m}$, and 71 ( 32 placebo/39 romosozumab) at $12 \mathrm{~m}$. For histomorphometry, 29 women (14 placebo/15 romosozumab) underwent quadruple fluorochrome labelling at $2 \mathrm{~m}$, and 70 (31 placebo/39 romosozumab) double labelling at $12 \mathrm{~m}$.

At 2 vs. $0 \mathrm{~m}$ bone formation rate/bone surface (BFR/BS) increased in cancellous and endocortical bone with romosozumab $(\mathrm{p}<0.001)$ with no change with placebo. From 0 to $2 \mathrm{~m}$, BFR/BS increased in cancellous bone by $+328 \%$ (romosozumab) versus $+79 \%$ (placebo; $\mathrm{p}=0.007$ ) and in endocortical bone by $+233 \%$ (romosozumab) versus $-34 \%$ (placebo; $\mathrm{p}<0.001$ ). At 2 and $12 \mathrm{~m}$, eroded surface/ bone surface significantly decreased in cancellous and endocortical bone with romosozumab vs. placebo. Over $12 \mathrm{~m}$, wall thickness, bone mass, trabecular and cortical thickness increased with romosozumab vs. placebo (all $\mathrm{p} \leq 0.03$ ), with normal bone texture/quality. At $2 \mathrm{~m}$, microCT showed a significant decrease in trabecular separation with romosozumab vs. placebo $(\mathrm{p}=0.046)$; at $12 \mathrm{~m}$, trabecular BMD, trabecular tissue BMD and trabecular bone volume/tissue volume increased with romosozumab vs. placebo (all $\mathrm{p}<0.05$ ). The significant increase in trabecular thickness $(p=0.001)$ and decrease in trabecular bone pattern factor $(\mathrm{p}=0.03)$ suggest an improvement of the microarchitecture with romosozumab vs. placebo. Romosozumab for $12 \mathrm{~m}$ led to an early, significant bone-forming effect associated with a sustained decreased bone resorption. These effects were associated with increased bone mass and improved microarchitecture. Reference: ${ }^{1}$ Cosman. N Engl J Med 2016.

Funding: Amgen Inc., Astellas Pharma, UCB Pharma.

Keywords: romosozumab, bone quality

\section{PLO14}

Osteocyte spreading is supported by Tgif1-mediated repression of PAK3

Simona Bolamperti ${ }^{2}$, Hiroaki Saito ${ }^{1}$, Antonio Virgilio Failla ${ }^{2}$, Hanna Taipaleenmäki ${ }^{1}$, Eric Hesse ${ }^{1,3}$

${ }^{1}$ Molecular Skeletal Biology Laboratory, Department of Trauma, Hand and Reconstructive Surgery, University Medical Center Hamburg-Eppendorf, Hamburg, Germany, ${ }^{2}$ Microscopy Imaging Facility, University Medical Center Hamburg-Eppendorf, Hamburg, Germany, ${ }^{3}$ Department of Anatomy and Cell Biology, Indiana University School of Medicine, Indianapolis, United States

Adherence and spreading of osteoblasts on bone surfaces and the formation of an organized lacuno-canalicular network by osteocytes are important processes for the maintenance of bone quality. We observed increased expression of the homeodomain protein TG-interacting factor 1 (Tgif1) during the spreading of osteoblasts on collagen I-coated surfaces in vitro, suggesting a role of Tgif1 during this process. In support of this hypothesis, calvarial osteoblasts obtained from mice bearing a germline deletion of Tgif1 (Tgif1 ${ }^{-1-}$ ) and osteocyte-like cells of the OCY454 cell line with a transient knockdown of Tgif1 were impaired in their capacity to adhere and spread $(\mathrm{p}<0.05)$. Furthermore, immunocytochemistry analyses demonstrated that Tgif1-deficient osteoblasts and osteocytes have an impaired capacity to form focal adhesions and cell protrusions, retaining a more spherical cell shape $(\mathrm{p}<0.01)$. Investigation of factors known to regulate cell spreading revealed an increased expression of the p21-activated kinase 3 (PAK3) in Tgif1-deficient osteocytes at the mRNA and protein level $(\mathrm{p}<0.01)$. In addition, gene reporter assays demonstrated that in the absence of Tgifl the activity of the PAK3 promoter was increased $(\mathrm{p}<0.01)$, suggesting a specific regulation by Tgif1. Mechanistically, inhibition of PAK3 expression in Tgif1-deficient osteocytes using siRNA restored the capacity of the cells to spread and to form focal adhesions, as well as cell protrusions $(\mathrm{p}<0.001)$. To confirm these observations in vivo, we analyzed the bones of Tgif1 $1^{-/-}$mice. Tgif1-deficient osteoblasts appeared to have a less cuboidal shape compared to littermate controls. Furthermore, osteocytes in the trabecular compartment of Tgif1 $1^{-\prime-}$ bones were irregularly shaped and unevenly distributed, representing an altered lacuno-canalicular network $(p<0.05)$. These findings indicate that Tgif1-mediated repression of PAK3 is important for osteoblasts and osteocytes to adhere and spread. This mechanism might contribute to the formation of an organized osteocyte network in bone.

Keywords: Osteocyte, Tgif1, PAK3, cell spreading

\section{PLO15}

Exogenous hyperthyroidism induces osteocytic osteolysis in male mice

Elena Tsourdi ${ }^{1,2}$, Katharina Jähn ${ }^{3}$, Franziska Lademann ${ }^{1,2}$, Eva M. $\overline{\text { Wölfel }}^{3}$, Björn Busse ${ }^{3}$, Stephan Blouin ${ }^{4}$, Paul Roschger ${ }^{4}$, Lorenz C. Hofbauer $^{1,2,5}$, Martina Rauner ${ }^{1,2}$

${ }^{1}$ Department of Medicine III, Technische Universität Dresden Medical Center, Dresden, Germany, ${ }^{2}$ Center for Healthy Aging, Technische Universität Dresden Medical Center, Dresden, Germany, ${ }^{3}$ Department for Osteology and Biomechanics, University Medical Center Hamburg-Eppendorf, Hamburg, Germany, ${ }^{4} 1$ st Medical Department, Ludwig Boltzmann Institute of Osteology at HanuschHospital of WGKK \& Trauma Center Meidling of AUVA, Vienna, Austria, ${ }^{5}$ Center for Regenerative Therapies Dresden, Dresden, Germany

Objective: Osteocytic osteolysis, a process where osteocytes remodel their perilacunar matrix, has been demonstrated during lactation. Our previous studies show that hyperthyroidism in mice results in a rapid bone turnover and a larger percentage of low mineralized bone. Here, we tested the hypothesis that hyperthyroidism may contribute to low bone mineralization through osteocytic osteolysis.

Methods: Twelve-week-old C57BL/6 male mice were rendered hyperthyroid by adding L-thyroxine $\left(\mathrm{T}_{4}\right)$ to drinking water $(1.2 \mu \mathrm{g} /$ $\mathrm{ml}$ ) for 4 weeks. Osteocyte lacunae were quantified using a silver precipitation stain, and osteocytic expression of the osteoclast marker tartrate-resistant acid phosphatase (TRAP) was investigated using a TRAP stain. Furthermore, the osteocyte-like cell line MLO-Y4 was exposed to 3,5,3'-triiodo-L-thyronine $\left(\mathrm{T}_{3}\right)$ for $24-72 \mathrm{~h}$ at different concentrations $(0.1-10 \mu \mathrm{M})$ and expression of osteoclast markers was assessed by real-time PCR.

Results: Hyperthyroid mice displayed a larger osteocyte lacunar area in tibial cortical bone $(+11 \%, \mathrm{p}<0.01)$ and trabecular bone $(+14 \%, \mathrm{p}<0.05)$ as assessed by 2D histology. In vertebral cortical bone of hyperthyroid mice, we found less canaliculi/osteocyte $(-15 \%, \mathrm{p}<0.01)$ and a larger percentage of empty osteocyte lacunae $(+48 \%, \mathrm{p}<0.05)$ as compared to euthyroid controls. Furthermore, hyperthyroid mice showed a higher percentage of osteocytes with TRAP activity in trabecular bone in comparison to controls $(34.5 \pm 15.5$ vs. $15.4 \pm 9.1 \%, \mathrm{p}<0.01)$. Similar results were obtained for osteocytes in cortical bone. Treatment of MLO-Y4 cells with $\mathrm{T}_{3}$ increased the expression of typical osteoclast markers in a time- and dose dependent manner, reaching a maximum at $10 \mu \mathrm{M}$ $\mathrm{T}_{3}$ and $72 \mathrm{~h}$. At these conditions, $\mathrm{T}_{3}$ enhanced mRNA levels of cathepsin $\mathrm{K}$ by fourfold $(\mathrm{p}<0.01)$, MMP13 by sixfold $(\mathrm{p}<0.01)$ and NFATc 1 by 2.5 -fold $(\mathrm{p}<0.01)$.

Conclusion: These results suggest that hyperthyroidism induces osteocytic osteolysis, which may contribute to low bone mineralization in hyperthyroid mice. 


\section{PLO16}

Cement lines mineralization is a bone tissue-age related phenomenon: a study in human femoral cortical bone

Petar Milovanovic ${ }^{1,2}$, Annika vom Scheidt ${ }^{1}$, Kathrin Mletzko ${ }^{1}$, George Sarau $^{3}$, Klaus Püschel ${ }^{4}$, Marija Djuric ${ }^{2}$, Michael Amling ${ }^{1}$, Silke Christiansen ${ }^{3}$, Björn Busse ${ }^{1}$

${ }^{I}$ Department of Osteology and Biomechanics, University Medical Center Hamburg-Eppendorf, Hamburg, Germany, ${ }^{2}$ Laboratory for Anthropology and Skeletal Biology, Faculty of Medicine, University of Belgrade, Belgrade, Serbia, ${ }^{3}$ Max Planck Institute for the Science of Light, Erlangen, Germany, ${ }^{4}$ Department of Forensic Medicine, University Medical Center Hamburg-Eppendorf, Hamburg, Germany

Whether the cement lines are mineral-rich or mineral-deficient was a matter of debate in the literature, particularly owing to the low thickness of these peripheral boundaries of osteons. While variations in osteonal mineralization reflect tissue age, there are no data on variations in cement lines' composition. We hypothesized that cement lines are highly mineralized regions, and their mineralization level is variable but related to osteon's tissue age. We analyzed the calcium content of osteons and their cement lines in a range of different osteonal tissue ages in femoral cortex from ten postmenopausal women with untreated osteoporosis and bisphosphonate-treated cases. Quantitative backscattered-electron imaging (qBEI) showed consistently higher calcium content in the cement lines than in their corresponding osteons $(29.46 \mathrm{wt} \% \pm 0.80$ vs. $26.62 \mathrm{wt} \% \pm$ $1.11 \mathrm{wt} \% ; \mathrm{p}<0.001)$. Micro-Raman spectroscopy complemented the qBEI data by showing a significantly higher $v 1$ phosphate/amide I ratio in the cement lines compared to the osteonal bone $(8.78 \pm 0.66$ vs. $6.33 \pm 0.58, \mathrm{p}<0.001)$, due to an increased phosphate peak and reduced amide I peak in cement lines. This was further confirmed by polarization insensitive $v 2$ phosphate/amide III ratio. Cement line mineralization was positively correlated with osteonal mineralization $(r=0.839, p=0.003)$, but the degree of discrepancy between cement line and osteonal calcium content tended to decrease with increased osteonal mineralization $(r=-0.709, \quad p<0.001)$, suggesting diverging mineralization dynamics in these osseous entities. The number of mineralized osteocyte lacunae per osteon bone area correlated positively with both osteonal and cement line calcium content $(\mathrm{p}<0.01)$. Considering that mineralization of cement lines relates strongly to the osteonal mineralization level, it may represent another tissue-age related phenomenon. Understanding of the cement lines' mineralization and their changes in aging and disease states is important for predicting crack propagation pathways and fracture resistance mechanisms in human cortical bone.

\section{PLO17}

Denosumab treatment improves bones hardness accompanied by lower osteocyte viability persisting during drug-holiday

Katharina Jähn ${ }^{1}$, Björn Jobke ${ }^{2}$, Eva Maria Wölfel ${ }^{1}$, Tobias Barth ${ }^{1}$, Christoph Riedel $^{1}$, Maya Hellmich ${ }^{3}$, Mathias Werner ${ }^{4}$, Björn Busse ${ }^{1}$

${ }^{I}$ Department of Osteology and Biomechanics, University Medical Center Hamburg-Eppendorf, Hamburg, Germany, ${ }^{2}$ Telemedicine Clinic, San Francisco, United States, ${ }^{3}$ Osteology and Metabolic Diseases, Immanuel Krankenhaus Berlin, Berlin, Germany, ${ }^{4}$ Histology/Pathology, Helios Klinikum Emil von Behring, Berlin, Germany

Antiresorptives are a gold-standard for treating osteoporosis, with Denosumab being the latest potent osteoclast inhibitor. Recent data highlighted the risk of vertebral fractures during drug-holiday from Denosumab osteoporosis therapy. Under physiological conditions, bone remodeling is coupled, and its spatiotemporal activation is guided by the mechanosensitive osteocyte network. One hallmark of osteoporosis is the death of osteocytes, weakening their network connectivity and regulatory functionality.

Iliac crest biopsies $(n=25$ during Denosumab, $n=10$ on drugholiday from Denosumab, $\mathrm{n}=11$ age-matched osteoporotic controls; $67 \pm 14$ years) were collected and histopathologically assessed. Therefore, biopsies were embedded into polymethyl methacrylate (PMMA). Structural and cellular histomorphometry was performed according to ASBMR guidelines. Histological sections were stained with toluidine blue or von Kossa van Gieson and analyzed with an Osteomeasure system. Biomechanical properties of the bone tissue were tested with a nanoindentation system equipped with a Berkovich tip (Agilent 200). Statistical tests were performed by ANOVA and post-hoc tests to compare the three groups.

Denosumab treatment (average treatment: $26 \pm 13$ months) showed higher hardness in comparison to age-matched controls with osteoporosis $(0.6 \pm 0.07-0.54 \pm 0.1 \mathrm{GPa} ; \mathrm{p}=0.046)$. No significant changes in cortical structural parameters were observed, yet a tendency of lower cortical thickness was seen with drug-holiday compared to control $(387.7 \pm 59.7$ vs. $647.6 \pm 212.6 \mu \mathrm{m}$; $\mathrm{p}=0.079)$. The trabecular BV/TV was lowest during drug-holiday $(10.48 \pm 3.41 \%$; average time: $15 \pm 12$ months) compared to Denosumab treatment $(15.93 \pm 6.45 ; \mathrm{p}=0.036)$. As expected, osteoclast numbers in trabecular bone were significantly lower with treatment in comparison to Denosumab-untreated individuals $(0.12 \pm 0.23$ vs. $0.72 \pm 0.8 / \mathrm{mm} ; \mathrm{p}=0.003)$. Interestingly, the number of empty osteocyte lacunae was highest with Denosumab treatment compared to osteoporotic controls $(106.23 \pm 38.45$ vs. $\left.64.4 \pm 17.4 / \mathrm{mm}^{2} ; \mathrm{p}=0.013\right)$ and remained elevated during drugholiday $\left(103.69 \pm 55.38 / \mathrm{mm}^{2} ; \mathrm{p}=0.012\right.$ to control).

Our results show that Denosumab treatment leads to harder bone tissue accompanied by lower osteocyte viability.

Keywords: osteoporosis, Denosumab, osteocytes, histomorphometry, drug-holiday

\section{PLO18}

Bone matrix mineralization after denosumab (DMAb) treatment discontinuation

Jacques Brown ${ }^{1}$, David W Dempster ${ }^{2,3}$, Susan Yue ${ }^{4}$, Sebastien Rizzo $^{5}$, Delphine Farlay ${ }^{5}$, Rachel B Wagman ${ }^{4}$, Xiang Yin ${ }^{4}$, Georges Boivin $^{5}$

${ }^{1}$ Laval University and $\mathrm{CHU}$ de Quebec-(CHUL) Research Centre, Ville de Québec, Canada, ${ }^{2}$ Columbia University, New York, United States, ${ }^{3}$ Helen Hayes Hospital, West Haverstraw, United States, ${ }^{4}$ Amgen Inc., Thousand Oaks, United States, ${ }^{5}$ INSERM, UMR 1033, Univ Lyon, Université Claude Bernard Lyon, Lyon, France

Objective: Assess the effects of DMAb discontinuation on bone matrix mineralization.

Methods: A non-interventional cohort study enrolled subjects who received 12 months of DMAb in one of two parent clinical trials and agreed to bone biopsies $\geq 12$ and $\leq 36$ months off-treatment (Brown et al., JBMR. 2011). Subjects could not have received any commercially available osteoporosis treatment except calcium/vitamin D. Mean degree of mineralisation of bone (DMB) and heterogeneity index (HI) were calculated for cancellous bone, cortical bone, endocortical + periosteal cortical sub-compartments, total bone (cancellous + cortical). Bone biopsies at 2 or 3 years in the FREEDOM trial (Dempster et al., ASBMR. 2016) were used as comparator groups.

Results: 15 subjects were enrolled. Mean (min, max) age: 62.1 $(54,73)$ years; years since menopause: $15.8(6,30)$; time since DMAb discontinuation: $25(21,29)$ months. Median $(\min , \max )$ total bone DMB was $1.080 \mathrm{~g} / \mathrm{cm}^{3}(1.001,1.123)$ vs. $1.055 \mathrm{~g} / \mathrm{cm}^{3}(0.985,1.118)$ and $1.081 \mathrm{~g} / \mathrm{cm}^{3}(0.953,1.142)$ in the FREEDOM PBO and DMAb 
groups, respectively, $p$ values $=0.029$ and 0.89 . Median $(\min , \max )$ total bone $\mathrm{HI}$ was $0.147 \mathrm{~g} / \mathrm{cm}^{3}(0.128,0.179)$ vs. $0.144 \mathrm{~g} / \mathrm{cm}^{3}(0.118$, $0.199)$ and $0.128 \mathrm{~g} / \mathrm{cm}^{3}(0.093,0.194)$ in the FREEDOM PBO and DMAb groups, $p$ values $=0.11$ and $<0.0001$. Similar results were observed in all bone compartments assessed.

Conclusion: After stopping DMAb for an average of 25 months, the DMB was similar to that observed after 2 or 3 years of DMAb treatment in FREEDOM, indicating a sustained benefit after discontinuation of DMAb. The HI was higher than that observed after 2 or 3 years of DMAb treatment, which may reflect a transient increase in remodeling activity. These data suggest no significant changes in the degree of mineralization were observed after DMAb cessation as bone remodeling returned to pretreatment levels.

Keywords: denosumab, bone matrix mineralisation, treatment discontinuation

Figure: Mean DMB and $\mathrm{HI}$ in Total Bone

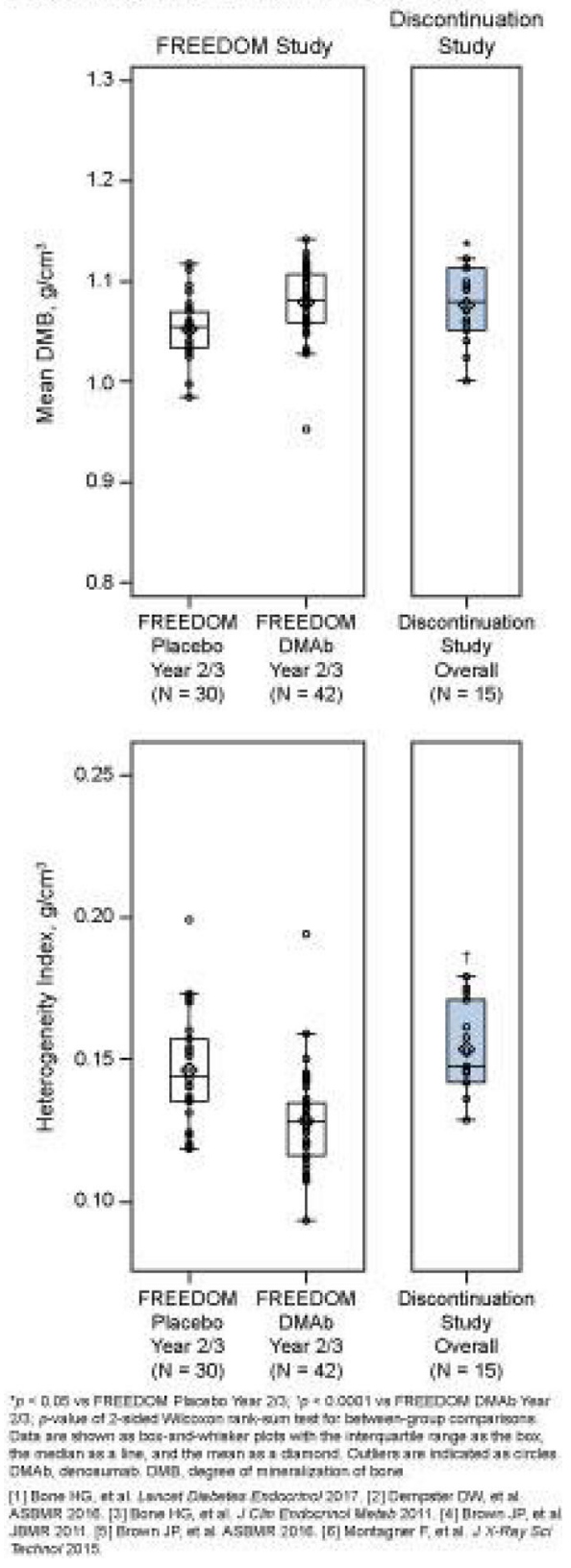

Plenary Oral Presentations 4: Energy metabolism

\section{PLO19}

Opg-Fc attenuates insulin-resistance and muscle weakness in a mouse model of diabetoporosis (Ppar $\beta$ KO)

$\underline{\text { Lucie Bourgoin }}^{1}$, Béatrice Desvergne ${ }^{2}$, Nicolas Bonnet ${ }^{1}$

${ }^{1}$ Department of Internal Medicine Specialties, Service of Bone Diseases, Geneva, Switzerland, ${ }^{2}$ Center of Integrative Genomics, Genopode, Lausanne, Switzerland

RANKL/OPG are key regulators of osteoclastogenesis. We previously demonstrated that RANK is expressed in skeletal muscle and liver, and that mouse overexpressing RANKL exhibit insulin resistance. We hypothesized that Opg-Fc is able to prevent bone loss, muscle weakness and insulin sensitivity in a diabetoporosis model. For this purpose, we treated Ppar $\beta-/-$ mice (KO) with Opg-Fc (4 mg/ $\mathrm{kg} /$ week) or vehicle (Veh) for 4 weeks. Muscle function was investigated by treadmill exercise, handgrip, microCT and infrared camera; gene expression by RT-qPCR and glucose by GTT and ITT. At 5 months of age, KO mice displayed a lower trabecular and cortical bone volume at the femur $(-33$ and $-14 \%$ vs. WT, p $<0.05)$ as well as a lower muscle limb volume, limb temperature, maximal speed and force, $-19,-1.6,-15$ and $-16 \%$ vs. WT $(\mathrm{p}<0.05)$. GTT and ITT AUC were higher in $\mathrm{KO},+38 \%$ and $+8 \%$ vs. WT ( $\mathrm{p}<0.05)$. In the soleus, Glut1, Fabp4, CyclinD1, Pgcla gene expression were downregulated whereas Myostatin and Fgf 21 were upregulated $(-29,-22,-20,-7,+33$ and $+172 \%$ vs. WT, $\mathrm{p}<0.05)$. In the liver, mRNA levels of gluconeogenic and stress markers like Pgcla, Fabp4, and Fgf21 were increased $(+262,+60$ and $+320 \%$ vs. WT, $\mathrm{p}<0.01)$. Opg-Fc increased BV/TV and TbN $(+107$ and $+40 \%$ vs. Veh, all $\mathrm{p}<0.05)$. Opg-Fc increased the force, muscle volume and temperature of the limb $(+6.7,+12$, $+1.8 \%, \mathrm{p}<0.08$ ). Moreover gene expression levels were normalized in soleus and liver. Opg-Fc decreased GTT and ITT AUC $(-36.7$ and $-22.3 \%$ vs. Veh, $p<0.05)$ arguing for a restoration of the insulin sensitivity, confirm by western in muscle and liver. In conclusion, Opg-Fc could rescue bone loss, muscle function, insulinresistance and gluconeogenesis in a diabetoporosis model. Hence RANKL could play a central role in the concomitant development of osteoporosis, sarcopenia and diabetes.

Keywords: Diabetes, opg

\section{PLO20}

Denosumab improves muscle function and glucose homeostasis

Nicolas Bonnet ${ }^{1}$, Emmanuel Biver ${ }^{1}$, Lucie Bourgoin ${ }^{1}$, Thierry Chevalley $^{1}$, Mélanie Hars ${ }^{1}$, Andrea Trombetti ${ }^{1}$, Serge Ferrari ${ }^{1}$

${ }^{I}$ Department of Internal Medicine Specialties, Service of Bone Diseases, Geneva, Switzerland

RANKL is a key regulator of osteoclastogenesis, but is also expressed in skeletal muscle and liver. Objective: Investigate if RANKL inhibitor Denosumab (Dmab) modifies muscle mass/function, and glucose homeostasis in mice overexpressing human RANKL (huRANKL). HuRANKL mice were treated with Dmab $(10 \mathrm{mg} / \mathrm{kg} /$ week) or vehicle (Veh) for 4 weeks. Muscle function was investigated by running and handgrip test and glucose by GTT, ITT and EH clamp. In addition, 11 post-menopausal women $(65.0 \pm 1.4$ years $)$ treated for osteoporosis with Dmab was evaluated by DXA and handgrip. They were matched to 49 controls for age, BMI, BMD and fracture. HuRANKL mice have severe osteoporosis and lower maximal speed and limb force, respectively -41 and $-11 \%$ vs. WT $(\mathrm{p}<0.05)$. Gastrocnemius and soleus mass were lower $(-29$ and $-57 \%$ vs. $\mathrm{WT}, \mathrm{p}<0.05)$ and liver mass higher $(+16 \%$ vs. WT, $\mathrm{p}<0.05)$. ITT 
AUC was higher, $+31 \%$ vs. WT $(\mathrm{p}<0.05)$ and GIR, an index of insulin sensitivity was lower $(-24.6 \%, \mathrm{p}<0.05) .2 \mathrm{D}\left[{ }^{14} \mathrm{C}\right]$-glucose incorporation indicate a lower glucose uptake in the soleus $(-51.9 \%$ vs. WT, $\mathrm{p}<0.05)$. Hepatic glucose production was higher $(+133 \%$ vs. WT, p < 0.05). Dmab increases limb force $(+34.7 \%$ vs. Veh, $\mathrm{p}<0.05$ ) and normalizes ITT (AUC $464 \pm 25.1$ vs. $353.9 \pm 8.1$ in Dmab, $\mathrm{p}<0.05)$ and hepatic glucose production $(4.3 \pm 3.2$ vs. $-6.2 \pm 2 \mathrm{mg} / \mathrm{kg} / \mathrm{min}$ in Dmab, $\mathrm{p}<0.05)$. In women, LSBMD, appendicular lean mass and handgrip decreased in controls $\left(-0.046 \pm 0.0712 \mathrm{~g} / \mathrm{cm}^{2}, \quad-0.22 \pm 0.34 \mathrm{~kg}, \quad-2.81 \pm 1.64 \mathrm{~kg}\right)$ but were maintained with Dmab $\left(0.012 \pm 0.12 \mathrm{~g} / \mathrm{cm}^{2}\right.$, $0.098 \pm 0.27 \mathrm{~kg}, 0.36 \pm 2.3 \mathrm{~kg}$, all $\mathrm{p}<0.05)$ over 3 years. In conclusion, overexpression of huRANKL causes bone loss, impairs muscle function and insulin sensitivity. Denosumab reversed these defects in mouse, but also had some positive effects on muscle function in osteoporotic women. Altogether these point towards a potentially new mechanism relating RANKL expression to fracture risk, i.e. not only by decreasing bone, but also muscle strength.

Keywords: Muscle, Diabetes, Denosumab

\section{PLO21}

Gut microbiome composition is associated with bone mineral density levels in healthy children of school age

Carolina Medina-Gomez ${ }^{1}$, Djawad Radjabzadeh ${ }^{1}$, Cindy G. Boer ${ }^{1}$, Joyce van Meurs ${ }^{1}$, Robert Kraaij ${ }^{1}$, Andre G. Uitterlinden ${ }^{1}$

\section{${ }^{I}$ ErasmusMC, Rotterdam, Netherlands}

Aim: Human gut microbiota is an important determinant of health and disease processes. Discoveries from recent microbiome studies may translate in actionable targets to treat malnutrition, diabetes obesity among other conditions. The potential role of the gut microbiome in the development of the musculoskeletal system remains to be explored in humans. We aimed studying the role of bacterial taxa present in the gut on bone mineralization in healthy children.

Methods: This study is embedded in the Generation R Study, a prospective multiethnic birth cohort in Rotterdam, The Netherlands. We included 2111 children (mean age $=9.84, \mathrm{SD}=0.3$ years) with total body DXA measurements (GE-Lunar iDXA) and 16S rRNA v3v4 microbiome profiles determined in stool. We assessed the association between total-body (less-head) BMD (TBLH-BMD) and taxa relative abundance (RA) using the MASSLIN approach. All analyses were adjusted for age, sex, BMI, 10 genomic principal components and technical covariates. Multiple testing was FDR corrected (significance FDR $<0.05$ ). Sensitivity analyses were carried as taxa presence/absence and linear effect of the taxa RA in the individuals (where present).

Results: RA from the Enterococcus and ErysipelotrichaceaeUCG003 genera, together with RA from Lachinospiraceae family and six of its constituent genera were associated with TBLHBMD (FDR < 0.05). All, but one genera (Hungatella), showed a positive association with TBLH-BMD. Sensitivity analyses were in line with these results.

Conclusion: Our results indicate that human gut microbiota profiles are associated with bone mineralization in children. Several of the identified genera hold species with relevant clinical implications i.e., (1) different taxa within Lachinospiraceae and Erysipelotrichaceae families are either highly immunogenic or correlated with inflammation; and (2) Enterococccus faecium: active component present in probiotics (Lactiferm) have been shown to increase bone strength in animal models; and together implicate a role of the gut microbiome in pediatric bone health.

Keywords: Microbiome; bone accrual

\section{PLO22}

How does high levels of exercise affect the risk of fracture?

$\underline{\text { Karl Stattin }}^{1}$, Ulf Hållmarker ${ }^{2,3}$, Johan Ärnlöv ${ }^{4,5}$, Stefan James ${ }^{2,6}$, Karl Michaëlsson $^{1}$, Liisa Byberg ${ }^{1}$

${ }^{1}$ Department of Surgical Sciences, Orthopaedics, Uppsala University, Uppsala, Sweden, ${ }^{2}$ Department of Medical Sciences, Uppsala University, Uppsala, Sweden, ${ }^{3}$ Department of Internal Medicine, Mora Lasarett, Mora, Sweden, ${ }^{4}$ Department of Neurobiology, Care Sciences and Society, Division of Family Medicine and Primary Care, Karolinska institutet, Stockholm, Sweden, ${ }^{5}$ School of Health and Social Studies, Dalarna University, Falun, Sweden, ${ }^{6}$ Uppsala Clinical Research Center, Uppsala, Sweden

It is not known how high levels of exercise affects the risk of fracture. To investigate this association, we studied 118,204 men and 71,757 women having 1991-2009 participated in a long distance cross-country skiing race and 505,194 non-participants from the general population, frequency-matched on age, sex and county of residence. Race participation, length of race, number of races run and finishing time were used as proxies for exercise. During a mean follow-up of 9.6 years, 53,177 any, 2929 hip, 3107 proximal humerus, 11,875 lower leg, 11,733 forearm and 2391 vertebral fractures were captured in national registers. Using time-updated exposure and covariate information, Cox proportional hazards regression showed a lower rate of hip [hazard ratio (HR) $0.75,95 \%$ confidence interval (CI) 0.67-0.83], proximal humerus (HR 0.90, 95\% CI 0.82-0.98) and lower leg fractures (HR 0.93, 95\% CI 0.89-0.97), a higher rate of any (HR 1.02, 95\% CI 1.01-1.05) and forearm fractures (HR 1.11, 95\% CI 1.06-1.15) and no difference in the rate of vertebral fracture (HR $0.97,95 \%$ CI $0.88-1.07)$ comparing race participants and non-participants. Compared to non-participants, hazard ratios were similar for participants regardless of length of race and number of races run. Among participants, a finishing time close to the median was associated with the lowest risk of fracture. In summary, high levels of exercise was associated with a lower rate of severe fractures such as hip, proximal humerus and ankle fracture but a higher rate of less severe fractures, such as any and forearm fracture.

\section{PLO23}

Role of lipocalin 2 in energy metabolism-bone crosstalk

Mattia Capulli ${ }^{1}$, Antonio Maurizi ${ }^{1}$, Marco Ponzetti ${ }^{1}$, Sara GeminiPiperni $^{1}$, Teti Anna ${ }^{1}$, Nadia Rucci ${ }^{1}$

${ }^{I}$ University of L'Aquila, L'Aquila, Italy

Lipocalin2 (Lcn2) is a small circulating protein acting in different cell types with different signalling pathways. The Lcn2 pleiotropic action is evidenced by the many names associated to it, among which super-reactive-protein-24 well describes its rapid upregulation in response to various stimuli. We demonstrated that, compared to WT, $\mathrm{Lcn} 2^{-/-}$mice presented with low tibial and femoral BV/TV\% $(-20 \% ; p=0.0006)$ and $\mathrm{TbN}(-15 \% ; p=0.007)$, and high TbSp $(+1.4$ fold; $p=0.026)$ at $1,3,6$ and 12 months of age. Cortical thickness and L4 vertebral trabecular bone were low in old Len2 $2^{-/-}$ mice $(-20 \% ; p=0.01)$. Lcn $2^{-1-}$ mice also showed low $\mathrm{ObN}$ and ObS/BS $(-58 \% ; p<0.0001 ;-46 \% ; \mathrm{p}=0.002$, respectively) and, subsequently, low BFR $(-48 \% ; p=0.009)$, while osteoclast variables were unremarkable $(p>0.05)$. In contrast, in vitro Lcn2 silencing with specific siRNAs did not affect osteoblast mRNA expression of ALP, Runx2, Coll1a1 and Ocn, nor ALP activity and matrix nodule mineralization $(p>0.4)$. These results suggested a non-autonomous osteoblast response to Lcn2 ablation. Given that Lcn $2^{-1-}$ mice showed higher body weight $(+1.2$ fold; $p=0.014)$ and nocturnal food intake $(+1.2 \mathrm{fold} ; p=0.002)$, we investigated 
whether their osteoblast impairment was due to altered energy metabolism. Lcn $2^{-/-}$mice showed lower fasted glycemia $(-25 \%$; $p=0.002)$ and hyperinsulinemia $(+1.7$ fold; $p=0.025)$. Glucose tolerance was higher $(p=0.0078)$ but insulin sensitivity was unaltered. However, in our model, hyperinsulinemia was not associated with high bone formation as expected. We therefore investigated other pathways known to link bone and energy metabolism and found that the expression of the glucose transporter Glut1, which is essential for osteogenesis and cannot support osteoblast differentiation in low glucose conditions, was significantly lower $(-80 \% ; p=0.04)$ in the $\mathrm{Lcn} 2^{-1-}$ bone, possibly explaining the in vivo impairment of bone formation and subsequent osteopenia. Taken together, these results unveil an important role of Lcn2 in bone metabolism, highlighting a link with glucose metabolism that is more complex than expected from the current knowledge (experiments approved by ethical authorities).

\section{PLO24}

The antidiabetic drug metformin improves the skeletal effects of plyometric exercise in ovariectomized rats

Mats Mosti ${ }^{1,2}$, Reinhold Erben ${ }^{3}$, Christiane Schüler ${ }^{3}$, Unni Syversen $^{1,4}$, Astrid Kamilla Stunes ${ }^{1,2}$

${ }^{1}$ Department of Clinical and Molecular Medicine, Norwegian University of Science and Technology, Trondheim, Norway, ${ }^{2}$ St. Olav's University Hospital, Trondheim, Norway, ${ }^{3}$ Department of Biomedical Sciences, University of Veterinary Medicine Vienna, Vienna, Austria,${ }^{4}$ Department of Endocrinology, St. Olav's University Hospital, Trondheim, Norway

Estrogen deficiency promotes bone loss and skeletal muscle dysfunction. Metformin is the most widely used drug in treatment of type 2 diabetes, and has been suggested to have beneficial skeletal effects.

We investigated the effects of metformin and plyometric jumping exercise (each alone and combined) in ovariectomized (OVX rats). Female Sprague Dawley rats, 12 weeks of age, were allocated to a sham-operated group (Sham), and four OVX groups: metformin (OVX-Met), exercise (OVX-Ex), combined metformin and exercise (OVX-MetEx) and a control group (OVX-Ctr), respectively ( $\mathrm{n}=12 /$ group). Metformin $(100 \mathrm{mg} / \mathrm{kg} / \mathrm{day})$ or vehicle (methylcellulose) were given by gavage for 8 weeks. Bone mineral density (BMD), was assessed by dual X-ray absorptiometry (DXA). Bone microarchitecture was analyzed using micro computed tomography. Bone histomorphometry was performed, and bone metabolism markers in plasma were analyzed by immunoassays.

OVX-MetEx rats jumped higher than OVX-Ex throughout the intervention ( 51 vs. $46 \mathrm{~cm}, \mathrm{p}<0.01$ ). Femoral BMD increased more in all intervention groups relative to $\mathrm{OVX}-\mathrm{Ctr}(\mathrm{p}<0.05)$, and was similar to sham-level in both exercise groups. Femoral BMD gain was higher in the OVX-MetEx group than in OVX-Met and Sham (15 vs. 10 and $8 \%, \mathrm{p}<0.05)$. OVX-MetEx had higher trabecular thickness relative to OVX-Ctr and OVX-Met ( $<<0.05)$, and higher trabecular bone volume than OVX-Ctr $(\mathrm{p}<0.05)$. Trabecular number, separation and bone density were restored to sham-level in the OVX-MetEx group. Bone mineralizing surface and osteoid surface were similar to sham in both metformin groups, as were osteoblast and osteoclast surface. Plasma levels of the bone resorption marker C-terminal telopeptide of type 1 collagen were comparable with sham in both exercise groups.

In conclusion, metformin seemed to normalize the accelerated bone turnover induced by estrogen deficiency, thereby enhancing the skeletal effects of plyometric exercise.

Keywords: Exercise, metformin, estrogen deficiency, bone

\section{Parallel Oral Presentations 5: Bone and cartilage biology}

\section{PO25}

Evidence supporting a vascular disease-muscle function relationship

Alexander Rodriguez ${ }^{1}$, Joshua Lewis ${ }^{2}$, David Scott ${ }^{1}$, Douglas $\mathrm{Kiel}^{3}$, John Schousboe ${ }^{4}$, Peter Ebeling ${ }^{1}$, Richard Prince ${ }^{5}$

${ }^{1}$ Bone and Muscle Health Research Group, Monash University, Melbourne, Australia, ${ }^{2}$ School of Medical and Health Sciences, Edith Cowan University, Perth, Australia, ${ }^{3}$ Institute for Aging Research, Harvard Medical School, Boston, United States, ${ }^{4}$ Division of Health Policy and Management, University of Minnesota, Minneapolis, United States, ${ }^{5}$ School of Medicine and Pharmacology, University of Western Australia, Perth, Australia

Objective: Investigate whether abdominal aortic calcification (AAC) is associated with changes in muscle function over 5 years.

Design: Prospective cohort of elderly women with AAC assessment at baseline and 5-year measures of muscle strength and mobility nested within the Calcium Intake Fracture Outcomes Study.

Methods: AAC24 scores [0-24] were semi-quantitatively assessed from lateral spine images captured during bone density testing at baseline. Severe AAC $\left[\mathrm{AAC}_{\mathrm{sev}}\right]$ was defined using established criteria [AAC24 > 5]. At baseline and follow-up, isometric grip strength was assessed using a dynamometer. Mobility was assessed by the Timedup-and-go (TUG) test. Using pre-defined criteria, muscle weakness was considered as grip strength $<22 \mathrm{~kg}$ (a threshold that identified clinically relevant weakness amongst older women) and poor mobility defined as TUG $>10.2 \mathrm{~s}$ (a threshold predictive of fractures). A subset of women had appendicular lean mass [ALM] determined by DXA at baseline and follow-up [ $\mathrm{n}=261]$.

Results: 1046 women [mean age $=74.9 \pm 2.6$ years; $\mathrm{BMI}=$ $27.1 \pm 4.4 \mathrm{~kg} / \mathrm{m}^{2}$ ] were included. $\mathrm{AAC}_{\mathrm{sev}}$ was evident in 193 [18.5\%] women. There were no cross-sectional associations between $\mathrm{AAC}_{\mathrm{sev}}$ and muscle weakness or poor mobility at baseline or followup. Average decline in grip strength after 5 years was greater in those with $\mathrm{AAC}_{\text {sev }}$ compared to those without $(3.6 \pm 3.7$ vs. $2.9 \pm 4.2 \mathrm{~kg}$; $\mathrm{p}=0.034)$. TUG or ALM changes did not differ $(\mathrm{p}>0.05) . \mathrm{AAC}_{\mathrm{ser}}$ at baseline was associated with greater 5-year declines in grip strength $(\beta=-0.776[-1.517,-0.035 ; p=0.040])$ independent of age, treatment allocation, diabetes, smoking, renal function, prevalent vascular disease, BMI and metabolic equivalents. Additional adjustment for ALM abrogated the association $(\beta=-1.080[-2.187$, $0.026 ; p=0.056]$ ). No association was evident for 5 -year declines in TUG or ALM.

Conclusion: In elderly women, $\mathrm{AAC}_{\mathrm{sev}}$ was associated with accelerated 5-year declines in handgrip strength but not mobility supporting other evidence that vascular disease in the elderly may have a direct effect on the loss of muscular strength independent of functional decline.

\section{PO26}

The key role of the Notch2 signal in breast cancer dormancy and tumour-initiation

Kashmala Carys ${ }^{1}$, Mattia Capulli ${ }^{1}$, Dayana Hristova ${ }^{1}$, Zoe Valbret ${ }^{1}$, Ronak Arjai $^{1}$, Francesco Masedu ${ }^{1}$, Antonio Maurizi ${ }^{1}$, Nadia Rucci ${ }^{1}$, Anna Teti ${ }^{1}$

\section{${ }^{1}$ University of L'Aquila, L'Aquila, Italy}

Breast cancer relapse after a prolonged disease-free period results from subtle changes in the microenvironment triggering the switch of dormant Breast Cancer Cells (BCCs) to tumor-initiating cells. In this 
context, we identified the Notch2 pathway to play a key role in BBC dormancy. Once injected into mouse tibias, single non-proliferating BBCs lodged nearby the endosteal surface, interacting with Spindleshaped N-cadherin ${ }^{\mathrm{HIGH}}$ Osteoblasts (SNOs). SNOs are inducers of the hematopoietic stem cell (HSC) quiescence and highly express Jagged1 (a Notch ligand). Here we show that in-vitro co-culture of human MDA-MB231 (MDA) or mouse 4T1 BCCs and SNOs impaired BCC proliferation (cell number, $-87 \% ; p=0.002$; $\mathrm{Ki} 67^{+} \mathrm{MDA},-60 \% ; p=0.0002$ vs. NON-SNO/BCC co-culture), suggesting SNO-induced BBC dormancy. Blocking Notch2 or the downstream enzyme $\gamma$-secretase prevents this impairment $(\mathrm{p}>0.2$ vs. NON-SNO/BCC co-culture). In immunocompromised mouse tibias injected with Notch $2{ }^{\mathrm{HIGH}} \mathrm{MDA}$, that after one month showed no bioluminescence signal or osteolysis (presumed dormancy), single Notch $2{ }^{\mathrm{HIGH}} \mathrm{Ki} 67^{-}$MDA located close to SNO enriched endosteum. Single treatment with a $\gamma$-secretase inhibitor dislodged Notch2${ }_{\mathrm{HIGH}} \mathrm{MDA}$ from the endosteum $(\mathrm{p}<0.0001)$ and increased distant organ colonization ( +3 fold of liver metastases; $p=0.0496)$ vs. vehicle. Notch $2{ }^{\mathrm{HIGH}} \mathrm{MDA}$ represented $1-5 \%$ of the total MDA population and were enriched in the HSC stem markers, CD34, c-kit, Tie2 and Cxcr4 $(+103,103,1$ 02, 10fold; $p<0.01$, respectively). Conversely, they did not display the classical MDA stem markers, $\mathrm{CD} 44^{\mathrm{HIGH}} / \mathrm{CD} 24^{\mathrm{LOW}}$, suggesting a unique stem phenotype similar to HSCs. Notch $2{ }^{\mathrm{HIGH}}$ MDA stemness and tumour-initiating ability were confirmed in culture by the generation of more $(+2$ fold; $p=0.00323)$ and larger primary mammospheres $(\mathrm{p}=0.0001)$ and more secondary mammospheres $(+15$ fold; $p=0.0033)$ vs. Not$\mathrm{ch} 2{ }^{\mathrm{LOW}}$ MDA, despite their lower proliferation in standard 2Dcultures $(-54 \% ; p=0.03)$. When injected intratibially in immunocompromised mice, Notch $2^{\mathrm{HIGH}}$ MDA in-bone growth $(-70 \%$; $p=0.02)$ and dissemination to distant organs $(-67 \% ; p=0.0001)$ were lower vs. Notch $2^{\mathrm{LOW}}$ MDA, suggesting that the bone microenvironment favoured dormancy vs. tumour-initiation. Key results were confirmed with mouse 4T1 BCCs in immunocompetent mice (experiments approved by ethical authorities).

\section{PO27}

Characterisation of the role of the fractalkine receptor CX3CR1 in inflammatory osteoclasts

Maria-Bernadette Madel ${ }^{1}$, Lidia Ibanez $^{1}$, Antoine Boutin ${ }^{1}$, Christophe

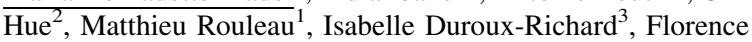
Apparailly $^{3}$, Gilles Chiocchia ${ }^{2}$, Henri-Jean Garchon ${ }^{2}$, Abdelilah Wakkach $^{1}$, Claudine Blin-Wakkach ${ }^{1}$

${ }^{1}$ LP2M, CNRS UMR 7370, Faculté de Médecine, Université Côte d'Azur, Université Nice Sophia Antipolis, Nice, France, ${ }^{2}$ INSERM U1173, Faculty of Health Sciences Simone Veil, University of Versailles Saint-Quentin-en-Yvelines, Montigny-le-Bretonneux, France, ${ }^{3}$ INSERM U1183, Institute for Regenerative Medicine and Biotherapies, University of Montpellier, Montpellier, France

Deregulated immune responses are associated with severe bone destruction in chronic inflammation as we recently demonstrated in Inflammatory Bowel Disease. Similar outcomes were reported for ovariectomy-associated bone loss. Recent findings by our team suggest that, besides bone resorption and depending on their origin, OCLs have an immune function by activating $\mathrm{T}$ cell responses through antigen presentation. Under normal conditions, OCLs induce immunosuppressive $\mathrm{CD}^{+}{ }^{+}$regulatory $\mathrm{T}$ cells (tolerogenic-OCLs/tOCLs), while during inflammation OCLs induce TNF $\alpha$-producing $\mathrm{CD}^{+}{ }^{+} \mathrm{T}$ cells (inflammatory-OCLs/i-OCLs). However, the precise role of i-OCLs and their underlying molecular mechanisms leading to inflammatory bone destruction are unknown. To characterise the different OCL subsets and to identify functional pathways, we performed comparative RNA-Seq analysis on t-OCLs and i-OCLs.
Transcriptomic profiling of the two OCL subsets identified a total of 1,484 genes that are significantly differentially expressed. Among these genes, CX3CR1 ( $\mathrm{p}$ value 0.001 ; $\log 2 \mathrm{FC} 3.2$ ), which we previously identified as marker for $20 \%$ of i-OCLs, as well as 12 genes known to interact with the CX3CR1 pathway were up-regulated in i-OCLs. Thus, we further investigated the role of CX3CR1 in i-OCLs using CX3CR1 $1^{\text {GFP/GFP }}$ mice, which lack functional CX3CR1. MicroCT analysis after ovariectomy (OVX) revealed significantly higher BV/TV ( $p$ value 0.0037) and cortical thickness ( $p$ value 0.0003 ) in OVX CX3CR1 $1^{\mathrm{GFP} / \mathrm{GFP}}$ mice compared to OVX WT mice. These findings indicate that CX3CR1 plays a substantial role in bone destruction. Consistent with this and compared to WT mice, bone marrow cells from CX3CR $1^{\mathrm{GFP} / \mathrm{GFP}}$ mice showed a decreased OCLs differentiation capacity in vitro. Analysis of the precise role of CX3CR1 in i-OCLs is still on-going. The characterisation of specific markers for i-OCLs, such as CX3CR1, provides essential therapeutic perspectives to target i-OCLs. Furthermore, our results will help to understand the role of CX3CR1 in i-OCLs and to unravel underlying molecular mechanisms leading to inflammatory bone destruction.

Keywords: Osteoimmunology, Osteoclasts, Inflammation

\section{PO28}

Perivascular fibrosis and upregulation of the TGF $\beta$ pathway in CLCN7-depedent Autosomal Dominant Osteopetrosis type 2 (ADO2)

Antonio Maurizi ${ }^{1}$, Capulli Mattia ${ }^{1}$, Annabel Curle ${ }^{1}$, Rajvi Patel ${ }^{1}$, Nadia Rucci ${ }^{1}$, Anna Teti ${ }^{1}$

${ }^{1}$ University of L'Aquila, L'Aquila, Italy

CLCN7-dependent ADO2 is an inherited bone disease, characterised by dense but fragile bones, due to defective osteoclast function. Despite ADO2 being classified as a bone disease, the CLC7 protein is widely expressed in the body, suggesting that other organs could be affected by its inactivating mutations. We tested this hypothesis in a mouse model carrying the heterozygous, ADO2-causing mutation, $C l c n 7^{G 213 R}$ (ethical approval n.564/2016-PR). Compared to WT mice, perivascular collagen, detected by the Masson's staining, was higher in ADO2 lungs $(+1.5$ fold; $\mathrm{p}=0.005)$, muscles $(+1.7$ fold; $\mathrm{p}=0.04)$ and kidneys $(+3$ fold; $p=0.01)$. Consistently, upregulation of Collal expression was detected in $\mathrm{ADO} 2$ lungs $(+6.7$ fold; $\mathrm{p}=0.001)$ and muscles $(+2.6$ fold; $\mathrm{p}=0.001)$, along with a higher number of $\mathrm{F} 4 / 80^{+} / \mathrm{ClC}^{+}$macrophages $(+2$ fold; $\mathrm{p}=0.005$ and +12 fold; $\mathrm{p}<0.001$, respectively), which are known to produce profibrotic mediators. RNA deep sequencing demonstrated an enrichment in the macrophage gene signature and an upregulation of the profibrotic Tgf $\beta$ pathway in ADO2 vs. WT lungs, muscles and kidney $(\mathrm{p}<0.05)$. Real-time RT-PCR showed upregulation of $T g f b 1$ and $T g f b 2$ in $\mathrm{ADO} 2$ lungs and muscles, respectively $(+1.5$ fold; $\mathrm{p}=0.01$ and +2.6 fold; $\mathrm{p}=0.0026)$, while both $T g f b 1$ and $T g f b 2$ were upregulated in ADO2 kidneys $(+2.4$ fold; $p=0.0008$ and +1.4 fold; $p=0.03$ ). In contrast, the expression of the antifibrotic $T g f b 3$ was unremarkable in all organs. Consistently, the TGF $\beta$-activated factors, $\alpha$-Sma and Grem1, were upregulated in ADO2 lungs $(+2.9$ fold; $\mathrm{p}<0.0001)$ and muscles $(+11.4$ fold; $\mathrm{p}=0.01)$. Compared to WT, in vitro $\mathrm{ADO} 2$ macrophages showed higher lysosomal $\mathrm{pH}(+1.64 \mathrm{pH}$ units; $\mathrm{p}<0.001)$, LC3bII accumulation $(+1.7$ fold $)$ and increase of p62 expression $(+2.7$ fold), suggesting altered autophagy, which is known to be induced by TGF $\beta$. These findings were confirmed by immunofluorescence and confocal imaging in in vivo $\mathrm{ADO} 2$ organs. Taken together, these results indicate that $\mathrm{ADO} 2$ is a systemic disease inducing perivascular fibrosis in various organs, possibly mediated by tissue macrophages and activation of the pro-fibrotic TGF $\beta$ pathway. 


\section{PO29}

Mechanisms of action of the sphingosine 1-phosphate metabolic pathway in spondyloarthritis

Alaeddine El Jamal ${ }^{1}$, Anne Briolay ${ }^{1}$, Saida Mebarek ${ }^{1}$, David Magne ${ }^{1}$, Leyre Brizuela $^{1}$, Carole Bougault ${ }^{1}$

${ }^{1}$ UMR5246, ICBMS, Univ Lyon, Lyon, France

Enthesis ectopic ossification is a distinctive feature of spondyloarthritis (SpA). We previously showed that levels of the bioactive lipid sphingosine 1-phosphate (S1P) were particularly high in SpA patients' serum, compared to healthy donors. Furthermore, we demonstrated that S1P producing enzymes were implicated in the mineralization capacity of cultured murine osteoblasts and chondrocytes. S1P can act intracellularly or be secreted and bind to one of its 5 receptors $\left(\mathrm{S}_{1} \mathrm{P}_{1-5}\right)$. We aimed to decipher, in entheseal cells, the mechanisms of action of S1P metabolic pathway leading to mineralization deregulation.

We have already noticed that during osteoblast and chondrocyte differentiation into mineralizing cells, S1P secretion significantly increased, suggesting an extracellular role for S1P. S1P receptors gene expression was thus analyzed. $\mathrm{S}_{1} \mathrm{P}_{1}$ and $\mathrm{S}_{1} \mathrm{P}_{3}$ mRNA were increased 20 folds in osteoblasts and 60 folds in chondrocytes. Specific antagonists were then used to block $\mathrm{S}_{1} \mathrm{P}_{1}$ (W146) or $\mathrm{S}_{3} \mathrm{P}_{3}$ (BML-241) or both (VPC23019). We also used the FDA approved drug Fingolimod (FTY720), which block $\mathrm{S}_{1} \mathrm{P}_{1}, \mathrm{~S}_{1} \mathrm{P}_{3}$ and the sphingolipid metabolism in general. In osteoblasts, alkaline phosphatase (AP) activity was increased $(+35 \%)$ after adding $1 \mu \mathrm{M}$ of S1P. Whereas antagonists had modest effects, Fingolimod significantly reduced mineralization (Alizarin Red staining, - 35\%), AP activity $(-20 \%)$ and osteocalcin gene expression $(-30 \%)$. In chondrocytes, the mineralization was reduced by $\mathrm{S}_{1} \mathrm{P}_{1}$ blockade $(-20 \%)$ and by Fingolimod $(-55 \%)$. VPC23019 and Fingolimod diminished AP activity $(-20$ and $-55 \%$, respectively). Osteocalcin mRNA decreased after the blockade of $\mathrm{S}_{1} \mathrm{P}_{1}(-10 \%)$, of $\mathrm{S}_{1} \mathrm{P}_{3}(-25 \%)$ and with Fingolimod $(-70 \%)$.

Our results indicate that in osteoblasts, S1P action may be restricted to the intracellular compartment. On the contrary, in chondrocytes, its action could be partly mediated by $\mathrm{S}_{1} \mathrm{P}_{1}$ and $\mathrm{S}_{1} \mathrm{P}_{3}$. Our work suggests that S1P metabolic pathway could be a potential therapeutic target in the treatment of SpA.

Acknowledgements: French Society of Rheumatology

Keywords: Spondyloarthritis, Sphingosine 1-phosphate, Mineralization

\section{PO30}

Lin28a overexpression protects chondrocyte from osteoarthritis phenotype

Yohan Jouan $^{1,2}$, Joanna Sanna ${ }^{1,2}$, Chahrazad Cherifi ${ }^{1,2}$, Augustin Latourte $^{2,3}$, Hang-Korng Ea ${ }^{1,2}$, Martine Cohen-Solal ${ }^{1,2}$, Eric Hay ${ }^{1,2}$

${ }^{1}$ Paris Diderot University, Paris, France, ${ }^{2}$ Inserm 1132, Paris, France, ${ }^{3}$ Hopital Lariboisière, Paris, France

Osteoarthritis is defined as all joint disease including cartilage breakdown and bone remodeling. Cartilage damage is promoted by an unbalance between anabolic and catabolic activity and apoptosis in osteoarthritic chondrocyte. Matrix lesions are irreversible due to the weak cartilage regeneration abilities.

Here we asked if Lin28a overexpression, an RNAs binding protein involved in tissues repair could induce dedifferentiation of osteoarthritic chondrocyte and regenerate cartilage.
In vitro assays were performed using organotypic femoral head culture (10 weeks old) $\mathrm{CreCo} 22^{\mathrm{ER}} \operatorname{Lin} 28 a^{c}(T g)$ female mice. Lin28a overexpression was induced by hydroxyl tamoxifene added in the culture medium. Chondrocyte activation was induced by Wnt3a; culture medium was collected for Western Blot analysis. Samples were processed for mRNA extraction or for histological analysis. In vivo $\mathrm{CreCol} 2{ }^{\mathrm{ER}} \operatorname{Lin} 28 a^{c}(\mathrm{Tg})$ male mice were treated by tamoxifen. One week after, osteoarthritis was induced surgically. $\operatorname{Lin} 28 a^{c}(T g)$ littermates were used as control, and left knee was sham operated. Cartilage (OA score and immunohistochemistry) and bone ( $\mu$ tomography) were analyzed 8 weeks after surgery.

Lin28a overexpression maintains cartilage integrity by reducing MMPs expression and increasing anabolic gene expression (2.94 fold vs. ctrl $\mathrm{p}<0.05)$ compare to WT explants. Reduced apoptosis (twofold vs. ctrl $\mathrm{p}<0.05$ ) and increased cell proliferation are also observed.

In vivo, bone architecture analysis reveals no difference in subchondral bone structure between $\mathrm{CreCo} 22^{\mathrm{ER}} \operatorname{Lin} 28 \mathrm{a}^{\mathrm{c}}(\mathrm{Tg})$ mice and $\operatorname{Lin} 28 a^{c}(T g)$ neither in sham and DMM knee. But in transgenic mice DMM promoted the osteophyte size (threefold vs. ctrl $\mathrm{p}<0.05$ ). Lin28a overexpression protects cartilage degradation (4 vs. 6 $\mathrm{p}<0.05)$ and reduces MMP13 expression (20\% decrease vs. ctrl $\mathrm{p}<0.05)$. Moreover, chondrocyte proliferation (2.7 fold vs. Ctrl $\mathrm{p}<0.05)$ is increased in transgenic mice whereas apoptosis is reduced.

We have demonstrated that Lin28a overexpression could be used to enhance cell reprogramming and cartilage regeneration in OA

Keywords: Cartilage, Regeneration, Osteoarthritis, Osteophyte

\section{Parallel Oral Presentations 6: Osteoporosis treatment}

\section{PO31}

The effect of vitamin MK-7 for 3 years on bone mineral density and microarchitecture in postmenopausal women with osteopenia

Sofie Hertz Rønn ${ }^{1}$, Torben Harsløf ${ }^{1}$, Steen Bønløkke Pedersen ${ }^{1}$, Bente Lomholt Langdahl ${ }^{1}$

${ }^{1}$ Department of Endocrinology and Internal Medicine, Aarhus University Hospital, Aarhus, Denmark

Background: Clinical studies suggest that vitamin K2 prevents bone loss. Vitamin $\mathrm{K}$ is a cofactor in the carboxylation of osteocalcin (OC). Carboxylated OC promotes mineralization of bone. Conventionally, bone loss is prevented by calcium and vitamin $\mathrm{D}$ that also decrease fracture risk. The aim of the study therefore was to investigate the effect of vitamin $\mathrm{K} 2$ as an add-on to calcium and vitamin D on osteocalcin, bone mass and -structure.

Methods: We conducted a randomized placebo-controlled doubleblinded clinical trial, including 142 postmenopausal osteopenic women who received vitamin K2 (375 $\mu \mathrm{g}$ MK-7) or placebo daily for 3 years. Both groups received vitamin D3 (38 $\mu \mathrm{g} /$ day) and calcium ( $800 \mathrm{mg} /$ day). We measured bone markers and bone mineral density and -structure by DXA and HRpQCT.

Results: After 1 year, undercarboxylated osteocalcin decreased in the MK-7-group $(-65.2 \pm 23.5 \%)$ (mean $\pm \mathrm{SD}$ ) compared to the placebo-group $(-0.03 \pm 38.5 \%), \mathrm{p}<0.01$. Analyses of bone turnover markers after 3 years are pending.

After 3 years, aBMD (total hip) did not change differently between the MK-7-group $(-1.5 \pm 2.5 \%)$ and the placebo-group $(-2.4 \pm 2.7 \%), p=0.09$. No differences were seen in changes in BMD (femoral neck); the MK-7-group $(-1.5 \pm 3.5 \%)$ and the 
placebo-group $(-1.0 \pm 5.0 \%), \mathrm{p}=0.84$, or (lumbar spine); the MK7 -group $(-1.8 \pm 3.9 \%)$ and the placebo-group $(-1.1 \pm 3.1 \%)$, $\mathrm{p}=0.57$.

We have previously reported improved microstructure with MK-7 after 1 year $^{1}$. However, changes in microstructure after 3 years were similar between the two groups. vBMD (tibia) changes similarly between the MK-7-group $(-2.2 \pm 3.4 \%)$ and the placebo-group $(-1.9 \pm 2.3 \%), p=0.98$. vBMD (radius) also changed similarly $(-2.4 \pm 5.1 \%$ in the MK-7 group versus $-1.9 \pm 4.9 \%$ in the placebo-group, respectively, $\mathrm{p}=0.85$ ).

Conclusion: Treatment with MK-7 $375 \mu \mathrm{g}$ daily as an add-on to calcium and vitamin D increased carboxylation of osteocalcin. However, treatment for 3 years did not affect bone mineral density, or bone microarchitecture as measured by HRpQCT.

\section{PO32}

Denosumab compared with risedronate in glucocorticoid-treated subjects: results from the final 24-month analysis of a randomized, double-blind, double-dummy study

Kenneth Saag ${ }^{1}$, Nicola Pannacciulli ${ }^{2}$, Piet Geusens ${ }^{3}$, Jonathan Adachi $^{4}$, Osvaldo Messina ${ }^{5}$, Jorge Morales-Torres ${ }^{6}$, Ronald Emkey ${ }^{7}$, Peter Butler ${ }^{2}$, Xiang Yin ${ }^{2}$, Willem Lems ${ }^{8}$

${ }^{I}$ Department of Medicine, University of Alabama, Birmingham, United States, ${ }^{2}$ Research and Development, Amgen Inc., Thousand Oaks, United States, ${ }^{3}$ Department of Internal Medicine, Maastricht University, Maastricht, Netherlands, ${ }^{4}$ Department of Medicine, McMaster University, Hamilton, Canada, ${ }^{5}$ Department of Rheumatology, Cosme Argerich Hospital, Buenos Aires, Argentina, ${ }^{6}$ Hospital Aranda de la Parra, Leon, Mexico, ${ }^{7}$ Clinical Research, Emkey Arthritis \& Osteoporosis Clinic, Wyomissing, United States, ${ }^{8}$ Department of Rheumatology, VU University Medical Center, Amsterdam, Netherlands

Denosumab $60 \mathrm{mg}$ subcutaneously Q6M increased spine and hip BMD significantly more than risedronate $5 \mathrm{mg}$ orally QD at 12 months in glucocorticoid-treated subjects (as previously reported). This analysis compared the BMD effects of denosumab vs. risedronate and further characterized denosumab safety in this population at 24 months.

This phase 3, randomized, double-blind, double-dummy study enrolled adults $\geq 18$ years receiving $\geq 7.5 \mathrm{mg}$ daily prednisone (or equivalent) for $<3$ months (glucocorticoid-initiating subpopulation) or $\geq 3$ months (glucocorticoid-continuing subpopulation). All subjects $<50$ years had history of osteoporotic fracture. Glucocorticoidcontinuing subjects $\geq 50$ years had lumbar spine (LS), total hip (TH), or femoral neck BMD T-scores $\leq-2.0$, or $\leq-1.0$ and history of fracture. Subjects were randomized 1:1 to denosumab $60 \mathrm{mg}$ subcutaneously Q6M or risedronate $5 \mathrm{mg}$ orally QD for 24 months. This analysis assessed denosumab superiority over risedronate for percentage change from baseline in LS and TH BMD at 24 months.

Of 795 randomized subjects, $590(74.2 \%)$ completed the 24-month study (glucocorticoid-initiating: 109/145 denosumab, 117/145 risedronate; glucocorticoid-continuing: 186/253 denosumab, 178/252 risedronate). Denosumab was superior to risedronate for increases from baseline in LS and TH BMD at all timepoints assessed through 24 months in each subpopulation (Figure). Adverse events, serious adverse events (including infection), and fractures were similar between groups.
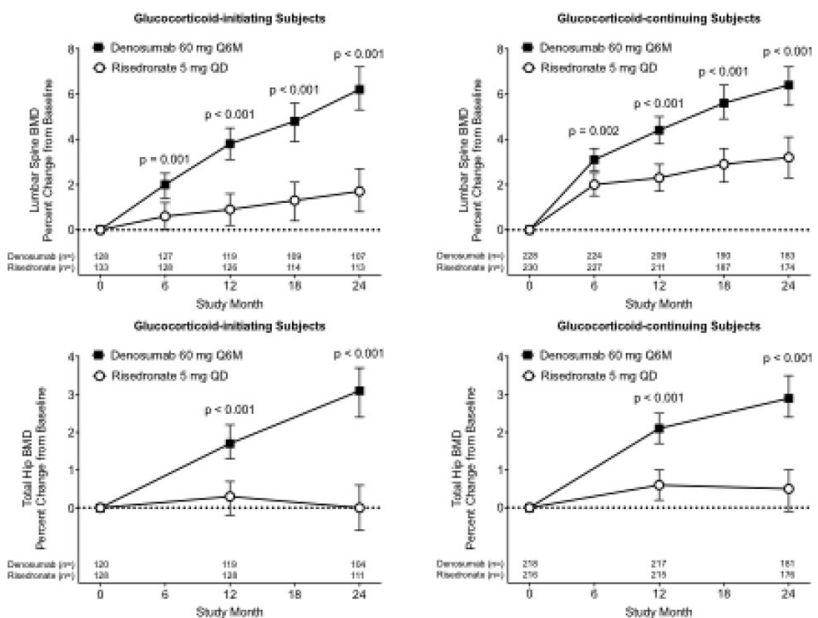

BMD: bone mineral density; Cl: confidence interval; Q6M: every 6 months; QD: once daily. Based on ANCOVA model adjusting for treatment, baseline BMD value, gender, machine type, and baseline BMD value-by-machine type interaction. For glucocorticoid-continuing subpopulation, duration of prior BMD value-by-machine type interaction. For glucocorticoid-continuing subpopula
gluoscorticoid use ( $<12$ vs $\geq 12$ months) was included as an additional covariate.

Figure BMD percentage change $(95 \% \mathrm{CI})$ from baseline through month 24

In conclusion, denosumab was superior to risedronate for increases in spine and hip BMD through 24 months. The overall safety profile was similar between groups. Denosumab may offer a valuable osteoporosis treatment option for patients receiving glucocorticoids.

Keywords: Glucocorticoid-induced osteoporosis (GIOP), denosumab

BMD percentage change $(95 \% \mathrm{CI})$ from baseline at 24

\begin{tabular}{|c|c|c|c|c|c|c|}
\hline & \multicolumn{3}{|c|}{ Glucocorticoid-continuing } & \multicolumn{3}{|c|}{ Glucocorticoid-initiating } \\
\hline & $\begin{array}{l}\text { Deno- } \\
\text { sumab }\end{array}$ & $\begin{array}{l}\text { Rise- } \\
\text { dronate }\end{array}$ & $\begin{array}{l}\text { Differ- } \\
\text { ence }\end{array}$ & $\begin{array}{l}\text { Deno- } \\
\text { sumab }\end{array}$ & $\begin{array}{l}\text { Rise- } \\
\text { dronate }\end{array}$ & Difference \\
\hline Lumbar & spine & $\begin{array}{l}6.4 \\
(5.5,7.2) \\
(\mathrm{n}=183)\end{array}$ & $\begin{array}{l}3.2 \\
(2.3,4.1) \\
(\mathrm{n}=174)\end{array}$ & $\begin{array}{l}3.2(2.0,4.3) \\
p<0.001\end{array}$ & $\begin{array}{c}6.2 \\
(5.3,7.2) \\
(\mathrm{n}=107)\end{array}$ & $\begin{array}{l}1.7(0.8,2.7) \\
(\mathrm{n}=113)\end{array}$ \\
\hline $\begin{array}{c}4.5(3.2, \\
5.8)\end{array}$ & & & & & $\mathrm{p}<0.001$ & \\
\hline $\begin{array}{l}\text { Total } \\
\text { hip }\end{array}$ & $\begin{array}{l}2.9 \\
(2.4,3.5) \\
(\mathrm{n}=181)\end{array}$ & $\begin{array}{l}0.5 \\
(-0.1 \\
1.0) \\
(\mathrm{n}=176)\end{array}$ & $\begin{array}{l}2.5(1.7,3.2) \\
p<0.001\end{array}$ & $\begin{array}{l}3.1(2.4,3.7) \\
(\mathrm{n}=104)\end{array}$ & $\begin{array}{l}-0.0 \\
(-0.6,0.6) \\
(\mathrm{n}=111)\end{array}$ & $\begin{array}{l}3.1(2.2,3.9) \\
\mathrm{p}<0.001\end{array}$ \\
\hline
\end{tabular}

$B M D$ bone mineral density, $C I$ confidence interval

\section{PO33}

Early increases in $\mathrm{N}$-terminal propeptide of type 1 procollagen (P1NP) with romosozumab therapy as an indicator for BMD response

Junichi Takada $^{1}$, Rajani Dinavah ${ }^{2}$, Akimitsu Miyauchi ${ }^{3}$, Etsuro Hamaya $^{4}$, Toshiyasu Hirama ${ }^{4}$, Cesar Libanati ${ }^{5}$, Yoichi Nakamura ${ }^{4}$, Cassandra E. Milmont ${ }^{2}$, Li Chen ${ }^{2}$, Andreas Grauer ${ }^{2}$

${ }^{1}$ Kitago Orthopedic Clinic, Sapporo, Japan, ${ }^{2}$ Amgen Inc., Thousand Oaks, United States, ${ }^{3}$ Miyauchi Medical Center, Osaka, Japan, ${ }^{4}$ Amgen Astellas BioPharma, Tokyo, Japan, ${ }^{5}$ UCB Pharma, Brussels, Belgium 
The bone formation marker, P1NP, may inform bone mineral density (BMD) response to teriparatide in treatment-naive patients with osteoporosis. ${ }^{1}$ We examined the utility of early P1NP changes to indicate BMD response in women pre-treated with bisphosphonates in the STRUCTURE trial (NCT01796301). ${ }^{2}$

Osteoporotic postmenopausal women with a fracture history, who had received oral bisphosphonates ( $\geq 3$ years) and alendronate (70 mg/week/1 year) before screening, were included. Patients received romosozumab $(210 \mathrm{mg} / \mathrm{month})$ or teriparatide $(20 \mu \mathrm{g} / \mathrm{day})$ for 12 months (m). BMD was assessed at 6 and $12 \mathrm{~m}$. P1NP measurement time points included baseline and $1 \mathrm{~m}$. The positive predictive value of a P1NP increase of $\geq 10 \mu \mathrm{g} / \mathrm{L}$ at $1 \mathrm{~m}^{1}$ and increase in BMD at 6 and $12 \mathrm{~m}$ was evaluated.

With romosozumab, 95\% of women $(191 / 202)$ had a $\geq 10 \mu \mathrm{g} / \mathrm{L}$ P1NP increase from baseline at $1 \mathrm{~m}$, versus 91\% (183/201) with teriparatide. Of these, $91 \%$ (romosozumab) had an increase in lumbar spine (LS) BMD of $\geq 3 \%$ at $12 \mathrm{~m}(82 \%$ at $6 \mathrm{~m})$ versus $66 \%$ (teriparatide, $12 \mathrm{~m}$ [52\% at $6 \mathrm{~m}])$. Total hip (TH) BMD increased by $\geq 3 \%$ with romosozumab in $46 \%$ of women at $12 \mathrm{~m}(38 \%$ at $6 \mathrm{~m})$ versus $18 \%$ at $12 \mathrm{~m}$ for women receiving teriparatide $(10 \%$ at $6 \mathrm{~m})$. Despite an increase in P1NP of $\geq 10 \mu \mathrm{g} / \mathrm{L}$ at $1 \mathrm{~m}, 12$ and $60 \%$ of teriparatide-treated women had no increase in BMD at LS and TH, respectively, at $12 \mathrm{~m}$. Conversely, only 3 and $18 \%$ of romosozumabtreated patients had no increase in LS and THBMD, respectively, at $12 \mathrm{~m}$.

These data suggest that following bisphosphonate pre-treatment, greater improvements in LS and THBMD within $12 \mathrm{~m}$ may be expected with romosozumab vs. teriparatide therapy, despite both therapies having similar early increases in P1NP. P1NP may be a more reliable indicator of a positive BMD response in romosozumabtreated compared with teriparatide-treated women.

References: ${ }^{1}$ Eastell, CMRO, 2006; ${ }^{2}$ Langdahl, Lancet, 2017

Funding: Amgen Inc., Astellas Pharma, UCB Pharma

Keywords: romosozumab, bone formation markers, BMD

\section{PO34}

Denosumab (DMAb) reduced bone remodeling, eroded surface, and erosion depth in cortical bone of iliac crest biopsies from postmenopausal women in the FREEDOM trial

Roland Chapurlat ${ }^{1}$, Nathalie Portero-Muzy ${ }^{1}$, Jean-Paul Roux ${ }^{1}$, Stephane Horlait ${ }^{2}$, David Dempster ${ }^{3}$, Andrea Wang ${ }^{2}$, Rachel Wagman $^{2}$, Pascale Chavassieux ${ }^{1}$

${ }^{1}$ INSERM UMR 1033, Université de Lyon, Lyon, France, ${ }^{2}$ Amgen Inc., Thousand Oaks, United States, ${ }^{3}$ Columbia University, New York, United States

Objective: Report the effects of DMAb vs. placebo (PBO) on cortical bone histomorphometry during FREEDOM measured by iliac crest biopsies.

Methods: 112 iliac crest bone biopsies were evaluable for cortical bone histomorphometry, 67 obtained at month 24 (37 PBO, 30 DMAb) and 45 at month 36 (25 PBO, 20 DMAb). Both cortices were analyzed, if available. Endocortical (Ec) eroded surface per bone surface (ES/BS), osteoclast surface (Oc.S/BS) and erosion depth (E.De) were assessed by an automatic-interactive method (Roux et al., Bone. 1995). Cortical porosity and Ec wall thickness (W.Th) were also measured. Dynamic remodeling parameters were assessed for Ec, periosteal, and intracortical envelopes.

Results: Ec structural variables, Oc.S/BS, ES/BS, and mean and maximal E.De were significantly lower in the DMAb group vs. PBO at months 24 and $36(p<0.0001$ and $<0.05$, respectively). There were no significant differences between groups for W.Th, cortical porosity, or cortical thickness. No extensive search for cortical fluorochrome labels was done. Cortical labels in at least one cortical envelope were observed in $13(43 \%)$ and 10 (50\%) of DMAb biopsies at months 24 and 36, respectively. Though envelope-specific dynamic bone formation and remodeling parameters could not be reliably assessed, overall labeling findings indicate that DMAb markedly reduced cortical bone turnover.

Conclusions: These data are consistent with DMAb mechanism of action, which inhibits osteoclasts throughout the skeleton. Lower Ec eroded surfaces with DMAb may reflect inhibited bone resorption and refilling of resorption spaces. Reduced cortical labeling and bone formation indices with DMAb may reflect refilling of resorption spaces that were present before fluorochromes were administered, and reduced activation of new remodeling sites. Reduced E.De is a novel finding for DMAb that may contribute to increased bone strength by reducing Ec bone loss and structural vulnerabilities associated with deep resorption cavities.

Keywords: denosumab, cortical bone, histomorphometry, remodelling

Summary of cortical histomorphometry parameters at months 24 and 36

\begin{tabular}{|c|c|c|c|c|}
\hline & \multicolumn{2}{|c|}{ Month 24} & \multicolumn{2}{|c|}{ Month 36} \\
\hline Parameter & $\begin{array}{c}\text { PBO } \\
(n=37)\end{array}$ & $\begin{array}{c}\text { DMAb } \\
(n=30)\end{array}$ & $\begin{array}{c}\text { PBO } \\
(\mathrm{n}=25)\end{array}$ & $\begin{array}{c}\text { DMAb } \\
(n=20)\end{array}$ \\
\hline $\begin{array}{l}\text { Ecoc S/BS } \\
(\%)\end{array}$ & $\begin{array}{c}0.26 \\
(0.00,0.59)\end{array}$ & $\begin{array}{c}0.00 \\
(0.00,0.00)^{*}\end{array}$ & $\begin{array}{c}0.14 \\
(0.00,0.22) \\
\end{array}$ & $\begin{array}{c}0.00 \\
(0.00,0.00)^{\dagger}\end{array}$ \\
\hline $\begin{array}{l}\text { Ec.ES/BS } \\
(\%)\end{array}$ & $\begin{array}{c}4.39 \\
(3.26,7.60)\end{array}$ & $\begin{array}{c}1.59 \\
(1.01,2.17)^{*}\end{array}$ & $\begin{array}{c}3.53 \\
(1.92,5.32)\end{array}$ & $\begin{array}{c}1.39 \\
(0.67,3.52)^{\dagger}\end{array}$ \\
\hline $\begin{array}{l}\text { Maximum } \\
\text { Ec. } \mathrm{E} . \mathrm{De}(\mu \mathrm{m})\end{array}$ & $\begin{array}{r}16.45 \\
(12.88 \\
21.61)\end{array}$ & $\begin{array}{c}11.43 \\
(9.00,13.11)^{*}\end{array}$ & $\begin{array}{r}17.40 \\
(12.13 \\
21.01)\end{array}$ & $\begin{array}{c}14.04 \\
(6.92,15.99)^{\dagger}\end{array}$ \\
\hline $\begin{array}{l}\text { Mean } \\
\text { Ec.E.De }(\mu \mathrm{m})\end{array}$ & $\begin{array}{c}10.26 \\
(8.32,13.34)\end{array}$ & $\begin{array}{c}7.10 \\
(6.03,8.69)^{*}\end{array}$ & $\begin{array}{c}10.14 \\
(7.39,12.87)\end{array}$ & $\begin{array}{c}7.91 \\
(4.94,9.65)^{\dagger}\end{array}$ \\
\hline $\begin{array}{l}\text { Mean } \\
\text { Ec.W.Th } \\
(\mu \mathrm{m})\end{array}$ & $\begin{array}{c}32.41 \\
(31.16 \\
33.83) \\
\end{array}$ & $\begin{array}{c}32.39 \\
(31.68,34.52)\end{array}$ & $\begin{array}{r}32.11 \\
(30.48 \\
33.05) \\
\end{array}$ & $\begin{array}{c}31.82 \\
(29.30 \\
33.41) \\
\end{array}$ \\
\hline $\begin{array}{l}\text { Cortical } \\
\text { thickness }(\mu \mathrm{m})\end{array}$ & $\begin{array}{r}749.3 \\
(572.0 \\
904.9) \\
\end{array}$ & $\begin{array}{c}753.6 \\
(521.3 \\
1022.0) \\
\end{array}$ & $\begin{array}{r}689.7 \\
(478.7 \\
920.4) \\
\end{array}$ & $\begin{array}{r}605.8 \\
(491.0 \\
799.3) \\
\end{array}$ \\
\hline $\begin{array}{l}\text { Cortical } \\
\text { porosity (\%) }\end{array}$ & $\begin{array}{c}6.43 \\
(4.56,9.34) \\
\end{array}$ & $\begin{array}{c}5.90 \\
(3.83,8.12)\end{array}$ & $\begin{array}{c}5.42 \\
(3.77,7.42)\end{array}$ & $\begin{array}{c}6.37 \\
(4.39,7.88) \\
\end{array}$ \\
\hline
\end{tabular}

\section{PO35}

Effects of teriparatide on hip and upper limb fractures in patients with osteoporosis: a systematic review and meta-analysis

Adolfo Díez-Pérez ${ }^{1,2}$, Fernando Marin ${ }^{3}$, Erik F. Eriksen ${ }^{4}$, David L. Kendler $^{5}$, John H. Krege ${ }^{6}$, Miguel Delgado-Rodríguez ${ }^{7,8}$

${ }^{1}$ Department of Internal Medicine, Hospital del Mar-IMIM-UAB, Barcelona, Spain, ${ }^{2}$ CIBERFES, Institute Carlos III, Barcelona, Spain, ${ }^{3}$ Lilly Research Center, Windlesham, United Kingdom, ${ }^{4}$ Department of Clinical Endocrinology, Morbid Obesity and Preventive Medicine, Oslo University Hospital, Oslo, Norway, ${ }^{5}$ University of British Columbia, Vancouver, Canada, ${ }^{6}$ Lilly Research Laboratories, Indianapolis, United States, ${ }^{7}$ Department of Preventive Medicine and Public Health, University of Jaén, Jaén, Spain, ${ }^{8}$ CIBERESP, Institute Carlos III, Madrid, Spain

In randomized clinical trials (RCTs) with teriparatide, the number of patients with incident hip fractures was small. We conducted a meta-analysis to evaluate the effects of teriparatide on hip and upper limb fractures compared to placebo or osteoporosis drugs in patients with osteoporosis.

A literature search was conducted for RCTs of teriparatide of at least 6-month duration, with the approved treatment indications and dose, and that reported non-spine fractures (hip, humerus, forearm, 
wrist). Study selection and data extraction were performed by two independent reviewers. Statistical procedures included Peto's method and Mantel-Haenszel with empirical correction, as most of the RCTs reported zero events in at least one of the treatment arms. The results are expressed as odds ratios (OR) with $95 \%$ confidence intervals (CI). Publication bias and heterogeneity were assessed with standard statistical tests.

Twenty-three RCTs were included, 19 with an active-controlled arm, 11 double-blind, representing data on 8591 subjects (3888 treated with teriparatide). Mean age was 67.0 years, median treatment duration 18 months (range 6-24 months). Thirty-four incident hip, 28 humerus, 31 forearm, and 73 wrist fractures were reported. Metaanalysis results showed an OR $(95 \%$ CI) for hip fractures of 0.44 $(0.22-0.87 ; p=0.019)$ in patients treated with teriparatide compared with controls (Figure). The effects on the risk of humerus [0.82 $(0.38-1.74)]$, forearm [0.53 (0.26-1.08)] and wrist [1.14 (0.71-1.83)] fractures were not statistically significant.

This meta-analysis provides evidence of efficacy of teriparatide in reducing osteoporotic hip fractures by $56 \%$. No significant effects were demonstrable for upper limb fractures.

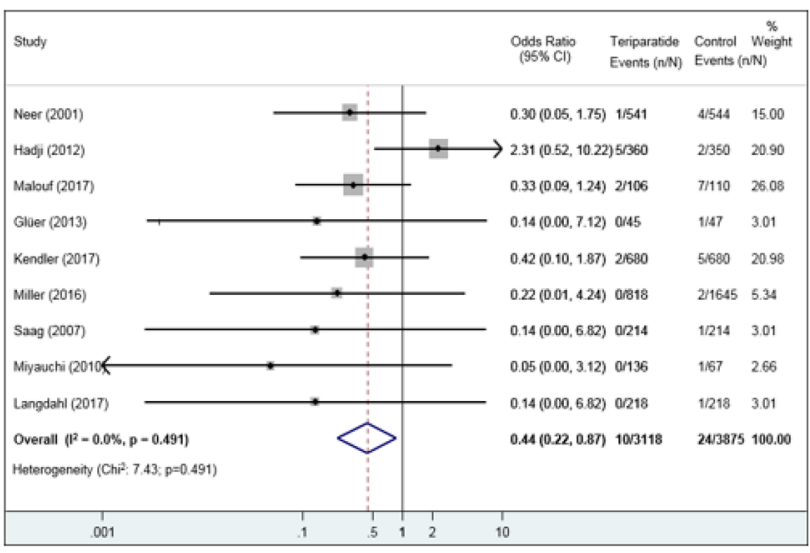

Figure Efficacy of teriparatide versus comparators for reducing hip fracture

\section{PO36}

Screening of high fracture risk in primary care to reduce fractures: the SALT Osteoporosis Study a randomized trial

Thomas Merlijn ${ }^{1}$, Karin Swart ${ }^{2}$, Coen Netelenbos ${ }^{3}$, Petra Elders ${ }^{4}$

${ }^{1}$ Department of General Practice and Elderly Care Medicine, Vu University Medical Center, Amsterdam, Netherlands, ${ }^{2}$ Department of General Practice and Elderly Care Medicine, VUmc Medical Centre, Amsterdam, Netherlands, ${ }^{3}$ Department of Internal Medicine, Endocrine Section, $V U$ University Medical Center, Amsterdam, Netherlands, ${ }^{4}$ Department of General Practice and Elderly Care Medicine, VU University Medical Center, Amsterdam, Netherlands

Objective: To study whether screening of fracture risk and subsequent treatment of older women in primary care can reduce fractures, in comparison to usual care.

Method: In a randomized trial in women 65-90 years we compared a screening programme to assess increased fracture risk with usual care. All women in 224 GP practices in the Netherlands received a questionnaire to assess clinical risk factors for fractures. We randomized all women with at least one clinical risk factor for fractures (previous fracture, parental hip fracture, low body weight, secondary causes for osteoporosis). Participants in the intervention group underwent bone densitometry and vertebral fracture assessment. The 10-year fracture probability was calculated with the fracture risk assessment tool (FRAX). Treatment with anti-osteoporotic medication was recommended if the 10-year fracture probability was above an age dependent threshold. The primary outcome was time to first fracture, analyzed with Cox proportional Hazard analysis. Self-reported fractures at 24 and 48 months were verified with the GP or hospital.

Results: We identified 11,331 women with $\geq 1$ risk factor for fractures. The mean follow up time was 3.9 years and follow up data was available in $99 \%$ of the women. In the intervention group 634 fractures occurred in 5673 participants and in the usual care group 639 fractures in 5542 women. Screening and subsequent treatment had no effect on time to first fracture (HR 0.96, 95\% CI 0.86-1.08). Among the participants who had an indication for bone sparing medication, 196 fractures occurred among 1426 participants in the intervention group vs. 166 fractures among 1353 participants in the control group (HR 1.11, 95\% CI 0.87-1.42).

Conclusion: Screening and treatment of older women with increased fracture risk in primary care did not reduce fractures. Our findings agree with two simultaneous trials and do not support screening of increased fracture risk in primary care.

\section{Oral Posters 1-Basic}

\section{P041}

Differential molecular profile of circulating extracellular vesicle (EV) cargos in mouse models of osteoporosis induced by oestrogen withdrawal or by mechanical unloading

Alfredo Cappariello ${ }^{1}$, Argia Ucci ${ }^{1}$, Nadia Rucci ${ }^{1}$, Anna Teti ${ }^{1}$

${ }^{1}$ DISCAB, University of L'Aquila, L'Aquila, Italy

EVs emerged as potent means of cell-cell communication. They are released in biological fluids, including blood, urines and saliva, and are considered new biomarkers of diseases. We hypothesize that circulating EVs shuttle biological messengers involved in bone metabolism in a disease-dependent manner, thus postulating that different osteoporotic conditions are characterized by different EV molecular profiles. To address this hypothesis, we induced osteoporosis in 9-week-old female mice by ovariectomy $(\mathrm{OVX}, \mathrm{n}=5)$ or hind-limb tail-suspension (HLTS, $n=6$ ). Sera were collected after 5 , 7 and 10 weeks, then circulating EVs were isolated and transcriptionally profiled by a real-time PCR "osteoporosis array" assessing 84 genes commonly involved in bone metabolism. The analysis showed differences in the EV transcripts between the two osteoporosis models, and at different time points within the same experimental group. Results, expressed as fold modulation vs. EVs from control mice (i.e. sham-operated for OVX and intact for HLTS), showed that the calcium-receptor $(+14.79, \mathrm{p}=0.038)$, the latenttgf $\beta$-binding-protein $(+4.17, \mathrm{p}=0.0037)$ and the collagenlal chain $(+98.81, \mathrm{p}=0.019)$ were the most upregulated mRNAs in OVX mice at 5, 7 and 10 week-time-points, respectively, while vascular-endothelial-growth-factorA was the most upregulated mRNA in HLTS $(+6.69, \mathrm{p}=0.026)$ at 7 week-time-point. Tgf $\beta$ $(-118.06, \mathrm{p}=0.00042)$ was instead the most downregulated mRNA in OVX mouse EVs at 5 week-time-point, while carbonic-anhydrase 2 mRNA downregulation was shared by OVX (7 weeks, - 113.77, $\mathrm{p}=0.0042 ; 10$ weeks, $-171.25, \mathrm{p}=0.0048)$ and HLTS (10 weeks, $-1173.55, p=0.018$ ) mouse EVs. Flow cytometry analysis also showed a transient increase of the EV-shuttled RANKL protein at 7 week-time-point, declining thereafter to level comparable to basal condition, both in OVX (Area Under Curve $(\mathrm{AUC})=+3.55$-fold vs. 
sham, $\mathrm{p}=0.0238$, Welch's $\mathrm{F}$ test) and in HLTS (AUC $=+3.37$-fold vs. intact, $\mathrm{p}=0.0298$ ) mice. These results suggest that liquid EV biopsies could represent a new diagnostic tool, unveiling specific molecular signatures in diverse types of osteoporosis (study approved by ethical authorities).

\section{P043}

Magnesium substituted hydroxyapatite nanoparticles enhance bone regeneration: in-vitro using osteoblast cells and in-vivo in a zebrafish jaw bone regeneration model

\section{Deepak Kumar Khajuria ${ }^{1}$, Dana Gigi ${ }^{1}$, Dalia David-Niv ${ }^{1}$, David} Karasik

${ }^{1}$ The Musculoskeletal Genetics Laboratory, The Azrieli Faculty of Medicine, Bar-Ilan University, Safed, Israel

We investigated the osteogenic potential of magnesium-substituted hydroxyapatite (Mg-HA) nanoparticles on the MC3T3-E1 osteoblast cell functions and in a zebrafish (ZF) jaw bone regeneration (JBR) model. Mg-HA nanoparticles were prepared and characterized. Cell proliferation assay, alkaline phosphatase (ALP) and alizarin red staining were used to confirm the effect of $\mathrm{Mg}$-HA nanoparticles on osteoblast proliferation, differentiation and mineralization, respectively. Reverse transcriptase polymerase chain reaction (RT-PCR) was used to measure the expression of the osteogenic genes like runtrelated transcription factor 2 (RUNX2), ALP and osteocalcin in response to $\mathrm{Mg}$-HA nanoparticles. $\mathrm{Mg}$-HA $\left(20 \mu \mathrm{gLL}^{-1}\right)$ nanoparticles significantly increased the expression of the RUNX-2, ALP, and osteocalcin as compared to HA nanoparticles $(p<0.05)$. Further, we performed mandibular resection $(\mathrm{n}=120)$ and sham-operations $(\mathrm{n}=20)$ in fish 12 months of age. Two days post-surgery, ZF were divided into seven groups and treated with various doses of HA and Mg-HA (80, 40 and $20 \mathrm{mg} / \mathrm{kg}$, once daily) nanoparticles supplemented into a ZF feed for up to 35 days. After 14, 28, or 35 days of treatment period, we evaluated the bone volume and bone mineral density (BMD) by microcomputed tomography $(\mu \mathrm{CT})$. The in-vivo study results showed that treatment with $\mathrm{Mg}-\mathrm{HA} \quad(80 \mathrm{mg} / \mathrm{kg})$ nanoparticles significantly enhanced ZF lower jaw bone volume as compared to HA treatment $(p<0.05)$. Further, therapy with $\mathrm{Mg}-\mathrm{HA}$ nanoparticles significantly increased BMD of lower-upper jaw and whole body as compared to HA nanoparticles $(p<0.05)$. The results of this study showed that Mg-HA nanoparticles stimulated the osteogenic differentiation of cultured osteoblast cells, enhanced bone volume and BMD in ZF model. Taken together, the results of our study strongly suggest that $\mathrm{Mg}-\mathrm{HA}$ nanoparticles appear to be more effective as a skeletal regenerative nanomedicine as compared to HA nanoparticles.

\section{P045}

Long noncoding RNAs: a new dimension in regulation of bone formation osteoblast differentiation from mesenchymal stromal cells

Coralee Tye ${ }^{1}$, Jonathan Gordon ${ }^{1}$, Kristiaan Finstadt ${ }^{1}$, Roland Elling ${ }^{2}$, Katherine Fitzgerald ${ }^{2}$, Janet Stein ${ }^{1}$, Gary Stein ${ }^{1}$, Jane Lian ${ }^{1}$

${ }^{1}$ Biochemistry, University of Vermont Larner College of Medicine, Burlington, United States, ${ }^{2}$ Medicine, University of Massachusetts Medical School, Worcester, United States

Long non-coding RNAs(lncRNAs) are critical for organ development, sex determination and lineage commitment-by regulating transcription, mediating chromatin interactions, RNA splicing, as miRNA sponges, and contributing to disease. Studies have associated lncRNAs affecting MSCs and osteoblasts by inhibiting miRNA, but in vivo functional characterization of lncRNAs is limiting for all tissues. We identified lncRNAs associated with specific stages of osteoblastogenesis, by global RNA-Seq using human and mouse primary mesenchymal stromal cells (hMSC; mMSC) during MSC differentiation (days 0-proliferation, 7-commitment, 14-ECM, 21-mineralization). During differentiation over 2000 annotated lncRNAs (mouse) and 5000 in human were identified. Only $22 \%$ of mouse lncRNA genes had conserved expression with human. In mouse 455 and 595 human IncRNAs were differentially expressed (DE $>$ twofold, FDR adjusted $\mathrm{p}<0.05$ ). We performed integrative analysis using lncRNA profiles and genome-wide binding of 4 histone modifications associated with transcription, activation (H3K4me3, $\mathrm{H} 3 \mathrm{~K} 27 \mathrm{ac}, \mathrm{H} 3 \mathrm{~K} 36 \mathrm{me} 3)$ and repression (H3K27me3) to determine regulatory modules influencing MSC commitment and differentiation at lncRNA genomic locations. Further intersection of lncRNAs with Runx 2 ChIP-Seq profiles revealed $>40 \%$ of lncRNAs genomic loci were bound by Runx2, thereby defining osteogenic-specific transcriptional regulation of lncRNA expression. Given the crosstalk between osteoblasts and macrophages, we selected the available $\mathrm{KO}$ mouse model lincRNA-Cox2, a broad-acting regulator of the inflammatory response, to characterize consequences of lincRNACox 2 depletion on bone homeostasis, where linc-Cox2 is proximal to the prostaglandin synthase gene, Pgts. Female KO mice exhibit a 2.5-3.7 fold increase in trabecular bone (TB) formation by 2 month and sustained at 6 month compared to WT bone (ScancoCT analyses, $\mathrm{n}=12 \mathrm{KO}$ and WT, $4 \mathrm{HET} /$ group) with no effect on cortical bone. The phenotype is caused primarily due to a loss of functional macrophages in bone marrow. These findings open novel dimensions for exploring lncRNAs in regulation of skeletal disorders.

\section{P047}

Pre-proenkephalin 1 is a new mechanoresponding gene involved in osteoblast differentiation

Nadia Rucci ${ }^{1}$, Antonio Maurizi ${ }^{1}$, Isabella Baldini ${ }^{1}$, Mattia Capulli ${ }^{1}$, Anna Teti ${ }^{1}$

${ }^{1}$ University of L'Aquila, L'Aquila, Italy

Pre-proenkephalin 1 (Penk1) belongs to the family of the typical opioid peptides. Penk1 gene encodes for a pro-peptide whose cleavage leads to 4 copies of met-enkephalin, 2 copies of met-enkephalin extended sequences and 1 copy of leu-enkephalin, which are classically known as opioid neuropeptides involved in the response to pain. Interestingly, we found Penk1 to be the most down-regulated gene in a microarray analysis performed in osteoblasts subjected to microgravity $(0.008 \times g)$ as a model of in-vitro mechanical unloading. Penk1 mRNA downregulation was confirmed in the bones of two invivo models of mechanical unloading: tail suspended mice $(-30 \%$, $\mathrm{p}=0.023)$ and mice injected with botulin toxin A $(-40 \%$, $\mathrm{p}=0.057$ ) (experiments approved by local ethical committee). Consistently, in human sera from healthy volunteers subjected to bed rest, we observed an inverse correlation between PENK1 levels and duration of bed rest (Pearson correlation test, $\mathrm{p}=0.0006, \mathrm{R}=0.6$ ). These results prompted us to investigate a role for PENK1 in bone metabolism. Interestingly, we found Penkl highly expressed in the marrow flushed-out bone, and its expression progressively increased in-vitro during osteoblast differentiation in osteogenic medium. Accordingly, osteoblast treatment with PP1, a c-Src inhibitor that stimulates osteoblast differentiation, increased Penk1 mRNA (1.8fold, $p=0.03$ ). Mouse primary osteoblasts expressed all the known opioid receptors (OPRM1, OPRD1, OPRK1 OGFR1), thus suggesting a paracrine/autocrine osteogenic role of PENK1. Silencing osteoblast Penkl by siRNAs decreased Alp $(-37 \%, \mathrm{p}=0.018)$ and Runx 2 $(-40 \%, \mathrm{p}=0.019)$ mRNAs, while it increased Sclerostin (7.5-fold, $\mathrm{p}=0.035)$ compared to osteoblasts treated with scrambled siRNA. Consistently, overexpression of Penkl by Amaxa nucleofection 
increased osteocalcin expression (sixfold, $\mathrm{p}=0.02$ ). A similar result was obtained in osteoblast cultures treated with met-enkephalin (fourfold, $\mathrm{p}=0.03$ ), while leu-enkephalin showed no effect. Taken together, these results highlight a role for PENK1 in osteoblast differentiation and in the regulation of the response of the bone to mechanical unloading.

\section{P049}

Novel role of Syndecan-3 (Sdc3) in maintaining bone mass

Francesca Brito $^{1}$, Addolorata Pisconti ${ }^{2}$, Gemma Charlesworth $^{1}$, Amanda Prior', George Bou-Gharios ${ }^{1}$, Rob van 't Hof ${ }^{1}$, Anna Daroszewska $^{1}$

${ }^{1}$ University of Liverpool/IACD, Liverpool, United Kingdom, ${ }^{2}$ University of Liverpool/IIB, Liverpool, United Kingdom

Syndecan-3 (Sdc3), a transmembrane heparan sulphate proteoglycan receptor, is highly expressed in periosteal osteoblasts during skeletal development, but its role in the adult skeleton is unknown. Sdc3 knock-out (Sdc3KO) mice have normal musculature and are resistant to diet-induced obesity. Here we analysed the role of Sdc3 in the adult skeleton.

Long bones of 3-month-old Sdc3KO (N = 12) and wild type (WT, $\mathrm{N}=13$ ) mice were analysed by $\mu \mathrm{CT}$ and dynamic histomorphometry. Osteoblasts were grown out of bone chips for mineralisation and gene expression studies.

At 3 months there was a decrease in bone volume (BV/TV, $\mathrm{p}<0.01)$, trabecular thickness $(\mathrm{Tb} . \mathrm{Th})$ and trabecular number $(\mathrm{Tb} . \mathrm{N})$ of 34,13 and $25 \%$ respectively in Sdc $3 \mathrm{KO}$ vs. WT $(\mathrm{p}<0.001)$. Cortical thickness was decreased in Sdc3KO vs. WT mice by $9 \%$ ( $p<0.001$ ). Three-point bending of the femur showed a 27 and $40 \%$ decrease in maximum force and work to fracture in the Sdc3KO $(\mathrm{p}<0.001)$. Histomorphometry revealed decreases of $50 \%$ in resorption surface, $40 \%$ in mineralising surface and $45 \%$ in bone formation rate $(\mathrm{p}<0.05)$ in $\mathrm{Sdc} 3 \mathrm{KO}$. There was a 60 -fold increase in bone marrow adipocyte number and area in Sdc3KO $(\mathrm{p}<0.001)$.

Alizarin red staining showed a reduction in mineralization in Sdc3KO osteoblast cultures $(\mathrm{N}=3)$ and fat cells were observed in contrast to WT. Osteoblast differentiation markers: Collal, Osteonectin, Runx2, Osteocalcin showed a 4x, 2x, 2x and 10x decrease respectively in Sdc3KO by qPCR. Smad6 expression was $2 \mathrm{x}$ higher suggesting inhibition of BMP signalling.

Our results suggest that the age-related low bone mass phenotype in $\mathrm{Sdc} 3 \mathrm{KO}$ mice is due to reduced bone formation, likely due to impaired differentiation and/or function of osteoblast-lineage cells and increased adipogenesis. These data provide evidence for the novel role of $\mathrm{Sdc} 3$ in regulating bone homeostasis, volume and marrow adiposity.

Keywords: Syndecan-3, osteoblasts, adipocytes, mineralisation, bone formation

\section{P051}

Rcor2 is a novel regulator of osteoblast differentiation and bone mass

Petri Rummukainen $^{1}$, Kati Tarkkonen ${ }^{1}$, Rana Al Majidi ${ }^{1}$, Lauri Saastamoinen $^{1}$, Amel Dudakovic ${ }^{2}$, Cristina Valensisi ${ }^{3}$, David Hawkins $^{3,4}$, Andre van Wijnen ${ }^{2}$, Riku Kiviranta ${ }^{1,5}$

${ }^{1}$ Institute of Biomedicine, University of Turku, Turku, Finland, ${ }^{2}$ Department of Orthopedic Surgery, Mayo Clinic, Rochester, United States, ${ }^{3}$ Division of Medical Genetics, University of Washington Seattle, Seattle, United States, ${ }^{4}$ Turku Centre for Biotechnology, Turku, Finland, ${ }^{5}$ Division of Endocrinology, Turku University Hospital, Turku, Finland
Despite the recent advances in understanding the complexity of epigenetic regulation during cell differentiation, epigenetic control of osteoblast maturation is still incompletely characterized. We identified Rcor2 as a significantly upregulated gene during MC3T3-E1 differentiation in our RNA-seq data. RCOR2 belongs to the CoREST/ RCOR family of proteins that regulate the action of histone lysinespecific demethylase 1 (Kdm1a/LSD1). ShRNA-mediated knockdown of Rcor2 in MC3T3-E1 cells and primary calvarial osteoblasts led to impaired osteoblastic differentiation demonstrated by decreased alkaline phosphatase and Von Kossa staining, and by differential gene expression profile in RNA-seq and quantitative PCR analyses. To assess the role of RCOR2 in bone formation in vivo, we generated global Rcor2KO and limb bud mesenchyme targeted conditional Rcor2cKO-Prrx1 knockout mouse models. Homozygous global Rcor2 deletion was embryonically lethal, whereas no bone phenotype was observed in heterozygous Rcor $2 \mathrm{KO}^{+/-}$mice by microcomputed tomography $(\mu \mathrm{CT})$. However, histomorphometric analysis showed a significant decrease $(40 \%)$ in BV/TV $(\mathrm{p}=0.028)$ in 12 week old Rcor $2 \mathrm{KO}^{+/-}$male tibias. Accordingly, we found a mild osteopenic phenotype in $\mu \mathrm{CT}$ analysis of Rcor2cKO-Prrx 1 mice examined at 6 , 12 and 26 weeks of age. At 12 weeks, female Rcor2cKO-Prrx 1 mice had $7 \%$ decreased tibial trabecular thickness $(p=0.010)$ and in 26 week old male mice the femur cortical bone volume was decreased by $13 \%(\mathrm{p}=0.002)$, accompanied by a trend towards decreased trabecular thickness $(-7 \%, \mathrm{p}=0.060)$. Whether this mild bone phenotype in Rcor2cKO-Prrx 1 mice would become more pronounced under challenged conditions such as ovariectomy, is under analyses. In conclusion, our study shows that Rcor2 is a novel gene highly expressed in mature osteoblast that is required for normal osteoblast differentiation in vitro. However, it is mostly dispensable for adult bone homeostasis in vivo, likely due to compensation by other members of the RCOR family.

Keywords: osteoblast, epigenetics, differentiation

\section{P053}

Fracture callus is responsive and adapts to individualized cyclic mechanical loading shown by time-lapsed in vivo imaging

Esther Wehrle ${ }^{1}$, Graeme R. Paul ${ }^{1}$, Duncan C. Betts ${ }^{1}$, Gisela A. Kuhn ${ }^{1}$, Ralph Müller ${ }^{1}$

${ }^{1}$ Institute for Biomechanics, ETH Zurich, Zurich, Switzerland

Objective: Mechanical stimulation is crucial for fracture healing. To understand the underlying mechanisms refined in vivo loading models are needed. Here, we combine state-of-the-art time-lapsed in vivo imaging with individualized mechanical loading protocols adapted to animal-specific callus properties.

Methods: Female 20-week-old mice $(\mathrm{n}=20)$ received a femur osteotomy and were scanned weekly (week 0-7; vivaCT40, ScancoMedical). Scans were registered consecutively and morphometric indices computed (thresholds: $395+645 \mathrm{mg} \mathrm{HA} / \mathrm{cm}^{3}$ ). At end of study, 3-point-bending was performed ( $\mathrm{n}=2$ /group). From week 4-7, individualized cyclic loading (8-16 N, $10 \mathrm{~Hz}, 3000$ cycles; 3x/week) was applied based on computed strain distribution in the callus using animal-specific finite element analysis. Controls received $0 \mathrm{~N}$ loading. Statistics: 2-way-ANOVA $(p<0.05)$.

Results: Both groups showed physiological healing from week 1-3 (Fig. 1). Loading maintained bone formation at significantly higher levels compared to controls, while bone resorption was significantly reduced (week 4-5). This led to linear increase in osseous callus volume from week $3-5(+43 \%)$, whereas in controls it significantly declined $(-27 \%)$. After 6 weeks, no significant differences in bone turnover were seen anymore indicating load adaptation. Nevertheless, loaded animals had $151 \%$ more bone in the defect with a significantly 
larger fraction of highly mineralized bone $\left(\mathrm{BV}_{645 / 395}\right.$ week 7: $91 \pm 2$ vs. $84 \pm 2 \%)$ and higher bending stiffness $(+34 \%)$.

Conclusions: This novel loading model combined with timelapsed in vivo micro-CT showed the remodeling phase to be highly responsive to animal-specific cyclic mechanical loading with significantly improved callus properties. Complementing this study with local cell-type-specific gene and protein expression analyses will enable to understand the mechano-molecular regulation of fracture healing.

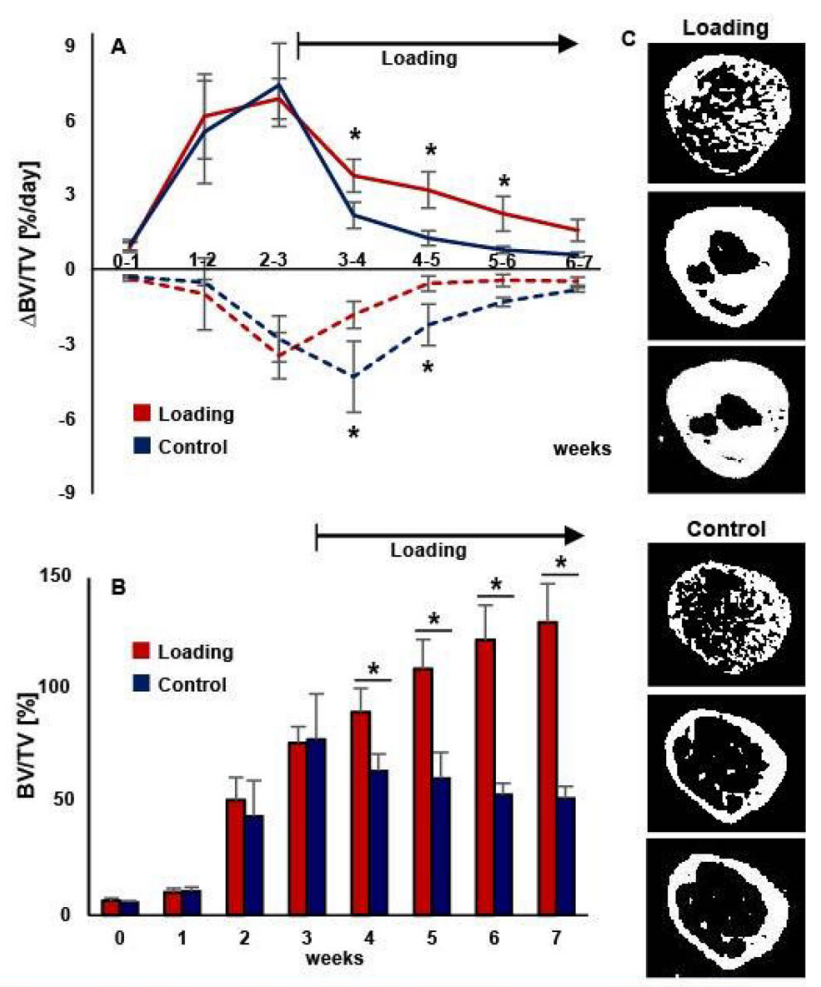

Figure 1. A: Formed, resorbed (solid, dashed line) bone volume (BV) in defect (TV). B: Bone volume (BV) normalized to defect volume (TV). C: Callus cross-sections of the loading, control group: week 3 (top), 5 (middle) and 7 (bottom). ${ }^{*}, p<0.05$.

Callus parameters assessed by time-lapsed in vivo imaging

\section{P055}

GDF11 locally determines axial skeletal patterning and systemically enhances bone formation

Joonho Suh ${ }^{1}$, Je-Hyun Eom ${ }^{1}$, Na-Kyung Kim ${ }^{1}$, Joo-Cheol Park ${ }^{2}$, Kyung-Mi Woo ${ }^{1}$, Jeong-Hwa Baek ${ }^{1}$, Hyun-Mo Ryoo ${ }^{1}$, Se-Jin Lee ${ }^{3}$, Yun-Sil Lee $^{1}$

${ }^{1}$ Department of Molecular Genetics \& Dental Pharmacology, Seoul National University, Seoul, Republic of Korea, ${ }^{2}$ Department of Oral Histology-Developmental Biology, Seoul National University, Seoul, Republic of Korea, ${ }^{3}$ Department of Molecular Biology \& Genetics, Johns Hopkins University School of Medicine, Baltimore, United States

Growth differentiation factor 11 (GDF11) regulates anterior-posterior (A-P) patterning of axial skeleton during development. Deletion of Gdf11 in mice leads to anterior homeotic transformations of vertebrae and cleft palate. Because $G d f 11$ is predominantly expressed in the tail bud around E9.5, it has been believed that GDF11 acts as a morphogen originating from the tail bud, to regulate A-P skeletal patterning. However, our findings show that locally expressed GDF11 determines positional fate of axial skeleton. In detail, we used a $C d x 2$ Cre transgene to remove $G d f 11$ expression in posterior regions below the umbilicus, which was confirmed by whole-mount in situ hybridization. Skeletal staining and micro-CT analysis revealed that both $C d x 2-C r e$; Gdf1 flox/flox and $G d f 11^{-1-}$ mice display 9 lumbar vertebrae, but $C d x 2-C r e$; Gdf11 $1^{\text {flox/flox }}$ mice show normal patterning phenotype above the umbilicus, including 7 vertebrosternal ribs and intact palate. Interestingly, we also found that GDF11 systemically increases bone formation. Micro-CT analysis of $\mathrm{P} 0$ mice revealed that bone volume (BV) and bone mineral density (BMD) are significantly diminished in $G d f 11^{-1-}$ mice $(P<0.001$ for both BV \& BMD), and mildly but significantly reduced in $\mathrm{Cd} x 2-\mathrm{Cr} e ; \mathrm{Gdfl} 1^{\text {flox/flox }}$ mice $(P<0.001$ for both BV \& BMD) compared with $w t$ mice, which contrast with increased bone formation in mice lacking myostatin (MSTN), a GDF11-homologous protein. Moreover, Micro-CT and DXA analysis showed that time-specific deletion of $G d f 11$ hinders bone formation in both E18.5 and 6-week old CAG-Cre-ER; $G d f 11^{f l o x / f l o x}$ mice. Finally, in vitro mineralization and resorption analysis demonstrated that osteoblast differentiation is inhibited, and osteoclastogenesis is enhanced in $G d f 11^{-1-}$ mice, while the opposite is observed in $\mathrm{Mstn}^{-1-}$ mice. All of our data strongly suggest that GDF11 locally regulates A-P patterning of axial skeleton, and systemically enhances bone formation as opposed to MSTN.

All animal experiments were approved by the Institutional Animal Care and Use Committee.

\section{P057}

Fam73b is essential for skeletal growth and the maintenance of bone mass and strength

Davide Komla-Ebri ${ }^{1}$, Apostolos Gogakos ${ }^{1}$, Penny Sparkes ${ }^{1}$, John G $\operatorname{Logan}^{1}$, Sanger Institute Mouse Pipelines ${ }^{2}$, Peter I Croucher ${ }^{3}, \mathrm{~J}$ H Duncan Bassett ${ }^{1}$, Graham R Williams ${ }^{1}$

${ }^{1}$ Molecular Endocrinology Laboratory, Imperial College London, London, United Kingdom, ${ }^{2}$ Wellcome Trust Sanger Institute, Cambridge, United Kingdom, ${ }^{3}$ Bone Biology Division, Garvan Institute of Medical Research, Sydney, Australia

Fam $73 b$ encodes mitoguardin 2 (Miga2), a protein that regulates mitochondrial fusion, morphology and function. We performed rapidthroughput skeletal phenotype analysis of knockout mice generated by the International Knockout Mouse Consortium at the Wellcome Trust Sanger Institute and identified $F a m 73 b$ as a novel genetic determinant of bone mass and strength. Sixteen-week-old female Fam $73 b^{-1-}$ knockout mice had weak and flexible bone with low mineral content, features that characterise the skeletal abnormalities in osteoporosis.

To determine the role of Miga2 in skeletal development and bone maintenance, we performed detailed phenotyping of $F a m 73 b^{-/-}$ mice. X-ray microradiography and micro-computed tomography demonstrated short stature throughout growth and in adulthood, decreased bone mineral content at post-natal days P14 $(-11.17 \%$ $\mathrm{p}<0.001 \mathrm{n}=15)$, P28 $(-8.66 \% \mathrm{p}<0.001 \mathrm{n}=14)$, P70 (females: $-3.05 \% \mathrm{p}<0.001 \mathrm{n}=11$; males: $-3.21 \% \mathrm{p}<0.01 \mathrm{n}=15)$ and P183 (females: $-4.15 \% \quad \mathrm{p}<0.001 \quad \mathrm{n}=10$; males: $-3.43 \%$ $\mathrm{p}<0.01 \mathrm{n}=12$ ), and decreased cortical bone thickness at P70 (females: $-5.91 \% \mathrm{p}<0.05 \mathrm{n}=11$; males: $-6.03 \% \mathrm{p}<0.05 \mathrm{n}=15$ ) and P183 (females: $-6.56 \% \mathrm{p}<0.01 \mathrm{n}=10$; males: $-4.22 \%$ $\mathrm{p}<0.05 \mathrm{n}=12)$ in Fam $73 b^{-1-}$ mice compared to wild-type. Threepoint bend biomechanical testing demonstrated that adult P183 Fam $73 b^{-1-}$ mice have markedly decreased bone strength and 
stiffness. Histomorphometric analysis of the tibial growth plate at P14 and tartrate-resistant acid phosphatase staining of tibial sections at P70 demonstrated no differences between $\mathrm{Fam}_{3} 3 \mathrm{~b}^{-1-}$ and wild-type mice, suggesting their abnormal phenotype may be due to defective osteoblasts rather than anomalies of chondrocyte or osteoclast function. Primary culture studies are underway to investigate further and preliminary data indicate that $\mathrm{Fam} 73 b^{-1-}$ osteoblasts have impaired proliferation compared to wild-type cells.

These data demonstrate Fam73b is necessary for skeletal growth and normal bone structure and strength, and suggest that a novel pathway involving mitochondrial fusion and function in osteoblasts is required for the establishment and maintenance of bone mass.

\section{P059}

$N$-acetylcysteine limits vascular calcification but preserves bone formation

Lucie E Bourne $^{1}$, Jessal J Patel ${ }^{1,2}$, Ellen Neven ${ }^{3}$, Patrick D’Haese ${ }^{3}$, Caroline Wheeler-Jones ${ }^{1}$, Isabel R Orriss ${ }^{1}$

${ }^{1}$ Comparative Biomedical Sciences, Royal Veterinary College, London, United Kingdom, ${ }^{2}$ School of Life and Medical Sciences, University of Hertfordshire, Hatfield, United Kingdom, ${ }^{3}$ Laboratory of Pathophysiology, Department of Biomedical Sciences, University of Antwerp, Antwerp, Belgium

Vascular calcification (VC), a significant risk factor for future cardiovascular events, involves hydroxyapatite deposition in the arteries. Historically believed to share similarities with bone mineralisation, emerging evidence suggests there are several key differences between these two processes. For example, antioxidants inhibit VC but promote bone formation. $\mathrm{N}$-acetylcysteine (NAC) displays direct and indirect antioxidant properties. This study investigated the effects of NAC on VC and bone mineralisation in vitro and in vivo. Human umbilical artery-derived vascular smooth muscle cells (VSMCs) and mouse osteoblasts were cultured in mineralising medium $(1 \mathrm{mM} \beta$-glycerophosphate, $1 \mathrm{mM}$ sodium phosphate, $50 \mu \mathrm{g} / \mathrm{ml}$ ascorbate) and treated with NAC $(0.5-5 \mathrm{mM})$ for up to 7 and 21 days, respectively. The effect of NAC on cell function, survival and gene expression was investigated using established assays. NAC potently increased in vitro bone formation $(\leq 10$-fold, $\mathrm{p}<0.001)$ but reduced VSMC calcification $(\leq 80 \%, p<0.001)$. NAC increased osteoblast TNAP activity, soluble collagen levels and gene expression (osteocalcin, osteopontin $) \leq$ threefold $(\mathrm{p}<0.001)$. Cell number and viability were unaffected. Conversely, NAC had no effect on VSMC TNAP activity but reduced VSMC apoptosis by $\leq 40 \%(\mathrm{p}<0.001)$. $\mathrm{VC}$ is associated with decreased expression of VSMC-associated genes ( $\alpha$-SMA, SMemb, SM22 $\alpha$ ). NAC prevented this reduction in calcifying VSMCs by $\leq$ threefold. NAC was administered daily $(300 \mathrm{mg} / \mathrm{Kg}$ ) to Wistar rats fed a warfarin/vitamin $\mathrm{K} 1$ enriched diet for 10 weeks $(n=9-10)$. VC and trabecular/cortical bone parameters were assessed using $\mu \mathrm{CT}$. Treatment with NAC decreased aortic calcification by up to $25 \%$ ( $\mathrm{p}<0.05$ ). Trabecular and cortical bone parameters were unaffected. However, a negative correlation between the level of aortic calcification and trabecular bone volume $(\mathrm{p}=0.0237)$ was observed. Taken together, our findings suggest that NAC exerts opposing effects on $\mathrm{VC}$ and bone mineralisation and could represent a potential therapy for $\mathrm{VC}$ without negatively affecting the skeleton. Current work investigates the mechanistic basis for these effects.

\section{P061}

Altered MicroRNA profile in osteoporosis caused by impaired WNT signaling
Riikka Mäkitie ${ }^{1}$, Matthias Hackl ${ }^{2}$, Riitta Niinimäki ${ }^{3}$, Sakari Kakko ${ }^{4}$, Johannes Grillari ${ }^{5}$, Outi Mäkitie ${ }^{1,6,7}$

${ }^{l}$ Folkhälsan Institute of Genetics and University of Helsinki, Helsinki, Finland, ${ }^{2}$ TAmiRNA GmbH, Vienna, Austria, ${ }^{3}$ Department of Children and Adolescents, Oulu University Hospital, and PEDEGO Research Unit, University of Oulu, Oulu, Finland, ${ }^{4}$ Internal Medicine and Clinical Research Center, University of Oulu, Oulu, Finland, ${ }^{5}$ Christian Doppler Laboratory on Biotechnology of Skin Aging, Department of Biotechnology, BOKU - University of Natural Resources and Life Sciences Vienna, Vienna, Austria, ${ }^{6}$ Children's Hospital, University of Helsinki and Helsinki University Hospital, Helsinki, Finland, ${ }^{7}$ Center for Molecular Medicine, Karolinska Institutet, and Clinical Genetics, Karolinska University Hospital, Stockholm, Sweden

Objective: A heterozygous missense mutation p.C218G in WNT1, a key WNT pathway ligand, leads to severe early-onset and progressive osteoporosis with multiple peripheral and spinal fractures. Despite the severe skeletal manifestations, conventional bone turnover markers are normal in mutation-positive patients. This study sought to explore the circulating miRNA pattern in patients with impaired WNT signaling.

Subjects and Methods: A cross-sectional cohort study on altogether 12 mutation-positive (median age 39 years, range 11-76 years) and 12 mutation-negative (35 years, range 9-59 years) subjects from two Finnish families with WNT1 osteoporosis due to the heterozygous p.C218G WNT1 mutation. We screened their serum samples with a custom-designed panel comprising 192 miRNAs using qPCR. Findings were compared between WNT1 mutation-positive and mutation-negative subjects. Sample quality was tested with spike-in controls. All studies were approved by the Research Ethics Board of Helsinki University Hospital.

Results: The pattern of circulating miRNAs was significantly different in the mutation-positive subjects as compared with the mutation-negative subjects. Of the 192 miRNAs, two were upregulated (miR-18a-3p, miR-223-3p; p values 0.042 and 0.043 , respectively) and six downregulated (miR-22-3p, miR-31-5p, miR34a-5p, miR-143-5p miR-423-5p, miR423-3p; p values 0.001, 0.053, $0.003,0.045,0.03,0.042$, respectively) in the WNT1 mutation-positive subjects. Three of these (miR-22-3p, miR-34a-5p, and miR-31$5 p)$ are known inhibitors of WNT signaling: miR-22-3p and miR-34a$5 \mathrm{p}$ target WNT1 mRNA and miR-31-5p is predicted to bind to WNT1 $3^{\prime}$ UTR. No correlation was found between subfamily division, age, or gender.

Conclusions: A unique miRNA profile reflects WNT1 mutation status. The findings suggest that a feed-back regulation between these miRNAs and WNT1 is disrupted due to the WNT1 mutation. These provide a novel finding of direct association between miR-31-5p and WNT1 and new insights into the pathogenesis of WNT-related bone disorders. These miRNAs could offer future potential in diagnosis and treatment of osteoporosis.

Keywords: WNT signaling, microRNAs, osteoporosis

\section{P063}

Slc38a10 is a novel genetic determinant of osteoblast proliferation and bone mineral density

Andrea Pollard ${ }^{1}$, Apostolos Gogakos ${ }^{1}$, John G Logan ${ }^{1}$, Davide Komla Ebri $^{1}$, Penny Sparkes ${ }^{1}$, Natalie C Butterfield ${ }^{1}$, Victoria D Leitch ${ }^{1}$, Sanger Mouse Pipelines ${ }^{2}$, Peter I Croucher ${ }^{3}$, JH Duncan Bassett ${ }^{1}$, Graham R Williams ${ }^{1}$

${ }^{1}$ Molecular Endocrinology Laboratory, Imperial College London, London, United Kingdom, ${ }^{2}$ Wellcome Trust Sanger Institute, Hinxton, 
United Kingdom, ${ }^{3}$ Bone Biology Division, Garvan Institute of Medical Research, Sydney, Australia

Low bone mineral density (BMD) is a key risk factor for osteoporosis and fracture, resulting in major societal and financial burdens to health services. Although BMD is highly heritable, over $85 \%$ of the phenotype variance remains unexplained. We hypothesised that highthroughput skeletal phenotyping of knockout mice generated by the International Knockout Mouse Consortium would identify new genetic determinants of bone structure and strength. Sixteen week-old female sodium-coupled neutral amino acid transporter 10 (Sl$c 38 a 10^{-1-}$ ) knockout mice were identified to have decreased body weight, bone length and BMD.

Comprehensive phenotyping of juvenile and adult Slc38a10 ${ }^{-1-}$ mice ( $\mathrm{n}=5$ per sex, per genotype) using X-ray microradiography and micro-computed tomography demonstrated short stature throughout postnatal growth and in adults $(\mathrm{P}<0.05$, ANOVA) with decreased bone mineral content $(\mathrm{P}<0.001$, Kolmogorov-Smirnov test), and reduced cortical $(\mathrm{P}<0.001$, ANOVA) and trabecular bone thickness $(\mathrm{P}<0.001$, ANOVA). Despite the clear deficit in skeletal growth, histology of Slc38a10 $0^{-1-}$ epiphyseal growth plates was normal. Histomorphometry revealed no difference in osteoclast numbers, but Slc38a10 ${ }^{-1-}$ mice had profound decreases in mineral apposition (40\%, $\mathrm{P}<0.05, \mathrm{t}$-test) and bone formation $(60 \%, \mathrm{P}<0.05$, t-test $)$ rates compared to wild-type mice. Slc 38 a $10^{-1-}$ primary osteoblasts had decreased proliferation $(\mathrm{P}<0.05$, t-test) compared to wild-type but produced extracellular matrix and mineralised normally once confluent.

Taken together, these data suggest that impaired skeletal growth and low BMD in Slc38a10 ${ }^{-1-}$ mice result primarily from an osteoblast defect. Slc38a10 is a sodium-coupled glutamine and aspartate transporter which has been implicated in amino acid sensing and transport and the regulation of mTORC1. Thus, Slc38a10 may contribute to a mechanism by which skeletal growth and mineralisation are regulated in response to nutrient availability. Identification of the molecular mechanisms underlying this process may identify new drug targets involved in the maintenance of BMD and bone strength.

Keywords: osteoblast, bone mineral density

\section{Oral Posters 2-Preclinical/Clinical}

\section{P065}

Organic matrix quality at actively forming trabecular surfaces is strongly associated with fragility fracture incidence independent of BMD and the clinical diagnosis

Eleftherios Paschalis ${ }^{1}$, Sonja Gamsjaeger ${ }^{1}$, Erik Eriksen ${ }^{2}$, Francis Glorieux $^{3}$, Frank Rauch ${ }^{3}$, David Dempster ${ }^{4}$, Hua Zhou ${ }^{4}$, Elizabeth Shane $^{4}$, Adi Cohen ${ }^{4}$, Rober Recker ${ }^{5}$, John Bilezikian ${ }^{4}$, Mishaela Rubin $^{4}$, Carolina Moreira ${ }^{6}$, Joseph Lane ${ }^{7}$, Imre Pavo ${ }^{8}$, Jan Stepan ${ }^{9}$, Socrates Papapoulos ${ }^{10}$, Wolfgang Brozek ${ }^{1}$, Peter Fratzl ${ }^{11}$, Klaus Klaushofer ${ }^{1}$

${ }^{1}$ Ludwig Boltzmann Institute of Osteology at the Hanusch Hospital of WGKK, and AUVA Trauma Center Meidling, 1st Medical Department Hanusch Hospital, Vienna, Austria, ${ }^{2}$ Oslo University Hospital and Institute of Clinical Medicine, University of Oslo, Oslo, Norway, ${ }^{3}$ Genetics Unit, Shriners Hospital for Children and McGill University, Montreal, Canada, ${ }^{4}$ Medicine and Pathology, College of Physicians and Surgeons of Columbia University, New York, United States, ${ }^{5}$ Creighton University School of Medicine, Omaha, United States, ${ }^{6} 5$ Endocrinology Division (SEMPR), Federal University of
Parana, Curitiba, Brazil, ${ }^{7}$ Orthopedic Surgery, Weill Medical College, Cornell University, New York, United States, ${ }^{8}$ Eli Lilly \& Co, Vienna, Austria, ${ }^{9}$ Institute of Rheumatology, and Charles University Faculty of Medicine 1, Prague, Czech Republic, ${ }^{10}$ Center for Bone Quality, Leiden University Medical Center, Leiden, Netherlands, ${ }^{11}$ Max Planck Institute of Colloids and Interfaces, Potsdam, Germany

Purpose: The etiology of fragility fractures (low or no trauma fractures irrespective of BMD values) incidence remains poorly understood. In this study we hypothesized that organic matrix quality can identify patients with fragile bone, defined by fracture history, irrespective of the clinical diagnosis.

Methods: Raman microspectroscopy was utilized to compare iliac crest biopsies from: (a) 108 healthy females (H; ages 1-59); (b) 130 females with a history of fragility fractures (Fx; ages 2-84); (c) 33 women with altered BMD but no history of fragility fractures (No Fx; ages 23-74). The Fx group included children and women diagnosed with mild osteogenesis imperfecta, idiopathic osteoporosis, postmenopausal osteoporosis, and postmenopausal osteoporosis coupled with chronic obstructive pulmonary disease (COPD). The NoFx group included premenopausal women with low BMD values, COPD, and hypoparathyroid subjects. The parameters measured at actively forming cancellous bone surfaces (indicated by fluorescent double labels) were: mineral/matrix ratio (MM; affecting stiffness and brittleness), glycosaminoglycan (GAGs; known negative modulators of mineral homeostasis), and pyridinoline (pyd; trivalent enzymatic collagen cross-link, associated with fiber stiffness, ultimate stress and post-yield energy, bending strength, and post-yield strength).

Results: Individual patient data were considered. By ANCOVA, LS, FN, and TH BMD values discriminated between $\mathrm{H}$ and either Fx or NoFx groups, but not between Fx and NoFx. MM did not differ among the 3 groups. GAGs content discriminated between $\mathrm{H}$ vs. Fx $(p<0.0001)$ and Fx vs. NoFx $(p=0.016)$ groups. Finally, Pyd content clearly $(\mathrm{p}<0.0001)$ discriminated patients among the 3 groups.

Conclusions: The results of the present study strongly suggest that bone matrix material properties are altered in patients who sustain fragility fractures, and highlight the potential utility of organic matrix quality, and in particular Pyd content at actively forming trabecular surfaces, as a new risk factor for fracture.

\section{P068}

Zoledronate every 18 months for 6 years in osteopenic postmenopausal women reduces non-vertebral fractures and height loss

Ian Reid $^{1}$, Anne Horne ${ }^{1}$, Borislav Mihov ${ }^{1}$, Angela Stewart ${ }^{1}$, Liz $\overline{\text { Garratt }}^{1}$, Mark Bolland ${ }^{1}$, Sonja Bastin ${ }^{1}$, Greg Gamble ${ }^{1}$

${ }^{1}$ University of Auckland, Auckland, New Zealand

Bisphosphonates prevent fractures in patients with osteoporosis, but their efficacy in women with osteopenia is unknown. Most fractures in postmenopausal women occur in osteopenic individuals, so if pharmaceutical intervention is to impact significantly on total fracture numbers, therapies with efficacy in osteopenic postmenopausal women are needed.

We report a double-blind trial of 2000 osteopenic, postmenopausal women, randomly assigned to receive 4 infusions of either zoledronate $5 \mathrm{mg}$, or normal saline at 18 -month intervals. Each was followed for 6 years. Monthly vitamin D supplements were provided but not calcium supplementation. The study has $80 \%$ power to detect a decrease in osteoporotic fractures of $30 \%$. 
Women were recruited using electoral rolls. Inclusion criteria were age $>65$ years, hip $\mathrm{T}$-score between -1.0 and -2.5 . Exclusion criteria were: lumbar spine T-score $<-3.0$, eGFR $<30 \mathrm{~mL} / \mathrm{min}$, major systemic disease, metabolic bone disease, or regular use of bone-active drugs in the previous year. Proportional hazards models were used

At baseline, age was 71 (SD 5) y, BMI 27 (5), femoral neck T-score $-1.5(0.5)$, and $95 \%$ were white. These characteristics were comparable in the two groups.

Non-vertebral fractures (excluding skull, face, hands and feet) occurred in 148 women in the placebo group and in 101 in the zoledronate group (ITT analysis, HR 0.66 [95\% CI 0.51, 0.85], $\mathrm{P}=0.0014$, Figure; NNT $=22$ ). Height loss, a surrogate for vertebral fracture, was $9.3(8.7,9.9) \mathrm{mm}$ in the placebo group and $7.4(6.9,8.0)$ $\mathrm{mm}$ in the zoledronate group $(\mathrm{P}<0.0001)$. There were 41 deaths in the placebo group and 27 in the zoledronate group, HR 0.65 , $\mathrm{P}=0.085$. Results of vertebral morphometry will be available at the meeting.

These results suggest this less intensive zoledronate regimen is effective for fracture prevention in osteopenia, possibly substantially broadening the target population for pharmaceutical intervention to prevent fractures.

\section{P069}

Structural geometry of bones is prominently associated with risk of fracture in children

Olja Grgic $^{1}$, Katerina Trajanoska ${ }^{2}$, Denise Heppe ${ }^{3}$, Carola Zillikens ${ }^{4}$, Vincent Jaddoe $^{3}$, Andre Uitterlinden ${ }^{4}$, Eppo Wolvius ${ }^{5}$, Tom Beck ${ }^{6}$, Fernando Rivadeneira $^{7}$, Carolina Medina-Gomez ${ }^{8}$

${ }^{1}$ Department of Maxillo-facial Surgery, Department of Internal Medicine, The Generation $R$ Study, Erasmus MC, Rotterdam, Netherlands, ${ }^{2}$ Department of Internal Medicine, Department of Epidemiology, Erasmus MC, Rotterdam, Netherlands, ${ }^{3}$ The Generation $R$ Study, Erasmus MC, Rotterdam, Netherlands, ${ }^{4}$ Department of Internal Medicine, Erasmus MC, Rotterdam, Netherlands, ${ }^{5}$ Department of Maxillo-facial Surgery, The Generation R Study, Erasmus MC, Rotterdam, Netherlands, ${ }^{6}$ Beck Radiological Innovations Inc., Baltimore, United States, ${ }^{7}$ Department of Internal Medicine, The Generation $R$ Study, Erasmus MC, Rotterdam, Netherlands, ${ }^{8}$ Department of Internal Medicine, Department of Epidemiology, The Generation R Study, Erasmus MC, Rotterdam, Netherlands

Objective: Low total body BMD (TB-BMD) is an established risk factor for fractures in healthy children. Bone strength also depends on the structural geometry of bones. Hip structural analysis (HSA) is a technique applied on hip DXA scans to measure bone geometry parameters. The aim of our study was to examine the association between femoral structural parameters including the geometryderived femoral stress index (FSI) and risk of incident fractures in children.

Methods: We studied 1848 children from the Generation R study, with whole body and hip DXA scans (GE-Lunar iDXA) at a mean age of 6.0 years. Hip DXA scans underwent HSA with derivation of FSI. This stress index considers both, bending and axial forces acting on the femoral neck and is adjusted for lean mass fraction. Fractures at any skeletal site were assessed using questionnaire reports obtained before a mean age of 9.8 years. Risk (odds) of fracture was estimated from logistic regression models adjusted for sex, age, height, weight and ethnicity.

Results: Incident fracture was observed in 139 children (7.5\%). A significant increase in the odds of incident fracture was observed for every standard deviation (SD) decrease in TB-BMD (OR 1.36, 95\% CI $1.04-1.77, \mathrm{P}=0.02$ ), femoral neck BMD (OR 1.34, 95\% CI $1.10-1.63, \mathrm{P}=0.004)$ and narrow neck BMD (OR 1.42, 95\% CI
$1.16-1.75, \mathrm{P}=0.001)$. Every increment of one SD in the FSI resulted in $31 \%$ increased odds of incident fracture (OR 1.31, 95\% CI $1.12-1.53, \mathrm{P}=0.001)$. After inclusion of any of the BMD measurements in the regression model, FSI remained significantly associated with risk of incident fractures.

Conclusion: Femoral and total body BMD parameters are associated with incident fracture risk in children. The stress index, which considers quantity and the distribution of bone in the femoral region is the biomechanical assessment best capturing fracture propensity in children.

\section{P071}

Dickkopf-1 (Dkk1) plays distinct pathogenic roles in estrogendeficiency vs. glucocorticoid-induced bone loss

Juliane Colditz $^{1,2}$, Sylvia Thiele ${ }^{1,2}$, Ulrike Baschant ${ }^{1,2}$, Christof Niehrs $^{3}$, Martina Rauner ${ }^{1,2}$, Lorenz Hofbauer ${ }^{1,2,4}$

${ }^{1}$ Department of Medicine III, Technische Universität Dresden, Dresden, Germany, ${ }^{2}$ Center for Healthy Aging, Technische Universität Dresden, Dresden, Germany, ${ }^{3}$ Institute of Molecular Biology, Mainz, Germany, ${ }^{4}$ DFG Research Center and Cluster of Excellence for Regenerative Therapies, Technische Universität Dresden, Dresden, Germany

The Wnt inhibitor Dickkopf-1 (Dkk1) is dysregulated in various bone disease with distinct underlying causes. Here, we investigated the role of Dkk1 in postmenopausal osteoporosis, which is characterized by high bone turnover, and GC-induced osteoporosis, a model of suppressed bone formation, and dissected which cell type contributes to its actions.

Phenotyping of female and male mice deficient of Dkk1 in osteoprogenitors (Dkk1 ${ }^{\mathrm{f} / \mathrm{f}}$;Osx-Cre) and in osteocytes (Dkk1 ${ }^{\mathrm{f} / \mathrm{f}}$;Dmp1Cre) was performed at 12 weeks of age. To induce estrogen deficiency 9-week-old females were ovariectomized. To induce bone loss with GCs, slow-release pellets ( $7.5 \mathrm{mg}$ prednisolone) were subcutaneously implanted into 24-week-old females. After 4 weeks, bone parameters were assessed.

Female and male Dkk1;Osx-Cre mice and Dkk1;Dmp1-Cre mice showed a 2-3-fold increase in bone volume compared to respective Cre-negative controls, which mainly resulted from increased bone formation. Dkk1 serum levels in Dkk1;Osx-Cre mice were markedly lower $(-77 \%)$ compared to controls, whereas they were not changed in Dkk1;Dmp1-Cre mice suggesting that most systemic Dkk1 derives from osteoprogenitors. However, both strains had reduced skeletal Dkk1 expression.

While GC treatment reduced vertebral bone volume $(-27 /-25 \%)$ and the bone formation rate $(-52 /-57 \%)$ in Crenegative littermates, both Dkk1 knock-out strains were protected from GC-induced bone loss and reduction of bone formation. Interestingly, neither Dkk1 knock-out strain was protected from ovariectomy-induced bone loss. Bone volume was equally reduced in Dkk1;Osx-Cre $(-48 \%)$ and Dkk1;Dmp1-Cre $(-52 \%)$ mice, compared to controls (-44/-43\%). Similar to their wildtype littermates, both strains showed a $48-55 \%$ increase in the bone formation rate.

In summary, Dkk-1 derived from osteogenic cells plays a crucial role in osteoblast biology and in the pathogenesis of GC-induced bone loss. However, it does not contribute to bone loss resulting from estrogen deficiency. Thus, the differential role of Dkk1 in bone diseases proposes a selective use of Dkk1 inhibitors depending on the type of osteoporosis. 


\section{P073}

Predicting the risk of hip fracture from DXA-based 3D finite element simulations

Carlos Ruiz Wills ${ }^{1}$, Simone Tassani ${ }^{1}$, Miguel A. González Ballester $^{1,2}$, Ludovic Humbert ${ }^{3}$, Luis Del Río ${ }^{4}$, Jérôme Noailly ${ }^{1}$

${ }^{1}$ BCN Barcelona Centre for New Medical Technologies, Universidat Pompeu Fabra, Barcelona, Spain, ${ }^{2}$ Catalan Institution for Research and Advanced Studies, Barcelona, Spain, ${ }^{3}$ Galgo Medical, Barcelona, Spain, ${ }^{4}$ CETIR Medical Centre, Barcelona, Spain

Introduction: Osteoporotic hip fracture is a major health burden in elderliness. Osteoporosis is diagnosed and monitored with dual-energy X-ray absorptiometry (DXA), but risk fracture prediction based on DXA remains poor.

Objective: Improve osteoporotic fracture prediction in the proximal femur, by virtually augmenting DXA information with biomechanical descriptors from subject-specific 3D finite elements (FE) models and simulations.

Methods: Patients with osteoporosis were selected: 85 fracture and 71 non-fracture cases. Post-fracture X-rays of 77 patients allowed localising fracture lines. 3D Subject-specific femoral shapes and volumetric bone mineral density distributions were obtained from hip DXA scans using the 3D-SHAPER software, and FE models were created. Bone Young's moduli were calculated from vBMD distributions.

Lateral falls were simulated by approaching the femur to a fixed plate. Movement velocity depended on patient's mass and height. Highest mechanical field values defined critical regions that were examined to identify biomechanical parameters for fracture case discrimination through ROC-AUC analyses. Support vector machine $(\mathrm{SVM})$ with fivefolds training-test validation (global $\mathrm{p}<0.05$ ) were used to further assess the classification power of these parameters.

Results: Simulated stress fields and fracture line localizations matched in $96 \%$ of the 77 post-fracture X-rays. In the trabecular bone of the neck region, statistical analysis for women showed that major principal stresses (MPS) led to a ROC-AUC of 0.99. SVM analyses further confirmed that trabecular bone MPS were the best descriptors for classification in non-fracture and fracture groups (Accuracy $=1.00$ ). Results suggest that fracture might be predicted from virtually enhanced DXA data, using biomechanical simulations.

Conclusions: Fall simulations, using DXA-derived 3D femur models, identified fracture location and regions of interest, in which trabecular bone MPS were excellent discriminators of fracture and non-fracture cases. MPS also stood for promising classification parameters for fracture risk prediction in osteoporotic patients.

Acknowledgements: CETIR-ASCIRES (Densi3D), MINECO (BioDXA-RTC-2014-2740-1, RYC-2015-18888), Chair QUAESUPF

\section{P075}

Altered bone microarchitecture in CFTR-deficient newborn piglets

Julien Braux ${ }^{1}$, Marie-Laure Jourdain ${ }^{1}$, Ignacio Caballero-Posadas ${ }^{2}$, Nathalie Winter ${ }^{2}$, Mustapha Si-Tahar ${ }^{3}$, Nikolai Klymiuk ${ }^{4}$, Sophie C. Gangloff $^{1}$, Jacky Jacquot ${ }^{1}$, Frédéric Velard ${ }^{1}$

${ }^{1}$ EA 4691 BIOS, University of Reims Champagne-Ardenne, Reims, France, ${ }^{2}$ UMR1282 ISP, INRA, Nouzilly, France, ${ }^{3}$ Centre d'Etude des Pathologies Respiratoires, UMR1100, Inserm, Tours, France, ${ }^{4}$ Institute of Molecular Animal Breeding and Biotechnology, Gene Center, Ludwig-Maximilians-Universitat Munchen, Munchen, Germany

People with cystic fibrosis exhibit growth defects and brittle bones. That observation has been attributed, to malnutrition and chronic pulmonary inflammation. We tested the hypothesis that disruption of the cystic fibrosis transmembrane conductance regulator (Cftr) gene directly affects bone microarchitecture and integrity by studying bone of newborn $\mathrm{Cftr}^{-/-}$pigs.

We examined femoral cortical and trabecular bones of $\mathrm{Cftr}^{-1-}$ newborn pigs using micro computed tomography $(\mu \mathrm{CT}$, Skyscan 1076, Bruker). Three-dimensional images (voxel size $17 \times 17 \times 17 \mu \mathrm{m})$ were reconstructed and analyzed (NRecon and CTAn softwares), respectively. The cortical bone porosity and structure were defined using a $3.4 \mathrm{~mm}$ wide region centered on the middle of the femur. A total of 37 newborn $\mathrm{Cftr}^{-/-}$piglets (24 males and 13 females) and 18 newborn $\mathrm{Cftr}^{+/+}$piglets $(8$ males and 10 females) was subjected to $\mu \mathrm{CT}$-scan.

Compared to newborn $\mathrm{Cftr}^{+/+}$pig controls, $\mathrm{Cftr}^{-1-}$ femoral bone exhibited significantly $(\mathrm{p}<0.05)$ lower total volume $(\mathrm{TV})$, bone volume (BV) and bone volume density (BV/TV) but only in females. However, the $\mathrm{Cftr}^{-/-}$bone mineral density (BMD) in trabecular and cortical tissues was significantly reduced in both sexes, compared to $\mathrm{Cftr}^{+/+}$piglets. Interestingly, focusing at the porosity of cortical bone in $\mathrm{Cftr}^{-l-}$ pigs as a determinant of bone fragility associated with high fracture risk, we observed higher closed porosity with a marked increase of closed pore surface in cortical bone of $\mathrm{Cftr}^{-1-}$ pigs $(+18.7 \%$ for males and $+48 \%$ for females, $\mathrm{p}<0.05)$. These results suggest a lower bone remodelling, lower interconnectivity within the vascular network, and increased bone fragility in $\mathrm{Cftr}^{-/-}$animals. No significant difference was observed in the open cortical porosity, whatever the gender.

Altogether, these data highlight the critical regulatory role of CFTR in bone development and maintenance, and suggest that some bone defects in people with cystic fibrosis are likely primary.

Keywords: Cystic Fibrosis; Bone disease; Pig model

\section{P077}

FSH is positively associated with vertebral bone marrow adiposity in postmenopausal women from the AGES-Reykjavik cohort

Annegreet G. Veldhuis-Vlug ${ }^{1,2}$, Gina N. Woods ${ }^{3,4}$, Sigurdur Sigurdsson $^{5}$, Susan K. Ewing ${ }^{6}$, Phuong T. Le ${ }^{1}$, Trisha F. Hue ${ }^{6}$, Kaipin $\mathrm{Xu}^{7}$, Vilmundur Gudnason ${ }^{8}$, Gunnar Sigurdsson ${ }^{8}$, Deborah M. Kado $^{3,9}$, Gudny Eiriksdottir ${ }^{5}$, Tamara Harris ${ }^{10}$, Xiaojuan $\mathrm{Li}^{7}$, Clifford J. Rosen ${ }^{1}$, Ann V. Schwartz ${ }^{6}$

${ }^{1}$ Center for Clinical and Translational Research, Maine Medical Center Research Institute, Scarborough, United States, ${ }^{2}$ Endocrinology and Metabolism, Academic Medical Center Amsterdam, Amsterdam, Netherlands, ${ }^{3}$ Department of Medicine, University of Caifornia San Diego, La Jolla, United States, ${ }^{4}$ VA San Diego Healthcare System, San Diego, United States, ${ }^{5}$ Icelandic Heart Association Research Institute, Reykjavik, Iceland, ${ }^{6}$ Department of Epidemiology and Biostatistics, University of California San Francisco, San Francisco, United States, ${ }^{7}$ Program of Advanced Musculoskeletal Imaging, Cleveland Clinic, Cleveland, United States, ${ }^{8}$ Faculty of Medicine, Icelandic Heart Association University of Iceland, Reykjavik, Iceland, ${ }^{9}$ Department of Family Medicine and Public Health, University of California San Diego, La Jolla, United States, ${ }^{10}$ National Institute on Aging, National Institutes of Health, Bethesda, United States

Bone marrow adiposity increases with age, particularly in women around menopause when estradiol levels decrease and follicle stimulating hormone (FSH) levels increase. Increased bone marrow adiposity is associated with osteoporosis and vertebral fractures. Understanding the factors that regulate bone marrow adiposity in older adults may lead to novel mechanisms for combatting age-related bone loss. Postmenopausal hormonal replacement therapy, increasing estradiol and decreasing FSH levels, decreases bone marrow adiposity. A recent study in mice showed that blocking FSH signaling 
with an antibody also decreases bone marrow adiposity following ovariectomy. The association between FSH and bone marrow adiposity in humans has not been previously reported.

We hypothesized that in humans higher FSH levels are associated with greater bone marrow adiposity. To test this hypothesis, we performed a cross-sectional, observational study in the AGESReykjavik cohort. In 237 women and 245 men, we measured vertebral (L1-L4) bone marrow adipose tissue (BMAT, \%) by magnetic resonance spectroscopy and serum FSH concentration by ELISA.

In older women (mean age 80.6 years) with a mean FSH of $71.5 \mathrm{IU} / \mathrm{L}$ and a mean vertebral BMAT of $55 \%$, mean vertebral BMAT was $0.65 \%$ greater for each $10 \mathrm{IU} / \mathrm{L}$ increment of FSH $(95 \%$ CI $0.19,1.10, p=0.006)$ in linear regression models adjusted for age, BMI and estradiol. In older men (mean age 82.4 years) with a mean FSH of $19.0 \mathrm{IU} / \mathrm{L}$ and a mean vertebral BMAT of $54 \%$, there was no evidence of an association between vertebral BMAT and FSH in unadjusted (beta $0.23,95 \% \mathrm{CI}-0.42,0.87, \mathrm{p}=0.49$ ) or adjusted (beta $-0.25,95 \% \mathrm{CI}-0.94,0.45, \mathrm{p}=0.48$ ) models.

In conclusion, higher FSH is associated with greater vertebral bone marrow adiposity in older women but not in men. Longitudinal studies are needed to determine whether higher FSH levels predict increases in bone marrow adiposity in older adults.

Keywords: FSH, marrow adiposity

First and second authors equally contributed

\section{P079}

Low bone mass in mice with conditional Wnt 1 deletion and an autosomal dominant WNT1 mutation causing early-onset osteoporosis

Nele Vollersen $^{1}$, Timur Alexander Yorgan ${ }^{1}$, Irm HermansBorgmeyer $^{2}$, Tim Rolvien ${ }^{1}$, Ralf Oheim ${ }^{1}$, Michael Amling ${ }^{1}$, Thorsten Schinke

${ }^{1}$ Institut für Osteologie und Biomechanik, University Medical Center Hamburg-Eppendorf, Hamburg, Germany, ${ }^{2}$ Center for Molecular Neurobiology, University Medical Center Hamburg-Eppendorf, Hamburg, Germany

Objective: Since mutations in WNT1 were recently found in individuals with early-onset-osteoporosis (EOOP), Wnt1 is suggested to be a key regulator of bone formation. We aimed at generating and investigating two independent mouse models with either an osteoblast-specific Wnt1 inactivation or carrying a Wnt1 R235W mutation causing autosomal dominant EOOP. The latter model was also established to test potential treatment options for Wnt1-dependent EOOP.

Methods: Wnt $1^{\mathrm{fl} / \mathrm{fl}}$ Runx2-Cre mice, as well as a knock-in mouse model harboring a mutation in Wnt1 causing a $\mathrm{R} 235 \mathrm{~W}$ amino acid exchange were characterized by $\mu \mathrm{CT}$ and histomorphometric analysis. Moreover, the effectiveness of intermittent PTH treatment was investigated in $W n t 1^{+/ R 235 W}$ mice.

Results: $W n t 1^{\mathrm{f} / \mathrm{fl}}$ Runx2-Cre mice displayed multiple skeletal fractures and decreased trabecular bone volume (BV/TV: Wnt $1^{\text {fllfl }}$ : $13.3 \pm 4.9 \%$, Wnt $1^{\text {flff }}$ Runx $2-C r e: 3.5 \pm 1.7 \%$; p value: 0.03$)$ and cortical thickness (Cort.Th: Wnt $1^{f l f t}: 199.3 \pm 12.6 \mu \mathrm{m}$, Wnt $1^{f l}$ ${ }^{f l}$ Runx2-Cre: $150.4 \pm 40.2 \mu \mathrm{m}$; p value: 0.001$)$ at 24 weeks of age. Moreover, mice heterozygous for the autosomal dominant mutation R235W displayed a decreased trabecular (BV/TV: $W n t 1^{+/+}$: $5.2 \pm 1.4 \%$,Wnt ${ }^{+/ R 235 W}: 2.7 \pm 1.3 \%$, p value: 0.008$)$ and cortical (Cort.Th: $W n t 1^{+/+}: 217.9 \pm 4.1 \mu \mathrm{m}, W n t 1^{+/ R 235 W}: 204.6 \pm 10.7 \mu \mathrm{m}$, p value: 0.03 ) bone mass at 52 weeks of age. Mice homozygous for the $\mathrm{R} 235 \mathrm{~W}$ mutation were viable and displayed an exacerbated bone phenotype (BV/TV: $1.9 \pm 0.6 \%$, Cort.Th: $152.3 \pm 15.2 \mu \mathrm{m}$ ). Importantly, Wnt $1^{+/ R 235 W}$ mice responded normally to the osteoanabolic influence of intermittent PTH treatment.
Conclusion: Both models displayed a low bone mass phenotype with decreased trabecular and cortical bone mass and should be considered as valuable tools for investigating the role of Wnt 1 in bone remodeling. The successful treatment of $W n t 1^{+/ R 235 W}$ mice with intermittent PTH suggests that osteoanabolic treatment by teriparatide is an appropriate therapeutic approach for individuals with Wnt1dependent EOOP.

Keywords: Mouse model, Wnt signaling, EOOP, PTH treatment

\section{P081}

Effect of the zinc finger protein 384 (ZNF384) gene in a family with osteoporosis

Melissa M Formosa $^{1}$, Dalila Palazzo ${ }^{1}$, Robert Formosa ${ }^{2}$, M. Carola Zillikens $^{3}$, Andre G Uitterlinden ${ }^{3,4}$, Fernando Rivadeneira ${ }^{3,4}$, Annemieke JMH Verkerk ${ }^{3}$, Angela Xuereb-Anastasi ${ }^{1}$

${ }^{1}$ Department of Applied Biomedical Science, Faculty of Health Sciences, University of Malta, Msida, Malta, ${ }^{2}$ Department of Medicine, Faculty of Medicine and Surgery, University of Malta, Msida, Malta, ${ }^{3}$ Department of Internal Medicine, Erasmus MC, Rotterdam, Netherlands, ${ }^{4}$ Department of Epidemiology, Erasmus MC, Rotterdam, Netherlands

Objectives: Osteoporosis is a skeletal disease having a strong genetic basis. The aim of the study was to identify the genetic cause of a highly penetrant form of osteoporosis in an extended Maltese family.

Methods: A 3-generation family of 29 relatives aged from 21 to 81 years was recruited. Osteoporosis was defined by lumbar and hip $\mathrm{T}$-scores and Z-scores derived from DXA measurements. A genomewide linkage scan followed by exome sequencing was performed. Pre-mRNA splicing aberrance was tested using an exon-trapping vector transfected in SAOS2 and hFOB cells.

Results: Multipoint parametric linkage with dominant inheritance identified two suggestive peaks with a LOD score of 2.1 mapping to chromosomes 12p13.33-12p13.1 and 19p13.2-q13.12. Exome variant filtering revealed the presence of rs146089604 in intron 7 of ZNF384 in all 7 affected relatives, having a MAF of $\leq 2 \%$ that is conserved in the 1000GP. According to in silico modelling, the $\mathrm{G}>\mathrm{A}$ variant located 32 base pairs upstream from the intron-exon junction abolished an exon splicing enhancer motif and branch site. Indeed, the A allele lead to exon 8 and part of intron 8 to be retained, both of which are spliced out in transcripts harbouring the $\mathrm{G}$ allele. Replication of rs146089604 in 1018 Maltese postmenopausal women strengthened the association with BMD and fracture susceptibility; women with the GA genotype had a twofold increased risk of low femoral neck (FN) BMD and fractures (OR 2.0 [95\% CI 1.1-3.8] and 2.0 [1.0-4.8] respectively). Only 2 women with the AA genotype were detected, both having a low FN BMD.

Conclusion: Observations suggest rs146089604 to be a causal variant. ZNF384 transactivates COL1A1 and matrix metalloproteinases, and suppresses BMP and canonical Wnt signalling. ZNF384deficient mice had increased bone formation and hence bone volume. Thus, impaired splicing mechanisms could alter ZNF384's function culminating in reduced bone quality.

Keywords: osteoporosis, ZNF384, fractures

\section{P083}

Anabolic stimuli prevent the decline of bone formation associated with long-term exposure to sclerostin-neutralizing antibodies

Maude Gerbaix ${ }^{1}$, Serge Ferrari ${ }^{1}$ 
${ }^{1}$ University Geneva Hospital (HUG)/Faculty of Medicine (UNIGE), Geneva, Switzerland

Sclerostin inhibition by neutralizing $\mathrm{Ab}(\mathrm{Scl}-\mathrm{Ab})$ in animals and humans leads to a massive increase, followed by a drastic downregulation, of predominantly modeling-based bone formation. Several mechanisms have been proposed, including upregulation of Sost and Dkk1, and/or exhaustion of osteoblasts precursor pool. We hypothesized that the bone forming response to Scl-Ab could be maintained by anabolic stimuli known to both downregulate Sost expression and promote Sost-independent bone formation.

Four month-old mice received Scl-Ab $(50 \mathrm{mg} / \mathrm{kg} / \mathrm{w})$ or vehicle for 7 weeks. At week 4, axial compression was applied on the left tibia ( $16 \mathrm{~N}$ to $\mathrm{Scl}-\mathrm{Ab}$ treated mice, $12 \mathrm{~N}$ to Veh mice, at $0.1 \mathrm{~Hz}, 7 \mathrm{~min}, 2$ days/week/3 weeks). A Subgroup of mice concomitantly received PTH $(40 \mu \mathrm{g} / \mathrm{kg} / \mathrm{day})$ from 4 to 7 weeks. Bone formation was evaluated by serum P1NP and microstructure by micro-CT.

In response to $\mathrm{Scl}-\mathrm{Ab}, \mathrm{P} 1 \mathrm{NP}$ peaked at 2 weeks and returned to veh levels by 4 weeks. Scl-Ab maximally increased BV/TV at distal femur and vertebrae $(+159,+187 \%$ respectively vs. Veh; $p<0.01)$ and femur Ct.Th $(+37 \%$ vs. Veh; p < 0.01$)$ by 4 weeks with no significant gain thereafter. PTH administration from week 4 sustained P1NP levels $(+23 \%$ vs. Scl-Ab alone at week $7 ; \mathrm{pNS})$ and further increased BV/TV at femur and vertebrae $(+16$ and $+20 \%$, respectively, vs. Scl-Ab alone; $\mathrm{p}<0.05)$, but not Ct.Th. Loading also further increased tibia BV/TV and Ct.Th in the Scl-Ab group $(+14$ and $+19 \%$ respectively vs. nonloaded tibia; $\mathrm{p}<0.05)$ and in the Scl$\mathrm{Ab}+\mathrm{PTH}$ group $(+49$ and $+12 \%$ vs. nonloaded tibia, $\mathrm{p}<0.05)$.

In summary, continuous exposure to $\mathrm{Scl}-\mathrm{Ab}$ leads to a rapid decline of bone formation, which can be rescued by both intermittent PTH and mechanical loading. These observations suggest that lining cells and/or osteoblasts precursors are still responsive to anabolic stimuli and could be further maintained by Sost independent mechanisms.

\section{P085}

CYP11B1 is a key adrenal steroidogenesis factor influencing skeletal maturation In children of school age

Olja Grgic ${ }^{1}$, Alessandra Chesi ${ }^{2}$, Yoseph Barash ${ }^{3}$, Carolina MedinaGomez $^{4}$, Babette Zemel ${ }^{5}$, Shana McCormack ${ }^{6}$, Enisa Shevroja ${ }^{7}$, Katerina Trajanoska ${ }^{8}$, Jenny Visser ${ }^{7}$, Leo Hofland ${ }^{9}$, Vincent Jaddoe ${ }^{10}$, Andre Uitterlinden ${ }^{7}$, Eppo Wolvius ${ }^{11}$, Struan Grant ${ }^{12}$, Fernando Rivadeneira $^{13}$

${ }^{1}$ Department of Maxillo-Facial Surgery, Department of Internal Medicine, The Generation $R$ Study, Erasmus MC, Rotterdam, Netherlands, ${ }^{2}$ Division of Human Genetics and Molecular Biology, The Children's Hospital of Philadelphia, Philadelphia, United States, ${ }^{3}$ Genomics and computational Biology, Perelman School of medicine; University of Pennsylvania, Philadelphia, United States, ${ }^{4}$ Department of Internal Medicine, Department od Epidemiology, The Generation $R$ Study, Erasmus MC, Rotterdam, Netherlands, ${ }^{5}$ Division of Gastroenterology, Hepatology and Nutrition, The Children's Hospital of Philadelphia, Philadelphia, United States, ${ }^{6}$ Division of Endocrinology and Diabetes, The Children's Hospital of Philadelphia, Philadelphia, United States, ${ }^{7}$ Department of Internal Medicine, Erasmus MC, Rotterdam, Netherlands, ${ }^{8}$ Department of Internal Medicine, Department of Epidemiology, Erasmus MC, Rotterdam, Netherlands, ${ }^{9}$ Department of Internal Medicine, ErasmusMC, Rotterdam, Netherlands, ${ }^{10}$ The Generation $R$ Study, Erasmus $M C$, Rotterdam, Netherlands, ${ }^{11}$ Deparmtent of Maxillo-facial Surgery, The Generation R Study, Erasmus MC, Rotterdam, Netherlands, ${ }^{12}$ Division of human genetics, The Children's Hospital of Philadelphia, Philadelphia, United States, ${ }^{13}$ Department of Internal Medicine, The Generation R Study, Erasmus MC, Rotterdam, Netherlands
Objective: Advanced or delayed physiological age influences health and disease processes. Skeletal age is a proxy of physiological age determined on carpal bones. We performed the first genome-wide association study (GWAS) meta-analysis on skeletal maturation in children of school age.

Methods: Meta-analysis included children from the Generation $\mathrm{R}$ Study (GenR: $n=3510$; age $=9.79 \pm 0.33$ ) multiethnic birth cohort in Rotterdam, NL and the multi-centric Bone Mineral Density in Childhood Study (BMDCS: $n=1048$; mean age $=11.34 \pm 1.79$ ) in the US. Skeletal age was assessed in X-ray (BMDCS) or DXA (GenR) images of the hand using the Greulich and Pyle method. Participants were genotyped with Illumina HumanHap 610K (GenR) and OmniExpressExome (BMDCS) arrays imputed to the 1000GP reference panel. Association between genotypes and SA was tested using linear regression (GenR) and linear mixed models (BMDCS) corrected for sex, age, BMI (and 10 PCs in GenR). Fixed-effects inverse variance meta-analysis using METAL was performed and significance set at $P<5 \times 10^{-8}$. Splicing expression quantitative trait loci (eQTL) analysis was performed in GTEx RNA-seq data using MAJIQ.

Results: One sole association signal for SA was identified mapping to $8 \mathrm{q} 24.3$ lead by a $C Y P 11 B 1$ synonymous coding variant (rs6410; $\operatorname{MAF}(\mathrm{T})=0.48 ;$ beta $=0.1 ; \quad P=1.1 \times 10^{-11}$ ) with in-silico predicted protein domain abolition affecting splicing regulation. GTEx local splicing variation eQTL analysis showed significant evidence $\left(\mathrm{P}=6.75 \times 10^{-15}\right)$ for a splicing change (skipping exon 4$)$ in $\mathrm{T}$-allele carriers.

Conclusion: We identified one novel locus robustly associated with skeletal age in children likely driven by a coding variant in CYP11B1 (or others in high LD) influencing splicing regulation and potentially resulting in differential skeletal maturation. While mutations in CYP11B1 cause congenital adrenal hyperplasia (monogenic disorder presenting with precocious puberty), our findings are the first to implicate a genetically-determined role of adrenal steroidogenesis in the process of pediatric skeletal maturation in healthy children.

\section{P087}

Cellular and molecular analysis of patients affected by Gorham-Stout disease

Michela Rossi ${ }^{1}$, Giulia Battafarano ${ }^{1}$, Eda Mariani ${ }^{1}$, Paola Sabrina Buonuomo $^{2}$, Ippolita Rana ${ }^{3}$, Alessandro Jenkner ${ }^{4}$, Rita De Vito ${ }^{5}$, Simone Pelle ${ }^{6}$, Matteo D'Agostini ${ }^{7}$, Andrea Bartuli ${ }^{2}$, Andrea Del Fattore $^{1}$

${ }^{1}$ Bone Physiopathology Group, Multifactorial Disease and Complex Phenotype Research Area, Bambino Gesù Children's Hospital, IRCCS, Rome, Italy, ${ }^{2}$ Rare Diseases and Medical Genetic Unit, Bambino Gesù Children's Hospital, IRCCS, Rome, Italy, ${ }^{3}$ UO Rare Diseases, Bambino Gesù Children's Hospital, IRCCS, Rome, Italy, ${ }^{4}$ Division of Immunology and Infectious Diseases Department of Pediatrics, Bambino Gesù Children's Hospital, IRCCS, Rome, Italy, ${ }^{5}$ Histopathology Unit, Bambino Gesù Children's Hospital, IRCCS, Rome, Italy, ${ }^{6}$ Casa di Cura Villa Aurora-San Feliciano, Rome, Italy, ${ }^{7}$ Clinical Laboratory, Bambino Gesù Children's Hospital, IRCCS, Roma, Italy

Gorham-Stout disease (GSD) is a very rare disorder characterized by progressive osteolysis and angiomatous proliferation. The most common symptoms are swelling, fractures, pain and impairment of affected regions. The etiology of GSD is unknown and the diagnosis is performed by exclusion criteria. We aim to investigate the bone remodeling alterations in GSD patients.

Bone biopsies and bone turnover markers were analysed. In vitro cell cultures were performed to evaluate alteration of morphology, differentiation, activity and gene expression of bone cells. 
Bone biopsy analysis revealed, in patients, fibrous tissue, increase of osteoclasts, vessels and osteocyte lacunae. In patient sera high levels of ICTP, VEGF-A, IL-6 and Sclerostin were observed. Patient's osteoclast precursors showed a twofold increased ability to differentiate into osteoclasts, with more nuclei per cell. About $75 \%$ of GSD osteoclasts displayed a more motile phenotype. A twofold increased ability to resorb bone was observed in GSD osteoclast cultures. Transcriptome analysis revealed an enrichment of PI3 kinase, EGF receptor and beta-arrestin pathways. To investigate the involvement of systemic factors in GSD, Healthy Donor (HD)-PBMC were treated with GSD sera and showed increased osteoclastogenesis compared to control sera-treated cells (Osteoclast number/field, HD: $17.24 \pm 2.38$; GSD: $26.27 \pm 2.43$. p < 0.02). Bone Marrow MSC isolated from a patient revealed a defect of osteogenic differentiation, as shown by reduced ALP activity and expression compared to HDMSC. Affected osteoblasts displayed reduced ability to form mineralized nodules. Transcriptome analysis revealed in GSD osteoblasts a modulation of pathways involved in bone morphogenesis and ossification and an increase of osteoclastogenic potential. Moreover, mature HD-osteoblasts treated with GSD sera showed decreased expression of $A L P$ and $C O L 1 a 2$ and increased RANKL/OPG ratio.

These results suggest that in GSD the skeletal alterations are related to bone cell autonomous defects and systemic factors, opening the way for the identification of new therapeutic approaches.

Keywords: Gorham-Stout; Osteoclast; BM-MSC

\section{Oral Posters 3-Basic}

\section{P042}

3D bone microstructure of the mandibular condyle correlates with masseter muscle mass in adult mice

$\underline{\text { Julián Balanta-Melo }}^{1,2,3}$, Viviana Toro-Ibacache ${ }^{1,4,5}$, María TorresQuintana $^{6}$, Kornelius Kupczik ${ }^{3}$, Sonja Buvinic ${ }^{1}$

${ }^{1}$ Institute for Research in Dental Sciences, Faculty of Dentistry, Universidad de Chile, Santiago de Chile, Chile, ${ }^{2}$ School of Dentistry, Universidad del Valle, Santiago de Cali, Colombia, ${ }^{3}$ Max Planck Weizmann Center for Integrative Archaeology and Anthropology, Max Planck Institute for Evolutionary Anthropology, Leipzig, Germany, ${ }^{4}$ Center for Quantitative Analysis in Dental Anthropology, Faculty of Dentistry, Universidad de Chile, Santiago de Chile, Chile, ${ }^{5}$ Department of Human Evolution, Max Planck Institute for Evolutionary Anthropology, Leipzig, Germany, ${ }^{6}$ Department of Pathology and Oral Medicine, Faculty of Dentistry, Universidad de Chile, Santiago de Chile, Chile

Background: Masticatory muscle activity is required for temporomandibular joint homeostasis. As we previously demonstrated in adult mice, the masseter muscle atrophy induced by botulinum toxin type A (BoNTA) produces bone loss in the mandibular condyle when assessed with bone histomorphometry after 14 days. However, covariation between skeletal muscle features and bone quality in the masticatory apparatus is still poorly understood. Therefore, we hypothesized that the 3D bone microstructure of the mandibular condyle correlates with the masseter muscle mass.

Methods: As approved by the ethics committee, adult BALB/c mice were randomly assigned to a control group without intervention $(\mathrm{n}=10)$, and a BoNTA group $(\mathrm{n}=8)$ that received one BoNTA injection in the right masseter and saline solution in the left masseter (intra-individual control). After 14 days, all mice were euthanized, and masseter muscles and mandibles were obtained. The mandibles were scanned with high-resolution X-ray Microtomography and bone morphometric parameters from each mandibular condyle were quantified.
Results: BoNTA intervention significantly reduced masseter mass $(-43 \% ; p<0.001)$. In the mandibular condyle of the BoNTA-injected side, bone volume fraction $(-11.3 \% ; p<0.001)$, and trabecular thickness $(-21.4 \% ; p<0.001)$ were significantly reduced. In contrast, trabecular separation was significantly increased $(7.7 \% ; p<0.05)$ if compared with both sides of the control group. Interestingly, there was a positive correlation between masseter mass and either bone volume fraction $(\mathrm{r}=0.742 ; p<0.001)$ or trabecular thickness $(\mathrm{r}=0.711 ; p<0.001)$, and a negative correlation with trabecular separation $(\mathrm{r}=-0.496 ; p=0.001)$.

Conclusions: These results support the hypothesis that the $3 \mathrm{D}$ bone microstructure is significantly correlated with masseter muscle mass in our model. Moreover, bone quality of the mouse mandibular condyle markedly reduces after masseter muscle atrophy induced by BoNTA intervention.

Keywords: mandibular condyle, masseter muscle, X-ray Microtomography

\section{P044}

High-resolution 3D X-ray imaging of the osteocyte lacunar network in the Chihuahua zebrafish model of osteogenesis imperfecta to assess cellular mechanisms associated with bone fragility

Imke A. K. Fiedler ${ }^{1}$, Hrishikesh A. Bale ${ }^{2}$, Katharina Jähn ${ }^{1}$, Antonella Forlino $^{3}$, Björn Busse ${ }^{1}$

${ }^{1}$ Department of Osteology and Biomechanics, University Medical Center Hamburg-Eppendorf, Hamburg, Germany, ${ }^{2}$ Carl Zeiss X-ray Microscopy, Pleasanton, United States, ${ }^{3}$ Department of Molecular Medicine, University of Pavia, Pavia, Italy

The Chihuahua (Chil+) zebrafish has been proposed as model system for the study of classical dominant osteogenesis imperfecta (OI). Previous assessment of bone quality in Chil+ showed a multi-scale phenotype similar to human OI, i.e. skeletal deformities and fractures associated with altered collagen composition and mineralization, leading to impaired local mechanical properties. A better understanding of the cellular mechanisms behind increased bone fragility in Chil+ is needed to identify potential drug targets and to improve treatment strategies of OI. This study aims to quantify the osteocyte-lacunar network as indicator for bone turnover in Chil+ and wild type (WT) zebrafish utilizing 3D X-ray microscopy (XRM).

Vertebrae of adult WT and Chil+ zebrafish were dissected and scanned with high-resolution 3D XRM at $0.75 \mu \mathrm{m}$ resolution. Osteocyte-lacunar properties were quantified in the distal, central and proximal regions of each vertebral body and pooled for quantification. 3D parameters included lacunar porosity (Lc.V/BV), lacunar number density (N.Lc/TV), and mean lacunar volume (Lc.V/N.Lc).

Data gained from one vertebral body per group indicate a less pronounced osteocyte-lacunar network and altered lacunar morphology in Chil+ vs. WT. The resulting 3D analysis of the XRM data showed lower lacunar porosity $(0.12 \pm 0.05$ vs. $0.35 \pm 0.09 \%)$, lower lacunar number density $\left(20,337 \pm 4223\right.$ voids $/ \mathrm{mm}^{3}$ vs. $51,651 \pm 12,521$ voids $\left./ \mathrm{mm}^{3}\right)$, and lower lacunar volume $(40 \pm 12$ vs. $73 \pm 98 \mu^{3}$ ) for Chil+ vs. WT ( $\leq 0.05$ for all parameters).

Quantification of 3D osteocyte-lacunar properties in Chil+ supports our previous 2D histological findings indicating a bone formation defect due to altered osteoblast activity, and weakened capability for bone tissue maintenance and repair due to an osteocyte network with reduced mechanosensitivity. High-resolution 3D imaging of the osteocyte-lacunar characteristics in Chil+ provides new insights into bone quality in zebrafish and moreover bares the potential to elucidate the pathology of human OI, fostering the design of new therapies.

Keywords: Osteogenesis imperfecta, zebrafish, osteocyte network, X-ray microscopy 


\section{P046}

Generation and characterization of zebrafish models of recessive osteogenesis imperfecta

Francesca Tonelli ${ }^{1}$, Laura Leoni ${ }^{1}$, Silvia Cotti ${ }^{1}$, Roberta Besio ${ }^{1}$ Roberta Gioia $^{1}$, Björn Busse ${ }^{2}$, Imke Fiedler ${ }^{2}$, Antonio Rossi ${ }^{1}$, Antonella Forlino ${ }^{1}$

${ }^{1}$ Department of Molecular Medicine, University of Pavia, Pavia, Italy, ${ }^{2}$ Department of Osteology and Biomechanics, University Medical Center Hamburg-Eppendorf, Hamburg, Germany

Osteogenesis imperfect (OI) is a heritable disorder characterized by bone deformity, skeletal fragility and short stature. CRTAP, proline 3-Hydroxylase 1 (P3H1) and Cyclophylin B are components of the collagen prolyl-3-hydroxylation complex, involved in the hydroxylation of specific proline residues in collagen type I $\alpha$ chains. Mutations in these proteins cause the recessive OI type VII, VIII and IX, respectively. We applied the CRISPR/Cas9 editing strategy to generate knock out models for crtap and $p 3 h 1$ (ethical approval 1/2013) and performed their characterization.

P3hl and Crtap homozygous mutants are smaller than WT $(\mathrm{p}<0.05)$ and show a delayed mineralisation in hyomandibular bone (HM), ceratohyal bone (CHB) and notochord (NC) both at 1 and 2 weeks post fertilization (wpf, $\mathrm{p}<0.05$ for crtap at both time points and for p3h1 at $2 \mathrm{wpf}$ ). Morphometric analysis of both models was performed at 8 wpf. p3hl mutants display decreased height of the head (HE, $\mathrm{p}=0.078$ ) and reduced distance from the snout to the most posterior point of operculum (SOL, $\mathrm{p}=0.032$ ). Furthermore, the distance from ventral to dorsal (HAA) is lower in mutants than in WT $(\mathrm{p}<0.05)$. At $8 \mathrm{wpf}$ in crtap knock out fish the head dimension is not significantly reduced, but the ratio SOL/HE is smaller in mutant compared to controls $(p<0.05)$, denoting the presence of a chunky head. Homozygous crtap are thinner than controls (HAA: $\mathrm{p}<0.05$ ). X-rays and $\mu \mathrm{CT}$ reveal disorganization of the vertebrae and skeletal deformities in adult mutants. In both mutant models collagen type I has abnormal electrophoretic migration, a typical OI feature.

We proved the goodness of our zebrafish models to reproduce the phenotype of OI type VII and VIII. Our goal will be to use them for drug screening in order to pave the way to new pharmacological treatments.

Keywords: Osteogenesis imperfecta, zebrafish, bone diseases

\section{P048}

LP4, a lactoferrin-derived small peptide reverses osteopenia via BMP2-OPG dependent pathway

Subhashis Pal ${ }^{1}$, Mohd Sayeed ${ }^{2}$, Amit Kumar ${ }^{2}$, Munesh Kumar Harioudh $^{2}$, Kalyan Mitra ${ }^{3}$, Jimut Kanti Ghosh ${ }^{2}$, Naibedya Chattopadhyay ${ }^{1}$

${ }^{I}$ Division of Endocrinology, Central Drug Research Institute, Lucknow, India, ${ }^{2}$ Division of Molecular and Structural Biology, Central Drug Research Institute, Lucknow, India, ${ }^{3}$ Electron Microscopy Unit, Central Drug Research Institute, Lucknow, India

Objective: Lactoferrin has been reported to have potent mitogenic and anti-apoptotic actions on osteoblasts. We here report identification of a small peptide fragment of lactoferrin having osteo-anabolic effect.

Results: We designed 3 small peptide fragments of lactoferrin and measured their effect on osteoblast differentiation. Out of three, one fragment, LP4 showed strongest osteogenic differentiation and the effect was significantly higher than lactoferrin. LP4 significantly enhanced BMP2, RunX2 and OPG expression and increased mineralization of bone marrow stromal cells. Noggin (BMP2 inhibitor) completely blocked the effect of LP4 on osteoblast differentiation and
OPG expression. This data suggests that LP4 mediates its effect via BMP2. We assessed the skeletal effects of LP4. First, we determined the effective dose in a rat femur osteotomy model and found that LP4 had significant bone regenerative effect at $6 \mu \mathrm{g} / \mathrm{kg}$ dose $(\mathrm{p}<0.01)$. Further, we treated skeletally mature osteopenic rats with LP4 for 90 days and significant bone restorative effect of LP4 was observed at $6 \mu \mathrm{g} / \mathrm{kg}$ dose. LP4 significantly enhanced serum PINP ( $\mathrm{p}<0.05)$, and partially suppressed serum CTX-1 $(\mathrm{p}<0.05)$. LP4 completely restored trabecular mineral apposition rate (MAR) and bone formation rate (BFR) up to the level of sham. We found that after 90 days LP4 treatment serum OPG level was higher $(\mathrm{p}<0.05)$ compared to vehicle treated animals.

Methodology: Skeletally mature Sprague-Dawley rats were used for femur osteotomy model and ovariectomy-induced osteopenia. Rat calvarial osteoblast cultures were used to assess osteoblast differentiation. $\mu \mathrm{CT}$, bone strength tester and ELISA kits were used for various measurements.

Conclusion: A small fragment of lactoferrin showed significant osteogenic activity likely via the induction of BMP2. LP4 also suppressed osteoclastic activation likely via the BMP2-OPG dependent mechanism. We conclude that LP4 could be a potential therapeutic agent for bone anabolic therapy.

Keywords: Lactoferrin, osteoanabolic, post-menopausal osteoporosis, bone morphogenetic protein 2 , fracture healing

\section{P050}

Inhibition of JAK2/STAT3 signaling impairs mesenchymal stem cell proliferation, osteogenic differentiation, and bone defect healing

Janak Lal Pathak ${ }^{1}$, Xin $\mathrm{Yu}^{2}$, Qilong Wan ${ }^{2}$, Zubing $\mathrm{Li}^{2}$

${ }^{1}$ Key Laboratory of Oral Medicine, Guangzhou Institute of Oral Disease, Stomatology Hospital of Guangzhou Medical University, Guangzhou Medical University, Guangzhou, China, ${ }^{2}$ Key Laboratory of Oral Biomedicine, Ministry of Education, School and Hospital of Stomatology, Wuhan University, Wuhan, China

JAK2/STAT3-signaling in osteoblast plays a role in bone formation. Mesenchymal stem cells (MSCs) undergo osteogenic differentiation during bone defect healing. However the role of JAK2/ STAT3-signaling in MSCs proliferation, osteogenic differentiation and bone defect healing is still a controversy. In this study, we aimed to analyze the effect of AG490, a JAK2-specific inhibitor, on MSCs proliferation, osteogenic differentiation, osteogenesis and bone defect healing. We used AG490 $(50 \mu \mathrm{M})$ to inhibit the JAK2/STAT3-signaling in mice bone marrow derived MSCs (BMSCs) culture and analyzed the effect on proliferation, apoptosis, and osteogenic differentiation in vitro. We further analyzed the effect of JAK2/STAT3inhibition on bone regeneration and femoral bone defect healing of mice in vivo. This study was approved by the ethical committee, Hospital of Stomatology, Wuhan University. Selected significance level was $\mathrm{P}<0.05$. AG490 did not affect BMSCs apoptosis and survival. AG490 inhibited BMSCs proliferation by 11 -fold at day 5 . AG490 inhibited Coll $\alpha$, Alp and Ocn mRNA expression by 20.3-, 4.1-, and 11.8-fold respectively at day 7. AG490 inhibited Col1 $\alpha 1$, Alp, Runx2 and Osx protein expression by 2.4-, 2.8-, 3.5- and 3.1-fold respectively at day 7 (Fig. 1). JAK2-inhibition reduced matrix mineralization at day 14 and 21 by 7-, and twofold respectively. JAK2inhibition strongly reduced number of ALP positive cells and ALP activity (Fig. 2). JAK2-inhibition reduced phosphorylation of STAT3, Akt, JNK and P38 by 2.08-, 1.54-, 4.76- and 3.23-fold respectively. Immunohistochemistry data showed that AG490 reduced number of pJAK2, pSTAT3, and ALP positive cells in bone defect area by 1.6-, $2.1-$, and 1.5-fold respectively at week 3 . Histological and $\mu$-CT data show that AG490 inhibited bone regeneration and bone defect healing. Our results clearly show JAK2/STAT3-inhibition reduced 


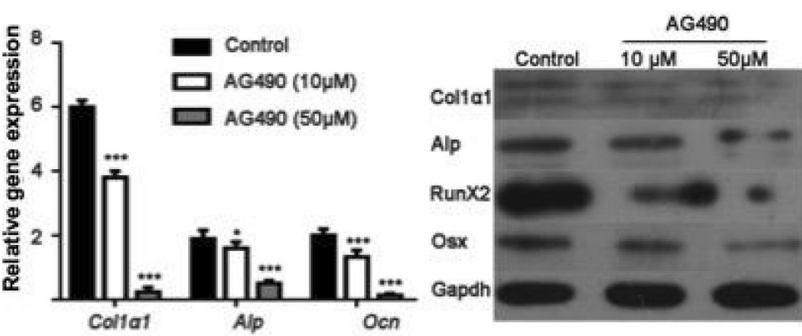

Fig. 1 Effect of AG490 on BMSCs proliferation and osteogenic differentiation

BMSCs proliferation, osteogenic differentiation, bone regeneration and bone defect healing (Fig. 3). This suggests that JAK2/STAT3signaling could be a possible target to enhance bone regeneration and bone defect healing.

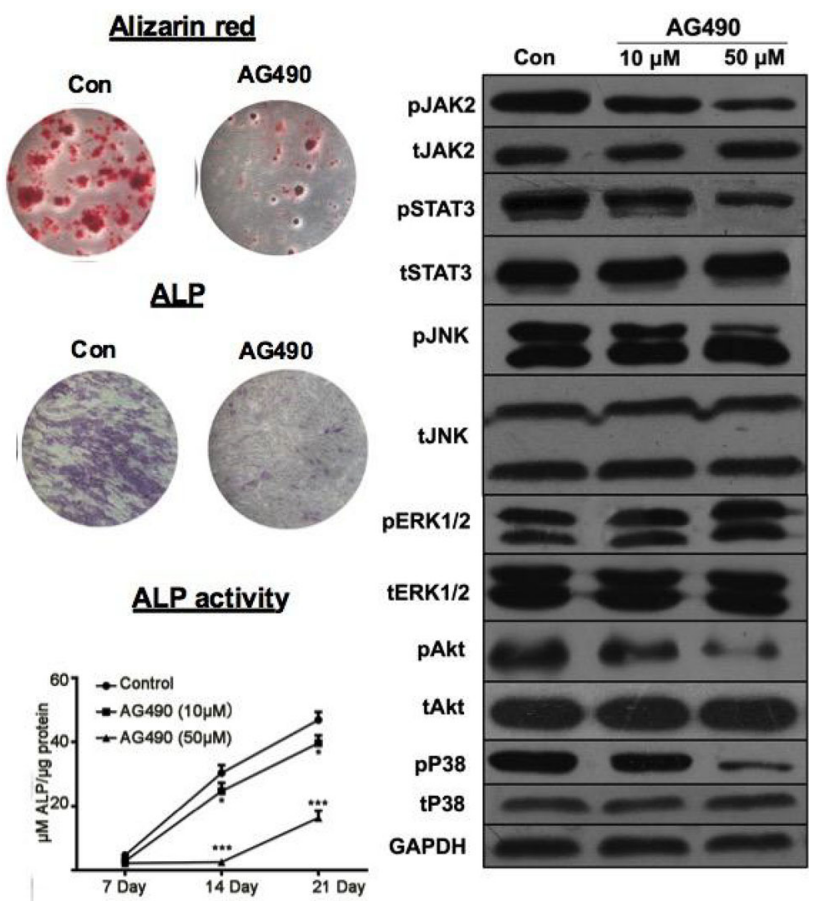

Fig. 2 AG490 inhibits matrix mineralization and JAK2/STAT3 signaling
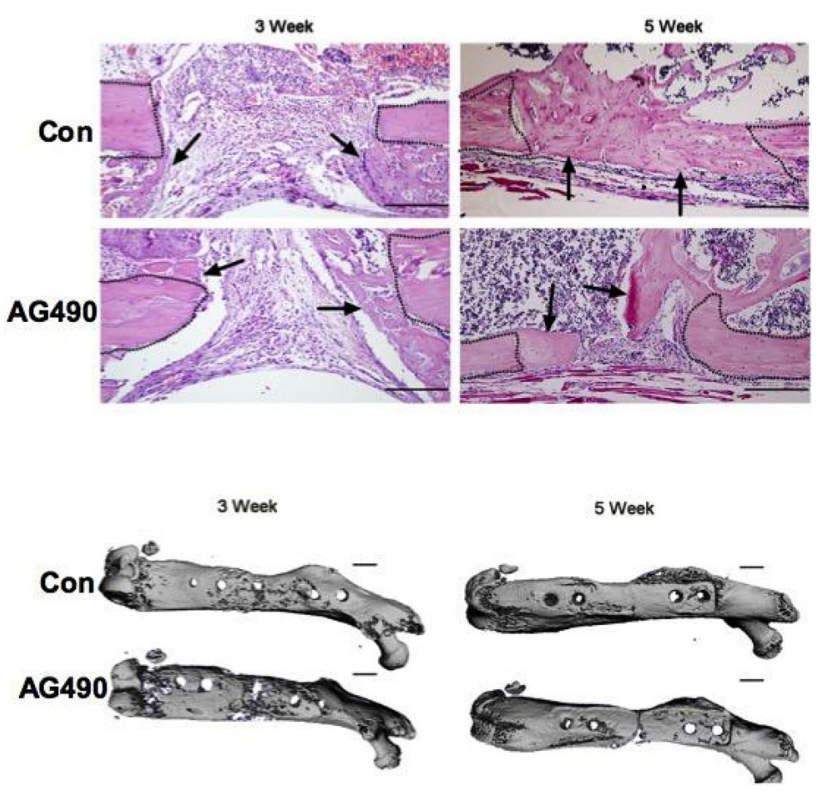

Fig. 3 AG490 inhibits bone regeneration and bone defect healing

\section{P052}

Modulation of subchondral bone turnover is associated with alteration of cartilage tissue quality

$\underline{\text { Patrick Ammann }^{1} \text {, Isabelle Badoud }}{ }^{1}$, Cedric Lavet ${ }^{1}$

${ }^{1}$ Service des maladies osseuses, Genève, Switzerland

Any alteration of osteochondral unit (cartilage or subchondral bone) could interfere with an optimal function of the joint. One obvious question is to understand whether alteration of subchondral bone turnover could affect cartilage quality.

Fifteen adult female rats where ovariectomized (OVX, $\mathrm{n}=10)$ or SHAM operated $(n=5)$. One group of OVX rats was treated for 8 weeks with pamidronate (APD) at dose of $0.6 \mathrm{mg} / \mathrm{kg}, 5$ days $/ \mathrm{month}$ $\mathrm{SC}$ to inhibit the increase of bone turnover. Bio-indentations (CSM, Switzerland) were performed at three different area submitted to different mechanical loading. Elastic modulus (MPa) and indentation depth $(\mathrm{mM})$ were recorded, using different load from 0.05 to $8 \mathrm{mN}$. Indentation depths were located in the upper third of the hyaline cartilage. Cartilage thickness was evaluated by microCT using (Hexabrix $\left.{ }^{\circledR}\right)$ as well as subchondral bone micro architecture. Blood and urine were collected to evaluate bone turnover using measurement of osteocalcin and deoxypyridinoline excretion. Values are mean \pm SEM; significance of differences $*$ vs. SHAM, ${ }^{\circ}$ vs. OVX (ANOVA).

Subchondral bone turnover and cartilage

\begin{tabular}{llllll}
\hline & $\begin{array}{l}\text { Pyridin/ } \\
\text { creat }\end{array}$ & $\begin{array}{l}\text { BV/TV } \\
(\%)\end{array}$ & $\begin{array}{l}\text { Tb.Th } \\
(\mathrm{mm})\end{array}$ & $\begin{array}{l}\text { Modulus } \\
(\mathrm{MPa})\end{array}$ & $\begin{array}{l}\text { Indent } \\
\text { depth } \\
(\mathrm{mm})\end{array}$ \\
\hline $\begin{array}{c}\text { SHAM } \\
(\mathrm{n}=5)\end{array}$ & $23.81 \pm 3.03^{\circ}$ & $48.0 \pm 3.0^{\circ}$ & $0.074 \pm 0.003^{\circ}$ & $2.72 \pm 0.22^{\circ}$ & $15.87 \pm 1.12^{\circ}$ \\
$\begin{array}{c}\text { OVX (n = 5) } \\
\text { OVX }+ \text { APD } \\
(\mathrm{n}=5)\end{array}$ & $68.63 \pm 11.02^{*}$ & $35.0 \pm 1.7^{*}$ & $0.063 \pm 0.002^{*}$ & $1.58 \pm 0.14^{*}$ & $23.7 \pm 1.22^{*}$ \\
\hline
\end{tabular}


Markers of bone turnover and bone microarchitecture of the subchondral bone were altered in OVX rats, APD fully prevented it. Hyaline and mineralized cartilage thicknesses were not affected by OVX nor by APD treatments. OVX alters cartilage tissue quality as indicated by a decreased of modulus and increased indentation depth. All these alterations were fully prevented by APD treatment

These results demonstrate that an increment of subchondral bone turnover is associated with degradation of the cartilage tissue quality independently of the estrogen deficiency. These results underline the crucial role plays by subchondral bone as "regulator" of cartilage quality

\section{P054}

Tissue engineered bone marrow modeling: multiple myeloma cell adipomimicry and drug resistance induced by bone marrow adipocytes

Michaela Reagan $^{1}$, Heather Fairfield ${ }^{1}$, Mariah Farrell ${ }^{1}$, Carolyne Falank

${ }^{1}$ Center for Molecular Medicine, Maine Medical Center Research Institute, Scarborough, United States

Myeloma cells have a dependency on the bone marrow (BM) microenvironment for survival and proliferation. Herein, we aimed to determine how BM adipose tissue (BMAT) engages in cross-talk with myeloma cells. We generated BMAT from human and mouse BMderived stromal cells using adipogenic media in 2D and 3D silk scaffold cultures, and used these for co-cultures with myeloma cells +/- chemotherapies.

We explored changes in cell-cell adhesion genes in vitro using qRT-PCR and found that VCAM-1 was increased in OPM2 cells after co-culture with BMAT, and this was further increased after addition of bortezomib, while other genes (e.g., N-cadherin) were not changed. Direct culture with BMAT rescued OPM2 cells from dexamethasoneand bortezomib-induced apoptosis (by flow cytometry) and cell death (by bioluminescent imaging). MM1S cells also showed significant dexamethasone resistance and decreased apoptosis when co-cultured with BMAT CM, and significant changes (e.g., elevated IL-6, IL-8 and IGFBP2) in their secreted cytokine profile. Our 3D BMAT models also induced OPM2 dexamethasone resistance (Fig. 1A,B).

We also observed the phenomenon of myeloma cell "adipomimicry". OPM2 cells expressed FABP4 and PPAR $\gamma$ and contained lipid droplets when co-cultured with BMAT (Fig. 1C). In both 2D and $3 \mathrm{D}$ co-cultures, we observed a reduction in BMAT lipid content using Oil-Red-O staining. Lastly, an adipokine array revealed significant increases in tumor supportive secreted factors (AgRP, IGF2, IL6, Lipocalin-2, RAGE, RANTES, and RBP4) from 3T3L1 adipocytes after transwell co-culture with myeloma cells. Overall, we propose a positive feedback loop between myeloma cells and BMAT with therapeutic targeting potential.
A
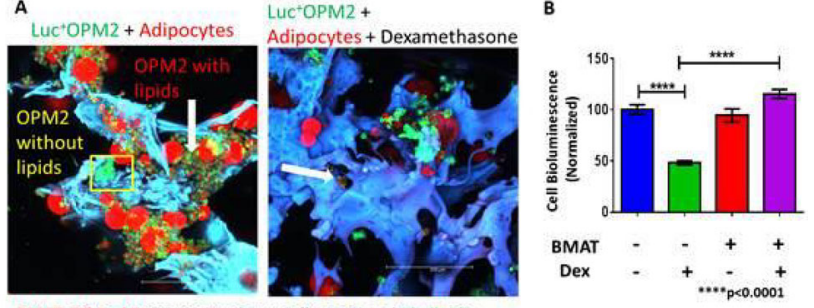

BMAT: Oil Red O; OPM2 Myeloma Cells: DAPI and Phalloidin silk Scaffold: Autoituriescente

\section{MM1S Cells Cultured with BMAT-CM Show Secretion of} Adipogenic Proteins: Adiponectin, Pref-1, Leptin, and

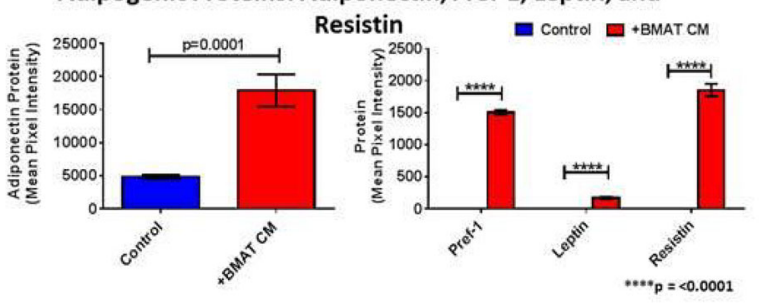

A) Tissue-engineered BMAT models can be used to study many diseases, such as myeloma. In myeloma-BMAT co-cultures, some myeloma cells accumulated lipid droplets when cultured with BMAT while others do not. B) Dexamethasone resistance was observed in $3 \mathrm{D}$ BMAT and myeloma 2 week co-cultures. A two-way ANOVA with posthoc testing was used for significance. C) MM1S cells cultured with BMAT-conditioned media had increased secretion of adipogenic proteins

Fig. 1 BMAT and myeloma cell interactions

\section{P056}

Differential effects of IKKa inhibition on trabecular and cortical bone in a prostate cancer xenograft model

$\underline{\text { Abdullah Aljeffery }}{ }^{1}$, Silvia Marino ${ }^{1,2}$, Marco Ponzetti $^{3}$, Nadia Rucci $^{3}$, Aymen I. Idris

${ }^{1}$ Department of Oncology and Metabolism, University of Sheffield, Medical School, Sheffield, United Kingdom, ${ }^{2}$ Division of Hematology/ Oncology, IU School of Medicine, Indianapolis, United States, ${ }^{3}$ Department of Biotechnological and Applied Clinical Sciences, University of L'Aquila, L'Aquila, Italy

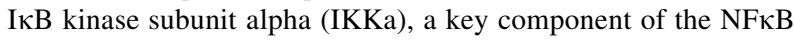
pathway, is implicated in prostate cancer progression and bone remodelling, but its role in the regulation of prostate cancer associated bone disease remains unknown. Here, we tested the effects of IKKa manipulation on prostate cancer cell behaviour in vitro and on osteolysis in a xenograft model of human prostate cancer bone metastasis. The pharmacological inhibition of IKKa using the novel and highly selective IKKa inhibitor SU1349 $(\mathrm{Ki}=16 \mathrm{nM})$ and stable knockdown of IKKa in PC3 cells reduced cell viability (33\%; $\mathrm{p} \leq 0.001)$, migration $(27 \% ; \mathrm{p} \leq 0.01)$ and invasion $(43 \% ; \mathrm{p}$ $\leq 0.01$ ), whereas IKKa overexpression was stimulatory (viability, $42 \%$; migration, $61 \%$; invasion, $51 \%, \mathrm{p} \leq 0.05$ ). In prostate cancer cell-osteoclast co-cultures, IKKa overexpression in PC3 enhanced RANKL-induced osteoclastogenesis $(69 \%, \mathrm{p} \leq 0.05)$, whereas SU1349 treatment $(0.1 \mu \mathrm{M})$ and IKKa knockdown reduced these effects $(93 \%, \mathrm{p} \leq 0.001)$. Exposure of osteoblasts to SU1349 $(0.01 \mu \mathrm{M})$ enhanced alkaline phosphatase activity $(34 \%, \mathrm{p} \leq 0.05)$ 
and bone nodule formation $(61 \%, \mathrm{p} \leq 0.01)$. Protein microarray analysis of tumour-derived factors in PC3 conditioned medium showed that these effects were associated with significant inhibition of various $\mathrm{NF \kappa B}$-mediated pro-inflammatory factors and reduction in $\mathrm{PC} 3$ conditioned medium induced $\mathrm{NF \kappa B}$ activation in osteoclast and osteoblast precursors $(\mathrm{p}<0.05)$. Interestingly, NFKB inhibition in osteoblast was accompanied by an increase in GSK3 phosphorylation $(p \leq 0.05)$, indicative of activation of the $\mathrm{Wnt} / \mathrm{b}$-catenin pathway. In vivo, administration of SU1349 $(20 \mathrm{mg} / \mathrm{kg} / 3$ timesweekly) in immuno-deficient BALB/c mice after intra-cardiac injection with human PC3 cells enhanced trabecular bone volume $(70 \%, \mathrm{p}<0.0001)$ and reduced osteoclast and increased osteoblast numbers $(\mathrm{p}<0.05)$. Paradoxically, SU1349 treatment reduced cortical bone volume $(45 \%, \mathrm{p}<0.0001)$, increased osteoclast and reduced osteoblast numbers $(\mathrm{p}<0.05)$. Thus, the novel IKKa inhibitor SU1349 is of potential therapeutic value in protecting against prostate cancer-induced osteolysis. however, exacerbation of bone loss in the cortical compartment may limit its usefulness as bone sparing agent.

\section{P058}

Expression of sperm associated antigen 4 (SPAG4) and its role in myeloma bone disease

Regina Ebert $^{1}$, Anja Seckinger ${ }^{2}$, Julia Dotterweich ${ }^{1}$, Sabine Zeck ${ }^{1}$, Jutta Meissner-Weigl ${ }^{1}$, Dirk Hose ${ }^{2}$, Franz Jakob ${ }^{1}$

${ }^{1}$ Orthopädisches Zentrum für Muskuloskelettale Forschung, Universität Würzburg, Würzburg, Germany, ${ }^{2}$ Medizinische Klinik V, Labor für Myelomforschung, Universitätsklinikum Heidelberg, Heidelberg, Germany

Multiple myeloma (MM) is a hematological disease characterized by proliferation of malignant plasma cells within the bone marrow. As a consequence, $90 \%$ of patients suffer from osteolytic bone loss, in turn promoting tumor growth in a "vicious cycle". Disease progression is fostered by direct cell-cell interaction between MM cells and mesenchymal stromal cells (MSCs)/osteogenic precursor cells (OPCs) within the tumor niche. To detect MM cell-specific interaction molecules on bone forming cells, we performed gene expression profiling for MSCs and OPCs after MM cell contact.

RNA from MSCs and OPCs (each $n=5$ ) was investigated after co-culturing for 1 day with the MM cell line INA-6 using Affymetrix U133 2.0 Plus Arrays. Differentially expressed genes included angiogenic factors as VEGF-A and the adhesion molecule ICAM-1, both of which are of importance in MM disease progression. Additionally, we identified SPAG4 (sperm-associated antigen 4) to be upregulated in MSCs and OPCs after contact with INA-6 cells. Transwell experiments revealed that this upregulation is predominantly dependent on direct cell-cell contact. Furthermore, PCR analyses showed that expression of SPAG4 was increased after coculture of MSCs and INA-6 cells under hypoxic conditions. Whole transcriptome analyses of B-memory cells $(n=5)$, polyclonal plasmablasts $(n=5)$, normal $(n=19)$ and malignant plasma cells from patients with monoclonal gammopathy of unknown significance $(n=62)$, asymptomatic $(n=259)$, symptomatic $(n=764)$, and relapsed/refractory MM $(\mathrm{n}=90)$ as well as MM cell lines $(n=54)$ revealed that SPAG4 is highly expressed in both, normal and malignant plasma cells and decreases with disease progression. High expression of SPAG4 (maxstat cutoff) is associated with significantly inferior event-free and overall survival $(\mathrm{P}<0.001$ and $\mathrm{P}=0.009$, respectively) in symptomatic MM patients.

These data suggest that SPAG4 may play a role in the crosstalk between MM and bone-forming cells. Further research is needed to clarify its role in MM bone disease.

\section{P060}

Dissection of osteogenic differentiated human mesenchymal stromal cell population heterogeneity using single cell gene expression analysis

Jeroen van de Peppel ${ }^{1}$, Rolf Vossen ${ }^{2}$, Henk Buermans ${ }^{2}$, Andre J. van Wijnen $^{3}$, Hans van Leeuwen ${ }^{1}$

${ }^{1}$ Internal Medicine, Erasmus MC, Rotterdam, Netherlands, ${ }^{2}$ Department of Human Genetics; Leiden Genome Technology Center, Leiden University Medical Center, Leiden, Netherlands, ${ }^{3}$ Orthopedic Surgery, Mayo Clinic, Rochester, United States

Heterogeneity of mesenchymal stromal cell (hMSCs) isolates due to poorly defined cell surface molecules, inter-donor variations and unpredictable differentiation capacity are some of the main hurdles preventing routinely and robust use of hMSCs in regenerative therapies. The aim of this study is to elucidate the molecular cell-to-cell differences of osteogenic differentiated human mesenchymal stromal cells based on the mRNA expression of known osteoblast marker genes and novel bone lineage-specific transcription factors (TFs).

Four days osteogenic differentiated hMSCs were sorted, lysed and single cell cDNA was generated for 288 individual cells in a $\mathrm{C1}^{\mathrm{TM}}$ Single-Cell Auto Prep System (Fluidigm). By using a Biomark HD (Fluidigm) we quantitatively detected the expression of 48 different genes in 288 individual cells in duplicate and generated RNAseq data from 64 individual cells.

Our results illustrate that the housekeeping genes were detected at similar expression levels in most of the cells in both qPCR and RNAsEq. Interestingly the TFs, studied in the single cell qPCR analyses, were expressed in a limited number of individual cells (Top15 TFs determined in 25-200 cells out of 288 cells). Although RNAseq analyses could determine up to 15,000 different genes per cell, only a very little number of genes were detected in each individual cell and bioinformatics analyses illustrated large gene expression variability between individual cells. Moreover, the RNAseq data indicated that some TFs were specifically expressed in a subsets of cells, however, the number of genes that were co-expressed within these subsets were very limited. Unsupervised clustering of the gene expression data of the individual cells could not identify subsets that mark a specific or unique cell population. Our single cell gene expression analyses illustrate a large heterogeneity within the osteoblast differentiating hMSC and further indicate that the characterization and selection of homogenous hMSCs subsets is challenging.

\section{P062}

Implication of microbiota in the emergence of inflammatory osteoclasts: protective effect of Saccharomyces boulardii CNCM I-745

Maria-Bernadette Madel ${ }^{1}$, Lidia Ibanez ${ }^{1}$, Antoine Boutin ${ }^{1}$, Rodolphe Pontier-Bres $^{2}$, Majlinda Topi ${ }^{1}$, Dorota Czerucka ${ }^{2}$, Matthieu Rouleau ${ }^{1}$, Abdelilah Wakkach ${ }^{1}$, Claudine Blin-Wakkach ${ }^{1}$

${ }^{1}$ LP2M, CNRS UMR 7370, Faculté de Médecine, Université Côte d'Azur, Université Nice Sophia Antipolis, Nice, France, ${ }^{2}$ Centre Scientific de Monaco, Monaco, Monaco

Bone destruction after estrogen-deficiency is linked to modifications in gut microbiota that increase osteoclastogenic cytokine production by activated-immune cells. This destruction is limited by bacterial probiotics. We recently identified a dialog between the gut and bone marrow (BM) in inflammatory bowel disease, a dysbiosisassociated pathology. Immune cells activated in the gut stimulate the differentiation of inflammatory-osteoclasts (i-OCLs) that differ from physiological OCLs by expressing CX3CR1 (fractalkine receptor) and activating inflammatory responses. CX3CR1 ${ }^{+}$i-OCLs are not 
restricted to IBD and also emerge after ovariectomy in mice. This raises the question of the participation of dysbiosis in controlling i-OCLs and whether probiotics may reduce their proportion. We focused on Saccharomyces boulardii-CNCM-I-745 (Sb), a yeast probiotic having gut protective effects that have never been evaluated in the context of bone degradation.

Ovariectomized (OVX) and SHAM-operated mice were gavaged $\pm S b 3$ days/week for 4 weeks before sacrifice. Saccharomyces cerevisiae $(\mathrm{Sc})$ was used in control groups. Micro-CT analysis revealed that $S b$ and $S c$ significantly limit the decrease in BV/TV (p: 0.003 ) and trabecular number (p: 0.002), and the increase in trabecular space (p: 0.01) observed after OVX, with $S b$ being the most efficient. Furthermore, they limit the CX3CR $1^{+}$i-OCLs augmentation observed in OVX mice ( $\mathrm{p}<0.001)$. $S b$, and to a lesser extent $S c$ limit the proportion of Ly6 $\mathrm{C}^{\mathrm{hi}}$ monocytes (representing OCL-progenitors), in particular CX3CR $1^{+}$Ly6C ${ }^{\text {hi }}$ monocytes $(\mathrm{p}>0.0001)$. Lastly, $S b$ conditioned medium strongly inhibits osteoclastogenesis in-vitro without affecting cell viability. Analysis of the microbiota and the mechanisms involved are still ongoing. Nevertheless, our results suggest that microbiota modulation by $S b$ reduces the recruitment of CX3CR $1^{+}$i-OCL-progenitors in the BM and their differentiation into OCLs. These results extend to $S b$ the beneficial effect of probiotics on bone destruction and provide novel information on the control of i-OCLs differentiation by signals arising from the gut/BM dialog. Keywords: Osteoclasts; Microbiota; Inflammation

\section{P064}

Osteoclast precursors pattern of receptors is modulated by Ivacaftor treatment in G551D-bearing cystic fibrosis patients

Dina Abdallah $^{1}$, Marie-Laure Jourdain ${ }^{1}$, Christine Guillaume ${ }^{1}$, Nicola Ronan $^{2}$, Yvonne Mc Carthy ${ }^{3}$, Evelyn Flanagan ${ }^{3}$, Barry Plant ${ }^{3}$, Sandra Audonnet $^{4}$, Sophie C. Gangloff ${ }^{1}$, Frédéric Velard ${ }^{1}$, Jacky Jacquot ${ }^{1}$

${ }^{1}$ Laboratoire Bios EA4691, Université de Reims Champagne Ardennes, Reims, France, ${ }^{2}$ Respiratory Cork Cystic Fibrosis Center, Cork University Hospital Group, Cork, Ireland, ${ }^{3}$ Cork Cystic Fibrosis Center, Cork University Hospital Group, Cork, Ireland, ${ }^{4}$ Plateforme cytométrie URCA Cyt, Université de Reims Champagne Ardennes, Reims, France

Rationale: Bone fragility and low bone mineral density often affect children and young adults with cystic fibrosis (CF) disease and is associated with significant morbidity due to vertebral fractures and decreased lung function. It has been recently reported that bone demineralization is improved by the CFTR potentiator ivacaftor in young patients carrying the G551-D CFTR mutation. Due to the presence of CFTR in monocytes, we hypothesized that ivacaftor may impact monocyte differentiation and activation toward bone-resorbing multinuclear osteoclasts.

Methods: We examined the expression level of M-CSFR and RANK receptors on blood monocytes from G551-D CF patients by flow cytometry, prior to and at 6 and 9 months after receiving CFTR potentiator ivacaftor.

Results: Compared to healthy controls, our first set of data demonstrates higher level of a double M-CSFR ${ }^{\text {high }} /$ RANK $^{\text {high }}$ subpopulation on monocyte of G551-D CF patients (1.5 vs. 91\%, $\mathrm{p}<0.05)$, which was reduced by in vivo ivacaftor treatment $(77 \%$ of double M-CSFR ${ }^{\text {high }} /$ RANK $^{\text {high }}$ cells after 9 months). In addition, expression of both M-CSFR and RANK receptors (more predominantly for the RANK receptor) reported by unit of monocytic cells was markedly decreased by in vivo ivacaftor treatment. Moreover, we examined ex vivo differentiation and activation of healthy monocytes into osteoclasts for a 14-days period with/or without the addition of Inh-172 drug, an inhibitor of CFTR chloride channel activity. Interestingly, osteoclasts derived from Inh172-treated healthy monocytes were largest, more adherent, and generate large pits and trenches of dentin resorption. In addition, osteoclasts derived Inh172-treated healthy monocytes released a low level of sphingosine 1-phosphate (S1P) (35\% decrease vs. basal condition), a key mediator in the directed migration of osteoblast/osteoclast precursors.

Conclusion: These data highlight the critical regulatory role of CFTR in M-CSFR and RANK receptors expression in monocytes providing new insights into the pathogenesis of $\mathrm{CF}$ bone disease. Keywords: Cystic fibrosis, bone disease, osteoclasts

\section{Oral Posters 4-Clinical}

\section{P066}

Genome-wide association meta-analysis of skull bone mineral density identifies novel associations at four loci and replicates 57 known BMD loci

Carolina Medina-Gomez ${ }^{1}$, Katerina Trajanoska ${ }^{1}$, John Kemp ${ }^{2}$, Kun $\mathrm{Zhu}^{3}$, Maria Nethander ${ }^{4}$, Gudmar Porleifsson ${ }^{5}$, Alessandra Chesi ${ }^{6}$, Ivana Nedeljkovic $^{1}$, Tarun Ahluwalia ${ }^{7}$, Raimo Joro ${ }^{8}$, Ruifang Li$\mathrm{Gao}^{9}$, Daniel S Evans ${ }^{10}$, Katharina E Schraut ${ }^{11}$, Benjamin Mullin ${ }^{3}$, Dennis Mook ${ }^{9}$, Linda Broer ${ }^{1}$, M. Carola Zillikens ${ }^{1}$, Carol Wang ${ }^{12}$, Cornelia M van Duijn ${ }^{1}$, Nathalie Van der Velde ${ }^{1}$, M. Arfan Ikram ${ }^{1}$, Babette Zemel ${ }^{6}$, Scott G. Wilson ${ }^{3}$, Timo Lakka ${ }^{8}$, James A. Wilson ${ }^{11}$, Klaus Bønnelykke ${ }^{7}$, Struan Grant ${ }^{6}$, Vincent Jaddoe ${ }^{1}$, Stuart H Ralston ${ }^{11}$, Jeroen Van Der Peppel ${ }^{1}$, Bram Van Der Eerden ${ }^{1}$, Unnur Styrkársdóttir ${ }^{5}$, Andre van Wijnen ${ }^{13}$, Claes Ohlsson ${ }^{4}$, Andre G. Uitterlinden $^{1}$, Jon H. Tobias ${ }^{14}$, David Karasik ${ }^{15}$, Dave M. Evans ${ }^{2}$, Cheryl L Ackert-Bicknell ${ }^{16}$, Fernando Rivadeneira ${ }^{1}$

${ }^{1}$ ErasmusMC, Rotterdam, Netherlands, ${ }^{2}$ University of Queensland Diamantina Institute, Brisbane, Australia, ${ }^{3}$ Sir Charles Gairdner Hospital, Nedlands, Australia, ${ }^{4}$ University of Gothenburg, Gothenburg, Sweden, ${ }^{5}$ deCODE Genetics, Reykjavik, Iceland, ${ }^{6}$ Children's Hospital of Philadelphia, Philadelphia, United States, ${ }^{7}$ COPSAC, Copenhagen Prospective Studies on Asthma in Childhood, Copenhagen, Denmark, ${ }^{8}$ University of Eastern Finland, Kuopio, Finland, ${ }^{9}$ Leiden University Medical Centre, Leiden, Netherlands, ${ }^{10}$ California Pacific Medical Center Research Institute, San Francisco, United States, ${ }^{11}$ University of Edinburgh, Edinburgh, United Kingdom, ${ }^{12}$ University of Western Australia, Crawley, Australia, ${ }^{13}$ Mayo Clinic, Rochester, United States, ${ }^{14}$ University of Bristol, Bristol, United Kingdom, ${ }^{15}$ Hebrew SeniorLife, Roslindale, United States, ${ }^{16}$ University of Rochester, Rochester, United States

Bone mineral density measured at the skull (Skull-BMD) holds the highest heritability $\left(\mathrm{h}^{2}>95 \%\right)$ across bone traits. Such restricted environmental component (i.e. scarce biomechanical stimuli) postulates it as an optimal trait to study the genetics of bone. We performed a genomewide association study (GWAS) meta-analysis using skull-BMD derived from total body DXA scans across 21 studies comprising $\sim 44,000$ individuals, with ages ranging from childhood to old age. Genetic data imputed to the 1KGP reference panel was tested in association models adjusted for sex, age, weight, height and genomic principal components. Inverse variance meta-analysis was performed and significance set at $\mathrm{P}<5 \times 10^{-8}$. LD-score regression was used to estimate heritability and genetic correlation with other traits together with gene prioritization and pathway and tissue enrichment analysis using DEPICT. SNP-based heritability was estimated as 0.31 (SE 0.03). In total, we identified 61 loci associated with skull-BMD, of which four, hold novel association signals (cytoband:closest_gene(s): 8q22.3:AZIN1; 8q23.1:EIF3E/EMC2; 12p11.22:CCDC91 and 12q13.12:RND1/WNT1/WNT10B) after conditioning for 415 genetic variants reported as associated with different bone traits. All the genes prioritized in our analysis are differentially expressed in murine osteoblasts, osteoclasts or both types of cell models; as well as in human RNA-seq bone tissue expression profiles. Pathway and enrichment 
analysis of the associated loci showed significant clustering within genesets implicated in skeletal development and WNT signaling; and overexpression in musculoskeletal tissues. Skull BMD had significant genetic correlation (shared heritability) with BMD measured at other skeletal sites (rho $>0.55$ ), fracture risk (rho $=-0.38$ ) and autoimmune conditions (rho $\sim-0.11$ ). Overall our results highlight the potential of skull-BMD as an optimal trait for the study of bone biology, sharing genetic determinants of fracture risk, the clinically relevant outcome of osteoporosis. Keywords: Skull-BMD, GWAS, fracture risk

\section{P067}

The arterial calcification defines the survival and the graft function of the kidney transplant receptors

Raúl García Castro ${ }^{1}$, Minerva Rodríguez García ${ }^{2}$, María Luisa Suárez Fernández ${ }^{2}$, Miguel Ángel Suárez Hevia ${ }^{3}$, Jesús María Fernández Gómez $^{3}$, Sara Barrio Vázquez ${ }^{4}$, Cristina Montes Alonso ${ }^{4}$, Jose Luis Fernández Martín ${ }^{4}$, Jorge Benito Cannata Andía ${ }^{4}$, Carlos Gómez Alonso $^{4}$

${ }^{1}$ Diálisis, Fundación Hospital de Jove, Gijón, Spain, ${ }^{2}$ AGC Nefrología, Hospital Universitario Central de Asturias, Oviedo, Spain, ${ }^{3} A G C$ Urología, Hospital Universitario Central de Asturias, Oviedo, Spain, ${ }^{4}$ UGC Metabolismo Óseo y Mineral. REDinREN. Universidad de Oviedo, Hospital Universitario Central de Asturias, Oviedo, Spain

Introduction: The alteration of bone and mineral metabolism in chronic kidney disease includes biochemical, bone and vascular changes linked to the patient's clinical condition.

In this study, radiological vascular calcification, as well as the concentration of calcium in arterial tissue are evaluated, considering the impact of both on the survival and graft function in kidney transplant recipients, and with vertebral fractures.

Material and Methods: Samples were obtained from distal branches of renal arteries of donors $(\mathrm{N}=70)$ and epigastric artery of receptors $(\mathrm{N}=99)$. The calcium content was determined in 60 samples of receptors and 67 donor samples by biochemical method by digestion with $0.6 \mathrm{~N} \mathrm{HCL}$ and quantification ( $O$-cresolphthalein complexone). Clinical, biochemical, and radiological data (Kaupilla index and the Genant classification, double-blind) of kidney transplant patients were collected. In the 6-year follow-up, patient survival, graft dysfunction and return to dialysis were analyzed.

Results: We have included 99 kidney transplant receptors ( $55 \pm 12$ years), $54 \%$ men, $46 \%$ women, time on dialysis $26 \pm 24$ months, vertebral fractures $17.2 \%$. The arterial calcium concentration $(\mathrm{CaA}, \mathrm{mcg} / \mathrm{mg}$ protein) did not have a normal distribution; conversion was made with logarithmic mean for statistical analysis (Table 1).

Biochemical and radiologic parameters

\begin{tabular}{|c|c|c|c|}
\hline & $\begin{array}{l}\text { Non-calcified } \\
(\mathrm{n}=47)\end{array}$ & $\begin{array}{l}\text { Calcification } \\
(\mathrm{n}=52)\end{array}$ & $\mathrm{p}$ \\
\hline $\begin{array}{r}\mathrm{CaA}(\mathrm{MD}) \\
(\mathrm{n}=61)\end{array}$ & $\begin{array}{l}13.52 \mathrm{mcg} / \mathrm{mg} \\
\quad(5.52-1759.63)\end{array}$ & $\begin{array}{l}18.82 \mathrm{mcg} / \mathrm{mg} \\
\quad(12.26-3891.54)\end{array}$ & $\begin{array}{r}0.001 \\
(1)\end{array}$ \\
\hline $\begin{array}{c}\mathrm{CaA}(\log \\
\mathrm{X} \pm \mathrm{SD}) \\
(\mathrm{n}=61)\end{array}$ & $\begin{array}{r}2.92 \pm 1.19 \mathrm{mcg} / \\
\mathrm{mg}(1.71-7.47)\end{array}$ & $\begin{array}{r}4.38 \pm 2.35 \mathrm{mcg} / \\
\mathrm{mg}(2.51-8.27)\end{array}$ & $\begin{array}{r}0.001 \\
(2)\end{array}$ \\
\hline $\begin{array}{l}\text { Fractures }(\%) \\
\quad(\mathrm{n}=87)\end{array}$ & $9 \%$ & $26 \%$ & $\begin{array}{r}0.03 \\
(3)\end{array}$ \\
\hline
\end{tabular}

Non-fractured $(n=72)$ Fractured $(n=15)$

Age $(X \pm S D) \quad 54 \pm 12(20-77) \quad 61 \pm 8(45-75) \quad 0.01(2)$
Table d continued

\begin{tabular}{|c|c|c|c|}
\hline & Non-fractured $(\mathrm{n}=72$ & Fractured $(n=15)$ & \\
\hline CaxP & $40 \pm 11(14-70)$ & $33 \pm 10(20-52)$ & $0.02(2)$ \\
\hline $\begin{array}{l}\mathrm{P}(\mathrm{X} \pm \mathrm{SD}) \\
\quad(\mathrm{n}=72)\end{array}$ & $\begin{array}{l}1.41 \pm 0.42 \mathrm{mmol} / \mathrm{l} \\
\quad(0.52-2.5)\end{array}$ & $\begin{array}{l}1.18 \pm 0.35 \mathrm{mmol} / \mathrm{l} \\
\quad(0.61-1.91)\end{array}$ & $0.02(2)$ \\
\hline $\begin{array}{l}\mathrm{CaA} \\
\quad(\log \mathrm{X} \pm \mathrm{SD}) \\
\quad(\mathrm{n}=44)\end{array}$ & $\begin{array}{l}3.34 \pm 1.71 \\
\quad(1.71-7.73)\end{array}$ & $\begin{array}{l}4.16 \pm 2.33 \\
\quad(2.49-8.07)\end{array}$ & $0.07(2)$ \\
\hline
\end{tabular}

$M D$ median, $X$ mean, $S D$ standard deviation, $C a$ serum calcium, $P$ serum phosphorous, 1 U-Mann Whitney, 2 T-Student, 3 X2

Overall, the presence of vascular calcification was associated with patient survival (Log Rank 8,006, $\mathrm{p}=0.005$ ), with graft dysfunction $(r=0.36, p=0.004)$, not with the dialysis restart. Considering the semiquantitative index of Kaupilla grouped, it has been possible to objectify an impact on the survival of the patient (Image 1)

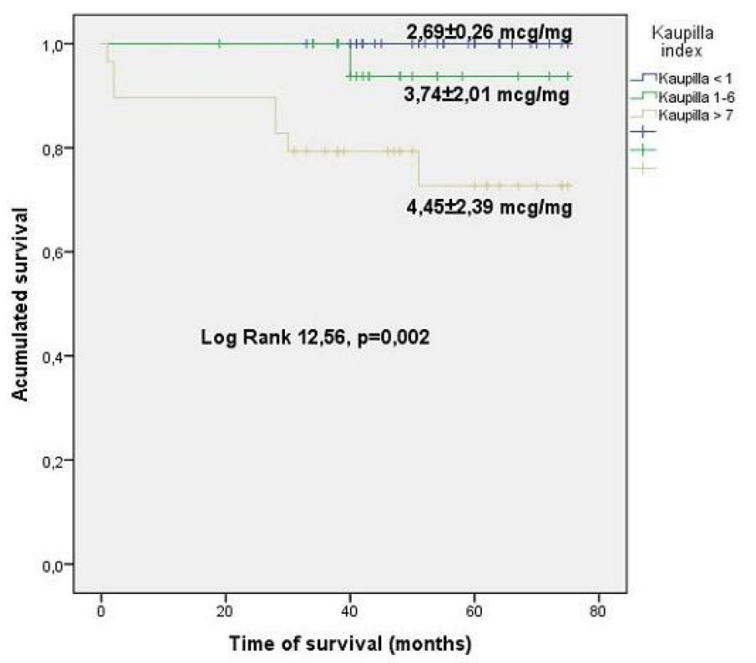

Image 1: Time of survival based on Kaupilla index and arterial calcium concentration $(X \pm S D)$

Conclusions: Vascular calcification prior to transplantation is an independent risk factor for mortality and a predictor of graft function.

The concentration of vascular calcium obtained at the time of transplantation can improve the sensitivity/prognostic specificity of vascular calcifications evaluated radiologically and allow therapeutic measures to be established earlier.

Keywords: Calcification, vascular, kidney, transplant, arterial

\section{P070}

Women at high risk of hip fracture based on FRAX respond to appropriate osteoporosis management: analysis from the SCOOP study of population screening

Eugene McCloskey ${ }^{1,2}$, Nicolas Harvey ${ }^{3}$, Helena Johansson ${ }^{4,5}$, Lee Shepstone $^{6}$, Elizabeth Lenaghan ${ }^{6}$, Cyrus Cooper ${ }^{3}$, John Kanis ${ }^{4,5}$, The SCOOP study team

${ }^{1}$ Oncology \& Metabolism, University of Sheffield, Sheffield, United Kingdom, ${ }^{2}$ Centre for Integrated research in Musculoskeletal Ageing (CIMA), University of Sheffield, Sheffield, United Kingdom, ${ }^{3}$ MRC Lifecourse Epidemiology Unit, University of Southampton, 
Southampton, United Kingdom, ${ }^{4}$ Centre for Metabolic Bone Diseases, University of Sheffield, Sheffield, United Kingdom, ${ }^{5}$ Australian Catholic University, Melbourne, Australia, ${ }^{6}$ School of Medicine, University of East Anglia, Norwich, United Kingdom

Targeting of treatment in interventional studies to reduce osteoporotic fractures has usually been based on low BMD and/or prior fracture rather than the absolute risk of fracture. The recently completed primary-care based SCOOP screening study targeted treatment to those at highest hip fracture risk using FRAX. We wished to examine the impact of the screening intervention on hip fracture risk according to baseline FRAX hip fracture probability.

The SCOOP study comprised a two-arm randomised controlled trial in women aged 70-85 years comparing a screening programme vs. usual management. In the screening arm, treatment was recommended in women identified to be at high risk of hip fracture by FRAX (including BMD). Age-dependent intervention thresholds were used ranging from $5.24 \%$ in $70-74$ year olds to $8.99 \%$ hip fracture probability in 85 year olds.

Of 12,483 eligible participants, 6233 women were randomised to screening, with treatment recommended in 898 (14.4\%); amongst these, anti-osteoporosis medication had been prescribed in over $70 \%$ by 6 months. In the screening arm, the number of incident hip fractures was lower than that in the control arm (218 vs. 164) reflecting a $28 \%$ reduction in hip fracture risk (hazard ratio $0.72,95 \% \mathrm{CI}$ $0.59-0.89, \mathrm{p}=0.002)$. As treatment was targeted to those at highest risk, the effect on hip fracture increased significantly with baseline FRAX hip fracture probability $(p=0.021$ for interaction); for example at the 10th percentile of baseline FRAX hip probability $(2.6 \%)$, hip fractures were not significantly reduced (HR 0.93 , $0.71-1.23)$ but at the 90 th percentile $(16.6 \%)$, there was a $33 \%$ reduction (HR 0.67, 0.53-0.84).

Women identified to be at high fracture risk based on FRAX hip fracture probability are responsive to appropriate osteoporosis management. Community-based screening using the FRAX tool is feasible and effective.

\section{P072}

Prevalence and association of sarcopenia in asthma and chronic obstructive pulmonary disease: the Rotterdam Study

Elizabeth Benz $\mathrm{I}^{1,2}$, Katerina Trajanoska ${ }^{1,2}$, Josje D Schoufour ${ }^{1,2}$, Lies Lahousse $^{2,3}$, Emmely De Roos ${ }^{2,3}$, Natalie Terzikhan ${ }^{2,3}$, Bruno Stricker $^{2,4}$, Oscar H Franco ${ }^{2,5}$, Guy Brusselle ${ }^{2,3}$, Fernando Rivadeneira $^{1,2}$

${ }^{1}$ Internal Medicine, Erasmus MC University, Rotterdam, Netherlands, ${ }^{2}$ Epidemiology, Erasmus MC University, Rotterdam, Netherlands, ${ }^{3}$ Respiratory Medicine, Ghent University Hospital, Ghent, Belgium, ${ }^{4}$ Inspectorate of Healthcare, The Hague, Netherlands, ${ }^{5}$ Netherlands Consortium on Healthy Aging (NCHA), Leiden, Netherlands

Introduction: Sarcopenia has been recently recognized as an independent geriatric syndrome. According to the international European Working Group on Sarcopenia in Older People (EWGSOP), it is defined as the presence of low muscle mass and reduced function. Using these criteria, there are few studies in COPD that have reported higher prevalence of this extra-pulmonary manifestation. Further, it is unclear whether the prevalence of sarcopenia is the same in Asthma; or whether it is related with chronic respiratory diseases. Therefore, our aim was to determine the prevalence of sarcopenia among individuals with COPD and Asthma.

Methods: We performed cross-sectional analyses among 4876 participants (aged 69.41 years $\pm 9.03,44 \%$ male) in a populationbased study, who had an interpretable lung function test and an available assessment of sarcopenia defined by EWGSOP. To measure the association between sarcopenia and COPD/Asthma, we used logistic regression models adjusted for age, sex, cohort, smoking, weight, physical activity and a large range of other confounders.

Results: Prevalence of sarcopenia (7.1\%, 95\% CI 5.5-9.1\%) and pre-sarcopenia $(\mathbf{1 1 . 7 \%}, 95 \%$ CI 9.6-14.2\%) was significantly higher $(\mathrm{p}<0.05)$ in participants with COPD than in participants without COPD or Asthma $\mathbf{3 . 1 \%}, 95 \%$ CI $2.6-3.7 \%$ and $\mathbf{4 . 5} \%, 95 \% \mathrm{CI}$ $3.8-5.2 \%$ respectively).The risk of presenting sarcopenia or pre-sarcopenia was approximately twice as high in participants with COPD than in participants with no chronic respiratory diseases (sarcopenia: OR 2.55, 95\% CI 1.78-3.64 and pre-sarcopenia: OR 1.9, 95\% CI 1.15-3.01). Similar results were observed for participants with asthma (Sarcopenia: OR 2.46, 95\% CI 1.35-4.47 and pre-sarcopenia: OR $\mathbf{1 . 8 3}, 95 \%$ CI $1.11-3.03$ )

Conclusion: The prevalence of sarcopenia and pre-sarcopenia is twice as high in participants with chronic respiratory diseases. This support the importance of early detection and treatment to reverse this clinical condition in this selected population.

Keywords: sarcopenia, low lean mass, COPD, asthma, prevalence

\section{P074}

Genetic basis of falling risk susceptibility

Katerina Trajanoska ${ }^{1,2}$, Felix Day ${ }^{1,3}$, Carolina Medina-Gomez ${ }^{1}$, $\overline{\text { Andre G. Uitterlinden }}^{1}$, John Perry ${ }^{1,3}$, Fernando Rivadeneira ${ }^{1,2}$

${ }^{1}$ Internal Medicine, Erasmus Medical Center, Rotterdam, Netherlands, ${ }^{2}$ Epidemiology, Erasmus Medical Center, Rotterdam, Netherlands, ${ }^{3}$ MRC Epidemiology Unit, University of Cambridge School of Clinical Medicine, Cambridge, United Kingdom

Introduction: Falls and fall-related injuries are growing healthcare problem in elderly people. Multiple factors can increase the risk of falling. We aimed to determine how much of an individual's fall susceptibility can be attributed to genetics.

Methods: We conducted the first genome-wide association study (GWAS) to evaluate the genetic variation in fall risk in 89,076 cases and 362,103 controls from a large general cohort study. Individuals were genotyped with cohort specific chip. Cases were defined as those who reported having experienced a fall in the past year. The model included age and sex as covariates and was analysed with BOLTLMM (v.2.3.1). Next, we tested the predictive value of polygenetic risk scores (PRS) across diverse significance thresholds in relation to falls using individual level data from a population based cohort. Finally, we used LD score regression to: (1) estimate falling risk heritability, (2) test for genetic correlation with other traits and (3) identify tissues where expression of genes related to fall-associated variants are enriched.

Results: We identified two novel fall loci mapping near PER4 on $7 \mathrm{p} 21.3\left(\mathrm{rs} 2709062\right.$, OR $\left.1.03, \mathrm{p}=3.4 \times 10^{-08}\right)$ and near TSHZ3 on $19 \mathrm{q} 12\left(\mathrm{rs} 2111530\right.$, OR $\left.1.03, \mathrm{p}=1.2 \times 10^{-08}\right)$. Falling risk heritability was modest (2.5\%). PRS $\left(10^{-2}-10^{-4}\right.$ thresholds) were significant explaining small proportion of the trait variance. Falls had strong positive genetic correlation with fracture risk $(\mathrm{rg}=0.35$, $\left.\mathrm{p}=5.0 \times 10^{-21}\right)$, insomnia $\left(\mathrm{rg}=0.42, \mathrm{p}=1.0 \times 10^{-16}\right)$, neuroticism $\left(\mathrm{rg}=0.26, \quad \mathrm{p}=2.7 \times 10^{-12}\right) \quad$ and ADHD $\quad(\mathrm{rg}=0.44$, $\left.\mathrm{p}=1.2 \times 10^{-03}\right)$; and strong negative genetic correlation with hand grip $\left(\mathrm{rg}=-0.24, \mathrm{p}=1.0 \times 10^{-05}\right)$, intelligence $(\mathrm{rg}=-0.12$, $\left.\mathrm{p}=1.2 \times 10^{-03}\right)$ and subjective well-being $(\mathrm{rg}=-0.29$, $\left.\mathrm{p}=6.4 \times 10^{-10}\right)$. Brain and in particular cerebellum tissue showed the highest gene expression enrichment $(\mathrm{FDR}<5 \%)$ for fall-associated variants.

Conclusions: Falling risk constitutes a heritable, heterogeneous and polygenic trait genetically correlated with fracture risk and grip strength. The cerebellum tissue enrichment of falls-associated variants corroborates the mediation of postural balance in the etiology of falls. Keywords: falling risk, GWAS, association, shared heritability 


\section{P076}

Children with Spinal Muscular Atrophy: a bone picture

Francesca Broggi ${ }^{1}$, Silvia Vai ${ }^{1}$, Giovanni Baranello ${ }^{2}$, Riccardo Zanin $^{2}$, Maria Luisa Bianchi ${ }^{1}$

${ }^{1}$ Istituto Auxologico Italiano IRCCS, Milano, Italy, ${ }^{2}$ Fondazione Istituto Neurologico C. Besta IRCCS, Milano, Italy

Spinal Muscular Atrophy (SMA) is a group of inherited neuromuscular diseases leading to progressive motor disability. Only few data on its skeletal consequences (low bone mineral density [BMD] and fragility fractures) are currently available.

We longitudinally studied bone metabolism (serum osteocalcin $[\mathrm{OC}]$, bone specific alkaline phosphatase [BSAP], C-terminaltelopeptide of collagen type I [CTx], parathyroid hormone [PTH], and 25-OH vitamin D [25OHD]), spine BMD and fractures in 28 children (age $37.4 \pm 31.6$ months, range 14-171) affected by SMA type 2 .

All children had two visits (V1, V2) at intervals of 12-37 months (mean \pm SD $19 \pm 6.5$ ). At V1, 9 children showed 25OHD deficiency $(13.9 \pm 4.3 \mathrm{ng} / \mathrm{ml})$, which persisted at $\mathrm{V} 2$ in 6 children, notwithstanding calcifediol treatment.

At V1, 3 children reported 1 previous peripheral fracture each, and vertebral fractures were found in 4 children (1, 1, 2, 4 fractures). At V2, an incident peripheral fracture was reported. Spine BMD Z-score was $-0.7 \pm 1.5$ at $\mathrm{V} 1$ and $-1.8 \pm 0.9$ at V2 (22 children showed BMD decrease)

All biochemical values were evaluated by D'Agostino-Pearson and Shapiro-Wilk normality tests. Using Pearson's and Spearman rank correlation tests, at V1 we found positive correlations between ALP and both OC $(r=0.89, p<0.001)$ and CTx $(r=0.92$, $\mathrm{p}<0.001)$, BSAP and CTx $(\mathrm{r}=0.58, \mathrm{p}=0.001)$, CTx and OC $(\mathrm{r}=0.46, \mathrm{p}=0.01)$, and a negative correlation between PTH and 25OHD ( $\mathrm{r}=-0.54, \mathrm{p}=0.001)$. At V2, the correlation between CTx and OC ( $r=0.94)$ was confirmed; ALP, BMC and BMD were significantly different with respect to V1 (Wilcoxon signed rank test).

In conclusion, we observed worsening bone problems even in very young SMA children, with significant correlations between bone turnover markers. The negative correlation between PTH and 25OHD and the regression of PTH and 25OHD vs. BMD $(\mathrm{p}=0.04$ and $\mathrm{p}<0.001$ respectively) shows the importance of avoiding vitamin $\mathrm{D}$ deficiency.

Keywords: Spinal Muscular Atrophy, 25-hydroxy-vitaminD, bone turnover markers, bone mineral density

\section{P078}

Grade 1 vertebral height loss is not associated with frailty in the Canadian Multicentre Osteoporosis Study (CaMos)

Tayyab S Khan ${ }^{1}$, George Ioannidis ${ }^{1}$, Alexandra Papaioannou ${ }^{1}$, Courtney Kennedy ${ }^{1}$, Claudie Berger ${ }^{2}$, Brian Lentle ${ }^{3}$, Jacques Brown ${ }^{4}$, Linda Probyn ${ }^{5}$, Christopher S Kovacs ${ }^{6}$, David A. Hanley ${ }^{7}$, Jerrilynn Prior $^{3}$, David Goltzman ${ }^{2}$, Stephanie M. Kaiser ${ }^{8}$, Suzanne N. Morin ${ }^{2}$, William D Leslie ${ }^{9}$, K. Shawn Davison ${ }^{10}$, Tanveer Towheed ${ }^{11}$, Wilma Hopman $^{11}$, Wojciech Olszynski ${ }^{12}$, Jonathan D Adachi ${ }^{1}$

${ }^{1}$ McMaster University, Hamilton, Canada, ${ }^{2}$ McGill University, Montreal, Canada, ${ }^{3}$ University of British Columbia, Vancouver, Canada, ${ }^{4}$ Laval University and $\mathrm{CHU}$ de Quebec-(CHUL) Research Centre, Quebec City, Canada, ${ }^{5}$ University of Toronto, Toronto, Canada, ${ }^{6}$ Memorial University of Newfoundland, St. John, Canada, ${ }^{7}$ University of Calgary, Calgary, Canada, ${ }^{8}$ Dalhousie University, Halifax, Canada, ${ }^{9}$ University of Manitoba, Winnipeg, Canada, ${ }^{10} \mathrm{~A}$ Priori Medical Sciences Inc, Vancouver, Canada, ${ }^{11}$ Queen's University, Kingston, Canada, ${ }^{12}$ University of Saskatchewan, Saskatoon, Canada
Morphometric vertebral fractures (MVF) on X-rays are classified by using the Genant method into grade 1 (20-25\%), grade 2 (25-40\%), and grade $3(\geq 40 \%)$ based on degrees of vertebral height loss. While grades $\geq 2$ are associated with fracture risk, the significance of grade $1 \mathrm{MVF}$ is controversial. We have previously demonstrated no association between any grade of MVFs with changes in health related quality of life (HR-QoL) after adjusting for covariates. The prospective relationship between grade $1 \mathrm{MVF}$ and frailty has not been assessed.

Data from CaMos were used to determine the association between baseline grade $1 \mathrm{MVF}$ and changes in CaMos Frailty Index scores over 5 years, before and after adjusting for covariates. Ethics approval was obtained at each CaMos participating centre, and all participants signed informed consent.

We included 1450 men and 3770 women whose mean age was 65.4 years and mean baseline Frailty Index score was $0.13 \pm 0.10$. At baseline, $4450(85.3 \%)$ had no MVF, 394 (7.6\%) had grade 1, 243 (4.7\%) had grade 2, and $133(2.6 \%)$ had grade 3 MVF. Multivariable linear regression analysis identified no statistically significant association between baseline grade $1 \mathrm{MVF}$ and 5-year changes in Frailty Index scores. Those with MVF grades 2 and 3 experienced significant increases in unadjusted Frailty Index scores over 5 years. These associations were eliminated following adjustment for confounders (Table 1).

Grade 1 MVF was not associated with increased frailty over 5 years. Further studies are needed to assess their utility as markers of bone fragility.

Table 1: Regression analysis-MVF grade and frailty

\begin{tabular}{lll}
\hline $\begin{array}{l}\text { Grade of } \\
\text { MVF } \\
\text { Normal }\end{array}$ & $\begin{array}{l}\text { Unadjusted } \\
\text { estimates }(95 \% \text { C.I. })\end{array}$ & $\begin{array}{l}\text { Adjusted estimates (95\% C.I.) } \\
\text { Reference }\end{array}$ \\
\hline 1 & $-0.0002(-0.013,0.012)$ & $-0.003(-0.011,0.006)$ \\
2 and 3 & $0.054 *(0.040,0.067)$ & $0.002(-0.008,0.012)$
\end{tabular}

*Statistically significant

\section{P080}

Location of first spinal fracture as determinant of future vertebral fracture risk

Fjorda Koromani ${ }^{1,2,3}$, Ling Oei ${ }^{1,2}$, Stephan Breda ${ }^{3}$, Enisa Shevroja ${ }^{4}$, Joyce van Meurs $^{1}$, Arfan Ikram ${ }^{2}$, JH Waarsing ${ }^{5}$, Frank van Rooij ${ }^{2}$, Carola Zillikens ${ }^{1}$, Andre Uitterlinden ${ }^{1,2}$, Gabriel Krestin ${ }^{3}$, Fernando Rivadeneira $^{1,2}$, Edwin Oei ${ }^{3}$

${ }^{1}$ Internal Medicine, Erasmus Medical Center, Rotterdam, Netherlands, ${ }^{2}$ Epidemiology, Erasmus Medical Center, Rotterdam, Netherlands, ${ }^{3}$ Radiology, Erasmus Medical Center, Rotterdam, Netherlands, ${ }^{4}$ Bone \& Joint, Lausanne University Hospital, Lausanne, Switzerland, ${ }^{5}$ Orthopaedics, Erasmus Medical Center, Rotterdam, Netherlands

Background: Prevalent vertebral fractures (VFs) predict future VFs. We aimed to examine if the location of the first fracture holds different risk liabilities in the incident VFs distribution.

Methods: Prevalent radiographic VFs were scored in 3885 subjects aged 55 years or older at baseline and after a mean follow-up of 4 years; using SpineAnalyzer ${ }^{\circledR}$ Quantitative Morphology (QM) and Algorithm Based Qualitative (ABQ) methods. The association between spinal location of prevalent and incident VFs was examined 
using logistic regression models adjusted for age, sex, BMI, FNBMD and presence of prevalent VFs.

Results: At least one incident VF was observed in $8.7 \%(\mathrm{QM})$ and $2.0 \%(\mathrm{ABQ})$ of the participants. As compared to participants without VFs, participants with prevalent VFs had increased risk of incident VFs (OR 4.0; 95\% CI 2.0; 8.2 for ABQ) and (OR 1.6; 95\% CI 1.2; 2.3 for $\mathrm{QM})$. Individuals with multiple fractures at baseline had three (OR $2.7 ; 95 \%$ CI $1.7-4.2$ for QM) to twelve (OR $12.4 ; 95 \%$ CI $4.8-31.9$ for $\mathrm{ABQ}$ ) times increased likelihood of suffering incident VFs. Among all vertebral body locations, subjects with VFs arising at T8 had (with both methods) the highest risk for incident VFs (OR 16.0; 95\% CI 2.5-102.2 for ABQ and OR 4.0; 95\% CI 2.0-7.9 for QM) as compared to participants without VFs. Fractures at T7 scored with QM (OR 2.4; 95\% CI 1.9-5.2) and at T12 scored with ABQ (OR 5.6; 95\% CI 1.5-20.8) were also associated with incident VFs. No other vertebral body locations were significantly associated with future VFs risk.

Conclusions: Prevalent VFs at T8 are strongly and robustly associated with increased risk for future VFs independent of BMD and number of prevalent VFs; providing bio-mechanical support, to the contention that patients with first VFs at T8 (center of the kyphotic curvature) require special attention, might restore normal vertebral architecture and spinal curvature.

Keywords: vertebral fractures, epidemiology

\section{P082}

The longitudinal association between type 2 diabetes and fractures in a large Dutch cohort of older women

$\underline{\text { Petra Elders }}{ }^{1}$, Thomas Merlijn ${ }^{2}$, Karin Swart ${ }^{1}$, Coen Netelenbos ${ }^{3}$

${ }^{1}$ General Practice and Elderly Care, VUmc medical centre, Amsterdam, Netherlands, ${ }^{2}$ General Practice and Elderly Care, Vumc Medical Centre, Amsterdam, Netherlands, ${ }^{3}$ Department of Internal Medicine, Endocrine Section, VUmc medical centre, Amsterdam, Netherlands

Background: Type 2 diabetes mellitus (T2DM) is associated with an increased fracture risk, despite higher bone mineral density (BMD). Glucose control might be of influence. We examined the association of T2DM and fractures during a 3-year follow-up period in a large primary care sample of older women.

Methods: Data were used from the SALT Osteoporosis Study, a randomized pragmatic trial among 11,331 women aged 65 years and older with one clinical risk factor for fractures in a primary care setting. Questionnaires were completed at baseline and after 18 and 36 months. Self-reported baseline TDM2 was verified with GP medical records, from which baseline $\mathrm{HbA} 1 \mathrm{c}$ concentrations were derived as well. The main outcome was fractures. Self-reported fractures were verified with medical records from the GP or hospital. Secondary outcomes were hip fractures, baseline vertebral fractures, baseline BMD of the hip and the lumbar spine, and falling. Baseline vertebral fractures assessment and BMD measurements were done in a subsample $(n=4310)$.

Results: In 1578 out of 11,331 women (13.9\%),TDM2 was identified. In these women mean $\mathrm{HbAlc}$ was $51.7 \mathrm{mmol} / \mathrm{mol} ; 66.8 \%$ had a $\mathrm{HbA} 1 \mathrm{c} \leq 53 ; 78.1 \%$ used oral anti diabetes drugs and $15.6 \%$ used insulin. 163 out of 1578 women with TDM2 sustained a fracture $(10.3 \%)$ versus 1101 out of 9537 women without DMII (11.5\%). T2DM was not associated with incident fractures of any type (adjusted HR 1.00, 95\% CI 0.84:1.19) or hip fractures (adjusted HR 1.28, 95\% CI 0.92:1.78). Women with T2DM had a significantly higher BMD of the hip (adjusted Beta $=0.04,95 \%$ CI 0.03:0.05) and BMD of the lumbar spine (adjusted Beta $=0.03,95 \%$ CI 0.02:0.04). No associations with baseline vertebral fractures or falling were observed. HbA1c among women with T2DM (analysed as continuous measure or as tertile categories) was not associated with any of the outcomes.

Conclusions: T2DM was not a risk factor for fractures in this primary care population.

\section{P084}

The effect of teriparatide and denosumab on circulating microRNAs related to bone metabolism in women with postmenopausal osteoporosis

Athanasios Anastasilakis ${ }^{1}$, Polyzois Makras ${ }^{2}$, Symeon Tournis ${ }^{3}$, Maria Pikilidou ${ }^{4}$, Konstantinos Makris ${ }^{3}$, Ilias Bisbinas ${ }^{1}$, Olga Tsave ${ }^{5}$, John Yovos ${ }^{5}$, Maria Yavropoulou ${ }^{5}$

${ }^{1}$ Department of Endocrinology, 424 General Military Hospital, Thessaloniki, Greece, ${ }^{2}$ Department of Endocrinology and Diabetes, 251 Hellenic Air Force \& VA General Hospital, Athens, Greece, ${ }^{3}$ Laboratory of Research of Musculoskeletal System 'Th. Garofalidis', University of Athens, Athens, Greece, ${ }^{4}$ Laboratory of Clinical and Molecular Endocrinology 1st Department of Internal Medicine, Aristotle University of Thessaloniki, Thessaloniki, Greece, ${ }^{5}$ Laboratory of Clinical and Molecular Endocrinology Department of Internal Medicine, Aristotle University of Thessaloniki, Thessaloniki, Greece

Expression of microRNAs (miRs) related to bone metabolism in the serum might be affected by anti-osteoporotic treatment. In order to address this issue we investigated the effect of two potent antiosteoporotic agents with opposite effects on bone metabolism, namely teriparatide and denosumab, on microRNA expression profile in the serum.

Patients and Methods: This is an observational, open label, nonrandomized clinical trial that included 60 postmenopausal women with osteoporosis treated with either teriparatide $(n=30)$ or denosumab $(\mathrm{n}=30)$ for 12 months.

Main outcome measures: Changes in the serum relative expression of selected miRs linked to bone metabolism at 3 and 12 months of treatment.

Secondary measurements: Associations of measured miRs with changes in bone mineral density (BMD) at 12 months and the bone turnover markers (BTM) C-terminal telopeptide of type I collagen ( $\beta$ $\mathrm{CTX}$ ) and procollagen type I N-terminal propeptide (P1NP) at 3 and 12 months.

Results: We found significantly decreased the relative expression of miR-33-3p at 3 months (fold change 0.71, p = 0.03) and of miR$133 \mathrm{a}$ at 12 months (fold change $0.76, \mathrm{p}=0.042$ ) of teriparatide treatment. BMD values at 12 months of teriparatide treatment were significantly and inversely correlated with miR-124-3p expression at 3 months $(\mathrm{p}=0.008)$, and this correlation remained robust after adjustment for possible confounders. The relative expression of miR24-3p and miR-27a was correlated with changes in BTM during teriparatide treatment and of miR-21-5p, miR-23a-3p, miR-26a-5p, miR-27a, miR-222-5p and miR-335-5p with changes in BTM during denosumab treatment.

Conclusions: Circulating miRs linked to bone metabolism are differentially affected by treatment with teriparatide and denosumab, possibly reflecting their different mechanism of action. Treatment with teriparatide affects the relative expression of mirRs related to the expression of RUNX-2 (miR-33) and DKK-1 gene (miR-133), suggesting that these pathways are regulated by teriparatide at both transcriptional and post-transcriptional level. 


\section{P086}

Burosumab, an anti-FGF23 monoclonal antibody, for X-Linked Hypophosphatemia (XLH): analysis by age from two phase 2 pediatric trials

Wolfgang Högler ${ }^{1}$, Thomas O. Carpenter ${ }^{2}$, Erik Imel ${ }^{3}$, Anthony A. Portale $^{4}$, Annemieke Boot ${ }^{5}$, Agnès Linglart ${ }^{6}$, Raja Padidela ${ }^{7}$, William van't Hoff $^{8}$, Gary S. Gottesman ${ }^{9}$, Meng Mao ${ }^{10}$, Alison Skrinar ${ }^{10}$, Javier San Martin ${ }^{10}$, Michael P. Whyte ${ }^{9}$

${ }^{1}$ Birmingham Children's Hospital, Birmingham, United Kingdom, ${ }^{2}$ Yale School of Medicine, New Haven, United States, ${ }^{3}$ Indiana University School of Medicine, Indianapolis, United States, ${ }^{4}$ University of California, San Francisco, San Francisco, United States, ${ }^{5}$ University of Groningen, Groningen, Netherlands, ${ }^{6}$ Hôpital Bicêtre, Le KremlinBicêtre, France, ${ }^{7}$ Royal Manchester Children's Hospital, Manchester, United Kingdom, ${ }^{8}$ Great Ormond Street Hospital, London, United Kingdom, ${ }^{9}$ Shriners Hospitals for Children, St Louis, United States, ${ }^{10}$ Ultragenyx Pharmaceutical Inc., Novato, United States

We evaluated efficacy and safety of burosumab, a fully human monoclonal antibody against FGF23, in children with XLH of different age groups, in two Phase 2 trials.

In study CL201, 52 children with XLH (5-12 years old [years], Tanner $\leq 2$ at baseline) were randomized $1: 1$ to receive subcutaneous (SC) burosumab every two $(\mathrm{Q} 2 \mathrm{~W})$ or four (Q4W) weeks for 64 weeks. Doses were titrated up to a maximum of $2 \mathrm{mg} / \mathrm{kg}$ to achieve serum phosphorus levels within 1.1-1.6 mmol/dL. In study CL205, 13 children with XLH, 1-4 years, received SC burosumab $0.8 \mathrm{mg} / \mathrm{kg}$ Q2W, or increased to $1.2 \mathrm{mg} / \mathrm{kg}$ if serum phosphorus remained low.

Results are reported by baseline age (years): $1-4,5-7,8-9$, and 10-12. Mean burosumab dose at week 40 in CL201 and CL205 was $\sim 1 \mathrm{mg}$ Q2W. Dose for Q2W or Q4W did not differ substantially by age. Regardless of regimen, mean serum phosphorus increased (range +0.1 to $+0.4 \mathrm{mmol} / \mathrm{dL}$ ) in all age groups by week 40 . Total Rickets Severity Score decreased from baseline to week 40 across all age groups, as did serum alkaline phosphatase (ALP). One subject in each study experienced a serious AE: hospitalization for fever/muscle pain resolving within a day (CL201) and a dental abscess (CL205). Other AEs were generally mild-moderate in severity. No clinically meaningful changes in serum calcium or iPTH occurred. No subjects discontinued therapy or developed hyperphosphatemia.

Treatment response was consistent across age groups. Burosumab was associated with increases in serum phosphorus, decreases in ALP, and improvements in rickets in children with XLH.

Bone efficacy by age (mean or LS mean change \pm SE)

\begin{tabular}{|c|c|c|c|c|}
\hline $\begin{array}{l}\text { Age at } \\
\text { baseline } \\
\text { (study) }\end{array}$ & $\begin{array}{l}1-<5 \text { years } \\
(205)\end{array}$ & $\begin{array}{l}5-<8 \text { years } \\
(201)\end{array}$ & $\begin{array}{l}8- \\
<10 \text { years } \\
(201)\end{array}$ & $\begin{array}{l}10-12 \text { years } \\
(201)\end{array}$ \\
\hline $\begin{array}{l}\text { Dose } \\
\quad \text { regimen } \\
\text { (n) }\end{array}$ & Q2W (13) & $\begin{array}{l}\text { Q2W (6) } \\
\text { and Q4W } \\
\text { (7) }\end{array}$ & $\begin{array}{l}\text { Q2W (12) } \\
\text { and Q4W } \\
\text { (12) }\end{array}$ & $\begin{array}{c}\text { Q2W (8) and } \\
\text { Q4W (7) }\end{array}$ \\
\hline
\end{tabular}

Serum ALP

\begin{tabular}{lllll} 
Baseline & $549 \pm 54$ & $446 \pm 23$ & $462 \pm 24$ & $466 \pm 27$ \\
Week 40 & $335 \pm 24$ & $384 \pm 29$ & $391 \pm 18$ & $412 \pm 27$ \\
Change & $-213 \pm 14$ & $-63 \pm 23$ & $-70 \pm 20$ & $-54 \pm 19$ \\
P value & $<0.0001$ & $<0.05$ & $<0.01$ & $<0.05$ \\
Total RSS & & & & \\
Baseline & $2.9 \pm 0.4$ & $1.2 \pm 0.2$ & $1.9 \pm 0.3$ & $2.2 \pm 0.2$ \\
Week 40 & $1.2 \pm 0.1$ & $0.9 \pm 0.1$ & $1.0 \pm 0.1$ & $0.8 \pm 0.1$ \\
Change & $-1.7 \pm 0.1$ & $-0.4 \pm 0.1$ & $-0.9 \pm 0.1$ & $-1.4 \pm 0.1$ \\
P value & $<0.0001$ & $<0.0001$ & $<0.0001$ & $<0.0001$ \\
\hline
\end{tabular}

\section{P088}

Effects of treatment with an angiotensin 2 receptor blocker and/ or vitamin $\mathrm{D}$ on parathyroid hormone and aldosterone: a randomized, placebo-controlled trial

Lise Sofie Bislev $^{1,2}$, Lene Langagergaard Rødbro ${ }^{1}$, Lars Rolighed ${ }^{3,4}$, Tanja Sikjær ${ }^{1}$, Lars Rejnmark ${ }^{1,2}$

${ }^{I}$ Department of Endocrinology and Internal Medicine, Aarhus University Hospital, Aarhus C, Denmark, ${ }^{2}$ Department of Clinical Medicine, Aarhus University, Aarhus C, Denmark, ${ }^{3}$ Department of Otolaryngology, Aarhus University Hospital, Aarhus C, Denmark, ${ }^{4}$ Department of Surgery P, Aarhus University Hospital, Aarhus C, Denmark

Objective: Emerging evidence support a positive, bidirectional and clinical relevant interaction between parathyroid hormone $(\mathrm{PTH})$ and the renin-angiotensin-aldosterone-system (RAAS). PTH receptors are present in the adrenal cortex, and angiotensin 2 as well as mineralocorticoid receptors in the parathyroid gland. A beneficial cardiovascular effect of the widely used RAAS inhibitors might also include a PTH lowering effect, as high PTH levels may be harmful to cardiovascular health.

We aimed to investigate whether short-term treatment with an angiotensin 2 receptor blocker (valsartan) independently of co-administration of vitamin D reduced PTH. Secondary end-points included effects of vitamin D on blood pressure and concentrations of renin and aldosterone.

Design and Methods: In a double-blind placebo-controlled trial, we included 81 otherwise healthy postmenopausal women with secondary hyperparathyroidism $(\mathrm{PTH}>6.9 \mathrm{pmol} / \mathrm{L})$ and vitamin $\mathrm{D}$ insufficiency $(25(\mathrm{OH}) \mathrm{D}<50 \mathrm{nmol} / \mathrm{L})$. Participants received twoweeks of treatment with valsartan $80 \mathrm{mg} /$ day, vitamin D3 $70 \mu \mathrm{g} /$ day, valsartan plus vitamin D3, or double-placebo.

Results: No women dropped out, and compliance was $99.4 \%$ for vitamin D and $98.9 \%$ for Valsartan. Valsartan treatment did not affect plasma PTH, although treatment reduced diastolic blood pressure $(\mathrm{p}=0.01)$ and the aldosterone/renin ratio $(\mathrm{p}<0.001)$. We found no associations between calciotropic hormones and renin or aldosterone. Vitamin D supplementation reduced PTH by $3.4 \%$ (interquartile range $-9.0-8.7$ ) compared to a $7.1 \%$ increase (interquartile range $-2.4-30.9)$ in the placebo group $(p=0.01)$, but did not affect blood pressure or concentrations of renin and aldosterone.

Conclusions: Independently of vitamin D, short-term valsartan treatment does not reduce PTH. Vitamin D reduced PTH but did not affect blood pressure or the RAAS. The study does not support a direct interaction between PTH and aldosterone or a causal effect of a vitamin D-mediated PTH reduction on blood pressure.

Keywords: Angiotensin 2 receptor blocker, Vitamin D, PTH, aldosterone, blood pressure

\section{Poster SNAPs 1}

\section{P001}

Protein Tyrosine Kinase 7 is a critical factor for osteoblast differentiation of human skeletal stem cells and its expression is decreased in patients with osteoporosis

Abbas Jafari $^{1,2}$, Weimin Qiu ${ }^{2}$, Majken S. Siersbaek ${ }^{2}$, Nicholas

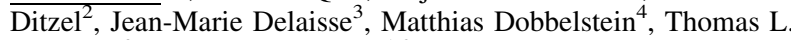
Andersen $^{3}$, Moustapha Kassem ${ }^{1,2}$

${ }^{1}$ The Novo Nordisk Foundation Center for Stem Cell biology (DanStem), Institute of Cellular \& Molecular Medicine, University of Copenhagen, Copenhagen, Denmark, ${ }^{2}$ Department of Endocrinology 
and Metabolism, Endocrine Research Laboratory (KMEB), Odense University Hospital \& University of Southern Denmark, Odense, Denmark, ${ }^{3}$ Department of Clinical Cell Biology, Vejle/Lillebaelt Hospital, Vejle, Denmark, ${ }^{4}$ Göttingen Center of Molecular Biosciences, University of Göttingen, Göttingen, Germany

Differentiation of human skeletal (mesenchymal) stem cells (hMSC) into bone-forming osteoblasts is a critical step in human bone remodeling. Compromised osteoblast differentiation is a major contributor to the bone loss causing osteoporotic fractures. Hence, new insight into this process is needed for the development of novel anabolic therapies. Protein Tyrosine Kinase 7 (PTK7) is a receptor tyrosine kinase that is highly expressed in cultured human MSC and in osteoblastic cells (e.g. osteoprogenitor canopy cells and mature osteoblastic bone lining cells) on the bone surfaces in iliac crest bone biopsies from healthy donors. PTK7 levels were increased during differentiation of hMSC toward osteoblast (OB) lineage. Silencing of PTK7 expression in ex vivo hMSC cultures, inhibited OB differentiation and in vivo heterotopic bone formation. PTK7 overexpression exerted opposite effect i.e. enhanced $\mathrm{OB}$ differentiation and bone formation. The regulatory effects of PTK7 on OB differentiation were mediated through stimulation of the non-canonical Wnt signaling, evidenced by increased expression of non-canonical Wnt ligands (e.g. Wnt5A, Wnt11) and increased activation status of different signaling molecules involved in this pathway (e.g. c-Jun, CamKII, CREB). Searching for the up-stream regulators of PTK7 that are relevant to bone biology, we found that TNF- $\alpha$ treatment led to a dose dependent inhibition of PTK7 expression and protein levels. In addition, histomorphometric analysis of the bone biopsies from patients with osteoporosis revealed significantly reduced levels of PTK7 in osteoblastic cells. Our data suggest a model where osteoporosis-related increased TNF- $\alpha$ inhibits PTK7 expression resulting in impaired bone formation due to compromised non-canonical Wnt signaling.

Keywords: PTK7, Human skeletal stem cells, Osteoblast, Noncanonical Wnt signaling, Osteoporosis

\section{P003}

Pharmacodynamic results from a phase $2 \mathrm{a}$, randomized, multicenter, open-label, dose-ranging study of asfotase alfa in adults with pediatric hypophosphatasia

Lothar Seefried $^{1}$, Priya Kishnani ${ }^{2}$, Scott Moseley ${ }^{3}$, Eric Watsky ${ }^{3}$, Michael Whyte $^{4,5}$, Kathryn Dahir ${ }^{6}$

${ }^{1}$ Orthopedic Department, University of Würzburg, Würzburg, Germany, ${ }^{2}$ Department of Pediatrics, Duke University Medical Center, Durham, United States, ${ }^{3}$ Alexion Pharmaceuticals, Inc., New Haven, United States, ${ }^{4}$ Center for Metabolic Bone Disease and Molecular Research, Shriners Hospital for Children, St. Louis, United States, ${ }^{5}$ Division of Bone and Mineral Diseases, Department of Internal Medicine, Washington University School of Medicine at BarnesJewish Hospital, St. Louis, United States, ${ }^{6}$ Division of Diabetes and Endocrinology, Vanderbilt University Medical Center, Nashville, United States

Hypophosphatasia (HPP) is a rare, inherited metabolic disease characterized by low tissue-nonspecific alkaline phosphatase (TNSALP) activity leading to extracellular accumulation of inorganic pyrophosphate (PPi) and pyridoxal 5'phosphate (PLP). Asfotase alfa (AA) is a recombinant human TNSALP replacement therapy approved for pediatric-onset HPP. A 13-week, Phase 2a, randomized, open-label, dose-response study (NCT02797821) evaluated AA in 27 adults (aged $\geq 18$ years) with pediatric-onset HPP; we report pharmacodynamic and safety/tolerability results. Patients diagnosed with HPP, Screening PPi $\geq 3.9 \mu \mathrm{M}$, and TNSALP gene mutation(s) or low Screening alkaline phosphatase with elevated PLP were randomized $1: 1: 1$ to AA $0.5,2.0$, or $3.0 \mathrm{mg} / \mathrm{kg}$ single dose at week 1, then 3 times/week (i.e., 1.5, 6.0, or $9.0 \mathrm{mg} / \mathrm{kg} /$ week) starting at week 3 for 7 weeks. Primary endpoint was plasma PPi change from baseline to week 9 (Day 61, last predose value; Table). Demographics included median age (range): 45.0 (18-77) years; median weight: 80.5 (48-121) kg; sex: $59 \%$ female; and race: $96 \%$ white. Adverse events (AEs) of interest included injection site reactions (21 patients), lipodystrophy (1 patient), ectopic calcifications (4 patients), and hypersensitivity (1 patient). Overall, 99\% (480/485) of AEs were mild/moderate, with no serious AEs or withdrawals. AA at 6.0 and $9.0 \mathrm{mg} / \mathrm{kg} / \mathrm{week}$ significantly decreased PPi and PLP from Baseline vs. $1.5 \mathrm{mg} / \mathrm{kg} / \mathrm{week}$ in adults with pediatric-onset HPP and was well tolerated.

\begin{tabular}{|c|c|c|c|c|}
\hline & $\begin{array}{l}\text { AA } \\
1.5 \mathrm{mg} / \\
\mathrm{kg} / \text { week } \\
(\mathrm{n}=8) \\
\text { LSM cha } \\
(\mathrm{SE})\end{array}$ & $\begin{array}{l}\text { AA } \\
6.0 \mathrm{mg} / \\
\mathrm{kg} / \mathrm{week} \\
(\mathrm{n}=10) \\
\text { ge from B }\end{array}$ & $\begin{array}{l}\text { AA } \\
9.0 \mathrm{mg} / \\
\mathrm{kg} / \text { week } \\
(\mathrm{n}=9) \\
\text { to week }\end{array}$ & $\begin{array}{l}\text { LSM difference } \\
\text { estimate between } \\
\text { dose groups }\end{array}$ \\
\hline $\mathrm{PPi}(\mu \mathrm{M})$ & $\begin{aligned}- & 2.6 \\
& (0.2)\end{aligned}$ & $\begin{aligned}- & 3.8 \\
& (0.2)\end{aligned}$ & $\begin{array}{r}-4.5 \\
(0.2)\end{array}$ & $\begin{array}{l}1.5 \text { vs. } 9.0 \mathrm{mg} / \\
\text { kg/week: } \\
-1.9 \\
(\mathrm{P}<0.0001) \\
1.5 \text { vs. } 6.0 \\
\mathrm{mg} / \mathrm{kg} / \text { week: } \\
-1.2 \\
(\mathrm{P}=0.0008)\end{array}$ \\
\hline $\begin{array}{c}\text { PLP (ng/ } \\
\text { mL) }\end{array}$ & $\begin{array}{r}-304 \\
(9.4)\end{array}$ & $\begin{array}{r}-333 \\
(8.2)\end{array}$ & $\begin{array}{r}-338 \\
(8.5)\end{array}$ & $\begin{array}{l}1.5 \mathrm{vs} .9 .0 \mathrm{mg} / \\
\text { kg/week: } \\
-34.0 \\
(\mathrm{P}=0.0128) 1 \\
.5 \mathrm{vs} .6 .0 \mathrm{mg} / \\
\mathrm{kg} / \\
\text { week: }-29.5 \\
(\mathrm{P}=0.0239)\end{array}$ \\
\hline
\end{tabular}

$L S M$ least squares mean, $B L$ baseline, $S E$ standard error

Keywords: alkaline phosphatase; metabolic bone disease; hypophosphatasia

\section{P005}

Elevated phosphate levels impair skeletal muscle cell differentiation in vitro

Adalbert Raimann $^{1}$, Susanne Greber-Platzer ${ }^{1}$, Alexander Dangl ${ }^{1}$, Monika Egerbacher $^{2}$, Gabriele Haeusler ${ }^{1}$, Peter Pietschmann ${ }^{3}$

${ }^{1}$ Department of Pediatrics and Adolescent Medicine, Medical University of Vienna, Vienna, Austria, ${ }^{2}$ University of Veterinary Medicine Vienna, Vienna, Austria, ${ }^{3}$ Center for Pathophysiology, Infectiology and Immunology, Medical University of Vienna, Vienna, Austria

Background: Hyperphosphatemic conditions such as chronic kidney disease are associated with severe muscle wasting and impaired life quality. Regeneration of muscle tissue is reliant on recruitment of myogenic progenitor cells, the effects of high phosphate loads on this process has not been investigated in detail so far. This study aims to clarify the direct effects of hyperphosphatemic conditions on skeletal myoblast differentiation in an established murine cell model system.

Material and Methods: C2C12 murine muscle progenitor cells were differentiated with physiological and pathological phosphate loads. Phosphate-induced changes in marker gene expression were 
quantified by RT-PCR. Furthermore, immunohistochemistry was performed to investigate nuclear positive cell counts under treatment. Cell viability and metabolic activity were measured by XTT and BrdU incorporation assays. All experiments were performed in $\geq 3$ independent runs.

Results: Inorganic phosphate directly induces ERK-phosphorylation in pre-differentiated $\mathrm{C} 2 \mathrm{C} 12$ myoblast cells. Phosphate concentrations resembling moderate and severe hyperphosphatemia (1.4-2.9 mmol/l) impaired the expression of differentiation markers Myogenin $(-61.0 \%, \quad \mathrm{p}<0.0001)$ and MyoD $\quad(-51.0 \%$; $\mathrm{p}<0.0001)$. While higher phosphate loads showed more pronounced effects, even moderately hyperphosphatemic conditions could significantly reduce Myogenin $(-22.5 \%, p=0.015)$, and Myf5 $(-33.3 \%, p=0.039)$ expression. Analogue effects were found on the protein level, where a significantly decreased count of Myogenin $(-42.0 \%, \mathrm{p}=0.004)$ and MyoD positive cells $(-25,7 \%, \mathrm{p}=0.002)$ was found in phosphate enriched medium. Increased phosphate concentration left metabolic activity and cellular proliferation rate unaffected.

Conclusion: Our data point to a phosphate-induced inhibition of myoblast differentiation without effects on cell viability. Strikingly, even phosphate levels corresponding to a serum value in the upper normal range significantly impaired marker gene and protein expression. Investigation of cellular responses during hyperphosphatemia may help to define serum phosphate cutoffs and modify existing treatment approaches of phosphate binders, especially in patients at risk for sarcopenia.

\section{P007}

Collagen, extracellular matrix and angiogenic biomarkers in octeoporosis in idiopathic pulmonary arterial hypertension Ludmila Ugay $^{1}$, Evgeniya Kochetkova ${ }^{2}$, Yulia Maistrovskaia ${ }^{1}$

${ }^{1}$ Pacific State Medical University, Vladivostok, Russian Federation, ${ }^{2}$ Multihealth, Vélizy-Villacoublay, France

Aim: To evaluate the relationship between circulating biomarkers of collagen metabolism, extracellular matrix and vascular remodeling with bone mineral density (BMDs) in idiopathic pulmonary arterial hypertension (iPAH) NYHA class III-IV. Pulmonary and hemodynamic parameters, BMD Z-scores at lumbar spine (LS) and femoral neck (FN), serum C-terminal telopeptide of type I collagen (CITP), metalloproteinase-9 (MMP-9) and its tissue inhibitor (TIMP-1), and complex MMP-9/TIMP-1, osteoprotegerin (OPG), endothelin-1 (ET1), vascular endothelial growth factor (VEGF), and soluble VEGF receptor-1 (sVEGFR1) were evaluated in 38 patients with $\mathrm{PAH}$ NYHA III-IV and 30 healthy volunteers.

CITP, MMP-9, TIMP-1 and MMP-9/TIMP-1 complex ET-1, VEGF and sVEGFR1 were higher in iPAH than in healthy while OPG were not statistically different compared to that of healthy.

The univariate analysis revealed a positive correlation between BMD Z-scores at both sites and 6-min walk test (FN: $p=0.000$; LS: $p=0.003$ ), and inverse relation with pulmonary vascular resistance (PVR) (FN: $p=0.0005$; LS: $p=0.003$ ) and mean pulmonary arterial pressure (mPAP) $(p=0.007)$. CITP, MMP-9 and TMIP-1 had positive correlations with $\mathrm{mPAP}$ and PVR, and negatively with LS and FN Z-scores.

ET-1 related to $\operatorname{mPAP}(p=0.001)$, cardiac index $(p=0.004)$ and PVR $(p=0.000), \mathrm{VEGF}$ and $\mathrm{sVEGFR} 1$ correlated with $\operatorname{mPAP}(p=0.006$ and $p=0.02$, respectively) and PVR ( $p=0.000$ and $p=0.000$, respectively). Negative correlation was observed between ET-1, sVEGFR1 and FN Z-scores $(p=0.004)$ and LS Z-scores $(p=0.035)$. VEGF indirectly correlated with FN BMD Z-scores $(p=0.000)$.

Positive correlations revealed between MMP-9, TIMP-1, MMP-9/ TMIP-1 complex, VEGF, sVEGFR1 and increased CITP.
Conclusion: Worsening endothelial dysregulation is associated with increased levels of osteopenia in $\mathrm{PAH}$; endothelial dysfunction may contribute to osteopenic syndrome development through mechanistic pathways associated with elevated ET-1 and angiogenic factors.

Keywords: iPAH, osteoporosis, endothelin-1, collagen, metalloproteinase, VEGF

This study was supported by a grant from Russian Science Foundation (No. 14-33-00009 П).

\section{P009}

Effects of postnatal osteoblast-specific suppression of platelet-derived growth factor receptors $\alpha$ and $\beta$ on bone metabolism

Cyril Thouverey $^{1}$, Joseph Caverzasio ${ }^{1}$, Serge Ferrari ${ }^{1}$

${ }^{1}$ Service of Bone Diseases, University Hospital of Geneva, Geneva, Switzerland

Platelet-derived growth factor (PDGF) BB, which can act through both PDGF receptors (PDGFR) $\alpha$ and $\beta$, is secreted by pre-osteoclasts in the bone microenvironment. Osteoblast lineage cells highly express PDGFR $\alpha$ and PDGFR $\beta$, suggesting that PDGF-BB/PDGFR signaling may directly regulate osteoblast development and function. To investigate the role of PDGFR $\alpha / \beta$ in osteoblast biology, we generated mice lacking PDGFR $\alpha$, PDGFR $\beta$ or both receptors in osteoblast lineage cells by breeding mice expressing the Cre recombinase under the control of an inducible Osterix promoter (Osx-Cre) with mice harboring floxed genes encoding PDGFR $\alpha$ or PDGFR $\beta$. The Cre expression and consequent Pdgfra or/and $P d g f r b$ inactivation were induced at 1.5 month of age by stopping doxycycline treatment. The bone phenotype of control (Osx-Cre), $\Delta P d g f r a, \Delta P d g f r b$ and $\Delta P d g f r a ; \Delta P d g f r b$ were assessed by micro-computed tomography at 4.5 months of age. All mutant mice were of normal weight and size. $\Delta P d g f r a$ mice did not exhibit any bone phenotype. $\Delta P d g f r b$ mice did not show any difference in cortical bone geometry but displayed increased trabecular bone volume at proximal tibiae $(+26 \%$, p $\leq 0.03)$ associated with elevated number of trabeculae $(+16 \%, \mathrm{p}$ $\leq 0.04) . \Delta P d g f r a ; \Delta P d g f r b$ mice exhibited a further increase in trabecular bone volume at proximal tibiae $(+50 \%, \mathrm{p} \leq 0.02)$ and augmented cortical bone volume at tibial midshafts $(+24 \%, \mathrm{p}$ $\leq 0.04$ ). In conclusion, suppression of PDGFR signaling in osteoblasts increases trabecular and cortical bone mass, and ongoing histomorphometric analyses will help to clarify mechanisms involved in PDGFR-mediated regulation of trabecular, endosteal and periosteal bone envelopes.

\section{P011}

Collagen orientation and mechanical competence of osteoporotic and bisphosphonate treated human bone

Kilian Stockhausen ${ }^{1}$, Katherina Lewandowski ${ }^{1}$, Katharina Jähn ${ }^{1}$, Michael Hahn $^{1}$, Michael Amling ${ }^{1}$, Björn Busse ${ }^{1}$

${ }^{I}$ Department of Osteology and Biomechanics, University Medical Center Hamburg-Eppendorf, Hamburg, Germany

The cortical microstructure represents an important factor of bone strength and collagen fiber orientation (CFO) contributes to the biomechanical competence of bone. Longitudinal fibers are found in regions supporting tensile loads, while transverse fibers correspond to regions under compression. During walking, bending forces load the anterior and anterolateral femoral cortex in tension, and the posteromedial and medial cortex in compression. Although CFO patterns have been investigated for healthy bone, possible CFO alterations in 
osteoporotic (OP) and bisphosphonate-treated (BP) bone remain unknown.

Mid-diaphyseal femoral bone specimen were acquired from 23 female individuals $\left(\mathrm{n}_{\mathrm{OP}}=13 ; 81.4 \pm 6.8\right.$ years and $\mathrm{n}_{\mathrm{BP}}=10$; $82.3 \pm 8.4$ years) after ethical approval. BP patients received a thirdgeneration $\mathrm{BP}$ alendronate for $6 \pm 1.6$ years. CFO properties were investigated using quantitative polarized light microscopy and expressed as a mean grayscale value (high values = transverse $\mathrm{CFO}$; low values = longitudinal CFO). Mechanical properties were assessed by reference point indentation. Non-parametric tests were employed to determine statistical significance $(\mathrm{p}<0.05)$.

In $\mathrm{OP}$, the lateral femur was characterized by more longitudinal CFO compared to all other anatomical axes $(57.13 \pm 11.27$ vs. $64.15 \pm 12.00$ (anterior), $65.68 \pm 15.76$ (posterior), $65.75 \pm 13.42$ (medial)). Following BP treatment, the medial femur showed more transversal CFO compared to the anterior and lateral axes $(63.82 \pm 14.32$ vs. $54.70 \pm 12.90$ (anterior), $52.18 \pm 9.41$ (lateral)). Medially, the total indentation distance was significantly smaller compared to the anterior and posterior axes $(50.49 \pm 2.67 \mu \mathrm{m}$ vs. $51.41 \pm 1.96 \mu \mathrm{m}$ (anterior), $51.70 \pm 2.14 \mu \mathrm{m}$ (posterior)). Between groups, CFO was significantly more transversal in the anterior axis with OP $(64.15 \pm 12.00$ vs. $54.70 \pm 12.90)$. No intergroup differences were found in the mechanical properties.

Our study shows that CFO of OP bone deviates from the physiological loading pattern and BP treatment resulted in a CFO closer resembling the healthy loading pattern. The collagen orientation analysis highlights that under BP treatment collagen is formed to resist habitual loading scenario suggesting a positive treatment effect regarding bone quality.

Keywords: Osteoporosis; Collagen Orientation; Bisphosphonate; Polarized Light

\section{P013}

Global vs. osteoprogenitor-specific Mct8 knockout exerts distinct effects on bone mass and turnover of male mice

Franziska Lademann ${ }^{1,2}$, Heike Heuer ${ }^{3}$, Elena Tsourdi ${ }^{1,2}$, Eddy Rijntjes $^{4}$, Josef Köhrle ${ }^{4}$, Lorenz C. Hofbauer ${ }^{1,2,5}$, Martina Rauner ${ }^{1,2}$

${ }^{1}$ Department of Medicine III, Technische Universität Dresden Medical Center, Dresden, Germany, ${ }^{2}$ Center for Healthy Aging, Dresden, Germany, ${ }^{3}$ Klinik für Endokrinologie, Universität Duisburg-Essen, Essen, Germany, ${ }^{4}$ Institut für Experimentelle Endokrinologie, Charité-Universitätsmedizin Berlin, Berlin, Germany, ${ }^{5}$ Center of Regenerative Therapies Dresden, TU Dresden, Dresden, Germany

Objective: Thyroid hormones $(\mathrm{TH})$ are crucial regulators of bone metabolism. Their import is guided through specific transporter proteins such as the monocarboxylate transporter 8 (Mct8). Due to the importance of $\mathrm{TH}$ in bone turnover, we tested the hypothesis that Mct8 is a critical mediator of $\mathrm{TH}$ actions in bone.

Methods: Twelve-week old male mice with a global Mct8-deficiency (M8, N = 12) or an osteoprogenitor-specific-deficiency (Mct8$\mathrm{fl} / \mathrm{fl}$;Osx-Cre, M8Cre+, $\mathrm{N}=8$ ) and their wildtype (WT, $\mathrm{N}=8$ )/crenegative littermate controls (M8Cre-, $\mathrm{N}=6$ ) were analyzed regarding their TH status, bone microarchitecture and bone turnover.

Results: M8 mice had elevated serum triiodothyronine $(+74 \%$, $\mathrm{p}<0.001)$, low thyroxine concentrations $(-52 \%, \mathrm{p}<0.01)$, and a low trabecular bone volume at the femur $(-71 \% \mathrm{p}<0.001)$ and lumbar spine $(-28 \%, \mathrm{p}<0.01)$ as compared to WT mice. M8 femora had thicker cortices $(+5.4 \%, \mathrm{p}=0.05)$. At the tissue level, osteoblast and osteoclast surfaces were increased in the femora $(+59 \% /+66 \%$, $\mathrm{p}=0.07 / \mathrm{p}<0.05)$ and vertebrae $(+90 \% /+54 \%, \mathrm{p}<0.05)$ of $\mathrm{M} 8$ mice indicating an accelerated bone turnover. Von Kossa/van Gieson staining revealed a threefold increased osteoid volume in M8 mice at both sites. Additionally, high FGF23 serum concentrations (+67\%, $\mathrm{p}<0.05)$ and a twofold longer maturation lag time suggest a mineralization defect in M8 mice. M8Cre + mice were euthyroid and displayed an increased trabecular bone volume of the femur $(+66 \%$, $\mathrm{p}<0.01)$ and spine $(+24 \%, \mathrm{p}<0.05)$ as compared to M8Cre- mice, while cortical BMD was decreased $(-2.2 \%, \mathrm{p}<0.05)$. In line with this, osteoblast surfaces were increased threefold in $\mathrm{M} 8 \mathrm{Cre}+$ ( $\mathrm{p}<0.05)$, whereas osteoclast parameters were unchanged.

Conclusion: Our data point to distinct effects of Mct8-deficiency on bone under euthyroid vs. hyperthyroid conditions. Further investigations are ongoing to unravel the underlying cellular processes.

Keywords: Thyroid hormone transporter, Mct8, bone mass, bone turnover, bone mineralization

\section{P015}

Patients on dialysis have markedly abnormal cortical hip parameters by dual-energy X-ray absorptiometry

Grahame Elder $^{1}$, Jasna Aleksova ${ }^{2}$

${ }^{1}$ Renal Medicine, Westmead Hospital, Sydney, Australia, ${ }^{2}$ Hudson Institute, Melbourne, Australia

Patients with chronic kidney disease (CKD) have fracture rates above the general population, higher post-fracture mortality and bone mineral density (BMD) by dual energy X-ray absorptiometry (DXA) is less informative. Micro-CT of bone trephines indicates reduction in cortical thickness and increased cortical porosity. Non invasive cortical assessment using micro-MRI and HRpQCT are not generally available. This study aimed to assess cortical parameters using DXA in dialysis patients. BMD and advanced hip analysis were performed using a Lunar iDXA. Normal ranges were determined from 2849 Lunar Prodigy femur scans of women (mean age $72.1 \pm 7.4$ years) and 1449 femur scans of men (mean aged $72.5 \pm 6.8$ years) enrolled in the Dubbo Osteoporosis Epidemiology Study (DOES). Compared to DOES results, 65 women aged $46 \pm 13$ years on dialysis about to undergo transplantation had Z-scores $-1.27 \pm 1.19$ vs. $-0.27 \pm 0.95$ and mean cortical thickness at the femoral neck of $2.53 \pm 1.52 \mathrm{~mm}$ vs. $5.13 \pm 1.79(\mathrm{p}<0.001)$, calcar $3.26 \pm 1.2 \mathrm{~mm}$ vs. $3.82 \pm 1.2(\mathrm{p}<0.001)$ with femoral shaft differences non-significant. Buckling ratios (indicative of cortical wall stability) were $8.01 \pm 4.57$ vs. $3.97 \pm 1.68(\mathrm{p}<0.001)$. For 90 men on dialysis aged $49 \pm 13$ years awaiting transplantation $Z$-scores were $-0.99 \pm 1.31$ vs. $0.31 \pm 1.09$. Femoral neck cortical width was $2.91 \pm 1.98 \mathrm{~mm}$ vs. $5.84 \pm 2.32(\mathrm{p}<0.001)$, calcar: $3.67 \pm 1 \mathrm{~mm}$ vs. $4.43 \pm 1.92(\mathrm{p}<0.001)$ and differences at the shaft non-significant. Buckling ratios were $10.03 \pm 11.27$ vs. $3.94 \pm 1.67$ $(\mathrm{p}<0.001)$. Renal osteodystrophy profoundly influences cortical bone, which is a major determinant of femoral strength and fracture risk. Despite potential limitations of DXA, cortical parameters are markedly abnormal in patients with CKD and should be assessed prospectively for usefulness in fracture prediction.

\section{P019}

Age at onset of walking in infancy is associated with hip shape in early old age

Alex Ireland $^{1}$, Stella Muthuri ${ }^{2}$, Fiona Saunders ${ }^{3}$, Anastasia Pavlova ${ }^{3}$, Rebecca Hardy ${ }^{2}$, Kathryn Martin ${ }^{3}$, Rebecca Barr ${ }^{3,4}$, Judith Adams ${ }^{5}$, Diana Kuh ${ }^{2}$, Richard Aspden ${ }^{3}$, Jennifer Gregory ${ }^{3}$, Rachel Cooper ${ }^{2}$

${ }^{I}$ School of Healthcare Science, Manchester Metropolitan University, Manchester, United Kingdom, ${ }^{2}$ MRC Unit for Lifelong Health and Ageing, University College London, London, United Kingdom, ${ }^{3}$ Aberdeen Centre for Arthritis and Musculoskeletal Health, University of Aberdeen, Aberdeen, United Kingdom, ${ }^{4}$ Medicines Monitoring 
Unit (MEMO), University of Dundee, Dundee, United Kingdom, ${ }^{5}$ Manchester Academic Health Science Centre and Radiology, Manchester Royal Infirmary, Manchester, United Kingdom

Bone shape and structure adapt in response to the muscle and reaction forces they experience during everyday movements. Onset of independent walking at $\sim 12$ months represents the first postnatal exposure of the lower limbs to the large forces associated with bipedal movements. Previous work has shown bone size and mass advantages in earlier-walking toddlers, which are also evident in adolescents and older adults. However, associations between early life loading and joint shape have not been explored.

We therefore examined associations between walking age and hip shape in later life in 1423 individuals (740 women) from the MRC National Survey of Health and Development (NSHD), a nationallyrepresentative British birth cohort. Walking age in months was obtained from maternal interview at age 2 years. Ten modes of variation in hip shape (HM1-HM10), described by statistical shape models, were ascertained from dual-energy X-ray absorptiometry (DXA) images taken at age 60-64 years.

In analyses adjusted for sex, height, body composition and socioeconomic position, earlier walking age was associated with higher HM1 and HM7 scores. Earlier walking was also associated with lower HM2 scores in women only (sex interaction $P$ value $=0.088$ ), and lower HM4 scores in men only (sex interaction $P$ value $=0.013)$. Taken together, in earlier walkers these modes describe a larger (HM4), flatter (HM2,4) femoral head, a wider (HM1,4,7), flatter (HM7) femoral neck, smaller neck-shaft angle (HM1,4), external rotation (HM2,7) and osteophytes (HM1).

These results suggest that age at onset of walking in infancy is associated with differences in hip shape in older age. In combination, hip modes suggest features of an osteoarthritic-like phenotype in earlier walkers. Unlike results of previous studies of walking age and bone mass, associations in this study were not affected by adjustment for lean mass. This suggests that associations may relate directly to skeletal loading in early life where joint shape changes rapidly.

\section{P021}

A new targeted mouse model for the rare bone disease Alkaptonuria: a disease exhibiting severe osteoarthropathy

Juliette Hughes ${ }^{1}$, Peter Wilson ${ }^{1}$, Ke Liu ${ }^{1}$, Hughes Andrew ${ }^{1,2}$, Hazel Sutherland $^{1}$, Lakshminarayan Ranganath ${ }^{1,2}$, James Gallagher ${ }^{1}$, George Bou-Gharios ${ }^{1}$

${ }^{1}$ Institute of Ageing and Chronic Disease, University of Liverpool, Liverpool, United Kingdom, ${ }^{2}$ Department of Clinical Chemistry, Royal Liverpool and Broadgreen University Hospital Trust, Liverpool, United Kingdom

Objectives: A novel alkaptonuria (AKU) mouse model with targeted homogentisate 1,2-dioxygenase (HGD) gene disruption has been generated. Homogentisic acid (HGA) levels and ochronosis, which causes severe osteoarthropathy in human AKU, will be assessed in HGD tm1a. The lacZ reporter construct will determine which adult tissues express HGD and when embryonic expression. The conditional HGD tm1d allele will investigate liver-specific HGD deletion.

Methods: Urinary and plasma HGA were measured using mass spectroscopy. Knee joint sections were Schmorl's stained to study ochronosis. Fresh adult tm1a tissues, frozen sections and time-mated embryos (E13.5-E16.5) were lacZ stained to localise HGD expression.

Tm1d MxCre +/- mice were injected with $\mathrm{pIpC}$ to induce exon removal via MxCre recombinase. Tm1d MxCre WT and tm1a mice were injected with $\mathrm{pIpC}$ as WT and AKU controls. HGA was measured in plasma and urine taken pre- and post-injection. HGD expression was analysed via qPCR.

Results: Homozygous tm1a show elevated HGA compared to heterozygous and WT controls (Fig. 1). Pericellular chondrocyte pigmentation of calcified articular cartilage is detected at 9 weeks (Fig. 2A). Numerous fully pigmented chondrocytes are seen at 26 and 40 weeks (Fig. 2B, 2C), with no pigmentation in heterozygotes (Fig. 2D). HGD is expressed in liver hepatocytes and kidney cortex proximal convoluted tubule cells. Embryonic HGD expression begins at E14.5.

Liver, but not kidney, HGD expression is knocked down by pIpC injection in $\mathrm{MxCre}+/-$ mice, resulting in plasma HGA increasing from 0 to $47 \mu \mathrm{mol} / \mathrm{L}$, almost to tm1a level (Fig. 3). Urinary HGA increases from 22 to $8621 \mu \mathrm{mol} / \mathrm{L}$, but not to the tm1a level.

Conclusion: The new model exhibits an AKU phenotype. HGD is in the liver and kidney, beginning at E14.5. Liver HGD was successfully knocked out in MxCre $+/-$ mice. The increase in plasma HGA suggests that kidney HGD expression alone is not sufficient to rescue the $\mathrm{AKU}$ phenotype.

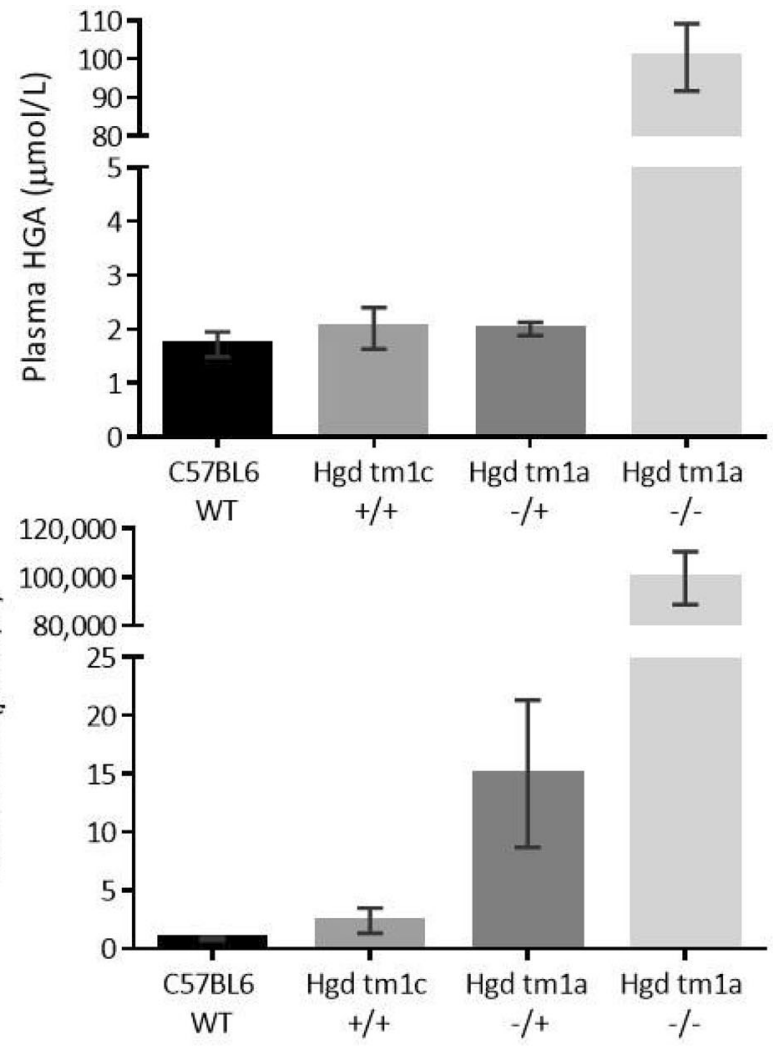

Figure 1 


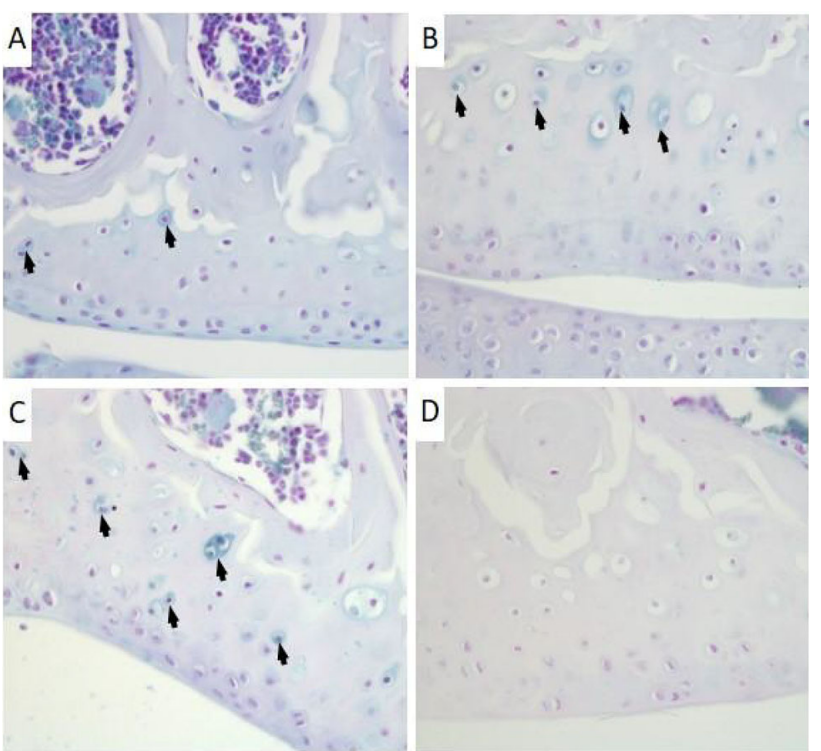

Figure 2 .

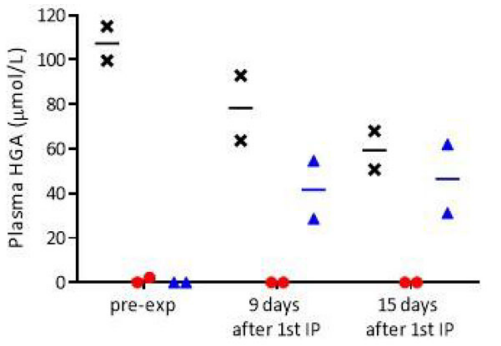

X Hgd tm1a - /

- Hgd tm $1 \mathrm{~d} f \mathrm{fl} / \mathrm{fl}:+/+$ MxCre WT

Hgd tmid $\mathrm{fl} / \mathrm{fl}:+/+$, MxCre +/-

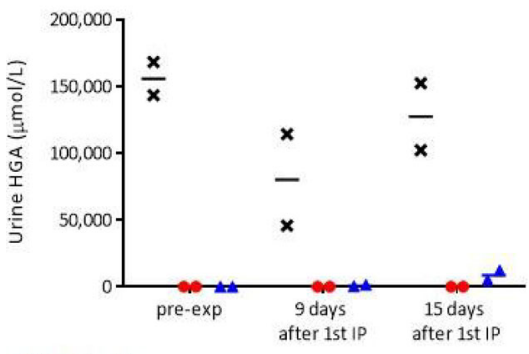

X Hgd tm1a -/-

Hgd tm1d fl/fl: $+/+$,

MxCre WT Hgd $\operatorname{tm} 1 \mathrm{~d} f \mathrm{fl} / \mathrm{fl}:+/+$

- MxCre +1-

Figure 3.

\section{P023}

Assessment of ruptured Achilles tendons by sonoelastography and risk for rupture of contralateral tendon

Ivo Dumic-Cule ${ }^{1}$, Gordana Ivanac ${ }^{1}$, Domagoj Lemac ${ }^{2}$, Boris Brkljacic $^{1}$

${ }^{1}$ Department of Diagnostic and Interventional Radiology, Clinical Hospital Dubrava, Zagreb, Croatia, ${ }^{2}$ Department of Orthopaedics and Trauma Surgery, Clinical Hospital Dubrava, Zagreb, Croatia

The concept of assessing the mechanical properties of the Achilles tendon after reconstruction using ultrasound shear wave elastography (SWE) and its positive correlation with standardized questionnaires has been recently introduced into the clinical practice. Since it was demonstrated that about $6 \%$ of patients with ruptured Achilles tendon experience the rupture of contralateral tendon in the future, the aim of this study was to estimate the risk for rupture of contralateral tendon in patients who underwent surgical reconstruction of ruptured Achilles tendon by using subjective questionnaires and ultrasound SWE.

Twenty-four patients who underwent surgical repair of the ruptured Achilles tendon and 12 aged matched healthy controls were examined with ultrasound SWE. Functional outcomes were assessed with American Orthopedic Foot and Ankle Society (AOFAS) scoring system and subjective rating system which we introduced and validated. Study was approved by ethical committee of the hospital and enrolled patients signed informed consent.

The elasticity of injured tendon was markedly decreased (by $42 \%$, $\mathrm{P}<0.01)$ compared to contralateral tendon of the patient, as expected. Both AOFAS score and our novel subjective assessment scale positively correlate with ultrasound SWE values in ruptured Achilles tendons. The elasticity of contralateral Achilles tendons in patients was $23 \%$ lower than among healthy individuals $(\mathrm{P}<0.05)$.

Irrespective of the lack of difference in the subjective feeling assessed by AOFAS, the contralateral tendon in the patients with reconstructed Achilles tendon has significantly lower elasticity than healthy individuals. Therefore, patients who already experienced the rupture are at significantly higher risk for rupture of contralateral Achilles tendon in the future.

\section{P025}

Targeted inactivation of Rin3 increases trabecular bone mass

Mahéva Vallet ${ }^{1}$, Antonia Sophocleous ${ }^{2}$, Anna E. Törnqvist ${ }^{3}$, Asim Azfer $^{1}$, Rob van't Hof ${ }^{4}$, Omar M.E. Albagha ${ }^{1}$, Stuart H. Ralston ${ }^{1}$

${ }^{1}$ Institute of Genetics and Molecular Medicine, University of Edinburgh, Edinburgh, United Kingdom, ${ }^{2}$ Department of Life Sciences, School of Sciences, European University Cyprus, Nicosia, Cyprus, ${ }^{3}$ Department of Internal Medicine at Institute of Medicine, University of Gothenburg, Gothenburg, Sweden, ${ }^{4}$ Institute of Ageing and Chronic Disease, University of Liverpool, Liverpool, United Kingdom

Background: Genetic variants in the RIN3 gene (Ras and Rab interactor protein 3) are strongly associated with Paget's disease of bone (PDB) and increased bone mineral density in children. Here, we investigate its role in bone metabolism using mice with targeted inactivation of $\operatorname{Rin} 3\left(\operatorname{Rin} 3^{-1-}\right)$, to further understand its potential role in bone metabolism.

Methods: Skeletal phenotyping was conducted in $14 \operatorname{Rin}^{-1-}$ mice and 11 wild type (WT) female littermates on a C57BL/6 $\times 129$ / OlaHsd background at 8 weeks of age. Changes in the trabecular and cortical bones were investigated in the femur, tibia and spine using microCT. Histomorphometric analyses were performed on the metaphysis of femurs, where mineralisation rate was measured by calcein double-labelling (4 days apart) and osteoclast phenotype by TRAcP staining.

Results: Trabecular bone volume was significantly higher in the femurs $\left(\operatorname{Rin}^{-1-}=12.5 \pm 0.6 \% ; \mathrm{WT}=10.1 \% \pm 0.4 ; P=0.001\right)$ and tibias $\left(\operatorname{Rin}^{-l-}=13.8 \pm 0.4 \%\right.$; WT $\left.=12.2 \pm 0.4 \% ; P=0.005\right)$ of Rin ${ }^{-1-}$ mice compared to WT. Similarly, trabecular number was increased in the femurs $\left(\operatorname{Rin} 3^{-/-}=0.014 \pm<0.01 \mu \mathrm{m}^{-1}\right.$; WT $=$ $\left.0.011 \pm<0.01 \mu \mathrm{m}^{-1} ; P<0.001\right)$ and tibias $\left(\operatorname{Rin} 3^{-/-}=0.016 \pm\right.$ $<0.001 \mu \mathrm{m}^{-1}$; WT $\left.=0.014 \pm<0.001 \mu \mathrm{m}^{-1} ; P<0.001\right)$. Histomorphometric analyses of trabecular bone confirmed these findings and additionally showed a decrease in active resorption surface in $\operatorname{Rin}^{-1-}$ mice compared to WT $\left(\operatorname{Rin} 3^{-1-}=24.1 \pm 1.3 \%\right.$; WT $=29.7$ $\pm 2.1 \% ; P<0.05)$. There was no significant differences in bone formation parameters or mineralisation between the genotype groups.

Conclusion: These findings illustrate that $\operatorname{Rin} 3$ is a negative regulator of trabecular bone mass. The data are consistent with a model whereby variants within the human RIN3 locus regulate susceptibility to PDB and bone mass by an effect on osteoclast activity. 


\section{P027}

Evidence that bi-allelic mutations in NPR3 result in a phenotype with tall stature, arachnodactyly, long halluces and multiple extra epiphyses in hands and feet

Eveline Boudin ${ }^{1}$, Tjeerd de Jong ${ }^{2}$, Tim Prickett ${ }^{3}$, Bruno Lapauw ${ }^{4}$, Kaatje Toye $^{4}$, Viviane Van Hoof ${ }^{5}$, Ilse Luyckx ${ }^{1}$, Aline Verstraeten ${ }^{1}$, Hugo Heymans ${ }^{6}$, Eelco Dulfer ${ }^{7}$, Lut Van Laer ${ }^{1}$, Ian Berry ${ }^{8}$, Angus Dobbie $^{9}$, Ed Blair ${ }^{10}$, Bart Loeys ${ }^{1}$, Eric Espiner ${ }^{3}$, Jan M Wit ${ }^{11}$, Wim Van Hul ${ }^{1}$, Peter Houpt ${ }^{2}$, Geert Mortier ${ }^{1}$

${ }^{1}$ Center of Medical Genetics, University of Antwerp, Edegem, Belgium, ${ }^{2}$ Department of Plastic and Reconstructive Surgery and Hand Surgery, Isala Clinics Zwolle, Zwolle, Netherlands, ${ }^{3}$ Department of Medicine, University of Otago, Christchurch, New Zealand, ${ }^{4}$ Department of Endocrinology and Unit for Osteoporosis and Metabolic Bone Diseases, Ghent University Hospital, Ghent, Belgium, ${ }^{5}$ Department of Clinical Chemistry, Antwerp University Hospital, Edegem, Belgium, ${ }^{6}$ Department of Pediatrics, Emma's Children's Hospital- Academic Medical Centre, Amsterdam, Netherlands, ${ }^{7}$ Department of Medical Genetics, University Medical Center Groningen, Groningen, Netherlands, ${ }^{8}$ Leeds Genetics Laboratory, St James's University Hospital, Leeds, United Kingdom, ${ }^{9}$ Yorkshire Clinical Genetics Service, Chapel Allerton Hospital, Leeds, United Kingdom, ${ }^{10}$ Oxford Centre for Genomic Medicine, Oxford University Hospitals NHS Foundation Trust, Oxford, United Kingdom, ${ }^{11}$ Department of Pediatrics, Leiden University Medical Center, Leiden, Netherlands

The natriuretic peptide (NP) signaling pathway has been implicated in many cellular processes including endochondral ossification and bone growth. More precisely, different mutations in the NPR-B receptor and the CNP ligand have been identified in patients with either short or tall stature. With this study we show that also the NPR$\mathrm{C}$ receptor (encoded by NPR3) is regulating linear bone growth.

Here, we report four individuals, originating from three different families, with a phenotype characterized by tall stature, long digits and extra epiphyses in hands and feet. In addition, aortic dilatation was observed in two of these families. Genetic analysis (mainly using whole exome sequencing) demonstrated the presence of bi-allelic loss-of-function mutations in NPR3 in all affected individuals. In these patients, we identified two missense mutations, p.Ser148Pro and p.Asp363Val, one nonsense mutation, p.Tyr508* and one frameshift mutation, p.Val83Glyfs*105.

Using in vitro cellular localization studies, we demonstrated that the missense mutations (p.Ser148Pro and p.Asp363Val) cause intracellular retaining of the NPR-C receptor with no expression on the plasma membrane. mRNA analysis demonstrated that the nonsense mutation (p.Tyr508*) results in nonsense mediated mRNA decay.

Since NPR-C is known to act as a clearance receptor for the natriuretic peptides (NP), we investigated the effect of the identified mutations on NP levels in blood. Biochemical analysis of the plasma from the proband in family 1 revealed a reduced NTproNP/NP ratio for all ligands and also high cGMP levels. These data strongly suggest a reduced clearance of natriuretic peptides by the defective NPR-C receptor and a compensatory increased activity of the NPR-A/B, two receptors with guanylyl cyclase activity.

In conclusion, this study demonstrates that loss-of-function mutations in NPR3 result in increased NPR-A/B signaling activity and cause a phenotype with enhanced bone growth and variable cardiovascular abnormalities.

\section{P029}

Tumour-derived extracellular vesicles affect the molecular profile of osteoblasts and stimulate endothelial functions
Riccardo Paone $^{1}$, Alexander Loftus ${ }^{1}$, Chris George ${ }^{1}$, Kirsty Shefferd ${ }^{1}$, Argia Ucci ${ }^{1}$, Simona Delle-Monache ${ }^{1}$, Alfredo Cappariello ${ }^{1}$, Maurizio Muraca ${ }^{2}$, Anna Teti ${ }^{1}$, Nadia Rucci ${ }^{1}$

${ }^{1}$ University of L'Aquila, L'Aquila, Italy, ${ }^{2}$ University of Padova, Padova, Italy

Extracellular vesicles (EVs) are membrane-bound cargoes of biologically active molecules shed by cells, which are emerging as mediators of several pathological processes, including cancer. We investigated EV-mediated communication between MNNG osteosarcoma cell line (HOS), osteoblasts and endothelial cells. In mouse osteoblasts, HOS EVs inhibited the expression of Cyclin D1 $(-75 \%, \mathrm{p}=0.02)$, Alp $(-35 \%, \mathrm{p}=0.002)$ and Osx $(-40 \%$, $\mathrm{p}=0.06)$, and enhanced the expression of Nos2 (15-fold, $\mathrm{p}=0.015)$. An increase of mRNA and protein expression of IL-1 $\beta$ (12-fold, $\mathrm{p}<0.001)$, IL-6 (10-fold, $\mathrm{p}=0.05)$, RANKL $(1.5$-fold, $\mathrm{p}=0.02)$ and LCN2 (fourfold, $p=0.01$ ) was also observed in HOS EVs-treated osteoblasts. A cytokine protein array on conditioned media (CM) collected from osteoblasts treated with HOS EVs showed a decrease of IGFBP2 $(-40 \%$, n.arrays $=2)$ compared to CM from untreated osteoblasts, and an increase of Pentraxin 3 (2.9-fold, n. arrays = 2), a positive modulator of tumour-associated inflammation. Moreover, the chemokines CXCL2, CCL2, CCL5, CCL20, Lix and MMP3, which were undetectable in untreated osteoblasts, were expressed by HOS EV-treated osteoblasts. HOS EV transcriptional profile showed the expression of genes involved in bone metabolism, such as $C L C N 7$, $T G F \beta 1$, Cathepsin K, IL-6 and VEGFA. Consistent with this latter result, HOS EVs significantly increased endothelial cell chemotaxis $(p=0.02$, AUC) and in vitro tube formation (threefold, $p=0.007$ ), evaluated by scratch assay and number of branching formed in HUVEC cell cultures. Consistently, in vivo angiogenesis, evaluated by the matrigel plug assay, was significantly enhanced by HOS-EVs (3.5fold, $\mathrm{p}=0.001$; n.mice $=5 /$ group; ethical approval n.173/2016$\mathrm{PR})$. In conclusion, these data allowed to elucidate (i) the transcriptional profile of osteosarcoma derived EVs and (ii) their impact on osteoblast and endothelial physiology.

\section{P031}

Type 2 diabetes, glucose and insulin metabolism in relation to hip bone size and bone turnover in elderly Swedish men and women

Adam Mitchell ${ }^{1}$, Tove Fall ${ }^{2}$, Håkan Melhus ${ }^{3}$, Alicja Wolk ${ }^{4}$, Karl Michaelsson $^{1}$, Liisa Byberg ${ }^{1}$

${ }^{1}$ Department of Surgical Sciences, Orthopaedics, Uppsala University, Uppsala, Sweden, ${ }^{2}$ Department of Medical Sciences, Molecular Epidemiology, Uppsala University, Uppsala, Sweden, ${ }^{3}$ Department of Medical Sciences, Clinical Pharmacogenomics and Osteoporosis, Uppsala University, Uppsala, Sweden, ${ }^{4}$ Institute of Environmental Medicine, Division of Nutritional Epidemiology, Karolinska Institutet, Stockholm, Sweden

Aims/hypothesis:Men and women with type 2 diabetes mellitus (T2DM) have a higher risk of hip fractures but the mechanisms are not fully understood. We aimed to investigate how T2DM, glucose and insulin were associated with femoral BMD, bone mineral area (BMA) and bone turnover markers-all fracture predisposing factors.

Methods: We used two cross-sectional cohorts: the Uppsala Longitudinal Study of Adult Men (ULSAM, $\mathrm{n}=452$, mean age 82 years) and the Swedish Mammography Cohort Clinical (SMCC, $\mathrm{n}=4713$, mean age 68 years). We identified those with T2DM, impaired fasting plasma glucose (IFG), and normal fasting glucose (NFG). Fasting plasma glucose and insulin were included as continuous exposure variables. BMD and BMA at the total hip and femoral shaft were measured using DXA. The bone turnover markers 
CrossLaps and osteocalcin were measured in women. Linear regression and spline models were applied.

Results: Men and women with T2DM, compared to those with NFG, had higher BMD at the total hip and femoral shaft but a lower BMA at the total hip $(-1.7 \%$; $95 \%$ CI $-3.2,-0.2$ and $-1.0 \%$; $95 \% \mathrm{CI}-1.6, \mathrm{v} 0.4)$ and the femoral shaft $(-2.0 \%$; $95 \% \mathrm{CI}-3.5$, -0.4 and $-0.6 \%$; $95 \%$ CI $-1.2,-0.01)$. Men and women with IFG compared with those with NFG tended to have lower BMA at the total hip or the femoral shaft. In spline curve analyses, increasing fasting glucose and fasting insulin were associated with a slightly lower BMA at the total hip and femoral shaft. T2DM was related with a lowering of CrossLaps $(-8.1 \%$; 95\% CI $-12.7,-3.6)$ and osteocalcin $(-15.2 \%$; 95\% CI - 19.0, - 11.2). Glucose and insulin were both inversely associated with CrossLaps and osteocalcin in women.

Conclusions:/interpretation: These cross-sectional results indicate that those with T2DM have a lower bone turnover and bone size, which could increase the risk of hip fracture.

\section{P033}

Suppression of p38 $\alpha$ MAPK signaling in osteoblasts impairs bone formation in RANKL-stimulated bone remodeling

Cyril Thouverey $^{1}$, Joseph Caverzasio ${ }^{1}$, Serge Ferrari ${ }^{1}$

${ }^{1}$ Service of Bone Diseases, University Hospital of Geneva, Geneva, Switzerland

Osteoporosis is the consequence of a failure of osteoblasts in refilling bone cavities dug out by osteoclasts despite the existence of various signals that couple bone resorption to bone formation. Osteoblastic p38 $\alpha$ MAPK signaling pathway positively regulates bone formation but can be diverted from its anabolic activity in condition of bone loss induced by estrogen withdrawal. We hypothesized that p38 $\alpha$ MAPK is important in osteoblasts to couple bone resorption to formation. To test this hypothesis, 8-week-old female mice lacking $\mathrm{p} 38 \alpha$ in osteoblasts $\left(\mathrm{Ocn}-\mathrm{Cre} ; \mathrm{p} 38 a^{\text {flf }}\right)$ and their control littermates $\left(p 38 a^{f l f}\right)$ were treated with 2 subcutaneous injections of $1 \mathrm{mg} / \mathrm{kg}$ recombinant RANKL in a 24-h interval to stimulate bone remodeling or its vehicle solution. Their bone microarchitecture and their serum levels of bone resorption/formation markers were measured 3 and 14 days after the second injection of RANKL. RANKL injections did not affect cortical bone mass but induced loss of $80 \%$ of initial trabecular bone mass in mice of both genotypes within 3 days. $p 38 a^{f l f}$ mice recovered $87 \%$ of their trabecular bone 14 days after RANKL injections while Ocn-Cre;p38 fif mice only recovered $36 \%$ of trabecular bone mass $(\mathrm{p} \leq 0.01)$. As expected, 3 days after RANKL injections serum levels of tartrate-resistant acid phosphatase (TRAP) were elevated by 2.3 -fold $(\mathrm{p} \leq 0.05)$ and carboxy-terminal collagen crosslinks $(\mathrm{CTX})$ by around fourfold $(\mathrm{p} \leq 0.05)$ in mice of both genotypes. Interestingly, at the same time-point, serum levels of aminoterminal propeptide of type I collagen (PINP) and osteocalcin were increased by respectively $2.2(\mathrm{p} \leq 0.01)$ and 1.5 -fold $(\mathrm{p} \leq 0.05)$ in $p 38 a^{f f f}$ mice but not in Ocn-Cre;p $38 a^{f l f}$ mice. In conclusion, our results show that bone formation in RANKL-stimulated bone turnover is impaired in mice lacking p38 $\alpha$ MAPK signaling in osteoblasts, and thus suggest that this kinase is a mediator of the coupling reaction linking bone resorption to formation.

\section{P035}

Secondary hyperparathyroidism and bone turnover after obesity surgery
Stephen Hewitt ${ }^{1}$, Jon Kristinsson ${ }^{2,3}$, Erlend Aasheim ${ }^{3}$, Ingvild Blom${ }_{\text {Høgest } \varnothing 1^{1} \text {, Eirik Aaseth }}{ }^{4}$, Erik Fink Eriksen ${ }^{1}$, Tom Mala ${ }^{3,5}$

${ }^{1}$ Department of Endocrinology, Morbid Obesity and Preventive Medicine, Oslo University Hospital and Institute of Clinical Medicine, University of Oslo, Oslo, Norway, ${ }^{2}$ Department of Gastrointestinal Surgery, Oslo University Hospital, Oslo, Norway, ${ }^{3}$ Department of Endocrinology, Morbid Obesity and Preventive Medicine, Oslo University Hospital, Oslo, Norway, ${ }^{4}$ Department of Medicine, Innlandet Hospital, Elverum, Norway, ${ }^{5}$ Department of Gastrointestinal Surgery, Oslo University Hospital and Institute of Clinical Medicine, University of Oslo, Oslo, Norway

Background: Secondary hyperparathyroidism (SHPT) and bone health is a concern after obesity surgery. Bone turnover is increased, but little data exists of the relationship between SHPT and bone turnover. We studied the prevalence of SHPT and indicators of bone turnover over 5 years after Roux-en-Y gastric bypass (RYGB), and if SHPT was associated with increased bone turnover.

Methods: 347 of $568(61 \%)$ patients operated with RYGB 2004-2008 at Oslo University Hospital who attended 5 year followup were eligible for study inclusion. All patients were invited for follow-up at 6 months, and 1,2 and 5 years postoperatively. Patients with missing data of parathyroid hormone, ionized calcium and $25-\mathrm{OH}$ vitamin $\mathrm{D}$ at 5 years $(\mathrm{n}=14)$, elevated serum creatinine $(n=10)$ and primary hyperparathyroidism $(n=4)$ were excluded. SHPT was defined as PTH $>7.0 \mathrm{pmol} / 1$ and increased bone turnover as bone specific alkaline phosphatase (ALP) above normal reference range ( $>31 \mathrm{U} / \mathrm{L}$ in women; $>41 \mathrm{U} / \mathrm{L}$ in men). Substitution of vitamin D (1000 IE/day) and calcium carbonate $(1000 \mathrm{mg} /$ day $)$ was recommended during follow-up. The study was approved by the regional ethical committee.

Results: Before surgery SHPT was found in $33 \%$ of 319 included patients ( 230 women). After 6 months, 1, 2 and 5 years the prevalence of SHPT was 18, 24, 28 and 35\%, respectively. Correspondingly, bone turnover was increased in $33,28,25$ and $36 \%$ of the patients. In patients with SHPT, bone turnover was increased in 43, 38, 46 and $67 \%$, compared with $31 \% \quad(\mathrm{p}=0.19), 24 \% \quad(\mathrm{P}=0.06), 20 \%$ $(\mathrm{P}<0.05)$ and $13 \%(\mathrm{P}=0.09)$ in patients without SHPT.

Conclusion: The prevalence of SHPT decreased the first half year after RYGB, and thereafter increased. Increased bone turnover appeared in the range $25-36 \%$ during the 5 years postoperatively, and was associated with SHPT.

Keywords: hyperparathyroidism, bone turnover, obesity, bariatric surgery

\section{P037}

Fatigue behaviour of non-osteocytic bone in swordfish and sitematched histomorphometric evaluation of bone structure with respect to cellular activities

Felix Nikolai Schmidt ${ }^{1}$, Flynn Walsh ${ }^{2}$, Christine Plumeyer ${ }^{1}$, Bernd Gludovatz $^{2}$, Robert O. Ritchie ${ }^{2,3}$, Björn Busse ${ }^{1,2}$

${ }^{1}$ Department of Osteology and Biomechanics, University Medical Center Hamburg-Eppendorf, Hamburg, Germany, ${ }^{2}$ Materials Sciences Division, Lawrence Berkeley National Laboratory, Berkeley, United States, ${ }^{3}$ Department of Mechanical Engineering, University of California San Francisco, Berkeley, United States

Bone remodeling is one key aspect to the fatigue behaviour of bone. While osteocytes are suggested as main orchestrators of remodeling in mammals, the sword of the swordfish is fully absent of osteocytes. Yet, it maintains resistance against fatigue and cycling loading throughout life. This study aims to investigate the role of nonosteocytic pathways associated with fatigue by performing site- 
matched histomorphometry, quantitative backscattered electron imaging and toughness testing of the sword.

Tip, mid and base of a 7 years-old sword were investigated. Total cement line length and canal diameter were assessed as measures for remodeling, while growth toughness was assessed as measure for fatigue resistance.

The sword exhibited a significant $(\mathrm{p} \leq 0.05)$ increase in growth toughness from the tip to base $(2.44 \pm 0.19$ vs. $3.61 \pm 0.43$ vs. $4.65 \pm 0.61 \mathrm{MPa} / \mathrm{m})$. The canal diameter significantly $(\mathrm{p} \leq 0.0001)$ increased from the tip $(12.51 \pm 8.25 \mu \mathrm{m}$-old bone) to the base $(28.59 \pm 16.66 \mu \mathrm{m}$-new bone), while the sum of cement lines length was lowest at the base $(1210 \pm 831.78 \mu \mathrm{m})$, and highest at mid-section $(5794.41 \pm 633.22 \mu \mathrm{m}), \mathrm{p} \leq 0.05$.

A low mean canal diameter in aged bone at the tip indicates secondary, concentric bone apposition as a compensation for accumulation of bone damage with tissue age. Indeed, the resistance to fatigue was highest in new bone and lowest in aged bone (tip). Histomorphometry revealed clear signs of remodeling, with highest remodeling activity (i.e. sum of cement line length) in the mid region of the sword. This study indicates that resistance to fatigue and fracture of the sword is partially maintained by non-osteocytic remodeling and non-cellular compensation processes which are dependent of the tissue age of the sword. Taken together the sword of swordfish represents a good model to study fatigue behaviour in osteoyte-deficient bone and for bone at different ages partially uncoupled of the animals age.

Keywords: Fatigue, non-osteocytic bone, non-mamalian model, fishbone, biomechanics

Results

\begin{tabular}{llll}
\hline$* * \mathrm{p}<0.05$ for all & Tip & Mid & Base \\
$\begin{array}{l}\text { loctions, *p }<0.05 \\
\text { for base vs. } \\
\text { other locations }\end{array}$ & & & \\
\hline $\begin{array}{l}\text { Growth toughness } \\
(\mathrm{MPa} / \mathrm{m})^{* *}\end{array}$ & $2.44 \pm 0.19$ & $3.61 \pm 0.43$ & $4.65 \pm 0.61$ \\
$\begin{array}{c}\text { Canal diameter } \\
(\mu \mathrm{m}) * *\end{array}$ & $12.51 \pm 8.25$ & $16.60 \pm 9.01$ & $28.59 \pm 16.66$ \\
$\begin{array}{l}\Sigma \text { of cement lines } \\
(\mathrm{mm}) *\end{array}$ & $3.99 \pm 3.02$ & $5.79 \pm 0.63$ & $1.21 \pm 0.83$ \\
& & & \\
\end{tabular}

\section{P039}

Bilirubin promotes down-regulation of RUNX2 and up-regulation of RANKL gene expression in bone explants and in osteoblastic and osteocytic cell lines

Silvia Ruiz-Gaspà ${ }^{1}$, Albert Parés ${ }^{1}$, Andrés Combalia ${ }^{1}$, Pilar Peris ${ }^{1}$, Ana Monegal $^{1}$, Núria Guañabens ${ }^{1}$

${ }^{1}$ Metabolic Bone Diseases Unit and Liver Unit, CIBERehd, Hospital Clinic, IDIBAPS, University of Barcelona, Barcelona, Spain

In vitro studies have shown that the retained substances of cholestasis have deleterious effects in human osteoblasts and osteocytic cells. Bilirubin (BIL) and lithocholic acid (LCA) induce alterations in the proliferation, differentiation and apoptosis of osteoblastic and osteocytic cells. However, their effects in human bone tissue and in bone cell lines have not been deeply analysed.

Aim: To investigate the effects of BIL, LCA and ursodeoxycholic acid (UDCA) in gene expression of human trabecular bone explants as well as in osteoblastic (SAOS2) and osteocytic cells (MLO-Y4/ MLO-A5).

Methods: Bone tissue harvested from trabecular bone fragments, SAOS2 and MLO-Y4/MLO-A5 cells were cultured and treated with BIL $(50 \mu \mathrm{M})$, LCA $(10 \mu \mathrm{M})$ and UDCA $(10 / 100 \mu \mathrm{M})$ for $24 \mathrm{~h}$. Gene expression of osteocalcin (BGLAP), Cbfa1 (RUNX2)/Osterix (OSX) and RANKL (TNFRSF11)/osteoprotegerin (TNFRSF11B) were quantified by real time PCR.

Results: BIL diminishes RUNX2 gene expression in bone tissue $(-37 \%)$, SAOS2 $(-75 \%)$, MLO-Y4 $(-56 \%)$ and MLO-A5 (-77\%), and increases RANKL expression in 60,27, 72 and $60 \%$, respectively $(\mathrm{p} \leq 0.02)$. In bone tissue and in osteoblastic and osteocytic cells, LCA increases gene expression of BGLAP (NS) and RANKL $(\mathrm{p} \leq 0.03)$. UDCA $100 \mu \mathrm{M}$ increases RUNX2 and OSX expression in bone tissue (78 and 82\%), MLO-Y4 (72 and 80\%) and SAOS2 (75 and 70\%) ( $\mathrm{p} \leq 0.03)$. In addition, UDCA $100 \mu \mathrm{M}$ significantly increases expression of BGLAP, OPG and RANKL in bone tissue and in osteocytic lines. UDCA $10 / 100 \mu \mathrm{M}$ counteracts the decrease in RUNX2 induced by BIL in bone tissue, SAOS2, MLO-A5 and MLO-Y4 cells.

Conclusion: The retained substances of cholestasis, particularly bilirubin, cause noxious effects on transcription factors of osteoblast differentiation and on osteoclastic activators in bone tissue and in osteoblastic and osteocytic cells. Ursodeoxycholic acid reverses the harmful effects of bilirubin. These results provide new insights into the low bone formation and at some stages, high resorption, associated with chronic cholestasis.

\section{Poster SNAPs 2}

\section{P002}

The role of iron in the development and activity of osteoclasts

Romina Cabra $^{1,2,3}$, Mark Siegrist $^{1}$, Silvia Dolder ${ }^{1}$, Willy Hofstetter ${ }^{1,2}$ ${ }^{1}$ Department of BioMedical Research, University of Bern, Bern, Switzerland, ${ }^{2}$ NCCR Transcure, Swiss National Centre of Competence in Research, Bern, Switzerland, ${ }^{3}$ Graduate School, University of Bern, Bern, Switzerland

Introduction: Iron is an essential trace element. Membrane iron transporters are critical to maintain systemic/cellular iron homeostasis to prevent metabolic disorders such as anemia and hemochromatosis. Recently, we described an increase in transcripts encoding Divalent Metal Transporter (DMT1), a membrane transporter for iron uptake, in osteoclastogenesis. To understand the role of iron and of DMT1 in osteoclasts (OC), we developed an in vitro iron uptake assay and generated osteoclast-specific DMT1-ko mice using the Cre/lox system, with Cre expressed under TRAP promoter.

Methods: Osteoclast progenitor cells (OPC) were cultured with MCSF, RANKL \pm iron chelator, DFO. After day 5, OC were harvested and incubated for up to $120 \mathrm{~min}$ with ${ }^{55} \mathrm{Fe}$-Transferrin. Bone mass and structure of DMT1 $(\mathrm{OC})^{\mathrm{f} / \mathrm{fl}} \mathrm{Cre}+$ lumbar vertebrae (L3-L5) were evaluated by microCT. OPC from DMT1 $(\mathrm{OC})^{\mathrm{f} / \mathrm{fl}} \mathrm{Cre}+$ were cultured with MCSF and RANKL to assess the effects of DMT1 deficiency on OC proliferation and development. Levels of DMT1 transcripts were determined in mixed and homogeneous OC cultures by RT-PCR.

Results: After 5 days, levels of transcripts encoding TfR1 and DMT1 were significantly increased in OC/DFO $(\mathrm{P}<0.0001)$. Iron uptake in OC/DFO was significantly increased compared to OC w/o DFO ( $\mathrm{P} \leq 0.05)$. Trabecular thickness in L5 vertebral bodies in male $\mathrm{DMT} 1(\mathrm{OC})^{\mathrm{f} / \mathrm{fl}} \mathrm{Cre}+$ mice was significantly decreased when compared to the Cre- control $(\mathrm{P} \leq 0.05)$. Levels of transcripts encoding DMT1 were decreased by $70 \%$ and $30 \%$ in OC derived from male and female 
DMT1 $(\mathrm{OC}){ }^{\mathrm{f} / \mathrm{f}} \mathrm{Cre}+$ mice, respectively. Development of osteoclasts, as assessed by XTT and TRAP, was not affected by the loss in DMT1.

Conclusion: Iron deficiency in vitro induces a significant upregulation of both TfR1 and DMT1 transcripts in OC. Osteoclast-specific DMT1-ko efficiency is sex-dependent. In male DMT1 $(\mathrm{OC})^{\mathrm{fl} / \mathrm{fl}} \mathrm{Cre}+$ mice, the decrease in DMT1 transcripts leads to a decrease in trabecular thickness. However, no changes in cell viability and osteoclastogenesis were detected in vitro.

\section{P004}

Treatment with zoledronic acid subsequent to odanacatib prevents bone loss in postmenopausal women with osteoporosis

Anne Sophie Sølling ${ }^{1}$, Torben Harsløf ${ }^{1}$, Bente Langdahl ${ }^{1}$

${ }^{1}$ Department of Endocrinology and Internal Medicine, Aarhus University Hospital, Aarhus, Denmark

Purpose: The development of odanacatib (ODN), a cathepsin K inhibitor, was discontinued due to an increased risk of cardiovascular events. As the treatment is considered reversible, participants from the LOFT trial (Long-Term Odanacatib Fracture Trial) in Aarhus, Denmark were offered treatment with zoledronic acid (ZOL).

Methods: Sixty-seven postmenopausal women (mean age 78) accepted treatment with ZOL $5 \mathrm{mg}$ and were followed for 12 months. Of these 39 had received ODN for 7 years and 28 had received placebo for 5 years and ODN for 2 years. Bone turnover markers (BTM) were measured 3, 6 and 12 months after ZOL, and DXA of spine and hip were performed at ZOL treatment and after 12 months.

Results: Within the entire study population, bone mineral density (BMD) at the lumbar spine increased significantly by $2.8 \pm 0.9 \%$ (mean \pm SEM) $(\mathrm{p}<0.01)$ from baseline to month 12 . There was no significant change in BMD at the total hip $(\mathrm{p}=0.17)$ or femoral neck $(\mathrm{p}=0.39)$. There was no difference in change in BMD from baseline to 12 months between the two groups at any site ( $p \geq 0.20$ for all). The BTMs were in the lower half of the reference range for postmenopausal women 3 months after ZOL. C-terminal collagen crosslinks increased by $107 \pm 9 \%(\mathrm{p}<0.001)$, procollagen type I $\mathrm{N}$-terminal propeptide by $102 \pm 16 \%$ ( $\mathrm{p}<0.001)$, osteocalcin by $32 \pm 6 \%(\mathrm{p}=0.001)$ and bone specific alkaline phosphatase by $79 \pm 37 \%(\mathrm{p}=0.001)$ between 3 and 12 months after ZOL. At month 12, BTM were still within postmenopausal reference range. Additionally, s-25-hydroxyvitamin D increased $(\mathrm{p}=0.059)$ and s-parathyroid hormone $(\mathrm{p}=0.007)$ and estimated glomerular filtration rate $(\mathrm{p}=0.014)$ both decreased significantly during the year following ZOL administration.

Conclusion: Treatment with ZOL $5 \mathrm{mg}$ maintained BTMs in the postmenopausal range and increased lumbar spine BMD in patients previously treated with ODN.

Keywords: osteoporosis, cathepsin K inhibitor, odanacatib, zoledronic acid

\section{P006}

Functional characteristics of a yogurt containing GOS obtained from enzymatic action on milk lactose: effect on calcium absorption

Mariana Seijo ${ }^{1}, \mathrm{C} \mathrm{Venica}^{2}$, Maria Luz Pita Martin de Portela ${ }^{3}$, C Bergamini $^{2}$, L Wolf ${ }^{2}$, Maria Cristina Perotti ${ }^{2}$, Susana N Zeni ${ }^{1}$

${ }^{1}$ Metabolic Osteopaties Laboratory. INIGEM (UBA/CONICET), Buenos Aires, Argentina, ${ }^{2}$ Instituto de Lactología Industrial (INLAIN). Facultad de Ingeniería Química. Universidad Nacional del Litoral/CONICET, Santa Fe, Argentina, ${ }^{3}$ Cátedra de Nutrición., Facultad de Farmacia y Bioquímica - UBA, Buenos Aires, Argentina
The galactooligosaccharides (GOS) are bioactive carbohydrates present in human milk that induce beneficial effects in breastfed infant intestinal microbiota. They could be incorporated in dairy products, such as yogurt, from enzymatic action on the milk lactose. This milk product consumption changes the gut microbiota composition which releases metabolites associated with increased mineral absorption that improve bone health.

Objective: to evaluate during growth, the prebiotic effect of a yogurt containing GOS on calcium absorption. Male weaning Wistar rats $(\mathrm{n}=10$ per group) received during 30 days AIN'93-G control diet $(\mathrm{CD})$ or diet based on the yogurt containing GOS produced by $\beta$ galactosidase added during its preparation (ED), and calcium and protein according to AIN'93-G.

Determinations: dietary consumption three times per week; body weight (BW) and faecal Lactobacilli (LB) colonies weekly; Ca absorption during the last 3 days of the experience and caecal $\mathrm{pH}$ and short-chain fatty acid (SCFAs) at the end of the study.

Result as mean \pm SD. No differences in dietary consumption or BW were found $(16.6 \pm 2.7$ vs. $16.4 \pm 0.6 \mathrm{~g}$ and $229 \pm 13$ vs. $214 \pm 5 \mathrm{~g}$, respectively). ED compared to $\mathrm{CD}$ group showed an increased in faecal LB colonies $(\mathrm{p}<0.05)$ from the first week of experience; a significant lower caecal $\mathrm{pH}(5.2 \pm 0.1$ vs. $6.8 \pm 0.1$; $\mathrm{p}<0.01$ ) and higher SCFAs production (propionic acid: $14.59 \pm 3.62$ vs. $4.33 \pm 1.01 \mathrm{mg}$; butyric acid: $14.45 \pm 3.02$ vs. $2.76 \pm 0.75 \mathrm{mg} ; \mathrm{p}<0.001)$ at the end of the experience. ED group also had a significant increase in $\mathrm{Ca}$ absorption percentage $(84.8 \pm 1.4$ vs. $78.3 \pm 9.4 \% ; \mathrm{p}<0.05)$ representing approximately $10 \%$ increase in $\mathrm{mg} /$ day of $\mathrm{Ca}$ absorption

Conclusion: These results evidence the functional characteristics of the product (lower caecum $\mathrm{pH}$, higher growing of LB colonies and high LCFA production) that could be the responsible, at least in part, of inducing the effect on $\mathrm{Ca}$ absorption which may beneficiate bone health.

Grant: PIP (CONICET) 11220130100199CO.

\section{P008}

4-PBA helps the clearance of the engorged osteogenesis imperfecta patients fibroblasts by stimulating both protein secretion and autophagy

Roberta Besio ${ }^{1}$, Giusy Iula ${ }^{1}$, Nadia Garibaldi ${ }^{1}$, Lina Cipolla ${ }^{2}$, Simone Sabbioneda ${ }^{2}$, Marco Biggiogera ${ }^{1}$, Joan C. Marini ${ }^{3}$, Antonio Rossi ${ }^{1}$, Antonella Forlino $^{1}$

${ }^{1}$ University of Pavia, Pavia, Italy, ${ }^{2}$ Consiglio Nazionale delle Ricerche, Pavia, Italy, ${ }^{3}$ National Institute of Health, Bethesda, United States

Mutant type I collagen molecules play a negative effect both on extracellular matrix structure and function and on cellular homeostasis, since are responsible for endoplasmic reticulum enlargement and activation of the unfolded protein response (UPR). To reduce cellular stress, fibroblasts from OI patients carrying mutations in the a1(I) and a2(I) collagen chains and characterized by UPR activation were treated with the FDA-approved chemical chaperone 4-phenylbutyric acid (4-PBA). 4-PBA decreased the activation of the UPR sensor PERK in 4 out of 5 cell lines with mutations in $\alpha 1$ chain, and in 2 out of 4 cell lines with mutations in $\alpha 2$ chain $(\mathrm{p}<0.05)$. Importantly, the drug rescued cell death in the majority of cases, as demonstrated by the decreased level of the terminal cleaved caspase 3 evident by western blot analysis and by the decreased expression of the annexin $\mathrm{V}$ marker detected by cytofluorimetry $(\mathrm{p}<0.05)$. In order to understand the molecular basis of 4-PBA effects we investigated its chaperone and histone deacetylase inhibitory abilities. 4-PBA increased protein secretion in all OI cells, as evaluated by protein labeling with ${ }^{35} \mathrm{~S}$-L-methionine and ${ }^{35} \mathrm{~S}$-L-cysteine, and enhanced 
collagen secretion in the cells with the strongest collagen intracellular retention, as evaluated by chase experiments $(\mathrm{p}<0.05)$. 4-PBA increased the level of the histone $\mathrm{H} 3$ acetylation and this was associated with the upregulation of the expression of the autophagic gene ATG5. Importantly mRNA data were supported by protein data which revealed increased LC3-II levels in all $\alpha 1$ and a2 mutant cells treated with 4-PBA ( $<<0.05)$, clearly indicating a stimulation of autophagy. Thus 4-PBA, favouring both protein secretion and autophagy, helps the clearance of the engorged cells with positive effects on cell homeostasis and a reduction of cell death.

Fondazione Cariplo 2016-0417; European Community n. 602300. Keywords: collagen, osteogenesis imperfecta, autophagy, chemical chaperone

\section{P010}

High fidelity of mouse models mimicking human genetic skeletal disorders resulting from mutations in 316 genes (Skeletal Dysplasia Society 2015 Nosology)

$\underline{\text { Robert Brommage }}^{1}$, Claes Ohlsson ${ }^{1}$

${ }^{1}$ Centre for Bone and Arthritis Research, University of Gothenburg, Gothenburg, Sweden

The 2015 Human Genetic Skeletal Disorders Nosology update (Bonafe L, Am J Med Genet A 167A:2869-2892; 2015) summarizes knowledge of human skeletal disorders with Mendelian inheritance. The 316 genes identified code for enzymes (33\%), signal transduction proteins $(17 \%)$, transcription factors $(16 \%)$, scaffolding proteins $(15 \%)$, cilia proteins $(7 \%)$, extracellular matrix proteins $(6 \%)$ and membrane transporters $(5 \%)$.

The goal of this analysis was to determine the extent to which mutant mouse models (spontaneous and chemical-inducted mutants, global and conditional gene knockout mice, and transgenic mice with gene overexpression or specific base-pair substitutions) show skeletal phenotypes similar to those observed in homologous human gene disorders.

The human X-linked gene ARSE (Arylsulfatase E) and small nuclear RNA U4ATAC, a component of the minor spliceosome, do not have mouse homologues. Mutations in $8(3 \%)$ mouse genes (Fgf10, Flna, Hlxb9, Lmd3, Nsd1, Smarcal1, Ttc21b, Wisp3) do not result in similar skeletal phenotypes observed with mutations of the homologous human genes.

Skeletal phenotypes resulting from mutations in 199 mouse genes $(63 \%)$ are similar to those observed in homologous human skeletal disorders. For 56 mouse genes (15\%) possible mutant bone phenotypes have not been investigated. Mutant mice are not presently available for $47(17 \%)$ of genes. Global gene knockouts of several of these genes result in early embryonic lethality, precluding skeletal analyses. Since many human disorders involve gain-of-function or dominant-negative mutations, future studies will undoubtedly utilize CRISPR/Cas9 technology to examine transgenic mice having genes modified to exactly mimic variant human sequences.

These comparisons indicate that homologous mutations in $96 \%$ of mouse genes examined (199 of 208) result in skeletal phenotypes mimicking human skeletal disorders. Mutant mice provide excellent models for drug development, with examples including Acvrl (Fibrodysplasia Ossificans Progressive), Casr (Familial Hypocalcemic Hypercalcemia), Notch2 (Hajdu-Cheney Osteolysis Syndrome) and Phex (Hypophosphatemic Rickets).

\section{P012}

Levels of soluble ST2 are unaffected by vitamin D supplementation
Vito Francic ${ }^{1}$, Martin Gaksch ${ }^{2}$, Verena Schwetz ${ }^{1}$, Christian Trummer $^{1}$, Marlene Pandis ${ }^{1}$, Felix Aberer ${ }^{1}$, Martin Grübler ${ }^{1}$, Nicolas D. Verheyen ${ }^{3}$, Winfried März ${ }^{4}$, Thomas R. Pieber ${ }^{1}$, Andreas Tomaschitz $^{3}$, Stefan Pilz ${ }^{1}$, Barbara Obermayer-Pietsch ${ }^{1}$

${ }^{1}$ Department of Internal Medicine, Division of Endocrinology and Diabetology, Medical University of Graz, Graz, Austria, ${ }^{2}$ Department of Laboratory Medicine, Paracelsus Medical University, Salzburg, Austria, ${ }^{3}$ Department of Internal Medicine, Division of Cardiology, Medical University of Graz, Graz, Austria, ${ }^{4}$ Synlab Holding Germany GmbH, Synlab Academy, Mannheim, Germany

Introduction: ST2 is the membrane-bound receptor of IL-33, while soluble ST2 (sST2) is the truncated form of the receptor found in the circulation and functions as a decoy receptor, thereby inhibiting the effects of IL-33. sST2 is a promising biomarker in cardiovascular disease and its levels have been associated with various adverse outcomes. A recent study found negative associations of SST2 with $25(\mathrm{OH}) \mathrm{D}$ and $1,25(\mathrm{OH})_{2} \mathrm{D}$, while in vitro studies showed $1,25(\mathrm{OH})_{2} \mathrm{D}$ can affect the secretion of sST2. In this study, we determined the effects of vitamin D treatment on serum sST2 levels in hypertensive, vitamin $\mathrm{D}$ deficient subjects.

Methods: sST2 levels were measured (by Human St2/IL-33 R Quantikine ELISA Kit; R\&D Systems) at baseline and follow-up in 185 hypertensive, vitamin D deficient $(25(\mathrm{OH}) \mathrm{D}<30 \mathrm{ng} / \mathrm{ml})$ participants of the Styrian Vitamin D Hypertension Trial (NCT02136771). The participants were treated with vitamin D (2800 IU daily) or placebo for 8 weeks. We used analysis of covariance (ANCOVA) with adjustments for baseline values to determine the differences in sST2 levels between the placebo and treatment groups.

Results: $25(\mathrm{OH}) \mathrm{D}$ and $1,25(\mathrm{OH})_{2} \mathrm{D}$ levels increased significantly with a mean treatment effect (95\% confidence interval) of $11.36 \mathrm{ng} /$ $\mathrm{mL} \quad(9.20-13.52, \quad \mathrm{P}<0.001)$ and $9.47 \mathrm{pg} / \mathrm{mL} \quad(4.91-14.02$, $\mathrm{P}<0.001)$, respectively. We observed no significant effect of vitamin D treatment on serum SST2 levels neither in the whole study cohort (treatment effect [95\% confidence interval], 1.01 [0.95-1.08], $\mathrm{P}=0.679$ ), nor in a subgroup of study participants with baseline 25(OH)D levels below $20 \mathrm{ng} / \mathrm{mL} \quad(\mathrm{N}=70 ; 1.01 \quad$ [0.91-1.13], $\mathrm{P}=0.821$ ).

Discussion: While $25(\mathrm{OH}) \mathrm{D}$ and $1,25(\mathrm{OH})_{2} \mathrm{D}$ levels rose significantly after supplementation, serum levels of sST2 remained unaffected, both in the whole study cohort and in participants with initial 25(OH)D levels below $20 \mathrm{ng} / \mathrm{mL}$. Our study provides the first information about a lack of dynamic effects of vitamin $\mathrm{D}$ treatment on systemic sST2 levels.

\section{P014}

Hyperbaric oxygen inhibits HMGB1/RAGE signaling pathway by upregulating Mir-107 expression in human osteoarthritic chondrocytes

$\underline{\text { Li-Jen Yuan }}^{1}$, Song-Shu Lin ${ }^{2}$, Yuan-Kun Tu${ }^{1}$, Chi-Chien Niu ${ }^{2}$, Chuen-Yung Yang ${ }^{2}$, Steve WN Ueng ${ }^{2}$

${ }^{1}$ Department of Orthopaedic Surgery, E-Da Hospital/I-Shou University, Kaohsiung City, Taiwan, Republic of China, ${ }^{2}$ Department of Orthopaedic Surgery, Chang Gung Memorial Hospital, Taoyuan, Taiwan, Republic of China

Objective: Expressions of high mobility group box 1 (HMGB-1) and receptor for advanced glycation end products (RAGE) is upregulated in osteoarthritic (OA) cartilages. HMGB-1 functions as a coupling factor between hypoxia and inflammation in arthritis. The inflammatory response is modulated by microRNAs. Mir-107 is downregulated in OA cartilage. In this study, we investigated the 
regulation of Mir-107/HMGB1/RAGE signaling pathway in OA chondrocytes after hyperbaric oxygen (HBO) treatment.

Methods: Chondrocytes were separated from the knees of OA patients. Control cells were maintained in $5 \% \mathrm{CO}_{2} / 95 \%$ air. Hyperoxic cells were exposed to $100 \% \mathrm{O}_{2}$ at 2.5 atmospheres absolute. MiRNA targets were identified using bioinformatics and luciferase reporter assay. Cellular protein or mRNA levels of HMGB-1, RAGE and inducible nitric oxide synthase (iNOS) were analyzed. Phosphorylation of p38MAPK, ERK, and JNK was evaluated using a phospho-kinase array kit. The secreted HMGB-1, nitric oxide (NO), and metalloproteases (MMPs) levels in conditioned media were quantified.

Results: Bioinformatics approaches show that $3^{\prime} \mathrm{UTR}$ of HMGB-1 mRNA contained the 'seed-matched-sequence' for hsa-miR-107, which was validated by dual-luciferase reporter assay. MiR-107 was remarkably induced $(\mathrm{p}<0.05)$ by $\mathrm{HBO}$ and a marked suppression of HMGB-1 ( $<<0.05)$ was observed simultaneously in OA chondrocytes. Knockdown of miR-107 upregulated HMGB-1 expression in hyperoxic cells. HBO treatment downregulated the mRNA levels of HMGB-1, RAGE, iNOS ( $\mathrm{p}<0.05)$. The protein levels of HMGB-1, RAGE, and iNOS $(p<0.05)$ and the secretion of HMGB-1 $(\mathrm{p}<0.05)$ were downregulated after HBO treatment. HBO upregulated the protein level of cytosolic I $\mathrm{I} B \alpha$ and decreased the nuclear translocation of NF- $\kappa \mathrm{B}$. Moreover, HBO downregulated the phosphorylation of p38MAPK, ERK, and JNK and significantly decreased the secretion of MMP-3, MMP-9 and MMP-13 ( $<<0.05)$.

Summary and Conclusions: HBO inhibits HMGB-1/RAGE signaling related pathways by upregulating Mir-107 expression in human OA chondrocytes

Keywords: Mir-107, HMGB-1, RAGE, osteoarthritis, hyperbaric oxygen

\section{P016}

BMD association studies and functional assays to uncover the role of FLJ42280 in osteoporosis

Neus Roca-Ayats ${ }^{1,2,3}$, Darío G. Lupiáñez ${ }^{4}$, Núria Martínez-Gil ${ }^{1,2,3}$, Marina Gerousi $^{1}$, Mónica Cozar ${ }^{1,2,3}$, Natàlia Garcia-Giralt ${ }^{5,6}$, Xavier Nogués ${ }^{5,6}$, Leonardo Mellibovsky ${ }^{5,6}$, Adolfo Díez-Pérez ${ }^{5,6}$, Susana Balcells $^{1,2,3}$, Daniel Grinberg ${ }^{1,2,3}$

${ }^{I}$ Dept. Genetics, Microbiology and Statistics, IBUB, Universitat de Barcelona, Barcelona, Spain, ${ }^{2}$ CIBERER, ISCIII, Barcelona, Spain, ${ }^{3}$ Institut de Recerca Sant Joan de Déu, Barcelona, Spain, ${ }^{4}$ Epigenetics and Sex Development Group, Berlin Institute for Medical Systems Biology, Max-Delbrück Center for Molecular Medicine, Berlin, Germany, ${ }^{5}$ Musculoskeletal Research Group, IMIM (Hospital del Mar Medical Research Institute), Barcelona, Spain, ${ }^{6}$ CIBERFES, ISCIII, Barcelona, Spain

Genome-wide association studies (GWAs) have repeatedly identified variants associated with bone mineral density (BMD) and osteoporotic fracture located in intronic regions of $F L J 42280$, a poorly studied gene of unknown function.

We aimed at exploring the genetic variability and functionality of this locus. Firstly, we resequenced the FLJ42280 genomic region in two extreme BMD groups from the BARCOS cohort of postmenopausal women; we compared the number and frequency of variants between the two groups, and analyzed their overlap with functional elements from ENCODE. We identified 110 variants, 59 of which were rare and low frequency $(\mathrm{MAF}<0.05)$. Overall, the number of variants was balanced between the two extreme groups and frequency differences of any single variant were below the statistical power of the design. Four variants were located in putative osteoblast enhancers. Taking into consideration both frequency and functionality, we selected 8 SNPs to genotype in the complete BARCOS cohort and test them for association with BMD and fracture. Significant differences were obtained for 2 upstream SNPs, rs4342521 and rs10085588, in lumbar spine BMD (p values $=0.0031$ and 0.0036 , respectively) and in osteoporotic fracture ( $\mathrm{p}$ values $=0.0391$ and 0.0355 , respectively). Functional studies are currently underway for rs 10085588 , located in a conserved putative regulatory element $4 \mathrm{~kb}$ upstream of the gene.

Additionally, we studied the functionality of an enhancer present in intron 2 of FLJ42280, which has been described as an active enhancer for Dlx5 and Dlx6 in embryonic mouse branchial arches (Birnbaum et al., Hum Mol Genet 21(22):4930-4938, 2012) and also found to contain histone marks of active enhancers in osteoblasts (ENCODE data). We performed circular chromosome conformation capture and sequencing (4C-seq) in mouse embryonic day (E) 12.5 developing limbs and a clear interaction between the enhancer and the Dlx5 promoter could be detected. Further functional studies are currently underway.

\section{P018}

The influence of non-enzymatic glycation on microdamage accumulation in bone after fatigue loading

Graeme Campbell ${ }^{1}$, Martin Bellin ${ }^{1}$, Felix Schmidt ${ }^{2}$, Gabriela Mielke $^{1}$, Björn Busse $^{2}$, Michael Morlock ${ }^{1}$, Gerd Huber ${ }^{1}$

${ }^{1}$ Institute of Biomechanics, Hamburg University of Technology, Hamburg, Germany, ${ }^{2}$ Department of Osteology and Biomechanics, University Medical Center Hamburg-Eppendorf, Hamburg, Germany

Patients with type II diabetes mellitus (T2DM) have an elevated fracture risk despite normal or high bone mineral density. This suggests that the mechanical properties of the bone are impaired; however, the specific mechanisms leading to fragility have not been elucidated. Advanced glycation endproducts (AGEs) may reduce collagen toughness in T2DM bone, therefore, the purpose of this study is to determine whether AGEs lead to increased micro-damage accumulation in bone subjected to fatigue loading within the physiological range.

Bovine cortical bone specimens were cut into $3 \times 3 \times 30 \mathrm{~mm}$ beams and divided into three groups: Control (CT) Fatigue loading (FL) and Fatigue + Ribose incubation (FLR) $(\mathrm{N}=5$ each). The FLR specimens were incubated in a D-ribose solution for 11 days to induce AGE formation. The FL and FLR specimens were then subjected to rotating 3-point fatigue bending loading $(100,000$ cycles, $2 \mathrm{~Hz}$, $20 \mathrm{MPa}$ max stress). The micro-damage was assessed with barium sulphate $\left(\mathrm{BaSO}_{4}\right)$ precipitation, and micro-computed tomography $(\mu \mathrm{CT})$ to determine the damaged volume per bone volume (DV/BV).

The micro-damage accumulation could be visualised $\mathrm{BaSO}_{4}$ precipitation (Fig. 1). Fatigue loading significantly increased DV/BV compared to the unloaded specimens $(+328 \%, \mathrm{p}=0.03)$. Ribose incubation further increased the micro-damage accumulation under fatigue loading compared to the unloaded $(+884 \%, \mathrm{p}<0.001)$ and FL groups $(+130 \%, \mathrm{p}<0.001)$.
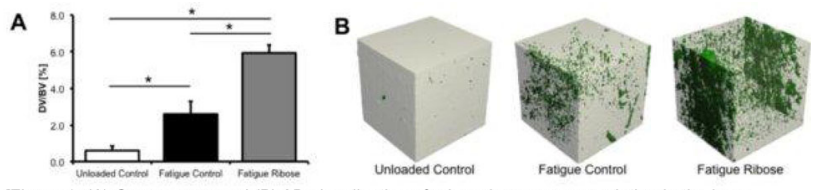

[Figure 1: (A) Group mean and (B) 3D visualisation of micro-damage accumulation in the bone specimens]

Increased micro-damage accumulation under physiological loading suggests that AGEs increase crack propagation and likely are significant contributors to bone fragility in T2DM. Testing on specimens from T2DM patients is required to confirm these findings.

Keywords: micro-damage, AGEs, micro-CT, fatigue loading 


\section{P020}

The effect of a vitamin D3 mediated PTH reduction on cardiovascular health in hyperparathyroid, vitamin D insufficient women: a randomized placebo-controlled trial

Lise Sofie Bislev ${ }^{1,2}$, Lene Langagergaard Rødbro ${ }^{1}$, Lars Rolighed ${ }^{3,4}$, Tanja Sikjær ${ }^{1}$, Lars Rejnmark ${ }^{1,2}$

${ }^{1}$ Department of Endocrinology and Internal Medicine, Aarhus University Hospital, Aarhus C, Denmark, ${ }^{2}$ Department of Clinical Medicine, Aarhus University, Aarhus, Denmark, ${ }^{3}$ Department of Surgery $P$, Aarhus University Hospital, Aarhus C, Denmark, ${ }^{4}$ Department of Otolaryngology, Aarhus University Hospital, Aarhus C, Denmark

Introduction: Emerging data reports a positive, bidirectional interaction between parathyroid hormone (PTH) and aldosterone. Although sparse investigated, adverse cardiovascular events of vitamin D insufficiency may partly be caused by the development of hyperparathyroidism. Accordingly we aimed to investigate the effect of a vitamin D3 mediated PTH-reduction on aldosterone and cardiovascular indices in vitamin D insufficient, hyperparathyroid women.

Method: In a double-blinded winter study we randomized 81 otherwise healthy postmenopausal women with secondary hyperparathyroidism (PTH $>6.9 \mathrm{pmol} / \mathrm{L})$ and 25-hydroxy-vitamin $D(25(\mathrm{OH}) \mathrm{D})$ levels $<50 \mathrm{nmol} / \mathrm{l}$ to 12 weeks of treatment with vitamin D3 $70 \mu \mathrm{g} /$ day or identical placebo.

Results: Vitamin D3 increased plasma $25(\mathrm{OH}) \mathrm{D}$ and $1.25(\mathrm{OH})_{2} \mathrm{D}$ by 219 and $42 \%$ respectively, compared to a decrease of 12 and $17 \%$ in the placebo group $(p<0.001)$. Treatment reduced PTH $15 \%$, while placebo (winter-season) increased PTH 5\% ( $<<0.001)$. Vitamin D3 did not affect blood pressure, vascular stiffness, glycemic state, heart rate, cardiac conduction or plasma concentrations of renin, angiotensin 2 or aldosterone. No associations between markers of the reninangiotensin-aldosterone system and levels of PTH was found. Vitamin D3 increased the renal excretion of calcium and magnesium with $31 \%(\mathrm{p}<0.05)$ and $10 \%(\mathrm{p}<0.01)$ respectively. Blood pressure and renal calcium excretion were positively correlated. Vitamin D3 treatment had a favorable effect on plasma levels of high-density lipoproteins (HDL) that decreased by $3 \pm 9 \%$ compared to a $7 \pm 12 \%$ decrease in the placebo group $(\mathrm{p}<0.05)$.

Conclusion: Our data do not support a direct interaction between PTH and aldosterone. We included hyperparathyroid women with low vitamin D levels, who assumable are most likely to benefit from a vitamin D supplement, but except from a favorable effect of HDL levels, lowering of PTH levels by normalization of vitamin D status had no beneficial effects on indices of cardiovascular health.

Keywords: Vitamin D, PTH, cardiovascular health, aldosterone, cholesterol

\section{P022}

Calcitriol may act on an alternate receptor during fetal development, since absence of calcitriol has different consequences than loss of the vitamin D receptor

Brittany A. Ryan ${ }^{1}$, K. Berit Sellars ${ }^{1}$, Beth J. Kirby ${ }^{1}$, René StArnaud $^{2,3}$, Christopher S. Kovacs ${ }^{1}$

${ }^{1}$ Memorial University of Newfoundland, St. John's, Canada,${ }^{2}$ McGill University, Montreal, Canada, ${ }^{3}$ Shriners Hospitals for Children, Montreal, Canada

Vitamin D receptor $(V d r)$ null fetuses have normal serum minerals, PTH, and skeletal morphology/mineralization. But $V d r$ null fetuses also have increased serum calcitriol, placental calcium transport, and placental expression of PTHrP and TRPV6.
We examined Cyp27b1 null fetal mice, which do not make calcitriol, to determine if loss of calcitriol has the same consequences. Cyp27b1 null and WT females (first-degree relatives) were mated to Cyp27b1 $1^{+1-}$ males. This generated Cyp27b1 null and Cyp27b1 $1^{+/-}$ fetuses from null dams, and $C y p 27 b 1^{+1-}$ and WT fetuses from WT dams.

Fetal and maternal serum were collected; intact fetuses were ashed; ash mineral content was measured by atomic absorption spectroscopy; tibiae and placentas were harvested; placental gene expression was assessed by qPCR. ${ }^{45} \mathrm{Ca}$ and ${ }^{51} \mathrm{Cr}$-EDTA were administered by intracardiac injection to pregnant dams and radioactivity transported to the fetuses was measured after $5 \mathrm{~min}$. Statistical analysis used ANOVA with Tukey's for multiple comparisons. Animal ethics approved these studies.

Calcitriol was undetectable in null fetuses, which also had (vs. littermates) normal serum calcium $(2.22 \pm 0.10$ vs. $2.29 \pm 0.07 \mathrm{mM})$, phosphorus $(2.10 \pm 0.11$ vs. $2.14 \pm 0.12 \mathrm{mM}$ ), FGF23 (49 \pm 8 vs. $53 \pm 7 \mathrm{pg} / \mathrm{mL})$, PTH, skeletal ash weight ( $17 \pm 0.4 \mathrm{vs} .17 \pm 0.4 \mathrm{mg})$, and ash calcium $(74.5 \pm 1.0$ vs. $76.2 \pm 1.5 \mathrm{mg} / \mathrm{g})$ and phosphorus. Placental calcium transport (104 \pm 5 vs. $111 \pm 9 \%)$, and placental expression of Pthrp $(0.85 \pm 0.32$ vs. $1.00 \pm 0.86)$ and Trpv6 $(0.95 \pm 0.39$ vs. $1.00 \pm 0.25)$, were no different between null and WT fetuses. Tibial lengths and morphology were normal.

In summary, loss of calcitriol did not alter fetal mineral or bone homeostasis, including placental calcium transport and placental gene expression. $V d r$ nulls differ by displaying increases in placental calcium transport and expression of TRPV6 and PTHrP. These findings confirm that calcitriol and VDR are not required during fetal development, but imply that high levels of calcitriol upregulate placental function in $V d r$ null fetuses by acting on an alternate ceptor.

\section{P024}

Prevalence and risk of vertebral fractures in primary hyperparathyroidism: a cross-sectional study

Henriette Ejlsmark-Svensson ${ }^{1}$, Lise Sofie Bislev ${ }^{2}$, Siv Lajlev ${ }^{2}$, Torben Harsløf ${ }^{2}$, Lars Rolighed ${ }^{2}$, Tanja Sikjær ${ }^{2}$, Lars Rejnmark ${ }^{2}$

${ }^{1}$ Endocrinology and Internal Medicine, Aarhus University Hospital, Aarhus, Denmark, ${ }^{2}$ Aarhus University Hospital, Aarhus, Denmark

Introduction: Prevalence of vertebral fractures (VFx) in patients with primary hyperparathyroidism (PHPT) remains uncertain. The presence of VFx is of importance to choice of treatment. We aimed to estimate the prevalence of VFx, study potential risk factors for VFx and compare bone mineral density (BMD) between PHPT- and osteoporotic patients with VFx.

Methods: Through The Danish Discharge register, we identified patients diagnosed with PHPT between 2005 and 2015. The diagnosis was biochemical verified. X-ray reports were reviewed by two investigators. Through our department's educational patients program, osteoporotic patients with VFx were identified and match on age and sex with PHPT patients.

Results: We identified 792 patients with PHPT, spine X-ray was available from 588 patients. VFx were present in $122(20.7 \%)$ patients, equally frequent among sex (77\% females). Fractured patients were older (70 [IQR 62-77] years. vs. 63 [IQR 55-71] years.) and had lower heights (163 [IQR 158-169] cm vs. 166 [IQR 160-172] $\mathrm{cm})$ compared to non-fractured patients ( $p<0.02$ for both). After stratification by sex, the prevalence of VFx were higher in males in younger age-group compared to females $(\mathrm{p}<0.01)$. Ionized-calcium and PTH did not differ between groups. BMD at total hip and forearm were lower in fractured compared to non-fractured patients, adjusted for age, sex and BMI, ( $<<0.03$ for both). Compared with osteoporotic patients with $\mathrm{VFx}(\mathrm{N}=108), \mathrm{BMD}$ at lumbar spine was higher in PHPT patients $(\mathrm{N}=108)\left(0.77 \mathrm{~g} / \mathrm{cm}^{2} ; 95 \%\right.$ CI $0.74-0.80 \mathrm{~g} /$ 
$\mathrm{cm}^{2}$ vs. $0.88 \mathrm{~g} / \mathrm{cm}^{2} ; 95 \%$ CI $\left.0.84-0.92 \mathrm{~g} / \mathrm{cm}^{2}\right)(\mathrm{p}<0.01)$. This was not changed by excluding patients with lumbar VFx $(\mathrm{p}<0.01)$.

Conclusion: Severity of PHPT does not seems to be associated with risk of VFx. The relatively high prevalence of VFx support a proactive screening of PHPT patients. Compared with osteoporosis; VFx seems to occur at a higher BMD in PHPT.

Keywords: Primary hyperparathyroidism, vertebral fractures

\section{P026}

ZNF687-induced Paget's disease of bone do not degenerate in Giant Cell Tumor in presence of pharmacological treatment

Federica Scotto di Carlo ${ }^{1,2}$, Giuseppina Divisato ${ }^{1}$, Nadia Petrillo ${ }^{1}$, Laura Pazzaglia $^{3}$, Maria Serena Benassi ${ }^{3}$, Teresa Esposito ${ }^{1,4}$, Fernando Gianfrancesco ${ }^{1}$

${ }^{1}$ Institute of Genetics and Biophysics, National Research Council of Italy, Naples, Italy, ${ }^{2}$ Department of Environmental, Biological and Pharmaceutical Sciences and Technologies (DiSTABiF), University of Campania Luigi Vanvitelli, Caserta, Italy, ${ }^{3}$ Laboratory of Experimental Oncology, Rizzoli Orthopedic Institute, Bologna, Italy, ${ }^{4}$ IRCCS INM Neuromed, Pozzilli (IS), Italy

Paget's disease of bone (PDB) is a bone remodelling disorder characterized by focal areas of increased bone resorption coupled with a disorganized bone formation. PDB can rarely degenerate into Osteosarcoma (OS/PDB) in $1 \%$ of cases, and less frequently in Giant Cell Tumor (GCT/PDB). Recently, we disclosed that the P937R mutation in the ZNF687 gene is responsible for Giant Cell Tumor arising on PDB bones. Since $80 \%$ of Italian GCT/PDB patients derive from South Italy, we hypothesized that ZNF687 mutation rate in PDB patients was higher in this region than elsewhere.

Interestingly, $33 \%$ of recruited patients hosted ZNF687 mutations, with the P937R identified in all familial cases that showed a more severe PDB. Our investigation of familial history of the ZNF687-mutated patients revealed that in four pedigrees PDB and GCT/PDB coexisted. Importantly, all probands of these families are still alive and report a stabilized clinical course of the disease as a result of zoledronate or clodronate treatment. On the other hand, in their affected and untreated relatives, the longstanding disease degenerated into GCT/PDB, leading them to death.

To fully elucidate ZNF687 role in GCT/PDB and other PDB neoplastic transformations, here we also performed its somatic molecular analysis on 42 tumor tissues (4 GCT/PDB, 28 OS/PDB, 8 Sarcoma/PDB, 1 Fibrosarcoma/PDB, and 1 Chondrosarcoma/PDB). We identified the same mutation (P937R) in all GCT/PDB cases that, to the best of our knowledge, did not undergo pharmacological treatment. Moreover, the P937R mutation was also identified in 1 Sarcoma/PDB patient, whereas a different mutation (R331W) was found in 1 OS/PDB sample.

These data strictly relate the causative role of ZNF687 to the GCT/ PDB pathogenesis that seems to be a degeneration of an untreated PDB.

\section{P028}

Risk factors of new adjacent and remote vertebral fracture following vertebroplasty for osteoporotic vertebral compression fracture: survivorship analysis of 205 patients

\section{$\underline{\text { Ye-Soo Park }}^{1}$, Jin-Sung Park ${ }^{1}$, Woong-Hwan Choi $^{2}$}

${ }^{1}$ Orthopaedic Surgery, Guri Hospital, Hanyang University College of Medicine, Guri City, Republic of Korea, ${ }^{2}$ Internal Medicine, Hanyang University College of Medicine, Seoul, Republic of Korea

Objectives: The purpose of this study was to investigate the natural history of new fracture following vertebroplasty for osteoporotic vertebral compression fracture (OVCF) and to evaluate the risk factors for adjacent vertebral fracture (AVF) and remote vertebral fracture (RVF) separately.
Methods: A retrospective study of 205 consecutive patients who underwent a vertebroplasty for OVCF with a minimum follow-up of 1 year was conducted. A separate survivorship analysis of AVF and RVF was performed using the Cox proportional hazards model.

Results: Of the 47 patients (22.9\%) with new vertebral fracture, AVF developed in 21 patients $(10.2 \%)$ and RVF in 26 patients $(12.7 \%)$. The median survival time was 4.0 months for AVF and 14.0 months for RVF. Multivariate analyses revealed that sagittal alignment imbalance and initial vertebroplasty at the thoracolumbar junction were independent risk factors for AVF (hazard ratio $=9.6$ and 4.7, respectively), whereas the significant risk factor for RVF was a sagittal alignment imbalance (hazard ratio $=11.5$ ). A low bone mineral density of the spine (T-score $\leq-3$ ) was shown to be a risk factor for AVF and RVF on univariate analyses, but not on multivariate analyses.

Conclusion: AVF developed at a median of 4 months and RVF at a median of 14 months. An initial vertebroplasty at the thoracolumbar junction was an independent risk factor for AVF, and patients with sagittal alignment imbalance had a high risk of AVF and RVF after vertebroplasty for OVCF.

Keywords: vertebroplasty, risk factor, thoracolumbar junction, sagittal alignment imbalance

\section{P030}

Sclerostin: a mediator between bone and vasculature?

Annelies De Maré ${ }^{1}$, Britt Opdebeeck ${ }^{1}$, Ellen Neven ${ }^{1}$, Patrick C. D’Haese $^{1}$, Anja Verhulst ${ }^{1}$

${ }^{1}$ University of Antwerp, Wilrijk, Belgium

The Wnt/ $\beta$-catenin signaling, one of the most important bone anabolic pathways, might also be a major player in the crosstalk within the bone-vascular-axis. When pathologically disturbed, this axis results in the concomitant occurrence of disturbed bone metabolism and vascular calcification (VC). A hallmark of these VCs is the transdifferentiation of vascular smooth muscle cells (VSMCs) towards bone-forming (osteochondrogenic) cells. In the current study we investigated parameters related to the $\mathrm{Wnt} / \beta$-catenin signaling cascade and its inhibitor sclerostin.

Rats were given $0.3 \mathrm{mg}$ warfarin/g diet to induce VC. Rats not receiving warfarin were included as controls. Rats were sacrificed at different time-points, i.e. after 4, 6, 8 and 10 weeks of warfarin treatment, to follow up the development of VC. At sacrifice; VC, aortic mRNA expression and immunohistochemistry, bone status and serum biochemistry were analyzed.

Results showed a time-dependent increase in VC in warfarin-treated rats. Aortic calcium concentration significantly differed from controls in 4 -wk treated rats $(\mathrm{p}=0.0286)$, reaching a 50 -fold increase in 10 -wk treated rats $(\mathrm{p}=0.0061)$. Furthermore, aortic mRNA levels of osteochondrogenic transdifferentiation markers (Sox9, $\mathrm{p}=0.0317$ and Cbfa1, $\mathrm{p}=0.0635$ ) and $\beta$-catenin (regulating target gene transcription, $\mathrm{p}=0.0159)$ were upregulated. Interestingly, aortic mRNA $(\mathrm{p}=0.0159)$ and protein levels of sclerostin, and also serum levels $(\mathrm{p}=0.0381)$ of this protein, were significantly upregulated in 10-wk treated rats compared to control rats. Finally, a mild but significant decrease in bone formation parameters was observed in 10-week warfarin-treated rats.

Our results support the hypothesis that VSMCs transdifferentiate towards osteochondrogenic cells and thereby also express genes/ proteins associated with the $\mathrm{Wnt} / \beta$-catenin signaling, including its inhibitor sclerostin. The latter thereby may act as a negative feedback protein to prevent excessive (vascular) calcifications, similar to its function in bone. Sclerostin might also spillover from the vessels to the circulation (high serum sclerostin levels) causing mild inhibition of bone formation.

Keywords: Vascular calcification, sclerostin, Wnt/ $\beta$-catenin signaling 


\section{P032}

Onset of bone remodeling awakens local osteoprogenitors

Thomas Levin Andersen ${ }^{1}$, Pia Risgaard Jensen ${ }^{1}$, Tanja Tvistholm Sikjaer $^{2}$, Lars Rejnmark ${ }^{2}$, Charlotte Ejersted ${ }^{3}$, Jean-Marie Delaisse ${ }^{1}$

${ }^{1}$ Clinical Cell Biology, University of Southern Denmark, Vejle, Denmark, ${ }^{2}$ Institute of Clinical Medicine, Aarhus University Hospital, Aarhus, Denmark, ${ }^{3}$ Department of Endocrinology, Odense University Hospital, Odense, Denmark

Flat osteoblast lineage cells interposed between the quiescent bone surfaces (QS) and the bone marrow have been recognized as an important source of osteoprogenitors. At the onset of bone remodeling, these osteoprogenitors either colonize the eroded surface generated by osteoclasts, thereby becoming reversal cells, or form a continuous canopy above remodeling surfaces. These canopy and reversal cells later transform into bone forming osteoblasts.

Here we report the changes in density, proliferation and gene expression profile of these local osteoprogenitors at the onset of bone remodeling.

Immunostainings and in situ hybridizations of specific markers were performed on transiliac biopsies from 11 healthy women (age 36-71 years).

Compared with osteoblast lineage cells of QS, the canopy and reversal cells showed a higher cell density (collectively $58 \pm 5.8$ vs $22 \pm 1.7$ nuclei $/ \mathrm{mm}$ surface, $\mathrm{p}<0.001)$ and were more proliferative $(\mathrm{Ki} 67$ immunoreactive) (OR vs. QS $>2.2, \mathrm{p}<0.002$ ). Canopy cells were more frequently positive for the early osteoblast lineage markers SMA (smooth muscle actin) and Tenascin C than osteoblast lineage cells of QS (OR vs. $\mathrm{QS}>11, \mathrm{p}<0.01)$. Upon contact with osteoclasts, both reversal and canopy cells more frequently expressed the early osteoblast lineage markers MMP-13 and fibronectin than those away from osteoclasts (OR vs. away of osteoclasts $>4.4, \mathrm{p}<0.005$ ) and than the osteoblast lineage cells of QS (OR vs. QS $>5, \mathrm{p}<0.001)$. Most osteoprogenitors, including reversal and canopy cells, were stained for CD271 (nerve growth factor receptor), Frizzled-9 (WNT receptor) and PDGF-R $\alpha$.

Collectively, these specific changes in cell density, proliferation and gene expression profile support that the onset of bone remodeling awakens the local osteoprogenitors which gradually differentiate into bone forming osteoblasts. Moreover, it highlights that the contact with osteoclasts plays a critical role in this process.

\section{P034}

Tryptophan hydroxylase 2: a potential bone mass regulator?

Yannick Sogl $^{1}$, Deeksha Malhan ${ }^{1}$, Sabine Stoetzel ${ }^{1}$, Polina Peeva ${ }^{2}$, Michael Bader ${ }^{2,3}$, Natalia Alenina ${ }^{2}$, Stefanie Kern ${ }^{1}$, Christian Heiss $^{1,4}$, Thaqif El Khassawna ${ }^{1}$

${ }^{1}$ Laboratory for Experimental Trauma Surgery, Faculty of Medicine, Justus-Liebig University Giessen, Giessen, Germany, ${ }^{2}$ Max Delbrück Center for Molecular Medicine (MDC), Berlin, Germany, ${ }^{3}$ Charité University Medicine, Berlin, Germany, ${ }^{4}$ Department of Trauma, Hand and Reconstructive Surgery, University Hospital of Giessen-Marburg, Giessen, Germany

Age related bone loss results from dysregulated bone metabolism. Serotonin influences bone remodeling differentially depending on its origin of synthesis. Understanding the effect of central serotonin loss on bone metabolism in relation to aging requires a longitudinal investigation. Therefore, we used young and old rats deprived of brain serotonin due to genetic deletion of Tryptophan hydroxylase 2 (TPH2), the rate limiting enzyme of serotonin synthesis in brain. Such understanding might suggest to target serotonin synthesis as therapeutic or prophylactic option for age related bone loss.

Forty wild type (WT) and Tph2-/- rats were euthanized at ( $\mathrm{W}=$ weeks) $6 \mathrm{~W}, 20 \mathrm{~W}, 30 \mathrm{~W}$, and $40 \mathrm{~W}$. Time points were chosen to study bone parameters before and after the peak bone mass age in rats. The samples were used for histomorphometrical analysis of matrix mineralization using Von Kossa/van Gieson stain. Osteoblasts and osteoclasts activity were examined using immunostainings.

Von Kossa/Van Gieson stain and histomorphometry showed lower mineralized bone matrix and higher non-mineralized bone matrix portions in $T p h 2-1-$ at $40 \mathrm{~W}$ compared with $30 \mathrm{~W}$. Intriguingly, both osteoblasts and osteoclasts area and number increased exponentially with age in Tph2-/- group. Whereas the WT showed a fluctuating pattern in osteoblast and osteoclast activity between time points. Further, WT showed more osteoclast at $20 \mathrm{~W}$ and $40 \mathrm{~W}$ when compared with the Tph2-l-.

Understanding the regulation of bone mass is important to treat systemic skeletal diseases. Loss of Tph2-/- indicated better bone parameters when compared with WT. Nonetheless, the discrepancies at the later time points suggest a more rapid age-related bone loss. The current results suggest a therapeutic potential of TPH 2 to regulate age-related bone loss. However, dysregulation in bone matrix mineralization requires further investigation of changes in collagen fibers arrangement and osteocytes morphology using histological methods. Furthermore, bone mineral density measurements using DXA are carried out.

\section{P036}

Analysis of bone form a case of Bruck syndrome caused by PLOD2 mutations reveals the abnormal collagen post-translational chemistry and cross-linking driving pathogenesis

Charlotte Gistelinck ${ }^{1}$, Maryann Weis ${ }^{1}$, Peter H. Beyers ${ }^{2}$, David R. Eyre $^{1}$

${ }^{1}$ Department of Orthopaedics and Sports Medicine, University of Washington, Seattle, United States, ${ }^{2}$ Departments of Pathology and Medicine, University of Washington, Seattle, United States

Bruck syndrome (BS) is a congenital disorder characterized by joint contractures, skeletal dysplasia and bone fragility, which overlaps clinically with Osteogenesis Imperfecta (OI). On a genetic level, BS is caused by biallelic mutations in either FKBP10 or PLOD2. PLOD2 encodes lysyl hydroxylase 2 (LH2), which is responsible for the hydroxylation of cross-linking lysine residues in fibrillar collagen telopeptide domains. This modification is essential for allowing collagens to form stable intermolecular cross-links in the ECM. Bone collagen has a unique pattern of such cross-links which are required for bone strength, resistance to micro-damage and crack propagation, and also an ordered deposition of mineral nanocrystals in the fibrillar collagen matrix.

To date, no direct studies of human bone from BS caused by PLOD2 mutations have been reported. We present here results from a case of BS in a 4-year-old Caucasian patient, caused by mutations in PLOD2. Bone collagen was biochemically characterized using SDS-PAGE, cross-link analysis and peptide mass-spectrometry. The results showed diminished hydroxylation of type I collagen telopeptide lysines, but normal hydroxylation at triple-helical sites. Consequently, mature trivalent cross-link levels, for which telopeptide hydroxylysines, are required, were greatly reduced. Instead, allysine aldol dimeric cross-links, which are not normally present in bone but are a feature in skin collagen, were prominent. Urine from the patient was also analysed, confirming the paucity of bone type I collagen pyridinoline-containing peptides, but a normal pyridinoline-containing cross-linked telopeptide from type II collagen. This indicates that in contrast to bone type I collagen, telopeptide lysines in the patient's growth plate cartilage type II collagen were normally hydroxylated. Taken together these findings shed light on the complex mechanisms that control the unique posttranslational chemistry and cross-linking of bone collagen, and how defects produce brittle bones and related connective tissue problems.

Keywords: Collagen, Osteogenesis Imperfecta, Bruck Syndrome, PLOD2 


\section{P038}

Long-term follow-up of the bone marrow adipose tissue distribution in ovariectomized rats reveals a clustering of adipocytes at the tibiae trabecular bone surface

Xavier Coutel ${ }^{1,2,3}$, Jérôme Delattre ${ }^{1}$, Pierre Marchandise ${ }^{1}$, Greet Kerckhofs $^{4,5}$, Guillaume Penel ${ }^{1,2,3}$, Cécile Olejnik ${ }^{1,2,3}$

${ }^{1}$ Université de Lille, EA4490 PMOI, Lille, France, ${ }^{2}$ Faculté de Chirurgie Dentaire, Lille, France, ${ }^{3}$ Service d'Odontologie, CHRU de Lille, Lille, France, ${ }^{4}$ Development and Regeneration, Skeletal Biology and Engineering Center, KU Leuven, Belgium, ${ }^{5}$ Division of Skeletal Tissue Engineering, Prometheus, KU Leuven, Belgium

Introduction: During osteoporosis, higher bone marrow adipose tissue (BMAT) is associated with lower bone density (Schwartz AV, 2015). However relationship between BMAT and bone loss is unclear. This study aims to analyze the 3D-relationship between BMAT and tibiae trabecular bone during ovariectomy in a long-term follow-up on mature rats.

Methods: Female Sprague-Dawley rats of 6-month-old $(n=45)$ were OVX or SHAM operated and tibiae were studied at 6 (baseline), 10 (M4) and 15 (M9) months. An advanced microCT imaging (Coutel $X, 2018$ ) allowed accurate alignment of both the BMAT distribution and bone micro-architecture as well as calculation of the distance of the BMAT from the bone surface.

Results: Bone microarchitectural parameters were altered in both M4and M9-OVX groups compared to the corresponding SHAM groups: BV/ TV $(-63,-65 \%, \mathrm{p}<0.0001)$, Tb.N $(-65,-68 \%, \mathrm{p}<0.0001)$, Tb.Th $(+7,+13 \%, \mathrm{p}=0.01)$, Tb.Sp $(+188,+238 \%, \mathrm{p}<0.0001)$.

BMAT parameters were increased in both M4- and M9-OVX groups compared to the corresponding SHAM groups: MaV/TV $(+34$, $+34 \%, \mathrm{p}<0.0001)$ and AdV/Ma.V $(+103,+483 \%, \mathrm{p}<0.0001)$.

At baseline, BMAT content (Ad per $\mu \mathrm{m}^{3}$ ) was higher at $80-100 \mu \mathrm{m}$ distance to trabecular bone in comparison to $0-20 \mu \mathrm{m}(+79 \%$, $\mathrm{p}=0,0004)$. A higher BMAT content was located close to trabecular bone surface $(0-20 \mu \mathrm{m})$ in both M4 and M9 OVX groups compared to baseline $(+92,+87 \%, \mathrm{p}=0.0002 / 0.0004)$ and compared to the corresponding SHAM groups $(+53,+75 \%, \mathrm{p}=0.0008 / 0.0002)$.

Conclusion: The present study demonstrate that trabecular bone loss occurring during ovariectomy is associated with BMAT clustering at bone surface in short and long-term processes. Based on the present results, additional studies in the paracrine role of BMAT mediators appears of great interest for better physiopathology understanding of bone loss and should lead to new treatments.

Keywords: Bone marrow adipose tissue, Bone microstructure, Osteoporosis, Rat model, microCT

\section{P040}

Chronic psychosocial stress disturbs the immune response and endochondral ossification after fracture

Melanie Haffner-Luntzer ${ }^{1}$, Sandra Förtsch ${ }^{2}$, Verena Fischer ${ }^{1}$, Katja Prystaz $^{1}$, Anita Ignatius ${ }^{1}$, Stefan O. Reber ${ }^{2}$

${ }^{1}$ Institute of Orthopedic Research and Biomechanics, University Medical Center Ulm, Ulm, Germany, ${ }^{2}$ Clinic for Psychosomatic Medicine and Psychotherapy, University Hospital Ulm, Ulm, Germany

Stress is known to disturb bone homeostasis and to activate the immune system. Previous studies demonstrated that a balanced inflammatory response after fracture is crucial for bone healing. Therefore, chronic stress may alter the inflammatory response after fracture, and thus, impair bone regeneration. To investigate this hypothesis, we used the chronic subordinate colony housing paradigm (CSC), a murine model for chronic psychosocial stress, the most important stressor in humans.

7-weeks-old male C57BL/6 mice were subjected to CSC for 19 days. Four mice were housed together with a dominant male mouse in order to induce chronic subordination. Single-housed (SHC) mice were used as controls. On day 20, the femur was osteotomized. Recruitment of immune cells to the site of fracture was assessed by FACS of the hematoma $24 \mathrm{~h}$ after fracture. Endochondral bone formation was analyzed by $\mathrm{qPCR}$ following lasercapturemicrodissection of callus tissue, histomorphometry, 3-point-bendingtesting and $\mu \mathrm{CT}$-analysis on days 10 and 21 after fracture. $\mathrm{n}=8$ /group. Student's $t$ test, $\mathrm{p} \leq 0.05$.

Mice subjected to CSC displayed significantly increased numbers of neutrophils in the hematoma [2.7\% of living cells (SHC) vs. $4.8 \%$ (CSC)], whereas T- and B-lymphocytes were significantly reduced (Tlymphocytes: $1.4 \%$ (SHC) vs. $0.4 \%$ (CSC); B-lymphocytes: 1.2 vs. $0.9 \%)$. Ten days after surgery, markers for cartilage-to-bone-transition (Runx2, VEGFa) were significantly lower expressed in callus chondrocytes. 21 days after fracture, CSC mice displayed significantly decreased bending stiffness $\left(1269 \mathrm{Nmm}^{2}\right.$ (SHC) vs. $855 \mathrm{Nmm}^{2}$ (CSC)) and mineralization in the fracture callus. Cartilage persisted longer in the callus of CSC mice, indicating disturbed endochondral ossification.

Concluding, chronic psychosocial stress results in a misbalanced immune response after fracture. The increase of neutrophils and decrease of lymphocytes $24 \mathrm{~h}$ after fracture may indicate a stronger initial innate immune reaction and a delayed shift to adaptive immune responses. Consequently, endochondral ossification was disturbed, leading to delayed fracture healing.

Keywords: fracture healing; stress

\section{NI Seminar}

\section{P089}

Associations between metabolic syndrome and bone mineral density, trabecular bone score in postmenopausal women with non-vertebral fractures

Vladyslav Povoroznyuk $^{1}$, Larysa Martynyuk ${ }^{1}$, Iryna Syzonenko ${ }^{1}$, Liliya Martynyuk ${ }^{1}$

${ }^{1}$ Department of Clinical Physiology and Pathology of Locomotor Apparatus, D. F. Chebotarev Institute of Gerontology NAMS Ukraine, Kyiv, Ukraine

This study is aimed to examine the associations of metabolic syndrome components, bone mineral density (BMD) and trabecular bone score (TBS) in menopausal women with non-vertebral fractures.

Materials and Methods: 1161 menopausal women aged 50-79 year-old were examined and divided into three groups: A included 419 women with increased body weight (BMI $\left.-25.0-29.9 \mathrm{~kg} / \mathrm{m}^{2}\right)$, B -442 female with obesity (BMI $>29.9 \mathrm{~kg} / \mathrm{m}^{2}$ ) and C-300 women with metabolic syndrome (diagnosis according to IDF criteria, 2005). It was used dual-energy X-ray absorptiometry (Prodigy, GE Medical systems, Lunar, Madison, WI, USA, 2005) for measuring of lumbar spine (L1-L4), femoral neck, total body and forearm BMD and bone quality indexes (last according to Med-Imaps installation). Data were analyzed using Statistical Package 6.0.

Results: A significant increase of lumbar spine (L1-L4), femoral neck, total body and ultradistal radius BMD was found in women with obesity and metabolic syndrome compared to the pre-obese ones $(\mathrm{p}<0.001)$. TBS was significantly higher in women with increased body weight compared to obese and metabolic syndrome patients. Analysis showed significant positive correlation between waist circumference, triglycerides level and BMD of lumbar spine and femur. Significant negative association between serum HDL level and BMD of investigated sites was established. The TBS (L1-L4) indexes positively correlated with HDL level. Low-trauma non-vertebral fractures occurred in $14.6 \%$ female with increased body weight, $17.4 \%$ of women with obesity and $21.3 \%$ of patients with metabolic syndrome.

Conclusion: Menopausal women with obesity and metabolic syndrome have a significantly higher BMD at all measured sites 
compared to females with pre-obesity. TBS is significantly lower in women with non-vertebral fractures and increased body weight or obesity. Despite the fact that BMD indexes were better in women with metabolic syndrome, the frequency of non-vertebral fractures was significantly higher in this group of patients.

\section{P091}

Description of cortical fibula structure in trained footballers using peripheral quantitative computed tomography (pQCT), with dynamometric correlates

Sergio Luscher ${ }^{1}$, Laura Marcela Nocciolino ${ }^{1,2}$, Nicolas Pilot ${ }^{2}$, Leonardo Pisani ${ }^{2}$, Gustavo Roberto Cointry ${ }^{1}$, Joern Rittweger ${ }^{3,4}$, Alex Ireland $^{5}$, Jose Luis Ferretti ${ }^{1}$, Ricardo Francisco Capozza ${ }^{1}$

${ }^{1}$ Centro de Estudios de Metabolismo Fosfocálcico (CEMFoC), Universidad Nacional de Rosario, Rosario, Argentina, ${ }^{2}$ Center of Musculoskeletal Biomechanical Studies (CEBOM), University Institute of Gran Rosario (IUGR),, Rosario, Argentina, ${ }^{3}$ Institute of Aerospace Medicine, German Aerospace Center (DLR), Cologne, Germany, ${ }^{4}$ Department of Pediatrics and Adolescent Medicine, University of Cologne, Cologne, Germany, ${ }^{5}$ School of Healthcare Science, Manchester Metropolitan University, Manchester, United Kingdom

Human fibula diaphysis is insensitive to disuse and its responses to exercise are scarcely studied. In this study, 18 serial pQCT scans of the whole fibula were taken and the strength of the external rotation of the feet was dynamometrically determined in dominant (kicking) leg of 15 male football players (4-8 years of training) and of 15 untrained controls aged $18-30$ years.

Trained individuals had higher bone mass (total and cortical BMC, cortical area) than untrained ones throughout the bone (about $+13 \%$, $\mathrm{p}<0.001$ ), with region-dependent differences in periosteal and endocortical perimeters, cortical thickness, and buckling ratio (distally, about +5 , $-2,+7,+7 \%$; proximally, $+12,-12,+2,+2 \%$, respectively; always $\mathrm{p}<0.001$ ). Cross-sectional moments of inertia for lateral bending (yMI) and torsion (pMI) were consistently larger throughout the bone in football players $(+26 /+32 \% ; \mathrm{p}<0.001)$, while that for A-P bending $(\mathrm{xMI})$ was greater proximally than distally (about +28 vs $+5 \%$; $\mathrm{p}<0.001$ ). Adjusting for total or cortical area reduced but did not eliminate these differences. The circularity of the cross-sections decayed distally (about $-7 \%, \mathrm{p}<0.05)$, in correlation with differences in xMI $(\mathrm{p}<0.01)$. As the volumetric cortical mineral density (vCMD) was fairly constant, bone strength indices $(\mathrm{yBSI}, \mathrm{xBSI}, \mathrm{pSSI}=\mathrm{vCMD} * \mathrm{MI})$ reflected the MI values. The values or differences in all design indicators correlated with the strength of the external rotation of the foot, with increasing adjustment ( $\mathrm{r}$ values) toward the middle of the distal region.

Football training appears to improve fibula resistance to buckling distally by enhancing cortical thickness, and resistance to lateral bending and torsion (throughout) and A-P bending (only proximally) through increases in the corresponding MI's. Effects on proximal bone (affecting cross-sectional circularity) would be related to the strength of the muscles inserted in the region rather than to the induced (homogeneous) enhancement in bone mass.

\section{P093}

Mesenchymal cell-derived juxtacrine Wnt1 signaling regulates osteoblast activity and osteoclast differentiation

Fan Wang ${ }^{1}$, Kati Tarkkonen ${ }^{1}$, Vappu Nieminen-Pihala ${ }^{1}$, Kenichi Nagano $^{2}$, Rana Al Majidi ${ }^{1}$, Tero Puolakkainen ${ }^{1}$, Petri Rummukainen $^{1}$, Jemina Lehto ${ }^{1}$, Anne Roivainen ${ }^{3}$, Fuping Zhang $^{4}$, Outi Mäkitie $^{5,6}$, Roland Baron ${ }^{2}$, Riku Kiviranta ${ }^{1,7}$
${ }^{1}$ University of Turku, Turku, Finland, ${ }^{2}$ Department of Oral Medicine, Harvard School of Dental Medicine, Boston, United States, ${ }^{3}$ Turku PET Centre and Turku Centre for Disease Modeling, University of Turku, Turku, Finland, ${ }^{4}$ Turku Center for Disease Modeling, University of Turku, Turku, Finland, ${ }^{5}$ Folkhälsan Institute of Genetics, Helsinki, Finland, ${ }^{6}$ University of Helsinki and Helsinki University Hospital, Helsinki, Finland, ${ }^{7}$ Department of Endocrinology, University of Turku and Turku University Hospital, Turku, Finland

Extensive human genetic evidence demonstrates that WNT1 mutations cause osteogenesis imperfecta (OI) and early-onset osteoporosis implicating WNT1 as a major regulator of bone metabolism. However, its main cellular source and mechanisms of action in bone remain elusive.

We generated global and limb bud mesenchymal cell-targeted deletion of Wnt1 in mice. Heterozygous deletion of Wnt1 resulted in mild trabecular osteopenia and impaired osteoblast function in vitro. Homozygous $\mathrm{Wnt} 1^{-1-}$ embryos were lost in utero. To bypass the embryonic lethality of $\mathrm{Wnt}^{-1-}$ mice and to identify the major source of Wnt1 in long bone, we targeted Wnt1 knockout to limb bud mesenchymal cells using Prrx1-Cre. Wnt $1_{\text {Prrx } 1}{ }^{-/}$mice exhibited spontaneous long bone fractures already at the age of 4 weeks mimicking the severe OI phenotype in humans with homozygous WNT1 mutations. Trabecular BV/TV was decreased by $70 \%$ in proximal tibia and cortical thickness was decreased by $47 \%$ in Wnt $1_{\text {Prrx } 1}{ }^{-1-}$ at 6 weeks by microCT. Histomorphometric analysis at 12 weeks showed the number of osteoclasts was increased by $38 \%$ and eroded surface was increased by $53 \%$ and decreased bone for-

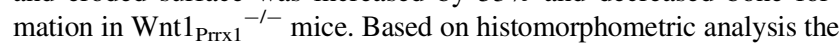
osteopenic phenotype was due to impaired osteoblast function and increased bone resorption. To address the mechanisms by which Wnt 1 promoted osteoblast and suppressed osteoclast differentiation, we performed co-cultures of Wnt 1 overexpressing and control cells with either osteoblast or osteoclast progenitor cells. In a co-culture system, we demonstrated that Wnt1 signals strictly in a juxtacrine manner requiring close proximity to its target cells to induce osteoblast differentiation. Moreover, Wnt1 directly suppressed osteoclastogenesis via cell-cell contact. These data supported the recent evidence of short-range signaling mode of Wnt proteins. We also identified the mesenchymal cells as the major source of Wnt1 in bone.

In conclusion, mesenchymal cell-derived Wnt1, acting in short range, is an essential regulator of bone homeostasis.

\section{P095}

Syndecan 3 deletion leads to premature bone ageing

Andrew Butcher ${ }^{1}$, Francesca Britto ${ }^{1}$, Gemma Charlesworth ${ }^{1}$, Amanda Prior $^{1}$, Adolorata Pisconti ${ }^{2}$, George Bou-Gharios ${ }^{1}$, Anna

Daroszewska', Rob van 't Hof'

${ }^{1}$ Department of Musculoskeletal Biology I, IACD, University of Liverpool, Liverpool, United Kingdom, ${ }^{2}$ Department of Biochemistry, IIB, University of Liverpool, Liverpool, United Kingdom

Syndecan $3(\mathrm{Sdc} 3)$ is a transmembrane heparan sulphate proteoglycan receptor primarily known for its role in the development and function of the central nervous and muscular systems. We have previously shown low trabecular bone volume and increased bone fragility in adult Sdc3 knockout (Sdc3-KO) mice compared to wild type (WT), suggesting a role of $\mathrm{Sdc} 3$ in bone development and homeostasis.

Here we studied whether Sdc3 regulates post-natal bone development and growth. We analysed the effects of Sdc3 deletion on bone in both 2-day-old pups and 3-month-old adults. We measured bone length of tibias of the 2-day-old-pups (WT $\mathrm{N}=23, \mathrm{KO} \mathrm{N}=21$ ) and 3 -month-old adults (WT $\mathrm{N}=15, \mathrm{KO} N=11$ ) by $\mu \mathrm{CT}$. The width of the entire growth plate and the hypertrophic region was measured in 
histological sections from the proximal tibia of 3 month old mice (WT $\mathrm{N}=8, \mathrm{KO} \mathrm{N}=4$ ).

We found reduced tibia length in 3-month-old Sdc3-KO mice compared to WT mice $(10.25 \pm 0.15$ vs. $10.64 \pm 0.04 \mathrm{~mm}$, $\mathrm{p}<0.001)$. However, surprisingly the 2-day-old Sdc3-KO pups showed increased bone length $(3.40 \pm 0.38$ vs. $3.16 \pm 0.27 \mathrm{~mm}$, $\mathrm{p}<0.05$ ). Analysis of the growth plates of the proximal tibia in adult mice showed reduced width of both the entire growth plate $(101.2 \pm 5.8$ vs. $118.3 \pm 9.6 \mu \mathrm{m}, \mathrm{p}<0.05)$ and the hypertrophic region $(29.4 \pm 4.34$ vs. $35.0 \pm 2.78 \mu \mathrm{m}, \mathrm{p}<0.05)$. The Sdc3-KO had a disorganised growth plate morphology with reduced, irregular columnar chondrocytes and a virtual absence of a primary spongiosa, suggesting premature ageing.

Our results show that Sdc3 plays a prominent role in bone development and growth in both neonatal and adult mice, and that this role differs depending on the development stage. Furthermore, the changed morphology and reduced width of the growth plate suggest that $\mathrm{Sdc} 3$ deletion contributes to a premature bone ageing phenotype. Keywords: Syndecan, Bone development, Growth plate

\section{P097}

Mechanical characteristics of mineralized lacunae and surrounding bone tissue in osteoporotic and healthy humans

Annika vom Scheidt ${ }^{1}$, Ezgi D. Yilmaz ${ }^{2}$, Kathrin Mletzko ${ }^{1}$, Jasmin Koldehoff $^{2}$, Eva Maria Wölfel ${ }^{1}$, Michael Amling ${ }^{1}$, Katharina Jähn ${ }^{1}$, Björn Busse $^{1}$

${ }^{1}$ Department of Osteology and Biomechanics, University Medical Center Hamburg-Eppendorf, Hamburg, Germany, ${ }^{2}$ Institute of Advanced Ceramics, University of Technology Hamburg, Hamburg, Germany

Maintenance of the mechanical characteristics of bone is orchestrated by mechanosensitive osteocytes residing in lacunae. With aging and osteoporosis, osteocytes die and lacunae mineralize (micropetrosis). This inhibits cellular communication, necessary for bone tissue maintenance. But it remains unclear how lacunar mineralization affects local mechanical properties of bone.

This study aimed to determine mechanical properties of mineralized lacunae and surrounding bone tissue in osteoporotic and healthy humans employing backscattered electron imaging and nanoindentation.

Six femoral cross-sections were obtained during autopsy (osteoporotic: $79.4 \pm 5.5$ years, female; healthy: $80.6 \pm 2.5$ years, 2 female, 1 male) following ethical guidelines. After formalin-fixation, dehydration and PMMA embedding, samples were ground and polished.

First, mineralized lacunae were identified with backscattered electron microscopy (BSE). Second, samples were subjected to nanoindentation (Berkovich tip, depth: $500 \mathrm{~nm}$, strain rate: $0.05 \mathrm{~s}^{-1}$ ) at three locations: (i) inside mineralized lacuna, (ii) near mineralized lacuna, (iii) near normal lacuna. After verification of indent position with BSE, 315 indents were included.

We found no significant differences between mechanical properties of bone tissue near mineralized or normal lacunae. However, calcified material inside mineralized lacunae showed greater hardness and higher elastic modulus compared to bone tissue near mineralized or normal lacunae for both groups (cf. Table $1, * \mathrm{p}<0.01$ ).

Mechanical properties of mineralized lacunae

\begin{tabular}{llll}
\hline & $\begin{array}{l}\text { Tissue inside } \\
\text { min. lacunae }\end{array}$ & $\begin{array}{l}\text { Tissue near } \\
\text { min. lacuna }\end{array}$ & $\begin{array}{l}\text { Tissue near } \\
\text { normal lacuna }\end{array}$ \\
\hline $\begin{array}{l}\text { Modulus [GPa] } \\
\text { Healthy }\end{array}$ & $32.9 \pm 4.3^{*}$ & $26.9 \pm 3.5$ & $26.2 \pm 4.9$ \\
\hline
\end{tabular}

Table $\mathbf{j}$ continued

\begin{tabular}{lccc}
\hline & $\begin{array}{c}\text { Tissue inside } \\
\text { min. lacunae }\end{array}$ & $\begin{array}{l}\text { Tissue near } \\
\text { min. lacuna }\end{array}$ & $\begin{array}{l}\text { Tissue near } \\
\text { normal lacuna }\end{array}$ \\
\hline $\begin{array}{l}\text { Osteoporosis } \\
\text { Hardness [GPa] }\end{array}$ & $29.2 \pm 6.4^{*}$ & $26.1 \pm 3.2$ & $25.9 \pm 3.2$ \\
Healthy & $1.69 \pm 0.36^{*}$ & $1.19 \pm 0.22$ & $1.18 \pm 0.27$ \\
Osteoporosis & $1.42 \pm 0.47^{*}$ & $1.16 \pm 0.19$ & $1.13 \pm 0.19$ \\
\hline
\end{tabular}

While characteristics of mineralized lacunae are similar in healthy and osteoporotic individuals, they are much more frequent with osteoporosis. Since we found locally altered mechanical properties in bone tissue with micropetrosis, abundant mineralized lacunae likely act as stress risers and thus contribute to impaired fracture resistance in osteoporosis.

Keywords: micropetrosis, mineralized lacunae, nanoindentation

\section{P099}

Significance of continuous gestational hypoglycaemia for foetal skeletal development in the rat

Vivi Flou Hjorth Jensen ${ }^{1,2,3}$, Anne-Marie Mølck ${ }^{2}$, Jens Lykkesfeldt ${ }^{3}$, Fiona Mcguigan $^{1}$, Kristina Åkesson ${ }^{1}$, Ingrid Brück B $\emptyset \mathrm{gh}^{2}$

${ }^{1}$ Department of Clinical Sciences Malmö, Clinical and Molecular Osteoporosis Research Unit, Lund University, Malmö, Sweden, ${ }^{2}$ Toxicology Development Projects, Novo Nordisk A/S, Maaloev, Denmark, ${ }^{3}$ Department of Veterinary and Animal Sciences, Section for Experimental Animal Models, University of Copenhagen, Copenhagen, Denmark

The present study investigates effects of continuous hypoglycaemia throughout gestation on foetal skeletal development, and importance of normoglycaemia vs. hypoglycaemia following organogenesis.

Pregnant rats received infusion with human insulin until gestation day 20 (GD20) or GD17 (group HI-GD20 and HI-GD17), termination on GD20. Controls received vehicle-infusion. Foetuses were doublestained for cartilage and calcified bone (Alcian blue/Alizarin Red), and skeletal ossification and malformations evaluated. Foetal bone mineral density (BMD) and long bone length were measured by $\mu 3 \mathrm{D}$ CT. All procedures involving live animals were ethically approved.

Insulin-infusion induced maternal hypoglycaemia to termination in group HI-GD20 and until GD17 in group HI-GD17, followed by normoglycaemia. Long bone length decreased in both groups (femur: 1.56 and 1.83 vs. $2.01 \mathrm{~mm}[\mathrm{p}<0.001, \mathrm{p}<0.05]$; tibia: 2.01 and 2.19 vs. $2.38 \mathrm{~mm}[\mathrm{p}<0.001, \mathrm{p}<0.05])$, shorter bones in HI-GD20 vs. HI-GD17 (femur: $\mathrm{p}<0.001$, tibia: $\mathrm{p}<0.05$ ). Ossification of sternebrae decreased in both groups (97 and 68\% of foetuses vs. 39\%, $\mathrm{p}<0.001)$, higher incidence in HI-GD20 vs. HI-GD17 ( $<<0.0001)$. In HI-GD20, additional bones displayed decreased ossification; e.g. vertebrae ( 81 vs. $11 \%, \mathrm{p}<0.0001)$. Sternebrae BMD decreased with hypoglycaemia (791 and 1278 vs. $1557 \mathrm{HU}, \mathrm{p}<0.001$ ), lower levels in HI-GD20 vs. HI-GD17 $(\mathrm{p}<0.001)$. Thoracic vertebrae BMD decreased in HI-GD20 (1215 vs. $1607 \mathrm{HU}, \mathrm{p}<0.05$ ), but not in HIGD17 (1435 vs. 1607 HU, p = 0.452). Both groups showed similar increases of skeletal abnormalities; thoracolumbar vertebrae $(8-10 \%$ of foetuses vs. $0 \%, \mathrm{p}<0.001)$, ribs $(6-10$ vs. $0 \%, \mathrm{p}<0.0001)$.

In conclusion, maternal hypoglycaemia throughout gestation decreases foetal skeletal growth and ossification/mineralisation. Reestablishing normoglycaemia in late gestation reduces this effect. Nevertheless, hypoglycaemia during organogenesis causes minor 
skeletal abnormalities regardless if followed by hypo-/normoglycaemia. This shows the importance of sufficient materno-foetal glucose supply during gestation on foetal skeletal development/growth, particularly during late gestation.

\section{Posters}

\section{P090}

miRNAs as biomarkers for osteoporotic vertebral fractures

Patryk Zarecki ${ }^{1}$, Matthias Hackl ${ }^{2}$, Johannes Grillari ${ }^{2}$, Miguel Debono $^{1}$, Richard Eastell ${ }^{1}$

${ }^{1}$ Department of Oncology \& Metabolism, University of Sheffield, Medical School, Sheffield, United Kingdom, ${ }^{2}$ TAmiRNA GmbH, Vienna, Austria

Rationale and hypothesis: Vertebral fractures are the hallmark of osteoporosis. Some microRNAs (miRNAs) can affect bone homeostasis, including bone remodelling and fracture healing by altering the gene expression in osteoblasts, osteoclasts and osteocytes. We hypothesise that miRNAs affect bone metabolism and their levels should differ between osteoporotic patients and healthy controls.

Objectives: We compared the levels of circulating miRNAs in older women with osteoporotic vertebral fractures, women with low bone mineral density (BMD) and healthy controls.

Methodology: The set of 20 circulating miRNAs was selected based on the literature and our previous studies. The serum levels of selected miRNAs were measured by a RT-qPCR (LightCycler ${ }^{\circledR} 480$ System, Roche) in 4 groups of older women: healthy controls $(n=40$, group 1); low BMD and no fractures $(n=35$, group 2$)$; vertebral fractures and low BMD without a treatment $(n=24$, group 3$)$; or receiving a treatment against osteoporosis $(n=17$, group 4$)$. The data quality was monitored using spike-in and hemolysis controls and was normalized to the RNA spike-in control. Statistical analysis was performed by a two-way ANOVA with Tukey post hoc test with statistical significance of $\mathrm{P}$ value: $\mathrm{P}<0.05$ as $*$; $\mathrm{P}<0.01$ as $* *$; $\mathrm{P}<0.001$ as $* * * ; \mathrm{P}<0.0001$ as $* * * *$.

Results: We found 7 miRNAs (hsa-miR-375, hsa-miR-532-3p, hsa-miR-19b-3p, hsa-miR-152-3p, hsa-miR-23a-3p, hsa-miR-335-5p, hsa-miR-21-5p) which were upregulated $(\mathrm{P}<0.05)$ in patients with vertebral fractures/low BMD against patients with low BMD/no fractures (twofold) and against healthy controls (1.5-fold), regardless of osteoporosis treatment. There were no significant differences in any miRNA level between low BMD/no fracture group and healthy controls.

Conclusion: These data suggest that some miRNA levels reflect the presence of osteoporotic vertebral fracture. They are unlikely to reflect low BMD, and more likely changes in bone quality or fracture healing. Osteoporosis treatments do not appear to have a large influence on miRNA levels.

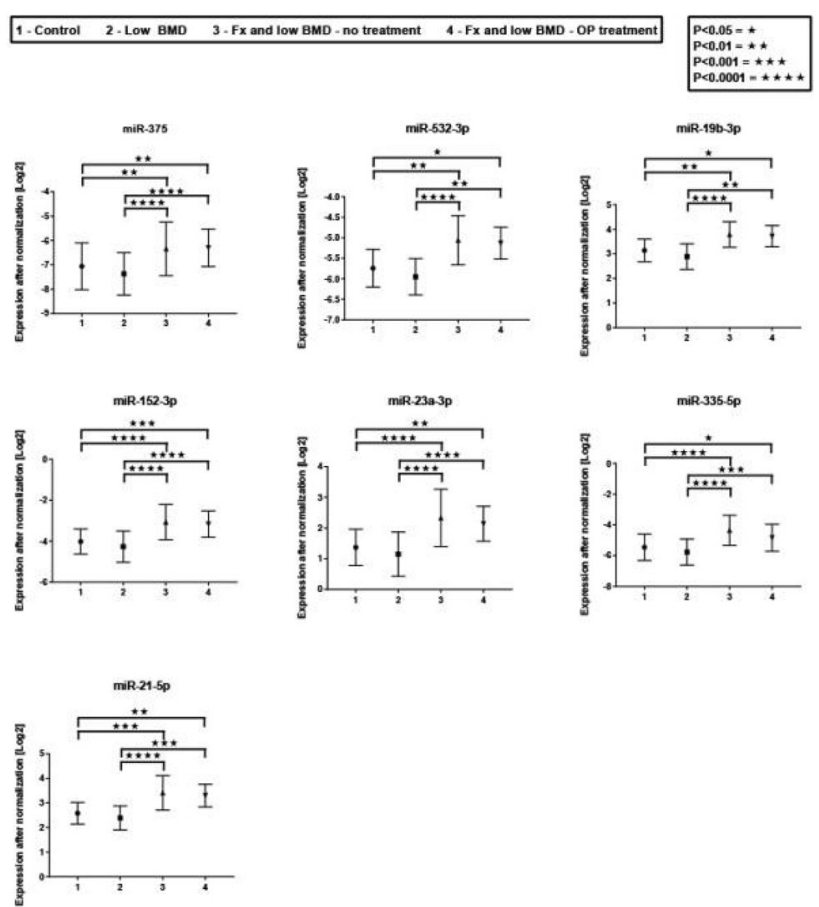

Increased expression levels of miRNAs in patients with vertebral fractures

\section{P092}

Correlation of circulating microRNA and bone turn over biomarkers in ankylosing spondylitis patients using next-generation sequencing

Chi-Chien Niu ${ }^{1}$, Song-Shu Lin ${ }^{1}$, Li-Jen Yuan ${ }^{2}$, Chuen-Yung Yang ${ }^{1}$, Steve WN Ueng ${ }^{1}$

${ }^{1}$ Department of Orthopaedic Surgery, Chang Gung Memorial Hospital, Taoyuan, Taiwan, Republic of China, ${ }^{2}$ Department of Orthopaedic Surgery, E-Da Hospital/I-Shou University, Kaohsiung City, Taiwan, Republic of China

Objective: MicroRNAs have emerged as important regulators in the post-transcriptional control of gene expression. The discovery of their presence not only in tissues but also in blood, together with their changes in expression in various pathological conditions. Wnt signaling is used to promote the osteogenesis of mesenchymal stem cells. Ankylosing spondylitis (AS) is a disease with excessive ossification structures. This study investigated the relationship between serum miRNAs and Wnt signaling factors in AS.

Methods: Peripheral blood samples were collected from 7 patients and 10 controls. An initial screening of miRNA expression by Illumina next generation sequencing (NGS) was performed. Real-time PCR was used to validate candidate miRNA. MiRNA targets were identified using bioinformatics assay. ELISA were used to measure the levels of serum osteocalcin and DKK-1.

Results: After the NGS sequencing, 5,528,879 clean reads per sample were generated and $16 \%$ of the clean reads were mapped to mature miRNA. About 1130 miRNAs were differentially expressed in AS when compared with control, including 588 upregulated and 542 downregulated miRNAs. MiRNAs with a mean fold change $>2$ or $<0.5$ were selected for further analysis. Among them, miR-29a-3p and miR-146a-5p were significantly upregulated $(p<0.05)$ in AS patients compared with controls. Real-time PCR identified the 
expression differences between AS and control. Bioinformatics approaches show that $3^{\prime}$ UTR of DKK-1 mRNA contained the 'seedmatched-sequence' for hsa-miR-29a-3p and $3^{\prime}$ UTR of osteocalcin for hsa-miR-146a-5p. ELISA data showed the serum levels of DKK-1 $(\mathrm{p}<0.01)$ and osteocalcin $(\mathrm{p}<0.01)$ were lower in AS patients than control. MiR29a-3p expression level was found to be negative correlated with DKK-1 level and miR-146a-5p expression level with osteocalcin level.

Summary and Conclusions: Our data showed that serum miRNA may have an application in the precise clinical description of AS.

Keywords: MicroRNA, Ankylosing spondylitis, NGS, osteocalcin, DKK-1

\section{P094}

Investigation into the role of CD146 (MCAM) in promoting the latter stages of metastases and tumor cell survival in the metastatic niche

Maren Tietgen ${ }^{1,2}$, Ulf Geisen ${ }^{1}$, Sanjay Tiwari ${ }^{1}$, Claus-Christian Glüer $^{1}$

${ }^{1}$ MOIN CC, UKSH Kiel, Kiel, Germany, ${ }^{2}$ Molecular Oncology, UKSH Kiel, Kiel, Germany

Introduction: The latter stages of skeletal metastases requires docking of tumor cells (TC) to the endothelium and extravasation into the bone microenvironment. Once established, it is postulated that the metastatic niche which includes mesenchymal stem cells (MSCs) provide survival signals for the TC which eventually then subvert the niche to create a proliferative environment. CD146 (MCAM) is a membrane glycoprotein that is expressed on TC, endothelial cells, pericytes and a sub-population of MSC. In this study we hypothesize that homophilic CD146 mediated cell contact (CD146-CD146) with endothelial, pericytes and MSCs mediates extravasation and survival of TC to the bone.

Method: Flow cytometric expression of CD146 was determined on primary bone marrow derived MSCs from mouse tibia, as well on TC lines. Co-cultures were performed by incubating cell culture supernatants from MSCs with breast cancer cell (BCC) lines. Ex vivo analyses of expression of CD146 was determined by immunohistochemistry on bone sections from mice harboring $\mathrm{BCC}$ bone metastases.

Results: Co-culture of BCC with MSC supernatant reveal that TC have increased survival/proliferation. Conversely, MSCs have reduced survival/proliferation when co-cultured in the TC supernatant (Fig. 1). Flow cytometric determination of CD146 expression on BCC lines reveal that a bone-homing variant of MDA-MB231 has the highest expression of CD146. Staining for CD146 expression on MSCs reveal localization of CD146 to be at the cell-cell contact junction, reminiscent of tight-junctions which demarcate the boundary between the apical and basolateral membrane domains of a cell and regulate of cell polarity (Fig. 2). Finally, ex vivo staining of bone metastases reveal abundant CD146 staining, which is strongest at the tumor invasive front (Fig. 3).

Conclusion: Taken together, how data provide preliminary support for a role of CD146 in promoting BCC bone metastases possibly through polarized mediated expression and adhesion of CD146 mediating cell-cell contact.

\section{P096}

Plasma levels of microRNAs hsa-miR-30d-5p and hsa-miR-21-5p correlate with physical activity of postmenopausal women

Tilen Kranjc $^{1}$, Barbara Ostanek ${ }^{1}$, Tomaž Kocjan ${ }^{2}$, Janez Preželj ${ }^{2}$, Janja Marc $^{1}$
${ }^{1}$ University of Ljubljana, Faculty of Pharmacy, Ljubljana, Slovenia, ${ }^{2}$ Dept. of Endocrinology, Diabetes and Metabolic Diseases, University Medical Centre Ljubljana, Ljubljana, Slovenia

Osteoporotic fractures occur due to poor bone quality and quantity, which progressively declines in postmenopausal women. The rate of decline can be reduced to some extent by maintaining healthy lifestyle with regular physical activity. MicroRNAs (miRNA) have been recently proposed as a biomarker of bone health. In this observational study we investigated the link between physical activity, which leads to better bone health, and microRNA biomarkers, which can correlate with bone quality and quantity.

The participants were postmenopausal women without ongoing therapy for osteoporosis. The population was balanced to remove age or body mass index bias for groups of patients with high $(>1.35)$ or low $(<1.35)$ Trabecular Bone Score (TBS). We screened plasma samples of 5 patients with reduced bone quality (TBS $<1.35$ ) and 5 patients with normal bone quality (TBS $>1.35$ ) for 800 miRNA molecules. Then we validated 12 top-expressing miRNA molecules by using qPCR. The validation cohort consisted of 113 normal TBS patients and 35 reduced TBS patients. The correlations were calculated as Pearson correlation coefficients.

The screening revealed 33 miRNA molecules which could be detected in plasma. We further validated 6 miRNAs with highest correlation with TBS and additional 6 miRNA molecules that were previously shown specific for osteoporotic population. We discovered that hsa-miR-30d-5p and hsa-miR-21-5p negatively correlate with weekly physical activity of the population $(\mathrm{r}=-0.24, \mathrm{p}=0.003$ and $r=-0.20, p=0.013$, respectively). We also discovered that patients with reduced levels of hsa-miR-21-5p more often used their hands to stand up from the seated position $(\mathrm{r}=0.20, \mathrm{p}=0.017)$, which is a sign of weakness and fragility.

Plasma levels of hsa-miR-30d-5p and hsa-miR-21-5p correlate with physical activity of postmenopausal women. This complies with previous studies which reported increased plasma levels of hsa-miR30d-5p and hsa-miR-21-5p in patients with osteoporotic fractures. These miRNAs could be used as biomarkers of bone homeostasis.

\section{P098}

Comparison of the reference interval for procollagen I N-propeptide and osteocalcin in men and women by two assays

Antonia Ugur $^{1}$, Fatma Gossiel ${ }^{1}$, Philip Nicklin ${ }^{1}$, Jennifer Walsh ${ }^{1}$, Kim Naylor $^{1}$, Richard Eastell ${ }^{1,2}$

${ }^{1}$ Academic Unit of Bone Metabolism, Oncology and Metabolism, University of Sheffield, Sheffield, United Kingdom, ${ }^{2}$ University of Sheffield, Mellanby Centre for Bone Research, Sheffield, United Kingdom

Bone turnover markers are increasingly used in clinical practice to monitor osteoporosis treatment. Recent evidence supports the use of targets that demonstrate greater than $90 \%$ sensitivity in showing response of female study subjects to treatment. One target for treatment is to suppress markers below the geometric mean for healthy premenopausal women. In 2017 the IFCC-IOF Working Group on bone marker standards, reiterated the need for further studies examining the relationship between assays for bone turnover markers before concluding whether recommendations of targets could be generalisable. Our aim was to establish gender-specific reference intervals for PINP and OC using two commonly-used assays.

We measured N-MID osteocalcin and Intact and Total PINP by IDS-iSYS and Roche Cobas e411, respectively on morning serum samples of 181 fasted healthy subjects; 51 men age 30-60 years old and 130 premenopausal women aged $30-45$ years old. 
PINP and OC reference interval by assay and gender

\begin{tabular}{|c|c|c|c|c|c|}
\hline & & \multicolumn{2}{|l|}{ PINP $\mu \mathrm{g} / \mathrm{L}$} & \multicolumn{2}{|c|}{ Osteocalcin $\mu \mathrm{g} / \mathrm{L}$} \\
\hline & & IDS-iSYS & $\begin{array}{l}\text { Roche } \\
\text { Cobas } \\
\text { e411 }\end{array}$ & $\begin{array}{l}\text { IDS- } \\
\text { iSYS }\end{array}$ & $\begin{array}{l}\text { Roche } \\
\text { Cobas } \\
\text { e411 }\end{array}$ \\
\hline $\begin{array}{l}\text { Male } \\
\qquad \mathrm{n}=51\end{array}$ & $\begin{array}{l}\text { Geometric } \\
\text { mean } \\
(95 \% \text { RI } \\
\text { LL-UL) }\end{array}$ & $\begin{array}{c}51.3(21.8- \\
120.9)\end{array}$ & $\begin{array}{l}49.0 \\
\quad(21.7- \\
110.4)\end{array}$ & $\begin{array}{l}19.1 * * * \\
\quad(9.7-37.5)\end{array}$ & $\begin{array}{l}22.9 \\
\quad(13.9- \\
37.6)\end{array}$ \\
\hline $\begin{array}{l}\text { Female } \\
\qquad \mathrm{n}=130\end{array}$ & $\begin{array}{l}\text { Geometric } \\
\text { mean } \\
\text { (95\% RI } \\
\text { LL-UL) }\end{array}$ & $\begin{array}{l}37.2 * * *,+\dagger \\
\quad(21.6-63.9)\end{array}$ & $\begin{array}{c}41.7^{\dagger \dagger} \\
(25.4- \\
68.5)\end{array}$ & 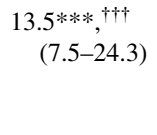 & $\begin{array}{r}17.8^{+\dagger \dagger} \\
(11.3- \\
27.9)\end{array}$ \\
\hline
\end{tabular}

$\uparrow$ Denote statistical significance: $p<0001$ for between ASSAY difference by gender, $\dagger \dagger p<001$ and ${ }^{\dagger \dagger} p<0001$ for between GENDER difference for each assay

Levels of PINP and OC were lower in premenopausal women than men with both assays, in keeping with reference intervals published from other geographical areas. Men have broader reference intervals than the women. The Roche N-MID OC assay has previously shown a higher reference interval than the IDS N-MID assay

The differences by assay underline the importance of assay standardisation or harmonisation and meanwhile of being aware of the assay used in clinical decision making. The differences by gender raise the question of whether targets for treatment response should be gender-specific or not

Keywords: Bone-turnover, osteocalcin, PINP, gender, assay

\section{$\mathbf{P 1 0 0}$}

Mass spectrometry based biomarkers discovery for pseudarthrosis and confirmation using immunostaining

Stefanie Kern ${ }^{1}$, Deeksha Malhan ${ }^{1}$, Felix Schulze ${ }^{2}$, Sabine Schulz ${ }^{3}$, Angela Rösen-Wolff ${ }^{2}$, Bernhard Spengler ${ }^{3}$, Markus Rupp ${ }^{4}$, Thaqif El Khassawna ${ }^{1}$, Christian Heiß ${ }^{1,4}$

${ }^{1}$ Experimental Trauma Surgery, Justus-Liebig-University Giessen, Giessen, Germany, ${ }^{2}$ Department of Pediatrics, University Hospital Carl Gustav Carus, Technical University Dresden, Dresden, Germany, ${ }^{3}$ Institute of Inorganic and Analytical Chemistry, JustusLiebig-University Giessen, Giessen, Germany, ${ }^{4}$ Department of Trauma, Hand and Reconstructive Surgery, University Hospital of Giessen-Marburg GmbH, Giessen, Germany

Bone regeneration after a fracture is a crucial issue in clinic and research. $5-10 \%$ of fractures result in non-unions. After second surgical revision of non-unions, bone morphogenetic proteins (BMP) are commonly applied. Although BMPs improve osteoblast bone formation, they also increase fibroblast proliferation and mineralization. However, using BMPs in pseudarthrosis, where fibroblasts are the abundant cell type, is debatable. The study aims at analyzing proteinlevel differences between mesenchymal stem cells and fibroblasts after osteogenic differentiation. Mass spectrometry based proteomics followed by bioinformatics analysis was used for biomarker discovery. Neurofibromin deficiency is associated in pseudarthrosis formation. Therefore, fracture callus of a neurofibromatosis-type-one (NF1) mouse model was performed to histologically validate biomarkers.

Human MSCs and fibroblasts harvested from reaming debris underwent osteogenic differentiation using BMP-2-stimulation.
Protein extraction, digestion and purification were performed before analysis with nano-HPLC-ESI-MS/MS. The relative gene expression of chosen biomarkers was carried out on total RNA extracted from differentiated cells. Pseudarthrotic tissue sections were histological examined using Movat's pentachrome staining. Immunohistochemical analysis of chosen biomarkers was performed on patient pseudarthrotic tissue and validated on NF1 knock-out mouse samples at day 10 after closed femoral fracture.

Osteogenic differentiated MSCs and fibroblasts showed 193 similar proteins out of 2470 total proteins. Collagen-alpha-2(I)-chain, calmodulin-1 (CALM1) and glycine-tRNA-synthetase (GARS) were significantly down-regulated and associated with neurofibromin in osteogenic differentiated fibroblasts when compared to differentiated MSCs. Interestingly, same genes showed lower relative expression $(\mathrm{p}<0.05)$, and less staining intensities in pseudarthrosis biopsies and NF1 KO-mouse samples.

The MS-based discovered biomarkers are potential diagnostic tools to decide the feasibility of osteoinductive local therapy. Establishing a correlation between biomarkers discrepancies in the tissue and their serum level concentration can provide information about the healing status of surgically revised non-unions. Cell-specific molecular analysis of pseudarthrotic tissue is the main outlook of the study. Keywords: Non-union, biomarker, proteomics, pseudarthrosis

\section{P101}

Circulating periostin and tartrate-resistant acid phosphatase $5 \mathrm{~b}$ as markers of activity in Paget's disease of bone

Nuria Guanabens ${ }^{1}$, Xavier Filella ${ }^{2}$, Helena Florez ${ }^{1}$, Arantxa Conesa ${ }^{3}$, Silvia Ruiz-Gaspa $^{4}$, Pilar Peris ${ }^{1}$, Ana Monegal ${ }^{1}$, Ferran Torres ${ }^{5}$

${ }^{1}$ Rheumatology, Hospital Clinic, University of Barcelona, IDIBAPS, Barcelona, Spain, ${ }^{2}$ Biochemistry and Molecular Genetics, Hospital Clinic, University of Barcelona, IDIBAPS, Barcelona, Spain, ${ }^{3}$ Rheumatology, Hospital General Universitario, Castellon, Spain, ${ }^{4}$ CIBERehd. Hospital Clinic. IDIBAPS. University of Barcelona, Barcelona, Spain, ${ }^{5}$ Biostatistics and Data Management Platform, Hospital Clinic, IDIBAPS, Barcelona, Spain

Periostin is a matricellular protein with a preferential location in cortical bone and periosteal tissue, and tartrate-resistant acid phosphatase $5 b$ (TRAP5b) is a marker of osteoclast numbers. In Paget's disease of bone (PDB) there is increased cortical thickening, periosteal apposition and increased osteoclast numbers.

Aim: To analyse if circulating periostin is a biomarker for PDB and if it is associated with disease activity, as well as whether TRAP5b is useful in the assessment of PDB.

Patients and Methods: We recruited 41 patients with PDB (13F/ $28 \mathrm{M} ; 71.1 \pm 11.8$ years). $70.7 \%$ had active disease, defined as total alkaline phosphatase higher than the upper normal range. Blood and urine samples were taken between 8:00 and 10:00 am after an overnight fast. Periostin and TRAP5b were measured in serum using commercial ELISA assays (Biomedica and IDS, respectively). Serum PINP, CTX, bone ALP and urinary NTX were measured. Reference values for periostin and TRAP5b were obtained from 38 healthy subjects.

Results: Serum periostin did not differ between patients and controls $(966 \pm 210$ vs. $992 \pm 175 \mathrm{pmol} / \mathrm{L}, \mathrm{p}=0.553)$, between patients with and without active disease $(967 \pm 171$ vs. $1052 \pm 176 \mathrm{pmol} / \mathrm{L}, \mathrm{p}=0.160$ ), involvement or not of long bones $(1022 \pm 146$ vs. $952 \pm 204 \mathrm{pmol} / \mathrm{L}, \mathrm{p}=0.209)$, monostotic or polyostotic disease $(990 \pm 982$ vs. $988 \pm 143 \mathrm{pmol} / \mathrm{L}, \mathrm{p}=0.897)$ and previous bisphosphonate treatment (964 \pm 199 vs. $1007 \pm 163 \mathrm{pmol} / \mathrm{L}, \mathrm{p}=0.471)$. There were no significant correlations between periostin and any of the assessed bone turnover markers, except for TRAP5b $(r=0.312, p=0.047)$. TRAP5b was 
significantly higher in patients than in controls $(4.41 \pm 1.77$ vs. $3.24 \pm 0.95 \mathrm{U} / \mathrm{L}, \mathrm{p}=0.0005)$, significantly correlated with PINP, CTX, bone ALP and NTX, and showed a high predictive capacity for PDB (ROC: 0.715 [95\% CI 0.600-0.829]), particularly when combined with age and gender (ROC: 0.919 [95\% CI 0.858-0.979]).

Conclusions: Serum periostin is not useful for assessing Paget's disease. By contrast, TRAP5b may be a useful marker in Paget's disease assessment.

Keywords: Paget's disease; periostin; TRAP5b

\section{P102}

Wnt3a and Wnt10b as biomarkers of changes in the regulation of bone metabolism in patients with Cushing's disease

Tatiana Grebennikova ${ }^{1}$, Zhanna Belaya ${ }^{1}$, Alexander Solodovnikov ${ }^{2}$, Alexander Ilyin $^{1}$, Larisa Nikankina ${ }^{1}$, Galina Melnichenko ${ }^{1}$

${ }^{I}$ The National Research Center for Endocrinology, Moscow, Russian Federation, ${ }^{2}$ Ural State Medical Academy, Ekaterinburg, Russian Federation

Background: Endogenous hypercortisolism due to Cushing's disease (CD) is complicated by low-traumatic fractures in $50 \%$ of cases.

Aims: To evaluate levels of Wnt-proteins related to bone remodeling regulation in serum samples from patients with $\mathrm{CD}$.

Materials and Methods: Fasting serum samples were taken and stored in aliquot at $\leq-80{ }^{\circ} \mathrm{C}$ from 42 consecutive subjects with clinically evident and biochemically confirmed active CD and 42 healthy volunteers matched by age, sex and body mass index (BMI). Evaluation of the levels of Wnt proteins (Wnt3a, Wnt10b) was measured by immunochemiluminescence assay using the WNT3 SEL818Hu (USCN) and the WNT10B SEL553Hu (USCN). Twentyfour hours urine free cortisol (24hUFC) (60-413 nmol/24 h) and bone turnover markers was measured by electrochemiluminescence assay on a Cobas 6000 Module e601 (Roche). At the time of enrollment all participants were questioned regarding any low traumatic fractures for the period of the disease. Patients underwent standard spinal radiographs in anterior-posterior and lateral positions of the vertebrae Th4-L4 (Axiom Icons R200 "Siemens").

Results: The median (Me Q25; Q75) age of patients with CD was $33(21 ; 43)$ years with no difference among the groups, $\mathrm{p}=0.936$; BMI - $29(23 ; 34) \mathrm{kg} / \mathrm{m}^{2}, \mathrm{p}=0.094$ and without differences by sex, $\mathrm{p}=0.254$. The median 24hUFC in subjects with CD -825 (301; 2077) $\mathrm{nmol} / 24 \mathrm{~h}$ was significantly higher as compared to the control group $(\mathrm{p}<0.001)$. We found increased levels of Wnt3a and Wnt10b in patients with CD: Wnt3a $0.15(0.04 ; 0.23) \mathrm{ng} / \mathrm{ml}$ in patients with $\mathrm{CD}$ vs. $0.04(0.01 ; 0.13) \mathrm{ng} / \mathrm{ml}$ in control group $(\mathrm{p}=0.017)$ and Wnt10b $2621(2226 ; 3688) \mathrm{pg} / \mathrm{ml}$ vs. 1917 (1721; 2549) pg/ml $(\mathrm{p}=0.008)$.

Conclusions: The serum level of Wnt3a and Wnt10b reflects the intensity of Wnt-signaling dysregulation, and therefore they may be considered as biomarkers of bone remodeling deterioration in hypercortisolism.

Keywords: Wnt/ $\beta$-catenin pathway, Cushing's disease, glucocorticoid induced osteoporosis, Wnt10b, Wnt3a

\section{P103}

Asfotase alpha: interference with ALP-detection system in immunoassays

$\underline{\text { Isabelle Piec }}^{1}$, William D. Fraser ${ }^{1}$

${ }^{1}$ Department of Medicine, University of East Anglia, Norwich, United Kingdom
Asfotase alpha (AsfA, Strensiq ${ }^{\text {TM }}$, Alexion Pharmaceutical) is the first FDA-Approved treatment of patients with hypophosphatasia, result of a mutation in the tissue-nonspecific alkaline phosphatase gene. Alkaline Phosphatase (ALP) is used as the measuring/signalling system in many routine assays for proteins, hormones and other small molecules. Because it contains the ALP active site, AsfA is able to catalyse the substrate as the antibody-conjugated ALP would. Therefore, AsfA present in a patient's sample may generate a false positive or a false negative result. We investigated whether the presence of AsfA within a sample induces interference in sandwich and competitive assays using base-plate and on routine automated analysers that uses or not ALP as a detection system.

AsfA was added to samples at concentrations of $0.08-5 \mu \mathrm{g} / \mathrm{mL}$. Manual ELISAs and COBAS6000 assays were tested. We observed no significant change in the absorbance of alkaline phosphatase measured using the RayBiotech assay for ALKP1, Normetanephrine (IBL) in urine samples (hydrolysed or not) nor adrenaline (IBL) at any AsfA concentration. However, AsfA significantly cross-reacted with the oxytocin assay (ENZO, p $<0.001$ ), in a concentration dependant manner on non-extracted samples. While the ALP activity showed a significant $(\mathrm{p}<0.0001)$ AsfA concentration-dependant increase in ALP, the concentration of the analytes of interest did not change by more than $2.4 \%$ from the original sample for assays run on the COBAS (TSH, LH, FSH).

Assays using a different detection system than ALP were not affected by the presence of AsfA in the samples. Most assays using ALP detection systems were sufficiently specific to not show interference with the AsfA; however, the oxytocin needed to be extracted to demonstrate this specificity. The presence of AsfA should be taken into consideration when analysing blood samples to avoid any risk of misinterpretation of false positive/negative results.

Keywords: Asfotase alpha, immunoassay, alkaline phosphatase, interference

\section{P104}

Bone turnover markers after the menopause: T-score approach

Fatma Gossiel $^{1}$, Hibatallah Altaher ${ }^{2}$, David Reid ${ }^{3}$, Christian Roux ${ }^{4}$, Dieter Felsenberg $^{5}$, Claus Glueer ${ }^{6}$

${ }^{1}$ University of Sheffield, Sheffield.ac.uk, United Kingdom, ${ }^{2}$ University of Sheffield, Sheffield, United Kingdom, ${ }^{3}$ University of Aberdeen, Aberdeen, United Kingdom, ${ }^{4}$ Université Paris-Descartes, Paris, France, ${ }^{5}$ Universitätsmedizin Berlin, Berlin, Germany, ${ }^{6}$ Universitätsklinikum Schleswig-Holstein, Kiel, Germany

Bone turnover increases at the menopause and is associated with accelerated bone loss. However, it is not known to what extent there is an imbalance between the processes of bone resorption and bone formation nor whether it is the rate of bone turnover or the bone balance that is most closely associated with the rate of bone loss. 657 healthy women ages 20-79 were recruited from five European cities (the OPUS Study). They were divided into two premenopausal age groups, 20-29 $(\mathrm{n}=129), 30-39$ years $(\mathrm{n}=183)$, and three postmenopausal groups, $1-10$ years $(n=91), 11-20$ years $(n=131)$ and $21+$ years since menopause $(n=123)$. Collagen type I C-telopeptide (CTX) and procollagen I N-propeptide (PINP) were measured in nonfasting serum using the iSYS-IDS automated immunoassay. These were used to calculate the overall bone turnover and the difference between bone formation and resorption (bone balance). The results from the women ages 30-39 years were used to calculate a standardised score (T-score). CTX and PINP levels were higher in women aged 20-29 and the three menopausal groups compared to 30-39 years $(\mathrm{p}<0.001)$. CTX and PINP levels were 80 and $33 \%$ higher in women 1-10 years since menopause, respectively. In this group of postmenopausal women, the bone turnover expressed as a 
T-score was $0.72(0.57-0.88,95 \% \mathrm{CI})$ and the bone balance was -0.37 ( -0.59 to -0.16$)$. There was greater rate of bone loss from the total hip in all the groups of women after the menopause compared to before. Bone loss after the menopause is associated with both an increase in bone turnover and a negative bone balance. Bone loss was associated with overall bone turnover.

\section{P105}

A new flow cytometry method to analyze Lamin A expression in circulating osteoprogenitor (COP) cells as a biomarker for musculoskeletal disease

Ahmed Al Saedi ${ }^{1,2}$, Piumali Gunawardene ${ }^{3}$, Lakshman Singh ${ }^{1,2}$, Pushpa Suriyaarachchi Suriyaarachchi ${ }^{3}$, Gustavo Duque ${ }^{1,2}$

${ }^{1}$ Melbourne Medical School (Western) - Faculty of Medicine, Dentistry and Health Sciences, The University of Melbourne, St Albans, Australia, ${ }^{2}$ Australian Institute for Musculoskeletal Science (AIMSS), St Albans, Australia, ${ }^{3}$ Sydney Medical School Nepean, The University of Sydney, Penrith, Australia

Background: Circulating osteoprogenitor (COP) cells are considered a surrogate of the bone marrow stem cell population. COP cell population is efficiently quantified using flow cytometry. Lamin A, one of the intermediate filaments of the nuclear lamina, plays a vital role in BMSC differentiation. Lamin A deficiency is associated with accelerated aging and alterations in the musculoskeletal system. Therefore, lamin A quantification in COP cells could constitute a robust biomarker for musculoskeletal diseases. However, this hypothesis has not been tested in the past due to lack of a reliable and feasible method.

Methods: A cross-sectional study was undertaken in 144 healthy volunteers in Western Sydney (20-90 year-old, 10 male and 10 female subjects per decade). Lamin A expression in COP cells was quantified by flow cytometry using a 6 colour panel. Targeted population was gated as $\mathrm{CD} 45+\mathrm{OCN}+\mathrm{Lamin} \mathrm{A}+$ and mean fluorescence intensity (MFI) values were generated by the flow cytometry software based on fluorescence intensity of gated cell populations. Protein samples also collected for Western Blotting

Results: Lamin A expression in COP cells did not change significantly with age. Lamin A was expressed in $8 \%$ of COP cells (mean value) with an expression range of $4-57 \%$ throughout the cohort. Lamin A geometric mean florescence intensity (G-MFI) in COP cells did not change significantly amongst different age groups (correlation coefficient $=0.056, p<0.523$ ). Additionally, there was no significant difference in the expression of lamin A G-MFI with respect to other variables like gender, BMI, falls and osteoporosis risk indicators.

Conclusion: In this study we have tested the feasibility of a new method to quantify Lamin A expression in COP cells. This new, accurate and easy to perform diagnostic method, will offer a novel platform for greater consistency in future research on the role of lamin $\mathrm{A}$ in aging and musculoskeletal disease.

\section{P106}

Development and characterization of an ELISA for the measurement of human Semaphorin 4D in plasma samples

Anna Laber ${ }^{1}$, Elisabeth Gadermaier ${ }^{1}$, Gabriela Berg ${ }^{1,2}$, Gottfried Himmler $^{1}$

${ }^{1}$ The Antibody Lab GmbH, Vienna, Austria, ${ }^{2}$ Biomedica Medizinprodukte GmbH \& Co KG, Vienna, Austria

Objective: Semaphorin 4D (SEMA4D, CD100) is a type I integral membrane glycoprotein that regulates key cellular functions. Shedding of SEMA4D near the cell membrane, e.g. through matrix metalloproteinases, leads to the release of the $120 \mathrm{kDa}$ biologically active soluble SEMA4D (sSEMA4D). In bone, SEMA4D is produced by osteoclasts and acts through its receptor plexin-B1 on osteoblasts to inhibit their differentiation and motility. As potential biomarker of bone turnover sSEMA4D may assist in the management of bone diseases. SEMA4D is also expressed on the surface of platelets, and it is shed during blood coagulation.

Methods: We selected two monoclonal anti-human SEMA4D antibodies, and characterized them by determining binding kinetics and affinities with biolayer interferometry, and by resolving epitopes with microarray technology using cyclized peptides. The antibodies were used to develop a sandwich ELISA. Human serum and plasma samples from apparently healthy subjects that were collected either in the conventional way, or in presence of the protease inhibitor TAPI-1, were measured.

Results: The two monoclonal antibodies bind to SSEMA4D with a $\mathrm{KD}$ of $5.8 \times 10^{-10} \mathrm{M}$ for the coating antibody, and of $3.79 \times 10^{-9} \mathrm{M}$ for the detection antibody. Both antibodies recognize conformational epitopes that involve amino acids AA30-AA34 (coating antibody), and AA238-AA241 (detection antibody). The assay covers a range from 0 to $2000 \mathrm{pmol} / \mathrm{L}$ with a quantification limit of $31 \mathrm{pmol} / \mathrm{L}$. Assay characteristics for EDTA, citrate and heparin plasma samples meet the standards of acceptance. There was no relevant difference between SSEMA4D in EDTA, citrate and heparin plasma (median 271-315 pmol/L). However, serum samples where coagulation has taken place showed significantly elevated sSEMA4D $(\mathrm{p}<0.05)$ due to clotting-induced shedding. Serum samples where protease activity was inhibited with TAPI- 1 showed sSEMA4D levels comparable to plasma.

Conclusion: Our novel ELISA provides an accurate tool for the quantitative determination of sSEMA4D in human plasma samples.

\section{P107}

A new, highly sensitive fluorescence immunoassay for the TGF- $\beta$ antagonist ASPORIN based on plasmonic microtiter plates

Gerhard Hawa $^{1}$, Teresa Jungwirth ${ }^{1}$, Albert Missbichler ${ }^{1}$, Adrian Prinz $^{2}$, Georg Bauer ${ }^{2}$, Christoph Mauracher ${ }^{2}$

${ }^{1}$ FIANOSTICS GmbH, Wiener Neustadt, Austria, ${ }^{2}$ STRATEC Consumables GmbH, Anif, Austria

Objective: ASPORIN, like decorin or biglycan is a member of the SLRP (small leucine-rich repeat proteoglycans/proteins) protein family. In addition to the well-known collagen binding properties of those SLRPs, ASPORIN is also a potent inhibitor of TGF- $\beta$ function thus having an impact on chondrogenesis. Several studies linked ASPORIN to the occurrence of Osteoarthritis (OA), the most prevalent form of arthritis in humans. Unfortunately, serum measurement for this interesting biomarker is hampered by the fact that currently available immunoassays for ASPORIN are not sensitive enough. Therefore we decided to use our recently developed high sensitivity fluorescence immunoassay platform based on plasmonic micro titer plates (MEF-MTPs) to develop a reliable ASPORIN assay. We hereby present a small pilot study in a blood donor cohort.

Methods: MEF-MTPs were generated by STRATEC Consumables proprietary injection molding and sputtering processes originating from Blu-Ray manufacturing. After coating of anti-ASPORIN capture antibody and blocking of unspecific binding, $50 \mu \mathrm{l}$ of fluorescence labeled detection antibody together with $10 \mu \mathrm{l}$ of human serum or plasma samples were incubated over night at RT in the dark. Signals were read using a standard fluorescence microplate reader.

Results: ASPORIN was not reliably detectable in human plasma, probably due to the calcium binding properties of this molecule. However, in human serum performance of the assay was acceptable (LOD 10 pM, intra/inter assay CVs: 3-7/8-11\%). In a collection 
of 58 samples of blood donors ( $31 \mathrm{f}, 27 \mathrm{~m}$ ) we found a significant trend towards decreasing serum levels with age and lower values in females than males (f: $34.7 \pm 12.5 \mathrm{pM}, \mathrm{m}: 46.1 \pm 17.6 \mathrm{pM}$ )

Conclusion: The developed assay is sufficiently reliable for the detection of ASPORIN in human serum. Further studies must be performed to demonstrate the clinical usefulness of this biomarker. Keywords: Osteoarthritis, Tumor Progression, ASPORIN, High Sensitivity

\section{P108}

Identification of serum biomarkers associated with osteoporosis in Mexican postmenopausal women

Mayeli Margarita Martinez Aguilar ${ }^{1}$, Diana Aparicio-Bautista ${ }^{2}$, Juan Pablo Reyes-Grajeda ${ }^{3}$, Eric Gustavo Ramírez Salazar ${ }^{4}$, Jorge Salmeron Castro ${ }^{5}$, Berenice Rivera Paredez ${ }^{6}$, Rafael Velazquez $\mathrm{Cruz}^{7}$

${ }^{1}$ Genomic of Bone Metabolism Laboratory, National Institute of Genomic Medicine, Mexico, Mexico, ${ }^{2}$ Proteomic, National Institute of Genomic Medicine, Mexico, Mexico, ${ }^{3}$ Protein Structure, National Institute of Genomic Medicine, Mexico, Mexico, ${ }^{4}$ CONACYT-INMEGEN, National Institute of Genomic Medicine, Mexico, Mexico, ${ }^{5}$ Public Policy Research Institute, National Institute of Health, Mexico, Mexico, ${ }^{6}$ Public Policy Research Institute, National Institute of Public Health, Mexico, Mexico, ${ }^{7}$ Genomics of Bone Metabolism Laboratory, National Institute of Genomic Medicine, Mexico, Mexico

Osteoporosis (OP) is a common bone metabolic disease characterized by low bone mass and deterioration of bone microarchitecture. The use of bone markers in the clinic is very useful for monitoring the therapy. However have some limitations including a lack of specificity for bone tissue and their inability to reflect osteocyte activity. Thus, the search for novel markers for OP is essential and will be beneficial for patients, particularly at the early stages. Therefore, this study aims to identify differentially expressed proteins that might serve as potential biomarkers for osteoporosis in Mexican population. The Ethics Committees of the participating institutions approved the study protocol. The samples were classified into three groups based in the T-score: Normal $(-1.0$ to +1$)$, Osteopenia $(-1.0$ to -2.5$)$, and Osteoporosis $(-2.5$ to -3$)$. A proteomic study was performed using 2D-DIGE followed by MALDI/TOF-TOF. A total of 365 spots were detected in 2D-DIGE gels, and six spots with FCs \pm 1.5 and $p$ values $\leq 0.05$ were selected and sent to digestion for the identification of peptides. The proteins identified were: KNG1 (FC $=2.19$, $\mathrm{p}=0.0031), \mathrm{GSN}(\mathrm{FC}=-3.25, \mathrm{p}=0.0086), \mathrm{CBR} 1(\mathrm{FC}=-2.7$, $\mathrm{p}=0.013), \operatorname{VDBP}(\mathrm{FC}=-2.57, \mathrm{p}=0.022$ and $\mathrm{FC}=-1.8$, $\mathrm{p}=0.019)$ and $\mathrm{CP}(\mathrm{FC}=-1.74, \mathrm{p}=0.0017$ and $\mathrm{FC}=-1.57$, $\mathrm{p}=0.05$ ) (Fig. 1). Validation by WB/ELISA demonstrated that $\mathrm{CP}$ decreases significantly between normal vs. osteopenic $(p=0.0079)$ and osteopenic vs. osteoporotic women $(\mathrm{p}=0.0311)$ and VDBP decreased significantly the levels between osteopenic $(p=0.0003)$, osteoporotic $(p<0.0001)$ and fracture compared to the group of healthy women. By Receiver Operating Characteristic analysis, CP and VDP distinguished osteoporosis from healthy women with an AUC value of $0.6061, p<0.001$ and $0.7744, p<0.001$, respectively. Our results support to CP and VDBP as potential biomarkers for the early diagnosis of OP in Mexican population.

\section{P109}

Correlation of bone turnover markers, vitamin D levels and steroidal hormones with bone density in healthy male population

Panagiotis Kokkoris ${ }^{1}$

${ }^{1}$ Hellenic Air Force General Hospital, Athens, Greece
Aim: The aim of the study is to find any possible factors which may influence bone density in a healthy young male population. More specific to examine if there is any correlation between bone turnover markers, vitamin D levels and steroidal hormones with BMD.

Materials-Methods: We examined 300 healthy young men. They all had a DXA scan in both the lumbar spine and the hip. Additionally we measured the following: Bone turnover markers (osteocalcin, alkaline phosphatase and P1NP for bone formation, and CTX for bone resorbsion), 25(OH) Vitamin D levels, steroidal hormones (testosterone and estradiol), and calcium and phosphorus levels.

Results: The mean age of the population was $33.9 \pm 7.7$ years, mean weight $84.1 \pm 10.8 \mathrm{~kg}$, and mean body mass index $26.1 \pm 4.2 \mathrm{~kg} / \mathrm{m}^{2}$. The mean bone mineral density was $1.26 \pm 0.15 \mathrm{~g} / \mathrm{cm}^{2}$ for the lumbar spine and $1.08 \pm 0.14 \mathrm{~g} / \mathrm{cm}^{2}$ for the hip. The mean values for the markers were: Osteocalcin $18.3 \pm 7 \mathrm{ng} / \mathrm{ml}$, alkaline phosphatase $63 \pm 17.2 \mathrm{IU} / \mathrm{l}, \quad \mathrm{P} 1 \mathrm{NP}$ $51.7 \pm 25 \mathrm{ng} / \mathrm{ml}, \quad$ CTX $0.36 \pm 0.18 \mathrm{ng} / \mathrm{ml}, \quad 25(\mathrm{OH})$ Vitamin D $21.7 \pm 9.2 \mathrm{ng} / \mathrm{ml}$, testosterone $5.5 \pm 2.3 \mathrm{ng} / \mathrm{ml}$, estradiol $43.6 \pm 17.3 \mathrm{pg} / \mathrm{ml}$, calcium $9.5 \pm 0.6 \mathrm{mg} / \mathrm{dl}$ and phosphorus $3.2 \pm 0.5 \mathrm{mg} / \mathrm{dl}$. As for the correlations we found the following: positive correlation between BMD and osteocalcin, P1NP and CTX respectively $(\mathrm{p}<0.05)$, negative correlation between $\mathrm{BMD}$ and alkaline phosphatase $(\mathrm{p}<0.05)$. We didn't find any correlation either positive or negative between BMD and $25(\mathrm{OH})$ Vitamin D, testosterone, estradiol, calcium and phosphorus. Additionally we found positive correlation between BMD and BMI $(p<0.05)$, and negative correlation between BMI and $25(\mathrm{OH})$ Vitamin D and testosterone.

Conclusion: Our study showed that in a relatively young healthy male population bone turnover markers are positively correlated with bone mineral density, while steroidal hormones, Vitamin D and calcium and phosphorus are not correlated with BMD. Additionally, body weight is positively correlated with BMD and negatively correlated with Vitamin D and testosterone.

\section{P110}

Mesenchymal stromal cells are sensitised to Wnt stimuli by plasma sprayed hydroxyapatite coatings

$\underline{\text { David Kuntin }}^{1,2,3}$, David Wood ${ }^{2}$, Niki Gosling ${ }^{3}$, Paul Genever ${ }^{1}$

${ }^{1}$ Biology, University of York, York, United Kingdom, ${ }^{2}$ University of Leeds, Leeds, United Kingdom, ${ }^{3}$ DePuy Synthes, Leeds, United Kingdom

Joint replacement stems may be coated in hydroxyapatite (HA), a calcium phosphate ceramic applied to the titanium by plasma spraying. HA is osteoconductive and promotes osteointegration. Optimal cellular response and bone healing can be achieved by in situ targeting of regenerative mesenchymal stromal cells (MSCs) through coating optimisation.

The Wnt pathway is heavily implicated in the regeneration of bone through proliferation and differentiation of MSCs. We hypothesise that Wnt signalling responds to HA surface characteristics, such as roughness and crystallinity, in a manner integral to bone healing following joint replacement.

To evaluate the effect of HA on Wnt signalling, a green-fluorescent, immortalised reporter MSC line was developed, expressing enhanced green fluorescent protein (EGFP) downstream of TCF/LEF. Reporter cells were seeded onto HA-coated titanium discs in the presence and absence of a canonical Wnt ligand (Wnt3a). Canonical Wnt pathway activation was measured by fluorimetry and compared with tissue culture plastic. Plasma sprayed HA coating properties, such as crystallinity (48-108\% compared to starting HA powder), roughness (arithmetic mean roughnesses $\left(\mathrm{S}_{\mathrm{a}}\right)$ of 10.4-16.6 $\mu \mathrm{m}$ ), and $\mathrm{Ca} / \mathrm{P}$ ratios (1.80-1.88) were measured using X-ray diffraction, laser profilometry, and scanning electron microscopy with energy- 
dispersive X-ray diffraction respectively. This surface property information is used to correlate changes in Wnt signalling to specific parameters and model Wnt response to them.

On tissue culture plastic, addition of ligand caused an eightfold increase in fluorescence, which was enhanced to 14-fold on HA coatings $(p<0.05)$. Notably, a twofold increase was observed between untreated tissue culture plastic and HA $(\mathrm{p}<0.05)$, suggesting that MSCs are not only sensitised to Wnt ligand on HA surfaces, but that they promote endogenous Wnt signalling. These findings are relevant to the impact of HA coatings on bone healing and long-term outcomes.

Keywords: MSC, Wnt, hydroxyapatite, bioceramic, hip

\section{P111}

Therapeutic potential of periosteum-derived mesenchymal progenitor cells in bone regeneration

Ana Belén González-Gil ${ }^{1}$, José María Lamo-Espinosa ${ }^{1}$, Emma Muiños-López ${ }^{2}$, Purificación Ripalda-Cemboráin ${ }^{1}$, Kai Stuckensen ${ }^{3}$, Gloria Abizanda ${ }^{4}$, Elena María Juan-Pardo ${ }^{5}$, Juergen Groll ${ }^{3}$, Dietmar W. Hutmacher ${ }^{5}$, Felipe Prósper ${ }^{4}$, Froilán Granero-Moltó ${ }^{1,2}$

${ }^{1}$ Orthopaedic Surgery and Traumatology, Clínica Universidad de Navarra, Pamplona, Spain, ${ }^{2}$ Cell Therapy, Clinica Universidad de Navarra, Pamplona, Spain, ${ }^{3}$ Functional Materials in Medicine and Dentistry, University of Würzburg, Würzburg, Germany, ${ }^{4}$ Cell Therapy, Clínica Universidad de Navarra, Pamplona, Spain, ${ }^{5}$ Regenerative Medicine, Queensland University of Technology, Brisbane, Australia

In the treatment of nonunions an attractive alternative to bone autografts is the use of a combination of autologous mesenchymal progenitors cells (MSCs), biomaterials and growth factors. Our goal was to determine the therapeutic potential of different sources of mesenchymal stem cells for the treatment of nonunions.

A diaphyseal critical size defect was performed. Six groups were created. A nonunion group (Control); (LBA group) live bone allograft; BMP2 group, rhBMP-2 in collagen sponge; PCL group, polycaprolactone scaffold; PMSCs group, PCL scaffold loaded with $5 \times 10^{6}$ periosteum-derived MSCs; and BMSCs group, PCL scaffold loaded with $5 \times 10^{6}$ bone marrow-derived MSCs. LBA and MSCs were derived from SD-GFP transgenic rats. The repair process was allowed for 10 weeks and analyzed by micro computed tomography (mCT), histology and immunohistochemistry. One-way ANOVA followed by Dunnett's test for single comparisons was used for statistical significance. Statistical significance was established for $\mathrm{p}<0.05$.

Control group did not show healing during follow up or by $\mathrm{mCT}$ and histological analysis. Treatment groups BLA and BMP2 showed full healing by week 10 . The repair callus was quantified by $\mathrm{mCT}$, Control group showed limited formation of bone $\left(11.47 \pm 2.01 \mathrm{~mm}^{3}\right)$ while both LBA and BMP2 groups showed increased bone formation by week 10 when compared with control group (LBA, $35.36 \pm 2.24$ $\mathrm{mm}^{3}$; BMP2, $\left.33.32 \pm 1.84 \mathrm{~mm}^{3}, \mathrm{p}=0.0022\right)$.In PCL treated groups a low number of animals showed radiographic healing: PCL group 0/6 animals; PMSCs group, 2/6 animals; BMSCs group, 0/6. Quantification showed that only PMSCs group produced a significant volume of bone when compared with the Control group (PMSCs, $\left.24.97 \pm 6.03 \mathrm{~mm}^{3}, \mathrm{p}=0.0411\right)$. PCL and BMSCs groups counts were similar to control group (PCL, $19.00 \pm 4.25 \mathrm{~mm}^{3}, \mathrm{p}=0.3095$; BMSCs, $12.88 \pm 2.38 \mathrm{~mm}^{3}, \mathrm{p}=0.9372$ ). Only the LBA group showed positive signal for GFP at week 10-post surgery.

In conclusion, PMSCs are suitable for mimetic autograft design.

Keywords: PMSCs, mimetic autografts

\section{P112}

Vitamin E ( $\alpha$-tocopherol) administration enhances the osseointegration of stainless steel implants by suppressing the immediate postoperative oxidative stress. An experimental study in rats

Matthaios Savvidis ${ }^{1}$, Ioannis Taitzoglou ${ }^{2}$, Kyriakos Papavasiliou ${ }^{3}$, Ioannis Mirisidis $^{4}$, Aristidis Veskoukis ${ }^{5,6}$, Ioannis Vrabas ${ }^{6}$, Nikiforos Galanis $^{3}$, Ioannis Kyrkos ${ }^{3}$

${ }^{1} 1$ st Orthopaedic Dpt, 424 General Military Hospital, Thessaloniki, Greece, ${ }^{2}$ Laboratory of Physiology, School of Veterinary Medicine, Aristotle University, Thessaloniki, Greece, ${ }^{3} 3 \mathrm{rd}$ Orthopaedic Department, Aristotle University, Papageorgiou General Hospital, Thessaloniki, Greece, ${ }^{4}$ Laboratory for Machine Tools and Manufacturing Engineering, Mechanical Engineering Department, Aristotle University, Thessaloniki, Greece, ${ }^{5}$ Department of Biochemistry and Biotechnology, University of Thessaly, Larissa, Greece, ${ }^{6}$ Exercise Physiology and Biochemistry Laboratory, Faculty of Physical Education and Sport Sciences, Aristotle University, Serres, Greece

Objective: Orthopaedic implants are widely used in everyday practice. The aim of this study was to assess the suppressing action of $\alpha$-tocopherol, a well-known anti-oxidative agent, on the postoperative oxidative stress and its subsequent enhancing action on the osseointegration procedure of stainless steel orthopaedic implants.

Methods: The study was performed in two groups, consisting of 15 adult male Wistar rats each. In the proximal metaphysis of each tibia, a specially designed stainless steel screw was implanted. Study group animals received $\alpha$-tocopherol ( $40 \mathrm{mg} / \mathrm{kg}$ once daily intraperitoneally for 28 days). Control group received saline. On day 29, all animals were euthanized, tibias were harvested, the serum levels of $\alpha$-tocopherol, malondialdehyde, protein carbonyls, glutathione (GSH), glutathione disulfide (GSSG), total antioxidant capacity (TAC) and catalase were evaluated and the ratio GSH/GSSG was calculated. The implants' pullout strength and removal torque were also assessed.

Results: All animals completed the study period and all harvested tibias were suitable for evaluation.

Results

\begin{tabular}{lllll}
\hline Parameter & Units & Study & Control & $\mathrm{P}$ \\
\hline Pullout strength & Newton & $124.870 \pm 20.729$ & $88.137 \pm 12.734$ & 0.000 \\
Torque & Newton/cm & $6.932 \pm 0.948$ & $4.778 \pm 0.907$ & 0.000 \\
GSH & $\mu \mathrm{mol} / \mathrm{g} \mathrm{Hb}$ & $1.966 \pm 0.387$ & $1.327 \pm 0.337$ & 0.000 \\
GSH/GSSG & - & $435.277 \pm 70.563$ & $87.334 \pm 25.187$ & 0.001 \\
Catalase & $\mathrm{U} / \mathrm{mg} \mathrm{Hb}$ & $155.638 \pm 44.625$ & $87.334 \pm 25.187$ & 0.000 \\
Malondialdehyde & $\mu \mathrm{mol} / \mathrm{g}$ protein & $0.068 \pm 0,023$ & $0.138 \pm 0,027$ & 0.000 \\
$\begin{array}{l}\text { Protein } \\
\quad \text { carbonyls }\end{array}$ & $\mathrm{nmol} / \mathrm{mg}$ protein & $0.164 \pm 0,04$ & $0.267 \pm 0,08$ & 0.002 \\
TAC & nmolDPPH/g & $15.9 \pm 3.2$ & $13.754 \pm 1.740$ & 0.008 \\
& \multicolumn{1}{c}{ protein } & & & \\
$\alpha$-Tocopherol & $\mathrm{mg} / \mathrm{L}$ & $3.897 \pm 4.056$ & $0.933 \pm 0.243$ & 0.011 \\
\hline & & & &
\end{tabular}

Conclusions: All parameters studied were statistically significantly different between groups, in favor of the animals receiving $\alpha$-tocopherol. Our results confirm our initial hypothesis that the postoperative administration of $\alpha$-tocopherol, enhances the osseointegration of orthopaedic implants, possibly by suppressing the postoperative oxidative stress

Keywords: $\alpha$-tocopherol, osseointegration, rat, implants 


\section{P113}

Hydrogen sulfide-loaded silk fibroin scaffold for bone tissue regeneration

Laura Gambari ${ }^{1}$, Emanuela Amore ${ }^{1}$, Brunella Grigolo ${ }^{1}$, Antonella Motta $^{2}$, Francesco Grassi ${ }^{1}$

${ }^{1}$ Lab Ramses, Istituto Ortopedico Rizzoli, Bologna, Italy, ${ }^{2}$ Department of Industrial Engineering, University of Trento, Trento, Italy

Augmentation of osteogenic differentiation and vasculogenesis are important requirements in the development of strategies for bone healing and regeneration. In the present study, we aimed to combine silk fibroin (SF), a biomaterial with good mechanical and osteoconductive properties, to hydrogen sulfide $\left(\mathrm{H}_{2} \mathrm{~S}\right)$, a recently recognized anabolic agent. SF was loaded with GYY4137 (GYY), an $\mathrm{H}_{2} \mathrm{~S}$ donor, obtaining SF-GYY $1.25 \%$ and SF-GYY 5\% w/w. Their biocompatibility and osteoinduction activity were assayed using human bone marrow mesenchymal stromal cells (hMSCs) in vitro. Cells were cultured on scaffolds using a perfusion bioreactor to engineer bonelike tissue constructs. Cytotoxicity and mortality were excluded by LDH assays and live and dead assay. Colonization and osteoinduction were evaluated by Haematoxylin\&Eosin and VonKossa/Alizarin RedS stains, respectively. PCR array for human osteogenesis and immunohistochemical analyses were performed to identify targets involved. $\mathrm{H}_{2} \mathrm{~S}$-loaded scaffolds supported cell adhesion, proliferation and viability. SF-GYY 5\% displayed higher and anticipated mineralization compared to SF alone and SF-GYY 1.25\%. Furthermore, it up-regulated genes involved in osteoblast differentiation and mineralization compared to SF alone: DLX-5 $(+681 \%$ compared to control SF; $p<0.0001)$ and COMP $(+46 \% ; \mathrm{p}<0.0001)$ were significantly increased; BMP6, BMPR2 and ACVR1 displayed a trend towards a increased expression. Extracellular matrix and cell adhesion molecules were also significantly increased: ITGA2 $(+1100 \%$; $\mathrm{p}<0.0001)$ and SERPINH1 $(+388 \%$; $\mathrm{p}<0.0001)$. Moreover, SFGYY scaffold showed significant up-regulation of VEGFA (D0: $+40 \%, \mathrm{p}<0.0001 ; \mathrm{D} 7:+149 \%, \mathrm{p}=0.376 ; \mathrm{D} 14:+416 \%$, $\mathrm{p}<0.0001)$. Coherently with mRNA, SF-GYY 5\% increased VEGF protein expression, along with ALP and CSE. Our findings demonstrate that $\mathrm{H}_{2} \mathrm{~S}$ incorporation is able to increase the osteogenic potency of hMSCs in $3 \mathrm{D}$ cultures and stimulate the production of the master gene for angiogenesis. Furthermore, we show a long lasting $\mathrm{H}_{2} \mathrm{~S}$ effect on hMSCs. Therefore, $\mathrm{H}_{2} \mathrm{~S}$ loaded scaffold hold good potential for bone healing and regeneration applications.

\section{P114}

Evaluation of fracture healing by Low Adhesive Scaffold Collagen (LASCol) in a rat femur osteotomy model

Toshiyuki Takemori ${ }^{1}$, Naomasa Fukase ${ }^{1}$, Saori Kunii ${ }^{2}$, Ryosuke Kuroda $^{1}$, Koichi Morimoto ${ }^{2}$

${ }^{1}$ Kobe University, Kobe, Japan, ${ }^{2}$ Kindai University, Kinokawa, Japan Introduction: Collagen is the most abundant extracellular matrix in bone and has been utilized as a scaffold to regenerate bone deficit. However, it is not always a desired performance yet. Recently, we succeeded in developing low adhesive scaffold type I collagen (SLASCol) (patent pending) from pigskin which has the ability to accelerate osteogenic differentiation. In this study, we utilized a rat femur fracture model to test the hypothesis that the implant of S-LASCol to fractured site would accelerate fracture healing.

Methods: All animal experiments were performed under the approval and guidance of the IRB and IACUC at the authors' institutions. Rat bone marrow stromal cells (rMSCs) were seeded in three different conditions: S-LASCol-coated, Atelocollagen-coated, or noncoated dish. After 3 days, mRNA expression levels of Alpl (alkaline phosphatase), Runx2 (runt-related transcription factor 2), BGP (osteocalcin) and $O s x$ (osterix) were assessed by real-time qPCR. Rat femur osteotomy model with a defect size of $1 \mathrm{~mm}$ was created, and we evaluated S-LASCol implant $(\mathrm{n}=19)$ and a control group $(\mathrm{n}=13)$. Radiographs were taken on 4 weeks after the surgery. Femurs on the operated side were evaluated by HE staining.

Statistical analysis: ANOVA with post hoc test was used to compare for continuous values.

Results: The mRNA expression levels of all osteogenesis-related genes of rMSCs cultured on the S-LASCol-coated dish increased significantly compared to the other groups. In the X-ray evaluation showed bone union in S-LASCol group $(n=9)$, whereas it was confirmed in control group $(\mathrm{n}=2)(\mathrm{p}=0.06)$. The HE staining showed bone union in S-LASCol group than in control group.

Conclusion: In vitro-study suggested that S-LASCol strongly promotes the differentiation of rMSCs into osteoblasts at very early stages of culture. In vivo-study indicated that S-LASCol augments bone union.

Funding: This work was supported by the Adaptable and Seamless Technology Transfer Program through target-driven R\&D, JST

\section{P115}

Human mesenchymal stromal cells in adhesion to cell-derived extracellular matrix and titanium: comparative kinome profile analysis

Marta Baroncelli ${ }^{1}$, Gwenny Fuhler ${ }^{2}$, Jeroen van de Peppel $^{1}$, Willian Zambuzzi $^{3}$, Johannes van Leeuwen ${ }^{1}$, Maikel Peppelenbosch ${ }^{2}$, Bram van der Eerden ${ }^{1}$

${ }^{1}$ Internal Medicine, Erasmus MC, Rotterdam, Netherlands, ${ }^{2}$ Gastroenterology, Erasmus MC, Rotterdam, Netherlands, ${ }^{3}$ Chemistry and Biochemistry, Universidade Estadual Paulista - UNESP, Sao Paulo, Brazil

The extracellular matrix (ECM) is essential to physically support cells and actively influence cell behaviour by modulating kinasemediated signalling cascades. Cell-derived ECMs have recently emerged in the context of bone regeneration, as they reproduce physiological tissue-architecture and ameliorate the promising properties of mesenchymal stromal cells (MSCs). Titanium scaffolds show good mechanical properties and porosity to facilitate cell adhesion, and thus have been routinely used for bone tissue engineering applications. The aim of this study was to analyze the kinomic signature of human MSCs in adhesion to an osteoblast-derived ECM that we have previously shown to be osteopromotive, and to compare it to MSCs on titanium. PamChip kinase array analysis revealed 63 phosphorylated peptides on ECM and 59 on titanium, with MSCs on ECM exhibiting significantly higher levels of kinase activity than those on titanium (Area -under-curve on ECM 34.1 vs. 27.6 on titanium, $P<0.0001$ ). MSCs on the 2 substrates showed a substantial overlap of kinome profiles, with the activation of similar signalling pathways, such as FAK, ERK and PI3K signalling, as confirmed by immunoblot analyses. Inhibition of PI3K signalling in cells significantly reduced cell adhesion to the ECM $(0.5$-fold, $P<0.001)$, and increased the number of non-adherent cells, both on ECM (2.4-fold, $P<0.001)$ and on titanium (1.9-fold). The increase in non-adherent cell number in response to PI3K inhibition was not due to induction of apoptosis by PI3K inhibitors. In summary, this study comprehensively characterized the kinase activity in MSCs on cell-derived ECM and titanium, highlighting the role of PI3K signalling in kinomic changes regulating osteoblast viability and adhesion. Kinome profile analysis represents a powerful tool to select pathways for a better understanding of cell behaviour. Osteoblast-derived ECM could be further investigated as coating for titanium scaffolds, to improve bone tissue engineering applications. 
Keywords: Kinome profiling; osteoblasts; extracellular matrix; titanium; cell adhesion; peptide array

\section{P116}

Histomorphometrical comparison of three different bone grafts: deproteinized bovine bone, bioglass and, synthetic hydroxyapatite

Miguel Angel Pellegrini ${ }^{1}$, Macarena Gonzales Chaves ${ }^{2}$, Ricardo Orzuza $^{2}$, Susana N Zeni ${ }^{1,2}$, Gretel G Pellegrini ${ }^{1,2}$

${ }^{I}$ Institute of Immunology, Genetics and Metabolism, Osteopathies Laboratory, University of Buenos Aires-CONICET, Ciudad Autónoma de Buenos Aires, Argentina, ${ }^{2}$ General and Oral Biochemistry, University of Buenos Aires, School of Dentistry, Ciudad Autónoma de Buenos Aires, Argentina

Bone graft implantation is required to guide bone repair. Deproteinized bovine bone putty (BB), bioglass (BG) and, synthetic hydroxyapatite $(\mathrm{SH})$ are frequently indicated as bone grafting materials due to their osteoconductive properties. We compared the bone healing response of $\mathrm{BB}, \mathrm{BG}$ and $\mathrm{SH}$ (Synergy, Odontit Implant Systems, Argentina) in a critical sized bone defect. We created a bone defect of $4 \mathrm{~mm}$ diameter in rat tibiae for implantation with each biomaterial $(\mathrm{N}=30$ rats). Samples were collected at 2 and 4 weeks for histological and histomorphometrical analysis of new bone formation (NBF) and remaining particles of each device (RP).

Results: Percentage of NBF (mean \pm SD): 2 weeks: Control group: $6.60 \pm 3.71 ; \quad \mathrm{BB}$ group: $23.23 \pm 3.89^{*} ; \quad \mathrm{BG}$ group: $18.35 \pm 5.23 *$; SH group: $26.27 \pm 9.30 *$; 4 weeks: Control group: $6.69 \pm 2.38$; BB group: $24.37 \pm 3.66^{*}$; BG group: $17.45 \pm 6.64 *$; SH group: $32.25 \pm 3.80 *$. Percentage of RP: 2 weeks: Control group: $0 \pm 0$; BB group: $5.04 \pm 1.39 *$; BG group: $3.03 \pm 2.31 *$; SH group: $4.46 \pm 2.87^{*} ; 4$ weeks: Control group: $0 \pm 0$; BB group: $4.45 \pm 2.35^{*} ; \mathrm{BG}$ group: $2.87 \pm 1.14^{*}$; $\mathrm{SH}$ group: $3.78 \pm 1.68^{*}$ $(* \mathrm{p}<0.05$ vs. control group; $\mathrm{p}=\mathrm{NS}$ between $\mathrm{BB}, \mathrm{BG}$ and $\mathrm{SH}$ groups). Although $\mathrm{SH}$ exhibited a trend towards increased NBF we did not find statistical significance among the three biomaterials. Bone healing at the implanted sites, was accompanied by a progressive inflammatory response consistent with the expected histological stages of bone repairing. All biomaterials were associated with trabecular bone formation. To date, there are no studies comparing the effect of these biomaterials in bone healing. While further studies need to be done, our results indicate that $\mathrm{BB}, \mathrm{BG}$ and $\mathrm{SH}$ exhibit similar characteristics in terms of osteoconduction. Furthermore, the three biomaterials were histologically substantially equivalent.

Keywords: Bone healing; biomaterials; critical sized bone defect; bovine bone; bioglass; synthetic hydroxyapatite

\section{P117}

Bone regenerative potential of adipose-derived stromal vascular fraction on a xenohybrid bone scaffold

Ilaria Roato ${ }^{1}$, Dimas Carolina Belisario ${ }^{1}$, Mara Compagno ${ }^{1}$, Laura Verderio $^{2}$, Anna Sighinolfi' ${ }^{2}$, Federico Mussano ${ }^{3}$, Tullio Genova ${ }^{4}$, Francesca Veneziano ${ }^{5}$, Gianni Pertici ${ }^{6}$, Giuseppe Perale ${ }^{6,7}$, Riccardo Ferracini $^{8}$

${ }^{I}$ Center for Research and Medical Studies, A.O.U. Città della Salute e della Scienza of Turin, Turin, Italy, ${ }^{2}$ Chemistry, Materials and Chemical Engineering 'Giulio Natta', Politecnico of Milan, Milan, Italy, ${ }^{3}$ Surgical Sciences, University of Turin, Turin, Italy, ${ }^{4}$ Life Sciences \& Systems Biology, University of Turin, Turin, Italy, ${ }^{5}$ Pathology Unit, A.O.U. Città della Salute e della Scienza of Turin, Turin, Italy, ${ }^{6}$ Industrie Biomediche Insubri SA, Mezzovico-Vira, Switzerland, ${ }^{7}$ University of Applied Sciences and Arts-SUPSI, Manno,
Switzerland, ${ }^{8}$ Surgical Sciences (DISC), Orthopaedic Clinic-IRCCS A.O.U. San Martino, Genoa, Italy

Adipose tissue-derived stem cells (ASCs) are a promising tool for treatment of bone diseases or skeletal lesions, thanks to their multilineage differentiating ability. Osteoarthritis, a disease characterized by articular cartilage degeneration and subchondral bone sclerosis, may benefit from non-surgical treatments based on intra-articular infusions of ASCs. One of the major limitations of ASCs is represented by the necessity to be isolated and expanded through in vitro culture, thus a strong interest was generated by the adipose stromal vascular fraction (SVF), the non-cultured fraction of ASCs.

We investigated and compared the bone regenerative potential of SVF and ASCs, taking advantage of their ability to grow on SmartBone ${ }^{\circledR}$ (SB), a xenohybrid bone scaffold. Both ASCs and SVF colonized and formed new tissue on SB, filling its periphery and bone lacunae over time. At 15, 30 and 60 days, we monitored the tissue growth through immunoistochemical staining: collagen I, osteocalcin and TGFb markedly stained the new tissue on SB. MicroCT analysis showed a progressive increase in mineralised tissue apposition by newly formed trabeculae. Indeed, their quantification analysis demonstrated that SVFs were significantly more efficient than ASCs $(3391 \pm 270.5$ vs. $1825 \pm 133.4, p<0.001)$ in inducing bone formation, when cultured on SB with osteogenic medium. In SVF cultures, we observed an increased secretion of soluble factors stimulating osteoblasts over time: VEGF (153.5-1278.1 pg/ml) and endothelin $1(0.43-1.47 \mathrm{pg} / \mathrm{ml})$.

In conclusion, the absence of manipulation of SVF in an in vitro culture could definitively represent a benefit for a larger use in clinical applications. Moreover, our data strongly support an innovative idea for a regenerative medicine based on solid scaffold functionalised with SVF to improve the precision of stem cells implant and the quality of new bone formation.

Keywords: Adipose tissue-derived stromal vascular fraction, bone scaffold, bone regeneration

\section{P118}

Investigation of the relationship between stem taper topography and the degree of corrosion and fretting in total hip arthroplasty

Kilian Stockhausen ${ }^{1}$, Christoph Riedel ${ }^{1}$, Dorothea Rothe ${ }^{2}$, Alex Victoria Belinski ${ }^{1}$, Felix Klebig ${ }^{2}$, Mustafa Citak ${ }^{2}$, Matthias Gebauer ${ }^{2}$, Michael Amling ${ }^{1}$, Björn Busse ${ }^{1}$

${ }^{I}$ Department of Osteology and Biomechanics, University Medical Center Hamburg-Eppendorf, Hamburg, Germany, ${ }^{2}$ Department of Orthopedic Surgery, Helios ENDO-Klinik Hamburg, Hamburg, Germany

Modularity in total hip arthroplasty (THA) is highly valued as it allows adaption to patient-specific anatomy and inter-operative flexibility. Primary THA belong to the most successful medical procedures, yet a certain percentage of revisions is inevitable. Besides more common causes, wear at the modular interface between stem and head taper is a potential cause of THA failure. This is predominately expressed in the form of mechanical fretting and chemical corrosion, and might depend on variations in surface topography of the stem tapers.

Forty-nine stem tapers of four different models (Alloclassic, CLS Spotorno, Aesculap Bicontact, SL-PLUS) were characterized regarding their surface topography using high-resolution 3D microscopy. Wear was defined as corrosion and fretting and quantified using an established scoring scheme.

Statistical tests employed ANOVA with Bonferroni correction, Mann-Whitney-U test for not normally distributed data, and Spearman's rank correlation as test of correlation.

Profile height and effective surface enlargement were significantly higher in the Alloclassic and CLS compared to the Bicontact and SL- 
PLUS stem taper. Contrarily, the profile width was significantly smaller (Table 1) $(\mathrm{p}<0.05)$.

Table 1: Topographic measurements of stem trunnions

\begin{tabular}{lllll}
\hline & Manufacturer & $\begin{array}{l}\text { Profile } \\
\text { height }(\mu \mathrm{m})\end{array}$ & $\begin{array}{l}\text { Profile width } \\
(\mu \mathrm{m})\end{array}$ & $\begin{array}{l}\text { Surface } \\
\text { enlargement }\end{array}$ \\
\hline Alloclassic & Zimmer & $65.58 \pm 3.64$ & $140.16 \pm 0.27$ & $1.86 \pm 0.07$ \\
CLS & Zimmer & $69.90 \pm 13.87$ & $140.05 \pm 0.50$ & $1.97 \pm 0.26$ \\
Bicontact & Braun & $10.41 \pm 1.29$ & $224.75 \pm 16.37$ & $1.08 \pm 0.03$ \\
SL-PLUS & Smith \& & $13.37 \pm 2.21$ & $206.90 \pm 11.82$ & $1.11 \pm 0.02$ \\
& Nephew & & & \\
\hline
\end{tabular}

Even though the surface topographies revealed great variations, the fretting and corrosion scores did not show inter-group differences. No correlation between surface topography and fretting and corrosion scores were observed.

Our results reveal a high variability of the surface topography of the implants with no global correlation to wear. Implant geometry, corresponding stress distribution and surgical technique must be considered to unravel the cause of wear at the head-neck-interface of the implant.

Keywords: Corrosion; Fretting; Total Hip Arthroplasty

\section{P119}

Comparison of healing effect on inflammatory bone loss between cultivated human dental pulp stem cells and their conditioned medium concentrate

In Sook Kim ${ }^{1}$, Soon Jung Hwang ${ }^{1,2}$

${ }^{1}$ Dental Research Institute, Seoul National University, Seoul, Republic of Korea, ${ }^{2}$ Department of Oral and Maxillofacial Surgery, School of Dentistry, Seoul National University, Seoul, Republic of Korea

Stem cell-cultured conditioned media (CM) for bone regeneration is regarded as a unique alternative to cell transplantation in bone regeneration due to abundant growth factors and chemokines which are secreted during their cultivation. This study aimed to investigate the efficiency on in vivo healing capacity of inflammatory bone loss in comparison between cell transplantation and the use of CM concentrate derived from identical cells. CM-concentrate was prepared from human dental pulp stem cells (hDPSC) after three dimensional culture on collagen sponge in serum-free media for 2 days. Inflammatory bone loss was induced by injection of lipopolysaccharide (LPS; $50 \mathrm{mg}$ ), weekly three times for 2 weeks at tooth germ area around second premolar of rats. After LPS injection, experimental groups were divided into three groups; CM alone, hDPSC $\left(5 \times 10^{5}\right.$ cells) alone and combination of $\mathrm{CM}$ and hDPSC (CM/hDPSC). Control groups include LPS-treated and none-treated group. Alveolar bone at the treated germ area were evaluated using microcomputed tomography analysis and histological staining 4 weeks after LPS injection. LPS induced significant bone loss at 2 weeks, which spontaneously recovered around 30\% level at 4 weeks. CM or hDPSC alone showed a $12 \%$ increase in new bone volume (BV), compared to LPS group. CM/hDPSC group resulted in higher BV over CM or hDPSC alone, without significance. These preliminary results showed that local treatment of $\mathrm{CM}$ has a comparable efficacy with stem cell transplantation on the bone healing of inflammatory bone loss, suggesting that $\mathrm{CM}$ would be an encouraging alternative in stem cellmediated tissue regeneration.

\section{P120}

Characterization of Mg-alloy degradation from in-vivo $\mu \mathrm{CT}$-data within the MgBone project

Timo Damm $^{1}$, Olga Will ${ }^{1}$, Mirko Gerle ${ }^{2}$, Claus-Christian Glueer ${ }^{1}$

${ }^{1}$ Department of Biomedical Imaging, Clinic of Radiology, UniversityHospital Schleswig-Holstein, Kiel, Germany, ${ }^{2}$ Clinic of oral and maxillofacial surgery, University-Hospital Schleswig-Holstein, Kiel, Germany

Introduction: Within living tissue degradable implant materials are subject to material- and site-specific processes. Bone healing and its underlying biochemical machinery is influencing (and at the same time influenced by) alloy composition. Within the MgBone project funded by the German Ministry of Education and Research theses complex mutual dependencies are investigated together with our partners.

Methods: Evaluation of in-vivo $\mu \mathrm{CT}$ data of 16 Sprague Dawley rats will be presented. Each animal received one of two different magnesium-gadolinium $(\mathrm{MgGd})$ alloy implant screws into one tibia and either a titanium (Ti) or a polyether-ether-ketone (PEEK) plastic screw of equal dimension as control implant material into the other tibia. Scans have been acquired directly post-OP and at week 1, 4 and 8. Weeks 12 and 16 will follow.

Results: Contradicting naive expectations, MgGd-alloy degradation in living tissue leads to increasing segmentation volumes of the implants using threshold-based methods. Relative segmentation volume increases of $(2.9 \pm 0.3) \%$ for $\mathrm{Mg} 10 \mathrm{Gd}$ and $(7.9 \pm 1.2) \%$ for Mg5Gd per week have been calculated. Decreasing mean densities do not fully compensate.

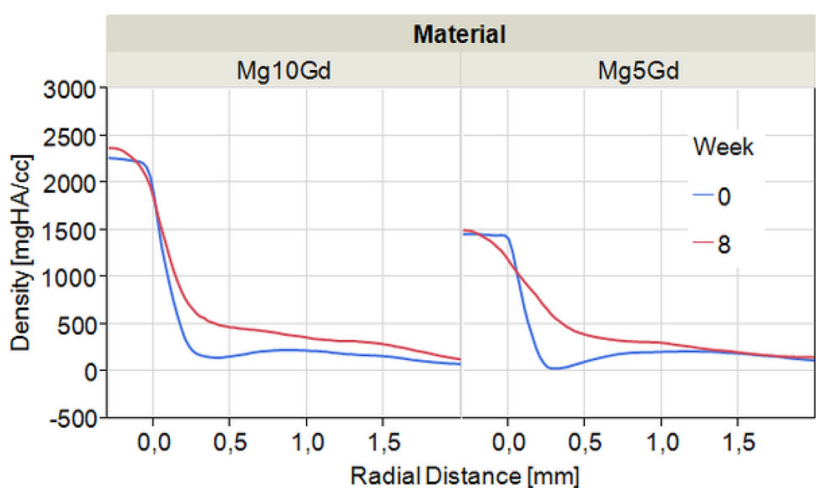

Individual density profiles collected on equidistant 3D shells around the implanted screw's surface

For the generation of density profile data, the baseline segmentations have been transported by image registration to their corresponding follow-up measurements to achieve a well matched profile basis. Comparing profiles of the baseline and follow-up data gives very specific insights into the BIC region, whereas the contribution of either degradation or bone healing into this zone will be clarified by additional modalaties like SR $\mu \mathrm{CT}$, SAXS or histology ex-vivo.

Keywords: Degradable Implants, In-Vivo $\mu \mathrm{CT}$, MagnesiumGadolinium (MgGd) alloy, Bone Healing, Bone-Implant-Contact (BIC)

\section{P121}

The impact of titanium dioxide-based scanning powder on L929 cells and human oral fibroblasts

Gunpreet Oberoi ${ }^{1,2}$, Anna Müller ${ }^{1,2}$, Andreas Moritz ${ }^{1,2}$, Hassan Shokoohi-Tabrizi $^{1,2}$, Christoph Kurzmann ${ }^{1,2}$, Hermann Agis ${ }^{1,2}$ 
${ }^{1}$ Department of Conservative Dentistry and Periodontology, School of Dentistry, Medical University of Vienna, Vienna, Austria, ${ }^{2}$ Austrian Cluster for Tissue Regeneration, Vienna, Austria

Digital impression has revolutionized the essence and workflow of dentistry. The aim of this study was to reveal the impact of titanium dioxide-based scanning powder used for intra oral scanning on the activity of L929 cells and human oral fibroblasts.

L929 cells and fibroblasts from human periodontal ligament and gingiva (PDLF, GF) were treated with scanning powder at concentrations of $30-0.00000003 \mathrm{mg} / \mathrm{ml}$ and the corresponding conditioned medium which was depleted of the particles. MTT and resazurinbased cytotoxicity assays, Live-Dead staining, and Annexin V/PI stainings were performed. Interleukin (IL)-6, and IL-8 expression by scanning powder treated fibroblasts was evaluated using immunoassays. Scanning powder composition was assessed using energydispersive X-ray spectroscopy (EDX).

We found that L929 were more sensitive to scanning powder than PDLF and GF. L929 showed reduced formazan and resazurin conversion upon treatment with scanning powder in a dose-dependent manner and lower impact of the corresponding conditioned medium. PDLF and GF were more resistant to the effects of the powder. Induction of cell death was found in L929 cells upon treatment with $30 \mathrm{mg} / \mathrm{ml}$ of scanning powder but not in PDLF and GF. No pronounced impact of the conditioned medium was seen in MTT staining, Live-Dead staining, and Annexin V/PI staining. IL-6 production was increased by the scanning powder, while there was a trend for a decrease of IL-8 production in PDLF and GF. SEM and EDX analysis revealed that scanning powder is a heterogeneous mixture predominantly containing titanium dioxide.

Scanning powder can reduce cell activity and induce cell death in L929 cells. Human oral fibroblasts are more resistant to these effects of the scanning powder, but they show an increase in the production of pro-inflammatory IL- 6 . These results provide novel insights into the response of oral tissue to titanium dioxide based scanning powder for intra oral scanning.

\section{$\mathbf{P 1 2 2}$}

Comparison of osteogenic efficacy between alendronate, zoledronate or risedronate-combined collagen sponge composites for high dose BMP-2 delivery

In Sook Kim ${ }^{1}$, Soon Jung Hwang ${ }^{2}$

${ }^{1}$ Dental Research Institute, Seoul National University, Seoul, Republic of Korea, ${ }^{2}$ Department of Oral and Maxillofacial Surgery, School of Dentistry, Seoul National University, Seoul, Republic of Korea

Local bisphosphonate (BP) have shown an outstanding synergy with recombinant human bone morphogenetic protein (rhBMP)-2 to heal bone defects. Our previous study demonstrated the manufactured collagen sponge (CS) composite combined with alendronate (ALN) led to excellent outcome with less adverse effects and higher boneforming efficacy in the treatment of high dose rhBMP-2. This study aimed to compare the osteogenic efficacy of CS composites prepared with addition of various BPs including ALN, zoledronate (ZOL) or risedronate (RIS) as a high dose rhBMP-2 carrier. Alkaline phosphatase activities of rat osteoblasts in vitro showed a synergistic effect of rhBMP-2 with BPs, while proliferation was inhibited, dependently of BP-doses. ALN-, ZOL-, or RIS-CS were loaded with rhBMP-2 $(40 \mu \mathrm{g})$ and analyzed for new bone formation using $8 \mathrm{~mm}$-critical sized calvarial defect model of rats, compared with non-loaded CS. Osteoclast activation in vivo was remarkably suppressed in all BPCS-BMP groups at initial period of 1 week. Total bone mass and bone density, calculated from micro-CT reconstructions at 4 weeks, were significantly greater in all BP-CS-BMP groups. Especially, all BP-
CS-BMP groups significantly decreased heterotopic ossification over CS-BMP group. Histology corroborated these findings and revealed dense spatial pattern of bone formation in BP-CS-BMP groups. The efficacy for rhBMP-2 delivery is the order of ALN-CS, ZOL-CS and RIN-CS. These results demonstrate that BP-CS composites has remarkable advantages in high dose-rhBMP-2 delivery over CS with less early bone resorption, less heterotopic ossification but early dense ossification, highlighting a practical delivery matrix of rhBMP-2.

\section{P123}

Electrospun shape memory mesh for in-vivo delivery of therapeutics to aid in fracture healing in a diabetic rodent model

Benjamin Braun ${ }^{1}$, Jordan Skelly ${ }^{1}$, Ben Zhang ${ }^{1}$, David Ayers ${ }^{1}$, Jie Song $^{1}$

${ }^{1}$ Orthopaedic Surgery, University of Massachusetts Medical School, Worcester, United States

Introduction: Impaired fracture healing is a significant orthopedic co-morbidity of diabetes. We propose expediting diabetic fracture healing with a novel degradable shape-memory mesh designed to self-wrap and deliver osteogenic therapeutics to fractured periosteal surface.

Methods: The shape-memory mesh composed of degradable amphiphilic triblock copolymer PLGA-PEG-PLGA (PELGA) and hydroxyapatite (HA) were prepared as recently reported. IACUC approval was obtained for all procedures. Nine Sprague-Dawley rats weighing 275-325 g (Charles Rivers Lab) received an intraperitoneal injection of streptozotocin $(60 \mathrm{mg} / \mathrm{kg})$ to induce diabetes, which was confirmed by blood glucose $>250 \mathrm{mg} / \mathrm{dl}$. The diabetic rats were divided into 3 groups. Group 1: Bilateral femoral fractures stabilized by intramedullary (IM) rods without mesh. 1-mm IM rods were inserted into femurs before they were fractured using a blunt guillotine/drop weight method. Group 2: Bilateral femoral fractures stabilized with IM rods and wrapped with the shape-memory mesh. Group 3: Bilateral femoral fractures stabilized with IM rods and wrapped with the shape-memory mesh pre-absorbed with 500-ng rhBMP-2. To place the mesh, an incision was made to expose the femur. The mesh, pre-programmed with a permanent shape, was placed over the fracture and allowed to wrap around the femur. The healing was monitored by longitudinal microCT $(n=6)$ at 3 and 6 weeks and systemic organ pathology was examined at 6 weeks.

Results and discussions: The electrospun HA-PELGA scaffold exhibit excellent shape-memory properties around physiological temperature. Diabetic induction was achieved with $100 \%$ success rate. The use of electrospun shape-memory mesh to deliver a single dose of 500-ng rhBMP-2 significantly expedited the diabetic fracture healing as evidenced by microCT. No systemic side effects were detected with the mesh or BMP-2. The low dose rhBMP-2 delivered to the fracture via the self-wrapping mesh allowed enhanced therapeutic efficacy while reducing the cost and minimizing adverse effects of BMP-2.

Keywords: Shape-memory, low-dose BMP-2, Diabetic Fracture
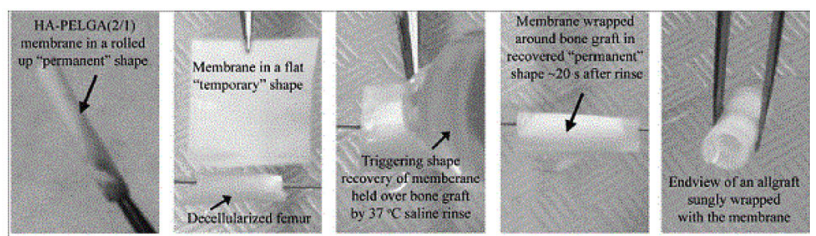

[Figure 1]

Fig. 1 


\section{P124}

KP-070, a novel benzamide derivative, protects ligature-induced alveolar bone erosion by inhibiting NFATc1-mediated osteoclastogenesis

Hye Jung $\mathrm{Ihn}^{1}$, Ju Ang Kim², Soomin $\mathrm{Lim}^{2}$, Jung Eun Kim ${ }^{3}$, Hong-In Shin $^{4}$, Eui Kyun Park ${ }^{2}$

${ }^{1}$ Institute for Hard Tissue and Bio-tooth Regeneration, Deagu, Republic of Korea, ${ }^{2}$ Patholgy and Regenerative Medicine, Kyungpook National University School of Dentistry, Deagu, Republic of Korea, ${ }^{3}$ Molecular Medicine, Kyungpook National University School of Medicine, Daegu, Republic of Korea, ${ }^{4}$ Pathology and Regenerative Medicine, Kyungpook National University School of Dentistry, Daegu, Republic of Korea

Suppression of osteoclast differentiation and bone resorption has been a primary therapeutic strategy in diverse osteolytic bone diseases including periodontitis and osteoporosis. In this study, we investigate the therapeutic potential of a novel benzamide-linked molecule, KP070, for preventing alveolar bone loss in mice with ligature-induced experimental periodontitis. KP-070 inhibited osteoclast formation by acting on both early and late stages of differentiation, and attenuated the induction of nuclear factor of activated T-cells, cytoplasmic 1 (NFATc1) and the expression of osteoclast-specific genes. In addition, KP-070 significantly suppressed the formation of actin rings and resorption pits. Analysis of the inhibitory action of KP-070 showed that it markedly suppressed receptor activator of nuclear factor- $\kappa \mathrm{B}$ ligand (RANKL)-induced extracellular signal-regulated kinase (ERK) and NF- $\kappa \mathrm{B}$ signaling cascades. Moreover, KP-070 prevented ligatureinduced alveolar bone erosion in mice by suppressing osteoclast formation. These findings demonstrate that KP-070 attenuated osteoclast differentiation and function as well as ligature-induced bone erosion by inhibiting RANKL-mediated ERK and NF- $\kappa \mathrm{B}$ signaling pathways.

\section{$\mathbf{P 1 2 5}$}

Biodegradable poly(lactide-co-glycolide) beads for multiple deliveries of Wnt agonists and antibiotics to treat bone infection

Song-Shu Lin ${ }^{1}$, Steve W. N. Ueng ${ }^{1}$, Shih-Jung Liu ${ }^{2}$, Err-Cheng Chan $^{3}$, Huang-Yu Chen ${ }^{2}$, Yung-Bin Kuo ${ }^{4}$, Chuen-Yung Yang ${ }^{1}$, ChiChien Niu ${ }^{1}$, Li-Jen Yuan ${ }^{5}$

${ }^{1}$ Department of Orthopaedic Surgery, Chang Gung Memorial Hospital, Taoyuan, Taiwan, Republic of China, ${ }^{2}$ Department of Mechanical Engineering, Chang Gung University, Taoyuan, Taiwan, Republic of China, ${ }^{3}$ Department of Medical Biotechnology and Laboratory Science, Chang Gung University, Taoyuan, Taiwan, Republic of China, ${ }^{4}$ GenProNex INC, Research and Development, Taoyuan, Taiwan, Republic of China, ${ }^{5}$ Department of Orthopaedic Surgery, E-Da Hospital/I-Shou University, Kaohsiung City, Taiwan, Republic of China

Objective: Antibiotic beads have been used as a drug delivery system for the treatment of surgical infections. Wnt agonist lithium has been used to promote the osteogenesis of mesenchymal stem cells (MSCs). However, the effect of lithium on the bactericidal efficiency of antibiotics is not clear.

Methods: The 50:50 poly(DL-lactide):co-glycolide (PLGA) was mixed with vancomycin and lithium bicarbonate $\left(\mathrm{Li}_{2} \mathrm{CaCO}_{3}\right)$ powder and hot compressing molded at $55{ }^{\circ} \mathrm{C}$ to form biodegradable beads. The beads were placed in $5 \mathrm{~mL}$ of phosphate buffered saline (PBS) and incubated at $37{ }^{\circ} \mathrm{C}$. The PBS was changed daily, and the removed buffer solutions were stored at $-70{ }^{\circ} \mathrm{C}$. The antibiotic and lithium concentration in each sample was determined by Dimension ${ }^{\circledR}$ EXL $^{\mathrm{TM}} 200$ Integrated Chemistry System.
Results: The released lithium was lasting for more than 10 days and most marked during the first $48 \mathrm{~h}$. The released lithium was shown to promote the osteogenesis of MSCs by increasing in osteocalcin mRNA, Runx 2 mRNA expression, in phosphorylation of GSK$3 \beta$ protein, and in Runx 2 protein expression $(\mathrm{p}<0.05)$. The concentration of vancomycin in each sample was well above the breakpoint sensitivity concentration for more than 14 days and most marked during the first $48 \mathrm{~h}$. The diameter of the sample inhibition zone ranged from 12 to $15 \mathrm{~mm}$, which was equivalent to $62.5-100 \%$ relative activity. The bactericidal effect of vancomycin was not significantly affected by $\mathrm{Li}^{+}$treatment. Taken together, this study offers a convenient method for multiple deliveries of Wnt agonists and antibiotics by biodegradable PLGA beads to meet the specific antibiotic requirements for osteomyelitis patients.

Keywords: PLGA, vancomycin, Wnt agonist, MSCs, osteomyelitis

\section{P126}

Impact of implantoplasty on surface roughness and human fibroblast growth in vitro

Mehrnaz Beheshti Maal $^{1}$, Stig Aanerød Ellingsen ${ }^{1}$, Janne Reseland ${ }^{2}$, Anders Verket ${ }^{1}$

${ }^{1}$ Institute of Clinical Dentistry, Oslo, Norway, ${ }^{2}$ Biomaterials, Institute of Clinical Dentistry, Oslo, Norway

Objective: Implantoplasty, mechanical smoothening of titanium implant surfaces, is an emerging treatment of peri-implantitis in dentistry. It is performed in order to facilitate oral hygiene and thus limit inflammatory bone resorption around implants.

The aim of the in vitro study was to characterize the extent of surface roughness following various implantoplasty protocols, and examine the impact on human cell growth.

Method: Six different rotating bur sequences were applied to coins of titanium grade IV with a diameter of $6 \mathrm{~mm}$ and a height of $2 \mathrm{~mm}$. The bur sequences included either diamond burs or carbide burs alone, or followed by either Arkansas stone bur or silicon burs. Machine-polished coins and rough-surface coins (sandblasted and acid-etched) were used as control groups. An optical profilometer was used to examine the surface topography in each group $(n=6)$. Human gingival fibroblasts (HGF) were cultured on the coins and effects of cell morphology and attachment were analyzed using confocal microscopy.

Results: The carbide bur sequence alone or in combination with either Arkansas or silicon burs rendered consistently lower mean Root mean square deviation (Sq) and Arithmetical mean height (Sa) values compared to the diamond bur sequence alone or in combination.

Treatments combined with the Arkansas stone bur provided lower mean $\mathrm{Sq}$ and $\mathrm{Sa}$ values as compared to diamond or carbide burs alone, but diamond or carbide burs followed by silicon burs rendered the lowest mean values of the test groups.

No significant differences were observed with respect to fibroblast morphology and adhesion on the various surfaces.

Conclusions: The carbide bur sequence followed by silicon burs provided the surface with the lowest $\mathrm{Sq}$ and $\mathrm{Sa}$ in the test groups. In this in vitro study the titanium surface alterations seemed not to influence the fibroblast growth.

Keywords: implantoplasty, peri-implantitis, fibroblasts, implants, surface

\section{P127}

Low adhesive scaffold type I collagen prepared from pig shinbone induces the osteogenic differentiation of rat bone marrow stromal cells 
Saori Kunii ${ }^{1}$, Koichi Morimoto ${ }^{1}$

${ }^{1}$ Kindai University, Kinokawa, Japan

Introduction: Bone consists of inorganic and organic compounds and is considered the most perfect architecture for supporting tissues. Collagen is a major component of bone matrices. However, bone collagen has not been utilized as a biomaterial scaffold because of its insolubility. These days, we succeeded in developing low adhesive scaffold type I collagen from pig skin (S-LASCol, patent pending). Similarly, we attempted to prepare bone LASCol (B-LASCol) from pig shinbone. In this study, we report that B-LASCol markedly facilitates osteogenic differentiation of rat bone marrow stromal cells (rMSCs).

Methods: B-LASCol was prepared from pig shinbone under mild conditions with protease. The purity and the molecular weight of B-LASCol were investigated by SDS-PAGE. Three types of culture dish, B-LASCol (own coated), Atelocollagen (commercially precoated), and non-coated (control) were used. Rat MSCs $\left(4 \times 10^{4}\right.$ cells/dish) were cultured on each coated-dish with osteogenic basal medium. The cell motility was monitored by time-lapse observation with a microscope. To evaluate osteogenic differentiation, we investigated a mineralization by alizarin red S staining. Moreover, the quantity of mRNA of osteogenesis-related genes (Alpl, BGP, Osx, and Runx2) was analyzed by RT-qPCR.

Results: Bone collagen was solubilized by our methods, and it was shown to keep the triple-helical structure. The molecular weight of B-LASCol was very similar to skin collagen. Rat MSCs on B-LASCol coated-dish formed multicellular aggregates, and each cell clearly showed alkaline phosphatase activity earlier than usual. Calcium deposits on the B-LASCol coated-dish showed increasing most earlier (Day 3-6). The mRNA of cells cultured on B-LASCol coated-dish much expressed than those on others.

Conclusions: B-LASCol was obtained from shinbone with a high yield. We demonstrated that B-LASCol has the acceleration effects of osteogenic differentiation.

Funding: This work was supported by the Adaptable and Seamless Technology Transfer Program through target-driven R\&D, AMED and JST

\section{P128}

Characterization of bone extracellular matrix produced by Recql4deficient osteoblasts

Tatiana Gritsaenko ${ }^{1}$, Valérie Pierrefite-Carle ${ }^{1}$, Jean-Marie Guigonis ${ }^{1}$, Sabrina Pisano ${ }^{2}$, François Orange ${ }^{3}$, Chantal Cros $^{1}$, Georges F. Carle ${ }^{1}$, Sabine Santucci-Darmanin ${ }^{1}$

${ }^{1}$ TIRO-MATOs UMR E-4320, CEA/UNS, Université Nice Sophia Antipolis/BIAM, Nice, France, ${ }^{2}$ IRCAN - CNRS UMR 7284, INSERM U1081, Université Nice Sophia Antipolis, Nice, France, ${ }^{3}$ Centre Commun de Microscopie Appliquée (CCMA), Université Nice Sophia Antipolis, Nice, France

The bone extracellular matrix (ECM) is a dynamic network of molecules secreted by osteoblasts (OB) and osteocytes that in turn regulate the behavior of all bone cells by modulating their proliferation, differentiation and function. As an example, senescent $O B$ create a defective microenvironment through an altered secretome, which in turn stimulates osteoclast function. Meanwhile, another study shows that exposure to a decellularized ECM deposited by young $\mathrm{OB}$ rescues replication potential of aged OB precursors. Taken together, these observations highlight the connection between $\mathrm{OB}$ secretome and bone cells behaviour.

Our laboratory is studying two murine models of bone loss potentially associated with altered $\mathrm{OB}$ secretome. Aiming to decipher the mechanisms underlying bone loss in our models, we planned to investigate the relationship between the OB-produced bone matrix and bone cells.

Here, we describe the methodologies used to produce, decellularize and characterize bone matrices synthesized in vitro by primary $\mathrm{OB}$ isolated from Recql4-deficient mice. recql4 $4^{-1-}$ mice exhibit a premature bone aging phenotype reminiscent of the one observed in patients harboring RECQL4 mutations. RECQL4 is a DNA helicase involved in genomic stability and its dysfunction has been associated with cellular senescence.

We first observed that bone matrix produced by recql4 ${ }^{-1-} \mathrm{OB}$ are less mineralized than those from recql4 ${ }^{+-}$control OB. We next analyzed protein and mineral composition, ultrastructure and stiffness of the resulting matrices using different approaches such as proteomic analysis, scanning electron microscopy with energy dispersive X-ray spectroscopy (SEM/EDX), SEM and atomic force microscopy (AFM). Our preliminary results will be presented and discussed.

We expect that the protocols we developed to analyze mineralized matrices will help to understand bone cells behavior in pathophysiological conditions such as premature bone ageing.

Keywords: mineralized matrices, Recq14 mouse model, premature ageing

\section{P129}

Original densitometric evidence of the independent influence of regional muscles' mass, strength and use and sex hormones upon bone mineral content (BMC) determination

Carlos Cure Cure ${ }^{1}$, Jose Luis Ferretti ${ }^{2}$, Pablo Cure ${ }^{3}$, Laura Marcela Nocciolino $^{2}$, Gustavo Roberto Cointry ${ }^{2}$, Alex Ireland ${ }^{4}$, Patricio López Jaramillo $^{5}$, Ricardo Francisco Capozza ${ }^{2}$

${ }^{1}$ Biomelab Research Center, Barranquilla, Colombia, ${ }^{2}$ Centro de Estudios de Metabolismo Fosfocálcico (CEMFoC), Universidad Nacional de Rosario, Rosario, Argentina, ${ }^{3}$ George Washington University, Washington D.C., United States, ${ }^{4}$ School of Healthcare Science, Manchester Metropolitan University, Manchester, United Kingdom, ${ }^{5}$ Fundación Oftalmológica de Santander (FOSCAL), Bucaramanga, Colombia

To define the independent influence of muscle mass, force and use and estrogens on BMC determination, we analyzed the relationships between DXA-assessed BMC and lean mass (LM) and hand-grip strength (HGS) in the upper limbs of 300 healthy Colombian residents with strictly defined rural $(\mathrm{R})$ working activities ( 83 men, $52.9 \pm 11.0$ years; 63 premenopausal women, $40.5 \pm 2.8$ years; 164 postmenopausal women, $55.7 \pm 8.0$ years) and 300 with urban (U) habits $(93,53.4 \pm 9.7$ years; $55,40.3 \pm 2.9$ years; $152,54.8 \pm 6.5$ years $)$.

$\mathrm{BMC}$ was higher in $\mathrm{R}$ than $\mathrm{U}$ men and premenopausal women ( $p<0.001)$. LM was higher in all $\mathrm{R}$ than $\mathrm{U}$ groups $(\mathrm{p}<0.001)$. HGS and LM correlated logarithmically in all groups together $\left(\mathrm{R}^{2}=0.58\right.$, $\mathrm{p}<0.001$ ) with no R/U difference and higher HGS and LM values for men than women. BMC correlated with HGS with variable significance for $\mathrm{R} / \mathrm{U}$ men, premenopausal and postmenopausal women (R: $\mathrm{r}=0.63, \mathrm{p}<0.001 ; \mathrm{r}=0.25, \mathrm{p}=0.01 ; \mathrm{r}=0.160, \mathrm{p}: \mathrm{ns} ; \mathrm{U}$ : $\mathrm{r}=0.45, \mathrm{p}<0.001 ; \mathrm{r}=0.30, \mathrm{p}<0.01 ; \mathrm{r}=0.26, \mathrm{p}<0.05)$, with higher slopes and intercepts for men than women $(\mathrm{p}<0.001)$ and higher intercepts for $\mathrm{R}$ than $\mathrm{U}$ in men $(\mathrm{p}<0.01)$ and premenopausal women $(\mathrm{p}<0.01)$.

The significant HGS-vs-LM correlation suggests that LM reflects the regional muscle force in healthy individuals, regardless of sex and working habit. The significant correlations between BMC and LM or HGS indicate that bone mass depends not only on the mass, but also on the strength of regional muscles. The higher intercepts of BMC-vsHGS correlations for men vs. women and for R vs. U men and premenopausal women suggest that bone mass is affected by the use of muscles in fertile men and women. Similar intercepts for R and $\mathrm{U}$ 
postmenopausal women may reflect the hormonal interference on this relationship.

These results represent the first derivation of the above 4 conclusions from a single DEXA study.

\section{P130}

Microarchitecture variation in the vicinity of the standard transiliac biopsy site assessed by microCT

Stéphane Blouin ${ }^{1}$, Barbara Misof ${ }^{1}$, Andrea Berzlanovich ${ }^{2}$, Gerlinde ${\text { Maria } \text { Gruber }^{3} \text {, Klaus Klaushofer }}^{1}$, Peter Fratzl ${ }^{4}$, Paul Roschger ${ }^{1}$

${ }^{1} 1$ st Medical Department, Ludwig Boltzmann Institute of Osteology at the Hanusch Hospital of WGKK and AUVA Trauma Centre Meidling, Vienna, Austria, ${ }^{2}$ Department of Forensic Medicine, Medical University of Vienna, Vienna, Austria, ${ }^{3}$ Department of Anatomy, Center for Anatomy and Cell Biology, Medical University of Vienna, Vienna, Austria, ${ }^{4}$ Department of Biomaterials, Max Planck Institute of Colloids and Interfaces, Vienna, Austria

Transiliac bone biopsy samples typically used for diagnosis and management of bone pathology are well characterized by histomorphometry and microCT. However, little is known about trabecular and cortical microarchitecture variations with age or in the region near to the standard biopsy site $(2 \mathrm{~cm}$ dorsal from spina iliaca superior anterior and $2 \mathrm{~cm}$ caudal from iliac crest).

Twelve $5 \times 5 \mathrm{~cm}$ iliac crest necropsy samples were obtained from control females under 65 years (median $=62$ years; $\mathrm{n}=6$ ) or over 90 years $($ median $=94$ years; $\mathrm{n}=6)$. MicroCT images (SCANCO 50, $90 \mathrm{kV}$ ) were acquired on the whole sample ( $24 \mu \mathrm{m}$ voxel resolution) for trabecular analysis and on a $10 \mathrm{~mm}$ broad vertical stripe including the standard site $(7.4 \mu \mathrm{m}$ voxel resolution) for cortical porosity analysis. We analysed the inner and outer cortex on a stack of $\mu \mathrm{CT}$ images representing a layer of $5 \mathrm{~mm}$, by setting a $500 \mu \mathrm{m}$ thick region of interest starting from the periosteal surface.

Trabecular microarchitecture variations: bone volume (BV/TV + $41 \% ; \mathrm{p}=0.004)$, thickness $(\mathrm{Tb} . \mathrm{Th}+18 \% ; \mathrm{p}=0.04)$ and number (Tb.N $+15 \% ; \mathrm{p}=0.01$ ) were higher in the younger age group. A general reduction in BV/TV $(-22 \% ; \mathrm{p}=0.001)$, Tb.Th $(-1 \%$; $\mathrm{p}=0.024)$ and Tb.N $(-6 \% ; \mathrm{p}<0.001)$ was measured in $6 \mathrm{~mm}$ caudal direction from the standard site, with progressive reduction with larger distances.

Cortical porosity variation: The porosity (Po.V/TV), due to mainly-vertical cortical canals, was lower in the inner cortex $(-362 \%, p=0.015)$ and outer cortex $(-337 \%, p=0.002)$ in the younger individuals, while the interpore space was higher in the inner cortex $(+11 \%, \mathrm{~ns})$ and outer cortex $(+24 \%, \mathrm{p}=0.002)$. In general, an increased porosity $(+50 \% ; \mathrm{p}=0.012)$ and a decreased interpore space $(-11 \% ; \mathrm{p}=0.034)$ was found in the outer cortex compared to the inner cortex.

Trabecular microarchitecture and cortical porosity of transiliac biopsy samples are highly dependent on age as well as on bone extraction site.

\section{P131}

Relationships between DXA-assessed bone, lean and fat masses in a large sample of non-obese children, men, and pre- and postmenopausal women considering the Tanner stage
Edgar Denova-Gutierrez ${ }^{1,2}$, Patricia Clark ${ }^{2,3}$, Ricardo Francisco Capozza $^{4}$, Laura Marcela Nocciolino ${ }^{4}$, José Luis Ferretti ${ }^{4}$, Rafael Velazquez-Cruz ${ }^{5}$, Berenice Rivera ${ }^{6}$, Alex Ireland ${ }^{7}$, Gustavo Roberto Cointry $^{3,4}$, Jorge Salmerón ${ }^{6,8}$

${ }^{1}$ Centro de Investigación en Nutrición y Salud, Instituto Nacional de Salud Pública, Cuernavaca, Mexico, ${ }^{2}$ Facultad de Medicina, Universidad Nacional Autónoma de México, Ciudad de México, Mexico, ${ }^{3}$ Unidad de Investigación en Epidemiología Clínica, Hospital Infantil de México Federico Gómez, Ciudad de México, Mexico, ${ }^{4}$ Centro de Estudios de Metabolismo Fosfocálcico (CEMFoC), Universidad Nacional de Rosario, Rosario, Argentina, ${ }^{5}$ Laboratorio de Genómica del Metabolismo Óseo, Instituto Nacional de Medicina Genómica, Ciudad de México, Mexico, ${ }^{6}$ Unidad Académica en Investigación Epidemiológica, Centro de Investigación en Políticas, Población y Salud. Facultad de Medicina, Universidad Nacional Autónoma de México, Ciudad de México, Mexico, ${ }^{7}$ School of Healthcare Science, Manchester Metropolitan University, Manchester, United Kingdom, ${ }^{8}$ Centro de Investigación en Salud Poblacional, Instituto Nacional de Salud Pública, Cuernavaca, Mexico

DEXA-assessed bone mineral content (BMC), lean mass (LM) and fat mass (FM) and anthropometric features were determined in 4,001 healthy, non-obese $(\mathrm{BMI}<30)$ Mexican children/adolescents $<19$ years $(n=720$, classified according to their Tanner stages $)$, and men $(n=864)$, premenopausal women $(n=1717)$ and postmenopausal women $(\mathrm{n}=700)$ aged $\geq 20$ years aiming to describe the associations of BMC with LM, FM, body weight, and body height taking into account the influence of age and sex hormones. The influence of LM, FM, weight, height and age on BMC was assessed by simple and multiple correlation analyses in every group.

Among boys' subgroups, the BMC(y)-vs-LM(x) curves were virtually parallel, with similar intercepts. A progressive displacement of values for each Tanner subgroup toward the upper-right region of the graph was observed. In contrast, in female subgroups, curves for Tanner 1-2 and 4-5 stages were virtually parallel but the intercepts were significantly higher for the latter by about $+300 \mathrm{~g}$ of BMC $(P<0.001)$. Tanner-3 curve 'bridged the gap' between the other two. Premenopausal and postmenopausal women showed parallel curves with a lower intercept for the latter $(P<0.001)$. A generally higher independent determining power (higher partial $\mathrm{r}$ values in multiple correlations) of LM on BMC than those of FM, weight, height, and age was shown, except in the postmenopausal women.

BMC-vs-LM relationships were described in the studied population, pointing out the influence of Tanner stage on the evolution of bone/muscle proportionality during puberty. Female Tanner-3 was identified as the transition stage between 'child' and 'adult' patterns of the BMC-vs-LM relationship. Results further support the higher determining power of LM on BMC than those of the other confounders studied, except in postmenopausal women.

For practical purposes, reference Z-scored charts of BMC-vs-LM relationships are provided separately for children at different Tanner stages, men, and pre- and post-MP women. 


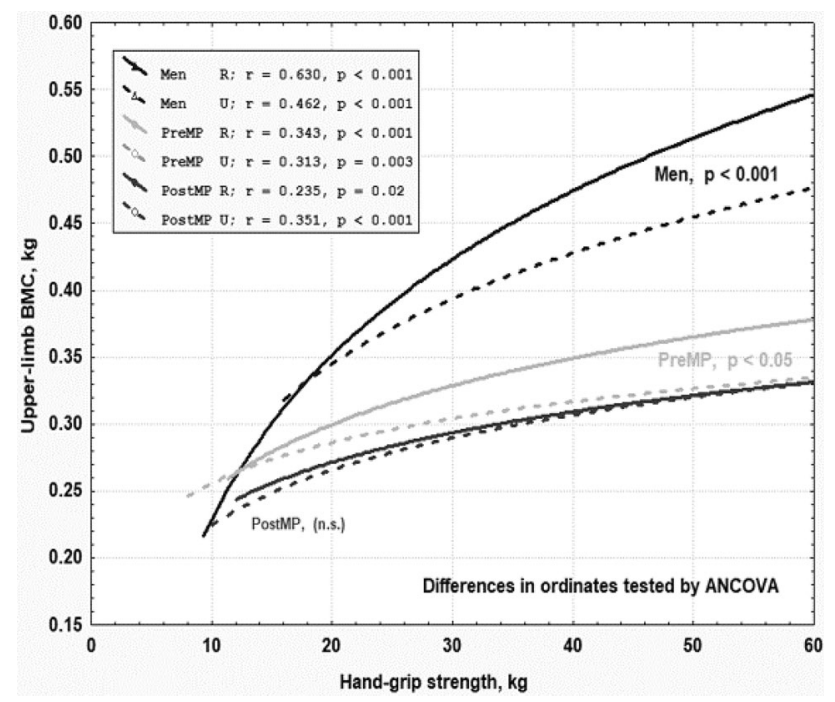

Figure

\section{P132}

Influence of egg production on tibiae bone properties of laying hens

Beryl Eusemann $^{1}$, Stefanie Petow ${ }^{1}$, Estefania Sanchez-Rodriguez ${ }^{2}$, Cristina Benavides-Reyes ${ }^{2}$, Nazaret Dominguez-Gasca ${ }^{2}$, Santiago Gonzalez-Lopez $^{3}$, Alejandro B. Rodriguez-Navarro ${ }^{2}$

${ }^{1}$ Institute of Animal Welfare and Animal Husbandry, Friedrich-Loeffler-Institut, Celle, Germany, ${ }^{2}$ Departamento de Mineralogia $y$ Petrologia, University of Granada, Granada, Spain, ${ }^{3}$ Departamento de Estomatologia, University of Granada, Granada, Spain

Laying hens offer an interesting model for studying bone pathologies such as osteoporosis. During the intensive egg laying period, due to high calcium demand for eggshell calcification, cortical bone reabsorption may occur causing a severe weakening of the skeleton that is considered a type of osteoporosis.

To determine how egg production affects bone quality, we conducted a comparative study (approved by an ethical committee) of bone properties in 62 weeks old laying hens with either high (WLA) or low (G11) laying performance (320 vs. 200 eggs/year). To characterize tibiae bone properties we measured bone dimensions and determined bone mechanical properties (three-point-bending tests), chemical composition and structural organization using attenuated total reflection-Fourier transform infrared spectroscopy, thermogravimetry and 2D X-ray diffraction.

Our results showed that low performing G11 hens had a higher degree of bone mineralization than WLA hens (see Table 1). Also, G11 cortical bone mineral had a greater crystallinity $(\mathrm{p}<0.05)$ and higher degree of carbonate substitution $(\mathrm{p}<0.01)$ which are all indicative of increased bone mineral maturity. This could indicate that bone metabolism (turnover rate) was greater in high performing hens due to the higher calcium demand for eggshell formation. Whole bone breaking strength was not statistically different between the two layer lines $(p>0.05)$. However, when correcting for bone cross-section area, G11 hens showed a higher bone strength $(\mathrm{p}<0.05)$ which correlates with their greater degree of mineralization. In conclusion, laying performance strongly influences bone properties which seems to be associated to differences in bone remodeling rates between lines.
Table 1: Degree of mineralization of cortical and medullary bone

\begin{tabular}{lllllll}
\hline & $\mathrm{N}$ & $\begin{array}{l}\text { WLA } \\
(\text { Mean } \pm \text { SEM })\end{array}$ & $\begin{array}{l}\text { N } \\
(\text { Mean } \pm \text { SEM })\end{array}$ & F & p \\
\hline $\begin{array}{c}\text { Cortical } \\
\text { bone }\end{array}$ & 24 & $2.75 \pm 0.03$ & 8 & $2.95 \pm 0.01$ & 18.867 & $<0.001$ \\
$\begin{array}{c}\text { Medullary } \\
\text { bone }\end{array}$ & 24 & $0.72 \pm 0.07$ & 8 & $1.37 \pm 0.11$ & $31.321<0.001$ \\
\hline
\end{tabular}

Keywords: osteoporosis, laying hens, laying performance

\section{P133}

Dose microstructural and mechanical change effect on change of stress distribution on the distal femur with osteoarthritic change?

Kwangkyoun $\mathrm{Kim}^{1}$

${ }^{I}$ Orthopedic Surgery, Konyang University, Daejeon, Republic of Korea

Objectives: The knee joints are weight bearing joints and have several motions in various directions. Loads applied to the distal femur are different depending on regions. The purpose of this study is to analyze regional differences of stress distribution and to evaluate the microstructural and mechanical property's effect on stress distribution on the distal femur.

Materials and Methods: Distal femur specimens were obtained from ten donors composed of ten women with OA (mean age of 65 years, ranging from 53 to 79). As controls, the normal distal femur was sampled from age and gender matched donors consisting of ten women (mean age of 67 years, ranging from 58 to 81). The areas of interest were six regions of the condyles of the femur (lateral-anterior, middle, posterior; medial-anterior, middle, posterior). A total of 20 specimens were scanned using the micro-CT system. Micro-CT images were converted to micro-finite element model using the mesh technique, and micro-finite element analysis was then performed for assessment of the mechanical properties.

Results: Stress is not different in anterior, middle and posterior section within each compartment regardless OA change $(p=0.237)$. Part of microstructure parameter, which are BV/TV, Tb.Th and SMI, are different in the inter-sectional comparison between Advanced OA group and control group $(\mathrm{p}=0.012)$. There are no difference in stress except middle-medial region between advanced OA group and control group $(p=0.285)$. In middle-medial region, control group is higher stress than advanced OA group (0.002).

Conclusion: In the control group, stress is distributed differently depend on region of distal femur, but Stress is evenly distributed with advanced OA change. Regional difference in microstructure are likely related to the adaptive bone remodeling for more even stress distribution in advanced OA.

Keywords: Microstructure, bone, stress, femur

\section{$\mathbf{P 1 3 4}$}

Delayed fracture healing in Mmp10 (Stromelysin 2) knockout mice

José Valdés-Fernández $^{1}$, Emma Muiños-López ${ }^{1}$, Tania López-

Martínez ${ }^{1}$, Purificación Ripalda-Cemboráin ${ }^{1}$, José Antonio

Rodriguez ${ }^{2}$, Jose Anrtonio Páramo ${ }^{2,3}$, Josune Orbe ${ }^{4}$, Felipe Prósper ${ }^{1,3}$, Froilán Granero-Moltó ${ }^{1,5}$ 
${ }^{1}$ Cell Therapy, Clínica Universidad de Navarra, Pamplona, Spain, ${ }^{2}$ Atherothrombosis Research Laboratory, CIMA-University of Navarra, Pamplona, Spain, ${ }^{3}$ Haematology, Clínica Universidad de Navarra, Pamplona, Spain, ${ }^{4}$ Atherothrombosis Research Laboratory, CIMA-University of Navarra, Pmaplona, Spain, ${ }^{5}$ Orthopaedic Surgery and Traumatology, Clínica Universidad de Navarra, Pamplona, Spain

Background: After a severe injury bone is able to develop a strong healing response recovering its original structure and functionality without forming scar tissue. Matrix metalloproteinases take a leading role in the remodelling of the extracellular matrix during this regenerative process, in consequence disorders affecting MMPs expression or activity could deeply affect the fracture-healing process. MMP-10 (Stroemlysin 2) is a matrix metalloproteinase highly similar to MMP3 (Stromelysin 1) with a wide spectrum of protein targets (fibronectin, elastin, gelatins, several types of collagen).

Purpose: To determine the role of MMP-10 during fracture repair process.

Methods: A closed fracture was performed in wild type (WT) and Mmp-10 knockout (Mmp10 ${ }^{\mathrm{KO}}$ ) C57BL/6 mice. Callus structure and composition were analysed at 7, 10, 14 and 21 days post fracture (dpf) by histology, immunohistochemistry and micro computed tomography (mCT).

Results: By mCT analysis we found a significant increase of the callus volume at 14-dpf in Mmp10 ${ }^{\mathrm{KO}}$ mice (WT, $17.13 \pm 4.56 \mathrm{~mm}^{3}$; $\left.\mathrm{Mmp} 10^{\mathrm{KO}}, 27.64 \pm 11.82 \mathrm{~mm}^{3}, \mathrm{p}=0.023\right)$. Interestingly, at 21-dpf the callus volume between both groups was similar (WT, $\left.13.59 \pm 3.48 ; \mathrm{Mmp} 10^{\mathrm{KO}}, 17.50 \pm 4.99, \mathrm{p}=0.1564\right)$. Segmentation of the callus at 14-dpf indicates that the differences in callus volume were located in soft tissue accumulation (WT, $6.375 \pm 3.839 \mathrm{~mm}^{3}$; $\mathrm{Mmp} 10^{\mathrm{KO}}, 12.270 \pm 8.100 \mathrm{~mm}^{3}, \mathrm{p}=0.0433$ ) without differences in bone tissue content. Histomorphometric analysis of the 7, 10, 14 and 21-dpf calluses showed increased content of cartilage in $\mathrm{Mmp} 10^{\mathrm{KO}}$ mice (WT, $5.995 \pm 6.256 \mathrm{~mm}^{2}$; Mmp10 ${ }^{\mathrm{KO}}, 18.290 \pm 10.770 \mathrm{~mm}^{2}$, $\mathrm{p}=0.0124)$. At 14-dpf TRAP staining showed an osteoclast accumulation in the interzone between cartilage and bone in Mmp10KO mice, suggesting that cartilage accumulation phenotype could be caused by a deficient osteoclast activity.

In conclusion, Lack of MMP-10 activity results in transient fracture healing impairment with a delay in cartilage resorption. This phenotype suggests that there is a compensation mechanism between different MMPs.

Keywords: MMP-10, bone repair, endochondral ossification, cartilage

\section{$\mathbf{P 1 3 5}$}

Autoantibodies in human growth correlate positively with body dimensions

Christoph Haudum $^{1,2}$, Waldemar Minich ${ }^{3}$, Julia Münzker ${ }^{1}$, Andrea Groselj-Strele $^{4}$, Ewald Kolesnik ${ }^{5}$, Ines Mursic ${ }^{5}$, Christian

Schwiebert $^{3}$, Tim Welsink ${ }^{3}$, Albrecht Schmidt ${ }^{5}$, Thomas Pieber ${ }^{1,2}$, Lutz Schomburg $^{3}$, Barbara Obermayer-Pietsch ${ }^{1,2}$

${ }^{1}$ Division of Endocrinology and Diabetology, Department of Internal Medicine, Medical University Graz, Graz, Austria, ${ }^{2} \mathrm{CBmed}$ GmbH Center for Biomarker Research in Medicine, Graz, Austria, ${ }^{3}$ Institut für Experimentelle Endokrinologie, Charité-Universitätsmedizin, Berlin, Germany, ${ }^{4}$ Center for Medical Research, Core Facility Computational Bioanalytics, Medical University of Graz, Graz, Austria, ${ }^{5}$ Division of Cardiology, Department of Internal Medicine, Medical University of Graz, Graz, Austria

Objective: Insulin-like growth factor 1 (IGF1) influences bone growth. Its receptor (IGF1R) has been shown to be involved in functional pathologies of body dimensions (e.g. in the Laron syndrome). We specifically designed an autoantibody (aAb) assay against the IGF1R, which is able to detect IGF1R-aAb in serum. We aimed to investigate the role of IGF1R-aAb in a large populationbased cohort of middle-aged volunteers and their potential effects on bone and anthropometric characteristics.

Methods: Biochemical and clinical parameters of 966 volunteers (55\% female and $45 \%$ male) of the BioPersMed cohort (Biomarkers in Personalized Medicine, Medical University Graz) were analysed to identify putative differences between IGF1R-aAb positive (binding index higher than 3 times background) and control subjects. Laboratory data in combination with DXA-derived measurements of bone density, dimensions and body composition were calculated in relation to increased IGF1R-aAb detection. Unpaired 2 tailed T-test was used to test for statistical significance.

Results: IGF1R-aAbs were identified in $6.0 \%$ of all volunteers ( $7.6 \%$ in females and $5.2 \%$ in males). IGF1R-aAb positive volunteers showed significantly higher bone density $(\mathrm{p}=0.001)$, different body composition $(\mathrm{p}=0.012)$ and a significant increase in body height $[+4.1 \mathrm{~cm}$ in men $(\mathrm{p}=0.002)$ and $+1.6 \mathrm{~cm}$ in women $(\mathrm{p}=0.1)]$, with a more pronounced effect in men. There was no difference in age or BMI between the groups. Mean age of the cohort was $57.7 \mathrm{y} \pm 8.1$ in women and $58.4 \mathrm{y} \pm 9.0$ in men.

Summary and Conclusions: With a considerable prevalence in our population-based cohort, IGF1R-aAbs may affect body height and dimensions by modulating bone growth. However, regulation, origin and relation to metabolic and hormonal pathways remain to be clarified. Therefore, research and monitoring of IGF1R-aAbs before puberty and during lifetime is a compelling approach to elucidate the mechanisms around IGF1R-aAbs and their potential effects.

\section{P136}

Increased Midkine serum levels in female fracture patients after menopause negatively affect osteogenic differentiation

Verena Fischer $^{1,2}$, Miriam Kalbitz ${ }^{2,3}$, Florian Gebhard ${ }^{2,3}$, Anita Ignatius $^{1,2}$, Astrid Liedert ${ }^{1,2}$, Melanie Haffner-Luntzer ${ }^{1,2}$ '

${ }^{1}$ Institute of Orthopedic Research and Biomechanics, Ulm University, Ulm, Germany, ${ }^{2}$ Center for Trauma Research Ulm (ZTF), Ulm University, Ulm, Germany, ${ }^{3}$ Department of Traumatology, Hand-, Plastic-, and Reconstructive Surgery, University Medical Center Ulm, Ulm, Germany

Impaired bone repair is frequently observed in osteoporotic patients. Pathomechanisms are still poorly understood. We found increased levels of the pro-inflammatory cytokines Midkine (Mdk) and Interleukin-6 (IL-6) in the serum and fracture hematoma of ovariectomized mice, indicating an increased inflammatory response contributing to disturbed bone repair. Here, we proofed clinical relevance by investigating Mdk and IL-6 in male and female fracture patients, and effects of fracture sera on osteogenic differentiation in vitro.

Serum Mdk and IL-6 levels were measured in 23 patients with isolated long-bone fracture at the day of surgery (d0), d14 and d42. Sub-group analysis was performed between age-matched men $(n=6)$ and women after menopause $(n=16) .26$ healthy donors served as controls. In vitro, human mesenchymal stem cells (MSC) underwent osteogenic differentiation for 10 days with $10 \%$ serum from controls or male/female patients $(n=6-10)$. Half of cells were incubated with an inhibitory Mdk-antibody (Mdk-Ab). Osteogenic differentiation was evaluated by qPCR-analysis and AP-staining. Depending on Shaphiro-Wilk-Normality-test: Student's $t$ test/Kruskal-Wallis test/ ANOVA/Fisher's-LSD, $\mathrm{p}<0.05$.

At all time points, fracture patients displayed significantly increased Mdk and IL-6 levels compared to controls ( $p<0.05$ ). Mdk, but not IL-6 levels, were significantly higher in female patients 
compared to males $(\mathrm{p}=0.03)$. In vitro, Bglap expression was significantly reduced in MSCs cultured with male or female serum compared to controls ( $\mathrm{p}<0.001)$. Alpl $(2.41 \pm 1.26$ vs. $4.24 \pm 3.54)$ and Bglap $(0.123 \pm 0.01$ vs. $0.162 \pm 0.06)$ expression were reduced comparing female to male sera. Mdk- $\mathrm{Ab}$ treatment significantly increased Alpl $(\mathrm{p}=0.0161)$ and Bglap $(\mathrm{p}=0.0201)$ expression in MSCs cultured with female sera. Mdk-Ab effect was not visible in males. This was confirmed by AP-staining.

Female patients after menopause displayed increased Mdk serum levels after fracture. Because Mdk is a negative regulator of bone formation, this could explain compromised bone repair. Confirming, osteogenic differentiation of MSCs incubated with serum from female patients was increased after Mdk-Ab treatment.

\section{P137}

Populations of colony-forming mesenchymal stromal cells are tolerant to antimicrobial silver ion concentrations by activation of the glutathione antioxidant pathway

Paul Souter $^{1}$, James Dodd ${ }^{2}$, Katie Blackwell ${ }^{2}$, Emma Tozer $^{2}$, Mike Hall $^{2}$, Jim Cunningham ${ }^{2}$, Darren Wilson ${ }^{2}$, Alan Horner ${ }^{2}$, Paul Genever $^{1}$

${ }^{1}$ Department of Biology, University of York, York, United Kingdom, ${ }^{2}$ Smith \& Nephew plc, Hull, United Kingdom

Orthopaedic trauma infection impacts patient wellbeing, healing rates and increases healthcare costs, therefore lowering the risk of implant infection is of benefit to all. The prevention of bacterial biofilm formation on the implant surface is a target to achieve clinical results, with the use of silver $(\mathrm{Ag}+)$ receiving increased attention. It was therefore the aim of this study to determine the effects of clinically relevant $\mathrm{Ag}+$ concentrations on the mesenchymal stromal cell (MSC) population and fracture healing. In vivo data revealed a rapid elution of $\mathrm{Ag}+$ from an intramedullary implanted pin, providing the potential to deliver a maximal intramedullary concentration $(7.8 \mu \mathrm{M})$ below that determined as toxic to MSCs in our in vitro viability test $\left(\mathrm{EC}_{50}=33 \mu \mathrm{M}\right)$. Released $\mathrm{Ag}+$ did not reside in either the plasma or femur at 28 days. MSC viability and proliferation was unaffected at the sub-toxic concentration of $10 \mu \mathrm{M} \mathrm{Ag}+$. At clonal density, the CFU-f capacity of MSCs was significantly reduced in the presence of $\mathrm{Ag}+$ as measured by CFU-f number $(10 \mu \mathrm{M} \mathrm{Ag}+, p<0.01)$, an observation that was replicated using CFU-Ob $(10 \mu \mathrm{M} \mathrm{Ag+,}$ $p<0.01$ ), suggesting however that a subpopulation of MSCs were able to survive and derive colonies from single cells. At a molecular level, surviving CFU-f demonstrated a significant upregulation of oxidative stress response genes. By qPCR, 20 genes demonstrated mean upregulation greater than twofold; of these, five showed significance compared to control (GCLM, NQO1, SQSTM1, TXN, TXNRD1, $p<0.05)$. There was also a twofold upregulation of CCL5, SRXN1, GPX3, GPX6, FTH1 and UCP2 $(p>0.05)$. Furthermore, a reduction in CFU-f number was observed at $5 \mu \mathrm{M} \mathrm{Ag}+$ when glutathione was blocked with BSO. These data have identified a population of MSCs that survive through activation of the glutathione antioxidant pathway and generate CFU-f under clinically relevant concentrations of silver.

Keywords: MSC, silver, glutathione

\section{P138}

IL-4 strongly inhibits osteogenic differentiation of human adipose stem cells under hypoxia

Angela Bastidas-Coral ${ }^{1}$, Jolanda Hogervorst ${ }^{1}$, Tim Forouzanfar ${ }^{2}$, Jenneke Klein-Nulend $^{1}$, Astrid Bakker ${ }^{1}$
${ }^{1}$ Oral Cell Biology, ACTA, UvA and VU, Amsterdam, Netherlands, ${ }^{2}$ Oral and Maxillofacial Surgery, VU University Medical Center, Amsterdam, Netherlands

The cytokines IL- 4 and IL- 6 are expressed in the first days of bone healing, when hypoxic conditions prevail. Hypoxia- and cytokineactivated pathways (e.g. mTORC1) may interact in mesenchymal stem cells (MSCs). To better predict the effects of cytokines on MSCaided bone healing, we investigated whether IL-4, IL-6, hypoxia, and their combination affect osteogenic differentiation and VEGF production by MSCs via the mTORC1 pathway.

Human adipose stem cells (hASCs) were cultured with IL-4, IL-6 ( 1 or $10 \mathrm{ng} / \mathrm{ml})$, and the combination thereof $(10 \mathrm{ng} / \mathrm{ml})$ for 3 days, under normoxia $\left(20 \% \mathrm{O}_{2}\right)$ and hypoxia $\left(1 \% \mathrm{O}_{2}\right)$, followed by 11 days of culture without cytokines under normoxia and hypoxia. Osteogenic differentiation was assessed by gene expression (qPCR), alkaline phosphatase (ALP) activity, and bone nodule formation (alizarin red). VEGF protein was measured by ELISA, and mTORC1 activation by western blot of pP70S6k. Only significant results $(\mathrm{p}<0.05)$ are reported.

Under hypoxia, IL-4 (10 ng/ml) decreased RUNX2 expression by 3.5- and 5.3-fold at day 2 and 7 respectively. At day 7, it decreased COL1 expression by 3.6-fold, ALP activity by 13.3 -fold, and VEGF protein by 2.0 -fold. It reduced bone nodule formation at day 14 . Under hypoxic conditions, IL-6 (10 ng/ml) increased RUNX2 by 3.2fold at day 2, while under normoxia it also enhanced bone nodule formation. The combination of IL- 4 and IL- 6 enhanced bone nodule formation under normoxia only, and ALP activity and VEGF expression under hypoxia only. The only condition enhancing phosphorylation of P70S6K in ASCs was IL-4 in combination with IL-6 when applied under hypoxia.

In conclusion, the effects of IL-4 and/or IL-6 on ASCs strongly depend on oxygen tension. IL-4 reduces osteogenic differentiation of ASCs under hypoxia, indicating that this cytokine inhibits bone healing and regeneration. These effects of IL-4 do not seem to be mediated via the mTORC1 pathway.

\section{P139}

Management of femoral fractures in an orthogeriatric ward $\underline{\text { Ferdinando D'Amico }}^{1}$, Rosetta Grasso ${ }^{2}$

${ }^{1}$ Geriatrics, Hospital of Patti - University of Messina, Patti, Italy, ${ }^{2}$ Geriatrics, Hospital of Patti, Patti, Italy

Objective: The Department of Geriatrics has recently created an integrated service of Orthogeriatrics where patients with femoral fractures are monitored from admittance to E.R. through Orthopedics, Geriatrics and Long-term-care Unit. In a 12-month time span 107 patients with femoral fractures have accessed this service. They have been assessed through a multidimensional examination including MMSE, CIRS, Barthel Index, MNA. 27 out of 97 needed pre-surgery treatment, 16 couldn't access surgery due to severe clinical conditions detected via CIRS (Severity index $>3$ and Complex Comorbidity index $\leq 4)$. Prior to the femoral fracture the Barthel Index showed an average score $\pm 70 / 100$. MNA score was $<24$. Standing position recovery started 3 days after prosthesis for 73 patients with femoral medial fracture, dismissed after a 15/25-day hospitalization. Elderly subjects recovering from osteosynthesis have regained the sitting position in 2-3 days and left the unit some 30/45 days after admittance. At dismissal 81 patients were prescribed drugs (Biphosphonate-Denosumab-Teriparatide) for the secondary prevention of osteoporosis.

Results: Among 81 subjetcs receiving surgery, those showing at admittance MMSE > 21/30 (38\%), CIRS > 3 (75\%), Barthel Index prior to trauma $<50(20 \%)$, MNA $<23(58 \%)$ determined a better outcome in terms of hospitalization length, efficiency of recovery and 
reduction of functional impairment. Those with extra effects like bedsores, malnutrition scores like albumina $<3 \mathrm{~g} / \mathrm{l}$, anemia $\mathrm{Hb}<$ $11 \mathrm{~g} / \mathrm{dL}$, transferrin $<150 \mathrm{~g} / \mathrm{dL}$, lymphocytes $<1200 \mathrm{~mm} 37 / \mathrm{UL}$ and infections contracted in hospital had a worse outcome (10-day longer hospitalization).

Conclusion: Successful surgery and functional recovery in elderly patients with femoral fracture are connected with: (1) general, functional, nutritional conditions prior to fracture; (2) comorbidity. There is also a relationship with severity and comorbidity CIRS indexes, malnutrition and mortality in acute phase (5\%). Considering the Barthel Index prior to trauma, the overall outcome was to reestablish the functional condition before the fracture.

\section{P140}

Growth rate of the tibia after implantation of selenium enhanced hydroxyapatite into it

Oleg Meriuts $^{1}$, Vladyslav Luzin ${ }^{1}$, Dmitry Astrakhantsev ${ }^{1}$, Ilya Prikhodchenko ${ }^{1}$

${ }^{1}$ LPR SE 'St. Luke State Medical University of Lugansk', Lugansk, Ukraine

Objectives: The study is aimed at testing of growth rate of the tibia after implantation of selenium enhanced hydroxyapatite into it.

Materials and Methods: The study involved 252 male rats with body weight of 135-145 g. The 1st group comprised intact animals, the 2 nd group comprised animals with $2.2 \mathrm{~mm}$ defect in the tibia, and the groups 3 through 6 comprised the animals with the same $2.2 \mathrm{~mm}$ defects filled with biogenic hydroxyapatite OK-015 enhanced with $0.00,0.15,0.3$, and $0.5 \%$ share of selenium. Upon expiration of observation terms (the 7 th, the 15 th, the 30 th, the 60 th, the 90 th, and the 180th day), tibiae were prepared for gross measurements.

Results: A plain defect in the bone is manifested with acceleration of longitudinal and appositional growth rate with peak on the 30th day. Implantation of pure hydroxyapatite resulted in acceleration of appositional growth rate as compared to the 2 nd group in the period from the 15th up to the 60th day. Selenium in the implants seems to reduce these effects. Mid-shaft width and thickness of the bones filled with $0.15 \%$ Se implants diminished as compared to the 3rd group by 7.47 and $5.82 \%$, and 4.55 and $6.38 \%$ by the 15 th and the 30th days respectively. For $0.3 \%$ Se implants the same measurements dropped by 9.77 and $9.13 \%$, and by 8.59 and $9.36 \%$ in the same terms. Finally, the same measurements of the bones with $0.5 \mathrm{Se}$ implants decreased by $11.49,11.97$, and $4.57 \%$ and by $10.61,14.47$, and $9.18 \%$ in the period from the 15 th up to the 60th day respectively.

Conclusions: Implantation of selenium enhanced hydroxyapatite reduces appositional bone growth rate related to formation of bone cuff around fracture point. The highest efficacy is yielded from the implants with $0.5 \%$ share of selenium.

Keywords: bone defect, hydroxyapatite, implantation, selenium

\section{P141}

Profiling osteogenic and angiogenic markers in cells engaged in early alveolar/mandibular bone formation

Jan Bobek ${ }^{1}$, Barbora Veselá ${ }^{1}$, Eva Švandová ${ }^{1}$, Hervé Lesot $^{1}$, Eva Matalová $^{1}$

${ }^{1}$ Institute of Animal Physiology and Genetics, Czech Academy of Sciences, Brno, Czech Republic

Challenging repair/regeneration issues in the facial region are related to nasal and mandibular bones. Mandibular/alveolar bone regeneration is related to dental implantology, when artificial implants need to be fixed in a solid bone structure. Better knowledge in the physiological development of this structure is a prerequisite for successful targeted therapies.

Condensation of pre-osteogenic cells, the first cellular event involved in initiation of intramembranous osteogenesis, has been widely studied. Less attention has been paid to the post-condensation signalling which take place when the mesenchymal anlage transforms into the initial complex bone structure.

This work aimed to investigate molecular background for both vascularization and osteogenesis at early stages of mandibular/alveolar bone formation. Comparing the situation at prenatal day 13 in the mouse lower jaw, when mesenchymal condensation becomes morphologically, and day 15, when the first bone cells differentiate was performed using osteogenic and angiogenic PCR-Arrays. Profiling the expression of 84 marker genes was performed.

Massive increase in osteogenic gene expression was documented for osteopontin, osteocalcin and Sost. Prominent changes were observed also for Bmp3, Col1a1, Col1a2, Col10a1, Ctsk, Mmp10, Phex and Vdr. Significantly altered expression was detected for 13 other osteogenic markers. The angiogenic array showed prominent changes in the expression of Itgb3, Fgf1, Cxcl1 and Mmp9. Significant differences were detected for Ctgf, Serpinf1, Angpt2, Epas1, Edn1, Vegfb, Thbs1, Sphk1, Mdk.

The simultaneous angiogenic and osteogenic profile indicates some novel interactions which may be critical for the post-condensation stage of intramembranous osteogenesis. Some of the molecules have not been even connected with the intramembranous bone. In general, these results illustrated molecular dynamics during the early stages of the alveolar/mandibular bone formation after mesenchymal condensation and point to the most prominent markers engaged in the onset of osteogenesis and vascularization during intramembranous ossification.

Supported by the Grant Agency of the Czech Republic 17-14886.

\section{P142}

Bone structural changes on neonatally thymectomized rats: a histomorphometric study

Wagner Garcez de Mello ${ }^{1,2}$, Noélle Egídia Watanabe Kiil ${ }^{2}$, Gisele Mitsui Kayahara ${ }^{3}$, Olívia Borghi Nascimento ${ }^{1}$, Narayana Guimarães Marqui ${ }^{1}$, Samuel Rodrigues Lourenço de Morais ${ }^{2}$, Marcelo Macedo Crivelini $^{3}$, João Cesar Bedran de Castro ${ }^{2}$

${ }^{1}$ Centro Universitário Toledo-UNITOLEDO, Araçatuba, Brazil, ${ }^{2}$ Department of Basic Sciences, School of Dentistry, Univ. Estadual Paulista-UNESP, Araçatuba, Brazil, ${ }^{3}$ Department of Pathology and Clinical Propaedeutic, School of Dentistry, Univ. Estadual PaulistaUNESP, Araçatuba, Brazil

This study evaluates the effects of neonatal thymectomy on skeletal sexual dimorphism and bone structural in prepubertal, puberty and adulthood rats. For this, Wistar newborns were divided into control and thymectomized groups, of both sexes, totaling four experimental groups ( $n=08$ per group). The animals were thymectomized at the third postnatal day, and euthanized at 20,40 and 120 days' postnatal life, corresponding respectively to the prepuberty, puberty and adulthood periods. Right femurs were collected, and the base of the femoral neck was used to quantitative histometric analysis. All experiments were approved by the Ethics Committee for Animal Research of the Univ. Estadual Paulista, Araçatuba, São Paulo, Brazil (CEAA-FOA/UNESP-2012-01081). Taken together, the data of the present study demonstrates that the animals belong to the control group presented sexually dimorphic differences regards to skeletal development, there during the prepubertal period but are consolidated after puberty and Thymus gland modulates, in part, these responses. At 120 days, the total bone area, which represents only a mineralized region of the tissue, was higher in control males when 
compared to the other groups, showing that both treatment $(p=0.0572)$ and sex $(p=0.0464)$, influenced the response, but without significant interaction between variables $(p=0.1995)$. The cortical region area showed statistically significant interaction $(\mathrm{p}=0.0075)$, where the cortical area of males control group was greater than control females group. We also observed a larger medullary space area in the male groups $(p=0.0018)$ compared to females groups, clearly demonstrating the sex influence on this bone specific area. Our results present that the neonatal thymectomy causes changes sex- and time-dependent on structural properties of bone tissue. These findings provide new insight into the dynamic complexity of bone homeostasis and suggest that the presence of Thymus during the perinatal period is important for normal skeletal development.

\section{P143}

Macroelemental composition of tibial proximal epiphysis in rats after 60-day application of sodium benzoate and formation of defect in tibia

Olga Fastova $^{1}$, Vladyslav Luzin ${ }^{1}$, Vitaly Morozov $^{2}$, Helen Morozova $^{2}$, Nadezhda Mosyagina ${ }^{1}$

${ }^{1}$ LPR SE “St. Luke State Medical University of Lugansk”, Lugansk, Ukraine, ${ }^{2}$ Belgorod National Research University, Belgorod, Russian Federation

Objectives: The aim of the study was to test chemical composition of tibial proximal epiphysis (TBE) in rats after 60-day sodium benzoate (SB) intake nd formation of defect in tibia.

Materials and Methods: The experiment involved 210 male rats with body weight of $200-210 \mathrm{~g}$. The 1 st group comprised animals that received daily per os $1 \mathrm{ml}$ of $0.9 \%$ solution of $\mathrm{NaCl}$, the 2nd and the 3rd groups received per os $1 \mathrm{ml}$ of SB in dosage of 500 or $1000 \mathrm{mg}$ per $\mathrm{kg}$ of body weight daily, the 4th group (D) comprised animals with defects in both tibiae made after SB was discontinued in groups 2 and 3 . The 5th and the 6th groups (DSB1 and DSB2) comprised the rats that received $\mathrm{SB}$ and had defects in tibiae also made after SB discontinue. Observation terms constituted 3, 10, 15, 24 , and 45 days after termination of experimental influences. Upon expiration of observation terms, TBE were prepared for chemical analysis.

Results: In DSB1, mineral share in TBE was lower than that of D on the 15 th day by $3.79 \%$, the Ca share by the $3 \mathrm{rd}$, the 15 th and the 24th days-by $4.32,4.68$ and $4.66 \%$ and $\mathrm{Ca} / \mathrm{P}$ by the 24 th day-by $5.30 \%$ (here and below $\mathrm{p}<0.05$ in all cases).

In DSB2, mineral share and $\mathrm{Ca} / \mathrm{P}$ in TBE were lower than that of D group from the 10th to the 45th days by 3.90, 4.28, 4.89, 3.87 and $3.66 \%$ and $5.25,7.52,4.78,9.96$ and $3.53 \%$. Ca share was lower than that of the controls from the 3rd through the 24th days by $6.38,5.34$, 6.86 and $6.97 \%$.

Conclusions: SB intake has adverse effects on macroelemental composition of TBE; intensity and duration of alterations depend on dosage of SB.

Keywords: bone, regeneration, sodium benzoate

\section{P144}

KIAA1199, a novel secreted factor of stromal (skeletal) stem cells that promotes adipogenesis and cellular cross-talk in bone marrow microenvironment

$\mathrm{Li} \mathrm{Chen}^{1}$, Kaikai Shi ${ }^{1}$, Weimin Qiu ${ }^{1}$, Greet Kerckhofs ${ }^{2}$, Thomas Levin Geiser Andersen $^{3}$, Moustapha Kassem ${ }^{1,4}$
${ }^{1}$ Department of Endocrinology and Metabolism, Endocrine Research Laboratory (KMEB), Odense University Hospital, Southern Denmark University, Odense, Denmark, ${ }^{2}$ Biomechanics Lab - Institute of Mechanics, Materials and Civil Engineering, UCLouvain, Louvainla-Neuve, Belgium, ${ }^{3}$ Department of Clinical Cell Biology (KCB), Institute of Regional Health Research, University of Southern Denmark, Odense, Denmark, ${ }^{4}$ The Danish Stem Cell Center (DanStem), University of Copenhagen, Copenhagen, Denmark

Objective: Bone marrow microenvironment contains many factors that maintain dynamic equilibrium between adipogenesis and osteogenesis needed for bone remodeling. In a quantitative proteomic screen, we identified KIAA1199 as a novel secreted factor in human bone marrow stromal (skeletal) stem cells (hMSC) during osteoblast differentiation. In the present study, we investigated the role of KIAA1199 in bone marrow adipocyte (AD) differentiation.

Methods: Production of KIAA1199 in human bone marrow was detected using in situ-hybridization. The effect of KIAA1199 on hMSC adipocyte differentiation was examined in hMSC that overexpress (hMSC-KIAA1199 ${ }^{\text {over }}$ ) (created by lentiviral gene transfer) or knockdown KIAA1199 (hMSC-KIAA1199 ${ }^{\text {down }}$ ) created by siRNA-mediated knock-down. Global gene expression analysis was performed by DNA microarrays. RT-qPCR, Western-blotting analysis and Oil Red O Staining were employed to examine at gene expression, protein level, signaling transformation and adipogenesis; molecular cloning and luciferase-reporter assay were used for promoter analysis.

Results: KIAA1199 is expressed and secreted by bone marrow cells, and reduced the expression and secretion levels during $\mathrm{AD}$ differentiation. hMSC-KIAA1199 ${ }^{\text {down }}$ exhibited deficient differentiation to AD differentiation, while hMSC-KIAA $1199^{\text {over }}$ enhanced AD differentiation as evidenced by quantitative Nile-Red staining of mature adipocytes and adipocyte gene expression analysis. Global gene expression analysis identified osteopontin (OPN) as one of the most regulated genes by KIAA1199 in hMSC. siRNA-mediated depletion of OPN or blocking its putative receptor integrin $\beta 1$ rescued the AD differentiation in hMSC-KIAA1199 ${ }^{\text {down }}$, and this effect was mediated through AKT and ERK MAP kinase signaling. Analysis of different deletions of OPN promoter revealed that SP1 transcription factor is the major element that mediated KIAA1199-mediated OPN transcription.

Conclusion: KIAA1199 is an example of a previously unidentified factor with novel function regulating bone marrow adipogenesis. Targeting osteopontin suggest that KIAA1199 plays a role in crosstalk of adipocytes, osteoblast and osteoclast in the bone marrow microenvironment.

Keywords: KIAA1199, secretion, bone marrow, adipocyte differentiation, osteopontin

\section{P145}

Bone marrow mesenchymal stem cell implantation for osteonecrosis of femoral head. A matched pair control study

Joon-Soon Kang ${ }^{1}$, Young-Sook Park ${ }^{2}$, Jun-Sung Park ${ }^{1}$

${ }^{1}$ Inha University Hospital, Incheon, Republic of Korea, ${ }^{2}$ Eulji Medical University Hospital, Seoul, Republic of Korea

To date, several trials have reported the use of bone marrow mesenchymal stem cells (BMMSC) implantation in osteonecrosis of femoral head (ONFH). However, the clinical outcomes were not conclusive. This study was designed to compare clinical and radiological results of stem cell implantation with traditional simple core decompression $(\mathrm{CD})$.

Method: We retrospectively studied 147 patients (172 hips) who underwent $\mathrm{CD}$ or BMMSC implantation after diagnosis of ONFH from 2004 to October 2014. Retrospectively matched pair comparison was done for 54 hips undergoing traditional core decompression (Group I) with 118 
hips having cell therapy (Group II). There were 112 men and 35 women. The mean age was 47.4 years at the time of surgery and the mean follow up period was 6.7 years (5-10 years). All cases were matched by age, stage, cause of osteonecrosis. Radiologically, the degree of deterioration of the ARCO stage was compared, and the Total hip arthroplasty (THA) conversion rate and VAS score were compared clinically.

Results: The percentage of good clinical results was $37 \%$ in Group I and 39\% in Group II. Conversion arthroplasty was performed in $50 \%$ in group I and $24 \%$ in group II. When analyzed by stage, the THA conversion rate was significantly lower in the BMMSC group in stage 2 ( $\mathrm{p}$ value $=0.02)$, but in stage 3 and 4 , the THA conversion rate did not differ between $\mathrm{CD}$ and BMMSC implantation. Based on the stage the extent of the deterioration of the ARCO stage did not differ between the two groups.

Conclusion: Imaging deterioration was the same in both groups, but the conversion rate to artificial joint surgery was less in the cell therapy group. This study suggests the possibility of bone marrow cell therapy in early stage of osteonecrosis.

\section{P146}

Dissociation of the inverse relationship of marrow adipocytes and osteoblasts in burns

Amina El Ayadi ${ }^{1}$, Ron Helderman ${ }^{2}$, David Herndon ${ }^{1}$, Celeste C Finnerty $^{1}$, Clifford J Rosen ${ }^{2}$, Gordon L Klein ${ }^{3}$

${ }^{1}$ Surgery, University of Texas Medical Branch and Shriners Burns Hospital, Galveston, United States, ${ }^{2}$ Maine Medical Center Research Institute, Scarborough, United States, ${ }^{3}$ Orthopaedic Surgery and Rehabilitation, University of Texas Medical Branch and Shriners Burns Hospital, Galveston, United States

Frequent reports describe an inverse relationship between marrow adipocytes and osteoblasts in conditions such as osteoporosis and osteopenia. The nature of the reciprocity has yet to be clarified but the question arises as to whether this relationship persists in other conditions, which would verify its physiologic importance. We examined the marrow adipocytes and serum osteocalcin concentration as a marker of osteoblastogenesis in burned rats to assess the reciprocity of the relationship in this condition. 8 wk-old Sprague Dawley rats received a $60 \%$ scald burn under isoflurane anesthesia and were sacrificed on day 4 postburn. 3 burned and 3 control rats had tibias removed, placed in 10\% formalin, transferred to PBS, decalcified, and had marrow adipocytes quantified. Osteocalcin was measured in serum $(\mathrm{n}=15$ burns, $\mathrm{n}=12$ controls) using a BGLAP ELISA kit (Aviva Biosystems, San Diego). Marrow stromal cells were stained for FOXO3a on day 1 post-burn.

Results: Adipocyte counts per $\mathrm{mm}^{2}=173.3 \pm 30.4$ (SD, control) vs. $55.7 \pm 21.8$ (burn), $\mathrm{p}=0.0055$. Serum osteocalcin was significantly lower in burns than controls, $4.53 \pm 1.93$ vs. $10.96 \pm 1.93 \mathrm{ng} /$ $\mathrm{ml}, \mathrm{p}<0.001$. Stain of the marrow stromal cells for FOXO3a in the nuclei ( $\mathrm{n}=5$ per group) revealed no differences between groups on day 1 post-burn. These data indicate that any inverse relationship between adipocytes and osteoblasts in the bone marrow are overridden by the massive energy requirements of the body post-burn and the massive inflammatory response could in itself explain the reduction of osteocalcin by the action of pro-inflammatory cytokines.

\section{P147}

Inhibition of $1,25(\mathrm{OH})_{2} \mathrm{D}_{3}$-enhanced intestinal calcium transport after exposure to high apical calcium is mediated by calcium-sensing receptor and fibroblast growth factor-23

Narattaphol Charoenphandhu ${ }^{1,2,3}$, Mayuree Rodrat ${ }^{1,2}$, Kannikar Wongdee $^{2,4}$
${ }^{1}$ Department of Physiology, Faculty of Science, Mahidol University, Bangkok, Thailand, ${ }^{2}$ Center of Calcium and Bone Research, Faculty of Science, Mahidol University, Bangkok, Thailand, ${ }^{3}$ Institute of Molecular Biosciences, Mahidol University, Nakhon Pathom, Thailand, ${ }^{4}$ Faculty of Allied Health Sciences, Burapha University, Chonburi, Thailand

Chronic exposure to high apical calcium concentration has been shown to diminish the 1,25-dihydroxyvitamin $\mathrm{D}_{3}\left[1,25(\mathrm{OH})_{2} \mathrm{D}_{3}\right]$-induced calcium absorption across the small intestinal epithelium, presumably to help prevent excessive calcium uptake. However, the underlying mechanism by which luminal calcium inhibits calcium absorption remains elusive. We hypothesized that the luminal calcium may be sensed by the apical calcium-sensing receptor (CaSR) and fibroblast growth factor (FGF)-23, which has been reported to antagonize several actions of $1,25(\mathrm{OH})_{2} \mathrm{D}_{3}$, may be an inhibitory paracrine mediator. Herein, we aimed to determine the in vitro transepithelial calcium transport across the intestinal epithelium-like Caco-2 monolayer exposed to $1,25(\mathrm{OH})_{2} \mathrm{D}_{3}$ and various concentrations of apical calcium (i.e., $10-30 \mathrm{mM} \mathrm{Ca}^{2+}$ ) or CaSR activator (cinacalcet). By using Ussing chamber technique and radioactive tracer, we found that exposure to high apical calcium diminished the $1,25(\mathrm{OH})_{2} \mathrm{D}_{3}$-induced calcium transport in a concentration-dependent manner $(P<0.05)$. This inhibitory effect of apical calcium could be mimicked by allosteric CaSR activator [i.e., transepithelial calcium fluxes of $20.00 \pm 2.78 \mathrm{nmol} / \mathrm{h} / \mathrm{cm}^{2}$ in $10 \mathrm{nM} 1,25(\mathrm{OH})_{2} \mathrm{D}_{3}$ group vs. $14.99 \pm 3.72$ and $13.85 \pm 2.54 \mathrm{nmol} / \mathrm{h} / \mathrm{cm}^{2}$ in 5 and $10 \mu \mathrm{M}$ cinacalcet groups, respectively; $P<0.05$ ], and was completely reversed by CaSR inhibitor (i.e., $2 \mu \mathrm{M}$ NPS2143). High apical calcium was found to upregulate the expression of FGF23 protein, which was later secreted into both apical and basolateral compartments. Neutralization of FGF23 in the apical compartment by specific FGF23 antibody successfully prevented the negative effect of apical calcium on $1,25(\mathrm{OH})_{2} \mathrm{D}_{3}$-induced calcium transport. In conclusion, CaSR and local production of FGF23 contributed to negative feedback regulation of $1,25(\mathrm{OH})_{2} \mathrm{D}_{3}$-enhanced calcium transport, and may help prevent superfluous calcium uptake as well as its hazardous consequences.

Keywords: Calcium transport; calcium-sensing receptor; cinacalcet; fibroblast growth factor-23; vitamin D

\section{P148}

Impairment of intestinal calcium absorption, bone mineral density and mechanical property in spontaneous hydronephrotic mice

Panan Suntornsaratoon $^{1,2}$, Nithipak Thammayon ${ }^{1,2}$, Narattaphol Charoenphandhu ${ }^{1,2,3}$

${ }^{1}$ Department of Physiology, Faculty of Science, Mahidol University, Bangkok, Thailand, ${ }^{2}$ Center of Calcium and Bone Research, Faculty of Science, Mahidol University, Bangkok, Thailand, ${ }^{3}$ Institute of Molecular Biosciences, Mahidol University, Nakhon Pathom, Thailand

Hydronephrosis is a distention of the renal calyceal system and pelvis with build-up urine and swelling. Kidney impairment may then lead to impairment of calcium and phosphate homeostasis and bone mineralization, all of which are under a regulation of circulating $1,25(\mathrm{OH})_{2} \mathrm{D}_{3}$ that is secreted by the renal proximal tubular cells. This study aimed to investigate intestinal calcium absorption, bone mechanical property, and alterations of calcium regulating-hormones in hydronephrosis. Spontaneous male hydronephrotic mice (ICR/ Mlac-hydro) ages of 1, 2, 3 and 6 months old were used. This work was approved by the Animal Care and Use Committee of the Faculty of Science, Mahidol University. Blood was collected for analyses of blood chemistry, levels of bone turnover markers, PTH, 1,25(OH $)_{2} \mathrm{D}_{3}$ and fibroblast growth factor-23 (FGF-23). Femora were collected for 
bone mineral density (BMD) and mechanical property analyses. Mlac-hydro mice had lower circulating PTH, $1,25(\mathrm{OH})_{2} \mathrm{D}_{3}$ and FGF23 while serum phosphate increased. Blood urea nitrogen/creatinine ratio was higher in 6-month Mlac-hydro mice, suggesting abnormal kidney function. Ussing chamber technique showed 3- and 6-monthold Mlac-hydro mice had lower duodenal calcium transport compared with control ( $11.58 \pm 1.76$ vs. $6.15 \pm 1.05$ in 3 months old $P<0.05$; $9.19 \pm 0.88$ vs. $5.00 \pm 0.95$ in 6 months old $P<0.01$ ). BMD analysis by micro-CT showed no change in 1-month old but decrease in $\mathrm{BMD}$ in 2, 3 and 6 months old. Impairment of bone mechanical property was observed in 3 and 6 months old with a reduction in maximum load, yield load and stiffness. Bone turnover markers analyses indicated low bone formation and resorption only in female, but not in male mice. Bone defects in females were similarly observed in male. In conclusion, hydronephrosis caused failure of $1,25(\mathrm{OH})_{2} \mathrm{D}_{3}$ synthesis, and hyperphosphatemia with hypoparathyroid. A reduction of $1,25(\mathrm{OH})_{2} \mathrm{D}_{3}$ level led to low intestinal calcium absorption and bone mineralization, which, in turn, impaired bone mechanical property.

\section{P149}

The effect of Vitamin D supplementation on plasma Non-oxidized PTH concentration

Stan Ursem ${ }^{1}$, Vito Francic ${ }^{2}$, Martin Gaksch ${ }^{3}$, Verena Schwetz ${ }^{2}$, Christian Trummer ${ }^{2}$, Marlene Pandis ${ }^{2}$, Felix Aberer ${ }^{2}$, Martin Grübler $^{2}$, Nicolas D. Verheyen ${ }^{4}$, Winfried März ${ }^{5}$, Thomas R. Pieber ${ }^{2}$, Andreas Tomaschitz ${ }^{4}$, Stefan Pilz ${ }^{2}$, Barbara Obermayer-Pietsch ${ }^{2}$, Annemieke C. Heijboer ${ }^{1,6}$

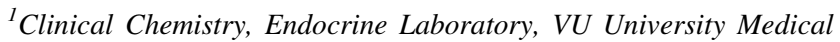
Center, Amsterdam, Netherlands, ${ }^{2}$ Division of Endocrinology and Diabetology, Medical University of Graz, Graz, Austria, ${ }^{3}$ Department of Laboratory Medicine, Paracelsus Medical University, Salzburg, Austria, ${ }^{4}$ Division of Cardiology, Medical University of Graz, Graz, Austria, ${ }^{5}$ Synlab Holding Germany GmbH, Synlab Academy, München, Germany, ${ }^{6}$ Department of Clinical Chemistry, Laboratory of Endocrinology, Academic Medical Center, Amsterdam, Netherlands

Background: PTH can be oxidized in vivo, rendering it biologically inactive. Non-oxidized PTH (n-ox PTH) may therefore give a better image of the hormonal status of the patient. Currently, PTH is measured by assays that do not discriminate between the oxidized and non-oxidized forms. While vitamin D supplementation decreases total PTH (tPTH) concentration, the effect on n-oxPTH concentration is unexplored. We aimed to investigate the effect of vitamin D supplementation on n-oxPTH concentration in comparison to TPTH and compare the correlations between parameters of calcium and phosphate metabolism with n-oxPTH and tPTH.

Methods: EDTA plasma samples of 108 vitamin D insufficient $(25(\mathrm{OH}) \mathrm{D}<75 \mathrm{nmol} / \mathrm{L})$ hypertensive patients who completed the Styrian Vitamin D Hypertension Trial (NCT02136771). Participants were treated with vitamin D (2800 IE daily) or placebo for 8 weeks. $\mathrm{N}$-oxPTH was measured using oxPTH affinity columns followed by 2nd generation PTH measurements in the eluate. ${ }^{1}$ We calculated the treatment effect and performed correlation analyses of n-oxPTH and tPTH concentrations at baseline with parameters of calcium and bone metabolism.

Results: After 8 weeks of vitamin D treatment, 25(OH)D concentrations increased, tPTH decreased by $9 \%$ and n-oxPTH by $7 \%$ (treatment effect: -0.90 and $-0.08 \mathrm{pmol} / \mathrm{L}$ ); $\mathrm{p}<0.001$ and $\mathrm{p}=0.025$, respectively. The percentage decrease, was not significant $(\mathrm{p}=0.51)$.
$\mathrm{N}$-oxPTH showed a significant correlation with PTH $(\mathrm{R}=0.555$, $\mathrm{P}<0.001)$ and osteocalcin (OC) $(\mathrm{r}=0.237, \mathrm{p}=0.014)$. tPTH and OC had no significant correlation $(r=0.108, p=0.268)$.

Conclusion: In hypertensive, vitamin $\mathrm{D}$ insufficient individuals with normal renal function, the extent of PTH oxidation differs individually. Both $\mathrm{TPTH}$ and n-oxPTH concentrations decreased (9 and $7 \%$, respectively) during vitamin $\mathrm{D}$ supplementation. Interestingly, only OC exhibited a significant correlation with n-oxPTH but not $\mathrm{PTTH}$, which remains to be explored.

${ }^{1}$ Ursem et al. CCLM, 2018

Keywords: parathyroid hormone, vitamin D, oxidative stress, boneturnover markers

\section{P150}

Bone and calcium metabolic changes during anti-resorptive treatment in early breast cancer

Victoria Gunmalm ${ }^{1}$, Anne Qvist Rasmussen ${ }^{1}$, Trine Lund-Jacobsen ${ }^{1}$, Michael Andersson ${ }^{2}$, Charlotte Brøns ${ }^{1}$, Peter Schwarz ${ }^{1}$

${ }^{1}$ Department of Endocrinology, Rigshospitalet, Copenhagen, Denmark, ${ }^{2}$ Department of Oncology, Rigshospitalet, Copenhagen, Denmark

Aromatase inhibitors (AI) increase bone mineral loss during adjuvant treatment for early breast cancer (EBC). Due to anti-neoplastic effect, zoledronic acid ( $\mathrm{Zol})$ is used in the adjuvant setting even though other anti-resorptive medications might be as bone effective.

We aimed to compare the bone and calcium metabolic effects of denosumab (Dmab) vs. Zol acid during initial 12 months of adjuvant AI treatment in EBC.

Method: Sixty EBC female patients who initiated adjuvant AI (oral Letrozol $2.5 \mathrm{mg}$ ) treatment were randomly included. Thirty patients were in oral Dmab $60 \mathrm{mg}$ and $30 \mathrm{in} \mathrm{Zol} 4 \mathrm{mg}$ iv. treatment every 6 months (mean age 66.7 (range 52-86) vs. 66.0 (range 52-78) years). Calcium metabolic measures, markers of bone turnover and DXA-scanning of spine and hip were performed.

Results: All patients had a T-score $<-1$ and $>-2.5$ at all sites at the beginning of therapy. No had suffered from a bone fracture. Baseline plasma (P) ionized calcium was $1.23 \pm 0.03$ vs. $1.23 \pm 0.04 \mathrm{mmol} / \mathrm{L}$ for the Dmab and the Zol group, respectively. Baseline P-PTH and P-vitamin D (25OHD) were within normal range for both groups. After 1 and 3 months of treatment, P-ionized calcium was $1.19 \pm 0.04$ vs. $1.19 \pm 0.06 \mathrm{mmol} / \mathrm{L}$, and $1.21 \pm 0.04$ vs. $1.23 \pm 0.05 \mathrm{mmol} / \mathrm{L}$ for the Dmab and Zol group, respectively. A corresponding P-PTH increase was observed 1 month after for both groups. P-ionized calcium and P-PTH remained normal in both groups at 6 and 12 months.

For the Dmab group P-CTX was suppressed from 1 month and hereafter vs. suppressed 70, 50, 17 and $20 \%$ at month 1, 3, 6 and 12 for the Zol group $(\mathrm{p}<0.001)$.

In Conclusion: We observed no difference in hypocalcemia and hyperparathyroidism during 12 months of treatment, despite a more pronounced suppression of the bone resorption marker P-CTX among Dmab treated EBC patients.

\section{P151}

LC-MS/MS based 25(OH)D status in a large central European outpatient cohort—gender and age specific differences

Markus Herrmann $^{1}$, Silvia Giuliani ${ }^{2}$, Verena Barbieri ${ }^{3}$, Angela Maria ${ }_{\text {Di Pierro }}^{3}$, Fabio Rossi ${ }^{3}$, Thomas Widmann ${ }^{4}$, Manuela Lucchiari ${ }^{3}$, Irene Pusceddu ${ }^{3}$, Stefan Pilz ${ }^{5}$, Barbara Obermayer-Pietsch ${ }^{5}$ 
${ }^{1}$ Clinical Institute for Medical and Chemical Laboratory Diagnostics, Medical University of Graz, Graz, Austria, ${ }^{2}$ Department of Clinical Pathology, Bolzano Hospital, Bolzano, Italy, ${ }^{3}$ Bolzano Hospital, Bolzano, Italy, ${ }^{4}$ Asklepios Clinic Triberg, Triberg, Germany, ${ }^{5}$ Department of Internal Medicine, Division of Endocrinology and Diabetology, Medical University of Graz, Graz, Austria

Background: Developed countries have a high prevalence of vitamin D deficiency. In previous studies $25(\mathrm{OH}) \mathrm{D}$ was predominantly measured by immunoassays, which are known for their variable analytical performance. The present study assessed serum $25(\mathrm{OH}) \mathrm{D}$ in a very large European outpatient cohort by liquid chromatography-tandem-mass-spectrometry (LC-MS/MS).

Material and Methods: 74,235 serum 25(OH)D results generated under routine conditions between 2015 and 2016 were extracted from the laboratory information system of the Department of Clinical Pathology at Bolzano Hospital (Italy). In 3801 cases parathyroid hormone $(\mathrm{PTH})$ was requested in parallel. Serum 25(OH)D was measured by a NIST-972 aligned commercial LC-MS/MS method. The distribution of serum $25(\mathrm{OH}) \mathrm{D}$ concentrations in males and females of different age groups, the prevalence of $25(\mathrm{OH}) \mathrm{D} 2$ and seasonal variability were studied.

Results: $25(\mathrm{OH}) \mathrm{D}$ testing was requested three times more often in females than in males. The average $25(\mathrm{OH}) \mathrm{D}$ concentration in the entire cohort was $68.6 \mathrm{nmol} / \mathrm{L}(7.5-1880 \mathrm{nmol} / \mathrm{L})$. Females had a $7 \mathrm{nmol} / \mathrm{L}$ higher average $25(\mathrm{OH}) \mathrm{D}$ concentration than males, which increased significantly with age. 37.9 and $28.3 \%$ of males and females, respectively, had a deficient $25(\mathrm{OH}) \mathrm{D}$ concentration of $<50 \mathrm{nmol} / \mathrm{L}$. 620 samples $(0.84 \%)$ had measureable amounts of $25(\mathrm{OH}) \mathrm{D} 2$. In samples with a normal PTH $25(\mathrm{OH}) \mathrm{D}$ was $20 \mathrm{nmol} / \mathrm{L}$ higher than in the entire cohort. Seasonal variation ranged between 20 and $30 \%$ and was most pronounced in young individuals. 25(OH)D2 remained constant throughout the year.

Conclusion: Vitamin D status in South Tyrol is rather good. Higher $25(\mathrm{OH}) \mathrm{D}$ concentrations in individuals with normal PTH suggest a functional deficit in a substantial number of subjects. Seasonal variation of serum $25(\mathrm{OH}) \mathrm{D}$ is a relevant aspect in young and middle-aged adults, but becomes less relevant in elderly subjects. $25(\mathrm{OH}) \mathrm{D} 2$ is of minor practical importance in South Tyrol.

Keywords: 25-hydroxy vitamin D, LC-MS/MS, outpatients, age, gender

\section{$\mathbf{P 1 5 2}$}

The relevance of vitamin D receptor gene Bsm I and Fok I polymorphism with bone mineral density in Han female in Changchun City

Mengmeng ZHANG ${ }^{1}$, Qianqian $\mathrm{Ma}^{1}$, Weixian $\mathrm{Mao}^{1}$, Yuan $\mathrm{Gao}^{1}$, Shikai Song ${ }^{1}$, Jiwei Yin $^{1}$

${ }^{1}$ Osteoporosis Treatment Center, The Fourth Hospital of Jilin University, Changchun, China

Objective: To study the polymorphisms of VDR gene Bsm I and Fok I in Han female aged 35-85 years old in Changchun City and to analyze the relationship with bone mineral density.

Methods: Polymorphisms of Bsm I and Fok I were analyzed by PCR-RFLP, and lumbar spine (L1-4) BMD were detected by Hologic Discovery-WA-type bone mineral density detector. 230 objects were divided into menopausal group and premenopausal group, then we observed the distribution characteristics of each genotype. The SPSS 19.0 software was used to analyze the experimental results.

Results: In the present study, the genotype distributions of Bsm I were $\mathrm{bb}(83.9 \%), \mathrm{Bb}(12.6 \%)$ and $\mathrm{BB}(3.5 \%)$. The $\mathrm{b}$ allele frequency was as high as $90.2 \%$ and the $\mathrm{B}$ allele frequency was $9.8 \%$. The genotype distributions of Fok I were ff $(18.3 \%)$, Ff $(43.9 \%)$ and FF $(37.8 \%)$. The frequency of $\mathrm{f}$ allele was $40.2 \%$ and the frequency of $\mathrm{F}$ allele was $59.8 \%$. The distribution of genotypes of two sites both were in accordance with Hardy-Weinberg law $\left(\chi^{2}=1.283, \mathrm{P}=0.125\right.$; $\left.\chi^{2}=0.96, p=0.342\right)$; There was no significant difference in the genotype distribution between the premenopausal group and the menopausal group $(\mathrm{P}>0.05)$. BMD for each genotype of Bsm I was no significant difference between premenopausal group and menopausal group $(\mathrm{P}>0.05)$. BMD of Fok I ff type was lower than FF type and Ff type, and there was significant difference in BMD between ff type and FF type in menopause group $(\mathrm{P}<0.05)$.

Conclusion: In this study about 230 cases Han women aged 35-85 years, the distribution of Bsm I genotype was mainly bb, B allele frequency was as high as $90.2 \%$, and there was no difference in BMD of each genotype; Fok I ff type had lower BMD, but there was no significant difference in premenopausal group, ff type was associated with lower BMD in postmenopausal women.

Keywords: VDR; Bsm I ; Fok I ; lumbar spine BMD; relevance

\section{P153}

Clinical profile of patients with chronic hypoparathyroidism and potential indications for treatment with parathyroid hormone

Antonia García-Martín ${ }^{1}$, M Carmen Serrano-Laguna ${ }^{1}$, María HayónPonce $^{1}$, M Dolores Avilés-Pérez ${ }^{1}$, Elena Torres-Vela ${ }^{1}$, Manuel Muñoz-Torres ${ }^{1}$

${ }^{1}$ Endocrinology, University Hospital Campus de la Salud, Granada, Spain

Chronic hypoparathyroidism it is a rare entity. Therefore clinical guidelines have been published to facilitate its management. In addition, recombinant PTH [rhPTH (1-84)] has been approved as additional therapy to calcium and vitamin $\mathrm{D}$ in these patients. In this context, our aims were to study the clinical profile of patients with chronic hypoparathyroidism followed in Endocrinology Department of Granada and to evaluate the indications for considering the use of rhPTH (1-84) according to the proposals by Brandi ML et al. (J Clin Endocrinol Metab 2016;101:2273-83). We carried out a cross-sectional observational study that included 51 patients with chronic hypoparathyroidism ( $86.3 \%$ posquirurgical, $78.4 \%$ females). Clinical, biochemical and therapeutic data are summarized in Table $1.31 .4 \%$ of patients presented hipocalcemia and $43.1 \%$ had hyperphosphatemia. Oral medications required to control the serum calcium or symptoms exceed $2.5 \mathrm{~g}$ of calcium in $21.6 \%$ of patients. Hypercalciuria, renal stones, or reduced glomerular filtration $(<60 \mathrm{ml} / \mathrm{min})$ were present in 9.8, 3.9 and $31.4 \%$ respectively. In contrast, one patient had calciumphosphate product $>55$ and none had nephrocalcinosis. In conclusion, treatment with rhPTH (1-84) could be indicated in a significant percentage of our patients with chronic hypoparathyroidism.

Keywords: Chronic hypoparathyroidism, parathormone

\section{P154}

The role of non-canonical Wnt signalling in osteosarcoma formation

Kazuhiko Matsuoka $^{1}$, Latifa Bakiri ${ }^{1}$, Özge Uluçkan ${ }^{2}$, Erwin F. Wagner $^{1}$, Genes, Development and Disease Group

${ }^{1}$ CNIO: Centro Nacional de Investigaciones Oncológicas, Madrid, Spain, ${ }^{2}$ Novartis Institutes of Biomedical Research, Basel, Switzerland

Objective: Osteosarcoma(OS) is the most frequent primary malignant bone tumor. Our objective is to determine the function of Wnt signalling and identify the molecular mechanisms operating during OS development.

Methods: H2k-c-fos-LTR $\left(\right.$ Fos $^{T \boldsymbol{g}}$ ) mice over-expressing Fos/AP-1 are a powerful genetically-engineered mouse model for OS, as 
previously reported. An inducible bone-specific Wntless (Wls) lossof-function OS model (Wls ${ }^{\Delta \mathrm{OB}}$-OS mice) was generated by combining $w l s$ floxed, Osx-Cre and Fos $^{T g}$ mice. Tumors were monitored longitudinally by micro-CT $(\mathrm{n}=8)$. Osteocalcin $(\mathrm{Ocn})$, sclerostin and lysyl oxidase like 2 (Loxl2) expression was assessed by immunohistochemistry $(\mathrm{n}=2)$. Gene expression was analyzed by quantitative RT-PCR $(n=5)$. Histological visualization of collagen fibers was performed by sirius red staining and polarized-light microscopy.

Results: In bone tumors from Fos $^{T g}$ mice, the canonical Wnt target genes, axin 2 and tnfrsf $11 b$ were decreased, while the non-canonical Wnt ligands $W n t 7 b$ and $W n t 9 a$ were increased 8 and fourfold respectively. Consistently, knockdown of c-Fos decreased $W n t 7 b$ and $W n t 9 a$, while c-Fos expression had the opposite effect in OS cell lines and MC3T3-E1 cells, respectively. In Wls ${ }^{\Delta \mathrm{OB}}$-OS bones, axin2 and collagen-modifying enzyme, loxl2 decreased upon wls gene inactivation. Importantly, tumor burden was decreased by $70 \%$ in $\mathrm{Wls}^{\Delta \mathrm{OB}}$ OS mice compared to Fos $^{\boldsymbol{T g}}$ mice, with an obvious effect on tumor

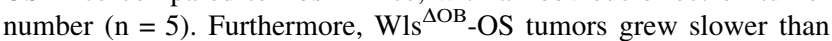
$F s^{T g}$ tumors in vivo and in vitro $(\mathrm{n}=17)$. While the majority of $\mathrm{OS}$ developing in Fos $^{\boldsymbol{T g}}$ mice appeared osteoblastic and expressed Ocn, sclerostin and Loxl2, OS from Wls ${ }^{\Delta \mathrm{OB}}$-OS mice were enriched in fibroblastic cells surrounded by collagen fibers.

Summary and Conclusions: Increased expression of Wnt7b and Wnt9a, which are important for bone and cartilage development, are a

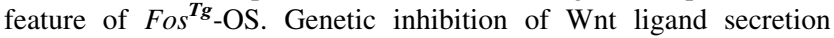
results in changes in tumor burden and OS histology associated with abnormal extracellular matrix deposition, indicating that non-canonical Wnt signalling is important for OS development.

\section{P155}

RANK expression in circulating tumor cells (CTCs): clinical utility in monitoring metastatic breast cancer patients (MBC) under Denosumab treatment

Francesco Pantano ${ }^{1}$, Elisabetta Rossi ${ }^{2,3}$, Michele Iuliani ${ }^{1}$, Giulia Ribelli $^{1}$, Sonia Simonetti ${ }^{1}$, Bruno Vincenzi ${ }^{1}$, Giuseppe Tonini ${ }^{1}$, Rita Zamarchi $^{2}$, Daniele Santini ${ }^{1}$

${ }^{1}$ Medical Oncology, Campus Bio-Medico University of Rome, Rome, Italy, ${ }^{2}$ Veneto Institute of Oncology IOV- IRCCS, Padua, Italy, ${ }^{3}$ Department of Surgery, Oncology and Gastroenterology, Oncology Section, University of Padova, Padova, Italy

Introduction: We evaluate the RANK expression on the surface of circulating tumor cells (CTCs) directly in metastatic breast cancer (MBC), under anti-RANKL (Denosumab) therapy as predictor of treatment response in $\mathrm{MBC}$ and as prognosticator of skeletal outcome.

Methods: An automated sample preparation and analysis system for enumerating CTCs (CellSearch System) was integrated with a specific mAb for detecting RANK-positive CTCs. CTCs from blood were analyzed at baseline and at day 2, 7, 14, 28 after the first Denosumab administration. A companion algorithm ( $\triangle \mathrm{AUC})$ was developed to express the difference between RANK-positive and RANK-negative CTC concentration-Time Area (AUC), as calculated according to the following formula:

$\triangle A U C=$ RANK-positive CTC AUC-RANK-negative CTC AUC Time-to-first-SRE, Time-to-Bone-Metastasis-Progression and Timeto-Visceral-Metastasis-Progression were estimated by Univariate COX Regression analysis, evaluating $\triangle \mathrm{AUC}$ as continuous variable and as a categorical variable (cut-off value: $\leq 0$ vs. $>0$ ). Moreover, to evaluate changes of CTCs' levels during treatment, we determined the Slope of the straight line connecting the pair T0-T1 of RANK-positive and RANK-negative CTCs, respectively. Time-to-first-SRE, Time-to-Bone-Metastasis-Progression and Time-to-Visceral-Metastasis-Progression were estimated by Kaplan-Meier analysis and the $p$ value calculated by log-rank test according to RANK-positive and RANK-negative CTC slope.

Results: We found significant association between longer Time-tofirst-SRE, Time-to-Bone-Metastasis-Progression and Time-to-Visceral-Metastasis-Progression, respectively, and positive $\triangle \mathrm{AUC}$, expression of persistence of RANK-positive CTCs during Denosumab treatment. Furthermore, the analysis of the Slope demonstrated a significant association between positive-Slope value of RANK-positive CTCs and longer Time-to-first-SRE, Time-to-Bone-MetastasisProgression and Time-to-Visceral-Metastasis-Progression, respectively.

Conclusion: The persistence of RANK-positive CTCs in MBC treated with Denosumab predicts therapy efficacy. Furthermore, more rapid is the decrease of RANK-positive CTCs at the $2^{\circ}$-day of treatment, more efficacious will be the therapy. Overall, we demonstrate the clinical utility of the RANK-integrated CTC test to evaluate SRE risk and bone metastasis in MBC under Denosumab therapy.

Keywords: Circulating Tumor Cells (CTCs); RANK; breast cancer; Denosumab

\section{P156}

A long-coding RNA MANCR, restricted in expression to metastatic breast cancer metastatic cells, induces cell death upon depletion

Kristen Tracy ${ }^{1}$, Coralee Tye ${ }^{1}$, Prachi Ghule ${ }^{1}$, Heidi Malaby ${ }^{2}$, Jason Stumpff ${ }^{2}$, Janet Stein ${ }^{1}$, Gary Stein ${ }^{1}$, Jane Lian ${ }^{1}$

${ }^{1}$ Biochemistry, University of Vermont Larner College of Medicine, Burlington, United States, ${ }^{2}$ Molecular Physiology and Biophysics and Vermont Cancer Center, University of Vermont Larner College of Medicine, Burlington, United States

Long non-coding RNAs (lncRNAs) are epigenetic regulators with essential roles in normal development of tissues and associated with breast cancer (BrCa). LncRNAs have diverse functions and have emerged as essential regulators of "genome structure", a characteristic of a cell's phenotype. LncRNAs mediate chromosomal interactions with histone modifying (HM) enzymes and other factors contributing to higher order gene regulation at specific genomic loci. We have profiled (by RNA-Seq), differentially expressed lncRNAs in normal-like mammary epithelial MCF10A cells compared to 4 cancer cell lines: pre-malignant MCF10AT1, malignant MCF10CA1a, ER + MCF-7 (luminal), and TNBC MDA-MB-231 (basal) cells. Given the critical need to prevent $\mathrm{BrCa}$ end stage-metastasis to lung and bone, we focused on the most upregulated lncRNA, LINC00704 detected in the two metastatic cell lines and absent in nearly all normal tissues. This lncRNA is highly expressed in PR- and ERtumors and associated with poor survival (30\%/10 year survival compared to $59 \%$ in patients with low LINC00704). We showed expression enrichment on mitotic chromatin with low expression in interphase cells (RNA-ISH). We named the lncRNA MANCR (mitosis associated nuclear RNA) and confirmed its transcriptional activation by presence of 3 activating HM markers spanning the MANCR gene. Functional effects of MANCR in metastatic cells were identified using 2 different GapmeRs (Exiqon, 90\% inhibition), resulting in 980 upregulated and 1240 downregulated transcripts (by RNA-Seq) in MANCR depleted MDA-MB-231 cells. GSEA revealed many categories related to cell cycle control and genome stability. We found knockdown of MANCR decreased proliferation, increased cell death by cleaved caspase 3 and flow cytometry, increased DNA damage by $\mathrm{gH} 2 \mathrm{AX}$, and cells showed defective cytokinesis (live imaging). Discovery of MANCR properties that are restricted to metastatic cells, support genomic stability of aggressive breast cancer, and its inhibition induces cell death, identifies MANCR as a viable therapeutic target. 


\section{P157}

LIGHT promotes osteolytic bone metastases in non-small cell lung cancer patients

Giacomina Brunetti ${ }^{1}$, Dimas Carolina Belisario ${ }^{2}$, Lucio Buffoni ${ }^{3}$, Silvia Colucci ${ }^{1}$, Giuseppe Ingravallo ${ }^{4}$, Carl Ware ${ }^{5}$, Maria Grano ${ }^{6}$, Riccardo Ferracini $^{7}$, Ilaria Roato ${ }^{2}$

${ }^{1}$ Basic and Medical Sciences, Neurosciences and Sense Organs, section of Human Anatomy and Histology, University of Bari, Bari, Italy, ${ }^{2}$ CeRMS, A.O.U. Città della Salute e della Scienza di Torino, Torino, Italy, ${ }^{3}$ Oncology, San Luigi Hospital, University of Turin, Orbassano, Italy, ${ }^{4}$ Emergency and Organ Transplantation, Pathology Section, University of Bari, Bari, Italy, ${ }^{5}$ Infectious and Inflammatory Disease Center, Sanford Burnham Prebys Medical Discovery Institute, La Jolla, United States, ${ }^{6}$ Emergency and Organ Transplantation, Section of Human Anatomy and Histology, University of Bari, Bari, Italy, ${ }^{7}$ DISC, S. Martino Hospital, University of Genova, Genova, Italy

LIGHT, a TNF superfamily member, is one of the cytokines produced by tumor and immune cells, particularly by activated T-cells, monocytes and neutrophils. Literature data report high LIGHT levels in erosive rheumatoid arthritis and multiple myeloma bone disease. We investigated the role of LIGHT in the osteolytic bone metastatic process induced by non-small cell lung cancer (NSCLC), which is an osteotropic tumor. We analysed by flow citometry LIGHT expression on CD4 and CD8 T cells, CD14 monocytes, CD16 neutrophils from peripheral blood of patients and controls, showing that LIGHT expression was significantly higher in monocytes from bone metastatic patients than non-bone metastatic ones $(9.32 \pm 5.8$ vs. $3.8 \pm 2.5, p<0.001)$. Since osteoclasts (OCs) derive from monocyte-macrophage lineage, we investigated the effect of anti-LIGHT added in OC cultures of both bone and non-bone metastatic patients. We detected a significant inhibition of osteoclastogenesis in both cultures of bone metastatic patients $(272 \pm 97$; $132 \pm 73, p<0.001)$ and non-bone metastatic ones $(137 \pm 56$; $86 \pm 48, p<0.001)$. The gene expression analysis on PBMCs from NSCLC patients showed that RANKL/OPG fold increase was higher in bone metastatic patients than in non-bone metastatic ones (1327.4 vs. 277.4, $p<0.05)$. We dosed LIGHT serum levels without detecting significant differences between NSCLC patients and controls. In human samples of bone metastases from NSCLC, we detected the expression of LIGHT by tumor cells.

Preliminary data on LIGHT KO mice showed that LIGHT-deficiency seems to have a protective action against tumor growth in bone. Indeed, we detected a reduced proliferation of LLC1 tumor cells injected in the tibia of LIGHT-KO mice compared to WT injected mice. In conclusion, our data obtained from both patients and murine model implicate LIGHT as potential therapeutic target in NSCLC.

Keywords: bone metastasis, osteoclast, LIGHT

\section{P158}

Inhibition of PARP1 potentiate the effect of the DNA damaging agent doxorubicin in osteosarcoma

Jung Ryul Kim ${ }^{1}$, Kyu Yun Jang ${ }^{1}$

${ }^{1}$ Orthopaedic Surgery, Chonbuk National University Medical School, Jeonju, Republic of Korea

PARP1 is early responsive for the DNA damage by inducing recruitment of phosphorylated $\mathrm{H} 2 \mathrm{AX}(\gamma \mathrm{H} 2 \mathrm{AX})$ and BRCA1/2. Therefore, these DNA damage response (DDR) molecules are used as indicators of DNA damage. However, recent reports have shown that these molecules are also involved in the resistance for the antitumor therapies and the progression of human cancers. However, the role of
DDR molecules in osteosarcoma is limited. In this study, we evaluated the expression of DDR molecules in 35 human osteosarcomas and investigated the effects of co-treatment of PARP inhibitor olaparib and doxorubicin in the proliferation of osteosarcoma cells. In 35 cases of osteosarcomas, the expression of PARP1, $\gamma \mathrm{H} 2 \mathrm{AX}$, and BRCA2 were significantly associated with shorter overall survival and relapse-free survival of osteosarcoma patients in univariate analysis. Multivariate analysis revealed the expression of $\gamma \mathrm{H} 2 \mathrm{AX}$ and combined expression pattern of PARP1, $\gamma \mathrm{H} 2 \mathrm{AX}$, BRCA1, and BRCA2 as independent poor prognostic indicator of osteosarcoma patients. In U2OS, SaOS2, and MG63 osteosarcoma cells, PARP inhibitor olaparib significantly inhibited proliferation of cells. Moreover, co-treatment of olaparib and doxorubicin significantly inhibited proliferation of osteosarcoma cells. Western blot analysis and flow cytometry analysis with annexin $\mathrm{V}$ and propidium iodide staining showed a significant increase of apoptosis of osteosarcoma cells with the combined use of olaparib and doxorubicin when compared with olaparib- or doxorubicin-treatment only. This study suggests a possibility that combined use of PARP inhibitor with doxorubicin, a DNA damaging agent, might be helpful for the treatment of osteosarcoma patients especially in the poor-prognostic subgroup of osteosarcoma expressing PARP1, $\gamma \mathrm{H} 2 \mathrm{AX}$, or BRCA1/2.

Keywords: PARP1, olaparib, doxorubicin, prognosis, osteosarcoma

\section{P159}

In-vitro effect of pharmaco-modulation of pyridazinone molecules on osteosarcoma cells

Aurélie Moniot ${ }^{1}$, Fabien Lamret ${ }^{1}$, Christine Guillaume ${ }^{1}$, Ingrid Allart-Simon $^{2}$, Janos Sapi ${ }^{2}$, Sophie C. Gangloff ${ }^{1}$, Stéphane Gérard ${ }^{2}$, Frédéric Velard ${ }^{1}$

${ }^{1}$ EA4691 BIOS, Université Reims Champagne-Ardenne, Reims, France, ${ }^{2}$ UMR CNRS 7312 ICMR, Université Reims ChampagneArdenne, Reims, France

Osteosarcoma is a primary bone cancer which mostly affects children and young adults (Mirabello L et al., 2009). Actual therapies are unfortunately insufficient to avoid relapse and metastasis, and patient survival remains at the same level since 20 years (Perkins SM et al., 2014). The use of new molecules for osteosarcoma therapy are crucial nowadays. Preliminary studies carried out by the laboratory have highlighted the anti-cancer effect of the pyridazinone-derived molecules named 516 and 532. The aim of the present work was to test the activity of a second generation of molecules with a pyridazinone structure, 5016 and 5032, in vitro, on human osteosarcoma cell lines (Saos-2 and MG-63). Using WST-1, we determined their IC50 on Saos-2 in vitro, which are $50 \mu \mathrm{M}$ for 5016 and $30 \mu \mathrm{M}$ for 5032. At these concentrations, metabolic activity decreased by 68 and $80 \%$ compared to the control $(\mathrm{n}=7, p<0.05)$ (against 50 and $35 \%$ for the maximum values obtained with the first generation molecules used at three fold stronger concentrations). These data were strengthened by: (i) treated cells' DNA quantity measurement which decreased by 48 and $39 \%$ with 5016 and 5032 (n=7,p<0.05) (as compared with 35 and $14 \%$ for 516 and 532), and (ii) decrease of cells number per field (phalloidin/DAPI) in presence of the molecules. Moreover, no alteration in cells morphology was seen after treatment. The 516 molecule showed an ability to alter gelatinolytic activity by $50 \%$ in MG-63 culture supernatants (zymography, $\mathrm{n}=7, p<0.05$ ). Thereby, our data confirm that our pyridazinone-based molecules, and especially the second-generation molecules, can be interesting hit to develop potential osteosarcoma therapy. The anti-invasive potential of these second-generation molecules will be evaluated on MG-63, as well as their toxicity on human primary osteoblasts, before considering in-vivo tests.

Keywords: Osteosarcoma, pyridazinone, cytotoxicity 


\section{P161}

Role of osteoblasts (OBs) in castration resistant prostate cancer (CRPC) progression

Giulia Ribelli ${ }^{1}$, Sonia Simonetti ${ }^{1}$, Michele Iuliani ${ }^{1}$, Bruno Vincenzi ${ }^{1}$, Giuseppe Tonini ${ }^{1}$, Daniele Santini ${ }^{1}$, Francesco Pantano ${ }^{1}$

${ }^{I}$ Campus Bio-Medico University, Rome, Italy

Introduction: Skeletal involvement in metastatic CRPC (mCRPC) is common and results in significant morbidity and mortality. The interaction of prostate cancer $(\mathrm{PCa})$ with the bone microenvironment contributes to cancer progression in the bone. Our aim is to elucidate the specific role of OBs in PCa progression using human in-vitro model.

Materials and Methods: Primary human OBs were obtained from healthy patients undergoing hip replacement. Bone mCRPC cells (C42B) GFP-tagged were used to perform direct co-cultures with OBs, while $C 4-2 B$ transfected with androgen receptor (AR) reporter gene to analyze AR activity.

C4-2B were treated with osteoblast conditional media (OCM) to investigate the indirect cross talk OBs/cancer cells. Proliferation and cell cycle of C4-2B cells were evaluated by flow cytometry analyses.

Results: We found that OBs inhibited AR activity of C4-2B cells both in direct and indirect co-cultures (Fig. 1A), downregulating AR expression at transcriptional and protein levels (Fig. 1B). We demonstrated, in the same time, that OBs stimulated cancer cell proliferation increasing the percentage of ki-67+ C4-2B cells in G1, S and G2-M phases (Fig. 2).
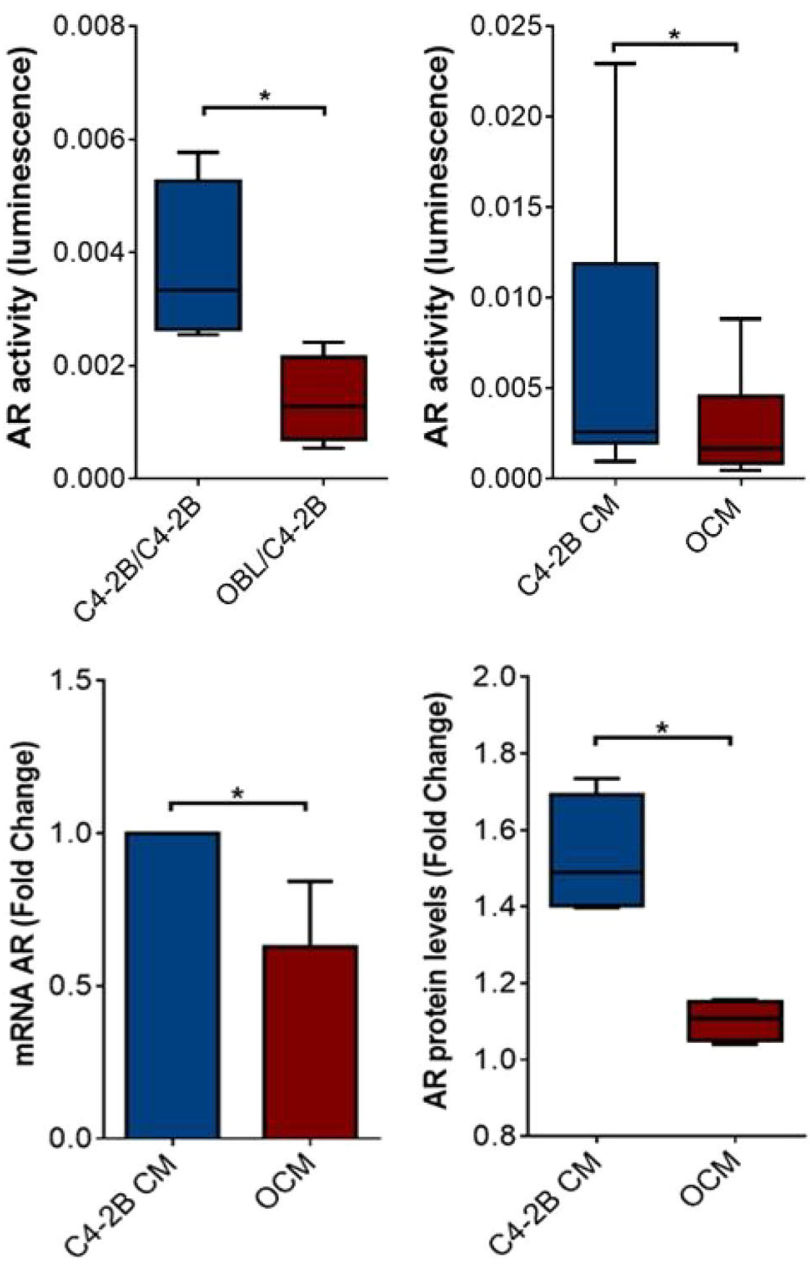

Fig. 1 OBs inhibit AR activity in C4-2B cells downregulating AR mRNA and protein levels

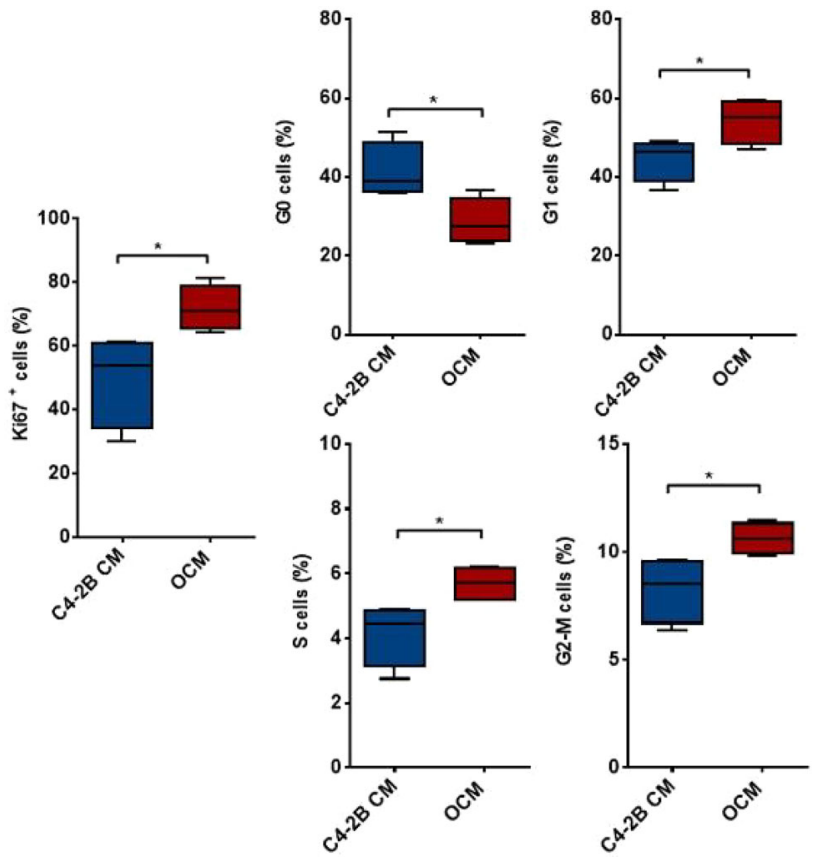

Fig. 2 OBs increase the percentage of proliferative cancer cells

Conclusions: Our results suggest that soluble factors secreted by OBs promoted CRPC cells proliferation through an androgen-independent mechanism.

Keywords: Castration Resistant Prostate Cancer; Osteoblasts; Androgen-Independence

\section{P162}

Increased osteoblastic activity suppressed proliferation of multiple myeloma plasma cells

Young-Hoon $\mathrm{Kim}^{1}$, Yoo-Kyung $\mathrm{Cho}^{2}$, Kee-Yong $\mathrm{Ha}^{2}$, Hyung-Youl Park $^{2}$

${ }^{1}$ Seoul St. Mary's Hospital, The Catholic University of Korea, Seoul, Republic of Korea, ${ }^{2}$ Orthopedic surgery, Seoul St. Mary's Hospital, The Catholic University of Korea, Seoul, Republic of Korea

Backgrounds and Objectives: Decreased proliferation of Multiple myeloma (MM) at fractured site has been noted in clinical situation regardless of the systemic disease activity. Therefore, this study was planned to investigate the impact of increased osteoblastic activity on the proliferation of $\mathrm{MM}$.

Design and Methods: For investigation of the effect of increased osteoblastic activity on survival and proliferation of MM cells, coculture under various conditions was adopted. MM cells (IM-9) were cultured in culture media (control) and co-cultured with human mesenchymal stem cells (hMSCs, SS16-P2, group I), osteoblasts (OBs) induced from hMSCs (group II) and bone morphogenic protein-2 (BMP-2, group III). Proliferation and apoptosis of cultured MM cells were assessed.

Results: In $72 \mathrm{~h}$ co-culture, the group II (relative optical density $0.8 \pm 0.2, \mathrm{n}=6)$ and III $(0.4 \pm 0.2)$ showed decreased ERK expression compared with those of the control $(1.6 \pm 0.4$ for OBs coculture, $1.3 \pm 0.2$ for BMP-2 condition) $(P<0.05)$. Lower expression of Ig $\mathrm{G}$ was noted in the group II $(0.1 \pm 0.02)$ and III $(0.5 \pm 0.05)$ compared with those of the control $(1.5 \pm 0.1$ and $1.6 \pm 0.09)(P<0.05)$. However, hMSCs co-culture condition did not show a significant decrease of Ig $G$ and ERK expression. 
Expression of caspase- 3 in the group II $(0.8 \pm 0.06)$ and group III $(1.01 \pm 0.07)$ were significantly higher than those of the control $(0.3 \pm 0.03$ and $0.3 \pm 0.04)(P<0.05)$. However, co-culture with hMSCs $(0.3 \pm 0.02)$ showed a significantly decreased expression of caspase- 3 compared to that of the control $(0.7 \pm 0.1)(P<0.05)$. FACS with annexin $\mathrm{V}$ method also showed corresponding results of higher apoptosis in the group II and III.

Conclusions: Even following studies including the mechanism of this phenomenon and other in vivo trials are required, this experiment present that different microenvironmental conditions in the bone marrow may influence the survival of MM plasma cells.

Keywords: myeloma, osteoblasts, mesenchymal stem cells, bone morphogenic protein

\section{P163}

Regulation of RANKL in human osteosarcoma cells

$\underline{\text { Nika Lovšin }}^{1}$, Klemen Kodrič ${ }^{2}$, Janja Marc ${ }^{1}$

${ }^{1}$ University of Ljubljana, Faculty of pharmacy, Ljubljana, Slovenia,

${ }^{2}$ Faculty of Pharmacy, University of Ljubljana, Ljubljana, Slovenia

Receptor activator of nuclear factor $\kappa \mathrm{B}$ ligand (RANKL) is a part of the RANKL/RANK/OPG system which plays a crucial role in bone resorption. RANKL misregulation has been implicated in bone diseases such as osteoporosis (OP) and osteoarthritis (OA). Changes in RANKL expression have also been associated with tumorigenesis and metastasis in several cancers. In clinical trials, monoclonal antibody against RANKL decreased the risk of metastasis development. Here, we hypothesized that, changes in expression of transcription factor cause mis-regulation of RANKL expression. To identify transcription factors that regulate RANKL promoter, we first bioinformatically analyzed the proximal promoter region of RANKL gene and then examined the impact of the most likely candidates on the activity of RANKL promoter by luciferase activity assays in vitro. Indeed, we identified several potential transcription factors that could regulate expression of RANKL in osteosarcoma cell. Silencing of transcription factor c-Myb resulted in lower activity of RANKL promotor and lowered RANKL expression in human osteosarcoma cells. Moreover, gain of function experiments revealed that in the presence of transcription factor c-Myb, the activity of proximal RANKL promoter was highly elevated. In addition, in the presence of c-Myb, RANKL expression increased.

Altogether, our data demonstrate that c-Myb overexpression activates RANKL promoter in vitro and could cause elevated levels of RANKL in cancer diseases.

Keywords: osteoporosis, RANKL promoter, c-Myb, RANKL expression, osteosarcoma

\section{P164}

Role of autophagy in the crosstalk between osteosarcoma and the bone microenvironment

Valérie Pierrefite-Carle ${ }^{1}$, Olivier Camuzard ${ }^{1}$, Sabine SantucciDarmanin ${ }^{1}$, Tatiana Gritsaenko ${ }^{1}$, Sophie Pagnotta ${ }^{2}$, Chantal Cros ${ }^{1}$, Fanny Burel-Vandenbos ${ }^{3}$, Dominique Heymann ${ }^{4}$, Georges F. Carle ${ }^{1}$

${ }^{I}$ TIRO-MATOs UMR E-4320, Université Nice Sophia Antipolis BIAM/DRF/CEA, Nice, France, ${ }^{2}$ Centre Commun de Microscopie Appliquée (CCMA), Université Nice Sophia Antipolis, Nice, France, ${ }^{3}$ Laboratoire d'Anatomopathologie, Hôpital Pasteur, CHU de Nice, Nice, France, ${ }^{4}$ INSERM UMR1232, Université de Nantes/Institut de Cancérologie de l'Ouest, Nantes, France

Osteosarcoma (OS) is a bone cancer of osteoblastic origin which mainly affects children. Approximatively $15-20 \%$ of the patients exhibit a metastatic OS at diagnosis, and $25-50 \%$ will eventually develop metastasis. The overall 5-year survival rate of these patients is about $20 \%$. Surgery and chemotherapy are the standard treatment regimens but developing novel, effective-targeted therapies to treat this complex disease remains a challenge. The balance between bone formation and bone resorption is affected in osteosarcomas. To date, very few studies have been performed to understand the interactions between OS cells and their complex microenvironment.

Autophagy is the major catabolic process of eukaryotic cells that degrades and recycles damaged macromolecules and organelles. Autophagy occurs at low level in all cells to ensure the homeostatic turnover of long-lived proteins and organelles and is upregulated under stressfull conditions. Recent publications provide evidence that this process is involved in some secretion mechanisms, and we and others have demonstrated that autophagy is a crucial mechanism for bone metabolism.

The objective of the project is to analyze the development of syngeneic OS tumor cells in an autophagy-deficient or competent microenvironment. To this aim, we used an OS cell line in a syngeneic mouse model of autophagy deficiency in osteoblasts (OB). Primary mouse $\mathrm{OB}$ are used as a source to generate bone extracellular matrix (ECM) and conditioned medium to culture OS cells. Using several ex vivo approaches, we first characterized the ECM generated by mutant OB. Our results suggest an increased level of ECM proteins such as collagen in mutant matrix, which could modify matrix stiffness. In addition, we show that osteosarcoma cells seeded on these mutant matrix exhibit an increased proliferation. The in vivo analysis of OS development following intratibial injection in WT and mutant mice is on going and will be presented.

Keywords: Autophagy, Osteosarcoma, Microenvironment

\section{P165}

ER exit site microautophagy recycles misfolded procollagen in osteoblasts

Shakib Omari ${ }^{1}$, Elena Makareeva ${ }^{1}$, Lynn Mirigian ${ }^{1}$, Anna RobertsPilgrim $^{1}$, Edward Mertz ${ }^{1}$, Michal Jarnik ${ }^{1}$, Laura Gorrell ${ }^{1}$, Jennifer Lippincott-Schwartz ${ }^{2}, \underline{\text { Sergey Leikin }}{ }^{1}$

${ }^{1}$ NICHD, National Institutes of Health, Bethesda, United States, ${ }^{2}$ Janelia Research Campus, Howard Hughes Medical Institute, Ashburn, United States

Osteoblast cell stress and malfunction caused by accumulation of misfolded procollagen in the Endoplasmic Reticulum (ER) contributes to bone fragility in osteogenesis imperfecta (OI) and other skeletal disorders. To understand how osteoblasts respond to such accumulation and enhance their ability to adapt to and reduce the cell stress, we examined effects of a Gly610 to Cys (G610C) substitution in the triple helix of type I procollagen proa2(I) chain. In G610C mice (MGI:3711122), we observed osteoblast adaptation to the cell stress by autophagic degradation of misfolded procollagen. We visualized intracellular trafficking and degradation of misfolded procollagen by live-cell imaging and correlative light and electron microscopy of MC3T3 and primary calvarial osteoblasts. The cells were transfected with fluorescently-tagged proa2(I) and fluorescent markers of different subcellular compartments. The G610C substitution increased the fraction of autophagic structures that contained the transfected pro $\alpha 2$ (I) chain from $\sim 30 \%$ to over $50 \%$ ( $p<0.001)$. These structures also contained ER exit site (ERES) but not ER lumen or membrane proteins. Blocking ERES formation by H89 decreased their number $(p<0.001)$, whereas Golgi dispersal by brefeldin A had no effect, suggesting autophagy initiation at ERESs. Inhibition of autophagosome-lysosome fusion by bafilomycin A1 did not affect lysosomal internalization of misfolded procollagen. A lysosomal membrane marker LAMP1 was internalized together with procollagen 
and not restricted to the lysosome surface. Apparently, misfolded procollagen triggered modification of ERES membranes by lipidated LC3, followed by lysosomal engulfment and degradation through a non-canonical, $\mu$-autophagy-like pathway. Reduced expression and osteocalcin-Cre based knockout of ATG5 (required for LC3 lipidation) exaggerated bone pathology in $\mathrm{G} 610 \mathrm{C}$ mice $(\mathrm{p}<0.05)$. In contrast, osteocalcin-Cre based ATG5 overexpression moderated the pathology $(\mathrm{p}<0.05)$. Overall, our observations reveal a novel ERES $\mu$-autophagy pathway and suggest that its pharmacological or dietary enhancement is a promising approach to treatment of OI and potentially other bone pathologies.

\section{P166}

Wnt1 is an Lrp5-independent bone-anabolic Wnt ligand

Julia Luther $^{1}$, Timur A Yorgan ${ }^{1}$, Tim Rolvien ${ }^{1}$, Lorenz Ulsamer ${ }^{1}$, Till Koehne $^{1,2}$, Daniela Mau ${ }^{1,3}$, Nelle Vollersen ${ }^{1}$, Stefan Teufel ${ }^{1,4}$, Mona Neven ${ }^{1}$, Sefanie Peters ${ }^{1}$, Michaela Schweizer ${ }^{5}$, Andreas Trumpp ${ }^{6}$, Sebastian Rosigkeit ${ }^{7}$, Ernesto Bockamp ${ }^{7}$, Stefan Mundlos ${ }^{3,8,9}$, Uwe Kornak $^{3,8,9}$, Ralf Oheim ${ }^{1}$, Michael Amling ${ }^{1}$, Thorsten Schinke ${ }^{1}$, JeanPierre David $^{1}$

${ }^{1}$ Institute for Osteology and Biomechanics (IOBM), University Medical Center Hamburg-Eppendorf, Hamburg, Germany, ${ }^{2}$ Department of Orthodontics, University Medical Center Hamburg-Eppendorf, Hamburg, Germany, ${ }^{3}$ Berlin-Brandenburg Center for Regenerative Therapies, Charité-Universitätsmedizin Berlin, Berlin, Germany, ${ }^{4}$ Institute of Experimental Musculoskeletal Medicine, Medical Faculty of the University of Münster, Münster, Germany, ${ }^{5}$ Center for Molecular Neurobiology Hamburg, University Medical Center Hamburg-Eppendorf, Hamburg, Germany, ${ }^{6}$ Division of Stem Cells and Cancer, Deutsches Krebsforschungszentrum (DKFZ), Heidelberg, Germany, ${ }^{7}$ Institute for Translational Immunology and Research Center for Immunotherapy, University Medical Center, Johannes Gutenberg University, Mainz, Germany, ${ }^{8}$ Institute of Medical Genetics and Human Genetics, Charité-Universitätsmedizin Berlin, Berlin, Germany, ${ }^{9}$ Max Planck Institute for Molecular Genetics, Berlin, Germany

WNT1 mutations in humans are associated with a new form of osteogenesis imperfecta and with early-onset osteoporosis suggesting a key role of WNT1 in bone mass regulation. However, clear insight into the mechanisms governing this process as well as proof-ofprinciple experiments to demonstrate the therapeutic potential of Wnt1 are still missing. We observed that pathologic heterozygous WNT1 mutations associated with low bone mass and high incidence of fractures due to low bone turnover was present in $8.5 \%$ of patients in a cohort of 83 cases with early onset osteoporosis. Importantly and providing direct in vivo evidence for the essential function of Wnt 1 in sustaining bone integrity, we show that inactivation of Wnt 1 in osteoblasts causes strong osteoporosis and spontaneous bone fractures in mice. To demonstrate the therapeutic potential of Wnt1, we used an inducible conditional transgenic model where Wnt1 expression in osteoblast can be timely controlled. Switching on Wnt1 promoted a rapid bone mass increase in young growing (3-12 week-old), adult ( 6 month-old) and even aging (1 year-old) mice. Importantly, switching on Wnt 1 for 1 week in young or even adult mice was enough to markedly increased osteoblast numbers and function demonstrating the rapidity of the bone anabolic effect. Strikingly and in contrary to current mechanistic models, loss of the Lrp5 co-receptor, previously assumed to transmit extracellular WNT signals during bone mass regulation, did not abolish the bone-anabolic effect of Wnt 1 as shown by the similar 4 time increased BV/TV and twofold increased BFR observed in Lrp5 positive or deficient mice over-expressing Wnt1. These data provide direct evidence that Wnt 1 function does not require the LRP5 co-receptor and identified Wnt1 as master regulator of bone mass formation and remodeling thus providing the basis for a novel class of drugs for the treatment of osteoporosis.

\section{P167}

Single-cell high content imaging predicts the functionality of cultured skeletal stem cells

Justyna Magdalena Kowal ${ }^{1}$, Hagen Schmal ${ }^{2}$, Arndt-Peter Schulz ${ }^{3}$, Moustapha Kassem ${ }^{1}$

${ }^{1}$ The Molecular Endocrinology \& Stem Cell Research Unit, Odense University Hospital, Odense, Denmark, ${ }^{2}$ Department of Orthopaedics and Traumatology and Department of Clinical Research, Odense University Hospital, Odense, Denmark, ${ }^{3}$ Department of Orthopaedics and Trauma Surgery, University Medical Center Schleswig-Holstein, Luebeck, Germany

Objectives: Bone marrow skeletal (stromal) stem cells (BM-MSC) are being tested in clinical trials for their efficiency in enhancing bone regeneration in non-healed fractures and bone defects. However, there is a lack of biomarkers of cultured BM-MSC to be employed in predicting their bone forming capacity.

Aim: To employ a novel method based on high-content single cell imaging to identify cellular characteristics predictive of BM-MSC functionality.

Methods: Human BM-MSC were isolated from bone marrow from male $(n=24)$ and female $(n=27)$ patients undergoing routine orthopedic surgeries. BM-MSC surface marker expression, cell proliferation and in vitro differentiation capacity were determined. Single-cell high-content imaging (Operetta) following staining for cytoskeleton and nuclei, was employed to determine cell morphology and texture.

Results: BM-MSC cell area and dimensions were highly predictive for proliferation ability $(\mathrm{p}<0.0001)$. Similar trends were found for the nuclear area and dimensions, where nucleus size negatively correlated with cell proliferation $(\mathrm{p}<0.0001)$. In addition, nucleus texture parameters correlated with cell proliferation. For osteoblast (OB) cell differentiation capacity, nucleus morphology was a better predictor than cellular morphology. Nucleus width to length ratio negatively correlated with $\mathrm{OB}$ differentiation (Pearson $\mathrm{r}=-0.53$, $\mathrm{p}<0.0001)$. In contrast, cells with higher nucleus width to length ratio (i.e. circular), were prone to adipogenic differentiation (Pearson $\mathrm{r}=0.31, \mathrm{p}=0.028)$. Furthermore, CD146, alkaline phosphatase and PTK7 (tyrosine-protein kinase-like 7) membrane markers correlated with OB capability and cells expressing those markers demonstrated decreased nucleus width to length ratio, corroborating the validity of high content imaging.

Conclusion: Combining surface marker expression and cellular/ nuclear morphology predicts the functionality of cultured BM-MSC. These parameters can be employed for screening of BM-MSC populations to choose cells suitable for bone regeneration.

Acknowledgement: Study was carried out as a part of Bonebank project founded by Interreg.

Keywords: human MSC, proliferation, osteogenesis, adipogenesis, high-content screening

\section{P168}

Osteoblast functionality in patients with Shwachman-Diamond syndrome

Annalisa Frattini ${ }^{1,2}$, Isabella Villa ${ }^{3}$, Roberto Valli ${ }^{1}$, Michela Signo ${ }^{3}$, Simona Bolamperti ${ }^{3}$, Maria Rita Pinto ${ }^{4}$, Marco Zecca ${ }^{5}$, Maria Rita Frau $^{6}$, Emanuela Maserati ${ }^{1}$, Francesco Pasquali ${ }^{1}$, Alessandro Rubinacci $^{3}$ 
${ }^{1}$ Human and Medical Genetics, Department of Medicine and Surgery, University of Insubria, Varese, Italy, ${ }^{2} I R G B$, National Council of Research, Milano, Italy, ${ }^{3}$ Bone Metabolism Unit, San Raffaele Scientific Institute, Milano, Italy, ${ }^{4}$ Department of Pediatric Hematology/ Oncology and Transfusion Medicine, IRCCS Bambino Gesù Hospital, Roma, Italy, ${ }^{5}$ Department of Pediatric Oncohematology, IRCCS Policlinico San Matteo, Pavia, Italy, ${ }^{6}$ Pediatric and Intensive Care Unit, San Francesco Hospital, Nuoro, Italy

Shwachman-Diamond syndrome (SDS) is a rare autosomal recessive multi-system disorder characterized by neutropenia, exocrine pancreatic insufficiency and skeletal abnormalities. The majority of SDS patients showed homozygous loss-of-function mutations in $S B D S$ gene.

Skeletal anomalies, reported in about half of SDS patients, include various features, as metaphyseal dysostosis, abnormal development of growth plates, delay of secondary center of ossification, early-onset low turnover osteoporosis, delayed bone age. Poor growth manifested by both short stature and/or abnormal weight gain is also common.

Although defects in growth plate may suggest altered chondrocytes functionality, the low turnover osteoporosis drove us to analyze the function of osteoblasts (OBs) derived from eight SDS patients (5 males, 3 females, with bi-allelic mutations in SBDS gene) and from five healthy donors ( 2 males, 3 females). All samples were obtained under written informed consent signed by the patients or their parents.

Western blot analysis showed a reduced/undetectable SBDS protein amount in all the patients.

The expression of $S B D S$ and of two genes involved in ribogenesis (EIF6 and EFL1), analysed by qRTPCR, showed a decreasing trend, that was significant $(\mathrm{p}<0.01)$ for $S B D S$.

The expression of genes involved in the differentiation and maturation of OBs (RUNX2, OSX, OPN, COL1A) was evaluated by qRTPCR in OBs primary culture. In basal culture condition, SDSOBs showed a similar behaviour compared to healthy OBs. When cultured in osteogenic medium, SDS-OBs showed a reduced ability to mineralize compared to control cells $(\mathrm{p}<0.05)$. At variance, $R U N X 2$, $O S X, O P N$ and $B S P$ mRNA showed a trend to increase, suggesting a positive feedback due to reduced protein synthesis.

In conclusion, according to the function of SBDS in ribosome biogenesis, we may hypothesize that in normal growing condition SDS-OBs are able to fulfill cellular needs, while in stressed condition they may not: a result consistent with the skeletal features of SDS.

\section{P169}

Azathioprine protects against poor bone health in mice with DSS induced inflammatory bowel disease

Stephanie Morgan ${ }^{1}$, Kirsty Hooper ${ }^{1}$, Katherine Halewood ${ }^{1}$, Elspeth Milne $^{2}$, Colin Farquharson ${ }^{2}$, Craig Stevens ${ }^{1}$, Katherine Staines ${ }^{1}$

${ }^{1}$ School of Applied Sciences, Edinburgh Napier University, Edinburgh, United Kingdom, ${ }^{2}$ Roslin Institute and R(D)SVS, University of Edinburgh, Edinburgh, United Kingdom

Individuals with inflammatory bowel disease (IBD) often present with poor bone health. The development of targeted therapies for this bone loss requires a better understanding of the underlying cellular mechanisms. Azathioprine is a commonly used drug for IBD management, however its mechanisms of action, in particular its effects on the skeleton, are not yet fully understood. Here, we have utilised the dextran sulphate sodium (DSS) model of colitis in mice to examine the effects of azathioprine on bone health. 10-week-old male wild-type mice $(n=6 /$ group) received $3.0 \%$ DSS in their drinking water for 4 days, following which they received tap water for a 14-day recovery period. Mice were treated by oral gavage daily throughout the experiment with clinically-relevant doses of azathioprine, or vehicle control. Micro-computed tomography assessment of vehicle- treated DSS mice revealed a worsened trabecular architecture compared to vehicle-treated control mice. Specifically, DSS treated mice exhibited decreased BV/TV (32\%), trabecular number $(12 \%)$ and trabecular thickness $(12 \%)$. In comparison, azathioprine treated mice were partially protected from the adverse effects of DSS, with no decreases in trabecular parameters observed. Histopathological analysis of the colon revealed no differences in the severity or extent of inflammation in azathioprine treated mice, in comparison to vehicletreated mice, suggesting that azathioprine has a direct protective effect against bone loss typical of IBD. Ongoing in vitro studies will determine the mechanism of action and this will impact on our understanding of bone protective agents given to patients with IBD. Keywords: Bone, IBD, osteoblast, micro-CT, azathioprine

\section{P170}

Hepcidin regulate biomineralization of bone by BMP Signaling in zebrafish

Yu Jiang ${ }^{1}$

${ }^{I}$ The Second Affiliated Hospital of Soochow University, Suzhou, China

Iron overload, as a risk factor for osteoporosis, can result in the upregulation of Hepcidin, and Hepcidin knockout mice display defects in their bone microarchitecture. However, the molecular and genetic mechanisms underlying Hepcidin deficiency-derived bone loss remain unclear. Here, we used CRISPR-Cas9, a versatile genomeediting tool, to generate a zebrafish hepcidin mutant. Iron overload and a mineralization delay were observed in osteoblast cells in hepcidin-/- zebrafish larvae, and these defects could be partially restored with a microinjection of hepcidin mRNA. Quantitative realtime PCR analyses revealed the down-regulation of the osteoblastspecific genes alp, runx $2 a$, runx $2 b$, and $s p 7$ in homozygous hepcidin mutant zebrafish. Luciferase reporter assays further showed that bone morphogenetic protein 2a (Bmp2a) enhanced the expression of runx $2 a$, while iron overload repressed its expression through bmp $2 a$ (independent of $h j v$ ). High-throughput transcriptome analysis of hepcidin-/- zebrafish revealed multiple pathways involved in osteoblast metabolism. Together, these findings show that iron overload derived from hepcidin deficiency represses bone formation, possibly through the BMP pathway, and affects runx2 in zebrafish.

\section{P171}

C5aR1 interacts with TLR2 and upregulates the immune cellrecruiting and osteoclast-inducing chemokine CXCL10 in osteoblasts Yvonne Mödinger ${ }^{1}$, Verena Fischer ${ }^{1}$, Melanie Haffner-Luntzer ${ }^{1}$, Anna Rapp ${ }^{1}$, Julia Pazmandi ${ }^{2}$, Markus Huber-Lang ${ }^{3}$, Anita Ignatius ${ }^{1}$

${ }^{1}$ Institute of Orthopeadic Research and Biomechanics, University of Ulm, Ulm, Germany, ${ }^{2}$ Ludwig Boltzmann Institute for Rare and Undiagnosed Diseases, Ludwig Boltzmann Institute, Vienna, Austria, ${ }^{3}$ Institute of Clinical and Experimental Trauma-Immunology, University Hospital Ulm, Ulm, Germany

The proinflammatory anaphylatoxin $\mathrm{C} 5 \mathrm{a}$ is generated by activation of the complement system, a crucial arm of innate immunity. There is evidence that the $\mathrm{C} 5 \mathrm{a} / \mathrm{C} 5 \mathrm{aR} 1$ axis is involved in physiological bone turnover and in inflammatory bone disorders, including osteoarthritis, periodontitis and fractures. C5a induces osteoblast migration and cytokine secretion. However, the underlying mechanisms remain elusive. Thus, we aimed to determine C5a-mediated signaling in osteoblasts and examined an interplay of C5aR1 with Toll-like receptor 2 (TLR2). Both receptors interact in immune cells 
and in context of infectious bone diseases, such as periodontitis, which is associated with bone loss.

Primary osteoblasts isolated from 8- to 12-week-old WT-mice and MC3T3-E1 cells were differentiated for 14 days. Cells were assessed for C5aR1 and TLR2 expression. Gene expression profiling after $4 \mathrm{~h}-$ exposure to C5a was performed by microarray and RT-PCR. Phosphorylation of mitogen-activated protein kinases (MAPK) in response to C5a or Pam3CSK4 (TLR2-agonist) was analyzed by immunoblotting. Immunoprecipitation was performed using a C5aR1antibody. CXCL10 was determined by ELISA. Statistics: Student's $t$ test or one-way ANOVA, $\mathrm{p}<0.05, \mathrm{n}=4-6$.

Our results revealed that in osteoblasts, C5a regulates genes involved in MAPK and transforming growth factor- $\beta$ (TGF- $\beta$ ) pathways. For instance, negative MAPK-regulators were downregulated (Dusp1, p $=0.009 ;$ Dusp 5, p = 0.055) and TGF- $\beta$-related genes were slightly upregulated $(T g f b i, \mathrm{p}=0.09, T g f b 1=0.19)$. C5a also induced an important TLR2-adaptor (Tirap, $\mathrm{p}=0.09$ ). Via immunoprecipitation, we showed that C5aR1 and TLR2 interact in osteoblasts. We investigated shared intracellular signaling pathways and found a C5aR1- and TLR2-dependent phosphorylation of p38 MAPK. This was enhanced and prolonged by receptor costimulation and led to an upregulation (Cxcl10, $\mathrm{p}=0.03)$ and secretion $(\mathrm{p}=0.0001)$ of CXCL10, compared to C5a-induced levels alone.

Concluding, our data show that C5a regulates genes, which are crucial for osteoblast function. Further, by inducing chemokine CXCL10, an immune cell-recruiting and osteoclastogenic mediator, osteoblasts could contribute to inflammation and bone resorption.

Keywords: Complement System, Osteoblast

\section{$\mathbf{P 1 7 2}$}

Activation of TRPC3 by ATP regulates the differentiation and functions of bone cells in bone remodelling

$\underline{\text { Yu-Mi Yang }}{ }^{1}$, Namju Kang ${ }^{2}$, Dong Min Shin ${ }^{1}$

${ }^{I}$ Department of Oral Biology, Yonsei University College of Dentistry, Seoul, Republic of Korea, ${ }^{2}$ Department of Oral Biology, BK21 PLUS Project, Yonsei University College of Dentistry, Seoul, Republic of Korea

Mechanical stress plays an important role in bone remodelling and maintenance. However, the mechanism of intracellular $\mathrm{Ca}^{2+}$ entry by TRPC 3 and mechanical stress in bone cells is not well known. This study investigated the role of TRPC 3 in $\mathrm{Ca}^{2+}$ signal transduction in bone remodelling using TRPC 3 knockout $(\mathrm{KO})$ mice. The deletion of TRPC3 markedly increased of the bone density of the femur $(\sim 1.39$ fold in 4-week old) in an age-dependent manner. After treatment with RANKL, TRPC3 KO bone marrow-derived monocytes/macrophages (BMMs) significantly reduced NFATc1 translocation to the nucleus, bone resorption activity, and multinucleated cells formation. The potency of osteoblastic differentiation in TRPC3 $\mathrm{KO}$ mice showed enhanced effects compared to wild-type mice by the methods of alkaline phosphatase and alizarin red staining. Mechanical stress induces extracellular ATP release. TRPC3 contributed to an ATPinduced increases of intracellular $\mathrm{Ca}^{2+}$ in osteoblasts $(\sim 2.33$-fold), providing a pathway for receptor-operated $\mathrm{Ca}^{2+}$ entry in TRPC3 KO osteoblastic cells. These results suggest that TRPC3 plays an important role in bone remodelling and receptor-operated $\mathrm{Ca}^{2+}$ entry. This work was supported by the National Research Foundation of Korea (NRF) grant funded by the Korea government (MOE) (2012R1A1A2007673, 2015R1D1A1A01057277).

Keywords: mechanosensitive calcium channels, calcium signalling, receptor-operated $\mathrm{Ca}^{2+}$ entry, TRPC3 $\mathrm{KO}$ mice, bone remodelling

\section{P173}

A human mesenchymal stem cell model of aging reveals paracrine signaling as a strategy to modulate osteogenesis

Arantza Infante $^{1}$, Clara I Rodríguez ${ }^{1}$

${ }^{1}$ Stem Cells and Cell Therapy Lab, BioCruces Health Research Institute, Barakaldo, Spain

Aging is a complex biological process, which involves multiple mechanisms with different levels of regulation. Senescent cells are known to secrete senescence-associated proteins, which exert negative influences on surrounding cells. Mesenchymal stem cells (MSCs), the common progenitors for bone, cartilage and adipose tissue (which are especially affected tissues in aging), are known to secrete a broad spectrum of biologically active proteins with both paracrine and autocrine functions in many biological processes.

In this report, we have studied the secreted factors (secretome) from human MSCs (hMSCs) and hMSCs-derived adipocytes which were induced to accumulate prelamin $\mathrm{A}$, the immature form of the nuclear lamina protein called Lamin A, known to induce premature aging syndromes in humans and in murine models. For this, we used two complementary proteomic approaches, antibody arrays and liquid chromatography-mass spectrometry (LC-MS). Antibody arrays were performed with a pooled secretome coming from 2 independent hMSCs donors, and for LC-MS, secretome coming from 4 independent hMSCs donors were independently processed and analyzed. Notably, in secretome from preA-hMSCs, we found a high proportion of differentially secreted proteins which had been previously identified as secreted by hMSCs undergoing osteogenesis. Many of these factors were related to extracellular matrix, adhesion and collagen metabolism. When functionally tested, the secretome from preAhMSCs resulted to enhance hMSCs adhesion to cell culture plate, and increased osteogenic differentiation (measured in terms of Runx2 expression and alkaline phosphatase activity) of hMSCs $(n=5)$ but not adipogenesis differentiation. Furthermore, this study revealed the essential role of a factor overexpressed in the secretome from preAhMSCs, IGFBP7, in osteogenesis of hMSCs.

Overall, the present work demonstrates that aged hMSCs, the progenitors of bone cells, can modulate osteogenesis by means of paracrine signaling via a secretome rich in osteogenesis-related proteins.

\section{P174}

Evidence that teriparatide highly increases $C X C R 4$ expression during mineralization in MC3T3-derived osteoblasts

Beatriz Larraz Prieto $^{1}$, Asim Azfer ${ }^{1}$, Esther Anguiano ${ }^{1}$, Angela Quiroga $^{1}$, Stuart H. Ralston ${ }^{1}$, Nerea Alonso ${ }^{1}$

${ }^{1}$ Rheumatology and Bone Disease Unit, University of Edinburgh, Edinburgh, United Kingdom

Teriparatide (1-34 fragment of PTH) is an anabolic agent that increases bone formation and reduces fracture rates. However, treatment costs are high and the response is variable. A two-stage GWAS in 442 patients with osteoporosis identified a SNP in chromosome 2 with a large effect size as a marker for teriparatide response at lumbar spine. This variant was found to be an eQTL for CXCR4, a chemokine receptor involved in the activation of canonical Wnt signalling. To validate this finding at functional level, MC3T3-E1 osteoblast-like cells were cultured with osteogenic media for 7 days, and then stimulated with $100 \mathrm{nM}$ PTH for $48 \mathrm{~h}$. Vehicle-treated cells were used as controls. RNA from 6 independent experiments was quantified by qPCR and data was analysed using the $\Delta \Delta \mathrm{Ct}$ method. We found that teriparatide significantly increased $C X C R 4$ mRNA levels at $24 \mathrm{~h}$ (fold change mean $\pm \mathrm{SEM})(6.06 \pm 1.02$ vs. $1.29 \pm 0.28$, 
$\mathrm{p}=0.001)$ and $30 \mathrm{~h}(5.91 \pm 1.07$ vs. $1.13 \pm 0.13, \mathrm{p}=0.003)$ compared to controls. A trend of high levels of CXCR4 expression was also detected at $48 \mathrm{~h}(3.36 \pm 0.87$ vs. $1.66 \pm 0.21)$.

To investigate the effect of PTH in CXCR4 expression during osteoblast differentiation, MC3T3-E1 cells were cultured during 21 days in osteogenic media, and mRNA was collected every 7 days. Cultures were stimulated with $100 \mathrm{nM}$ PTH or vehicle $24 \mathrm{~h}$ before sample collection. We found that PTH increase $C X C R 4$ expression by $7.48 \pm 1.61$ fold at early stages of differentiation compared to control $\left(\mathrm{p}=6 \times 10^{-4}\right)$, and it continues highly expressed until the osteoblasts are completely functional $(6.65 \pm 1.63$ vs. $0.88 \pm 0.14$, $\mathrm{p}=0.01$ at 14 days; $5.16 \pm 1.22$ vs. $0.6 \pm 0.11, \mathrm{p}=0.004$ at 21 days).

We demonstrate that CXCR4 is a response gene for PTH during osteoblast differentiation, especially at early stages. These findings, together with the GWAS results, suggest that $C X C R 4$ could be a mediator of PTH anabolic effect. Further work is currently ongoing to fully understand its role in PTH response.

\section{$\mathbf{P 1 7 5}$}

Cell surface glycoprotein CD24 marks bone marrow derived hMSCs with reduced proliferation and differentiation in vitro

Jeroen van de Peppel ${ }^{1}$, Adriana Arruda Matos ${ }^{1}$, Tanja Strini ${ }^{1}$, Wenda Verschoor $^{1}$, Amel Dudakovic ${ }^{2}$, Gerben Schaaf ${ }^{3}$, Andre J. van Wijnen $^{2}$, Hans van Leeuwen ${ }^{1}$

${ }^{1}$ Internal Medicine, Erasmus MC, Rotterdam, Netherlands, ${ }^{2}$ Orthopedic Surgery, Mayo Clinic, Rochester, United States, ${ }^{3}$ Pediatrics, Erasmus MC, Rotterdam, Netherlands

Mesenchymal stem/stromal cells (MSCs) are an excellent biological source for bone regenerative therapies, tissue engineering, and treatment of post-menopausal osteoporosis. To date, treatment of osteoporosis using bone marrow-derived MSCs is not yet standard clinical practice. Donor variation among patients, cell heterogeneity and unpredictable capacity for differentiation are among the current shortcomings of hMSCs for their application in regenerative cell therapies.

Previous gene expression profiling studies in our lab identified the cell surface glycoprotein CD24 which was among the highest differentially expressed cell surface genes between osteogenic and adipogenic differentiation hMSCs. Therefore our hypothesis was if CD24 may well serve for the selection of a more homogeneous subpopulation with increased osteoblast function.

In non-differentiated hMSCs, CD24 cell surface expression ranged from 2 to $10 \%$ and was $2-4$ fold increased upon osteogenic differentiation. Unexpectedly, CD24 positive cells sorted from nondifferentiated as well as osteogenic differentiated cells led to reduced mineralization and adipocyte differentiation capacity. Comparative RNAseq analyses of CD24pos and CD24neg sorted cells indicate that only a limited number of genes is differentially expressed (27 genes twofold higher, 16 genes twofold lower, 1\%FDR) and co-expressed with CD24. Among the downregulated genes we mainly found gene ontology terms that were associated with cell cycle regulation. Cell proliferation analyses confirmed that $\mathrm{CD} 24$ pos cells have a reduced number of proliferating cells.

Although the cell surface glycoprotein CD24 is specifically upregulated upon osteogenic differentiation of hMSC, the CD24pos cells illustrated a reduced differentiation capacity and proliferation in vitro. Since several genes associated to extracellular matrix were increased in the CD24 expressing cells, we hypothesize that CD24 expressing cells are prematurely differentiated and unable to generate a functional extracellular matrix that can mineralized in vitro.

\section{P176}

Kruppel-like factor 10 (KLF10) as a mediator of shear stress in osteoblast like MC3T3-E1 cells

Norbert Hassler ${ }^{1}$, Martha Blank ${ }^{1}$, Silvia Spitzer ${ }^{1}$, Klaus Klaushofer ${ }^{1}$, Franz Varga ${ }^{1}$

${ }^{1}$ Ludwig Boltzmann Institute of Osteology at the Hanusch Hospital of WGKK and AUVA Trauma Centre Meidling, 1st Medical Department of Hanusch Hospital, Vienna, Austria

Bone tissue cells like osteoblasts are sensitive to shear stress induced by flow of culture medium. The zinc finger protein KLF10 is a member of the Kruppel-like family of 17 transcription factors and belongs to the transforming growth factor (TGF)-beta inducible proteins. KLF10 influences cell differentiation and is involved in human diseases like breast cancer and osteoporosis. Our aim was to investigate the influence of shear stress on RNA expression of Klf10 and stress related genes.

We cultured preosteoblastic MC3T3-E1 cells in a flow chamber at permanent fluid shear stress of $0.02 \mathrm{dyn} / \mathrm{cm}^{2}$ (dynamic cultures) and at stationary conditions (static cultures) for 1 and 3 days. Latter cultures served as controls. After extraction of RNA, the expression of Klf1O and selected genes known to respond to shear stress has been determined by real time-qPCR. Additionally, we subjected static cultures to a forced expression of Klf10 to determine the influence of KLF10 on the expression of stress related genes in the absence of culture medium flow.

Compared to static cultures (control) we found a significant upregulation of $\operatorname{KlflO}(2.3 \pm 0.4$ fold $)$ in dynamic cultures together with substantially up-regulated stress and inflammation related genes like Nitric oxide synthase 2 (Nos2; $36.9 \pm 9.4$ fold), Lipocalin 2 (Lcn2; $16.1 \pm 4.5$ fold $)$, Dentin matrix protein 1 (Dmp1; $33.6 \pm 2.3$ fold $)$, Early growth response 1 and 2 (Egrl; $2.7 \pm 0.4$ fold and Egr2; $10.3 \pm 1.1$ fold). Forced expression of Klf10 in static cultures induced a significant up-regulation of Nos2 (7.2 \pm 1.7 fold) and $L c n 2$ (76.7 \pm 20.6 fold), whereas the expression of Dmp1, Egrl, and Egr2 stayed at the level of corresponding controls.

KLF10 modulated the expression of Nos 2 and Lcn2 in dynamic cultures, whereas the elevated expression of Dmp1, Egrl, and Egr2 indicated the action of a shear stress transducing pathway where KLF10 is not involved.

Keywords: Klf10, shear stress, dynamic culture, Nos2, Dmp1

\section{P177}

Characterization of extracellular vesicles obtained from murine mesenchymal D1 cell cultures

Martha Blank $^{1}$, Norbert Hassler ${ }^{1}$, Stéphane Blouin ${ }^{1}$, Peter Pichler ${ }^{2}$, Goran Mitulovic $^{3}$, Silvia Spitzer ${ }^{1}$, Klaus Klaushofer ${ }^{1}$, Franz Varga ${ }^{1}$

${ }^{1}$ Ludwig Boltzmann Institute of Osteology at the Hanusch Hospital of WGKK and AUVA Trauma Centre Meidling, 1st Medical Department of Hanusch Hospital, Vienna, Austria, ${ }^{2}$ University of Vienna, Department of Analytical Chemistry, Vienna, Austria, ${ }^{3}$ Medical University of Vienna, Core Facility Proteomics, Clinical Institute of Laboratory Medicine, Vienna, Austria

Extracellular vesicles (EVs) are spherical particles shed by cells to interact with other cells and are found in body fluids like blood and urine. The EVs released by bone forming cells are thought to be involved in the initiation of bone mineralization by formation of hydroxyapatitic nuclei. Our aim was to isolate EVs from osteoblastlike cells and further characterize them with different physicalchemical methods.

Murine mesenchymal D1 cells have been cultured for 17 days without and in presence of beta-glycerophosphate (beta-GP). We 
isolated EVs from the supernatant of cell cultures before and after the mineral deposition that was tracked by alizarin red staining. EVs isolated from cell cultures without beta-GP treatment were used as a control. Characterization of EVs was done by immune blotting of vesicle marker like heat shock protein 70 (HSP70), energy dispersive $\mathrm{X}$-ray scattering (EDX), Fourier transformed infrared (FTIR) spectroscopy and mass spectrometry.

Isolation of EVs was verified by immune blotting of the exosome marker HSP70 and qualitatively confirmed by mass spectrometry with the finding of EV-associated markers, e.g. HSP70, CD63, CD81 and annexins, as well as, bone related proteins, e.g. bone morphogenetic protein 1 and periostin. EDX revealed that the calcium amount of EVs of beta-GP treated cells before mineral deposition was significantly higher (2.3-fold \pm 0.6$)$ compared to untreated control. FTIR spectroscopic analysis showed that the phosphate content normalized to protein amount of EVs isolated from treated cells after mineral deposition was significantly decreased ( 0.7 -fold \pm 0.1$)$.

Current study showed differences in matrix vesicle composition of EVs isolated from treated and untreated cell cultures, especially the calcium and phosphate content was different. This suggests that EVs play an important role in the mineralization of the extracellular matrix.

Keywords: Extracellular vesicle, energy dispersive X-ray scattering, Fourier transformed infrared spectroscopy, mass spectrometry, HSP70

\section{$\mathbf{P 1 7 8}$}

Effects of icaritin on osteogenic differentiation of $\mathrm{C} 2 \mathrm{C} 12$ cells

Dan-Bi Park ${ }^{1}$, Hee Su Lee ${ }^{2}$, Seong-Hee Ko

${ }^{1}$ Pharmacology, College of Dentistry and Research Institute of Oral Science, Gangneung-Wonju National University, Gangneung, Republic of Korea ${ }^{2}$ Oral anatomy, College of Dentistry and Research Institute of Oral Science, Gangneung-Wonju National University, Gangneung, Republic of Korea

The most current therapies for osteoporosis have focused on inhibiting bone resorption by osteoclasts. To develop the new anabolic agents for treatment of osteoporosis that have fewer risks compared to conventional therapies, we searched the natural products that derived from traditional Asian medicines which have been used for treatment of bone related diseases. Icaritin is a flavonoid glycoside derived from herb Epimedium which has beneficial effects on bone formation. Several studies have shown that icaritin stimulates osteoblast differentiation. To determine the mechanism that icaritin promote osteogenic differentiation, we examined the effects of icaritin on the osteogenic differentiation of $\mathrm{C} 2 \mathrm{C} 12$ cell, undifferentiated non osteogenic cells and the underlying signal pathway. For determining the effects of icaritin on proliferation, we did MTT assay of $\mathrm{C} 2 \mathrm{C} 12$ cells. To evaluate whether icaritin could promote the osteogenic differentiation of $\mathrm{C} 2 \mathrm{C} 12$ cells, mRNA expression of runtrelated transcription factor-2 (Runx2), osteocalcin ( $\mathrm{OCN})$, alkaline phosphatase (ALP) and osterix was determined by quantitative real time PCR analysis. To characterize the underlying mechanisms, we examined the effects of inhibitors of extracellular signal-regulated kinase 1/2 (ERK1/2), c-JUN N-terminal kinase (JNK) and p38 mitogen-activated kinase (MAPK) on icaritin-mediated mRNA expression of Runx-2 and OCN.

Icaritin significantly increased $\mathrm{C} 2 \mathrm{C} 12$ cell proliferation. mRNA expression of Runx2, OCN, ALP and osterix after $72 \mathrm{~h}$ culture with icaritin was significantly increased. Icaritin-mediated effects on mRNA expression of Runx-2 and OCN were dramatically attenuated by treatment with specific inhibitors of MAPKs, U0126 (ERK inhibitor), SP600125 (JNK inhibitor) and SB203585 (p38 kinase inhibitor). These results suggest that Icaritin not only stimulates the maturation of committed osteoblast progenitors, but also induces the commitment of undifferentiated non-osteogenic cells into osteoblasts by increasing expression of Runx2 through ERK, JUN, p38 pathway activation.

Keywords: osteoblast differentiation, $\mathrm{C} 2 \mathrm{C} 12$ cell line, icaritin

\section{P179}

Wnt/ $\beta$-catenin signaling mediates HDAC inhibitor-induced osteoblast differentiation

Jeong-Hwa Baek ${ }^{1}$, Jae-Ran Seo ${ }^{1}$, Kyung Mi Woo ${ }^{1}$, Hyun-Mo Ryoo ${ }^{1}$, Yun-Sil Lee ${ }^{1}$, Hyun Jeong Kim ${ }^{1}$, Jin Chung ${ }^{2}$

${ }^{I}$ Seoul National University School of Dentistry, Seoul, Republic of Korea, ${ }^{2}$ Busan National University School of Dentistry, Yangsan, Republic of Korea

Histone deacetylase inhibitors (HDIs) influence a broad repertoire of biological processes, including cellular proliferation, differentiation, and cell death, via inhibiting the acetylation of both histone and non-histone proteins. Currently, HDIs are used as anti-cancer and anti-epileptic drug; however, positive effects for bone regeneration have recently been reported. Mechanisms by which HDIs are believed to stimulate osteoblast differentiation include the acetylation of Runx 2 and osterix; however, a further understanding of additional mechanisms is needed. In this study, the underlying mechanisms of HDIs' enhancement of osteogenic differentiation was investigated by focusing on the regulatory role of $\mathrm{Wnt} / \beta$-catenin signaling and the Smurf1 expression. To this end, we used trichostatin A (TSA), suberoylanilide hydroxamic acid (SAHA), and MS275 as HDIs, along with cultured $\mathrm{C} 2 \mathrm{C} 12$ cells and primary cultured mouse calvarial cells (MC cells). To elucidate the effects of HDIs on osteoblast differentiation, $\mathrm{C} 2 \mathrm{C} 12$ cells were incubated with HDIs and a sub-minimal concentration of bone morphogenetic protein 2 (BMP2) followed by alkaline phosphatase (ALP) staining, ALP activity assays, and quantitative reverse transcription-polymerase chain reaction (qRTPCR) for bone marker genes. $\mathrm{C} 2 \mathrm{C} 12$ cells were treated with only HDIs for select parts of the experiment to verify the molecular mechanisms of HDIs. Experimental results demonstrated that $\mathrm{C} 2 \mathrm{C} 12$ cells differentiated into osteoblasts when incubated with HDIs and BMP2, but not when treated with BMP2 alone. HDIs activated Wnt/ $\beta$-catenin signaling, with HDIs inducing the Wnt10A gene to the highest extent. In addition, HDIs repressed Smurf1 expression and these effects were dependent on $\mathrm{Wnt} / \beta$-catenin signaling. Taken together, these results suggest that HDIs enhance osteoblast differentiation through the increased expression of Wnt10A and the activation of $\mathrm{Wnt} / \beta$-catenin signaling and subsequent decrease in Smurf1 expression.

Keywords: Histone deacetylase inhibitors, Osteoblast, Wnt10A, Smurf1

\section{P180}

Circadian clock components in human dental pulp-derived cells under hypoxic conditions

Klara Janjic $^{1,2}$, Christoph Kurzmann ${ }^{1,2}$, Andreas Moritz ${ }^{1,2}$, Hermann Agis ${ }^{1,2}$

${ }^{1}$ School of Dentistry, Medical University of Vienna, Vienna, Austria, ${ }^{2}$ Austrian Cluster for Tissue Regeneration, Vienna, Austria

The circadian clock is responsible for synchronizing physiological processes such as regeneration to cycling changes in the environment. Current literature suggests a potential connection between the circadian clock and hypoxia. Hypoxia is a prevailing condition during dental pulp regeneration after trauma. The presence and role of the 
circadian clock in dental pulps of adults, in particular under hypoxia, has not been described yet. Under the hypothesis that hypoxia could have an effect on the circadian clock during dental pulp regeneration, we aimed to reveal the production of circadian core clock components in human dental pulp-derived cells (DPC) and their modulation by hypoxia or the hypoxia mimetic agent L-Mimosine (L-MIM).

Human DPC were prepared in monolayer and spheroid cultures and treated with hypoxia or L-MIM. mRNA levels of the mammalian core circadian clock components CLOCK, BMAL1, CRY1-2 and PER1-3 were measured with qPCR. Protein levels of significantly modulated genes, CLOCK, CRY1, CRY2 and PER3, were measured with Western Blot.

All measured components were detected at mRNA levels in both culture models. CLOCK, CRY1, CRY2 and PER3 were downregulated at mRNA levels after hypoxia and L-MIM treatment. CLOCK, CRY1, CRY2 and PER3 were produced, but not modulated at protein levels. Results of further time course experiments suggest that downregulations at mRNA levels are based on a decreased amplitude while the response differs at the protein level from the response on the mRNA level.

Taken together, these results confirm that core circadian clock components are expressed in human DPC of adults and that hypoxic conditions can affect the production. Future studies will focus on the specific role of the circadian clock system in dental pulp regeneration. Keywords: Circadian clock, Dental Pulp, Hypoxia, Regeneration, Spheroids

\section{P181}

Skp2 inhibits osteogenesis by promoting ubiquitin-proteasome degradation of Runx 2

Gatha Thacker ${ }^{1}$, Yogesh Kumar ${ }^{1}$, Mohd. Parvez Khan ${ }^{2}$, Nidhi Shukla $^{1}$, Isha Kapoor ${ }^{1}$, Jitendra Kumar Kanaujiya ${ }^{1}$, Savita Lochab ${ }^{1}$, Shakil Ahmed ${ }^{3}$, Sabyasachi Sanyal ${ }^{1}$, Naibedya Chattopadhyay ${ }^{2}$, Arun Kumar Trivedi ${ }^{1}$

${ }^{1}$ Biochemistry, CSIR-Central Drug Research Institute, Lucknow, India, ${ }^{2}$ Division of Endocrinology and Center for Research in Anabolic Skeletal Targets in Health and Illness (ASTHI), CSIR-Central Drug Research Institute, Lucknow, India, ${ }^{3}$ Molecular and Structural Biology Division, CSIR-Central Drug Research Institute, Lucknow, India

Osteogenic transcription factor Runx2 is essential for osteoblast differentiation. Activity of Runx 2 is tightly regulated at post-transcriptional as well as post-translational level. However very less E3 ubiquitin ligases are known to regulate Runx 2 stability by ubiquitin mediated proteasomal degradation pathway.

Here, for the first time we demonstrate that Skp2, an SCF family E3 ubiquitin ligase negatively target Runx 2 by promoting its polyubiquitination and proteasome dependent degradation. Coimmunoprecipitation studies revealed that Skp2 physically interacts with Runx 2 both in a heterologous as well as physiologically relevant system. Functional consequences of Runx2-Skp2 physical interaction was then assessed by promoter reporter assay. We show that Skp2mediated downregulation of Runx 2 led to reduced Runx2 transactivation and osteoblast differentiation. On the contrary, inhibition of Skp2 restored Runx2 levels and promoted osteoblast differentiation. We further show that both the proteins are co-expressed and show inverse relation in vivo such as lactating, ovariectomized and estrogen-treated ovariectomized animals.

Taken together, our data demonstrate Skp2 target Runx2 for ubiquitin mediated degradation and hence negatively regulates osteogenesis. Therefore, the present study provides a plausible therapeutic target for osteoporosis or CCD.
Keywords: Runx2, Osteoblast, Skp2, Polyubiquitination, Osteogenesis

\section{P182}

Elevated extracellular calcium ions promote proliferation and migration of mesenchymal stem cells via increasing osteopontin expression

Jeong-Tae $\mathrm{Koh}^{1,2}$, Mi Nam Lee ${ }^{1,2}$, Hee-Su Hwang ${ }^{1,2}$, Sin-Hye $\mathrm{Oh}^{1,2}$, Jung-Woo Kim $^{1,2}$, Ju Han Song ${ }^{1,2}$, Yun-Chan Hwang ${ }^{2,3}$, Je-Hwang $\mathrm{Ryu}^{1,2}$

${ }^{1}$ Department of Pharmacology and Dental Therapeutics, Chonnam National University School of Dentistry, Gwangju, Republic of Korea, ${ }^{2}$ Research Center for Biomineralization Disorders, Gwangju, Republic of Korea, ${ }^{3}$ Department of Conservative Dentistry, Chonnam National University School of Dentistry, Gwangju, Republic of Korea

Background: Supplementation of mesenchymal stem cells (MSCs) at sites of bone resorption is required for bone homeostasis because of the non-proliferation and short lifespan properties of the osteoblasts. Calcium ions $\left(\mathrm{Ca}^{2+}\right)$ are released from the bone surfaces during osteoclast-mediated bone resorption. However, how elevated extracellular $\mathrm{Ca}^{2+}$ concentrations would alter MSCs migration in the proximal sites of bone resorption is largely unknown. In this study, the effects of extracellular $\mathrm{Ca}^{2+}$ on MSCs phenotype were examined.

Methods: Primary mouse bone marrow MSCs (BM-MSCs) or C3H10T1/2 cells were cultured with various concentration of $\mathrm{Ca}^{2+}$, and then cell proliferation, differentiation, migration, RT-PCR Western blot, and enzyme-linked immunosorbent (ELISA) assays were performed. For more clarifying $\mathrm{Ca}^{2+}$ effects under in vivo mimic condition, mouse calvarial bone slices were cultured with RANKLactivated osteoclasts. After that, calcium, ELISA and proliferation assays were done.

Results: The elevated extracellular $\mathrm{Ca}^{2+}$ (within $10 \mathrm{mM}$ ) promoted cell proliferation, matrix mineralization, and migration of BMMSCs or C3H10T1/2. The calcium-treated MSCs increased the expression and secretion of osteopontin (OPN). In calvarial tissue cultures, the bone resorption conditioned medium contained high level of $\mathrm{Ca}^{2+}$ and OPN protein compared to control groups. Treatment of the conditioned medium increased proliferation of BMMSCs.

Conclusion: These findings suggest that the dynamic changes in $\mathrm{Ca}^{2+}$ concentrations in the microenvironments of bone remodeling surfaces modulate MSCs phenotype and thereby contribute to bone regeneration.

\section{P183}

Sprouty 2 in intramembranous ossification

Eva Svandova ${ }^{1}$, Barbora Vesela ${ }^{1}$, Adela Kratochvilova ${ }^{1}$, Maria Hovorakova $^{2}$, Renata Peterkova ${ }^{2}$, Herve Lesot ${ }^{1}$, Eva Matalova ${ }^{1,3}$

${ }^{1}$ Institute of Animal Physiology and Genetics, CAS, v.v.i., Brno, Czech Republic, ${ }^{2}$ Institute of Experimental Medicine CAS, v.v.i, Prague, Czech Republic, ${ }^{3}$ University of Veterinary and Pharmaceutical Sciences, Brno, Czech Republic

Sprouty 2 (SPRY2) acts as a negative feedback regulator of multiple receptor tyrosine kinases including receptors for growth factors such as FGFs. Among the Sprouty family, Spry2 was identified as a non-redundant component promoting skeletal development. Spry2 functions were investigated in several developmental processes, e.g. lung branching and kidney morphogenesis, and also in limb development including osteogenesis. In long bones originating from pre-cartilagenous condensation via endochondral ossification, 
the effect of Spry2 was associated particularly with chondrocytes (Joo et al. 2017) which is supported by the requirement of Spry2 for temporospatial joint development (Purcell et al. 2012).

This study was designed to investigate the impact of Spry2 deficiency on osteogenic molecular profile in the developing alveolar/mandibular bone, an example of the intramembranous bones lacking the intermediary cartilaginous step. Spry2 knock-out mice (courtesy from Prof. O. Klein) were used and the bone samples were dissected at the day 15 of embryonic development, when the first osteoblasts, osteoclasts and osteocytes had differentiated within the jaw. After RNA isolation and cDNA preparation, wild type and knock-out samples were compared using Osteogenic PCR Array (Qiagen).

The preliminary results indicated increased gene expression for Fgfr1, Phex, Sox9, Tgfb2 and biglycan in the Sprouty 2 deficient samples. These findings support the hypothesis that Spry2 would have a role not only in regulation of chondrogenesis but also osteogenesis. Further experiments are running in progress to search for details related to more precisely specify the osteogenic impact of Sprouty 2. Keywords: osteogenesis, intramembranous, Spry2-I-

References: Joo et al. 2017: Bone. https://doi.org/10.1016/j.bone.2016.04.023,

Purcell et al. 2012: Journal of Dental Research. https://doi.org/10.1177/0022034512438401

Supported by the Grant Agency of the Czech Republic 14-37368G.

\section{$\mathbf{P 1 8 4}$}

Differentiation and mineral deposition of murine mesenchymal D1 cells

Martha Blank $^{1}$, Norbert Hassler ${ }^{1}$, Silvia Spitzer ${ }^{1}$, Klaus Klaushofer ${ }^{1}$, Franz Varga ${ }^{1}$

${ }^{1}$ Ludwig Boltzmann Institute of Osteology at the Hanusch Hospital of WGKK and AUVA Trauma Centre Meidling, 1st Medical Department of Hanusch Hospital, Vienna, Austria

A regulated mineralization process is indispensable for healthy bone tissue and ensures a strong and robust structure of bone. This process can be divided into two phases-the first one including the initiation of mineralization followed by a fast increase of mineral within days and a second phase characterized by a slow rise lasting months up to years. Often, established cell lines are used to approximate the mineralization process in-vitro. The aim of our study was to investigate this first short period of mineralization by using cell cultures of murine mesenchymal D1 cells as an in-vitro system and study their differentiation. Murine D1 cells have been cultured for 17 days without and in presence of beta-glycerophosphate (beta-GP). Afterwards, RNA was isolated and expression of selected osteoblastic and osteocytic markers was determined by real time-qPCR to check the status of differentiation. Mineral deposition was tracked by alizarin red staining.

Only in presence of beta-GP, D1 cell cultures started to accumulate mineral after 7 days while untreated cells did not start depositing mineral. After major mineral deposition (10 days) they showed a significant higher expression of differentiation markers of the mature osteoblasts Runx2 (2.7 \pm 0.1 fold) osterix ( $S p 7)$ ( $2.3 \pm 0.64$ fold $)$ and Dmpl ( $7.7 \pm 0.38$ fold) compared to treated cells before mineral deposition. Moreover, osteocalcin was highest expressed when major mineral deposition was detected and Sost 1 expression was at a maximum at day 17.

Runx2 and osterix expression suggested that D1 cells were differentiated into osteoblastic-like cells at day 10 at the onset of mineral deposition. High levels of Sost 1 and Dmpl expression indicated that at the end of culturing time D1 cells were already differentiated into an osteocytic-like cell state. In conclusion, we established an in-vitro system capable to study mineral deposition within the stated period of time.

Keywords: Differentiation, mineral deposition

\section{P185}

Comprehensive analysis of osteoclastogenic proteome reshaping reveals a novel regulator of osteoclast differentiation and function Cecilia Facchi ${ }^{1}$, Enrico Milan ${ }^{1,2}$, Maria Scolari ${ }^{1}$, Simone Cenci ${ }^{1,2}$ ${ }^{1}$ Genetics and Cell Biology, San Raffaele Scientific Institute, Milan, Italy, ${ }^{2}$ Vita-Salute San Raffaele University, Milan, Italy

Osteoclasts (OCs) are unique bone marrow monocyte (BMM)derived syncytia ensuring skeletal homeostasis through regulated bone resorption. We deployed two unbiased approaches to comprehensively characterize the proteome reshaping of OCgenesis in order to identify new regulatory mechanisms and putative targets for skeletal disorders. We compared the proteome of mouse purified $\mathrm{BMMs}$ and BMM-generated OCs through stable isotope labelling with amino acids in cell culture (SILAC)-based quantitative comparative proteomics, subsequently validated by label-free massspectrometry quantification. Both approaches identified established OC markers, e.g. TRAP and cathepsin $\mathrm{K}$, among the proteins most upregulated in mature OCs, validating our strategy. Moreover, proteome analysis by cellular compartment demonstrated reorganization of specific organelles upon OCgenesis, including lysosomal reshaping and increased mitochondrial mass, accounted for by higher expression of enzymes of the TCA cycle and oxidative phosphorylation. Finally, both proteomic analyses identified proteins distinctively expressed upon OCgenesis, previously not associated with OC function. These included ubiquitin (Ub) carboxy-terminal hydrolase L1 (UCHL1), a deubiquitinating enzyme. UCHL1 is a key component of the Ub proteasome system (UPS) required for axonal integrity implicated in neurodegeneration. UCHL1 was $\sim 9$ times more abundant by SILAC in OCs as compared to BMMs. Quantitative RT-PCR and immunoblotting analyses revealed a significant rise of UCHL1 transcript and protein expression (fourfold and $>10$-fold, respectively) early in OCgenesis ( 3 days post RANKL). Attesting to a relevant role of UCHL1 in OC homeostasis, immunofluorescence revealed progressive accumulation of $\mathrm{Ub}^{+}$protein aggregates during OCgenesis, and pharmacological inhibition of UCHL1 potently suppressed OC differentiation and resorptive activity in vitro. Overall, our work: (i) offers a powerful strategy for the identification of novel regulators of OC activity; (ii) uncovers the coordinated reorganization of organelle composition and metabolic activities upon OCgenesis; and (iii) reveals a previously unrecognized key role of the UPS component, UCHL1 in OC differentiation and function.

Keywords: mitochondria; osteoclast; proteomics; ubiquitin; UCHL1

\section{P186}

Fra1 dependent IgG1 secretion by bone marrow B cells induces osteoporosis

Bettina Grötsch ${ }^{1}$, Anja Lux ${ }^{2}$, Anna-Carin Hoffmann ${ }^{3}$, Falk Nimmerjahn $^{2}$, Wei Xiang ${ }^{3}$, Hans Ulrich Scherer ${ }^{4}$, Georg Schett ${ }^{1}$, Aline Bozec ${ }^{1}$

${ }^{1}$ Rheumatology and Immunology, University Hospital Erlangen, Department of Medicine III, Erlangen, Germany, ${ }^{2}$ University Erlangen-Nuremberg, Division of Genetics, Erlangen, Germany, ${ }^{3}$ Department Biology, University of Erlangen-Nuremberg, Division of Biochemistry, Erlangen, Germany, ${ }^{4}$ Leiden University Medical Center, Leiden, Netherlands 
The main function of osteoclasts, derived from monocyte-macrophage precursors, is to resorb the bone matrix. Thereby, an excess of osteoclast activity is commonly associated with osteoporosis. During the course of rheumatoid arthritis, osteoclastogenesis is tightly controlled by immune cells. Indeed IgG immune complex binding to Fc receptors could enhance osteoclast differentiation and bone loss during rheumatoid arthritis development. However the regulation of the IgG immune complexes within the bone niche in absence of inflammatory trigger is not well understood. We used two independent models triggering IgG1 secretion by $\mathrm{B}$ cells, the $\mathrm{T}$ dependent immunization and the genetic mouse model of Fral deficiency in B cells to study the effect of IgG1 and IgG1 immune complexes on osteoclasts. We could show that the increase in bone marrow IgG1 was accompanied by decreased bone volume and trabecular thickness due to increased osteoclast numbers in both models. Mechanistically, deletion of Fra1 in B cells increases the formation of IgG1 immune complexes, which induce strong signaling via the FcRg/ITAM motif to increase osteoclast differentiation in absence of inflammatory triggers. By in vivo and in vitro experiments using $F c g R 3-/-$ and FralFcgR3 double knock out mouse models, we could demonstrate that the IgG1 immune complex induced osteoclast differentiation is indeed dependent on FcgR3 signaling. Thus, we provide an unprecedented role of $\mathrm{IgG}$ triggered osteoclast differentiation via the FcgR3 signaling pathway in pre-osteoclasts and highlight how transcription factors could interfere in the tightly controlled B cellosteoclast crosstalk. Understanding the tight signaling between osteoclasts and B cells will provide new perspectives of how to control immune cell induced osteoporosis.

Keywords: B cells, Osteoclasts, immune complex, Fra1

\section{P187}

Osteoclasts differentiated from iPS cells as a test system for gene therapeutic approaches for autosomal recessive osteopetrosis

Uta Rössler $^{1,2,3}$, Floriane Hennig ${ }^{1,2}$, Harald Stachelscheid ${ }^{2,3}$, Manfred Gossen $^{2,4}$, Zsuzsanna Izsvák ${ }^{5}$, Uwe Kornak ${ }^{1,2,6}$

${ }^{1}$ Institute for Medical Genetics and Human Genetics, Charité Universitätsmedizin Berlin, Berlin, Germany, ${ }^{2}$ Berlin-Brandenburg Center for Regenerative Therapies, Charité - Universitätsmedizin Berlin, Berlin, Germany, ${ }^{3}$ Berlin Institute of Health, Berlin, Germany, ${ }^{4}$ Institute of Biomaterial Science, Helmholtz-Zentrum Geesthacht, Teltow, Germany, ${ }^{5}$ Max-Delbrück-Center for Molecular Medicine in the Helmholtz Society, Berlin, Germany, ${ }^{6}$ Max Planck Institute for Molecular Genetics, Berlin, Germany

Autosomal recessive osteopetrosis (ARO) is a rare disease showing an obstruction of the bone marrow cavity due to the accumulation of bone tissue. Therefore, ARO patients suffer from anemia, immune deficiency, variable central nervous system problems, and usually die during childhood. A frequent cause are mutations in the CLCN7 gene that is crucial for osteoclast-mediated bone resorption. Currently, the only curative treatment for ARO is allogenic hematopoietic stem cell transplantation, which still harbors significant risks. Here, we report on an alternative gene therapy approach for ARO based on additive gene transfer by the Sleeping Beauty (SB) transposon.

Proof of concept was obtained in K562 cells, in which we achieved $25 \%$ stable expression of the CLCN7 cDNA from the integrated transposon that was increased to $95 \%$ after puromycin selection. In order to demonstrate the therapeutic effect of the $C L C N 7$ expression from the SB transposon vector, we opted for induced pluripotent stem cells (iPSCs) and generated an iPS cell line derived from an ARO patient harboring CLCN7 mutations. First, we established an efficient protocol to differentiate iPSCs into osteoclasts and proved their identity via fluorescent staining and quantitative RT-PCR of osteoclast markers. In contrast to the healthy osteoclasts, patient- derived osteoclasts were incapable of generating resorption pits on dentine slices. After optimization of the transfection method, we reached transfection rates of up to $85 \%$ and stable integration of the SB construct in $21 \%$ of the iPSCs. The effect on $\mathrm{ClC}-7$ protein expression and resorption activity is currently being investigated in osteoclasts derived from the transposon-containing iPSCs. This approach will be compared to a safe harbor integration of the rescue construct mediated by CRISPR/Cas9. The establishment of additive gene transfer strategies is the first step in the direction of personalized treatment for ARO patients improving success rates and prognosis.

Keywords: Osteopetrosis, iPSCs, osteoclasts, gene therapy

\section{P188}

In vivo deletion of Gulp1 resulted in high trabecular bone mass phenotypes in male mice

Soon-Young Kim ${ }^{1,2}$, Gun-Il Park ${ }^{1,2}$, Eun-Hye Lee ${ }^{1,2}$, Seung-Yoon Park $^{3}$, Yeon-Ju Lee ${ }^{1,2}$, Suk-Hee Lee ${ }^{1,2}$, Seung-Hoon Lee ${ }^{1,2}$, Hye Jung $\mathrm{Ihn}^{4}$, Eui Kyun Park ${ }^{4}$, Jung-Eun Kim ${ }^{1,2}$

${ }^{1}$ Department of Molecular Medicine, School of Medicine, Kyungpook National University, Daegu, Republic of Korea, ${ }^{2}$ CMRI, BK21 Plus KNU Biomedical Convergence Program, Daegu, Republic of Korea, ${ }^{3}$ Department of Biochemistry, School of Medicine, Dongguk University, Gyeongju, Republic of Korea, ${ }^{4}$ Department of Pathology and Regenerative Medicine, School of Dentistry, Kyungpook National University, Daegu, Republic of Korea

Engulfment adaptor phosphotyrosine-binding (PTB) domain containing 1 (GULP1) was originally identified as an adaptor protein involved in the engulfment of apoptotic cells via phagocytosis. Although GULP1 is widely expressed in various tissues, including the brain, muscle, testis, and bone, the function of GULP1 has not been well studied. This study investigated the role of GULP1 in the regulation of bone mass in vivo using Gulp1 knockout mice. Endogenous mRNA expression of Gulp1 was observed in primary osteoblasts and MC3T3-E1 osteoblastic cells, but not in osteoclasts. Gulp1 knockout mice have exhibited a significant increase in trabecular bone mass of the femur and tibia as well as the lumbar vertebrae, compared with wild-type littermates in males. However, the higher bone mass was not observed in female mice. While the dynamic bone formation of osteoblasts in Gulp1 knockout mice did not differ from that in wildtype mice, the number of fully differentiated osteoclasts and the surface area of the resorption pit in bone slices were lower in Gulp1 knockout mice than in wild-type mice. Actin ring and microtubule formation in osteoclasts were also inhibited in Gulp1 knockout mice. It was known that testosterone directly inhibits osteoclast formation and bone resorption at physiological concentrations. Interestingly, the serum level of testosterone in male Gulp1 knockout mice was 2.5-fold higher compared with wild-type mice and the mRNA expressions of Cyp2b9 and $2 \mathrm{~b} 10$ in the liver, which regulate testosterone metabolism, were significantly down-regulated in male Gulp1 knockout mice. Taken together, Gulp1 deficiency suppresses osteoclast function, which appears to be a result from reduced metabolism of testosterone due to downregulation of Cyp2b, finally resulting in high bone mass.

\section{P189}

Mild inhibition of Cathepsin K paradoxically stimulates the resorptive activity of osteoclasts co-cultured with osteoblast lineage cells

$\underline{\text { Dinisha Cyril Pirapaharan }}^{1}$, Kent Soe ${ }^{2}$, Preety Panwar ${ }^{3}$, Sandra Bjerre Christensen $^{4}$, Per Kjærsgaard-Andersen ${ }^{5}$, Jonna Skov Madsen ${ }^{6}$, Dieter Brömme ${ }^{3}$, Jean-Marie Delaisse ${ }^{1}$ 
${ }^{1}$ Clinical Cell Biology, Vejle Hospital/Lillebaelt Hospital, Institute of Regional Health Research, University of Southern Denmark, Vejle, Denmark, ${ }^{2}$ Clinical Cell Biology, Institute of Regional Health Research, University of Southern Denmark, Vejle, Denmark, ${ }^{3}$ Biochemistry and Molecular Biology, Faculty of Medicine, University of British Columbia, Vancouver, BC, Canada, ${ }^{4}$ Clinical Cell Biology, Vejle Hospital/Lillebaelt Hospital, University of Southern Denmark, Vejle, Denmark, ${ }^{5}$ Department of Orthopedic Surgery, Vejle Hospital/ Lillebaelt Hospital, Vejle, Denmark, ${ }^{6}$ Clinical Immunology and Biochemistry, Vejle Hospital/Lillebaelt Hospital, Institute of Regional Health Research, University of Southern Denmark, Vejle, Denmark

Odanacatib (ODN) is a specific inhibitor of Cathepsin K (CatK), the protease responsible for collagenolysis during osteoclastic bone resorption. Clinical trials have shown that ODN prevents bone loss at high doses, but paradoxically, the lowest dose induced accelerated bone loss compared with the placebo group. The present study throws light on this paradox. To investigate the low dose stimulation in vitro, we generated osteoclasts from human peripheral blood, cultured them on bone slices and assessed resorption in response to increasing doses of ODN. High doses decreased resorption, but lower doses induced up to $50 \%$ increased resorption compared with controls. An inhibitor of non-CatK cysteine-proteases prevented the ODN-induced low dose stimulation, thereby suggesting that the induction results from an interplay between CatK and other cys-proteases. Collagen fibers exposed to CatK in the presence of other proteases (Cat L, B and MMPs) showed up to threefold enhanced degradation compared to CatK alone. Next, we obtained osteoblastic lineage cells (OBs) from outgrowths of human trabecular bone and co-cultured them with matured osteoclasts in the absence of serum and RANKL. We first found that the presence of OBs promoted bone resorption, and that GM6001, an MMP inhibitor, counteracted this effect but not so in the absence of OBs. This indicates that MMPs of OBs may contribute to resorption. Importantly, when subjecting the co-cultures to increasing doses of ODN, a low dose-induced stimulation of resorption was achieved and this stimulation was four times higher than in cultures of osteoclasts alone.

We conclude, that a slight inhibition of CatK in osteoclast cultures may stimulate bone resorption, and even more so in co-cultures with OBs. This reproduces the low dose-induced resorption of the clinical study. Our data support a mechanism where a low dose of ODN results in generating synergy between collagenolytic proteases.

Keywords: Osteoclast, odanacatib

\section{P190}

Age-related increased osteoclastogenesis is due to increased phosphorylation of $\mathrm{p} 38$ and enhanced expression of V-ATPase

Jacqueline Lim ${ }^{1}$, Francesca Brito ${ }^{1}$, Gemma Charlesworth ${ }^{1}$, Rob van 't $\overline{\text { Hof }}^{1}$, Anna Daroszewska ${ }^{1}$

${ }^{1}$ Musculoskeletal Biology I, Institute of Ageing and Chronic Disease, University of Liverpool, Liverpool, United Kingdom

Osteoporosis, the most common age-related metabolic bone disease, is characterised by increased bone resorption, which is due to highly active osteoclasts. Osteoclast differentiation and activity is dependent on many signalling pathways including MAPK-ases and NFATc1. Osteoclastogenesis increases with ageing, however the underlying intrinsic factors enhancing age-related osteoclastogenesis are incompletely understood.

Here we sought to investigate the underlying mechanisms responsible for age-related increased osteoclastogenesis.

Osteoclasts were differentiated from bone marrow macrophages of young adult (3-month-old) and aged (12-14-month-old) C57B16 mice in vitro using M-CSF and RANKL and RANKL-dose and -time response assays were performed. Reverse-phase-array and Western
Blotting were performed on protein extracts and gene expression was assessed by qPCR.

M-CSF and RANKL-induced osteoclast formation was significantly greater in aged vs. young mice. Osteoclast precursors from aged mice showed a twofold increased sensitivity to RANKL at $10 \mathrm{ng} / \mathrm{ml}(\mathrm{p}<0.05), 30 \mathrm{ng} / \mathrm{ml}(\mathrm{p}<0.01)$ and $100 \mathrm{ng} / \mathrm{ml}(\mathrm{p}<0.05)$ of RANKL stimulation compared to young mice $(\mathrm{N}=3)$. The reverse-phase-array showed an increase in phospho-p38 in aged osteoclasts vs. young osteoclasts $(\mathrm{N}=2)$ and this was confirmed on Western Blotting $(\mathrm{N}=2)$. The area under the curve of the RANKL time response was $70 \pm 7 \%$ greater in old vs. young osteoclasts. The maximum phosphorylation of $\mathrm{p} 38$ was $18-75 \%$ higher at $15-20 \mathrm{~min}$ in old vs. young osteoclasts. On qPCR a twofold increase of V-ATPase d0 subunit expression was seen in mature old vs. young osteoclasts $(\mathrm{N}=3)$. V-ATPase is an indirect target of phospho-p38 via NFATc1 and the d0 subunit plays a role in cell fusion and is essential for acidification of the resorbing osteoclast lacunae.

Our results indicate that ageing leads to increased phosphorylation of p38, which induces expression of V-ATPase d0, which likely contributes to increased age-related osteoclastogenesis and bone resorption.

Keywords: Osteoclastogenesis, ageing, p38, V-ATPase, RANKL

\section{P191}

Transcription factor Sry regulates RANKL gene expression by binding to its proximal promoter region

Klemen Kodrič ${ }^{1}$, Janja Zupan ${ }^{1}$, Tilen Kranjc ${ }^{1}$, Radko Komadina ${ }^{2}$, Vid Mlakar $^{1}$, Nika Marija Lovšin ${ }^{1}$, Janja Marc ${ }^{1}$

${ }^{1}$ Chair of Clinical Biochemistry, University of Ljubljana, Faculty of Pharmacy, Ljubljana, Slovenia, ${ }^{2}$ Department for Research and Education, General and Teaching Hospital Celje, Celje, Slovenia

Aim of the study: RANKL is the most important regulator of osteoclastogenesis and has been associated with bone diseases, including osteoporosis. Its involvement in bone turnover is well understood; however, regulation of RANKL expression has not yet been completely explained. Most of the known transcription factors influence RANKL expression through its distal regulatory regions, while its proximal promoter region remains mostly unexamined. Our previous results revealed that the male-specific transcription factor Sry could prevent bone resorption and osteoporosis in males. The aim of present study was to examine if Sry could prevent bone resorption by regulating the RANKL gene expression through its proximal promoter region.

Methods: RANKL proximal promoter region was cloned into pGL3-basic reporter vector. Sry was cloned into pcDNA3-FLAG expression vector. The influence of Sry on activation/repression of RANKL gene proximal promoter was tested using luciferase assays. Direct interaction between RANKL proximal promoter and Sry was tested using EMSA. The impact of Sry overexpression on the regulation of RANKL gene expression was tested in human primary osteoblasts using real-time PCR.

Results: Results of our luciferase assays confirmed that Sry decreased the activity of the RANKL proximal promoter by $30 \%$. Results of EMSA confirmed that Sry directly binds to RANKL promoter region. Overexpression of Sry decreased the level of RANKL expression in human primary osteoblasts.

Conclusion: Our results suggest that the male specific transcription factor Sry regulates RANKL gene expression by binding to its proximal promoter region, revealing the possible mechanism of bone loss prevention in males.

Keywords: RANKL, Sry, osteoporosis 


\section{P192}

Sestrin 2 regulates osteoclast differentiation via the interaction with p62 and TRAF6

$\underline{\text { Namju Kang }}{ }^{1}$, Sue Young $\mathrm{Oh}^{1}$, Inik Chang ${ }^{1}$, Dong Min Shin ${ }^{1}$

${ }^{1}$ Department of Oral Biology, BK21 PLUS project, Yonsei University College of Dentistry, Seoul, Republic of Korea

Sestrin 2 (Sesn2), an autophagy inducer, interacts with p62 and Keap1 and prevents oxidative stress via Keap1-Nrf2 pathway. In addition, previous reports showed that p62 interacted with TRAF6 and leads to the activation of osteoclast differentiation. However, the role of Sesn2 in osteoclast differentiation is unknown. In the present work, we investigated the effects of Sesn 2 in osteoclast differentiation with Sesn2 knock-out (Sesn2-1-) mice. The bone mass of Sesn2-/mice increased more than that of wild type mice by $\mu \mathrm{CT}$ analysis. Also, the formation of multinuclear osteoclasts was inhibited in Sesn2-I- mice. Furthermore, NFATc1 and osteoclastogenesis-related gene expression were significantly diminished in Sesn2-/mice during osteoclast differentiation. RANKL-induced TRAF6 downstream pathways were delayed in osteoclasts of Sesn2-/- mice. The interaction of p62 and TRAF6 also decreased in Sesn2-/-mice. These results suggest that Sesn2 regulates osteoclast differentiation through interaction with p62 and TRAF6.

Keywords: Sestrin 2, p62, TRAF6, osteoclast differentiation, Sesn2-/- mice

\section{P193}

Inhibition of CDK8 suppresses osteoclastogenesis and promotes osteoblast differentiation in vitro

Mehdi Amirhosseini ${ }^{1}$, Johan Flygare ${ }^{2}$, Anna Fahlgren ${ }^{1}$

${ }^{I}$ Department of Clinical and Experimental Medicine (IKE), Linköping University, Linköping, Sweden, ${ }^{2}$ Department of Molecular Medicine and Gene Therapy, Lund University, Lund, Sweden

The Mediator complex, a master coordinator of transcription and cell lineage development, is suggested to play role in osteoblast differentiation (Liu 2016). We therefore asked if small molecule inhibitors of CDK8, the regulatory subunit of the Mediator complex, modulate osteoblast and osteoclast differentiation. We investigated effects of two selective CDK8 inhibitors (Senexin B and Compound2) on osteoclast and osteoblast differentiation in vitro.

Mouse bone marrow macrophages (BMMs) were cultured with or without the CDK8 inhibitors. We found that osteoclast differentiation was significantly suppressed by $1 \mu \mathrm{M}$ of both inhibitors. The inhibitors also reduced resorbed area on bovine bone chips by osteoclasts. qPCR showed a downregulation in p27 mRNA in BMM at 8 and $48 \mathrm{~h}$ by both inhibitors $(1 \mu \mathrm{M})$, and a transient decrease in $\mathrm{p} 21$ at $8 \mathrm{~h}$ by Senexin B compared to controls. p21 and p27 are both crucial for RANKL-induced osteoclast differentiation (Okahashi 2001; Sankar 2004). NFATc1 and DC-STAMP, two major regulators of osteoclast formation, were downregulated at $48 \mathrm{~h}$ by both inhibitors.
Both CDK8 inhibitors were able to increase ALP activity in primary mouse osteoblasts. We further demonstrated increased calcium deposition by osteoblasts with $1.5 \mu \mathrm{M}$ of the CDK8 inhibitors. No significant changes were induced by treatment in Runx 2 and Osterix mRNA levels. However, Stat1, which is reported to inhibit Runx2 nuclear translocation (Kim 2003), was significantly downregulated, compared to controls by CDK8 inhibitors.

DNA synthesis rate of BMM and osteoblasts were not affected by $1.5 \mu \mathrm{M}$ of the inhibitors, indicating that CDK8 inhibition exerts its effect on bone tissue through mechanisms other than regulation of cell cycle and proliferation.

Our data suggest CDK8 inhibition to be a promising approach to induce anti-catabolic and pro-anabolic effects on bone tissue in metastatic bone destruction, inflammatory osteolysis and bone implant loosening.

Keywords: Osteoclast; Osteoblast; CDK8

\section{P194}

Tartrate resistant acid phosphatase $5 \mathrm{a}$ is secreted during osteoclastogenesis and correlates to CTX-I levels in serum

Laia Mira-Pascual ${ }^{1}$, Christina Patlaka ${ }^{1}$, Staffan Paulie ${ }^{2}$, Tuomas Näreoja ${ }^{1}$, Pernilla Lång ${ }^{1}$, Göran Andersson ${ }^{1}$

${ }^{1}$ Karolinska Institutet, Huddinge, Sweden, ${ }^{2}$ Mabtech AB, Stockholm, Sweden

TRAP $5 b$ is used as a marker to estimate bone resorption rate and number of osteoclasts (OCs); 5a has been suggested to serve as an inflammatory marker. However, its relevance to bone remodeling has not been shown. This study was conducted to establish a timeline of TRAP production and processing of $5 \mathrm{a}$ to $5 \mathrm{~b}$ in differentiating OCs and to correlate that to CTX-I, in serum.

CD14+ human blood monocyte (PBMC) cells were stimulated with M-CSF and RANKL. TRAP activity staining and ICC were analyzed at different stages of OC differentiation, and TRAP 5a and $5 \mathrm{~b}$ protein concentrations were measured in medium and lysate using a two-step 5a/5b ELISA. Serum levels of TRAP isoforms were assessed in healthy men and correlated to CTX-I levels.

In lysates, TRAP isoforms were progressively increasing, with predominance of $5 \mathrm{~b}$. In medium, 5b levels increased over 10 times, but also $5 \mathrm{a}$ was secreted. In serum, there was a positive correlation between TRAP isoforms themselves (p-value: 0.007) and to CTX-I (p-value: 0.027) (Fig. 1A). This suggests that both isoforms are secreted from differentiating OCs during bone turnover. TRAP activity staining confirmed the appearance of multinucleated TRAP positive cells with varying activity levels. By ICC, TRAP 5a specific staining was apparent in many of the multinucleated and mononuclear cells, while total TRAP signal was lower in the large multinucleated cells, indicating effective secretion of the TRAP 5b isoform (Fig. 1B). A few mononuclear cells stained double-positive for both isoforms, indicating that TRAP processing starts already at a late mononuclear stage. 

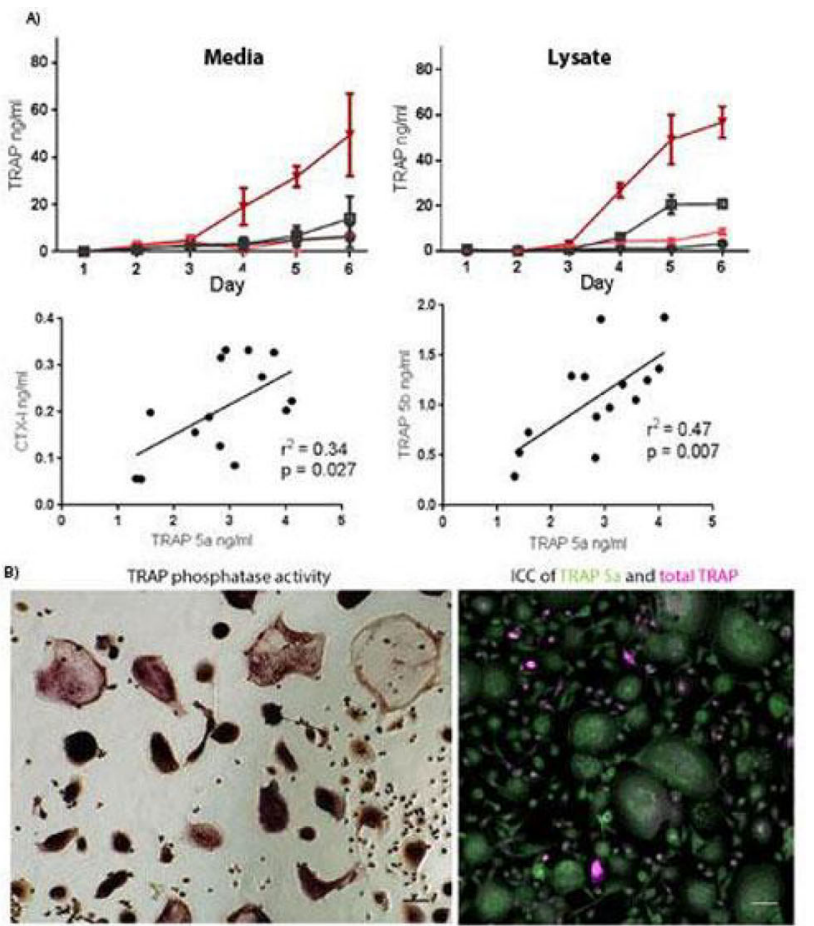

Figure 1. A) TRAP isoform distribution in media and lysates of CD14+ PBMCs and correlation of isoforms and 5 a to $C T X-1$ in human serum. PBMCS were seeded on plastic at density of $1.5 \times 105$ cells/well, alltures were terminated, medium was collected and bysates extracted daily, and finally both were measured with the two-step EUSA. Human sera were measured with the two-step EUSA and CTX-1 kit (DS). TRAP 5 a MCSF (-) , TRAP 5 a MCSF+RANKL ( -9$)$, TRAP $5 b$ MCSF ( $*)$, TRAP $5 b$ MCSF+RANKL $(*)$. B) TRAP staining of M-CSF and RANKL stimulated CD 14+PBMCs. PBNCs were seeded in plastic or glass at $1.5 \times 10^{\circ}$ cells/well and stained at day 6 with TRAP phosphatase activity staining and in ICC with TRAP 5 a specific mAb and a polyclonal antibody recognizing both isoforms. Scale bar IHC $100 \mu \mathrm{m} I C C 50 \mu \mathrm{m}$, $5 \mathrm{a}$; green; $5 \mathrm{a}+5 \mathrm{~b}$; magenta; equal staining both antibodies; white

\section{P195}

Heterogeneity of macrophage progenitor RAW 264.7 cell line

Laia Mira Pascual ${ }^{1}$, Tuomas Näreoja ${ }^{1}$, Göran Andersson ${ }^{1}$, Pernilla Lång $^{1}$

${ }^{1}$ Division of Pathology, Department of Laboratory Medicine, Karolinska Institutet, Stockholm, Sweden

The macrophage progenitor RAW 264.7 cell line may be differentiated into an osteoclast (OC) like cell; however, efficacy of this process is low. We hypothesize that this may be due to a fraction of these cells being unable to differentiate, and therefor, we wanted to enhance differentiation of the RAW line and understand the hallmarks of decision points in differentiation of these cells to OC or macrophage direction.

To characterize our parental RAW cell line we sub-cloned cell lines from it and measured their inherent TRAP gene expression to illustrate the heterogeneity of RAW population (Fig. 1). We examined this observation on mRNA level of TRAP, CtsK and MMP9 and found that the response to receptor activator of nuclear factor kappaB ligand (RANKL)-stimulus was significantly lower (Fig. 1) than with the RAW cells, but also that levels in $\mathrm{H} 9$ were high even without RANKL and in J8 levels remained low despite RANKL. To establish the significance of this observation we quantified the emergence of multinuclear TRAP positive cells in cultures of the sub-clones. The H9-line developed significantly more TRAP + OCs compared to RAW cells (H9, 350; RAW, 20: p < 0.001) on day 2 of stimulation (Fig. 2). On day 4 the RAW line had caught up the difference, while the line least likely to differentiate, J8, had not (Fig. 2).
The $\mathrm{J} 8$ cell line is unable to obtain similar level of OC functionality as the other two lines. To determine have they begun to irreversibly differentiate towards macrophage lineage or do they retain higher level of stemness, we exposed the cell lines to osteoprotegerin and with macrophage colony stimulating factor combined with lipopolysaccharide to measure macrophage differentiation. In summary, we present the timeline of decision points for fully committed differentiation to OCs or macrophages.
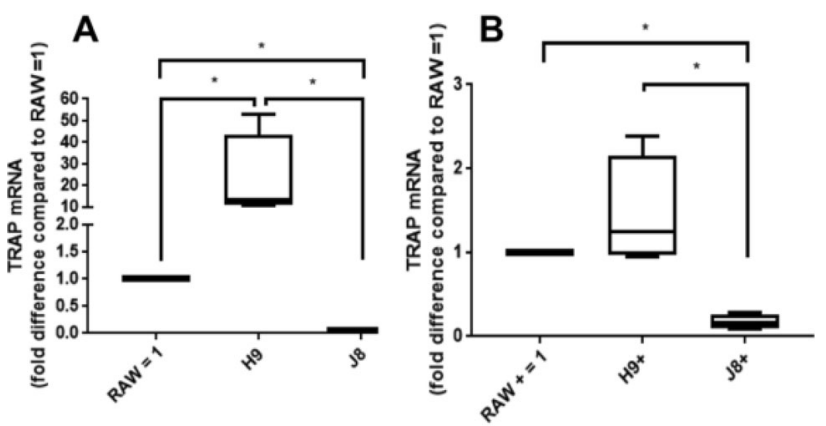

Figure 1. Quantitative PCR of TRAP expression in (A) un-stimulated and (B) $10 \mathrm{ng} / \mathrm{ml}$ RANKL stimulated RAW, H9 and J8 cells. mRNA expression is shown relative to parental RAW cell line. Statistical evaluation one-way ANOVA with Tukey's multiple comparisons test $\left({ }^{*}=p<0,05\right)$.

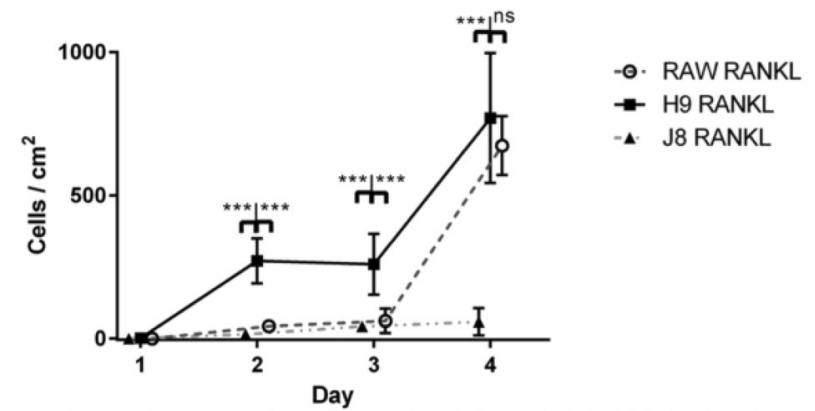

Figure 2. Osteoclastogenesis of $10 \mathrm{ng} / \mathrm{ml}$ RANKL stimulated parental RAW cells and selected clones, $\mathrm{H} 9$ and J8.

Cultures were stained simultaneously on days 1-4. Statistical evaluation one-way ANOVA with Tukey's multiple comparisons test $\left(^{* * *}=p<0,001\right)$.

\section{P196}

RACK1 regulates osteoclast function via its interaction with c-Src Sol Yi ${ }^{1}$, Ji Hee Kim ${ }^{1}$, Jin Hee Park ${ }^{1}$, Etteum Jeong ${ }^{1}$, Soo Young Lee ${ }^{1}$ ${ }^{1}$ College of Natural Sciences, Ewha Womans University, Seoul, Republic of Korea

The scaffolding protein receptor for activated C-kinase 1 (RACK1) mediates RANKL-dependent activation of p38 MAPK in osteoclast precursors. However, the role of RACK1 in mature osteoclasts has remained unclear. Here we report that the interaction between RACK1 and c-Src is critical for osteoclast function. A RACK1 mutant protein (RACK1 Y228F/Y246F) was unable to interact with c-Src. The mutant retained its ability to differentiate into osteoclasts; however, RANKL-mediated cytoskeleton integrity, bone resorption activity, and c-Src's phosphorylation were significantly decreased. These findings will not only clarify the role of RACK1 as a key regulator of osteoclast function but will also help in the development of new anti-resorption therapies designed to prevent bone loss-related diseases. 


\section{P197}

Assessment of fluid flow through the osteocyte lacuno-canalicular network in whole human osteons of different type

Alexander F. van Tol ${ }^{1}$, Andreas Roschger ${ }^{1}$, Junning Chen ${ }^{1,2}$, Felix Repp $^{1}$, Philip Kollmannsberger ${ }^{3}$, Paul Roschger ${ }^{4}$, Peter Fratzl ${ }^{1}$, Richard Weinkamer ${ }^{1}$

${ }^{I}$ Biomaterials, Max Planck Institute of Colloids and Interfaces, Potsdam, Germany, ${ }^{2}$ College of Engineering, Mathematics and Physical Sciences, University of Exeter, Exeter, United Kingdom, ${ }^{3}$ Center for Computational and Theoretical Biology, Universität Würzburg, Würzburg, Germany, ${ }^{4}$ Ludwig Boltzmann Institute of Osteology at the Hanusch Hospital of WGKK and AUVA Trauma Centre Meidling, Vienna, Austria

Osteocytes use a complex network of cavities and sub-micrometer wide canals, the osteocyte lacuno-canalicular network (OLCN), to respectively house their cell bodies and to connect to other osteocytes. The architecture of the OLCN is thought to be related to the multiple functions of the network including (i) mechano-sensation via the detection of fluid flow through canaliculi and lacunae induced by mechanical loads; (ii) transport of nutrients and signaling molecules; (iii) mineral deposition and resorption (osteocytic osteolysis). We aimed to assess the influence of the network topology in different types of osteons on interstitial fluid flow through the OLCN.

From the midshaft femoral bone of a healthy middle-aged woman (EK\#1757/2013) 8 normal osteons and 9 so-called osteon-in-osteons (type II osteons) were selected. After rhodamine staining the canalicular network architecture was determined using confocal laser scanning microscopy and image analysis [1]. To assess the fluid flow and pressure patterns induced by dynamic loading assuming a constant cross-section of the canaliculi, a system of equations analogous to electric circuits was solved.

The connectivity of the OLCN in osteon-in-osteons was locally strongly reduced with only a few bridges connecting the inner smaller osteon with the outer osteon. Fluid flow velocities attained maximum values in these bridges while the fluid flow velocity in the rest of the osteon was on average 65 times lower. The corresponding pressure pattern displayed a stepwise pressure increase over the bridging regions. In contrast fluid flow patterns in normal osteons were homogeneous over the whole osteon and the pressure showed a gradual radial dependency. A correct assessment of the mechanosensitivity of osteons, therefore, depends strongly on whether the average or the maximum fluid flow velocity is the key trigger of mechano-transduction in osteocytes.

References: [1] Repp et al., Bone Reports 6, 101 (2017).

Keywords: mechano-sensation, canalicular network, fluid flow, osteon-in-osteon, confocal microscopy

\section{P198}

Effect of mechanical loading on osteocyte activity

Mylène Zarka ${ }^{1}$, François Etienne ${ }^{2}$, Morgane Bourmaud ${ }^{1}$, Christophe Helary $^{3}$, François Rannou ${ }^{2}$, Eric Haÿ ${ }^{1}$, Martine Cohen-Solal ${ }^{1}$

${ }^{1}$ Inserm UMR1132, Hôpital Lariboisière; Univ Paris Diderot, Sorbonne Paris Cité, Paris, France, ${ }^{2}$ Inserm UMR-S1124, Université Paris Descartes, Sorbonne Paris Cité, Paris, France, ${ }^{3}$ Sorbonne Universités UPMC Univ Paris 06, CNRS, Collège de France, Laboratoire Chimie de la Matière Condensée de Paris UMR7574, Paris, France

Purpose: Osteocytes are major regulators of bone homeostasis and control bone remodeling in response to mechanical loading. These mechanosensitive cells produce soluble factors capable of modulating the activity of bone cells in response to different tensile forces. To date, the signal transduction pathways induced by mechanical loading in osteocytes are not clearly elucidated. We hypothesized that YAP and TAZ may be involved since both play an important role in mechanotransduction and they interact with several regulated signaling pathways during osteoblastogenesis. The objective of our study is to characterize the response of osteocytes in response to mechanical loading.

Methods: MLO-Y4 osteocyte cell lines were cultured in concentrated collagen hydrogels for 14 days. Osteocytes were submitted to mechanical loading using the Flexcell Compression System ${ }^{\circledR}$ with a regimen of sinusoidal compression at $1 \mathrm{~Hz}, 40 \mathrm{kPa}$ during $9 \mathrm{~h}$.

Results: Compared to unloaded cells, loading induced an increase of the expression of target genes known to be modulated by shear stress in osteocyte (E11GP38 and COX2, $\times 3$ fold increase $\mathrm{p}<0.005)$. Mechanical compression induced a large increase in the expression of osteoprotegerin $(\mathrm{OPG}, \times 4$ fold increase $\mathrm{p}<0.005)$ associated with a decrease in the expression of RANK ligand (RANKL, 25\% of decrease $\mathrm{p}<0.0005$ ), suggesting a negative role for osteoclastogenesis. Immunofluorescence revealed the mechanical compression increased nuclear translocation of YAP/TAZ by two fold, this being confirmed by western blot and gene expression analysis of YAP/TAZ target genes (ANKRD1, $\times$ threefold increase $\mathrm{p}<0.001)$. In addition, mechanical compression reduced osteocyte apoptosis (50\% decreased apoptosis).

In conclusion, our model allowed us to highlight gene modifications induced by compression strains in osteocytes and the concomitant activation of YAP/TAZ pathway. These results show the contribution of YAP/TAZ signaling in the mediation of mechanical loading through osteocytes and in the regulation of bone remodeling. Keywords: osteocyte, YAP/TAZ, mechanotransduction

\section{P199}

Osteocyte numbers and morphology correlate with the shear force at the bone-implant interface

Sabine Stoetzel ${ }^{1}$, Ute Wild ${ }^{1}$, Christoph Biehl ${ }^{2}$, Deeksha Malhan ${ }^{1}$, $\overline{\text { Carolin Dewald }}^{3}$, Stefan Maenz ${ }^{3}$, Klaus D. Jandt ${ }^{3}$, Katrin S. Lips ${ }^{1}$, Thaqif El Khassawna ${ }^{1}$, Christian Heiß ${ }^{2}$

${ }^{1}$ Experimental Trauma Surgery, Justus-Liebig University Giessen, Gießen, Germany, ${ }^{2}$ Department of Trauma, Hand and Reconstructive Surgery, University Hospital of Giessen-Marburg GmbH, Campus Giessen, Gießen, Germany, ${ }^{3}$ Otto Schott Institute of Materials Research, Chair of Materials Science, Friedrich Schiller University, Jena, Germany

Material design and composition influence the biomechanical and osseointegration properties of implants. Osteocytes respond to mechanical stress and influence anabolic or catabolic processes of bone. Functionalized surface of implants influence cell proliferation at the bone implant interface. Therefore implant coating can increase the amount and quality of the new formed bone.

Methods: Male Sprague Dawley rats were assigned to one of three groups. (1) Pure titanium implant (Ti) served as control, (2) titanium implant coated with gelatin $(\mathrm{Gel})$ or (3) titanium implant coated with hyaluronic acid (HA). Implants were designed as intramedullary nail of the tibia. Rats were euthanized after 3 or 8 weeks of healing. Operated tibiae were analyzed biomechanically (implant pull out test) and histologically (cross sections stained with modifies MassonGoldner and silver nitrate counterstain). Area of newly formed bone around the implant, number of osteoclasts, osteocytes, and osteoblasts were histomorphometrically defined.

Results: After 8 weeks of healing both coated implants were more rigidly anchored to bone than the uncoated ones $(\mathrm{p} \leq 0.05)$. Area of newly formed bone increased from week 3 to week 8 of healing. However, no differences were seen between the groups. Interestingly, numbers of spindle shaped osteocytes increased with a trend with 
healing progression. Whereas numbers of spherical osteocytes and empty lacunas decreased. Determination of osteoclast and osteoblast numbers as well as investigation of the matrix ultrastructure using scanning electron microscopy are currently being analyzed.

Bone quality is represented by osteocyte numbers and osteocytes morphology, which benchmarks the osteocytes vitality. In addition to bone mass, bone quality influences the bones load-bearing capacity. Under pathological conditions such as osteoporosis bone mass and quality are negatively affected. Implants with bioactive coating could improve new build bone quality after osteoporotic fractures.

Keywords: bone-implant-interface, coated implants, osteocytes, osseointegration

\section{P200}

Uhrf1 deficiency attenuates genome-wide DNA methylation and consequent specific gene expression in chondrocytes in vivo

Michiko Yamashita ${ }^{1}$, Noritaka Saeki ${ }^{1}$, Yuta Yanagihara ${ }^{1}$, Yuichiro Sawada $^{1}$, Jiwon Lee $^{1}$, Tadahiro Iimura ${ }^{1}$, Yuuki Imai ${ }^{1}$

${ }^{1}$ Proteo-Science Center, Ehime University, Toon, Japan

Transcriptional regulation can be tightly orchestrated by epigenetic regulators. Among these, ubiquitin-like with PHD and RING finger domains 1 (Uhrf1) is reported to have diverse epigenetic functions, including regulation of DNA methylation. However, the physiological functions of Uhrf1 in skeletal tissues remain unclear. Here we show that limb mesenchymal cell-specific Uhrf1 conditional knockout mice (Uhrf $\left.1^{\Delta L i m b / \Delta L i m b}\right)$ exhibit remarkably shortened long bones that have morphological deformities due to dysregulated chondrocyte differentiation and proliferation. RNA-seq performed on primary cultured chondrocytes obtained from $U h r f 1^{\text {LLimb/LLimb }}$ mice showed abnormal chondrocyte differentiation. Especially, Mmp13 expression was significantly increased in both mRNA and protein levels in Uhrf $1^{\Delta \text { Limb/ALimb }}$ mice. Volcano plot of RNA-seq revealed that Uhrf1 deficiency induced up-regulation rather than down-regulation of gene expression. Among significantly up-regulated 328 genes in Uhrf1 deficient chondrocytes, only 13 genes were overlapped with up-regulated genes in hematopoietic stem cells lacking Uhrf1, suggesting that transcriptional regulation mediated with Uhrf1 is cell type specific. In addition, genome-wide DNA methylation sequencing (MBD-seq) displayed that DNA methylation levels of Uhrf $1^{\text {ALimb/ }}$ $\triangle$ Limb chondrocytes were decreased across the genome, but especially in the promoter regions. Furthermore, integrative analyses using RNA-seq and MBD-seq revealed that Uhrf1 deficiency decreased genome-wide DNA methylation and increased gene expression through reduced DNA methylation in the promoter regions of 28 genes, including $H s p b 1$, which is reported to be an IL-1-related gene and to affect chondrocyte differentiation. Hspb1 knockdown in Uhrf ${ }^{\text {LLimb/ALimb }}$ chondrocytes can normalize abnormal expression of genes involved in chondrocyte differentiation such as Mmp13. These results indicate that Uhrf1 governs cell-type specific transcriptional regulation by controlling the genome-wide DNA methylation status and regulating consequent cell differentiation and skeletal maturation. Keywords: Uhrf1, DNA methylation, RNA-seq, MBD-seq, Hspb1

\section{P201}

Proteoglycan synthesis defects in a Cant1 knock-out mouse model of Desbuquois dysplasia type 1

Chiara Paganini ${ }^{1}$, Rossella Costantini ${ }^{1}$, Luca Monti ${ }^{1}$, Anna Lundari ${ }^{1}$, Marco Biggiogera ${ }^{2}$, Jean-Marc Schwartz ${ }^{3}$, Celine Huber ${ }^{4}$, Valerie Cormier-Daire ${ }^{4}$, Beth Gibson ${ }^{5}$, Katarzyna A. Pirog ${ }^{5}$, Antonella Forlino $^{1}$, Antonio Rossi ${ }^{1}$
${ }^{I}$ Department of Molecular Medicine - Unit of Biochemistry, University of Pavia, Pavia, Italy, ${ }^{2}$ Department of Biology and Biotechnology, University of Pavia, Pavia, Italy, ${ }^{3}$ Faculty of Biology, Medicine and Health, University of Manchester, Manchester, United Kingdom, ${ }^{4}$ Department of Genetics and INSERM UMR1163, Hopital Necker Enfants Malades, Paris, France, ${ }^{5}$ Institute of Genetic Medicine, Newcastle University, Newcastle upon Tyne, United Kingdom

Mutations in the CANT1 gene cause Desbuquois dysplasia type 1 (DBQD1) a recessive chondrodysplasia characterized by growth retardation, multiple dislocations and hand deformities. CANT1 encodes for a calcium activated nucleotidase of the ER/Golgi that preferentially hydrolyzes UDP; due to its function and localization a role in proteoglycan metabolism has been inferred. We have studied the role of the nucleotidase in proteoglycan synthesis in cartilage and chondrocytes from a Cant 1 knock-out mouse which reproduces the growth defects reported in DBQD1 patients. Reduced proteoglycan synthesis in knock-out cells compared with wild-types was detected by ${ }^{35} \mathrm{~S}$-sulfate metabolic labeling of rib chondrocytes (6408 and $16,308 \mathrm{dpm} / \mu \mathrm{g}$ protein, respectively $\mathrm{P}<0.01)$. Gel filtration chromatography of glycosaminoglycans released from proteoglycans after beta-elimination demonstrated that their hydrodynamic size was reduced in knock-out chondrocytes compared with the controls. Interestingly sulfation studies by HPLC disaccharide analysis demonstrated that glycosaminoglycan chains both in cartilage and in chondrocytes from knock-out animals were oversulfated compared to wild-types (94 and $86 \%$ of sulfated disaccharides, respectively; $\mathrm{P}<0.001$ in cartilage and 92 and $73 \%$ respectively, $\mathrm{P}<0.001$ in cultured medium). TEM analysis of knock-out cartilage showed in chondrocytes dilated vacuoles containing electron dense material suggesting a role of CANT1 in protein secretion and/or in ER stress. Pulse-chase labeling of cells with ${ }^{35} \mathrm{~S}$-sulfate demonstrated reduced proteoglycan secretion in mutant cells compared with the controls $(\mathrm{P}<0.05)$. However, intracellular retention in knock-out cells does not cause ER stress and does not elicit an UPR since microarray analysis did not show any altered expression of genes involved in these pathways and BiP expression was normal in western blots of chondrocyte lysates. In conclusion, CANT1 affects proteoglycan synthesis at different levels including glycosaminoglycan chain length, sulfation and secretion in the extracellular matrix.

Work supported by the European Community (FP7, "Sybil" project, grant n.602300).

Keywords: cartilage, proteoglycan, skeletal dysplasia

\section{P202}

Derivation and characterisation of a unique chondrocyte population from human Embryonic Stem Cells

Lauren Jevons $^{1}$, Franchesca Houghton ${ }^{1}$, Rahul Tare ${ }^{1}$

${ }^{1}$ Centre for Human Development, Stem Cells and Regeneration, University of Southampton, Southampton, United Kingdom

Osteoarthritis is the most common joint disease caused by the degradation of articular cartilage. This has profound effects on mobility and causes debilitating pain in affected individuals. Current reparative and restorative treatments for osteoarthritis require multiple invasive surgeries and generate fibrous repair tissue rather than hyaline cartilage. Human Embryonic Stem Cells (hESCs) grow indefinitely, are capable of differentiating into all somatic cell types of the three germ layers and have perceived immunoprivileged characteristics; hESCs therefore constitute a promising alternative cell type for cartilage generation, given the limitations associated with the application of human articular chondrocytes ${ }^{1}$ and bone marrowderived stem cells ${ }^{2}$ in regenerative medicine strategies for cartilage repair. The aim of this study is to direct the differentiation of hESCs into the chondrogenic lineage and characterise the resulting 
chondrocyte population to ascertain its efficacy for application in cartilage repair.

A 14-day directed differentiation protocol involving the temporal addition of growth factors, namely WNT-3a, Activin-A, FGF-2, BMP-4, Follistatin, NT4, GDF5 and TGF- $\beta 3$, was used to direct the differentiation of hESCs into chondrocytes. Analyses of temporal expression of lineage-specific genes by qPCR demonstrated successful differentiation of the pluripotent hESC population, via the primitive streak-mesendoderm and mesoderm developmental stages, into the chondrogenic lineage. In day $14 \mathrm{hESC}$-derived chondrocytes, the key pluripotency marker OCT4 was not detectable by immunoblotting, and there was a significant decrease in the expression of both SOX2 and NANOG $(\mathrm{p}<0.01)$, coupled with a $\sim 10$ fold increase in the expression of SOX9 $(\mathrm{p}<0.05)$. The hESCderived chondrocytes also exhibited robust immunostaining for SOX9 in the nuclei and deposited extracellular matrix rich in Type II Collagen.

hESC-derived chondrocytes constitute a unique cell population with the potential to transform strategies for cartilage regeneration. References:

1. Osteoarthritis Cartilage 2002;10:432-63

2. Journal of Biomaterials Applications 2015;29:824-36

Keywords: Chondrocytes, human Embryonic Stem Cells, pluripotency, cartilage

\section{P203}

Cardamonin attenuates IL-1 $\beta$ and urate crystal-induced inflammation in joint cells

Chun Jung Huang ${ }^{1}$, Shao Chi Chen ${ }^{1}$, Chih Chien Wang ${ }^{2}$, Feng Cheng $\mathrm{Liu}^{3}$, Yi Jen Peng ${ }^{1,4}$

${ }^{1}$ Graduate Institute of Pathology and Parasitology, National Defense Medical Center, Taipei City, Taiwan, Republic of China, ${ }^{2}$ Department of Orthopedics, Tri-Service General Hospital, Taipei City, Taiwan, Republic of China, ${ }^{3}$ Rheumatology/Immunology and Allergy, TriService General Hospital, Taipei City, Taiwan, Republic of China, ${ }^{4}$ Department of Pathology, Tri-Service General Hospital, Taipei City, Taiwan, Republic of China

Cardamonin, a chalcone isolated mostly from Zingiberaceae plants has been reported to exert anti-inflammatory and anti-oxidant properties. Osteoarthritis (OA) is a common degenerative joint disorder with progressive cartilage destruction and joint dysfunction. Interleukin (IL)- $1 \beta$ is one of the potent cytokine identified in osteoarthritic synovial fluid and triggers a succession of cartilage catabolic effects. Gouty arthritis (GA) arises an acute inflammatory response results from the precipitation of monosodium urate (MSU) crystals in joint. The uptake of MSU by macrophages activates NOD-like receptors containing a PYD 3 (NLRP3) inflammasome, thus triggering IL-1 $\beta$ production. Osteoarthritis and gouty arthritis both ended up in joint destruction and pain. The aim of this study was to investigate the protective effects of cardamonin on chondrocytes and macrophages in OA and GA models respectively. Cardamonin inhibits the overexpression of COX-2, MMPs and NOS- 2 in IL- $1 \beta$ induced chondrocytes by western blots $(\mathrm{p}<0.05)$, as well as the secretion of PGE2, NO, and matrix metalloproteinases (MMPs) $(p<0.05)$. IL-1 $\beta$ induced activation of NF- $\kappa B$ in chondrocyte was attenuated by cardamonin treatment. Cardamonin restored the IL- $1 \beta$-induced glycosaminoglycan degradation in ex vivo articular cartilage. Meanwhile, cardamonin can reduce COX-2 expression and IL- $1 \beta$ secretion by inhibiting inflammasome pathway of NLRP3 and mature caspase-1 in MSUstimulated J744.1 macrophages ( $p<0.05$ ). In conclusion, this study demonstrates that cardamonin may be of value in treatment of osteoarthritis and gouty arthritis through its anti-nitrosative and antiinflammatory activities.

Keywords: cardamonin, chondrocyte, macrophage, monosodium urate, IL-1 $\beta$

\section{P204}

Early insulin treatment partially improves bone mechanical properties of Goto-Kakizaki type-2 diabetic rats

$\frac{\text { Kannikar Wongdee }}{1,2}$, Ratchaneevan Aeimlapa $^{2,3}$, Narattaphol Charoenphandhu ${ }^{2,3,4}$

${ }^{1}$ Faculty of Allied Health Sciences, Burapha University, Chonburi, Thailand, ${ }^{2}$ Center of Calcium and Bone Research, Mahidol University, Bangkok, Thailand, ${ }^{3}$ Department of Physiology, Faculty of Science, Mahidol University, Bangkok, Thailand, ${ }^{4}$ Institute of Molecular Biosciences, Mahidol University, Nakhon Pathom, Thailand

Type 2 diabetes mellitus (T2DM) has been shown to impair bone strength due to low bone mineral density and impaired bone microstructure, e.g., reduction of trabecular thickness, osteoblast surface, and bone formation rate. In addition, aberrant production and alignment of collagen can lead to decreased bone strength. Although insulin administration is the treatment of choice for T2DM patients with poor response to antidiabetic drugs or poor glycemic control, whether early insulin treatment in T2DM rats is effective in restoring mechanical bone strength remains elusive. In the present study, we determined the effects of insulin on bone mechanical properties of adolescent female Goto-Kakizaki (GK) T2DM rats by using microcomputed tomography ( $\mu-\mathrm{CT})$ and three-point bending test. Rats were divided into 2 groups, i.e., untreated GK rats (control) and 12-week insulin-treated rats. This study has been approved by the institutional animal ethic committee. Although daily insulin treatment was able to reduce blood glucose from 151.91 to $96.00 \mathrm{mg} / \mathrm{dL}(P<0.001)$, but it could not alter cortical bone mineral density (BMD) and bone mechanical properties, i.e., maximum load, yield load, yield displacement and energy absorption. Interestingly, the stiffness was significantly higher in insulin-treated GK rats as compared with the control rats $(308.90$ vs. $345.10 \mathrm{~N} / \mathrm{mm} ; P<0.05)$, suggesting that insulin partially improved bone strength. In conclusion, despite being an effective treatment for T2DM, insulin could partially improve mechanical properties of GK T2DM rats. Therefore, early prevention of T2DM is probably the better way to protect against diabetic osteopathy.

Keywords: Goto-Kakizaki rats, insulin, three-point bending, type 2 diabetes mellitus (T2DM)

\section{P205}

Bone formation markers are reduced in patients with diabetes. Results of a 24-h study

Katrine Hygum ${ }^{1}$, Jakob Starup-Linde ${ }^{1}$, Torben Harsløf ${ }^{1}$, Bente L. Langdahl $^{1}$

${ }^{I}$ Department of Endocrinology and Internal Medicine, Aarhus University Hospital, Aarhus C, Denmark

Aim: Diabetes mellitus is associated with a decreased bone turnover. The aim of the study was to compare the 24-h variation in bone turnover markers (BTMs) in patients with type 1 diabetes (T1D) and type 2 diabetes (T2D), and healthy controls.

Methods: Five patients with T1D, five patients with T2D, and five controls were included. None suffered from conditions affecting bone. Meals and physical activity were standardized. Blood samples were drawn hourly and with a 30-min interval following meals, and every 
three hours during the night. BTMs were analyzed subsequently. ANOVA repeated measurements and area under the curve (AUC) statistics were used.

Results: Osteocalcin and procollagen type I N-terminal propeptide (P1NP) were significantly different between the three study groups during the study period $(\mathrm{P}<0.01$ for all) whereas $\mathrm{C}$-terminal crosslinked telopeptide and parathyroid hormone were not different between groups. P1NP (Fig. 1) and osteocalcin were lower in patients with diabetes compared to controls.

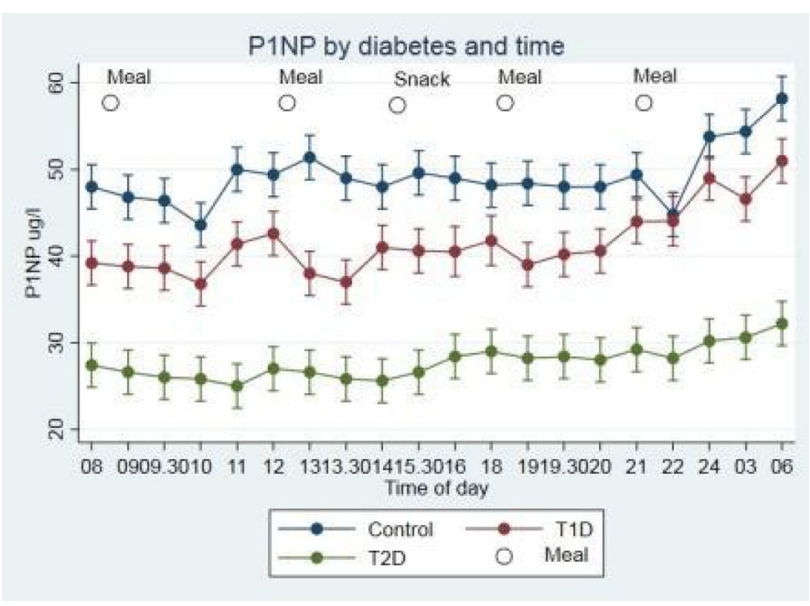

Fig. 1 P1NP by time and type of diabetes

The AUC (mean $\pm \mathrm{SE}$ ) for P1NP was reduced in patients with T2D compared to controls $(626 \pm 72.6$ vs. $1106 \pm 115 \mu \mathrm{g} / \mathrm{l}$, $\mathrm{P}<0.01)$ and similarly decreased for osteocalcin $(278 \pm 20.9$ vs. $488 \pm 50.6 \mu \mathrm{g} / \mathrm{l}, \mathrm{P}<0.01)$. Sclerostin trended to be higher in T2D, however the AUC was non-significantly higher in patients with T2D compared with controls $(26 \pm 3.8$ vs. $19 \pm 1.4 \mathrm{ng} / \mathrm{ml}, \mathrm{p}=0.08)$.

Conclusion: We show for the first time that the 24-h level of bone formation is different in patients with T1D and T2D compared with healthy controls. We propose that this is caused by altered bone signaling or impaired incretin response in diabetes.

Keywords: Diabetes, bone turnover

\section{P206}

Attenuation of glucocorticoid signalling in osteoblasts protects from high-fat diet-induced obesity, insulin resistance and bone loss

Sarah Kim ${ }^{1}$, Holger Henneicke ${ }^{1,2}$, Sylvia Gasparini ${ }^{1}$, Lee Thai ${ }^{1}$, Markus Seibel $^{1}$, Hong Zhou ${ }^{1}$

${ }^{I}$ Bone Research Program, ANZAC Research Institute, The University of Sydney, Sydney, Australia, ${ }^{2} D F G$-Center for Regenerative Therapies, Technische Universität Dresden, Dresden, Germany

Overconsumption of energy-dense diets is a major public health challenge due its causal association with obesity, diabetes and poor skeletal health. Most animal studies examining diet-induced obesity and diabetes have focused solely on combined high-energy, high-fat diets. We therefore aimed to define whether the adverse health outcomes are due to the high energy density or high fat content of these diets. Since osteoblasts co-regulate overall energy balance under the control of glucocorticoids (GCs), we also examined whether abrogating GC signalling in osteoblasts protects mice from diet-induced metabolic and skeletal disturbances.
We utilised transgenic $(\mathrm{tg})$ mice in which GC signalling has been disrupted in osteoblasts via targeted overexpression of the GC-inactivating enzyme, $11 \beta$-hydroxysteroid dehydrogenase type 2 . Sevenweek-old male tg mice and their wild-type (WT) littermates were fed ad libitum for 18-weeks one of the following diets: high-energy, standard-fat (fat $=14 \%$ total-energy, $16.3 \mathrm{~kJ} / \mathrm{g}$ ); high-energy, highfat (fat $=43 \%$ total-energy, $16.3 \mathrm{~kJ} / \mathrm{g}$ ); standard-energy, high-fat (fat $=43 \%$ total-energy, $13.8 \mathrm{~kJ} / \mathrm{g}$ ) or standard-chow $(\mathrm{fat}=14 \%$ total-energy, $13.8 \mathrm{~kJ} / \mathrm{g}$ ). At endpoint, body composition, glucose handling and bone mass were measured.

High-energy feeding, regardless of dietary fat content resulted in significantly increased fat mass in WT mice compared to WT chowfed mice along with fasting hyperglycaemia and reduced insulin sensitivity. Both high-energy diets induced significant tibial trabecular and cortical volume loss to a similar extent. WT mice on the standard-energy, high-fat diet remained lean and insulin sensitive but displayed pronounced trabecular and cortical bone loss (Fig. 1A). Notably, tg mice were protected from high-energy diet-induced excessive fat accrual, insulin resistance, glucose intolerance and bone loss (Fig. 1B), despite consuming the same amount as their WT littermates on either high-energy or standard-energy, high-fat diet.

Conclusion: High energy density rather than high fat content induces metabolic dysfunction in male mice, whereas high dietary fat results in bone loss. Both effects are mediated by GC signalling in osteoblasts.
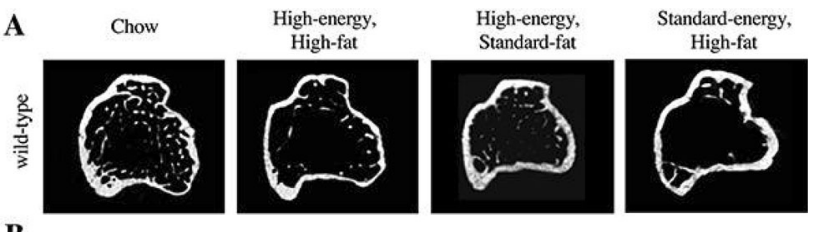

B
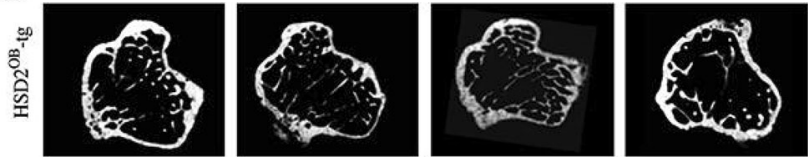

Figure

\section{P207}

Characterization of Bone Marrow Adipocytes in the ovariectomy model shows an evolving phenotype that can contribute to altered bone remodeling

Tareck Rharass ${ }^{1}$, Severine Delplace ${ }^{1}$, Damien Leterme ${ }^{1}$, Anne Resonet $^{1}$, Pierre Marchandise ${ }^{2}$, Pierre Hardouin ${ }^{1}$, Christophe Chauveau $^{1}$, Stephanie Lucas ${ }^{1}$

${ }^{1}$ EA-4490 PMOI laboratory, Littoral Côte d'Opale University ULCO, Boulogne sur Mer, France, ${ }^{2}$ EA4490 PMOI laboratory, Lille University, Lille, France

Objectives: Bone Marrow Adipocytes (BMA) are sensed to interfere with bone remodeling in osteoporosis but their activities and regulation remain poorly examined. This study addressed the phenotype of BMA through their adipokine expression pattern in ovariectomized mice and the regulation by glucose concentration of this pattern in vitro.

Methods: Using real-time-PCR, femoral and tibial isolated BMA were compared to perigonadal adipocytes after 4 and 14 weeks following ovariectomy (ethical approval CEEA75-2016071815452669). Indeed BV/TV decline, BMA accrual and glucose intolerance 
together worsen more during this time lapse in ovariectomized mice compared to sham-operated mice. Human Mesenchymal Stem Cells (MSC, Lonza) were differentiated and maturated into adipocytes in either $5 \mathrm{mM}$ (LG) or $25 \mathrm{mM}$ glucose (HG) for 21 days or only maturated in $25 \mathrm{mM}$ glucose for 1 week (LG/HG) to test if chronic hyperglycemia regulates BMA function.

Results: Following ovariectomy, expression levels of adipogenic transcriptional factors and classical adipokines (e.g. adiponectin, -25 fold, $\mathrm{p}<0.05)$ are lower in BMA compared to perigonadal adipocytes. However, RANKL and the Wnt-signaling inhibitors sFRP1 and sFRP4 mRNA levels are more expressed in the BMA (up to $+200,+6.2$ and +11.6 fold respectively, $\mathrm{p}<0.05$ ). Moreover, their expression in the BMA increase between 4 and 14 weeks (by $+30,+3.9$ and +8.4 fold, respectively, $\mathrm{p}<0.05)$ while adiponectin mRNA is only +1.7 fold upregulated. Immunohistochemistry shows adiponectin and sFRP4 expression in BMA. Compared to plasmas, the tibial $\mathrm{BM}$ fluids contain less adiponectin protein but measurable levels of sFRP4 protein which increase after 14 weeks of ovariectomy. In MSC-derived adipocytes, sFRP4 mRNA is upregulated by +50 and $+70 \%$ in $\mathrm{HG}$ and $\mathrm{LG} / \mathrm{HG}$ conditions $(\mathrm{p}<0.05)$ compared to LG exposure while adipogenesis is unaltered.

Conclusions: Mature BMA are a paracrine source of several factors involved in osteoblastogenesis and osteoclastogenesis and could contribute to the bone loss of postmenopausal osteoporosis partly in a glucose-regulated manner.

\section{P208}

Levels of sclerostin in serum and femoral vascular tissue and its relationship with vascular calcification in patients with and without type 2 diabetes

Sheila González-Salvatierra ${ }^{1}$, Cristina Novo-Rodríguez ${ }^{2}$, Francisco Andújar-Vera $^{3}$, Cristina García-Fontana ${ }^{3}$, Teresa Márquez-

Hernández $^{4}$, Silvia Lozano-Alonso ${ }^{5}$, Sonia Morales-Santana ${ }^{6}$, Pedro Rozas-Moreno $^{7}$, Antonia García-Martín ${ }^{8}$, Francisco O'valleRavassa ${ }^{9}$, Juan De Dios Luna-Del Castillo ${ }^{10}$, Manuel MuñozTorres $^{11}$, Beatriz García-Fontana ${ }^{8}$

${ }^{1}$ Instituto de Investigación Biosanitaria de Granada (Ibs.Granada); Department of Medicine, University of Granada, Granada, Spain, ${ }^{2}$ Endocrinology and Nutrition Unit, Hospital Universitario Campus de la Salud, Instituto de Investigación Biosanitaria de Granada (Ibs.Granada), Granada, Spain, ${ }^{3}$ Instituto de Investigación Biosanitaria de Granada (Ibs.Granada), Granada, Spain, ${ }^{4}$ Instituto de Investigación Biosanitaria de Granada (Ibs. Granada); Department of Medicine, University of Granada, Granada, Spain, ${ }^{5}$ Service of Angiology and Vascular Surgery, Universitario Campus de la Salud, Granada, Spain, ${ }^{6}$ Proteomic Research Service, Fundación para la Investigación Biosanitaria de Andalucía Oriental- Alejandro Otero, Instituto de Investigación Biosanitaria de Granada (Ibs.Granada), Granada, Spain, ${ }^{7}$ Endocrinology Division, Hospital General de Ciudad Real, Ciudar Real, Spain, ${ }^{8}$ Endocrinology and Nutrition Unit, Hospital Universitario Campus de la Salud, Instituto de Investigación Biosanitaria de Granada (Ibs.Granada); CIBERFES Instituto de Salud Carlos III, Granada, Spain, ${ }^{9}$ Department of Pathological Anatomy, University of Granada, Granada, Spain, ${ }^{10}$ Department of Biostatistical, University of Granada, Granada, Spain, ${ }^{11}$ Endocrinology and Nutrition Unit, Hospital Universitario Campus de la Salud, Instituto de Investigación Biosanitaria de Granada (Ibs.Granada); CIBERFES Instituto de Salud Carlos III, Department of Medicine, University of Granada, Granada, Spain

Introduction: Sclerostin is a glycoprotein expressed mainly by osteocytes, which acts as inhibitor of bone formation. Recent studies have shown an increase in serum levels of sclerostin in subjects with type 2 diabetes (T2D) and cardiovascular disease (CVD) suggesting an additional role of this protein at the vascular level. The hypothesis that sclerostin could be expressed by vascular smooth muscle cells (VSMCs) under calcifying conditions could justify this elevation. However, there are few data at the tissue level.

Objective: Correlation between serum sclerostin levels in nondiabetic and T2D patients with CVD and the transcriptional and immunohistochemical expression level in femoral vascular tissue of T2D patients with atherosclerosis and non-atherosclerotic vascular tissue from healthy donors.

Methods: Serum sclerostin levels were determined in 78 T2D subjects with CVD and in 55 non-diabetic subjects by commercial ELISA kit (Biomedica). Sclerostin expression was determined by RTqPCR from $400 \mathrm{ng}$ of total RNA from 45 sections of atherosclerotic femoral arterial tissue from T2D subjects as well as from healthy donors. Immunohistochemical detection of sclerostin was performed on sections of paraffined vascular tissue using 1:50 antisclerostinspecific antibody (Sigma Aldrich) and chromogenic detection. (Project approved by Ethical Committee from Granada).

Results: Serum sclerostin levels were significantly higher in T2D subjects with CVD compared to non-diabetic subjects (58.29 \pm 26.36 vs. $39.27 \pm 12.71 \mathrm{pmol} / \mathrm{L}, \mathrm{p}<0.001)$. Increased expression of sclerostin at the transcriptional and immunohistochemical level was observed in atherosclerotic femoral artery of T2D patients compared with the femoral artery of healthy donors.

Conclusions: The increase in serum sclerostin associated with CVD in T2D could be related to an increased expression of this protein by vascular tissue. This suggests the involvement of sclerostin in the calcified process of the atherosclerotic plaque.

Keywords: Sclerostin, vascular calcification, atherosclerosis, type 2 diabetes, bone-formation inhibition

\section{P209}

NGF/BDNF and Osteocalcin/Oxytocin/IL-6 mRNA levels are upregulated in bone and muscle after cold stress challenge in mice

Claudia Camerino $^{1}$, Elena Conte ${ }^{2}$, Maria Rosaria Carratù ${ }^{1}$, Adriano Fonzino $^{2}$, Domenico Tricarico ${ }^{2}$

${ }^{1}$ Department of Biomedical Sciences and Human Oncology, University of Bari, Bari, Italy, ${ }^{2}$ Department of Pharmacy-Drug Sciences, University of Bari, Bari, Italy

NGF/BDNF, Osteocalcin (Ost) and Oxytocin (Oxt) are multipurpose molecules that exert a protective function on bone, reproduction and cognition under thermogenic challenge. Oxt and Ost are also required for muscle regeneration, the latter triggering the increase of IL-6 in myofibers and BDNF in brain. Here, we evaluated the mRNA levels of $N g f, B d n f$, Ost, Oxt and their receptors ( $p 75 n t r$, Ntrk1, Gprc6a, Oxtr), as well as of uncoupling protein 1 (Ucp1) and Il-6 in bone, soleus (SOL) and tibialis (TA) muscles from 3 monthsold mice exposed to cold stress $(\mathrm{CS})$. Mice $(\mathrm{n}=15)$ were divided into: controls maintained at room temperature $\left(\mathrm{RT}=23{ }^{\circ} \mathrm{C}\right)$, exposed to $\mathrm{CS}$ at $\mathrm{T}=4{ }^{\circ} \mathrm{C}$ for $6 \mathrm{~h}$ and 5-days. $\mathrm{CS}$ exposure to 5-days enhanced $N g f$, but not its receptors, and Ucpl genes in bone. Ucpl and $N g f$ genes were significantly upregulated by 2 and 1.5 folds respectively in TA after 6 h CS. Ntrk1 was significantly upregulated by 4 and 22 folds respectively in the SOL muscle after $6 \mathrm{~h}$ and 5 days $\mathrm{CS}$, while p75Ntr was downregulated in both muscles after $6 \mathrm{~h} \mathrm{CS}$. Oxt was upregulated by fivefold following 5-days CS in bone. Oxtr and $I l 6$ genes were upregulated respectively by 1 and 1.5 folds after 5 days CS in SOL. Ost increased by 16-fold in bone, Gprc6A was unaffected. Gprc6A was upregulated respectively by 3.5 and twofolds after $6 \mathrm{~h}$ and 5 days CS in TA. Bdnf increased by 9.5 -fold in bone after 5-days CS while it was not affected in muscle. Our study confirms that Ngf and Ucpl are activated in thermogenic tissues, while $O x t$ and $O s t$ exert phenotype-dependent regenerative and protective 
effects towards muscle through up-regulation of their receptors, Oxtr in slow-twitch and Gprc6A in fast-twitch muscles. IL-6 up-regulation in muscle after CS is consistent with the concept of a coordinated axis between bone and muscle.

\section{P210}

Effect of estrogen deficiency on development of skeletal changes induced by type 1 diabetes in rats

Aleksandra Janas ${ }^{1}$, Ewa Kruczek ${ }^{1}$, Joanna Folwarczna ${ }^{1}$

${ }^{1}$ Department of Pharmacology, School of Pharmacy with the Division of Laboratory Medicine in Sosnowiec, Medical University of Silesia, Katowice, Sosnowiec, Poland

Both type 1 and type 2 diabetes lead to an increased fracture risk. Although postmenopausal osteoporosis often occurs concurrently with diabetes, little is known about interactions between estrogen deficiency and hyperglycemia in terms of effects on the skeletal system in vivo. The aim of the study was to investigate the effects of estrogen deficiency on the development of biochemical, microstructural and mechanical changes induced by type 1 diabetes (T1D) in the rat skeletal system.

The experiments were carried out on 3-month-old female Wistar rats, divided into 4 groups $(\mathrm{n}=8-10)$ : non-ovariectomized (NOVX) control rats, ovariectomized (OVX) control rats, T1D NOVX rats and T1D OVX rats. T1D was induced by a single injection of streptozotocin $(60 \mathrm{mg} / \mathrm{kg}$ i.p.), 3 days after the bilateral ovariectomy was performed. The experiment was terminated 5 weeks after the ovariectomy. Serum bone turnover markers (CTX-I and osteocalcin), bone mass and mineralization, histomorphometric parameters (OsteoMeasure system) and mechanical properties (Instron apparatus) of cancellous and compact bone were determined. The results were statistically evaluated by ANOVA tests.

Estrogen deficiency induced typical osteoporotic changes in OVX control rats, with increased bone resorption and formation, leading to worsening of microstructure (BV/TV, Tb.Sp, Tb.N) and mechanical properties of cancellous bone of the tibial metaphysis. Streptozotocin administration in NOVX rats induced T1D with decreased bone mass, increased bone resorption and decreased bone formation, which also led to deterioration of microarchitecture (BV/TV, Tb.Th) and strength of cancellous bone. In estrogen-deficient rats, T1D also increased bone resorption and decreased bone formation serum markers, and further worsened cancellous bone mechanical parameters in relation to the OVX controls. In relation to T1D NOVX rats, bone strength slightly decreased, and histomorphometric changes were not altered.

In conclusion, the unfavorable skeletal changes induced by T1D in female rats were only slightly intensified by estrogen deficiency.

Keywords: diabetes, osteoporosis, rats

\section{P211}

Association between bone mineral density and serum uric acid in postmenopausal women with diabetic nephropathy

Simeon Monov ${ }^{1}$, Daniela Monova ${ }^{2}$, Elena Miloshova ${ }^{3}$, Russka Shumnalieva $^{1}$

${ }^{1}$ Department of Internal Medicine, Clinic of Rheumatology, Medical University - Sofia, Sofia, Bulgaria, ${ }^{2}$ Department of Internal Medicine, Medical University - Sofia, Medical Institute, Sofia, Bulgaria, ${ }^{3}$ Medical Institute, Sofia, Bulgaria

Introduction: Oxidative stress has been implicated in the pathogenesis of osteoporosis. Uric acid, a potent antioxidant substance, has been associated with bone mineral density (BMD).
Objective: The objective of the study was to examine the association between serum uric acid and BMD in postmenopausal women with diabetic nephropathy.

Material and Methods: This cross-sectional study involved 78 postmenopausal women with diabetic nephropathy, 48-65 years of age. BMD measured at the lumbar spine and total hip region with dual energy X-ray absorptiometry (DXA) Annual rate of change in BMD (A\% $\triangle \mathrm{BMD}$ ) was calculated. The serum uric acid (SUA) levels were obtained at each DXA visit. Partial correlation and regression analyses were applied to determine the associations.

Results: Cross-sectional data analyses revealed that BMD was positively correlated with SUA in postmenopausal women with diabetic nephropathy after adjustment for age, body mass index, systolic blood pressure, diastolic blood pressure, hip circumference, cigarette smoking, alcohol consumption, milk intake, physical activity, fracture history, years after menopause, total protein, total bilirubin, triglycerides, total cholesterol, high-density lipoprotein cholesterol, fasting blood glucose, $\mathrm{HbA1c}$, plasma C-reactive protein, serum alkaline phosphatase serum calcium, and estimated glomerular filtration rate.

Conclusion: The BMD was linearly associated with SUA levels within the normal physiologic range of postmenopausal women with diabetic nephropathy. Higher SUA levels had a protective effect on bone loss in postmenopausal osteoporosis.

Keywords: diabetic nephropathy, uric acid, bone mineral density

\section{P212}

Calcium intake and BMD, bone fractures and cardiovascular hospitalizations in women with type 2 diabetes in a 5 -years prospective study

Luiza Napiórkowska ${ }^{1}$, Hanna Wichrowska ${ }^{1}$, Magdalena Walicka ${ }^{1}$, Edward Franek $^{1,2}$

${ }^{1}$ Department of Internal Diseases, Endocrinology and Diabetology, Central Clinical Hospital of the Ministry of the Interior and Administration, Warsaw, Warsaw, Poland, ${ }^{2}$ Department of Human Epigenetics, Mossakowski Medical Centre, Polish Academy of Sciences, Warsaw, Poland

Material and Methods: 221 women with type 2 diabetes were included. Intake of calcium was estimated using a detailed questionnaire. The patients were divided into two groups: I $(n=126)$ intake less than $400 \mathrm{mg} /$ day and group II $(\mathrm{n}=95)$-intake greater than $400 \mathrm{mg} /$ day. In both groups BMD of lumbar spine, femoral neck and distal radius at baseline and after 5 years of follow-up were measured using DEXA. The concentration of calcium, PTH and $\mathrm{HbA1c}$ was evaluated. The number of osteoporotic fractures and the frequency of hospitalization for cardiovascular reasons were assessed.

Results: The mean age of study participants was $65( \pm 7.7)$ years, BMI $32.5( \pm 5.0) \mathrm{kg} / \mathrm{m}^{2}$, mean HbA1c concentration $7.2( \pm 1.2) \%$ and average duration of diabetes $11.8( \pm 7.0)$ years. There was no difference between both groups in terms of those parameters at the baseline. In group II BMD of distal radius was significantly higher (33\% radius T-score $0.71 \pm 1.07$ vs. $-1.12 \pm 1.05 \%, \mathrm{p}<0.005)$. Femoral neck and lumbar spine BMD was comparable in both groups. BMD of femoral neck and distal radius decreased significantly during observation while BMD of lumbar spine has not changed. There was no correlation between the calcium intake and the occurrence of bone fractures but a positive correlation between the duration of diabetes and incidence of fractures was found. The number of hospitalizations for cardiovascular reasons was comparable in groups with higher and lower calcium intake.

Conclusions: The intake of calcium above $400 \mathrm{mg}$ /day may positively affect BMD of distal radius, however, it does not influence BMD of femoral neck and lumbar spine, frequency of osteoporotic fractures and number of cardiovascular hospitalisations in women with type 2 diabetes. The frequency of osteoporotic fractures positively correlates with the duration of diabetes. 


\section{P213}

Phloridzin, an apple polyphenol, may exert unfavorable effects on bone and muscle in an experimental model of type 2 diabetes in rats

Piotr Londzin ${ }^{1}$, Arkadiusz Waligóra ${ }^{1}$, Szymon Siudak $^{1}$, Urszula Cegieła $^{1}$, Maria Pytlik ${ }^{1}$, Aleksandra Janas ${ }^{1}$, Joanna Folwarczna ${ }^{1}$

${ }^{I}$ Department of Pharmacology, School of Pharmacy with the Division of Laboratory Medicine in Sosnowiec, Medical University of Silesia, Katowice, Sosnowiec, Poland

It is believed that apple fruits contain components with healthpromoting effects, including antidiabetic. One of the most known apple compounds is phloridzin, a glucoside of phloretin. Phloridzin is present in apple leaves, bark, and, in lower amounts, in fruits. Phloridzin and phloretin were reported to exert some favorable skeletal effects in estrogen-deficient rats and mice. The aim of the study was to investigate the effects of phloridzin on bone and muscle in rats with type 2 diabetes (T2D).

The experiments were performed on mature female Wistar rats. T2D was induced by a high-fat diet (HFD) and streptozotocin (STZ; $40 \mathrm{mg} / \mathrm{kg}$ i.p.) administered 2 weeks after the introduction of HFD. The rats were divided into: control rats (fed a standard laboratory diet), HFD/STZ control rats, and HFD/STZ rats receiving phloridzin (20 or $50 \mathrm{mg} / \mathrm{kg} /$ day p.o. for 4 weeks; $\mathrm{n}=10-12$ at the start). Administration of phloridzin (once daily by oral gavage) started 1 week after the STZ injection. Muscle mass and strength (grip test), and bone mass, density, histomorphometric parameters and mechanical properties were determined.

The HFD/STZ rats developed hyperglycemia, with statistically significant decreases in the muscle mass and strength and profound osteoporotic changes. Femoral bone density and trabecular thickness decreased, and the bone marrow cavity/diaphysis transverse crosssection area ratio increased. Mechanical properties of cancellous bone (the proximal tibial metaphysis) significantly worsened. Phloridzin did not affect blood glucose concentrations. Phloridzin at a dose of $20 \mathrm{mg} / \mathrm{kg}$ markedly augmented the unfavorable effects of diabetes on the muscle mass and strength, and decreased compact bone formation (however with ambiguous effects on bone strength), whereas, at a dose of $50 \mathrm{mg} / \mathrm{kg}$, it did not affect most of the investigated parameters.

Results of the study indicate the possibility of unfavorable effects of phloridzin on the musculoskeletal system in conditions of hyperglycemia.

Keywords: phloridzin, diabetes, osteoporosis

\section{P214}

Osteocytes regulate bone marrow fat during progression of osteoporosis in large and small animal models

Thaqif El Khassawna ${ }^{1}$, Deeksha Malhan ${ }^{1}$, Diaa Eldin S. Daghma ${ }^{1}$, Sabine Stözel $^{1}$, Stefanie Kern ${ }^{1}$, Fathi Hassan ${ }^{1}$, Markus Rupp ${ }^{1,2}$, Christian Heiss ${ }^{1,2}$

${ }^{1}$ Experimental Trauma Surgery, Justus Liebig University of Giessen, Giessen, Germany, ${ }^{2}$ Department of Trauma, Hand and Reconstructive Surgery, University Hospital of Giessen-Marburg GmbH, Campus Giessen, Giessen, Germany

Osteocytes act as mechanosensors to control bone volume. Osteocytes role in fat cell metabolism also remains unclear. The current study aims is to correlate osteocyte morphology and adipocytes accumulation in bone marrow with the progression of osteoporosis in sheep model.

Thirty-one female merino-land sheep (5.5 years age) were randomly divided into: Control, Ovariectomy (OVX), OVX with dietary deficiency (OVXD), and OVXD with steroid injection (OVXDS). The animals were euthanized after (month $=\mathrm{M}$ ) $0 \mathrm{M}, 3 \mathrm{M}$, and $8 \mathrm{M}$. Dualenergy X-ray absorptiometry DXA, serum analysis, molecular analysis, and histological analysis were carried out. The direct influence of osteocytes on adipocyte transition was examined through immunostaining of BMP7, UCP1, PGC1 alpha, and PRDM16.

DXA revealed increased body fat in OVXDS (p-value $\leq 0.05$ between the time points) and negatively correlated with Bone Mineral Density (BMD) with treatment progression. Significant decrease in BMD was seen in OVXDS at $3 \mathrm{M}$ and $8 \mathrm{M}$ (p-value $\leq 0.05$, and 0.026 , respectively). ECM degradation and trabecular thinning in OVXDS hallmarked osteoporotic bone status. Count of empty lacunae and adipocytes increased with treatment severity and duration. Immunostaining BMP7, UCP1, PGC1 alpha, and revealed a positive signal in spherical shaped osteocytes. Interestingly, PRDM16 signal location shifted from only in adipocytes at $0 \mathrm{M}$, to adipocytes and osteocytes at $3 \mathrm{M}$ to mainly in osteocytes at $8 \mathrm{M}$.

The OVXDS treatment showed the osteoporotic bone status after $3 \mathrm{M}$. Fat cells markers signals were located within osteocyte vicinity after $8 \mathrm{M}$, thereby indicating a regulatory mechanism. The study showed for the first time the frequent presence of fat cells markers within spherical shaped osteocytes rather than spindle shaped osteocytes. Further small animal models of osteoporosis are being investigated to get a deeper insight through more detailed treatment. Understanding the role of osteocytes in regulating fat metabolism might suggest cell specific therapeutic targeting to prevent osteoporotic fractures.

\section{P215}

Diabetes Mellitus: a synonym to functional hypoparathyroidism

Amit Saraf ${ }^{1}$

${ }^{1}$ Orthopaedics, Teerthanker Mahavir University, Moradabad, India

Background: Poor control of blood glucose levels in patients of Diabetes Mellitus often results in low bone mineral density. The reason for this decrease in bone mass is at present uncertain. We evaluated correlation of calcium metabolism with blood glucose levels and normal renal function.

Method: Total of 130 diabetic patients (35 Type 1, 95 Type 2) were enrolled in this study. In all patients plasma calcium $(\mathrm{Ca})$, serum phosphate (PO4), serum parathyroid hormone (PTH), and 24-h urinary calcium ( $\mathrm{uCa}$ ) were determined under both poor and improved control (for at least 7 days) as ascertained by four blood glucose determinations daily.

Results: Improvement of blood glucose level was associated with reduction of uCa both in Type 1 ( $6.7 \pm 1$ vs. $5.0 \pm 0.9 \mathrm{mmol} / \mathrm{day})$ and in Type 2 patients $(4.3 \pm 0.4$ vs. $3.1 \pm 0.4 \mathrm{mmol} /$ day $)$. It was found that considerably more Type 1 patients ( 15 out of 35 ) had PTH values below the detection limit $(1.5 \mathrm{pmol} / \mathrm{l})$ during poor than during improved control (4 out of 35). Type 2 patients also showed this difference but to a lesser extent. 33 out of 95 type 2 patients had PTH level below detection limit during poor control as compared to 5 patients during good control. Comparison between two types of diabetes showed that in Type 1 under poor control, Ca and PTH were lower, while uCa was higher, and after improved control, only uCa continued to be higher.

Discussion and Conclusion: Increased $\mathrm{uCa}$ excretion and decreased PTH levels are associated with uncontrolled blood glucose levels (especially in Type 1 diabetes). Therefore decreased serum PTH levels in uncontrolled Diabetes Mellitus may be one of factors leading to reduced bone mass. Hence it is justified to call Diabetes Mellitus a synonym to functional hypoparathyroidism.

Keywords: Mellitus, synonym, hypoparathyroidism 


\section{P216}

Chemical composition of dentin of the lower incisor in rats of various ages after excessive palm oil intake and administration of garciniae cambogia extract

Kamilya Ismailova ${ }^{1}$, Vladyslav Luzin $^{1}$, Yuliya Gayvoronskaya ${ }^{1}$, Maksim Grishchuk $^{1}$

${ }^{1}$ LPR SE “St. Luke State Medical University of Lugansk”, Lugansk, Ukraine

The aim of the study is to analyze chemical composition of dentin of the lower incisor (DI) in rats of different ages after excessive palm oil (PO) intake and administration of Garciniae cambogia extract (GE).

Material and Methods: The experiment involved 216 rats (immature, mature and senile). The 1st group comprised intact animals, the 2nd-the animals that received intragastric PO in dosage of $30 \mathrm{mg} / \mathrm{kg}$, and the 3rd-PO and intragastric GE in dosage of $0.25 \mathrm{mg} /$ $\mathrm{kg}$. The animals were withdrawn from the experiment by the 1 st to the 60th day after 6-week of PO intake. DI were prepared for atomic absorption spectrometry.

Results: Excessive intake of PO resulted in instability of chemical composition of the of DI. In immature animals $\mathrm{Ca}$ and $\mathrm{F}$ ratio decreased as compared to the control values from the 1 st to the 60th days by $4.63,5.06,5.49$ and $5.99 \%$ and by $5.13,5.52,5.81$ and $6.81 \%$ respectively (here and below $\mathrm{p}<0.05$ in all cases). In mature animals the same values changed in the same way by $4.31,4.28,5.54$ and $6.72 \%$ and by $4.01,4.96,6.62$ and $7.45 \%$ and in senile animals-by $4.92,5.28,5.87$ and $7.98 \%$ and by $6.74,7.43,7.71$ and $8.77 \%$. Administration of GE reduced negative effects of $\mathrm{PO}$ as compared to the 2nd group. After GE administration on the 60th days in immature rats $\mathrm{Ca}$ ratio increased as compared to the control by $5.21 \%$, in mature rats by $4.89 \%$, and in elderly-by $4.04 \%$.

Conclusions: Long-term excessive intake of PO results in instability of chemical composition of the of DI. Terms and intensity of alterations depend on age of experimental animals. Administration of GE reduced negative effects of PO on the chemical composition of DI.

Keywords: palm oil, lower incisor, dentin, chemical composition

\section{$\mathbf{P 2 1 7}$}

Development of novel CRISPR/Cas9-based gene therapeutic approaches for osteopetrosis

Floriane Hennig $^{1,2}$, Uta Rössler ${ }^{1,2,3}$, Dimitrios L. Wagner ${ }^{4}$, Thorsten Braun $^{5}$, Sven Geissler ${ }^{1,6}$, Harald Stachelscheid ${ }^{1,3}$, Manfred Gossen ${ }^{1,7}$, Uwe Kornak ${ }^{1,2,8}$

${ }^{1}$ Berlin-Brandenburg Center for Regenerative Therapies, Charité Universitätsmedizin Berlin, Berlin, Germany, ${ }^{2}$ Institute for Medical Genetics and Human Genetics, Charité - Universitätsmedizin Berlin, Berlin, Germany, ${ }^{3}$ Berlin Institute of Health, Berlin, Germany, ${ }^{4}$ Institute of Medical Immunology, Charité - Universitätsmedizin Berlin, Berlin, Germany, ${ }^{5}$ Department of Obstetrics and Gynecology, Charité - Universitätsmedizin Berlin, Berlin, Germany, ${ }^{6}$ Julius Wolff Institute and Center for Musculoskeletal Surgery, Charité - Universitätsmedizin Berlin, Berlin, Germany, ${ }^{7}$ Institute of Biomaterial Science, Helmholtz-Zentrum Geesthacht, Teltow, Germany, ${ }^{8}$ Max Planck Institute for Molecular Genetics, Berlin, Germany

Autosomal recessive osteopetrosis (ARO) is a severe hereditary bone disease caused by lacking osteoclast-mediated bone resorption. Some of the pathogenic mutations are located in the CLCN7 gene coding for the chloride channel $\mathrm{ClC}-7$ that is crucial for bone resorption. In consequence of non-functional osteoclasts, dense and fragile bone is built that, in turn, causes bone marrow failure in early childhood. As osteoclasts develop from hematopoietic stem cells (HSCs), allogeneic HSC transplantation is a curative treatment for ARO. However, this therapy is associated with high mortality and often no suitable donors are available. Somatic gene therapy offers new treatment options. Ex vivo CRISPR/Cas9-based genome editing in patient-derived HSCs followed by autologous stem cell transplantation could be used to cure ARO.

Here, we established novel CRISPR/Cas9-based gene therapeutic approaches for $C L C N 7$-related osteopetrosis. We developed a general additive gene transfer strategy that is applicable to all types of $C L C N 7$ mutations. By using recombinant adeno-associated virus serotype 6 donor delivery and Cas 9 ribonucleoproteins with synthetic guide RNAs, wild type CLCN7 cDNA can be introduced into the safe harbour region $A A V S 1$. In cord blood HSCs, targeted integration of the donor construct into the AAVS1 locus was achieved in over $65 \%$ of the treated cells. Towards a physiological approach, we are working on patient-specific mutation correction. Therefore, we generated patient-specific, osteopetrotic induced pluripotent stem cells (iPSCs). Single base pair editing was performed by delivering Cas9 ribonucleoproteins and single-stranded donor oligonucleotides to the cells. Quantification of fluorescent reporters by flow cytometry revealed up to $3 \%$ monoallelic and $60 \%$ biallelic correction in iPSCs. Currently, we are investigating the phenotypical rescue of our gene therapeutic approaches in our established iPSC-based osteopetrosis disease model. Collectively, we showed highly efficient CRISPR/ Cas9-based genome editing in HSCs and iPSCs forming the basis for new gene therapeutic treatments of ARO.

\section{P218}

Lysine-specific demethylase 1 regulates osteoblast differentiation through modulating $\mathrm{H} 3 \mathrm{~K} 4 \mathrm{me} 1$ levels at specific gene promoters

Kati Tarkkonen ${ }^{1}$, Petri Rummukainen ${ }^{1}$, Amel Dudakovic ${ }^{2}$, Rana AlMajidi $^{1}$, Cristina Valensisi ${ }^{3}$, David Hawkins ${ }^{3,4}$, Andre Van Wijnen ${ }^{2}$, Riku Kiviranta ${ }^{1,5}$

${ }^{1}$ Institute of Biomedicine, University of Turku, Turku, Finland, ${ }^{2}$ Orthopedic Research, Mayo Clinic, Rochester, United States, ${ }^{3}$ Division of Medical Genetics, University of Washington Seattle, Seattle, United States, ${ }^{4}$ Turku Centre for Biotechnology, University of Turku, Turku, Finland, ${ }^{5}$ Division of Endocrinology, Turku University Hospital, Turku, Finland

Regulation of histone methylation is an important step in controlling local chromatin structure and gene expression during mesenchymal stem cell differentiation. Several members of the Jumonji-domain containing histone demethylases (KDMs) have been implicated in osteoblast differentiation, but the role of the monoamine oxidase family of KDMs comprised of $\mathrm{Kdm} 1 \mathrm{a} / \mathrm{LSD} 1$ and $\mathrm{Kdm} 1 \mathrm{~b} /$ LSD2 is poorly known. In our RNA-seq analysis of mouse MC3T3E1 cells, we identified lysine-specific demethylase Lsd1 to be highly expressed during osteoblast differentiation. Inhibition of LSD1 activity either by shRNAs or by inhibitors RN-1 or GSK-LSD1 led to impaired differentiation and mineralization of MC3T3-E1 and primary calvarial osteoblasts in vitro, demonstrated by reduced alkaline phosphatase and von Kossa staining and decreased expression of osteoblastic genes. To study the role of LSD1 in modulating chromatin state, we performed ChIP-seq analysis of LSD1 and H3K4 at different methylation states (me1/me2/me3) in MC3T3-E1 cells during osteoblast differentiation. LSD1 knockdown did not affect global H3K4 methylation levels, but we found LSD1 binding to correlate with reduced $\mathrm{H} 3 \mathrm{~K} 4 \mathrm{me} 1$ levels at specific gene promoters and putative enhancer sites. More than $50 \%$ of LSD1 binding was at promoter regions with dynamic changes during cell differentiation. Up to $20 \%$ of the LSD1 ChIP-seq peaks contained RUNX2 binding motif. Interestingly, we also showed that RUNX2 and LSD1 interact 
in a co-immunoprecipitation assay. In conclusion, our data suggest that LSD1 modulates promoter H3K4 methylation status in osteoblasts and it could be recruited to it's target sites at least in part via interaction with RUNX2, leading to establishment of chromatin state favoring osteogenic differentiation. Our study also indicates that the context specific actions of LSD1 may result from the cell-lineage specific protein-protein interactions. This offers new options to modulate LSD1 activity in a cell-type specific manner, which could provide novel approaches for e.g. developing cancer therapeutics. Keywords: Histone demethylase, Osteoblast, ChIP-seq

\section{P219}

Functional studies of two variants found in the resequencing of SOST Núria Martínez-Gil ${ }^{1,2,3}$, Neus Roca-Ayats ${ }^{1,2,3}$, Natalia GarciaGiralt $^{4,5}$, Xavier Nogués ${ }^{4,5}$, Leonardo Mellibovsky ${ }^{4,5}$, Adolfo DíezPérez $^{4,5}$, Daniel Grinberg ${ }^{1,2,3}$, Susana Balcells ${ }^{1,2,3}$

${ }^{I}$ Dpt. Genetics, Microbiology and Statistics, IBUB, Universitat de Barcelona, Barcelona, Spain, ${ }^{2}$ CIBERER, Barcelona, Spain, ${ }^{3}$ Institut de Recerca Sant Joan de Déu, Barcelona, Spain, ${ }^{4}$ Musculoskeletal Research Group, IMIM (Hospital del Mar Medical Research Institute), Barcelona, Spain, ${ }^{5}$ CIBERFES, Institute Carlos III, Barcelona, Spain

SOST encodes the sclerostin protein, which acts as extracellular inhibitor of the Wnt signaling pathway. To better understand the role of this gene in BMD determination and fracture susceptibility, we resequenced it in a truncate selection (BMD extreme groups) from the BARCOS cohort. We found 2 interesting variants (rs17882143 and rs570754792) within the coding region and the upstream region, respectively, and these were genotyped in the complete BARCOS cohort. The SNP rs17882143 was tested for association with BMD and nominal significant differences were obtained for lumbar spine BMD (LS-BMD) under a dominant model ( $\mathrm{p}$-value $=0.0352$ ). This SNP is a missense change that substitutes an amino acid within the signal peptide of the sclerostin protein. To assess whether the change might affect the subcellular localization of the mutated protein or its abundance, western blot studies were performed and these showed that the amount of sclerostin protein excreted into the extracellular space was the same for the WT and the mutated protein. Regarding the rare variant rs570754792, it was found in heterozygosity in only three women all 3 below the 50 percentile of BMD in the BARCOS cohort. This variant lies in the SOST extended TATA box. To test its effect, we performed reporter gene (luciferase) assays with constructs containing each of the variant alleles. Our results showed significant differences in luciferase activity between the two alleles tested, where the minor allele displayed lower transcription capacity.

In conclusion, we found a significant association of a missense SOST SNP with LS-BMD but failed to uncover a functional basis for this association, thereby questioning its causality. Additionally, we provided functional evidence for a rare variant located in the SOST TATA box sequence carried by women with low LS-BMD.

\section{$\mathbf{P 2 2 0}$}

A genetic risk score for whole body BMD associates with the risk of pediatric fracture

Carolina Medina-Gomez ${ }^{1}$, Olja Grgic ${ }^{1}$, Katerina Trajanoska ${ }^{1}$, Andre ${ }_{\text {G. Uitterlinden }}{ }^{\text {, Vincent }}$ Jaddoe $^{1}$, Fernando Rivadeneira ${ }^{1}$

${ }^{l}$ ErasmusMC, Rotterdam, Netherlands

Background: Low bone density has been associated with higher risk of fractures in healthy children. However, this association might be confounded by calcium intake, fat mass or levels of physical activity. To study genetically-determined differences in BMD in children, we constructed a genetic score (GS) of total-body (TB-) BMD SNPs in children and tested for association with risk of fracture.

Methods: We studied 2504 children from the Generation R study with genome-wide genotyping (Illumina 660K) imputed to 30 million SNPs (HRC panel), and fracture information. Fractures at any skeletal site were assessed using questionnaire reports obtained before a mean age of 9.8 years. We constructed genetic scores consisting of the sum of BMD-decreasing alleles $(0,1,2)$ of 89 variants (from 78 TB-BMD loci) and of 215 eBMD SNPs (from 151 heel ultrasound loci). Risk (odds) of fracture was estimated from logistic regression models adjusted for sex, age and 10 genomic principal components.

Results: Fracture was observed in 364 children (14.5\%). A significant increase in the odds of fracture was observed for every BMD-decreasing allele (OR: $1.0295 \%$, CI 1.01-1.4; P = 0.01). In other words, having 5 more BMD-decreasing alleles would convey in average $12 \%$ increased odds of sustaining a pediatric fracture. Prevalence of fracture increased across the (low) BMD-GS bins $(\mathrm{P}=0.04)$. The highest prevalence was observed in children with the highest GS-tertile $(17.3 \%, \mathrm{n}=312)$, followed by children in the middle tertile $(14.6 \%, \mathrm{n}=1975)$ and those in the lowest tertile $(9.7 \%, \mathrm{n}=217)$. The heel ultrasound GS was not associated with paediatric fracture risk $(\mathrm{P}=0.60)$.

Conclusions: These findings support a causal (unconfounded) relationship between low BMD and increased risk of fracture in children; and imply that a considerable fraction of the differences in bone fragility occurring before peak-bone mass accrual are genetic in origin.

Keywords: pediatric fracture, TB-BMD, genetic risk score

\section{P221}

Bone-specific microRNA profiles in elderly diabetes patients with and without prevalent and prospective hip fractures

Ines Foess $^{1}$, Petra Kotzbeck ${ }^{1}$, Vito Francic ${ }^{1}$, Christoph Haudum ${ }^{1}$, Andrea Groselj-Strele ${ }^{2}$, Jutta Claudia Piswanger-Sölkner ${ }^{1}$, Harald Dobnig $^{1,3}$, Thomas Pieber ${ }^{1}$, Astrid Fahrleitner-Pammer ${ }^{1}$, Barbara Obermayer-Pietsch $^{1}$

${ }^{I}$ Department of Internal Medicine, Division of Endocrinology and Diabetology, Medical University of Graz, Graz, Austria, ${ }^{2}$ Core Facility Computational Bioanalytics, Medical University of Graz, Graz, Austria, ${ }^{3}$ Schilddrüsen/Endokrinologie/OsteoporoseInstitut Dobnig GmbH, Graz, Austria

Objective: Fragility fractures are major events in elderly patients, especially in type 2 diabetes mellitus (T2DM) based on impaired bone quality and/or metabolism. In these patients, insufficient fracture risk prediction via classical methods (e.g. bone density measurements) require new diagnostic approaches, such as bone-specific microRNAs. In this study, we aimed to define microRNA profiles for fracture risk prediction in T2DM.

Methods: In a multicenter cohort of 249 T2DM and 301 nonT2DM nursing home patients, fractures were prospectively recorded over a 2-year period. Mobility of the patients was documented as well as their medical history. Laboratory parameters including bone turnover were measured from blood samples and serum microRNAs were determined by sequencing. Target sequence prediction was used to identify candidate genes regulated by these microRNAs.

Results: The patients were on average $84.4 \pm 6.3$ years old and had a body mass index (BMI) of $25.4 \pm 4.7$. Prospective hip fractures occurred in $34 \%$ of patients with T2DM compared to $15 \%$ in the nonT2DM group $(p<0.0001)$. Non-T2DM patients without fracture were significantly more mobile than non-T2DM with fractures $(p<0.01)$. Vitamin D levels were generally very low and slightly different between non-T2DM patients with and without fracture ( $\mathrm{p}<0.01$ ), but not between T2DM and non-T2DM patients. 
MicroRNA sequencing in groups with and without fracture revealed differences in the profiles of the groups.

Conclusion: In elderly T2DM patients at high fracture risk, we found differently expressed microRNAs prior to prospective hip fractures. Therefore, microRNAs might be promising biomarkers for determining fracture risk in elderly patients, especially in patients with T2DM, where conventional diagnostic tools do not allow for sufficient risk prediction.

Keywords: microRNA, biomarker, diabetes, Vitamin D, mobility

\section{$\mathbf{P 2 2 2}$}

Autosomal recessive osteogenesis imperfecta caused by a novel homozygous COL1A2 mutation

Alice Costantini ${ }^{1}$, Symeon Tournis ${ }^{2}$, Anders Kämpe ${ }^{1}$, Noor Ul Ain ${ }^{1}$, Fulya Taylan $^{1}$, Artemis Doulgeraki ${ }^{3}$, Outi Mäkitie ${ }^{1,4,5}$

${ }^{1}$ Department of Molecular Medicine and Surgery and Center for Molecular Medicine, Karolinska institutet, Stockholm, Sweden, ${ }^{2}$ Laboratory for Research of the Musculoskeletal System 'Th. Garofalidis', KAT Hospital, National and Kapodistrian University of Athens, School of Medicine, Athens, Greece, ${ }^{3}$ Department of Bone and Mineral Metabolism, Institute of Child Health, "Aghia Sophia" Children's Hospital, Athens, Greece, ${ }^{4}$ Folkhälsan Institute of Genetics and University of Helsinki, Helsinki, Finland, ${ }^{5}$ Children's Hospital, University of Helsinki and Helsinki University Hospital, Helsinki, Finland

Osteogenesis imperfecta (OI) is a skeletal dysplasia characterized by brittle bones and extra-skeletal manifestations. The disease phenotype varies greatly. Most commonly, OI arises from monoallelic mutations in one of the two genes encoding type I collagen, COL1AI and $C O L 1 A 2$ and is inherited as an autosomal dominant trait.

Here we describe a consanguineous family with autosomal recessive OI caused by a novel homozygous glycine substitution in COL1A2, NM_000089.3: c.604G $>$ A, p.(Gly202Ser), detected by whole-genome sequencing. The index patient is a 31 -year-old Greek woman with severe skeletal fragility. She had mild short stature, low bone mineral density of the lumbar spine and blue sclerae. She had sustained multiple long bone and vertebral fractures since childhood and had been treated with bisphosphonates for several years. She also had an affected sister with similar clinical manifestations. Interestingly, the parents and one sister, all carriers of the COL1A2 glycine mutation, did not have manifestations of OI.

In summary, we report on autosomal recessive OI caused by a homozygous glycine to serine substitution in COL1A2, leading to severe skeletal fragility. The mutation carriers lacked OI manifestations. This family further expands the complex genetic spectrum of OI and underscores the importance of genetic evaluation for correct genetic counselling.

Keywords: autosomal recessive; osteogenesis imperfecta; COL1A2; type I collagen; whole-genome sequencing

\section{P223}

MicroRNA profiling of primary human osteoblasts and osteosarcoma cell lines reveals transcriptional regulators of oncogenesis and of osteoblastic differentiation

Brendan Norman ${ }^{1}$, Peter Wilson ${ }^{1}$, Mohd Osman ${ }^{1}$, Norman Roberts ${ }^{1,2}$, Lakshminarayan Ranganath $^{1,2}$, James Gallagher ${ }^{1}$

${ }^{I}$ Institute of Ageing \& Chronic Disease, University of Liverpool, Liverpool, United Kingdom, ${ }^{2}$ Liverpool Clinical Laboratories, Royal Liverpool and Broadgreen University Hospital Trust, Liverpool, United Kingdom
MicroRNAs (miRNAs) are 20-24 nucleotide complexes which are becoming increasingly recognised as key epigenetic regulators of transcriptional events throughout the cycle of osteoblast differentiation and maturation. In this study we characterised miRNA profiles of primary human osteoblasts (HOBS) and three osteosarcoma cell lines, MG-63, TE85 and Saos2, representing sequential stages of osteoblast differentiation.

HOBS were cultured from bone explants from three donors. Osteosarcoma cell lines were from lab stocks which have been extensively characterised for markers of osteoblastic differentiation. MiRNA was isolated from cell culture homogenates using miRNeasy Mini Kit (Qiagen). MiRNA profiling was performed with an Affymetrix GeneChip scanner $30007 \mathrm{G}$ on an Affymetrix miRNA 4.0 array.

In total, 2587 miRNA were profiled across samples. As shown in the heatmap (Fig. 1) and in the group clustering from principal components analysis (PCA), the clearest difference in miRNA profiles was between HOBS and cell lines, with 157 differentially expressed miRNAs $(\mathrm{p}<0.05$, fold change $>2)$. PCA revealed that component 1 , which distinguished HOBS from cell lines, accounted for $48 \%$ of the variation and comprised oncogenic miRNAs such as those associated with Wnt signalling.

Additionally, 85 miRNA were differentially expressed $(\mathrm{p}<0.05$, fold change $>2$ ) between the cell lines. PCA component 3 , which accounted for $9 \%$ of the variation, captured the sequential order of osteoblast differentiation between the cell lines. The miRNA contributing most to this sequence of differentiation were hsa-miR-1555p, hsa-miR-212-3p, hsa-miR-708-5p and hsa-miR-143-3p.

In conclusion, this experimental approach revealed microRNA associated with osteosarcoma oncogenesis and with osteoblastic differentiation.

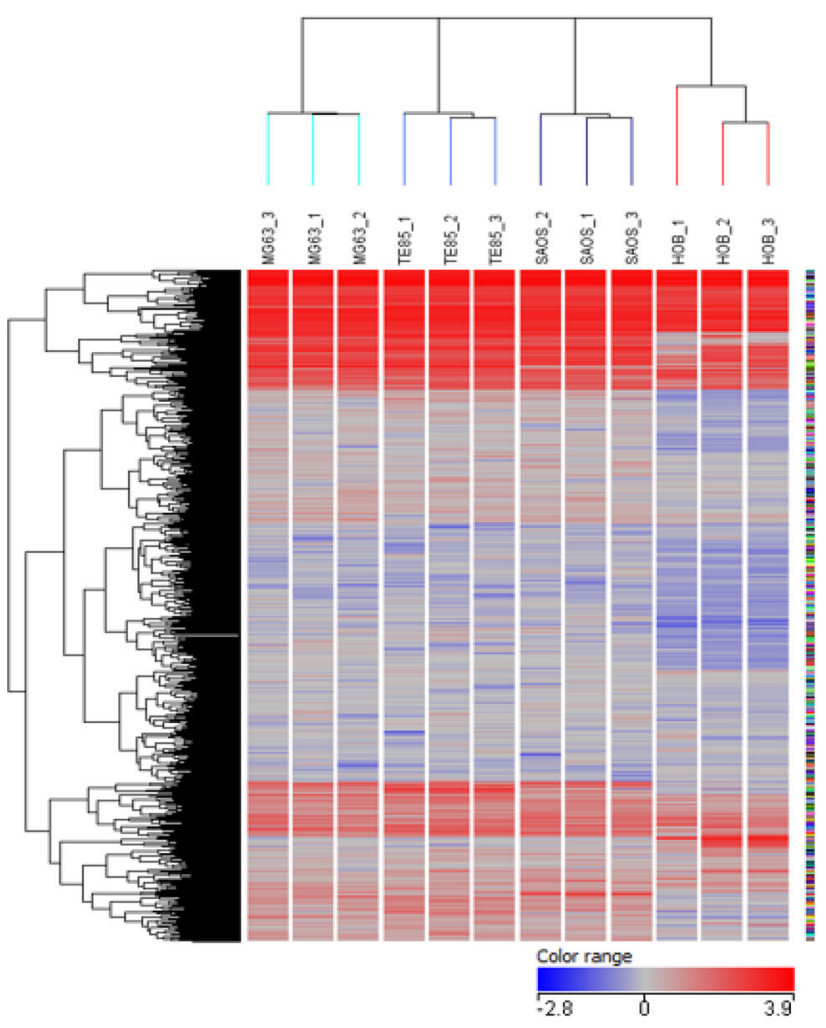

Heatmap of miRNA expression across cells under study. Vertical tree represents hierarchical clustering between individual miRNA.

Fig. 1 


\section{$\mathbf{P 2 2 4}$}

Incidence of mutations in the TNSALP, GGPS1 and CYP1A1 genes in patients with atypical femoral fractures

Pilar Peris ${ }^{1}$, Eva González-Roca ${ }^{2}$, Sebastian C. Rodríguez-García ${ }^{1}$, $\mathrm{M}^{\mathrm{a}}$ Del Mar López-Cobo ${ }^{3}$, Ana Monegal ${ }^{1}$, Nuria Guañabens ${ }^{1}$

${ }^{I}$ Rheumatology, Hospital Clinic, University of Barcelona, IDIBAPS, Barcelona, Spain, ${ }^{2}$ Immunology, Hospital Clinic, University of Barcelona, IDIBAPS, Barcelona, Spain, ${ }^{3}$ Molecular Biology Core Laboratory, Hospital Clinic, University of Barcelona, IDIBAPS, Barcelona, Spain

Atypical femoral fractures (AFF) are uncommon and often related to prolonged bisphosphonate (BP) treatment. Isolated AFF cases are linked to mutations of Tissue-Nonspecific Alkaline Phosphatase (TNSALP), as a presentation of hypophosphatasia in adults. Mutations in the geranylgeranyl pyrophosphate synthase (GGPPS) enzyme which can be inhibited by BPs, and in the enzyme of the cytochrome P450 superfamily (CYP1A1), related to the metabolism of several drugs, have also been associated with some AFF cases. Our aim was to analyse the clinical characteristics and incidence of TNSALP, GGPS1 and CYP1A1 gene mutations in AFF.

Methods: 17 women with AFF (aged $71 \pm 10$ years) were included. Sanger sequencing for the TNSALP, GGPS1 and CYP1A1 genes was performed in all patients. We analysed the ALP substrates (vitamin B6 and PEA), bone turnover markers, bone mass, previous antiosteoporotic treatments and the clinical characteristics of the patients and the fractures.

Results: $3 / 17$ patients $(17.6 \%)$ presented heterozygote mutations in TNSALP (p.Gly288Ala) and CYP1A1 (p.Arg136His, p.Val409Ile) genes, respectively. The patient with the TNSALP mutation presented increased vitamin B6 and reduced serum ALP levels. Patient with the CYP1A1 mutations had glucocorticoid-induced osteoporosis treated with. All patients were previously treated with BPs (94\% alendronate) for $85.5 \pm 38$ months, and nearly $50 \%$ also received glucocorticoids. AFF was bilateral in $35 \%$ of cases and $76 \%$ had previous fragility fractures.

Conclusions: Mutations in the CYP1A1 or TNSALP gene may be related to the development of AFF in some patients receiving BPs. Evaluation of ALP substrates in patients with low ALP levels allows the identification of hypophosphatasia. The role of CYP1A1 mutations in AFF needs further study.

\section{P225}

Hypophosphatasia: search for coding mutations and large genomic deletions of the ALPL gene in a large cohort of South Italy

Luigia Cinque $^{1}$, Antonio S Salcuni ${ }^{2}$, Domenico Trombetta ${ }^{3}$, Flavia Pugliese $^{4}$, Agostino Gaudio ${ }^{5}$, Luisa Politano ${ }^{6}$, Agnese Caiola ${ }^{7}$, Claudia Battista ${ }^{4}$, Teresa Mattina ${ }^{8}$, Giuseppa L Mandarà ${ }^{9}$, Piera Vicchio $^{10}$, Elisabetta Pasquini ${ }^{11}$, Annamaria Colao ${ }^{12}$, Mauro Celli ${ }^{13}$, Arturo Ferrara ${ }^{14}$, Cosimo Giannini ${ }^{15}$, Rosaria M Ruggeri ${ }^{16}$, Alfredo B Scillitani ${ }^{4}$, Vito Guarnieri ${ }^{1}$

${ }^{I}$ Division of Medical Genetics, IRCCS Casa Sollievo della Sofferenza, San Giovanni Rotondo, Italy, ${ }^{2}$ Department of Medical Sciences, University of Cagliari, Cagliari, Italy, ${ }^{3}$ Laboratory of Oncology, IRCCS Casa Sollievo della Sofferenza, San Giovanni Rotondo, Italy, ${ }^{4}$ Endocrinology, IRCCS Casa Sollievo della Sofferenza, San Giovanni Rotondo, Italy, ${ }^{5}$ Internal Medicine, Policlinico Universitario ' $G$. Rodolico', Catania, Italy, ${ }^{6}$ Cardiomyology and Medical Genetics, Campania University 'Luigi Vanvitelli', Napoli, Italy, ${ }^{7}$ Pathology, AO S. Anna and S. Sebastiano, Caserta, Italy, ${ }^{8}$ Medical Genetics, University of Catania, Catania, Italy, ${ }^{9}$ Medical Genetics, Maria Paternò Arezzo Hospital, Ragusa, Italy, ${ }^{10}$ Pediatrics and Neonatology, G. Jazzolino Hospital, Vibo Valentia, Italy, ${ }^{11}$ Metabolic and
Muscular Unit, Meyer Children's Hospital, Firenze, Italy, ${ }^{12}$ Endocrinology, University of Naples "Federico II", Napoli, Italy, ${ }^{13}$ Pediatrics, Sapienza University of Rome, Rome, Italy, ${ }^{14}$ Medical Genetics, Villa Sofia-Cervello, Palermo, Italy, ${ }^{15}$ Pediatrics, SS. Annunziata Hospital, Chieti, Italy, ${ }^{16}$ Endocrinology, AOU Policlinico G. Martino, Messina, Italy

Hypophosphatasia (HPP, MIM146300) is a genetic inherited disorder caused by inactivating mutations of the ALPL gene, encoding for the alkaline phosphatase enzyme. Though classified as a autosomal recessive disease, it is not unusual to find only one mutated allele. HPP may manifest with a high phenotypic variability, not just in patients carrying different mutations but also in patients with the same variant; this makes challenging the diagnosis, as well as the patient management and the genetic counselling.

Here we report our experience on a large cohort of patients with clinical and biochemical diagnosis of HPP, mainly belonging to the Middle and South Italy (the 90\%), consisting of 27 apparently sporadic cases and 7 familial cases.

Screening of the coding region of the ALPL gene (NM_000478.5) revealed 19 variants differently recurrent in 34 patients $(55.8 \%)$ : 6 variants were novel (p.M219I, p.F290L, p.D109E, p.V95M, p.H472R, p.K322Rfs*45); 3 patients with variable phenotype carried a double variant. The most representative variant, the p.R152H, classified as a harmless polymorphism and having a reported MAF $=0.01(1 \%)$, instead in our cohort was frequent up to the $18 \%$ (6 out of 34 patients), thus suggesting an actual pathogenic role. Sanger sequencing allowed us to deduce a pattern of homozygosity region suggestive of a possible Large Genomic Deletion (LGD). Thus we selected frank clinical cases, negative for coding mutations that we analyzed by classic RTqPCR and DdPCR. Our preliminary results showed the presence of LGDs as secondary underestimated mechanism of ALPL gene inactivation. These results may have implications for the genetic counselling.

\section{P226}

Common gene signature for osteoporosis, diabetes mellitus and peripheral neuropathy by translational bioinformatic approach

Francisco Andújar Vera ${ }^{1}$, Cristina García Fontana ${ }^{1}$, Sheila González Salvatierra ${ }^{2}$, Antonia García Martín ${ }^{3}$, Manuel Muñoz Torres ${ }^{4}$, Beatriz García Fontana ${ }^{5}$

${ }^{I}$ Instituto de Investigación Biosanitaria de Granada (Ibs.GRANADA), Granada, Spain, ${ }^{2}$ Instituto de Investigación Biosanitaria de Granada (Ibs.GRANADA); Department of Medicine. University of Granada, Granada, Spain, ${ }^{3}$ Endocrinology and Nutrition Unit, Hospital Universitario Campus de la Salud. Instituto de Investigación Biosanitaria de Granada (Ibs.GRANADA), Granada, Spain, ${ }^{4}$ Endocrinology and Nutrition Unit, Hospital Universitario Campus de la Salud. Instituto de Investigación Biosanitaria de Granada (Ibs.GRANADA); CIBERFES. Instituto de Salud Carlos III; Department of Medicine. University of Granada., Granada, Spain, ${ }^{5}$ Endocrinology and Nutrition Unit, Hospital Universitario Campus de la Salud. Instituto de Investigación Biosanitaria de Granada (Ibs.GRANADA); CIBERFES. Instituto de Salud Carlos III, Granada, Spain

Background: The link between osteoporosis and several diseases such as diabetes mellitus and peripheral neuropathy is well known. Nowadays high-throughput genome studies provides such extensive information avoiding the identification of the most relevant disease related-genes. To reduce the target genes, is better to study the common gene signature and the signaling pathways involved in osteoporosis, diabetes mellitus and peripheral neuropathy. This signature confirms known associations and identifies novel links between genes and diseases.

Methods: Translational bioinformatics approach was performed to choose the common gene signatures using the following 
bioinformatics tools: the discovery platform DisGeNET, enrichment analysis software FunRich, a candidate gene prioritization platform ToppGene suite and NetworkANalyst online tool to visualize common disease related-genes as a network.

Results: The analysis revealed that AR, MAPK1, TNF, LMNA, PRKAR1A, PTK2B, APOE, IGF1R and IFNG were potential common gene signature. The gene enrichment analysis showed that these genes were involved in phenotype processes related to cardiovascular $(22 \%)$, peripheral nervous system $(15 \%)$ or skeletal $(22 \%)$ disturbances. Also, the network analysis showed that these genes were important nodes with significant interacting partners, in agreement with the gene rank prioritization by ToppGene tool. In addition, a close relationship between the genetic signature of the three studied diseases and atherosclerosis was observed.

Conclusions: Our analysis provides novel insights into the links between osteoporosis, diabetes mellitus and peripheral neuropathy identifying 9 genes as potential common therapeutic targets.

Keywords: Gene signature, bioinformatics, diabetes mellitus, osteoporosis, peripheral neuropathy

\section{P227}

Single nucleotide polymorphisms in the OCT1 gene are associated with potential vascular calcification in non-diabetic, prediabetic and diabetic patients

Natascha Schweighofer $^{1,2}$, Christoph Haudum ${ }^{1,2}$, Albrecht Schmidt ${ }^{3}$, Caterina Raffaela Colantonio $^{3}$, Ines Mursic ${ }^{3}$, Thomas R. Pieber ${ }^{1,2}$, Barbara Obermayer-Pietsch ${ }^{1,2}$

${ }^{I}$ Department of Internal Medicine, Division of Endocrinology and Diabetology, Medical University of Graz, Graz, Austria, ${ }^{2}$ CBmed GmbH, Center for Biomarker Research in Medicine, Graz, Austria, ${ }^{3}$ Department of Internal Medicine, Division of Cardiology, Medical University of Graz, Graz, Austria

Increased cardiovascular events and a high risk for vascular calcification are known to be associated with type 2 diabetes (T2DM). We were recently able to associate polymorphisms in $O C T 1$ with cardiovascular risk factors and with a therapy-dependent increased risk of cardiovascular death.

In this study, we investigated whether SNPs in OCT1 were associated with cardiovascular parameters such as pulse wave velocity (PWV), echocardiographic parameters and intima/media thickness (IMT) in non-diabetic, prediabetic and T2DM patients with elevated cardiovascular risk.

Data from the BioPersMed cohort $(\mathrm{n}=1025)$, a prospective cohort study of asymptomatic patients at cardiovascular risk were analysed. T2DM, prediabetes, non-diabetes were defined according to ADA criteria. Determination of SNP genotypes was done by GSA array (Illumina Inc., USA). Pulse wave analysis was done using a SphygmoCor device (Atcor Medical, Australia), carotid intima/media thickness and echocardiographic measurements were performed using the Vivid 9 device (GE Healthcare Austria GmbH \& Co OG, Austria).

In a preliminary analysis, we focused on associations with score systems for cardiovascular risk (PROCAM and Framingham), as well as PWV and IMT.

An association with the Framingham score was seen for SNPs rs $35888596(\mathrm{p}=0.012)$ and $\mathrm{rs} 112476023(\mathrm{p}=0.015)$ in nondiabetics and $\mathrm{rs} 461473(\mathrm{p}=0.018)$ and $\mathrm{rs} 806383(\mathrm{p}=0.03)$ in prediabetics. PROCAM score was associated with SNPs rs112476023 (p = 0.023$)$ in non-diabetics and rs662138 $(\mathrm{p}=0.045)$ in T2DM.

A significant association with PWV was seen for rs 12208357 ( $\mathrm{p}=0.02$, non-diabetics), $\mathrm{rs} 12208357(\mathrm{p}=0.009$, prediabetics $)$ and rs662138 ( $\mathrm{p}=0.034, \mathrm{~T} 2 \mathrm{DM})$. Associations with IMT were seen in diabetics for $r s 806383(\mathrm{p}=0.034)$, rs2197296 $(\mathrm{p}=0.027)$, rs622342 $(\mathrm{p}=0.008)$, and rs662138 $(\mathrm{p}=0.030)$, in prediabetics for $\mathrm{rs} 34130495$ $(\mathrm{p}=0.048)$ and $\mathrm{rs} 622342(\mathrm{p}=0.049)$ and in T2DM for $\mathrm{rs} 2282142$ $(\mathrm{p}=0.008), \mathrm{rs} 2282143(\mathrm{p}=0.009)$ and $\mathrm{rs} 112476023(\mathrm{p}=0.019)$.

These associations might implicate a consistent role of $O C T 1$ variants in the modulation of vascular calcification processes and thus contribution to cardiovascular risk beyond T2DM.

\section{P228}

WNT16 genetic variation in fracture-prone children and osteoporosis $\underline{\text { Minna Pekkinen }}^{1,2}$, Sara Mäkitie $^{1}$, Mervi Mäyränpää $^{3}$, Helena Valta $^{3}$, Outi Mäkitie ${ }^{1,3}$

${ }^{1}$ Folkhälsan Institute of Genetics and University of Helsinki, University of Helsinki, Finland, ${ }^{2}$ Children's Hospital, University of Helsinki, Helsinki, Finland, ${ }^{3}$ Children's Hospital, Helsinki University Central Hospital and University of Helsinki, Helsinki, Finland

Introduction: WNT16 has been shown to associate with bone mineral density (BMD), bone strength, and osteoporotic fractures in several genome-wide association studies. WNT signaling stimulates the differentiation of osteoblast precursors and plays a major role in skeletal development and homeostasis. WNT16, a ligand to the signaling pathway, has a crucial role in cortical bone homeostasis. Decreased cortical bone density is associated with non-vertebral osteoporotic fractures. Practically no data exists regarding the significance of WNT16 in childhood-onset osteoporosis and fractures. We therefore wanted to explore the role of WNT16 genetic variation in fracture-prone children and early-onset osteoporosis.

Subjects and Methods: WNT16 gene was screened by Sanger sequencing in 46 pediatric patients with early-onset osteoporosis, in 60 pediatric patients with multiple fractures and 95 healthy controls.

Results: We found all together 12 variants in WNT16, of which one was a novel change. In spite of the large amount of genetic variation found in WNT16, no actual mutations were found. One of the changes rs 10668066 were associated statistically significant with BMD and early onset-childhood osteoporosis.

Conclusions: Despite recent observations demonstrating that WNT16 genetic variation impacts cortical bone thickness, BMD, bone strength, and risk of fractures in mice and humans, our findings indicate that WNT16 mutations or variations are not a common cause of increased fractures in children.

\section{P229}

Association between polymorphisms in neuropeptide $\mathrm{Y}$, and neuronal nitric oxide synthase (NOSI) gene polymorphisms and bone response to hormone therapy in postmenopausal Korean women

$\underline{\text { Jung Gu Kim}}{ }^{1}$, Hoon $\mathrm{Kim}^{1}$, Jong Hak Kim${ }^{2}$

${ }^{1}$ Seoul National University Hospital, Seoul, Republic of Korea,${ }^{2}$ Ewha Womans' University Hospital, Seoul, Republic of Korea

This study aimed to investigate the association between polymorphisms in neuropeptide Y (NPY), its receptor, and neuronal nitric oxide synthase (NOSI) genes and bone response to hormone therapy (HT) in postmenopausal Korean women. A total of seven polymorphisms in the neuropeptide Y (NPY) gene (rs3037354, rs17149106 and rs16147), NPY receptor 2 (NPY2R) gene (rs2880415, rs6887715), NOS1 gene (rs41279104, rs9658356) were analyzed in 448 postmenopausal Korean women receiving sequential estrogen-progestogen therapy (EPT) for 1 year. EPT involved 30-day cycles of receiving $0.3-\mathrm{mg}$ a daily dose of conjugated equine estrogen from day 1 to day 30 of the cycle and 5-mg daily dose of medroxyprogesterone acetate from day 1 to day 12 of the cycle. BMD at the lumbar spine (LS) and femoral neck (FN) was determined by dual energy X-ray absorptiometry. Among SNPs measured, NPY rs3037354 ( $\mathrm{P}=0.045)$, and rs16147 $(\mathrm{P}=0.02)$, and NOSI 
rs9658356 polymorphisms were associated with annual percent changes in BMD at FN after 1 year of HT respectively. The BMD at FN in the participants with del/del genotype of rs3037354 and TT genotype of rs16147, and NOSI rs9658356 increased significantly compared to other genotypes (4.4, 4.1 , and $4.6 \%$ respectively). When a non-responder was defined as a woman who had lost more than $3 \%$ of BMD per year after HT, NPY2R rs6887715 was associated with the risk of non-response of HT. The TT genotype of NPY2R rs6887715 demonstrated a 1.6-times higher risk of non-response at $\mathrm{FN}(\mathrm{P}=0.04)$, compared with the non-TT genotype. In conclusion, the NPY rs3037354 and rs16147 polymorphisms may be related with bone response at FN after HT, and the NPY2R rs6887715 polymorphism may be associated with risk of nonresponse to HT in postmenopausal Korean women.

\section{P230}

Osteoclast-derived autotaxin connects inflammation to bone erosion in arthritis

Irma Machuca-gayet ${ }^{1}$, Sacha Flammier ${ }^{1}$, Fanny Bourguillault ${ }^{1}$, François Duboeuf $^{1}$, Gabor Tigyi ${ }^{2}$, Fabienne Coury ${ }^{1}$, Olivier Peyruchaud $^{1}$

${ }^{1}$ Faculté de Medicine Laennec Lyon, Inserm1033 University Claude Bernard Lyon1, Lyon, France, ${ }^{2}$ Departement of Pharmaceutical Sciences, University of Tennessee Health Science Center, Memphis, United States

Bone erosions are hallmarks of rheumatoid arthritis (RA) correlating with disease severity, intractable pain and poor functional outcome. Osteoclasts are responsible for systemic bone loss and focal erosions in RA. Here we found that osteoclasts present at inflammatory sites express autotaxin (ATX). ATX is a secreted enzyme that converts lysophosphatidylcholine (LPC) into lysophosphatidic acid (LPA). ATX has been reported to control synovial inflammation. We asked if osteoclasts derived ATX could interfere with bone resorption under inflammatory conditions. Using ATX specific inhibitors, we first determined that osteoclast-produced autotaxin (ATX) was a crucial factor controlling osteoclast resorption activity in vitro.

To validate these results, we generated mice with conditional genetic ablation of ATX in osteoclasts $\left(D A T X^{\text {Ctsk }}\right.$ mice) that were challenged using an LPS inflammation-induced bone loss model. LPS injections induced a $43 \%$ decrease of the trabecular BV/TV in control mice but no significant decrease in DATX ${ }^{\text {Ctsk }}$ mice BV/TV. Results indicate that mice lacking ATX in mature osteoclasts were protected from LPS-induced bone loss. ATX is upregulated in the synovial liquid of patients with RA in response to TNF. Though, we challenged DATXCtsk and Control mice with $\mathrm{K} / \mathrm{BxN}$ serum transfer to induce severe arthritis. DATX ${ }^{\mathrm{Ctsk}}$ mice showed significant protection from systemic bone loss, and remarkably displayed almost no local bone erosion, indicating that osteoclast derived ATX was instrumental in the arthritic bone destruction. Transgenic $h \mathrm{TNF}$ mice that develop aggressive arthritis with joint swelling and bone erosions, were treated with anti-autotaxin drug (BMP22). The drug induced a $28 \%$ increase in the trabecular bone mass (BV/TV) and a $50 \%$ decrease in the extent of bone erosion $(\mathrm{BS} / \mathrm{BV})$ at the calcaneum. These results establish that ATX is a new factor produced by osteoclast that controls inflammationinduced bone loss. ATX might be a promising therapeutic target for the prevention of bone erosion occurrence in RA.

\section{P231}

Circulating mesenchymal stem cells are increased in patients with Ankylosing Spondylitis

Chul-Soo Cho ${ }^{1}$, Ki-Jo Kim², In-Woon Baek ${ }^{3}$, Wan-Uk Kim ${ }^{4}$
${ }^{I}$ Rheumatology, Yeouido St. Mary's Hospital, The Catholic University of Korea, Seoul, Republic of Korea, ${ }^{2}$ Rheumatology, St. Vincent Hospital, The Catholic University of Korea, Suwon, Republic of Korea, ${ }^{3}$ Rheumatology, Yeouido St. Mary's Hospital, Seoul, Republic of Korea, ${ }^{4}$ Rheumatology, Seoul St. Mary's Hospital, The Catholic University of Korea, Seoul, Republic of Korea

Objectives: An increased recruitment of circulating mesenchymal stem cells (cMSCs) has been shown to promote fracture healing. However, the role of cMSCs in abnormal bone formation in ankylosing spondylitis (AS) is still elusive.

Methods: Seventy-four male AS patients and 25 age-matched healthy controls were enrolled, and their peripheral bloods were subjected to flow cytometric analysis to count the number of cMSCs, which are positive for MSCs markers CD13, CD29, and negative for hematopoietic markers CD34 and CD45. The clinical and laboratory data were obtained from medical records, and serum bone specific alkaline phosphatase (BAP) and VEGF levels were measured.

Results: Compared to healthy controls, serum levels of BAP and VEGF were significantly increased in AS patients, respectively (median; 24 vs. $13 \mu \mathrm{g} / \mathrm{L}$ and 1066 vs. $892 \mathrm{pg} / \mathrm{mL}$, both $P<0.001$ ). Importantly, cMSCs number was significantly higher in AS patients than in controls (median; 428 vs. 227 cells $/ \mathrm{mL}, P=0.026$ ), and it was positively correlated with serum BAP $(\gamma=0.323, \mathrm{P}=0.004)$ and VEGF levels $(\gamma=0.279, P=0.015)$. When patients were divided into 2 groups according to the presence of syndesmophyte, cMSCs number was significantly higher in AS patients with syndesmophyte than in those without (556 vs. 143 cells $/ \mathrm{mL}, P<0.001)$. These differences and associations were not altered by adjusting for age, disease duration, C-reactive protein, and use of medications.

Conclusion: Increased numbers of MSCs in circulation could contribute to abnormal bone formation in AS patients, which are presumably mediated in part by the action of VEGF through enhancing the mobilization and/or osteogenic differentiation of MSCs.

Keywords: Ankylosing spondylitis, Mesenchymal stem cells, VEGF

\section{P232}

Associations between trabecular bone score and vertebral fractures in patients with axial spondyloarthritis

Hyoung Rae Kim ${ }^{1}$, Kwi Young Kang ${ }^{2}$

${ }^{1}$ Catholic University of Korea, Incheon, Republic of Korea,${ }^{2}$ The Catholic University of Korea, Seoul, Republic of Korea

Objectives: To compare trabecular bone scores (TBS) between axial spondyloarthritis (axSpA) patients with and without vertebral fractures and investigate associations between TBS and vertebral fractures.

Methods: Two hundred and fifty-five patients fulfilling the imaging arm of the Assessment of SpondyloArthritis International Society axSpA criteria were enrolled. TBS and bone mineral density (BMD) were assessed using dual-energy X-ray absorptiometry. Vertebral fracture of the thoracic and lumbar spine was defined according to the Genant criteria. Osteoporosis risk factors, inflammatory markers, disease activity scores, and spinal structural damage were also assessed. Multivariate logistic regression analysis was performed to identify factors associated with vertebral fractures.

Results: Of 255 axSpA patients, $28(11 \%)$ had 31 vertebral fractures. The mean TBS was $1.39 \pm 0.11$ and $1.30 \pm 0.13$ in patients without and with vertebral fractures, respectively $(<0.001)$. BMD in the femoral neck was lower in patients with vertebral fractures $(p=0.027)$, but BMDs in the lumbar spine and total hip were not. In the multivariate analyses, low TBS and the presence of syndesmophytes were significantly associated with vertebral fractures, independently of BMD (OR $[95 \% \mathrm{CI}]=3.6[1.2-11.1]$ and $3.3[1.0-10.7]$, respectively). For the total 
hip, TBS has a better discriminatory value than BMD for prediction of vertebral fractures in axSpA patients $(\mathrm{p}=0.034)$.

Conclusion: TBS values are lower in axSpA patients with vertebral fractures. Low TBS and syndesmophytes were independently associated with prevalent vertebral fractures. TBS has better predictive value to BMD for the discrimination of vertebral fractures and could help to detect axSpA patients with vertebral fractures.

\section{P233}

Disruption of glucocorticoid signalling in osteoblasts attenuates surgically induced osteoarthritis

Jinwen $\mathrm{Tu}^{1}$, Zhe $\mathrm{Ji}^{1}$, Peng Zhang ${ }^{1}$, Holger Henneicke ${ }^{1,2}$, Jingbao $\mathrm{Li}^{1,3}$, Christopher Little ${ }^{4}$, Markus Seibel ${ }^{1}$, Hong Zhou ${ }^{1}$

${ }^{1}$ Bone Research Program, ANZAC Research Institute, The University of Sydney, Sydney, Australia, ${ }^{2} D F G$-Center for Regenerative Therapies, Technische Universität Dresden, Dresden, Germany, ${ }^{3}$ Key Laboratory for Space Bioscience \& Biotechnology, School of Life Sciences, Northwestern Polytechnical University, Xi'an, China, ${ }^{4}$ Kolling Institute of Medical Research, The University of Sydney, Sydney, Australia

Background and aims: Effective treatments for osteoarthritis are limited with the benefit of intraarticular injections of glucocorticoids remaining highly controversial. In this study, we aimed to investigate the impact of endogenous glucocorticoid signalling on the development of bone and cartilage damage in a murine model of surgically induced osteoarthritis.

Methods: We utilized transgenic (tg) mice in which glucocorticoid signaling was disrupted in mature osteoblasts via targeted overexpression of the glucocorticoid-inactivating enzyme, $11 \beta$ hydroxysteroid dehydrogenase type 2 . Osteoarthritis was induced in 22-week-old male tg mice (tg-OA) and their wild-type littermates (WT-OA) by surgical destabilization of the medial meniscus (DMM). Sham operated mice served as controls (WT-Sham \& tg-Sham). Knee joints were harvested 16 weeks after surgery, followed by quantitative micro-CT analysis, histologic scoring and histomorphometric quantification of joint damage.

Results: Development of joint damage was more pronounced in mice with intact osteoblastic glucocorticoid signalling. Thus, both WT$\mathrm{OA}$ and tg-OA animals developed features of subchondral bone sclerosis, cartilage degeneration and osteophyte formation. However, when assessed by micro-CT and histomorphometric analysis, subchondral bone volume in the medial tibial plateau (MTP) was significantly increased in WT-OA compared to tg-OA mice. Cartilage erosion was also significantly attenuated in the medial femoral condyle and MTP of tg-OA mice compared to their WT-OA littermates. Osteophytes were smaller in the MTP of tg-OA compared to WT-OA mice.

Conclusion: Glucocorticoid signalling in mature osteoblasts and osteocytes promotes the development of subchondral-bone hyperplasia, cartilage erosion and osteophyte formation in a mouse model of surgically induced osteoarthritis.

Keywords: Glucocorticoid signalling in osteoblasts/osteocytes, osteoarthritis, DMM model

\section{P234}

Traditional Chinese Medicine formula Bi-Qi capsule alleviates rheumatoid arthritis-induced inflammation, synovial hyperplasia, and cartilage destruction in rats

Janak Lal Pathak ${ }^{1}$, Dongmei Zhang ${ }^{2}$, Kai Wang ${ }^{3}$, Baoli Li $^{2}$

${ }^{1}$ Key Laboratory of Oral Medicine, Guangzhou Institute of Oral Disease, Stomatology Hospital of Guangzhou Medical University, Guangzhou Medical University, Guangzhou, China, ${ }^{2}$ Department of Traditional Chinese Medicine, Tianjin Medical University General Hospital, Tianjin, China, ${ }^{3}$ Department of International Medicine, Geriatric Disease Research Institute, Tianjin Hospital, Tianjin, China

Traditional Chinese Medicine (TCM) formula Bi-Qi capsule (BiQi) is commonly prescribed drug to treat rheumatoid arthritis (RA). However, the mechanism of the Bi-Qi-mediated amelioration of RA pathogenesis is still a mystery. Collagen induced arthritis (CIA) in rats is an established model clinically resembling to human RA. In this study we investigated the effect of Bi-Qi on the pathogenesis of CIA-rats. CIA-rats were gavaged with high $(0.9 \mathrm{~g} / \mathrm{kg}$-body-weight/day) or moderate-dose of Bi-Qi ( $0.6 \mathrm{~g} / \mathrm{kg}$-body-weight/day) or subcutaneously methotrexate injected (MTX, $1.67 \mathrm{mg} / \mathrm{kg}$-bodyweight/week) in abdomen for 4 weeks. Effects of the treatment on RA-pathogenesis were extensively studied. The study protocol was approved by Animal Experimental Ethical Panel of Tianjin Medical University. Differences were considered significant if $P<0.05$. Inflamed and swollen ankles and joints as well as high arthritic score were observed in arthritic rats (Fig. 1), while Bi-Qi or MTX treatment alleviated these symptoms. Bi-Qi-high-dose, moderate-dose, and MTX treatment reduced CIA-induced arthritic score by 13,22 , and $15 \%$ respectively (Table 1 ).

Table 1 Arthritic score in CIA-rats

\begin{tabular}{llllll}
\hline $\begin{array}{l}\text { Group } \\
(\mathrm{n}=8)\end{array}$ & 2 weeks & 3 weeks & 4 weeks & 5 weeks & 6 weeks \\
\hline Healthy & 0 & 0 & 0 & 0 & 0 \\
$\begin{array}{l}\text { Arthritic } \\
\begin{array}{l}\text { BQ-high } \\
\text { dose }\end{array}\end{array}$ & $4.38 \pm 0.35$ & $4.50 \pm 0.53$ & $4.44 \pm 0.32$ & $4.38 \pm 0.44$ & $4.25 \pm 0.27$ \\
$\begin{array}{c}\text { BQ- } \\
\text { moderate } \\
\text { dose }\end{array}$ & $4.50 \pm 0.46$ & $4.56 \pm 0.42$ & $4.25 \pm 0.46$ & $4.13 \pm 0.44$ & $3.69 \pm 0.37 \Delta \Delta \Delta$ \\
MTX & $4.44 \pm 0.42$ & $4.44 \pm 0.32$ & $4.31 \pm 0.26$ & $4.06 \pm 0.56$ & $3.63 \pm 0.35 \Delta \Delta \Delta$ \\
\hline
\end{tabular}

CIA elevated serum TNF- $\alpha$ and IL-18 concentration by 67 and $25 \%$. Only Bi-Qi-moderate-dose decreased CIA-induced TNF- $\alpha$ and IL- 18 . Protein levels of cartilage oligomeric matrix protein (COMP) and osteopontin (OPN) in serum, synovium, and cartilage were elevated in arthritic rats, while Bi-Qi alleviated these effects. Bi-Qi-moderate-dose reduced CIA-induced serum COMP and OPN level by 48 and $57 \%$ respectively (Fig. 2). Synovial hyperplasia, inflammatory cells infiltration in synovium and high-degree of cartilage degradation were observed in RA, and Bi-Qi or MTX alleviated this effect. Bi-Qi-moderate-dose reduced CIA-induced neutrophils, lymphocytes and plasma cells infiltration in synovium by 44 , 65 and $62 \%$. Bi-Qi-moderate-dose showed the highest effect to mitigate CIA-related clinical complications. Our findings showed that Bi-Qi alleviates CIA-induced inflammation, synovial hyperplasia, cartilage destruction (Fig. 3), and majority of CIA-pathogenesis. This provides fundamental evidence for the anti-arthritic properties of Bi-Qi and corroborates the use of $\mathrm{Bi}-\mathrm{Qi} \mathrm{TCM}$ formula for RA treatment. 


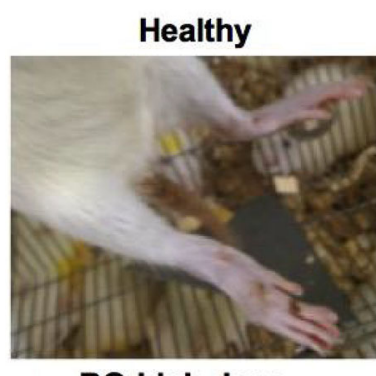

BQ-high dose

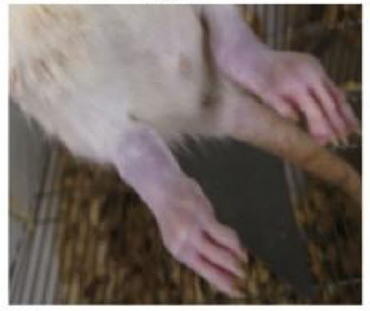

MTX

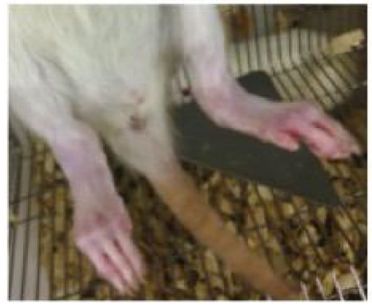

Figure 1 CIA-caused paw swelling and inflammation is alleviated by Bi-Qi treatment
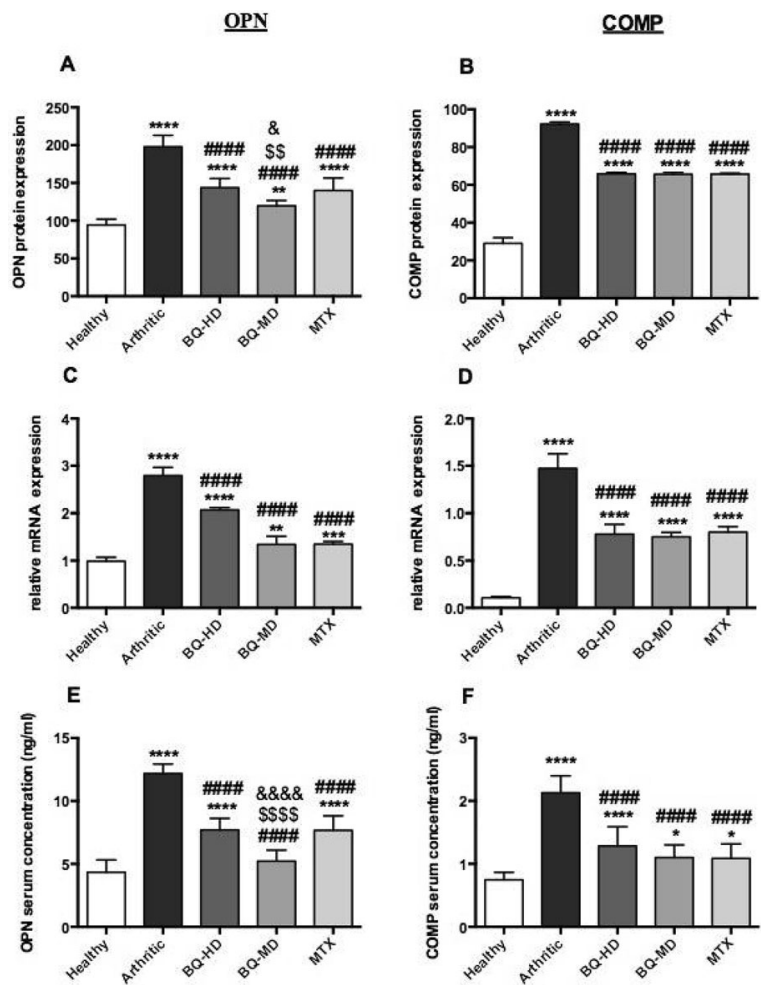

Figure 2 CIA enhances COMP and OPN and Bi-Qi alleviates this effect
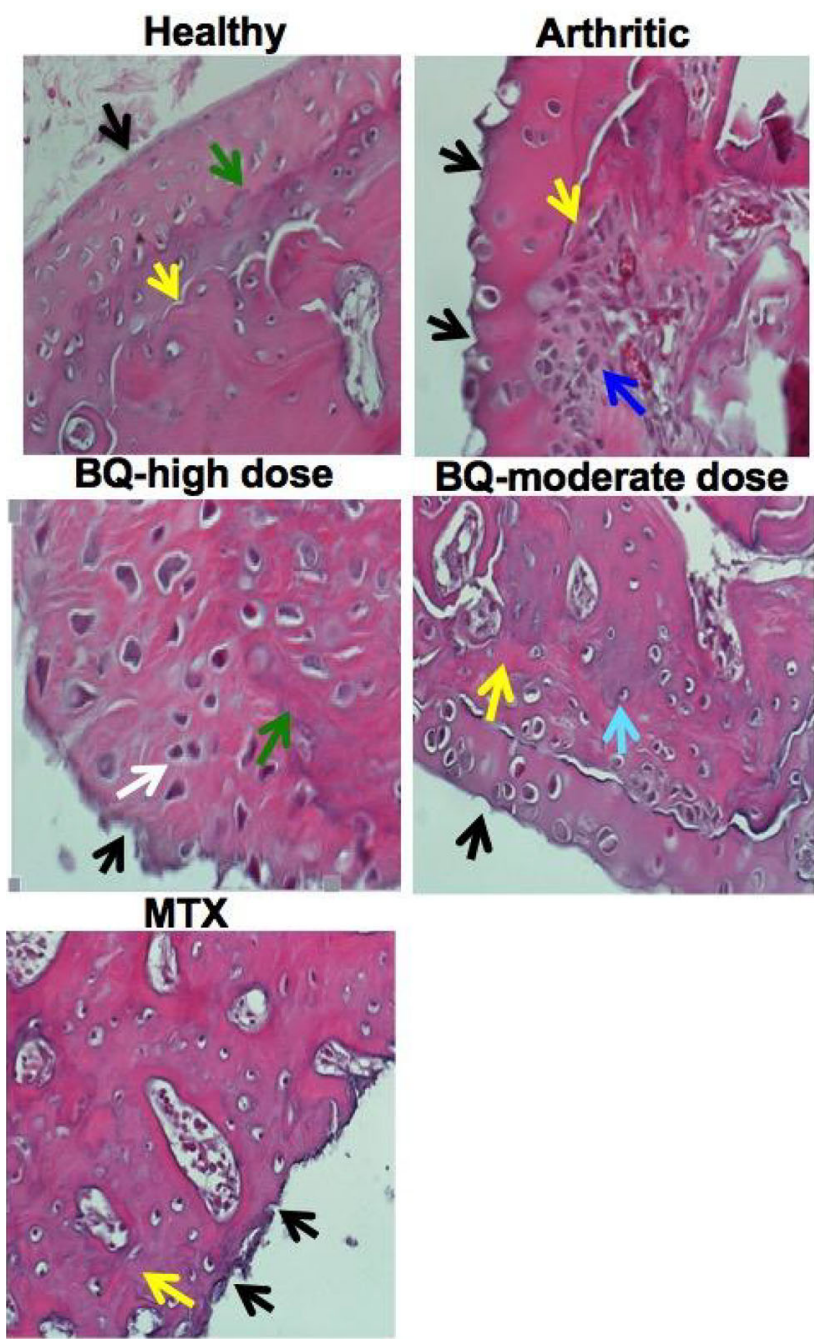

Figure 3 CIA causes cartilage destruction and Bi-Qi treatment alleviates this effect

\section{P235}

Trends in incidence of hip fracture in patients with Rheumatoid Arthritis in Spain: A national observational cohort study

Ramón Mazzucchelli $^{1}$, Natalia Crespí Villarías ${ }^{2}$, Elia Perez Fernandez $^{3}$, Alberto García-Vadillo ${ }^{4}$, Olalla Guzón ${ }^{3}$, Javier Quirós ${ }^{3}$, Marina Peña ${ }^{3}$, Cristina Macía Villa ${ }^{5}$, Jose Luis Morell ${ }^{6}$, Hilda Godoy $^{7}$, Carmen Barbadillo ${ }^{7}$, Manuel Fernandez Prada ${ }^{8}$, Virginia Villaverde $^{9}$, Concepción Morado ${ }^{10}$, Angeles Herranz ${ }^{11}$

${ }^{1}$ Rheumatology Unit, Hospital Universitario Fundación Alcorcón, Madrid, Spain, ${ }^{2}$ Centro de Salud de La Rivota, Alcorcón, Spain, ${ }^{3}$ Hospital Universitario Fundación Alcorcón, Alcorcón, Spain, ${ }^{4}$ Hospital Universitario La Princesa, Madrid, Spain, ${ }^{5}$ Hospital U. Severo Ochoa, Leganés, Spain, ${ }^{6}$ Hospital U. Ramón y Cajal, Madrid, Spain, ${ }^{7}$ Hospital U. Puerta de Hierro, Majadahonda, Spain, ${ }^{8} \mathrm{H}$. U. Guadalajara, Guadalajara, Spain, ${ }^{9}$ H. U. Móstoles, Móstoles, Spain, ${ }^{10}$ H. U. Clínico San Carlos, Madrid, Spain, ${ }^{11} H$. U. del Henares, Madrid, Spain 
Background: There have been significant changes in the treatment of patients with rheumatoid arthritis (RA) and in the treatment of osteoporosis. The potential impact of these strategies on important outcomes as the incidence of hip fracture in RA is unknown.

Objectives: To analyze the incidence and trend of hospital admissions for hip fracture in patients with RA, in Spain.

Methods: This is a retrospective population based study. We have analyzed a national administrative database that includes a Minimum Basic Data Set (MBDS) of the income of patients with RA. Period: 1999-2015. The hip fracture cases were identified by the presence in primary and secondary diagnosis of ICD9 820.0-820.29 codes. The population at risk was estimated through the population census of the National Institute of Statistics. The adjusted rates of hip fracture were calculated. The trend was analyzed using Generalized Linear Models (GLM).

Results: Of a total of 338.343 admissions of patients with RA, $6.656(2 \%)$ was due to hip fracture, $5.608(84.2 \%)$ in women and $1.048(15.7 \%)$ in men. The mean age was 77.54 (SD 9.6). Mean age increased linearly during the study period [in period 1999-2002 was 75.3 years (SD 9.3), and in period 2010-2015 of 79.08 years (SD 9.6)]. There were a total of $326(4.9 \%)$ deaths during admission. The age-adjusted hip fracture rate was $243.66 / 10^{5}$ inhabitants $*$ year (245.24 in women and 198.05 in men). This rate increased from $150.11 / 10^{5}$ hab * year in 1999 , to 303.12 in 2015 , both in women and in men. Through GLM, an increase in the fracture rate of 3.1 is estimated.

Conclusions: In Spain, during the period from 1999 to 2015, although the mean age has increased, the incidence of hip fracture has not been reduced. We estimate an increase of $3.1 \%$ annual.

\section{P236}

Osteophytes a characteristic pathology with osteoarthritis revisited

Katharina Jähn $^{1}$, Gustavo Davi Rabelo ${ }^{1}$, Felix Klebig ${ }^{2}$, Mustafa Citak $^{2}$, Björn Busse ${ }^{1}$

${ }^{I}$ Department of Osteology and Biomechanics, University Medical Center Hamburg-Eppendorf, Hamburg, Germany, ${ }^{2}$ Helios ENDOKlinik Hamburg, Hamburg, Germany

Osteoarthritis (OA) is a progressive disorder of the joints affecting about $11 \%$ of the $>60$ years-old population, with a characteristic gradual destruction of joint cartilage and a locally imbalanced bone formation contributing to the development of joint-associated bony spurs. Osteophytes have a significant clinical impact, as they can be a major source of pain and contribute to the loss of joint function. As osteophyte formation is not limited to OA, the question remains whether osteophytes are solely a pathological phenotype, or whether the local bone formation holds the potential for therapeutic targets.

The ultrastructure of osteophytes in tibial plateau specimen of OA patients submitted to arthroplasty ( $\mathrm{n}=23$ patients; $65 \pm 9$ years) was assessed. Samples were anonymously analyzed in as approved by the local ethics commission (WF-020/17). High-resolution computed tomography (CT) was performed on specimens with $82 \mu \mathrm{m}$ (X-treme $\mathrm{CT}$, Scanco) and on selected regions with $15 \mu \mathrm{m}$ resolution $(\mu \mathrm{CT} 40$, Scanco). Histological assessment was performed on polymethyl methacrylate (PMMA) embedded specimen, cut and stained with toluidine blue, safranin $\mathrm{O}$, or a modified silver precipitation. Quantitative parameters were determined using Image J. Statistical analysis was done with parametric tests using GraphPad.

$\mathrm{CT}$ analysis revealed a significantly different bone structure in osteophytes (BV/TV $0.18 \pm 0.05 \%)$ compared to the adjacent subchondral bone $(0.22 \pm 0.06 \%)$ and control area $(0.31 \pm 0.13 \%$; $\mathrm{p}=0.0001$ ). Bone mineral density was lowest in the osteophyte, compared both control areas (BMD $791.1 \pm 29.33$ to $820.3 \pm 21.38$ and $\left.829.1 \pm 35.42\left[\mathrm{mg} \mathrm{HA} / \mathrm{cm}^{3}\right] ; \mathrm{p}=0.0001\right)$. Histology revealed high levels of bone formation in osteophytes. Quantitative analysis of the osteocyte network demonstrated higher osteocyte numbers per bone area in osteophytes $443.7 \pm 64.23 / \mathrm{mm}^{2}$ compared to subchondral $260.6 \pm 85.15 / \mathrm{mm}^{2}$ and to control area $326.7 \pm 89.26 / \mathrm{mm}^{2}$ $(\mathrm{p}=0.007)$.

Our results show that tibial osteophytes reflect young bone tissue with low mineral content affecting the function of OA joints.

Keywords: osteoarthritis, osteophytes, bone formation, osteocytes, histology

\section{P237}

Achievement of imaging remission among patients with rheumatoid arthritis in clinical remission and their characteristics

Yeon-Ah Lee ${ }^{1}$, Ji-Young Choi ${ }^{1}$, Ran Song ${ }^{2}$, Seung-Jae Hong ${ }^{1}$, SangHoon Lee $^{2}$, Hyung-In Yang ${ }^{2}$

${ }^{1}$ Rheumatology, Kyung Hee University Hospital, Seoul, Republic of Korea, ${ }^{2}$ Rheumatology, Kyung Hee University Hospital at Gandong, Seoul, Republic of Korea

Background/Purpose: Therapeutic goal of rheumatoid arthritis (RA) is to achieve disease remission. However, several remission criteria have not always equated to the complete absence of true inflammation. Power Doppler Ultrasonography (PDUS) has been demonstrated to detect subclinical synovitis. The aim of this study was to elucidate the achievement rates of imaging remission and examine characteristics associated with achievement status among RA patients in clinical remission.

Methods: This study was conducted in 90 RA patients who attained clinical remission, defined by DAS28ESR remission criteria. PDUS was performed in 16 joints and 2 tendons, including both first to third metacarpophalangeal, second and third proximal interphalangeal, radiocarpal (RC), second and third metatarsophalangeal joints and extensor carpi ulnaris tendons and graded on the basis of a dichotomous assessment (presence/absence). The clinical and laboratory data of patients with imaging remission were compared with only clinical remission.

Results: Rate of imaging remission was $51.1 \%$ in patients with clinical remission. Forty four patients $(48.9 \%)$ were PDUS positive. $\mathrm{PD}$ was detected most frequently in right $\mathrm{RC}$ joint $(\mathrm{n}=38,42.4 \%)$. In addition, eleven patients have arthralgia on not evaluation joint, PDUS positivity was $91 \%$. PDUS positive patients had higher evaluator global assessment score $(\mathrm{p}<0.001)$, and all clinical disease activity score. Rheumatoid factor was correlated with imaging remission with positivity and level. Overall, Patients could not reach imaging remission tend to more tender and swollen joint count, higher patient global assessment score, higher health assessment questionnaire (HAQ) score. They use more nonsteroidal antiinflammatory drug (NSAID).

Conclusion: Only $51.1 \%$ of the patients with RA in clinical remission had PDUS defined imaging remission. Patients who were in imaging remission have lower disease activity, lower pain score and less frequent use of NSAIDs, compared to patients with only clinical remission

\section{P238}

Therapeutic effect of Metformin in ischemic osteonecrosis via Angiopoietin1 induced osteoblastic differentiation

Jung Ryul Kim ${ }^{1}$

${ }^{1}$ Orthopaedic Surgery, Chonbuk National University Medical School, Jeonju, Republic of Korea 
Introduction: Angiogenesis is well-known physiological process to promote differentiation of osteoblastic differentiation. In this study, Angiopoietin1 expression was examined in U2OS cells treated with 821 of US Food and Drug Administration-approved drugs by ELISA. Among drugs to induce the expression of Angipoietin1, Metformin was selected as a novel drug candidate, which is currently used as the first-line medication for the treatment of type 2 diabetes.

Methods: ELISA for Angiopoietin1 and alkaline phosphatase assay was carried out in U2OS and MG63 cells treated by Metformin. The effects of Metformin on osteoblastic differentiation were evaluated by Alizarin Red S staining analysis and the expression of proteins related to bone formation and differentiation by Western blotting. Furthermore, the suppression activity of Metformin to osteoclastic differentiation was assessed by Western blotting of IL-6 related proteins expression, TRAP staining/activity analysis in Raw264.7 cells. Finally, animal experiment adapting ischemic osteonecrosis rat model was performed and analyzed by radiographs and histological method.

Results: Angiopoietin1 was induced and alkaline phosphatase activity was increased by Metformin treatment in U2OS and MG63. Wound healing and migration assay showed that osteoblastic cell mobility was increased by Metformin treatment in U2OS and MG63 cells. Metformin upregulates expression of protein markers for osteoblast differentiation in U2OS and MG63 cells. Metformin inhibits osteoclast differentiation in Raw264.7 cells. Metformin protects ischemic necrosis in epiphysis of femoral head through maintaining osteoblast/osteocyte function and vascular density but inhibiting osteoclast activity in epiphyseal bone.

Conclusions: Metformin displayed the augment of increased Angiopoietin 1 expression and the inducestion of osteoblastic differentiation ion U2OS and MG63 but inhibited osteoclastic differentiation in Raw264.7 leading to exert the therapeutic effect in ischemic osteonecrosis rat model, which suggests that Metformin could be a potential bone regeneration agent.

Keywords: Metformin, Angiopoietin1, Ischemic Osteonecrosis, Osteoblastic Differentiation

\section{P239}

Rheumatoid arthritis patients with higher disease severity and subclinical carotid plaque experience more cardiovascular events despite a favorable conventional cardiovascular risk profiles

Yeon-Ah Lee $^{1}$, Ran Song ${ }^{2}$, Seung-Jae Hong ${ }^{1}$, Sang-Hoon Lee ${ }^{2}$, Hyung-In Yang ${ }^{2}$

${ }^{1}$ Rheumatology, Kyung Hee University Hospital, Seoul, Republic of Korea, ${ }^{2}$ Rheumatology, Kyung Hee University Hospital at Gandong, Seoul, Republic of Korea

Background: Increased cardiovascular (CV) disease in rheumatoid arthritis (RA) patients cannot be fully explained by conventional CV risk factors. The systemic inflammatory burden in RA may bring about its high $\mathrm{CV}$ event rate. This study investigated whether the extent of subclinical atherosclerosis, RA associated features, and other conventional $\mathrm{CV}$ risk factors are associated with later development of CV diseases in RA patients.

Methods: C-IMT was measured in $126 \mathrm{RA}$ and $85 \mathrm{OA}$ patients as controls who had no experience of CV events. Demographic data and $\mathrm{CV}$ risk factors are assessed. Thereafter, these patients have been followed-up and examined the $\mathrm{CV}$ event rate during 7 years.

Results: Although CV risks were fewer in RA than in OA, the mean and max C-IMT did not show a significant difference between two diseases groups. During follow-up, 21 patients experienced CV events. The incidence of CV events was higher in RA than OA (15.0 vs. $3.5 \%, p=0.004)$. But, the conventional $\mathrm{CV}$ risk factors such as $\mathrm{DM}$, hypertension and high BMI were fewer in RA than in OA (10.3,
27.7, 34.1 vs. 28.2, 55.2, 57.6\%, p=0.000). More CV events occurred in RA patients who initially showed the presence of subclinical plaques. The duration of $\mathrm{CV}$ event-free survival was shorter in RA patients with carotid plaque than those without (10 vs. 31 months, $\mathrm{p}=0.051$ ). The RA patients who developed CVD later had more bony erosions, higher positivity for rheumatoid factor or antiCCP, higher doses of steroid and higher levels of ESR and CRP, than those who did not.

Conclusion: Despite a favorable conventional CV risk profile, RA patients had a significantly higher incidence rate of CVD than OA patients. RA itself was an independent risk factor for CVD. Especially, RA patients with carotid plaque, seropositivity, bony erosion, higher ESR and CRP are at higher risk of CVD.

\section{P240}

Frequencies of ligament ossification in patients with ankylosing spondylitis according to several ligaments around spine by whole spine CT scan

Ran Song ${ }^{1}$, Yeon-Ah Lee ${ }^{2}$, Seung-Jae Hong ${ }^{2}$, Hyung-In Yang ${ }^{1}$, SangHoon Lee ${ }^{1}$

${ }^{1}$ Kyung Hee University Hospital at Gandong, Seoul, Republic of Korea, ${ }^{2}$ Kyung Hee University Hospital, Seoul, Republic of Korea

Background: Ankylosing spondylitis (AS) is inflammatory arthritis with ligament ossification in spine. We use mSASSS to evaluate progression of ossification which method calculate amount of ossification in ant. longitudinal ligament. But there has not been known which ligaments are ossified most commonly. We performed whole spine CT scan in AS patients to analyze the frequency of ligaments ossification according to the sites of spine.

Methods: We enrolled the 119 patients who were diagnosed as AS by modified NY criteria. The patients had been performed whole spine CT between the ages of 40 and 45 years. We analyzed the frequency of ossification in ant. and post. longitudinal ligaments, ligamentum flavum, ligaments around facet joints, interspinous ligaments, supraspinous ligaments and annulus fibrosus according to $\mathrm{C}, \mathrm{T}$ and $\mathrm{L}$ spine.

Results: The most frequent ossification site was lateral side of annulus fibrosus in T spine (68.9\%). Even regardless of C, T, L spine, ossification of lateral side in annulus fibrosus was also the most frequent site (157 sites). Second common ligaments ossified were ant. longitudinal ligaments (142 sites) and third common ligaments were ligaments around facet joints (109 sites). The ligaments of $\mathrm{T}$ spine were the most common ligaments ossified (252). The ligaments in $\mathrm{L}$ spine were second common ligaments ossified (177). Interestingly ossification in the ligaments around $\mathrm{L}$ spine did not developed, but developed in $\mathrm{T}$ spine in 27 patients $(22.7 \%)$. This is very important finding because ossification in the ligaments around $\mathrm{T}$ spine is not included in mSASSS score. Ossification in ant. longitudinal ligaments was strongly statistically correlated with ossification in ligaments around facet joints in $\mathrm{L}$ spine $(\mathrm{p}=0.00 . \mathrm{r}=0.547)$.

Conclusion: Ligaments around $\mathrm{T}$ spine were most commonly ossified in AS and we need to find new method to evaluate of ossification in $\mathrm{T}$ spine.

Keywords: ankylosing spondylitis, ligament ossification, whole spine CT

\section{P241}

Development of a modular microfluidic Joint-on-a-Chip platform for reliable in vitro modelling of the human joint 
Astrid Bakker ${ }^{1}$, Jolanda Hogervorst ${ }^{1}$, Joanneke Kweekeboom ${ }^{2}$, Simon Mastbergen $^{3}$, Andreea Ioan-Facsinay ${ }^{2}$

${ }^{1}$ Oral Cell Biology, ACTA, UvA and VU, Amsterdam, Netherlands, ${ }^{2}$ Rheumatology, Leiden University Medical Center, Leiden, Netherlands, ${ }^{3}$ Rheumatology \& Clinical Immunology, UMC Utrecht, Utrecht, Netherlands

There is a dire need for systems that accurately mimic human joint physiology and pathophysiology comprising all key joint tissue cells, including immune cells. Therefore we aimed to deliver proof of concept for the development of a modular human Joint-on-a-Chip platform for research and drug development in osteoarthritis.

Separate cultures of human primary osteoblasts, osteoclasts (induced from peripheral blood mononuclear cells), chondrocytes, synovial fibroblasts, macrophages, T-cells, mast cells, and synovial adipocytes were grown on co-culture compatible culture media for a minimum of $72 \mathrm{~h}$. Cells were assessed for cell-specific phenotypic and functional markers using currently available methods, e.g. Alkaline-Phosphatase activity (osteoblasts), TRACP/multinucleation (osteoclasts), gene expression (chondrocytes), cytokine/chemokine secretion (immune cells and synoviocytes), and fatty acid release (adipocytes). In addition, osteoblasts were cultured on Micronit inserts under perfusion inside a Micronit fluidics device for 3 days. Osteoblasts and osteoclasts were co-cultured under static conditions on Micronit tissue culture inserts for 21 days.

Standard operating procedures were established for all cell type cultures grown under similar basic culture conditions i.e. DMEM:F12, L-glutamine, pyruvate, $3.15 \mathrm{mg} / \mathrm{l}$ glucose, $10 \%$ Fetal Clone-III serum (Hyclone) and antibiotics, while maintaining phenotypic stability for 3 days. Mast cells in monoculture required additional supplementation of IL-6 and stem cell factor to the medium, which resulted in an > 10-fold increase in CD117 expression at day 3 compared to day 0. Multinuclear TRACP positive cells formed readily on osteoblast-containing Micronit inserts. Osteoblast survived a 3-day culture period under perfusion while maintaining their characteristic morphology.

Feasibility of a multi-cell type joint-on-a-chip platform has been established. This novel advanced-in vitro-system will be further developed to be applicable for improved research and enhanced drug development appealing to the need for experimental animal model replacement. The platform will be applicable not only to osteoarthritis, but to a wide variety of medical indications, including osteoporosis.

\section{P242}

Immunosuppressive mesenchymal stromal cells derived from human induced pluripotent stem cells use the PD-L1/PD1 axis and induce human regulatory $\mathrm{T}$ cells

Clémence Roux ${ }^{1}$, Gaelle Saviane ${ }^{1}$, Jonathan Pini ${ }^{1}$, Nourhene Belaïd ${ }^{1}$, Gihen Dhib ${ }^{1}$, Christine Voha ${ }^{1}$, Lidia Ibanez ${ }^{1}$, Antoine Boutin ${ }^{1}$, Nathalie Mazure ${ }^{2}$, Abdelilah Wakkach ${ }^{1}$, Claudine Blin-Wakkach ${ }^{1}$, Matthieu Rouleau ${ }^{1}$

${ }^{1}$ Faculty of Medicine, LP2M UMR 7370 CNRS UNS, Nice, France, ${ }^{2}$ Faculty of Medicine, IRCAN, Nice, France

Mesenchymal stromal cells (MSCs) isolated from bone marrow (BM) or other tissues are considered as a promising source of cells to modulate immune functions on cells from innate and adaptive immune systems. Nevertheless, their clinical use remains restricted (few number of cells recovered from these tissues, limited in vitro expansion, absence of a full phenotypic characterization, few insights on their in vivo fate, problematic of rejection, long term impact on the targeted immune responses). Standardized MSCs derived in vitro from human Induced Pluripotent Stem (huiPS) cells could remediate to some of these issues and should be considered, as well as BMMSCs, as a valuable tool for therapeutic approaches. Their immune functions need to be fully characterized. We generated multipotent MSCs derived from huiPS cells (huiPS-MSCs), and focusing on their immunosuppressive activity, we showed that human $\mathrm{T}$ cell activation in co-culture with huiPS-MSCs was significantly reduced. This immunosuppressive activity on CD4-positive and CD8-positive T cells engage the PD-L1/PD1 axis since it was partially reversed when PD-L1 blocking antibodies were used. Furthermore, we observed the generation of CD4-positive CD25-high CD127-low FoxP3-positive regulatory $\mathrm{T}$ cells. We further characterized these regulatory $\mathrm{T}$ cells as well as their function. Our results highlight the multiple arsenal of immunosuppressive mechanisms potentially used by huiPS-MSCs, and the concomitant generation of human regulatory $\mathrm{T}$ cells we characterized could be considered as a particular valuable tool for the induction of a long term immune tolerance.

Keywords: Induced Pluripotent Stem cells, Mesenchymal Stromal Cells, human $\mathrm{T}$ cell immunosuppression, PD-L1, regulatory $\mathrm{T}$ cells

\section{P243}

Lipid profile in Rheumatoid Arthritis patients and its relation to disease activity

\section{Poonji Gupta $^{1}$}

\section{${ }^{1}$ ENT, Teerthanker Mahavir Medical College, Ghaziabad, India}

Background: Cardiovascular morbidity and mortality are enhanced in Rheumatoid Arthritis (RA), which may be attributable to dyslipidemia. The dyslipidemia observed in RA appears to be dependent on disease activity, but only a few studies in the world literature are there, providing definite correlation and mechanisms. In this study we prospectively assessed the correlation of lipid profile with the disease activity.

Material and Methods: A total of 60 patients who fulfilled the "Revised Criteria for the Classification of Rheumatoid Arthritis 1987" were included in this study. The patients who satisfied at least 4 out of 7 criteria were included in the study. The serum was collected from rheumatoid arthritis patients for the determination of lipid values which are triglycerides (TG), total cholesterol (TC), high density lipoprotein (HDL), low density lipoprotein (LDL). Disease activity was assessed by using DAS 28 ESR score. Disease activity was then correlated to the lipid profile of the patients using co-efficient of correlation.

Results: Out of 60 patients, $42(70 \%)$ patients had very active disease activity at the time of presentation. $16(26.7 \%)$ patients had moderately active and $2(3.3 \%)$ had inactive disease. Patients with very active disease were found to have low levels of LDL, HDL and $\mathrm{TC}$ as compared to patients with inactive disease. However the reduction in HDL levels was significantly higher than TC levels. Levels of TG were found to be higher in patients with very active disease.

Conclusions: By causing greater reductions in HDL, RA does increases cardiovascular mortality and morbidity. In patients with RA it is more important to measure both total cholesterol and HDL and to use their ratio for the calculation of absolute cardiovascular disease risk. HDL, LDL, TC can be used as corroborating markers of disease activity in RA.

\section{P244}

Development of medullary bone in laying hens during eggshell formation 
Nazaret Domínguez Gasca ${ }^{1}$, Heather M McCormack ${ }^{2}$, Alejandro B Rodríguez Navarro $^{1}$, Robert H Fleming ${ }^{2}$, Ian Dunn ${ }^{2}$

${ }^{1}$ Mineralogy and Petrology, University of Granada, Granada, Spain, ${ }^{2}$ Roslin Institute, University of Edinburgh, Edinburgh, United Kingdom

During reproduction, female birds need to mobilize large amounts of calcium ( $>2 \mathrm{~g}$ ), equivalent to $10 \%$ of the total body calcium, for the rapid calcification of the eggshell. They develop a special type of bone, the medullary bone, that serves as a calcium reservoir and provides an adequate calcium supply for eggshell mineralization. Medullary bone is a highly metabolically active bone with a high turnover rate (less than 3 days) as its formation and resorption is synchronized with eggshell formation process.

To better understand the mineralization of medullary bone during eggshell calcification, tibiae samples, from laying hens sacrificed at 66 weeks of age, corresponding to different stages of eggshell formation were analyzed. All experiments were approved by an ethical committee. We have conducted an in vitro detailed study using a wide array of analytical techniques such as attenuated total reflectionFourier transform infrared spectroscopy (ATR-FTIR), Raman spectroscopy, X-ray diffraction (DRX), inductively coupled plasma optical emission spectrometry (ICP-OES), optical (OM) and scanning electron microscopy (SEM) in order to better understand the processes that control the formation, mineralization and structural organization of this material. The results showed that during the eggshell formation cycle, there are well defined changes in the mineralization, chemical composition and crystallinity of the medullary bone. Specifically, the relative amount of carbonate in the mineral $(\mathrm{R}=-0.452 ; \mathrm{p}=0.04)$ as well as its crystallinity $(\mathrm{R}=-0.397$; $\mathrm{p}=0.02$ ) decreases during eggshell formation. In conclusion, medullary bone offers an unique model for studying pathological situations characterized by a high calcium demand and/or fast remodeling rates.

Keywords: Laying hens, medullary bone, eggshell, egg cycle

\section{P245}

Soybean meal induces intestinal inflammation and bone resorption in adult zebrafish scale

Marta Carnovali ${ }^{1}$, Giorgia Millefanti ${ }^{2}$, Alessia Rainero ${ }^{2}$, Giuseppe Banfi $^{3,4}$, Massimo Mariotti ${ }^{3,5}$

${ }^{1}$ Gruppo Ospedaliero San Donato Foundation, Milan, Italy, ${ }^{2}$ Department of Medicine and Surgery, University of Insubria, Varese, Italy, ${ }^{3}$ IRCCS Galeazzi Orthopedic Institute, Milan, Italy, ${ }^{4}$ Vita-Salute San Raffaele University, Milan, Italy, ${ }^{5}$ Department of Biomedical, Surgical and Dental Sciences, University of Milan, Milan, Italy

Inflammatory bowel disease is linked to several health issues, including low bone mineral density. Danio rerio (zebrafish) is a good model to study intestinal inflammation since gastrointestinal and immune systems are closely related to that of mammalians. Zebrafish is a powerful model to study bone metabolism using the scale as readout model.

Soybean-meal (SBM) induces intestinal inflammation because of saponin. Adult zebrafish were fed with SBM (50\% commercial food and $50 \%$ defatted soybean flour with high saponin content) for 5 weeks (T1-T5). After 3 weeks SBM induces acute intestinal inflammation with increased intestinal mRNA expression of pro-inflammatory cytokines (Fig. 1, IL-1 $\beta$, IL-6, IL-8, IL-10, TNF- $\alpha$, $* \mathrm{p}<0.05 ; * * \mathrm{p}<0.01 ; * * * \mathrm{p}<0.001)$ returning to baseline at 4 weeks of treatment. Histological intestine analysis show increased number of immune cells in SBM mid intestine at T3 and T4 $(\mathrm{p}<0.01)$ without any morphological alterations.
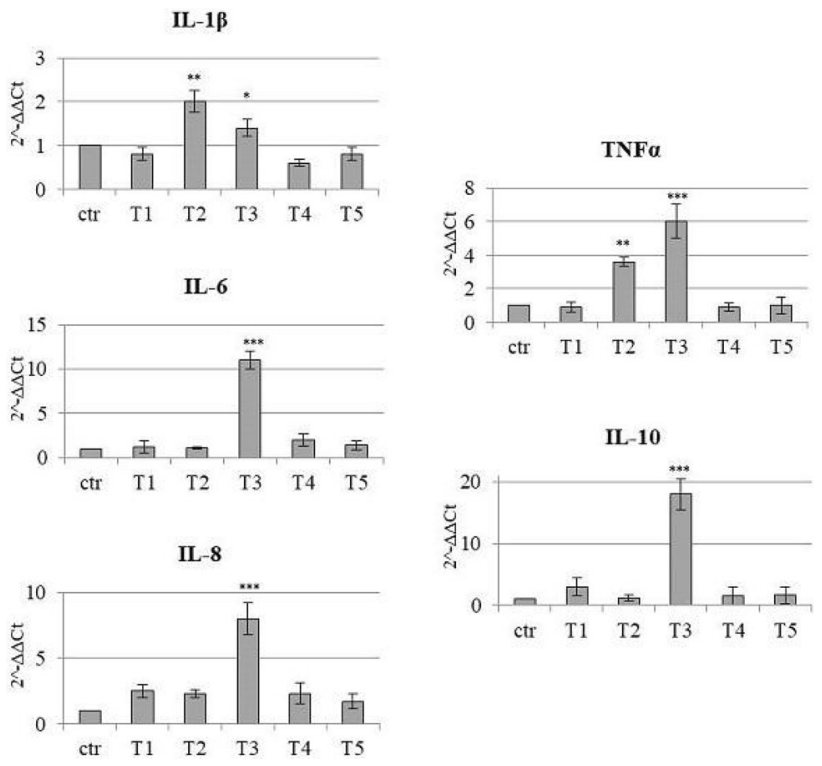

Fig. 1 Increased pro-inflammatory cytokines expression

Acute intestinal inflammation correlates with transitory osteoporosis-like phenotype in scales with bone resorption lacunae (Fig. 2A, T3) associated with intense osteoclastic tartrate-resistant acid phosphatase (TRAP) activity (Fig. 2B). Osteoclast activity decreases at $\mathrm{T} 4$ and the resorption lacunae shrink until closing at T5.
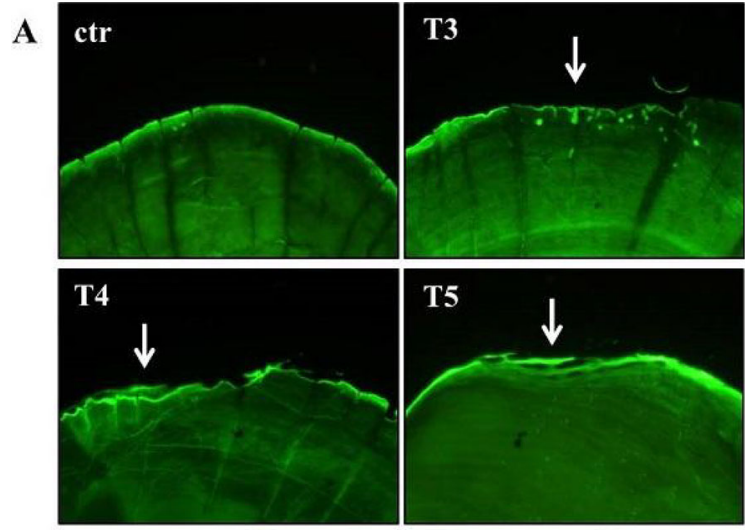

B

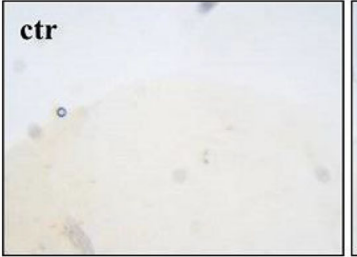

T3

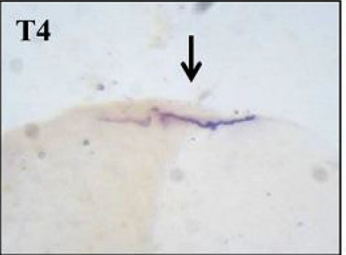

T5

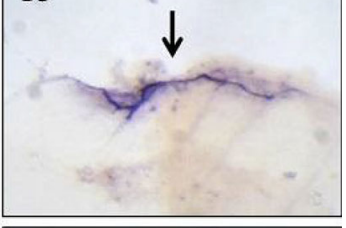

0

Fig. 2 Resorption lacunae (A) and TRAP activity (B) 
This model can contribute to elucidate in vivo the molecular mechanisms of bone disease induced by acute intestinal inflammation in human IBD patients.

Ethical approval: Fondazione Istituto Insubrico di Ricerca per la Vita (Gerenzano, VA, Italy); ASL Varese (Italy) (Prot. No. 014AVB0020033).

Keywords: Zebrafish, inflammation, osteoporosis

\section{P246}

Screening for osteogenic compounds using the zebrafish as a model Jan Willem Bek ${ }^{1}$, Charlotte Gistelinck ${ }^{2}$, Hanna De Saffel ${ }^{1}$, Paul Coucke $^{1}$, Andy Willaert ${ }^{1}$

${ }^{1}$ Ghent University, Ghent, Belgium, ${ }^{2}$ University of Washington, Seattle, United States

Objectives: The pharmacological therapy of choice in fragile bone disorders is based on bisphosphonates, which inhibit osteoclast-mediated bone resorption. However, this only prevents deterioration of existing bone. Therefore drugs are required that combine anti-resorptive and anabolic action, thus improving bone quality. Although some compounds have already shown promising effects in mouse models, these results are highly variable due to the limited number of animals that can effectively be screened in these models. Therefore, there is a need to assess the effect of promising new osteogenic drugs in larger sets of animal models for fragile bone disorders, using a standardized higher throughput workflow.

Methods: In this work we assessed the feasibility of different readouts for mineralization and bone formation in zebrafish larvae, combined with administration of osteogenic compounds. The zebrafish is considered as an excellent tool for high-throughput compound screening because of the low cost, external fertilization and the generation of large numbers of rapidly developing transparent embryos that can be treated with compounds via the water.

Results and Conclusion: A compound that has previously been shown to increase bone formation in zebrafish larvae, $1 \alpha, 25$-dihydroxyvitamin D3 (calcitriol), was used as a positive control in the development of a phenotypic readout $(15 \%$ increase. $p<0.05)$. These readouts include in vivo monitoring of larval mineralized structures through alizarin red staining in combination with transgenic reporter lines for osteoblasts and osteoclasts. This analysis is supplemented with measurements of alizarin red fluorescence through spectrophotometry. These methods will enable future screens of promising osteogenic compounds in zebrafish models for fragile bone disorders.

Take home message: The zebrafish is a platform that allows for quick screening for osteogenic compounds.

\section{P247}

Zebrafish type I collagen mutants faithfully recapitulate human type I collagenopathies

Andy Willaert ${ }^{1}$, Charlotte Gistelinck ${ }^{2}$, Ronald Y Kwon ${ }^{2}$, Fransiska Malfait $^{1}$, Sofie Symoens ${ }^{1}$, Matthew P. Harris ${ }^{3,4}$, Katrin Henke ${ }^{3,4}$, Shannon Fisher ${ }^{5}$, Patrick Sips ${ }^{1}$, Brecht Guillemyn ${ }^{1}$, Jan Willem Bek ${ }^{1}$, Petra Vermassen ${ }^{1}$, Hanna De Saffel ${ }^{1}$, Maryann Weis ${ }^{2}$, Anne De Paepe $^{1}$, David R. Eyre ${ }^{2}$, Paul Coucke ${ }^{1}$

${ }^{1}$ Center for Medical Genetics, Ghent University, Gent, Belgium, ${ }^{2}$ Department of Orthopaedics and Sports Medicine, University of Washington, Seattle, United States, ${ }^{3}$ Department of Genetics, Harvard Medical School, Boston, United States, ${ }^{4}$ Department of Orthopaedic Research, Boston Children's Hospital, Boston, United States, ${ }^{5}$ Department of Pharmacology \& Experimental Therapeutics, Boston University School of Medicine, Boston, United States
The type I collagenopathies are a group of heterogeneous connective tissue disorders, that are caused by mutations in the genes encoding type I collagen and include specific forms of Osteogenesis Imperfecta (OI) and the Ehlers-Danlos syndrome (EDS). These disorders present with a broad disease spectrum and large clinical variability of which the underlying genetic basis is still poorly understood. In this study, we systematically analyzed skeletal phenotypes in a large set of zebrafish, with diverse mutations in the genes encoding type I collagen, representing different genetic forms of human OI, and the first zebrafish model of human EDS, which harbors characteristic defects in the soft connective tissues. Furthermore, we provide insight into how zebrafish and human type I collagen are compositionally and functionally related, which is relevant in the interpretation of human type I collagen related disease models. Our studies reveal a high degree of inter-genotype variability in phenotypic expressivity that closely correlates with associated OI severity. Further, we demonstrate the potential for select mutations to give rise to variable phenotypic penetrance, mirroring the clinical variability associated with human disease pathology. Therefore, our work suggests the potential for zebrafish to aid in identifying unknown genetic modifiers and mechanisms underlying the phenotypic variability in OI and related disorders. This will improve diagnostic strategies and enable the discovery of new targetable pathways for pharmacological intervention.

\section{P248}

A rodent long bone fracture/fixation model with improved clinical relevance

Benjamin Braun ${ }^{1}$, Jordan Skelly ${ }^{1}$, David Ayers ${ }^{1}$, Jie Song ${ }^{1}$

${ }^{1}$ Orthopaedic Surgery, University of Massachusetts Medical School, Worcester, United States

Introduction: Contemporary models to study bone fixation and healing fall short of replicating high-energy injuries. We propose an open reduction internal fixation (ORIF) fracture model that combines high-energy long bone fractures with clinically relevant reduction/fixation. We hypothesize that our fracture/fixation model is more clinically generalizable than previous models.

Methods: Institutional animal care and use committee (IACUC) approved all procedures. Skeletally mature male Sprague-Dawley rats were assigned to control (fracture following intramedullary (IM) rod insertion) or ORIF groups. Control animals had a $1 \mathrm{~mm} \times 25$ $\mathrm{mm}$ rod inserted into the femoral canal and were brought to the fracture guillotine. A transverse fracture was created with a $1100 \mathrm{~g}$ weight dropped from $16 \mathrm{~cm}$ above. ORIF group: animals were brought to the guillotine and a transverse fracture was created prior to surgery. Blunt dissection was carried down to the femur. A four-hole plate is applied to the femur and fixed with $1.5-\mathrm{mm}$ cortical screws (Fig. 1d). MicroCT $(\mathrm{n}=5)(2,4,8$ weeks), histological analyses $(\mathrm{n}=2)(2,4,8$ weeks $)$, and torsion testing $(60 \% \mathrm{~min})$ of the retrieved femurs were carried out at 8 -week endpoints $(n=5)$.

Results: Transverse fractures on rat femurs were generated with $>90 \%$ success. Histology revealed differential callus formation in the ORIF vs. control groups. Toluidine blue staining and MicroCT showed asymmetrical cartilaginous callus predominantly on the periosteal surface of the fracture in the control group whereas the callus transversely formed along the fracture in the ORIF group. Neither the ORIF nor control groups achieved restoration of torsional integrity by 4 weeks.

Discussion: We created a rat femur fracture/fixation model that is more clinically relatable than control method, which is characterized with unstable fixation, resulting in motion-induced formation of asymmetric periosteal cartilaginous callus. Our ORIF method generated stable fixation and resulted in earlier bony callus formation along 
the transverse fracture line. This fracture/fixation model recapitulates more realistic soft-tissue trauma and subsequent surgical intervention. Keywords: Fracture, Trauma, Animal Model

\section{P250}

Clinical implications and potential role of the novel myokine irisin as a biomarker for sarcopenia

Hye-Sun Park ${ }^{1}$, Hyun Chang Kim ${ }^{2}$, Hoon Choi ${ }^{3}$, Sung-Kil Lim ${ }^{1}$

${ }^{I}$ Internal Medicine, Yonsei University/College of Medicine, Seoul, Republic of Korea, ${ }^{2}$ Preventive Medicine, Yonsei University, Seoul, Republic of Korea, ${ }^{3}$ Obstetrics and Gynecology, InJe University/ College of Medicine, Seoul, Republic of Korea

The previously reported associations of irisin with many clinical conditions including sarcopenia, fatty liver, and osteoporosis were inconsistent. To clarify the association of circulating irisin with muscle mass, liver steatosis, and bone mass and to evaluate irisin as a biomarker for sarcopenia. This is a prospective cross-sectional study at an university clinic, enrolling postmenopausal women. Quadriceps cross sectional area (QcCSA), bone mineral density (BMD), liver attenuation (measured in Hounsfield units (HU)) were assessed using quantitative computed tomography in 153 postmenopausal women. Muscle strength and physical performance were evaluated by handgrip test and short physical performance battery, respectively. Serum irisin was measured by a commercially available enzyme-linked immunosorbent assay kit. In addition, 147 young women were recruited as a reference group to define cut-off values for sarcopenia. The primary outcome was an association of irisin with sarcopenia, and the possible role of irisin as a biomarker for sarcopenia. Secondary outcome was the relationship between irisin and clinical conditions such as fatty liver, osteoporosis, diabetes, and renal impairment. Circulating irisin was positively correlated with QcCSA/body weight (BW) and liver HU even after adjusting for multiple covariates, and the serum level was significantly lower in the sarcopenia group $(\mathrm{QcCSA} / \mathrm{BW}<-2 \mathrm{SD}$ of the mean values for young women) than in the presarcopenia $(-2 \mathrm{SD} \leq \mathrm{QcCSA} / \mathrm{BW}<$ $-1 \mathrm{SD})$ or control groups ( $1 \mathrm{SD} \leq \mathrm{QcCSA} / \mathrm{BW}<2 \mathrm{SD})$. Logistic regression models showed that the relationship between circulating irisin and prevalence of sarcopenia remained significant after adjusting for confounding factors (per $1.0 \mathrm{ng} / \mathrm{mL}$ decrease of irisin, odds ratio $=1.95,95 \%$ confidence interval $1.33-2.87, p$-value $=$ $0.001)$. There was no significant association of serum irisin with BMD, kidney function, and insulin resistance. In conclusion, serum irisin may be used as a biomarker for sarcopenia, and we showed the potential for the development of irisin-based early screening and staging tool for sarcopenia.

\section{P251}

Psoas cross-sectional area as a predictor of mortality and a simple diagnostic method of sarcopenia in hip fracture patients age over 50

${ }^{1}$ Department of Orthopaedic Surgery, Chung-Ang University, Seoul, Republic of Korea, ${ }^{2}$ Department of Orthopaedic Surgery, Seoul National University Bundang Hospital, Seongnam, Republic of Korea, ${ }^{3}$ Kyung Hee University Medical Center, Seoul, Republic of Korea

Purpose: The objective of the current study is (1) to determine the association between PCA and mortality in elderly hip fracture patients and (2) to identify of usefulness of the PCA as a diagnostic tool for sarcopenia.

Methods: PCA was measured on L4-5 level of axial image in 571 hip fracture patients age over 50 underwent operation between 2012 and 2017. The PCA with the lowest quintile was considered sarcopenic, and the effect of decreased PCA (sarcopenia) on mortality was analyzed using the Kaplan-Meier method and the Cox proportional hazard ratio. For identifying usefulness of the PCA as a diagnostic tool for sarcopenia, the association between PCA and appendicular lean mass and Receiver operating characteristic (ROC) curve was analyzed.

Results: Among 571 patients, 434 (76.0\%) were female and 137 (24.0\%) were male. Mean age of patients was 79.5 (range 50-103) years in female and 75.3 (52-95) years in male. With Kaplan-Meier method, mean survival time of female patients with lowest quintile of PCA (sarcopenia) was 47.4 months while that of the remaining female patients was 73.1 moths $(\mathrm{p}=0.004)$. With Cox proportional hazard analysis incorporating age and ASA score, lowest quintile of PCA was also associated with mortality with odds ratio (OR) of 1.701 $(\mathrm{p}=0.04)$ in female patients. In male patients, A weak correlation $(r=0.326)$ was found between PCA and appendicular lean mass in female patients $(\mathrm{p}<0.001)$, while moderate association $(0.496)$ was found in male patients $(\mathrm{p}<0.001)$. C-statistics of female patients was 0.42 and that of male patients was 0.32 .

Conclusion: In female hip fracture patients age over 50, decreased psoas muscle cross-sectional area was a significant predictor of mortality. The correlation between PCA and appendicular lean mass was present, however, the correlation is not high enough to use PCA as a diagnostic tool for sarcopenia.

\section{P252}

Myostatin/Follistatin signalling in cultivated muscle derived stem cells

Sigrid Mueller-Deubert ${ }^{1}$, Regina Ebert ${ }^{1}$, Konstantin Horas ${ }^{1}$, Melanie Krug $^{1}$, Sabine Zeck ${ }^{1}$, Franz Jakob

${ }^{1}$ Orthopedic Center of Musculoskeletal Research, University of Würzburg, Wuerzburg, Germany

Objectives: There is increasing evidence for tight interaction between muscle and bone regeneration and activity is crucial for the maintenance of bone health. The TGF-b signaling pathway has a pivotal role in muscle cell function, regeneration and interaction with bone tissue.

Methods: Biopsies from skeletal muscle were collected from different donors and cultivated in stem cell medium to obtain cellpopulations with mesenchymal precursor characteristics (musclederived stem cells, MDSC). To induce differentiation, cells were grown in osteogenic, adipogenic or myogenic medium. Differentiation was approved by appropriate staining. After different time courses total RNA was isolated and RealTime PCR was performed.

Results: Expression of muscle stem cell markers showed that MDSC can be isolated and expanded in vitro. Under differentiation conditions, these cells are able to differentiate towards the osteogenic, adipogenic and myogenic pathways respectively. Myogenic differentiation capacity declines with ageing, both in vivo (age of donors) and in vitro (number of passages). The expression of adipogenic markers increased with time in culture, myogenic differentiation could strongly inhibit this phenomenon. Paradoxically application of follistatin, a myostatin inhibitor, to myogenically differentiated MDSCs from old donors is also able to increase the expression of adipogenic markers. However, in MDSCs from young donors this effect cannot be seen. It has been observed, that myogenic differentiation strongly enhances the expression of myostatin, a negative regulator of skeletal muscle. In higher passages this effect is less prominent. Follistatin treatment reduces myostatin expression, both in untreated and in myogenic differentiated MDSCs from young donors. In cells from old donors myostatin expression is not altered by follistatin application. 
In conclusion MDSCs are multipotent precursors, which upon in vivo and in vitro aging spontaneously express adipogenic markers. Unexpected alterations of follistatin/myostatin signaling during aging require further research.

Keywords: Muscle-derived stem cells, myostatin, follistatin

\section{$\mathbf{P 2 5 3}$}

Muscle adaptations to swimming exercise in the adult zebrafish model

Maiwulanjiang Mamuti ${ }^{1}$, Imke Fiedler ${ }^{1}$, Annika vom Scheidt ${ }^{1}$, Katharina Jähn ${ }^{1}$, Björn Busse ${ }^{1}$

${ }^{1}$ Department of Osteology and Biomechanics, University Medical Center Hamburg-Eppendorf, Hamburg, Germany

The functional link between muscle and bone in health and pathology is of current research interest. Novel therapeutic approaches are sought out to battle the concurrent progression of sarcopenia and osteoporosis. Anabolic physical exercise can increase both muscle and bone strength, body coordination and balance, and more importantly lead to measurable fracture risk reduction in humans. Zebrafish have been widely used in developmental biology and might also represent as advantageous model to study the musculoskeletal functions during adulthood. To test the hypothesis that swimming exercise leads a muscle adaptation in adult zebrafish, we subjected one group to increased physical exercise for 4 weeks in a swim tunnel experiment, as recently established. A second group was housed in a standard aquarium tank and served as control group. In our previous study, we found that bone of the vertebral column adapts to the swimming exercise with increased bone mass, increased mineralization and higher osteoblast numbers. The goal of this study is to quantify the cellular and structural adaptations of the zebrafish muscular system along the vertebral column. Muscle fiber histology was assessed on transverse sections stained with hematoxylin and eosin. The skeletal muscle adaption to swimming exercise resulted in a higher number of muscle fibers compared to non-exercised control fish (exercise: $1323.64 \pm 71.04 / \mathrm{mm}^{2}$ and control: $472.80 \pm 36.64 /$ $\mathrm{mm}^{2}$ ), and higher percentage of fibers containing central nuclei within the exercise group $(4.80 \pm 0.77 \%)$ compared to control $(0.92 \pm 1.11 \%)$, while the average size of the muscle fibers was found to be smaller in the exercise group compared to control $\left(675.18 \pm 42.302\right.$ vs. $\left.1726.73 \pm 213.13 \mu \mathrm{m}^{2}\right)$. This study gives first insights to the muscular adaptations of adult zebrafish to swimming exercise. Zebrafish represent a novel model organism to investigate appropriate prevention strategies for musculoskeletal diseases.

Keywords: physical exercise; muscle adaptations; zebrafish model; histology; muscle fibers

\section{$\mathbf{P 2 5 4}$}

Modulation of MMPs-TIMPs expression in cytokine-stimulated fibroblasts using cyclooxygenase 2 inhibitor

Jihye Kim ${ }^{1}$, Youngmi Kang ${ }^{1}$, Seonghwan Moon ${ }^{1}$, Byungho Lee ${ }^{1}$, Hwanmo Lee ${ }^{1}$, Kilhan Lee

${ }^{1}$ Orthopadic Surgery, Yonsei University/College of Medicine, Seoul, Republic of Korea

Introduction: Hypertrophy of ligamentum flavum (LF) induces narrowing of spinal canal which develops neurogenic claudication. Several mechanisms of LF hypertrophy have been suggested. Among them inflammatory cytokine play a crucial role in LF hypertrophy by increasing collagen synthesis. Cyclooxygenase-2 (COX-2) pathway shares inflammatory reaction from infection and arthritis. Selective COX-2 inhibitor (COX-2si) might modulates collagen synthesis via suppressing inflammatory pathway. Hence, the current study examined the effect of COX-2si in collagen synthesis of inflammatory cytokine induced LF cells.

Materials and Methods: Fibroblast and LF cells were harvested and cultured. Inflammatory cytokines (TNF-a) were utilized to stimulate inflammatory reaction of LF cells. Then COX-2si was administered to stimulated fibroblasts and LF cells. Collagen synthesis, RT-PCR for COX-2 and various collagens, were performed.

Results: Fibroblasts stimulated by inflammatory cytokines showed $50 \%$ decrease in fibronectin expression in translational level $(p<0.05)$. Stimulated fibroblasts with COX-2si demonstrated downregulation of TIMP isoforms and MMPs. Specially, pre-treated fibroblasts with COX-2si were suppressed TIMPS expression about $50 \%(p<0.05)$.

Summary and conclusion: In stimulated fibroblasts and LF cells, COX-2si provided therapeutic mechanism in reducing collagen synthesis. Hence COX-2si might be useful in preventing LF hypertrophy, which opens new therapeutic, preventive measures in symptomatic lumbar spinal stenosis.

\section{P255}

Risk factors of sarcopenia in Ukrainian postmenopausal women

Vladyslav Povoroznyuk $^{1}$, Nataliia Dzerovych ${ }^{1}$, Roksolana Povoroznyuk

${ }^{1}$ Department of Clinical Physiology and Pathology of Locomotor Apparatus, D.F. Chebotarev Institute of Gerontology AMS Ukraine, Kyiv, Ukraine

The aim of the study was to study the risk factors [dietary protein intake, vitamin D, growth hormone $(\mathrm{GH})$, total and free testosterone level (TT, FT)] of sarcopenia in postmenopausal women.

Materials and Methods: To evaluate the connection between appendicular lean mass (ALM) and dietary protein intake we have examined 63 women aged 52-89 years (age-68.46 \pm 9.26 years). For the purpose of studying the correlation between skeletal muscles and vitamin D level 87 women aged $45-83$ years were examined (age-66.29 \pm 8.35 years). To study the correlation between skeletal muscles and GH, TT, FT level, 42 healthy women aged 60-86 years (age $-70.62 \pm 6.97$ years) were examined.

To assess the dietary habits of women, we used a 3-day sampling method. Lean mass was evaluated using DXA (Prodigy, USA). Strength of skeletal muscle was evaluated using springy carpal dynamometer, the functional capacity of skeletal muscle- «fourmeter» test. To determine the level of $25(\mathrm{OH}) \mathrm{D}, \mathrm{GH}, \mathrm{TT}$ and FT electrochemiluminescent method was used.

Results: Women of 80-89 years consuming less than $1.0 \mathrm{~g}$ of protein per $1 \mathrm{~kg}$ of body weight accounted for more than a half of their group $(57.1 \%)$. Significant correlations among dietary protein, amino acids and ALM index values $(\mathrm{p}<0.01)$ were determined.

We determined a significant correlation between lean mass $(\mathrm{p}=0.05)$ and vitamin $\mathrm{D}$ level; skeletal muscle functionality $(\mathrm{p}=0.04)$ and vitamin D level.

Significant correlation between ALM and GH level (60-74 years: $r=0.36$; $60-89$ years: $r=0.31)$, between strength of skeletal muscle and TT (75-89 years: $r=0.55 ; 60-89$ years: $r=0.32)$, FT 
(75-89 years: $r=0.31)$, GH (75-89 years: $r=0.35 ; 60-89$ years: $r=0.32)$ levels; between skeletal muscle function and TT (75-89 years: $r=0.46)$, FT (75-89 years: $r=0.48)$ levels was found.

Conclusion: Significant correlation between parameters of lean mass, skeletal muscle strength, functionality and dietary protein intake, vitamin D, GH, TT and FT level was determined in postmenopausal women.

\section{P256}

The relationship between the changes of pulmonary function and whole body non-fat mass in Korean elderly population

\section{$\underline{\text { Young-Sang Kim}}^{1}$, Bom-Taeck Kim ${ }^{2}$, Seung Geon Park ${ }^{1}$}

${ }^{1}$ CHA University, Seongnam, Republic of Korea, ${ }^{2}$ Ajou University School of Medicine, Suwon, Republic of Korea

Objective: It was not well known whether the pulmonary function is affected by body composition or vice versa. This study is conducted to evaluate the relationship between the changes of pulmonary function and whole body non-fat mass in Korean elderly population.

Methods: A total of 411 subjects were followed up for 2 or 3 years. Pulmonary function and body composition were measured repeatedly. The pulmonary function indices and non-fat mass proportion were categorized into quartiles. The lowest quartiles were regarded as low levels, and the others were as normal levels. The changes of pulmonary function were investigated according to non-fat mass indices. In addition, the changes of body weight and non-fat mass were investigated according to low and normal pulmonary function. This retrospective study was performed with accordance with the ethical standards.

Results: The FVC and FEV1 were lower in low non-fat group than in normal group of both men and women at baseline. In contrast, FEV1/FVC was not different between the 2 groups. The changes of FVC $(+0.09$ and $-0.08 \mathrm{~L}$ in low and normal group, respectively) and FEV1 $(-0.02$ and $-0.12 \mathrm{~L}$ in low and normal group, respectively) were significantly higher in low non-fat group of men than in normal group. The changes of ...The changes of pulmonary function were not different in women between low and normal group. The changes of ...The changes of body weight $(-0.4$ and $+0.5 \mathrm{~kg}$ in low and normal FEV1 group, respectively) was significantly lower in low FEV1 group of men than in normal group. However, the changes of non-fat mass were not significantly different between low and normal FEV1 groups in men.

Conclusion: The subjects with low non-fat mass had lower pulmonary function. In addition, Korean elderly men with low pulmonary function tended to lose body weight in 2.5 years followup.

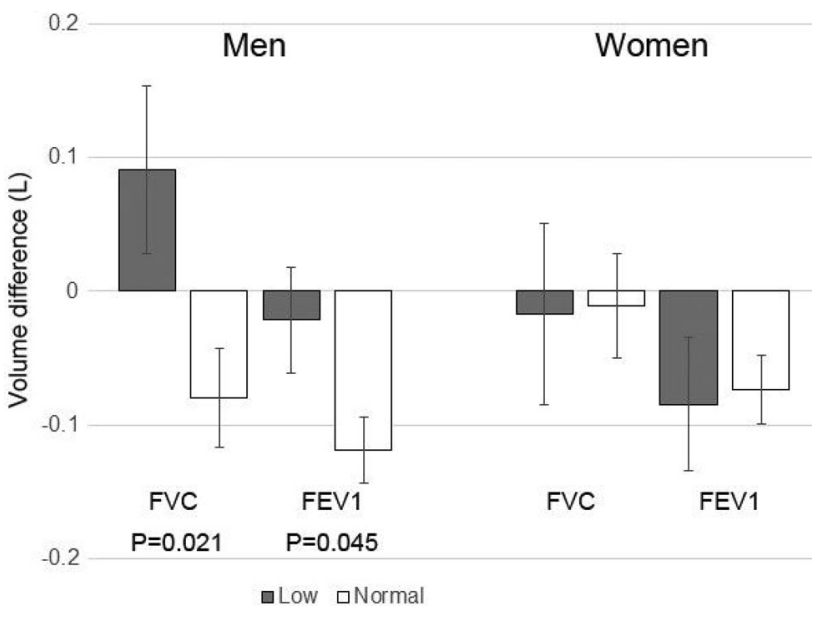

The changes of pulmonary function according to non-fat mass

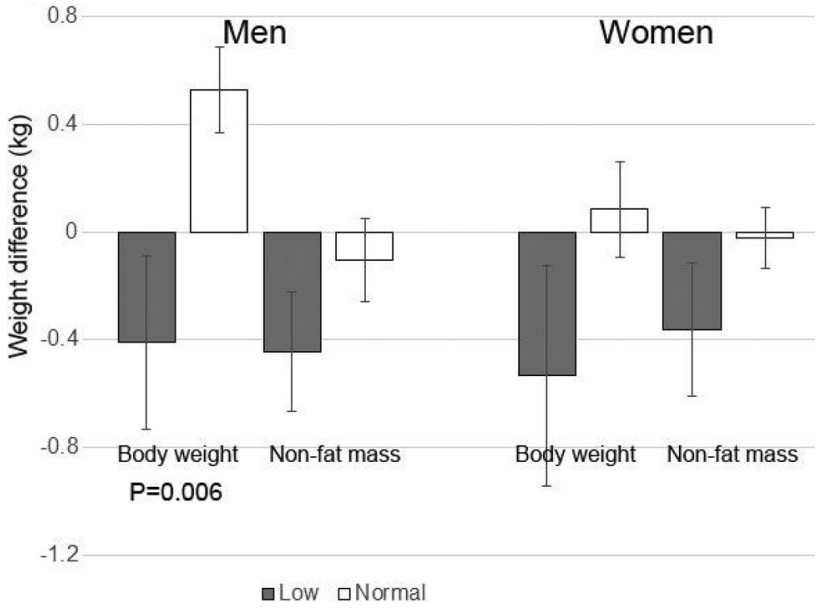

The changes of body weight and non-fat mass according to FEV1 levels

\section{$\mathbf{P 2 5 7}$}

The association between muscle mass deficits estimated from BIA and lumbar spine BMD in Korean male adults

Hee-Jeong Choi ${ }^{1}$, Han-Jin $\mathrm{Oh}^{1}$, Byeong-Yeon $\mathrm{Yu}^{2}$

${ }^{1}$ Family Medicine, Eulji University Hospital, Daejeon, Republic of Korea, ${ }^{2}$ Family Medicine, Konyang University Hospital, Daejeon, Republic of Korea

Background: Bone mineral density (BMD) is influenced by many factors. Among them, relative contributions of muscle mass or fat mass to BMD remains a contentious issue. The aim of this study is to investigate the association between muscle mass deficits (MMD) that are estimated from bioelectrical impedance analysis (BIA) and lumbar spinal BMD in male adults 50-75 years of age. 
Methods: This cross-sectional study included 1586 men who visited a health promotion center for a routine check-up. The lumbar spine BMD was measured by dual energy X-ray absorptiometry. Body composition analysis was performed using BIA. The MMD, which is the difference between ideal fat-free mass and real fat-free mass of the subject, is expressed as the muscle control $(\mathrm{kg})$ on the result sheet. The subjects were divided into three groups according to the tertile of the MMD.

Results: The mean age of the subjects was $56.6 \pm 5.5$ years. Fiftyfive subjects belonged to the osteoporosis (T-score $<-2.5$ ). All body indices and lumbar spine BMD decreased as MMD grade increased ( $\mathrm{P}$ for trend $<0.001)$. MMD had the strongest influence on $\mathrm{BMD}$ when age and all other variables were included in the regression model $(\mathrm{P}<0.005)$. The adjusted odds ratios for osteoporosis of Group 2 (MMD $\leq 3.05 \mathrm{~kg}$ ) and Group 3 (MMD > $3.05 \mathrm{~kg}$ ) were 3.11 (95\% CI 1.18-8.18) and 4.08 (95\% CI 1.03-16.12), respectively.

Conclusion: MMD estimated by BIA showed a significant association with BMD and could be regard as an independent risk factor for osteoporosis in male adults 50-75 years. These finding support that intervention such as physical activity or lifestyle changes may simultaneously modify both muscle and bone health in this group. Keywords: bone density, body mass index, muscles, osteoporosis, male

\section{P258}

The relationship between muscle parameters and balance abilities evaluated by a new device for elderly people

Myung Jun Shin ${ }^{1}$, Tae Sung Park ${ }^{2}$, Yun Kyung Jeon ${ }^{3}$, In Joo Kim ${ }^{3}$

${ }^{1}$ Rehabilitation Medicine, Pusan National University Hospital, Busan, Republic of Korea, ${ }^{2}$ Biomedical Research Institute, Pusan National University Hospital, Busan, Republic of Korea, ${ }^{3} E n-$ docrinology and Metabolism, Pusan National University Hospital, Busan, Republic of Korea

Falls are a major health problem for the elderly and their impact is an important public health issue. One of the causes of falling, sarcopenia is an important health condition. However, it is not easy to measure the muscle mass or function of outpatient clinics by estimate walking speed, grip strength or body composition. Balance test which is a new, easy way to evaluate patients' ability to maintain their posture. The higher the score, the more difficult it was to maintain balance.

We evaluated the correlation between muscle mass, function and balance test score. A total of 29 healthy elderly people (men $=14$, women $=15$ ) in their $70 \mathrm{~s}$ were recruited to perform a balance test when subjects stands still, and with eyes closed.

Grip strength, Skeletal muscle mass index (SMI), leg extension power and gait speed were significantly lower in women $(\mathrm{P}<0.001$ respectively), and the balance score was significantly higher $(\mathrm{P}<0.001)$.

The balance score was significantly higher in women than in men as well as when they opened their eyes $(14.3 \pm 6.5$ vs. $10.8 \pm 4.1$, $\mathrm{P}=0.036)$ and closed their eyes. $(23.9 \pm 10.7$ vs. $15.8 \pm 6.2$, $\mathrm{p}=0.040)$.

In women, the balance score was significantly correlated with grip strength $(p=0.024)$ and the leg extension power $(p=0.018)$, but the correlation disappeared with thy closed their eyes $(\mathrm{p}=0.152$, and $p=0.844$, respectively). SMI was not correlated with balance score. In men, the balance score was significantly related with SMI, but not with grip strength, and leg extension power with or without eye opening. However, skeletal muscle mass index was significantly correlated with balance score $(\mathrm{p}=0.033)$.
The balance score was related with muscle function or mass, but the relationship has gender difference. More study is warrant to evaluate the usefulness of balance score.

Keywords: balance, muscle, gender difference

\section{P260}

Association between bone mineral density, bone remodeling markers and diet inflammatory index in women

Olga Cvijanovic Peloza ${ }^{1}$, Gordana Kendel Jovanovic ${ }^{2}$, Sandra Pavicic Zezelj $^{2}$, Sanja Klobucar Majanovic ${ }^{3}$, Tanja Celic ${ }^{1}$, Mirna Bobinac ${ }^{4}$, Dragica Bobinac ${ }^{1}$

${ }^{1}$ Department of Anatomy, Medical Faculty of the University of Rijeka, Rijeka, Croatia, ${ }^{2}$ Teaching Institute of Public Health of Primorskogoranska County, Rijeka, Croatia, ${ }^{3}$ Department of Endocrinology, Diabetes and Metabolic Diseases, Medical Faculty of the University of Rijeka, Rijeka, Croatia, ${ }^{4}$ Clinical Hospital Rijeka, Rijeka, Croatia

Dietary intake has been shown to affect bone health via both proinflammatory and anti-inflammatory mechanisms. Some dietary constituents such as zinc, magnesium and calcium have anti-inflammatory effect and are beneficial for the bone physiology. The aim was to analyze bone mineral density (BMD), bone remodeling markers, serum vitamin $\mathrm{D}$ values and overall intake of the dietary minerals (calcium, magnesium, zinc and sodium) with respect to the dietary inflammatory index (DII) score.

Women $(\mathrm{N}=400)$ included in this study were divided in subgroups according to the adherence to DII score. Q1 indicated participants having the lowest DII values and highest adherence to anti-inflammatory diet, while Q4 were participants having pro-inflammatory diet. Kruskal-Wallis test was used to analyze the adherence of the bone status parameters to the DII score as well as the adherence of dietary minerals to DII score. Multiple regression analyses was employed to test the relationship between BMD and bone remodeling markers with the DII score and age. $\mathrm{P}<0.05$ was considered as statistically significant.

Our results revealed that women whose dietary habits were more in anti-inflammatory quartiles, had significantly higher values of the lumbar spine BMD $(\mathrm{P}=0.01)$, left hip BMD $(\mathrm{P}<0.01)$ and bone alkaline phosphatase $(\mathrm{P}<0.01)$. They also had significantly higher intakes of dietary calcium, magnesium and zinc, $\mathrm{P}<0.01$, respectively.

Multiple regression analyses revealed that age and DII were significantly and inversely correlated to left hip BMD, while deoxypyridinoline was just proportional to age and DII, $\mathrm{P}<0.01$, respectively.

Study results suggest that anti-inflammatory diet is associated with high BMD and younger age.

Keywords: bone mineral density, bone remodeling markers, dietary inflammatory index, women, age

\section{P261}

Impact of mild and moderate/severe vertebral fractures on physical activity: A five-year prospective study based on a cohort of older women in the UK

Usama Al-Sari ${ }^{1}$, Jonathan Tobias ${ }^{1}$, Emma Clark ${ }^{1}$

${ }^{1}$ University of Bristol, Bristol, United Kingdom

Background/Objectives: Little is known about the long-term impact of VFs on physical activities. There is also uncertainty over the clinical significance of mild VF. Therefore, the aim of the present study was to evaluate the prospective association between baseline $\mathrm{VF}$ and future physical activity. 
Design: A 5-year prospective study.

Setting: Mixed community and secondary care in the UK.

Participants: 700 women aged $>50$ (mean age 68.2) with baseline spine images provided data on self-reported physical activities after 5.4 years follow-up.

Measurements: VFs were identified at baseline on radiographs or DXA-based Vertebral Fracture Assessment (VFA) by a Quantitative Morphometric approach and defined as a moderate/severe VF ( $\geq 25 \%$ height decrease) or mild VF (20-24.9\% height decrease). Physical activity data were collected at follow-up by self-completion questionnaires. Multivariate logistic regression was used to determine the association between presence of VF and various physical activities whilst adjusting for potential confounders.

Results: 286 women without, 58 with mild, and 69 with moderate/severe VF were recruited to this study. Those with mild and moderate/severe fracture were older than women without fracture (mean age of 69.5 and 72.2 years vs. 66.8) and had more concomitant diseases at baseline. At 5.4 years follow-up, women with moderate/severe fracture reported shorter walking duration compared to those without fracture, even after adjusting for potential confounders (OR 2.96, 95\% CI 1.11-7.88, $\mathrm{P}=0.030$ ). No association was seen between presence of mild VFs and reduced physical functioning at follow-up.

Conclusion: This is the first study of older women from the UK that explored the prospective association between VF and physical activity duration. Both moderate/severe and mild VF are inversely related to subsequent activity.

\section{P262}

Quantitative computed tomography discriminates between individuals with low areal bone mineral density with and without vertebral fractures

Margaret Paggiosi ${ }^{1}$, Miguel Debono ${ }^{2}$, Jennifer S Walsh ${ }^{1}$, Richard Eastell

${ }^{I}$ The Mellanby Centre for Bone Research, Department of Oncology and Metabolism, The University of Sheffield, Sheffield, United Kingdom, ${ }^{2}$ Endocrinology and Acute Medicine, Sheffield Teaching Hospitals NHS Foundation Trust, Sheffield, United Kingdom

Spinal quantitative computed tomography (QCT) has the advantage that it can measure volumetric bone mineral density (vBMD) and exclude the vertebral posterior elements.

We (i) evaluated the ability of vBMD to discriminate between individuals with low areal bone mineral density (aBMD) by dualenergy X-ray absorptiometry (DXA) with and without vertebral fractures and (ii) compared this to the discriminatory ability of aBMD and trabecular bone score (TBS).

We studied postmenopausal women (age $=68.8 \pm 6.1$ years) with an aBMD T-score $<-1.0$ at either the hip or lumbar spine and either (i) $\geq 1$ vertebral fracture (Group $1, \mathrm{n}=39$ ) or (ii) no vertebral fractures and age- and aBMD-matched to those in Group 1 (Group 2, $\mathrm{n}=34$ ). Areal BMD was measured by DXA (QDR 4500A, Hologic) and TBS (Medimaps) was derived following DXA scan reanalysis. Volumetric BMD of L1-L3 was determined by QCT using a GE Lightspeed 64 VCT and Mindways QCT Pro software. Differences in bone measurements between Group 1 and 2 were examined using General Linear Univariate Modelling approaches. The discriminatory ability (area under the curve (AUC)) of aBMD, TBS and vBMD was assessed using receiver operator characteristic (ROC) analysis. ROCs were also compared. $\mathrm{P}<0.05$ indicated statistical significance.
Group differences, AUCs and ROC comparisons

\begin{tabular}{|c|c|c|c|c|c|}
\hline $\begin{array}{l}\text { Spine } \\
\text { measure- } \\
\text { ment }\end{array}$ & $\begin{array}{l}\text { Mean } \\
\text { (SE) }\end{array}$ & $\begin{array}{l}\text { Group } \\
\text { differences } \\
\text { (p value) }\end{array}$ & $\begin{array}{l}\text { AUC } \\
(\mathrm{p} \\
\text { value })\end{array}$ & $\begin{array}{l}\text { vBMD } \\
\text { ROC } \\
\text { comparison } \\
\text { (p value) }\end{array}$ & $\begin{array}{l}\text { aBMD } \\
\text { ROC } \\
\text { comparison } \\
\text { ( } p \text { value) }\end{array}$ \\
\hline $\begin{array}{c}\text { vBMD L1- } \\
\text { L3 }(\mathrm{mg} \\
\left.\mathrm{HA} / \mathrm{cm}^{3}\right)\end{array}$ & $\begin{array}{c}\text { Group 1 = } \\
71.844 \\
(6.100) \\
\text { Group 2 }= \\
94.593(4.015)\end{array}$ & $\begin{array}{l}-18.660 \\
(0.01)\end{array}$ & $\begin{array}{l}0.759 \\
(0.001)\end{array}$ & - & 0.005 \\
\hline $\begin{array}{l}\text { aBMD } \\
\quad \text { L1-L4 } \\
\left(\mathrm{g} / \mathrm{cm}^{2}\right)\end{array}$ & $\begin{array}{l}\text { Group } \\
\quad 1=0.787 \\
(0.013) \\
\text { Group 2 }= \\
0.805(0.015)\end{array}$ & $\begin{array}{l}-0.021 \\
(0.5)\end{array}$ & $\begin{array}{l}0.592 \\
(0.3)\end{array}$ & 0.005 & - \\
\hline $\begin{array}{l}\text { TBS } \\
\qquad \begin{array}{l}\text { L1-L4 } \\
\left(\mathrm{mm}^{-1}\right)\end{array}\end{array}$ & $\begin{array}{l}\text { Group } \\
\quad 1=1.236 \\
\quad(0.015) \\
\text { Group } \\
2=1.261 \\
(0.016)\end{array}$ & $\begin{array}{l}-0.038 \\
(0.196)\end{array}$ & $\begin{array}{l}0.617 \\
(0.08)\end{array}$ & 0.03 & 0.6 \\
\hline
\end{tabular}

In our study design, aBMD was similar for both groups. However, vBMD was significantly lower in Group 1 and could discriminate between those with and without vertebral fractures.

In conclusion, spinal QCT may discriminate well between individuals with and without vertebral fractures as it provides a 3D measure of bone mineral density and excludes the vertebral posterior elements.

Keywords: Discriminatory ability, vertebral fracture, quantitative computed tomography

\section{P263}

High rates of abnormal serum protein electrophoresis (SPEP) in patients $\geq 50 \mathrm{yrs}$ with fragility fractures admitted to a Midwestern Tertiary Academic Center

\section{Naga Yalla ${ }^{1}$, Chinenye Udokwu ${ }^{2}$, Elizabeth $\operatorname{Lin}^{3}$}

${ }^{I}$ Division of Bone and Mineral Diseases, Division of Endocrinology, Metabolism and Lipid Research, Washington University, St Louis, United States, ${ }^{2}$ Division of Endocrinology, Metabolism and Lipid Research, Washington University, St Louis, United States, ${ }^{3}$ Division of Bone and Mineral Metabolism, Division of Endocrinology, Metabolism and Lipid Research, Washington University, St Louis, United States

Introduction: The purpose of this study is to determine the prevalence of laboratory disorders affecting bone and mineral metabolism in adults $\geq 50$ years of age admitted to a Midwestern tertiary academic center with fragility fractures between September 2016 to September 2017.

We retrospectively screened clinical records of 488 consecutive patients $(>50$ years old) admitted to a large academic center because of low trauma fractures over 1 year. We selected fracture patients who also had a complete metabolic profile, and other laboratory tests, including corrected serum calcium, serum parathyroid hormone $(\mathrm{PTH}), 25 \mathrm{OHD}$, thyroid stimulating hormone, and SPEP with immunofixation electrophoresis.

Results: A total of 328 patients met inclusion criteria and were further analyzed. Of them, $74.1 \%$ were female, $86.5 \%$ Caucasian, with mean age $77.7 \pm 11.1$ years. Fracture history included $12.6 \%$ at upper extremity, $10.7 \%$ lower extremity, $19.5 \%$ femur, $36.9 \%$ hip, $9.8 \%$ pelvis, and $19.2 \%$ vertebrae; $11.6 \%$ had multiple fractures. 
Of the 328 subjects, $62.8 \%$ were vitamin D deficient or insufficient $(25 \mathrm{OHD}<30 \mathrm{ng} / \mathrm{ml})$ and $35 \%$ had high PTH. Renal insufficiency was common, as $68.7 \%$ of patients having CKD stage 3 or higher. Importantly, evidence of monoclonal gammopathy (abnormal peak or positive immunofixation) was found in $16.8 \%$ of subjects. The frequency of SPEP abnormalities was higher in patients with vertebral fractures $(27.7 \% ; \mathrm{p}=0.025)$.

Conclusion: Previous studies focused on select fractures in outpatient settings. Here we show a surprisingly high rate of monoclonal gammopathy in patients admitted to the hospital for an acute fracture. The prevalence of SPEP abnormalities we observed is significantly higher than previously reported in subjects $>50$ (3.2\%, with an increase to $8.3 \%$ in men and $6.0 \%$ in women $>80$ years). Hence, our data corroborate the notion of an increased prevalence of monoclonal gammopathies in patients with osteoporosis.

Keywords: SPEP, Monoclonal Gammopathy, Osteoporosis, Secondary Causes Osteoporosis

Frequency of Laboratory Abnormalities in 328 patients

\begin{tabular}{|c|c|}
\hline Test (reference interval) & Prevalence \\
\hline 25-OH vitamin D (30-100 ng/mL) & $\begin{array}{l}26.73 \text { Mean (14.85 } \\
\quad \text { SD) }\end{array}$ \\
\hline PTH (10-65 pg/mL) & $\begin{array}{l}71.66 \text { Mean }(67.90 \\
\text { SD) }\end{array}$ \\
\hline $\mathrm{Cr}(0.60-1.10 \mathrm{mg} / \mathrm{dL})$ & 1.39 Mean (5.16 SD) \\
\hline Corrected serum calcium $(8.6-10.3 \mathrm{mg} / \mathrm{dL})$ & $\begin{array}{l}9.421 \text { Mean }(4.82 \\
\text { SD) }\end{array}$ \\
\hline Phosphorus (2.3-4.5 mg/dL) & 3.29 Mean (2.75 SD) \\
\hline Alkaline phosphatase (40-130 units/L) & $\begin{array}{l}81.62 \text { Mean }(46.55 \\
\text { SD) }\end{array}$ \\
\hline TSH (0.30-4.20 mcI unit/ml) & 2.45 Mean (2.46 SD) \\
\hline $\begin{array}{l}\text { Monoclonal gammopathy (SPEP/ } \\
\text { immunofixation) }\end{array}$ & $55(16.8 \%)$ \\
\hline Estimated GFR & $\begin{array}{l}\text { 51.86 Mean }(48.55 \\
\text { SD) }\end{array}$ \\
\hline
\end{tabular}

\section{P264}

The utility of TBS-adjusted BMD T-score in the discrimination of the major osteoporotic fractures in the postmenopausal women of the Rotterdam and the OsteoLaus studies

Enisa Shevroja $^{1,2}$, Fjorda Koromani ${ }^{2}$, William D. Leslie ${ }^{3}$, Olivier Lamy $^{1}$, Fernando Rivadeneira ${ }^{2}$, Didier Hans ${ }^{1}$

${ }^{1}$ Center of Bone Diseases, University Hospital Lausanne, Lausanne, Switzerland, ${ }^{2}$ Departments of Internal Medicine and Epidemiology, Erasmus Medical Center, Rotterdam, Netherlands, ${ }^{3}$ Department of Internal Medicine, University of Manitoba, Manitoba, Canada

Introduction: Leslie et al. 2017 proposed an alternative approach for using trabecular bone score (TBS) in clinical practice based upon a "risk-equivalent" offset adjustment to the bone mineral density (BMD) T-score.

Objective: To test whether TBS-adjusted BMD T-score outperforms the unadjusted BMD T-score alone in the discrimination of major osteoporotic fractures (MOF).

Methods: Our study included 3018 women (age 46-99 years) from the OsteoLaus $(\mathrm{N}=1314)$ and the Rotterdam $(\mathrm{N}=1704)$ Studies. All women had DXA scans (Hologic Discovery or GE-Lunar Prodigy) of LS and femoral neck (FN). TBS-adjusted BMD T-score of FN, total hip (TH) or LS were calculated using the formulas provided by Leslie et al. 2017. Contingency $2 \times 2$ tables had groups divided according to the WHO osteoporosis definition (BMD T-score $\leq-2.5$, or TBSadjusted BMD T-score $\leq-2.5$ ) and MOF presence. Sensitivity and specificity of BMD T-score and TBS-adjusted BMD T-score were then calculated.

Results: In total, 268 women had a prevalent MOF. Women who fractured were older, had lower BMD, TBS and TBS-adjusted BMD. TBS-adjusted BMD T-score had higher sensitivity but lower specificity compared to unadjusted BMD T-score in all three regions (Table 1).

Table 1. The distribution of major osteoporotic fractures

\begin{tabular}{|c|c|c|c|c|}
\hline \multirow{2}{*}{$N=3,018$} & \multicolumn{3}{|c|}{ Unadjusted BMD T-score } & \multicolumn{2}{c|}{ TBS-adjusted BMD T-score } \\
\cline { 2 - 5 } & $\begin{array}{c}\text { Sensitivity } \\
(95 \% \mathrm{Cl})\end{array}$ & $\begin{array}{c}\text { Specificity } \\
(95 \% \mathrm{Cl})\end{array}$ & $\begin{array}{c}\text { Sensitivity } \\
(95 \% \mathrm{Cl})\end{array}$ & $\begin{array}{c}\text { Specificity } \\
(95 \% \mathrm{Cl})\end{array}$ \\
\hline LS & $24 \%$ & $88 \%$ & $37 \%$ & $80 \%$ \\
& $(14 \% ; 34 \%)$ & $(87 \% ; 89 \%)$ & $(29 \% ; 45 \%)$ & $(78 \% ; 82 \%)$ \\
\hline FN & $22 \%$ & $93 \%$ & $29 \%$ & $87 \%$ \\
& $(12 \% ; 32 \%)$ & $(92 \% ; 94 \%)$ & $(19 \% ; 39 \%)$ & $(86 \% ; 88 \%)$ \\
\hline Hip & $17 \%$ & $96 \%$ & $20 \%$ & $93 \%$ \\
& $(7 \% ; 27 \%)$ & $(95 \% ; 96 \%)$ & $(10 \% ; 30 \%)$ & $(92 \% ; 94 \%)$ \\
\hline Lowest & $62 \%$ & $83 \%$ & $76 \%$ & $75 \%$ \\
& $(54 \% ; 70 \%)$ & $(81 \% ; 85 \%)$ & $(70 \% ; 82 \%)$ & $(73 \% ; 77 \%)$ \\
\hline
\end{tabular}

Conclusion: This study illustrates how the adjustment of BMD by TBS contributes to MOF discrimination by increasing the sensitivity. As BMD has been shown to have low sensitivity, this need in the field might be filled by the information provided by TBS. This approach postulates itself as a helpful strategy to improve patient care in regions where intervention guidelines are based solely on the BMD T-score.

Keywords: osteoporosis, TBS

\section{P265}

Accurate BMD Calibration in QCT requires consideration of scanner specific X-ray field inhomogeneity

Klaus Engelke $^{1,2}$, Faulkner Ken ${ }^{3}$, Bernd Stampa ${ }^{2}$, Thomas Fuerst ${ }^{3}$, Harry Genant ${ }^{4}$

${ }^{1}$ Inst. of Medical Physics, FAU Erlangen-Nürnberg, Erlangen, Germany, ${ }^{2}$ Bioclinica, Hamburg, Germany, ${ }^{3}$ Bioclinica, Inc, Newark, United States, ${ }^{4}$ Radiology, University of California San Francisco, San Francisco, United States

Aim: Describe BMD calibration variations among CT-scanners and evaluate effectiveness of in-scan phantom calibration.

Methods: In 12 multicenter clinical trials of anti-osteoporotic medication, the European Spine Phantom (ESP) was scanned with the Mindways calibration phantom on 78 different CT scanners. ESP scans were performed to (a) determine short-term reproducibility, (b) measure field inhomogeneity (FI) between the location of the calibration phantom and 'ESP vertebrae' and (c) derive FI corrections (FIC). All CT scanners were regularly calibrated to water and air according to manufacturer recommendations. Mean trabecular BMD of the three ESP vertebrae was analyzed.

Results: Thirty-eight different scanner models from five manufacturers were included. Six different ESPs were used; nominal BMD slightly differed from the specifications of 50,100 , and $200\left[\mathrm{~g} / \mathrm{cm}^{3}\right]$. Among scanners, HU values of the three vertebrae were highly linear but absolute values varied considerably. Precision computed from 
repeat scans was $<1 \%$. After calibration using the Mindways phantom differences from nominal ESP BMD values varied from - 50 to $+28 \%$ (mean $-5.2 \pm 9.2 \%$ ) indicating considerable FI differences among scanners. Exclusion of Siemens Emotion and Cardiac models, decreased differences to $-4.0 \pm 8.0 \%$. After applying linear FIC obtained from each ESP scan, differences between calibrated and nominal BMD values decreased to $-0.2 \pm 1.4 \%$.

Conclusions: Despite regular calibration, HU values of trabecular BMD varied considerably among scanners. Calibration using an inscan calibration phantom does not guarantee accurate BMD results if FI is neglected. After linear FIC as applied here small accuracy errors remain. Short-term precision of modern CT scanners, as measured with the ESP, was $<1 \%$.

\section{Table 1}

\begin{tabular}{llll}
\hline $\begin{array}{l}\text { Vertebra (nominal BMD } \\
\left.\left[\mathrm{g} / \mathrm{cm}^{3}\right]\right)\end{array}$ & $(50.5-51.2)$ & $\begin{array}{l}\text { L2 } \\
(100.6-102.5)\end{array}$ & $\begin{array}{l}\text { L3 } \\
(196.5-201.4)\end{array}$ \\
\hline Uncalibrated HU/HU & $45-77 / 0.71$ & $107-150 / 0.23$ & $214-284 / 0.26$ \\
$\% \mathrm{CV}$ (SD) & $(1.1)$ & $(0.23)$ & $(0.50)$ \\
Difference from nominal & $-50 \%$ to & $-26 \%$ to & $-17 \%$ to \\
BMD & $+29 \%$ & $+20 \%$ & $+6 \%$ \\
Difference from nominal & $-5.4 \%$ to & $-1.1 \%$ to & $-1.0 \%$ to \\
BMD after FIC & $+1.5 \%$ & $+4.5 \%$ & $+0.2 \%$ \\
\hline
\end{tabular}

Keywords: QCT, calibration, field inhomogeneity, scanner variations

\section{P266}

The change of injected cement after Percutaneous Vertebroplasty (PVP) and Percutaneous Balloon Kyphoplasty (KP) on osteoporotic vertebral fractures

\section{Jin Hwan Kim ${ }^{1}$, Jung Hoon Kim ${ }^{1}$}

${ }^{1}$ Department of Orthopedic Surgery, Inje University, Ilsan Paik Hospital, Goyang, Republic of Korea

Purpose: To compare the radiologic results of the change of vertebral bodies and injected cements between percutaneous vertebroplasty (PVP) and balloon kyphoplasty (KP).

Material and Method: We targeted 103 patients (171 vertebrae) who were available for more than 5-year follow-up time point, from a total group of 436 patients who were treated with PVP and KP for osteoporotic vertebral fracture between January 2000 and December 2009; 69 patients (121 vertebrae) were treated by PVP, 34 patients (50 vertebrae) by KP. We analyzed the radiologic outcomes focused on the changes of injected cements and vertebral bodies as well as anterior vertebral height and kyphotic angle on pre- and postoperation.

Result: The mean follow-up period was 7.2 years. After PVP, 63 patients $(91.3 \%)$ among 69 patients were stable. But we found 2 cases of radiolucent line with decreased bone density in the adjacent area of cement and 4 cases of cement cracks accompanied with vertebral collapse. Anterior vertebral height was improved $0.3 \mathrm{~mm}$, (from $17.4 \mathrm{~mm}$ to $17.7 \mathrm{~mm}$ ), kyphotic angle was reduced $0.6^{\prime}$ (from $12.3^{\prime}$ to $\left.11.7^{\prime}\right)$. New fractures were documented in 22 patients [33 vertebrae, $(31.8 \%)$; adjacent fractures were observed in 11 vertebrae $(33 \%)$, nonadjacent fractures in 22 vertebrae (67\%)]. After KP, 21 patients $(61.8 \%)$ in 34 patients were stable. But we found 7 cases of radiolucent line with decreased bone density in the adjacent area of cement and 6 cases of cement cracks accompanied with vertebral collapse. New fractures were documented in 14 patients (21 vertebrae, $42 \%$ ); adjacent fractures were observed in 11 vertebrae (52.4\%), nonadjacent fractures in 10 vertebrae (47.6\%).

Conclusion: As the radiologic results, the vertebral bodies and injected cement were maintained more stable after PVP compared to KP.

Keywords: percutaneous vertebroplasty, percutaneous balloon kyphoplasty, bone cement, osteoporotic vertebral fracture

\section{P267}

Systematic screening by DXA lateral vertebral morphometry is associated with a high prevalence of vertebral fractures in Duchenne Muscular Dystrophy

Shuko Joseph $^{1,2}$, Sheila Shepherd ${ }^{1}$, Marina Di Marco ${ }^{3}$, Jennifer Dunne $^{2}$, Martin McMillan ${ }^{1}$, Iain Horrocks ${ }^{2}$, SF Ahmed ${ }^{1}$, SC Wong ${ }^{1}$

${ }^{1}$ The University of Glasgow, Developmental Endocrinology Research Group, Glasgow, United Kingdom, ${ }^{2}$ The Department of Paediatric Neurology, Paediatric Neurosciences Research Group The Royal Hospital for Children, Glasgow, United Kingdom, ${ }^{3}$ West of Scotland Genetic Services, Queen Elizabeth University Hospital, Scottish Muscle Network, Glasgow, United Kingdom

Background: The prevalence of vertebral fractures (VF) in Duchenne Muscular Dystrophy (DMD) is currently unknown as systematic spine imaging is rarely performed.

Objective: To determine the prevalence of VF in DMD and factors associated with VF.

Method: A prospective study utilising systematic screening with DXA lateral vertebral morphometry was performed in all 47 eligible boys. 6/47 were excluded due to spinal instrumentations and movement artefacts. Presence of VF was determined by semi-quantitative Genant method by two independent observers.

Results: Of 41 boys with median age of 9.9 years (range 5.0, 18.3), 37 (90\%) had been on glucocorticoid (GC) for a median of 3.8 years $(0.2,13.4)$. Four were GC naive. Of the $41,8(19.5 \%)$ had a total of $43 \mathrm{VF}$. Of the 8 boys with VF, $4(50 \%)$ had VF previously diagnosed due to presentation with back pain. Of the $43 \mathrm{VF}, 11$ (26\%), $21(48 \%)$ and $11(26 \%)$ were grade 1, 2 and 3, respectively. Of the 8 boys with VF, only $2(25 \%)$ had back pain. Bone turnover was low with median bone alkaline phosphatase SDS and median c-terminal telopeptide SDS of $-2.0(-5.7,0.2)$ and $-2.0(-4.1,0.1)$, respectively. There were no differences in mobility status $(\mathrm{p}=0.70)$, 25hydroxy vitamin $\mathrm{D}(\mathrm{p}=0.87)$ and lumbar spine $(\mathrm{LS})$ bone mineral apparent density (BMAD) SDS $(\mathrm{p}=0.73)$ in those with or without VF. Odds of VF increased by 1.5 times (95\% CI 1.03 to 2.20 , $\mathrm{p}=0.04$ ) for every 1-year increase in GC exposure after adjusting for GC dose in hydrocortisone equivalent, mobility status, back pain and LS BMAD SDS.

Conclusion: In this young cohort of DMD boys with relatively short duration of GC exposure, the prevalent VF rate was approximately $20 \%$ using systematic spine imaging. In the majority, this was associated with no symptoms. DXA bone mineral content was not discriminatory for VF in this group of individuals.

\section{P268}

Impact of the new trabecular bone score algorithm using a different correction model based on soft tissue thickness on incident atraumatic fracture risk prediction

$\underline{\text { Enisa Shevroja }}^{1,2}$, Olivier Lamy ${ }^{1}$, Berengere Aubry-Rozier ${ }^{1}$, Elena Gonzalez Rodriguez $^{1}$, Delphine Stoll ${ }^{1}$, Didier Hans ${ }^{1}$ 
${ }^{1}$ Center of Bone Diseases, University Hospital Lausanne, Lausanne, Switzerland, ${ }^{2}$ Departments of Internal Medicine and Epidemiology, Erasmus Medical Center, Rotterdam, Netherlands

Introduction: Trabecular bone score (TBS) is an indirect index of bone microarchitecture measured from lumbar spine (LS) DXA images. TBS iNsight ${ }^{\circledR} \mathrm{v} 3.0$ and older versions account for soft tissue variability by integrating a correction for body mass index $\left(\mathrm{TBS}_{\mathrm{BMI}}\right)$ in the TBS algorithm. A residual negative correlation has been reported between TBS and BMI. To deal with this issue, a new TBS correction based on the tissue thickness estimated by DXA $\left(\mathrm{TBS}_{\mathrm{TH}}\right)$, is developed.

Objective: To explore and compare the validity of $\mathrm{TBS}_{\mathrm{TH}}$ and $\mathrm{TBS}_{\mathrm{BMI}}$ in fracture prediction.

Methods: This study was embedded in the OsteoLaus cohort. Binary logistic regressions (M1: adjusted for age, osteoporotic treatment; M2: M1, LS-BMD; M3: osteoporotic treatment, FRAX ${ }_{\mathrm{BMD}}$ MOF) were used to obtain the risk estimates for MOF per SD decrease in $\mathrm{TBS}_{\mathrm{BMI}}$ or $\mathrm{TBS}_{\mathrm{TH}}$. The areas under-the-receiver-operating-characteristic curves (AUC) for the models adjusted for age, LS-BMD (M0); $\mathrm{M} 0+\mathrm{TBS}_{\mathrm{BMI}}$; and $\mathrm{M} 0+\mathrm{TBS}_{\mathrm{TH}}$ were calculated.

Results: During 2.5 years, among 1331 women (mean age $=$ $64.6 \pm 7.5$ years; mean $\left.\mathrm{BMI}=25.9 \pm 4.5 \mathrm{~kg} / \mathrm{m}^{2}\right), 128$ fractured BMI correlated negatively with $\mathrm{TBS}_{\mathrm{BMI}}(\mathrm{r}=-0.21)$ and positively with $\mathrm{TBS}_{\mathrm{TH}}(\mathrm{r}=+0.26)$. Lower TBS was significantly associated with increased risk for incident fractures, but $\mathrm{TBS}_{\mathrm{TH}}$ was shown to be a better predictor of fracture risk than $\mathrm{TBS}_{\mathrm{BMI}}$ (Table 1). The AUC $(95 \% \mathrm{CI})$ for M0 was $0.620(0.569 ; 0.670)$; M0 + TBSBMI 0.650 (0.601; 0.700); and M0 + TBSTTH 0.659 (0.610; 0.709).

Table 1. The results of the logistic regression analysis

\begin{tabular}{|c|c|c|c|c|c|c|}
\hline & \multicolumn{2}{|c|}{ Model 1} & \multicolumn{2}{|c|}{ Model 2} & \multicolumn{2}{|c|}{ Model 3} \\
\hline & $\mathrm{TBS}_{\mathrm{BMI}}$ & $\mathrm{TBS}_{\pi \mathrm{H}}$ & $\mathrm{TBS}_{\mathrm{BM}}$ & $\mathrm{TBS}_{T H}$ & $\mathrm{TBS}_{\mathrm{BM} I}$ & $\mathrm{TBS}_{\pi \mathrm{TH}}$ \\
\hline OR & 1.38 & 1.39 & 1.50 & 1.73 & 1.49 & 1.69 \\
\hline$(95 \% \mathrm{Cl})$ & $(1.13 ; 1.68)$ & $(1.14 ; 1.69)$ & $(1.21 ; 1.86)$ & $(1.35 ; 2.22)$ & $(1.20 ; 1.63)$ & $(1.31 ; 2.17)$ \\
\hline
\end{tabular}

Conclusion: The residual negative correlation between BMI and $\mathrm{TBS}_{\mathrm{BMI}}$ disappeared with the new $\mathrm{TBS}_{\mathrm{TH}}$. Furthermore, the superiority of TBS $\mathrm{TH}_{\mathrm{TH}}$ Vs. TBS $\mathrm{TBMI}_{\mathrm{BM}}$ in MOF risk prediction was demonstrated. Larger studies, with longer follow-up would be helpful to further validate our findings.

Keywords: Osteoporosis, TBS

\section{P269}

Bone mineral density and fragility fractures in lung or heart transplant recipients: a longitudinal study

Carla Caffarelli ${ }^{1}$, Maria Dea Tomai Pitinca ${ }^{2}$, Valentina Francolini ${ }^{2}$, Mario Alessandri ${ }^{2}$, Ranuccio Nuti ${ }^{2}$, Stefano Gonnelli ${ }^{2}$

${ }^{1}$ University of Siena, Siena, Italy, ${ }^{2}$ Department of Medicine, Surgery and Neuroscience, University of Siena, Siena, Italy

Bone loss and bone fractures are common complications after lung or heart transplantation. The loss of bone mass is particularly relevant in the year following transplantation. Many factors contribute to the pathogenesis of osteoporosis after organ transplantation, such as bone disease preceding transplantation, immunosuppressive medications, nutritional and lifestyle factors.
This study aimed to assess the bone mineral density and the incidence of vertebral fractures before and after lung and heart transplantation.

This retrospective observational study analyzed 197 electronic medical records of patients who underwent lung transplantation $(\mathrm{N}=122 ; 75$ men, 47 women $)$ and heart transplantation $(\mathrm{N}=75 ; 52$ men, 23 women) at Siena University Medical Center between January 2000 and June 2016.

Clinical, DXA scans and radiographic fractures obtained in the 12 months preceding transplant and at least one posttransplant evaluation were recorded.

The prevalence of a pretransplant osteopenia or osteoporosis was 42.8 and $27.0 \%$, respectively for heart candidates and 52.7 and $30.9 \%$ respectively for lung candidates. In all subjects, posttransplant BMD decreased significantly at the femoral neck but not at the lumbar spine in the first year, with subsequent stabilization.

Out of 122 lung transplant recipients, 18 patients (14.9\%) had fractures pre transplantation. Moreover, 29 (23.8\%) lung recipients developed a vertebral fracture within 18 months after transplantation. Similarly, out of 75 heart transplant recipient, 4 patients $(5.3 \%)$ had fractures pre transplantation, and 11 patients $(14.7 \%)$ heart recipient developed a vertebral fracture within 18 months after transplantation.

In conclusion, bone loss and high fracture rates have been observed in organ transplant recipients, particularly during the early posttransplant period, when doses of glucocorticoids and calcineurin inhibitors are high. Therefore, early evaluation of bone mass and prevention of bone loss should be considered in all patients undergoing organ transplantation.

\section{P270}

An innovative non-ionizing technique for bone status assessment: results of a multicenter clinical study comparing rems and DXA at femoral neck

Carla Caffarelli ${ }^{1}$, Giovanni Airoli ${ }^{2}$, Loredana Cavalli ${ }^{3}$, Gerolamo Bianchi $^{4}$, Maria Luisa Brandi ${ }^{3}$, Luisella Cianferotti ${ }^{3}$, Marco Matucci Cerinic $^{5}$, Francesco Conversano ${ }^{6}$, Marco Di Paola ${ }^{6}$, Davide Gatti ${ }^{7}$, Giuseppe Girasole ${ }^{4}$, Andrea Giusti ${ }^{4}$, Monica Manfredini ${ }^{2}$, Maurizio Muratore $^{8}$, Ranuccio Nuti ${ }^{1}$, Paola Pisani ${ }^{6}$, Eugenio Quarta ${ }^{8}$, Maurizio Rossini $^{7}$, Ombretta Viapiana ${ }^{7}$, Stefano Gonnelli ${ }^{1}$

${ }^{1}$ Department of Medicine, Surgery and Neurosciences, University of Siena, Siena, Italy, ${ }^{2}$ Department of Neurosciences and Rehabilitation, Carlo Poma Hospital, Mantova, Italy, ${ }^{3}$ Department of Surgery and Translational Medicine, Metabolic Bone Disease Unit, University of Florence, Florence, Italy, ${ }^{4} S C$ Rheumatology, ASL3 genovese, Genoa, Italy, ${ }^{5}$ Department of Experimental and Clinical Medicine, SOD Rheumatoligy, University of Florence, Florence, Italy, ${ }^{6}$ Institute of Clinical Physiology, National Research Council, Lecce, Italy, ${ }^{7}$ Department of Medicine, Rheumatology Unit, University of Verona, Verona, Italy, ${ }^{8}$ O.U. of Rheumatology, 'Galateo' Hospital, San Cesario di Lecce, Lecce, Italy

Background: Diagnosis and management of osteoporosis are routinely based on DXA outcomes and evaluation of clinical risk factors. Recently, researchers in this field have turned their attention to the investigation of ultrasonographic approaches for osteoporosis diagnosis directly applicable on proximal femur and lumbar spine.

Objective: To evaluate diagnostic accuracy of REMS (Radiofrequency Echographic Multi Spectrometry) technology in assessing the bone status at femoral neck through the comparison with DXA.

Material and Methods: A total of 1707 postmenopausal women aged 51-70 years were enrolled in seven Italian referral centers for osteoporosis management. REMS accuracy in osteoporosis evaluation was measured by performing in each clinical center two consecutive densitometric examinations for patient: one by using DXA device and 
the other one by REMS technology. All DXA reports affected by errors (according to the last ISCD guidelines) were discarded from the data analysis and REMS examinations reporting deviations from the correct selection of focus or scan depth values were also excluded.

Results: By analysing the diagnostic agreement between DXA and REMS diagnostic output in discriminating osteoporotic vs. non osteoporotic patients, REMS approach showed a sensibility of $85.6 \%$ and a specificity of $90.1 \%$. These parameters, when considering only DXA and REMS acquisitions perfectly adherent to guidelines, achieved values of 94.4 and $95.6 \%$ respectively. Moreover, densitometric values provided by the two techniques showed an high degree of Pearson's correlation, with $\mathrm{r}=0.94, \mathrm{p}<0.001$.

Conclusions: On the basis of the obtained results we can conclude that REMS has been shown to be an accurate non-ionizing technology able to assess the bone status at femoral neck and to discriminate osteoporotic women from healthy ones as classified by femoral DXA, showing an high correlation with DXA measurements.

\section{P271}

Three dimensional comparisons between QCTPro and MIAF Femur for the proximal femur measurements

Ling Wang $^{1}$, Oleg Museyko ${ }^{2}$, Keenan Brown ${ }^{3}$, Xiaoguang Cheng ${ }^{1}$, Klaus Engelke ${ }^{2}$

${ }^{1}$ Department of Radiology, Beijing Jishuitan Hospital, Beijing, China, ${ }^{2}$ Institute of Medical Physics, University of Erlangen, Erlangen, Germany, ${ }^{3}$ Mindways Software, Austin, United States

Introduction: Compared to DXA, QCT is less standardized. The aim of this study is to compare the proximal femur measurements between QCTPro and MIAF-Femur.

Methods: CT datasets of the hip $(120 \mathrm{kVp}, 125 \mathrm{mAs}, 1 \mathrm{~mm}$ slice thickness, standard bod kernel, Mindways calibration phantom) from 155 subjects aged 60-81 years (80 females and 75 males) were analyzed with QCTPro CTXA Hip and MIAF-Femur. Integral, cortical and trabecular vBMD, volume, and bone mineral content (BMC) of the femur neck (FN), trochanter (TR), inter-trochanter (IT), and total hip $(\mathrm{TH})$ volumes of interest (VOIs) were measured. As inconsistencies between cortical vBMD with aBMD were recently reported for QCTPro [1, 2], so called raw data and data corrected for partial volume artifacts (corrected data) were both used for the comparisons with MIAF measurements.

Results: Correlations $\left(\mathrm{R}^{2}\right)$ of integral and trabecular bone parameters of all VOIs between QCTPro and MIAF ranged from 0.51 to 0.91, Correlations between QCTPro corrected cortical vBMD and MIAF were negative for TH, FN and IT VOIs, whereas QCTPro raw data correlated positively with MIAF measurements for all VOIs. Integral, cortical and trabecular vBMD values of all VOIs of QCTPro were statistically higher than corresponding MIAF, except for FN and TR integral vBMD.

Conclusion: Integral and trabecular bone parameters are highly correlated between QCTPro and MIAF. With respect to volumetric cortical measurements, it is recommended to use QCTPro raw data, because corrected data correlated negatively with MIAF cortical vBMD and as shown before with aBMD [2].

Keywords: MIAF; QCTPro; 3D comparison

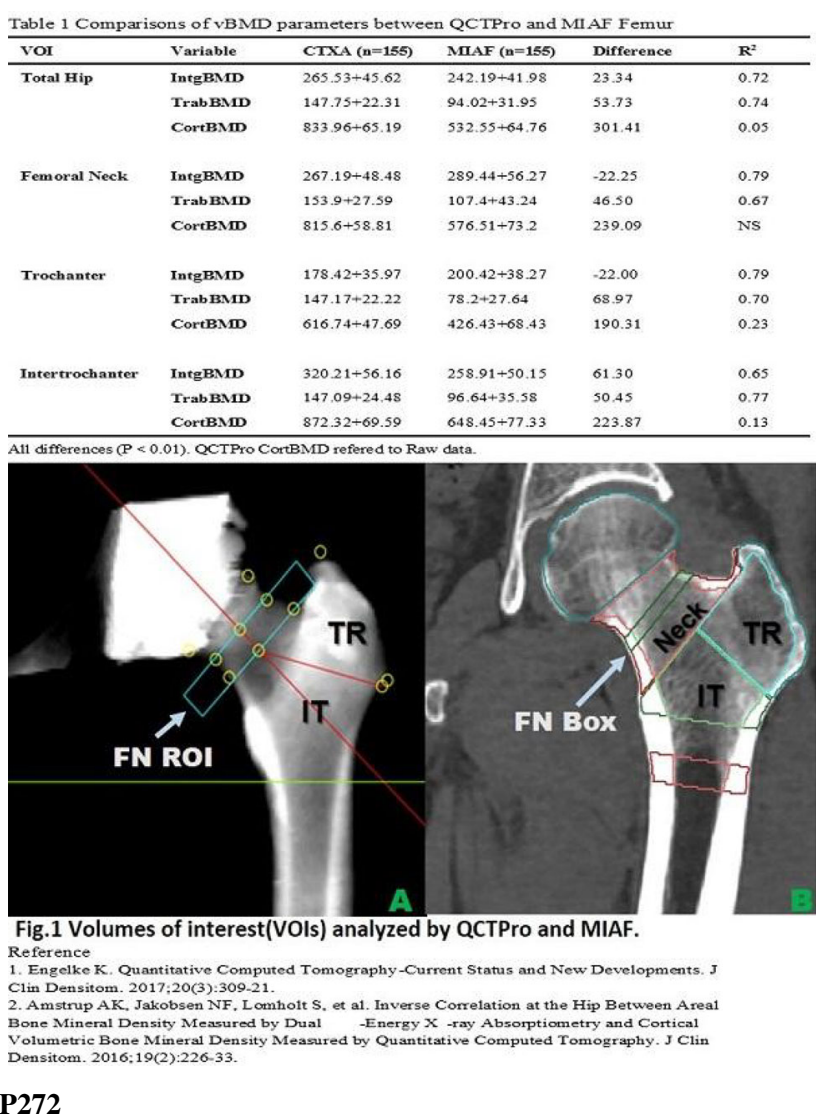

Fast versus accurate estimation of Colles' fracture load of the distal radius by homogenized finite element analysis based on lowand high-resolution HR-pQCT

\section{Denis E. Schenk $^{1}$, Philippe K. Zysset ${ }^{1}$}

${ }^{1}$ Institute for Surgical Technology and Biomechanics, University of Bern, Bern, Switzerland

Introduction: Radius fractures reveal an early onset of osteoporosis. A double section scanning protocol using HR-pQCT encompasses Colles' fracture site, which mechanical properties can be computed accurately using non-linear homogenized finite element (hFE) analysis. As processing time and reproducibility are important for clinical applications, the aim of this work was to compare the predictions of radial mechanical properties between fast, low resolution (LR: $82 \mu \mathrm{m}$ ) and accurate, high resolution (HR: $61 \mu \mathrm{m}) \mathrm{hFE}$, with respect to experimental data.

Materials and Methods: In a previous study, the $20 \mathrm{~mm}$ most distal section of 21 human cadaveric radii were scanned with HR-pQCT with LR and HR protocols. For assessment of stiffness and strength, they were dissected out and tested under compression up to failure. The fast LR hFE model is based on a single isotropic phase, while the accurate HR model distinguishes two cortical and trabecular bone phases with distinct fabric-based orthotropic material properties (Fig.). Stiffness and ultimate load obtained from the FE analyses were fitted to the corresponding experimental results. 


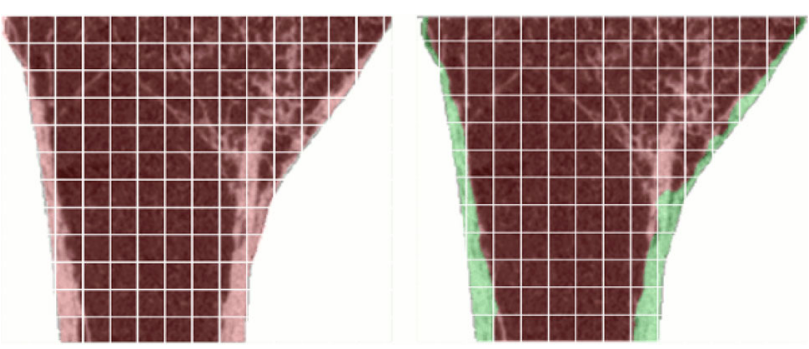

Figure Fast, monophase LR, isotropic (left) and accurate, two-phase HR, orthotropic (right) models

Results: The non-linear hFE simulation achieved a coefficient of determination $\mathrm{R}^{2}=0.971$ and $\mathrm{R}^{2}=0.974$ for fast and accurate prediction of strength and $R^{2}=0.873$ and $R^{2}=0.900$ for prediction of stiffness, respectively. Mean total processing time per sample for image processing and FE analysis was approximately $4 \mathrm{~min}$ for the fast and $60 \mathrm{~min}$ for the accurate model.

Discussion: The fast and accurate models achieved similar predictions of stiffness and strength. The additional processing time used for the accurate protocol doesn't significantly improve the results in view of clinical applications.

\section{P273}

Is a thickness-based corrective model of soft tissue effects better for TBS?

François De Guio ${ }^{1}$, Franck Michelet ${ }^{1}$, Doris $\operatorname{Tran}^{1}$, Didier Hans ${ }^{2}$

${ }^{1} R \& D$ Department, Medimaps, Canéjan, France, ${ }^{2}$ Center of Bone Diseases, Bone and Joint Department, Lausanne University Hospital, Lausanne, Switzerland

Objective: Trabecular bone score (TBS) is a textural parameter based on DXA spine scans providing an indirect measure of trabecular microarchitecture. To account for soft tissue variability between individuals, TBS computation currently integrates a correction based on BMI.

However, in some datasets acquired using Hologic systems, a residual significant negative correlation between TBS and BMI was reported. We aim to develop and test a new soft tissue corrective model to overcome this problem, while preserving or improving the clinical performance of TBS.

Methods: The new correction is based on the tissue thickness estimated by the DXA machine. We acquired scans of ex-vivo human vertebrae with varying thicknesses of tissue equivalent material. Then, the TBS-thickness relationship was used to derive a model that was applied on patient cohorts: two population-based cohorts of women $(\mathrm{N}=6742$, age $=61 \pm 9)$ and men $(\mathrm{N}=1343$, age $=56 \pm$ 11) and one case-control study (44 fractures, 145 controls, age = $64 \pm 9)$. We calculated the TBS-BMI correlation coefficients for the current and new corrections. We also evaluated the OR per SD increase of TBS in a logistic regression model to explain the prevalence of fractures in the case-control study.

Results: In women, TBS-BMI correlation coefficient changes from -0.24 (current TBS) to 0.08 (new TBS). Similarly, in men, it evolves from -0.26 to 0.08 . For the case-control study, OR rises from 2.4, $\mathrm{p}=0.001$ (current TBS) to $3.1, \mathrm{p}=0.0006$ (new TBS) adjusted for age, BMD and BMI.

Conclusion: With the new model for soft tissue effects in Hologic systems, the TBS-BMI correlation was no longer negative but becomes significantly positive, and lower than the BMD-BMI correlation. The new approach seems to also improve the clinical performance of TBS. Larger prospective studies are needed to confirm the clinical performance.

Disclosures: All authors are employees of Medimaps company that develops TBS iNsight software.

\section{P274}

A new approach for lumbar vertebrae location and morphological characteristics on Chinese lumbar DXA images

Wenmin Guan ${ }^{1}$, Wei $\mathrm{Yu}^{1}$, Evelyn $\mathrm{Hsieh}^{2}$

${ }^{1}$ Radiology, Peking Union Medical College Hospital, Chinese Academy of Medical Sciences and Peking Union Medical College, Beijing, China, ${ }^{2}$ Internal Medicine, Yale School of Medicine, New Haven, United States

Introduction: Although ISCD has introduced several approaches for vertebrae identification on lumbar DXA images, there remain individuals whose lumbar vertebrae cannot be labeled with certainty. We designed the present study to systematically evaluate lumbar vertebrae morphologic features, and present a new approach named the "--"-shaped L5.

Methodology: 1125 lumbar PA DXA images were retrospectively reviewed. For each patient, data were collected regarding the morphological characteristics of L1-L5, the proportion of patients presenting with "H"-shaped L4 and "- "-shaped L5, the position of the most superior portions of the iliac crests, the lowest vertebra with ribs and the longest transverse processes. Chi-squared analyses were used to compare proportions across age strata.

Results: In L1-L5, the angles of two connection lines between center points of spinous process and bilateral pedicels were increasing from L1 to L5. In L1-L4, these were like "V". The angles of the V were gradually increasing from L1 to L4. And "--"shaped L5 can be found in $80.3 \%$ individuals. We found L4 was predominantly " $\mathrm{H}$ "shaped (73.3\%) using Sydney's method, however the proportion of individuals with "H"-shaped L4 decreased steadily after 50 years of age. But "-_"-shaped L5 has no significant difference in proportions across all age strata $(p=0.063)$. The most superior portions of the iliac crests laying the intervertebral space between L4 and L5 was the most common (78.3\%). The lowest ribs were observed at T11, T12 and L1, with T12 being the most common (83.9\%). The longest transverse processes were observed at L2, L3 and L4, with L3 being the most common $(79.6 \%)$.

Conclusions: The "- "-shaped L5 should be considered as a routine reference tool for lumbar DXA image analysis, and maybe particularly helpful for lumbar vertebrae location among patients especially over 50 years of age.

Keywords: Dual energy X-ray absorptiometry; lumbar; morphology; postmenopausal woman

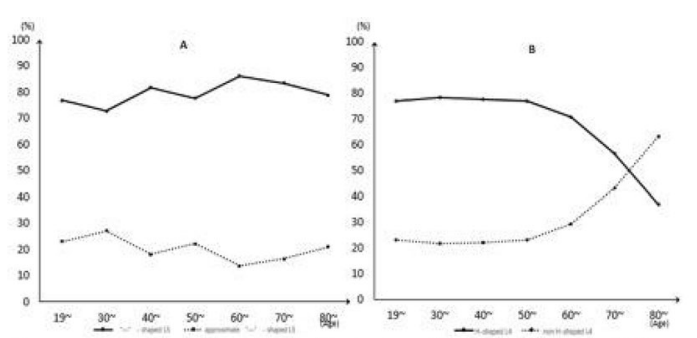

The curve indicates proportions of "-" "-shaped L 5 and " $\mathrm{H}$ "-shaped $\mathrm{L} 4$ across age strata. 


\section{P275}

The importance of distal forearm DXA in PHPT: a retrospective study of 33 patients

Theodor-Eugen Oprea ${ }^{1}$, Sorina Martin ${ }^{1,2}$, Anca Elena Sirbu ${ }^{1,2}$, Alice Albu $^{1,2}$, Carmen Barbu ${ }^{1,2}$

${ }^{I}$ Endocrinology, Elias Emergency Hospital, Bucharest, Romania, ${ }^{2}$ Carol Davila University of Medicine and Pharmacology, Bucharest, Romania

Background: Primary hyperparathyroidism (PHPT) leads to increased bone turnover, low bone mineral density, and increased fracture risk. For reasons not yet understood the balance achieved between increased resorption and formation depends not only upon the severity of the PHPT, but also upon skeletal location. Bone mineral density may be reduced particularly at sites of predominantly cortical bone, such as distal forearm. However, a dual X-ray absorptiometry (DXA) evaluation at the forearm is not routinely performed.

Materials and Methods: We retrospectively reviewed a series of 33 patients with PHPT in whom a DXA scan had been performed between January 2016 and November 2017. For data analysis we used IMB SPSS Statistics 21 software; we reported as significant a $p$ value $<0.05$.

Results: We obtained a strong significant negative correlation between serum PTH levels and distant forearm $\mathrm{T}$ and Z scores $(p<0.05)$. The mean $\mathrm{T}$ scores were $-2.935 \pm 0.39$ in the distal forearm, $-2.121 \pm 0.28$ in the spine and $-1.709 \pm 0.22$ in the total proximal femurs. The mean $\mathrm{Z}$ scores were $-1.916 \pm 0.37$ in the distal forearm, $-1.132 \pm 0.31$ in the spine and $-0.883 \pm 0.18$ in the total proximal femurs. There is also a statistically significant difference between $\mathrm{T}$ and $\mathrm{Z}$ scores of the distal forearm and lumbar spine vs. hip, PHPT affecting more the first two bone sites $(p<0.01)$.

Conclusions: Although it is not routinely performed, DXA analysis of the distal forearm, besides lumbar spine and hip, should be mandatory for a more accurate assessment of the severity of the bone loss and fracture risk in PHPT, as International Society for Clinical Densitometry recommends, due to the significant lower $\mathrm{T}$ and $\mathrm{Z}$ scores on distal radius, which seem to be inversely related with serum PTH levels.

Keywords: PHPT, DXA, osteoporosis, distal forearm

\section{P276}

Trabecular Bone Score (TBS) in postmenopausal women: an observational study in Italy

Cristiana Cipriani ${ }^{1}$, Jessica Pepe ${ }^{1}$, Luciano Nieddu $^{2}$, Vittoria Danese ${ }^{1}$, Veronica Cecchetti $^{1}$, Pietro Donato ${ }^{1}$, Valentina Piazzolla ${ }^{1}$, Luciano Colangelo ${ }^{1}$, Chiara Sonato ${ }^{1}$, Federica Biamonte ${ }^{1}$, Maurizio Angelozzi $^{1}$, Salvatore Minisola ${ }^{1}$

${ }^{1}$ Internal Medicine and Medical Disciplines, Sapienza University of Rome, Rome, Italy, ${ }^{2}$ Faculty of Economics, UNINT University, Rome, Italy

Objective: Aim of the study was to measure Trabecular Bone Score (TBS) in a cohort of postmenopausal women in Italy and to evaluate its role in defining the risk for fragility fractures.

Methods: We studied 390 postmenopausal women (mean age \pm SD $64 \pm 8$ years) aged $\geq 50$ and older. Subjects were contacted by general practitioners and referred to the Bone Unit of the Policlinico Umberto I. Hospital, Sapienza University of Rome. Questionnaires for FRAX ${ }^{\circledR}$ calculation were administered to all participants; bone mineral density (BMD) was measured at lumbar spine, femoral neck and total hip by DXA (Hologic QDR 4500A, USA). Site-matched LS TBS data were extracted from the DXA images using TBS iNsight software. The protocol was approved by the Ethics Committee of the Hospital.
Results: We observed a significant association between mean TBS values and age $(\mathrm{R}:-0.488 ; \mathrm{p}<0.0001)$. The analysis of the curve describing the relationship between TBS values distribution and age showed a significant break-point at 57 years. This indicates that mean TBS values were significantly higher in women $<57$ compared to those $>57(\mathrm{p}<0.0001)$. There was a significant negative association between risk for vertebral and major fractures and TBS (OR 0.8 e 0.6 , respectively; $\mathrm{p}<0.05$ ). The association was no longer significant after adjustment for risk factors included in FRAX and for BMD.

Conclusions: Our study reports for the first time TBS data in a wide cohort of postmenopausal women in Italy showing a significant decrease of TBS after the age of 57. TBS is significantly associated with fracture risk (particularly with vertebral fractures), but it is not an independent predictor compared to clinical risk factors and BMD. A wider cohort of subjects will be of help in realizing a database for defining country-specific normal values of TBS.

Keywords: TBS, postmenopausal, Italy

P277

Role of bone scan for predicting impending complete fracture among incomplete atypical femoral fractures

Young-Kyun Lee ${ }^{1}$, Youjin Lee ${ }^{2}$, Yong-Chan $\mathrm{Ha}^{3}$, Kyung-Hoi Koo ${ }^{1}$

${ }^{1}$ Seoul National University Bundang Hospital, Seongnam, Republic of Korea, ${ }^{2}$ National Cancer Center, Goyang, Republic of Korea, ${ }^{3}$ Chang-Ang University, Seoul, Republic of Korea

Background: Although bone scan might be useful to detect incomplete atypical femoral fractures (AFFs) earlier than radiographs, there is no study on predicting further progression to a complete fracture among incomplete AFFs. Our purposes are to determine whether bone scan detect impending complete fracture among incomplete AFFs.

Methods: We reviewed 18 patients (20 AFFs), who underwent bone scan at the diagnosis of incomplete AFF and were not treated with prophylactic fixation. A diagnosis of impending complete fracture was made, when the femur completely fractured within 6 months after the scan. We correlated radioisotope uptake with the impending complete fracture to calculate sensitivity, specificity, positive predictive value and negative predictive value of bone scan.

Results: Thirteen AFFs $(65 \%, 13 / 20)$ showed a positive uptake in bone scan. Among the 13, only one femur was completely fractured within 6 months. None of the 7 femurs without uptake in bone scan fractured. In diagnosing impending complete fracture, the sensitivity of bone scan was $100 \%$ and negative predictive value was $100 \%$. However, the specificity $(36.8 \%)$ and positive predictive value $(7.7 \%)$ were quite low.

Conclusion: Bone scan has no significant role in detecting the impending complete fracture, and a positive uptake does not mean the necessity of prophylactic fixation of incomplete AFF.

\section{P278}

Trabecular bone score (TBS) in Spanish adult men: the camargo cohort study

José M. Olmos ${ }^{1}$, José L. Hernández ${ }^{1}$, Josefina Martínez ${ }^{1}$, Jesús Castillo $^{2}$, José M. Olmos-Martínez ${ }^{1}$, Jesús González-Macías ${ }^{3}$

${ }^{1}$ Hospital Universitario M. Valdecilla, Santander, Spain, ${ }^{2}$ Centro de Salud. Camargo, Camargo, Spain, ${ }^{3}$ Medicina y Psiquiatría, Universidad de Cantabria, Santander, Spain 
Objectives: (a) To describe TBS values in men aged 50 years and older of our area, (b) analyze their relationship with bone mineral density (BMD) values in lumbar spine (LS-BMD), femoral neck (FNBMD) and total hip (TH-BMD).

Patients and Methods: We studied 1004 men aged 50-91 years $(65 \pm 9)$ included in a population-based study of osteoporosis screening and other bone metabolic diseases (the Camargo cohort study). BMD was measured by DXA (Hologic QDR 4500). TBS was evaluated using TBS iNsight ${ }^{\circledR}$ v2.1 (Med-Imaps, Pessac, France). TBS results were also analyzed after stratifying the participants according to the values of BMD in the spine and hip (normal, osteopenia, and osteoporosis). The study was approved by the local Ethics Committee.

Results: The mean values of TBS and BMD were as follows: TBS: $1.345 \pm 0.131 ; \quad$ LS-BMD: $\quad 1.020 \pm 0.157 \mathrm{~g} / \mathrm{cm}^{2} ; \quad$ FN-BMD: $0.818 \pm 0.122 \mathrm{~g} / \mathrm{cm}^{2}$; TH-BMD: $0.976 \pm 0.129 \mathrm{~g} / \mathrm{cm}^{2}$. Men with densitometric osteoporosis showed a lower TBS value than men with osteopenia or with normal BMD (Table). TBS values were inversely related to age $(\mathrm{r}:-0.169, \mathrm{p}<0.0001)$ and body mass index (r: $-0.490, \mathrm{p}<0.0001$ ), whereas they did directly with LS-BMD (r: 0.344; $\mathrm{p}<0.001$ ), FN-BMD (r: 0.139; $\mathrm{p}<0.001$ ) and TH-BMD (r: $0.154 ; \mathrm{p}<0.001)$.

TBS in normal, osteopenic or osteoporotic men

\begin{tabular}{llll}
\hline BMD & Normal & Osteopenia & Osteoporosis \\
\hline TBS & $1.384 \pm 0.126 *$ & $1.338 \pm 0.130 *$ & $1.271 \pm 0.112$ \\
\hline
\end{tabular}

$* \mathrm{p}<0.001$ compared to men with osteoporosis

Conclusions: TBS values of adult males from our area are similar to those described in other countries. TBS is lower in patients with densitometric osteoporosis than in those with osteopenia or normal BMD. Although the TBS and BMD values are significantly correlated, the degree of relationship is poor.

Supported by a grant from the Instituto de Salud Carlos III (PI15/ 00521), that included FEDER funds from the EU.

\section{P279}

Metabolism of bone tissue in peri- and postmenopausal women with type 1 diabetes mellitus

\section{$\underline{\text { Sain Safarova }}^{1}$}

${ }^{1}$ Internal Disease, Azerbaija Medical University, Baku, Azerbaijan

Objective: Evaluation of the effect of body changes in women with type 1 diabetes on the state of bone mineral density and its metabolic rate. Determine changes of directionality in serum markers of bone remodeling and bone mineral density in the peri- and postmenopausal periods in this disease.

Materials and Methods: 57 women with type 1 diabetes were included into the study. Evaluated anthropometric data (height, weight, BMI). The average age of patients with type 1 diabetes was $56.3 \pm 0.9$ лет years, the duration of diabetes was $17.1 \pm 0.8$ years, BMI was $25.8 \pm 0.3 \mathrm{~kg} / \mathrm{m}^{2}$, HBA1c was $7.56 \pm 0.2 \%$. The control group consists from 43 women. Studies parameters of phosphorus-calcium metabolism $\left(\mathrm{Ca}, \mathrm{Ca}^{2+}, \mathrm{P}\right)$, level of bone formation (ALP, PINP) and resorption (b-CTx) markers. The bone mineral density (BMD) was determined on a DXA densitometer at the lumbar spine and femoral neck.
Results: The results obtained indicate inconsistency in bone remodeling processes changes in women with diabetes (35.5 vs $16.6 \%$, $\mathrm{p}<0.001)$ with predominant bone formation changes. In postmenopausal women, a positive correlation was established between the duration of $\mathrm{CD} 1$ and the level of $\mathrm{b}-\mathrm{CTx}(\mathrm{r}=0.465, \mathrm{p}=0.001)$. Also, a negative correlation was found between T-score of the femoral neck and diabetes duration: $\mathrm{r}=-0.416, \mathrm{p}=0.04$. A significant correlation was found between the T-score of the lumbar spine and the level of b-CTX $(\mathrm{r}=-0.452, \mathrm{p}=0.002)$

Conclusion: BMD in the postmenopause demonstrate an earlier and accelerated bone mass loss, especially in the lumbar spine of women with type 1 diabetes. The bone mass loss is significantly accelerated during the late perimenopause and continues at a similar pace in the early years of postmenopause with decrease loss intensity in future.

Keywords: diabetes, bone mineral density

\section{P280}

A comparative study of changes of TBS between menopausal women with TSH suppressive therapy for differentiated thyroid cancer with healthy postmenopausal women

Yun Kyung Jeon ${ }^{1}$, Keunyoung $\mathrm{Kim}^{2}$, Myung Jun $\mathrm{Shin}^{3}$, Kyoungjune Park $^{2}$, Seong-Jang Kim ${ }^{4}$, In Joo Kim ${ }^{1}$

${ }^{1}$ Endocrinology and Metabolism, Pusan National University Hospital, Busan, Republic of Korea, ${ }^{2}$ Nuclear Medicine, Pusan National University Hospital, Busan, Republic of Korea, ${ }^{3}$ Rehabilitation Medicine, Pusan National University Hospital, Busan, Republic of Korea, ${ }^{4}$ Nuclear Medicine, Yangsan Pusan National University Hospital, Busan, Republic of Korea

Objective: Aimed to examine the changes of trabecular bone score (TBS) and bone mineral density (BMD) in postmenopausal women with thyrotropin (TSH) suppressive therapy for treatment of papillary thyroid cancer (PTC) after total thyroidectomy compared with healthy control postmenopausal women.

Methods: We enrolled a total of 38 postmenopausal patients (median age: 59 years; interquartile range [IQR]: 57-65 years) undergoing TSH suppressive therapy with levothyroxine after total thyroidectomy. Ninety-four postmenopausal women (median age: 60 years; interquartile range [IQR]: $56-63$ years) matched for age and body mass index were recruited as healthy control group. Dual-energy X-ray absorptiometry (DXA) was performed. The serial data of BMD and TBS of the lumbar spine were generated from DXA during average 4.3-year follow up period.

Results: The proportion of both osteopenia and osteoporosis was not significantly different between PTC and control group at the beginning. However, progression rate of normal to osteopenia or osteopenia to osteoporosis was more common in TSH suppression group than control group (78.9 vs. $54.3 \%, p=0.009)$. There was slightly decreasing trend in TBS and BMD during follow up, but there was no statistical significance. The median change ratio of TBS was significantly higher in PTC group (median 0.054; IQR: 0.029-0.118) than in control group (median: 0.020; IQR: 0.011-0.038, $p=0.039$ ). Even though TBS was higher than 1.35, the patients who were under TSH suppression tend to progress to osteopenia or osteoporosis compared with normal control group $(p=0.008)$.

Conclusions: This data suggests the more proportion of patients with TSH suppression progress to osteopenia or osteoporosis and the changes of BMD or TBS was minimal, but the ratio of TBS was significantly higher in a group of TSH suppression. 
Keywords: Trabecular bone score; bone mineral density, dual energy $\mathrm{X}$-ray absorptiometry; differentiated thyroid cancer

\section{P281}

Platisespondilia/complicated osteoporosis

Maria Del Pilar Ahijado Guzman ${ }^{1}$, Raul Maria Veiga Cabello², Miguel Cantalejo Moreira ${ }^{1}$, Justo Ruiz Ruiz ${ }^{3}$, Antonio Zapatero Gaviria $^{3}$

${ }^{1}$ Unit of Rheumatology, Htal Universitario de Fuenlabrada, Fuenlabrada, Spain, ${ }^{2}$ Service of Rheumatology, Htal Universitario Central de la Defensa, Madrid, Spain, ${ }^{3}$ Service of Internal Medicine, Htal Universitario de Fuenlabrada, Fuenlabrada, Spain

Introduction: Defects in the type II collagen gene are described. These predispose humans to various phenotypic combinations in families with skeletal dysplasia, and/or disease by causing deposits of microcrystals and/or early osteoarthritis and/or synovial osteochondromatosis. Flat vertebra is defined as the vertebrae with flattening of the vertebral body, also with irregular surface or with nodules of Schmorl (flat vertebra), in isolation or two a maximum of two vertebral bodies, to distinguish it from Scheuermann's disease. (Image 1 and 2) RX flat dorsal ... RX flat dorsal ...

On the other hand, vertebral size is an independent risk factor for osteoporotic vertebral fractures.

Objectives: The purpose of this is to demonstrate the possibility of "dorsal flat vertebra" in relation to complicated osteoporosis.

Methods: Patients attending physician since 1994, in whom Type II collagen disease or vertebral dysplasia was suspected, were selected for the study. Their medical history was taken. Also available radiographs, including dorsal lateral spine X-rays, were assessed for a flat vertebra, by triple-observer (two rheumatologists and one radiologist), according to the defined criteria. In all selected patients, a DEXA osteoporosis screening was performed, being chest trauma exclusion criteria.

Results: The 84 patients assessed included 43 women and 41 men, with a mean age of 47.1 years, mean weight of $84.6 \mathrm{~kg}$ in men and $74.4 \mathrm{~kg}$ in women, and mean height $168.8 \mathrm{~cm}$ in men and $159 \mathrm{~cm}$ in women. None presented T score suggestive of Osteoporosis by DEXA.

Conclusions: The association of dorsal flat vertebrae, according to the defined criteria, is observed as a form of vertebral dysplasia in the context of alterations of type II collagen. Therefore, in this genetic alteration, the vertebral size does not seem to be associated with an increased risk osteoporosis or vertebral fracture, although more detailed studies are necessary.

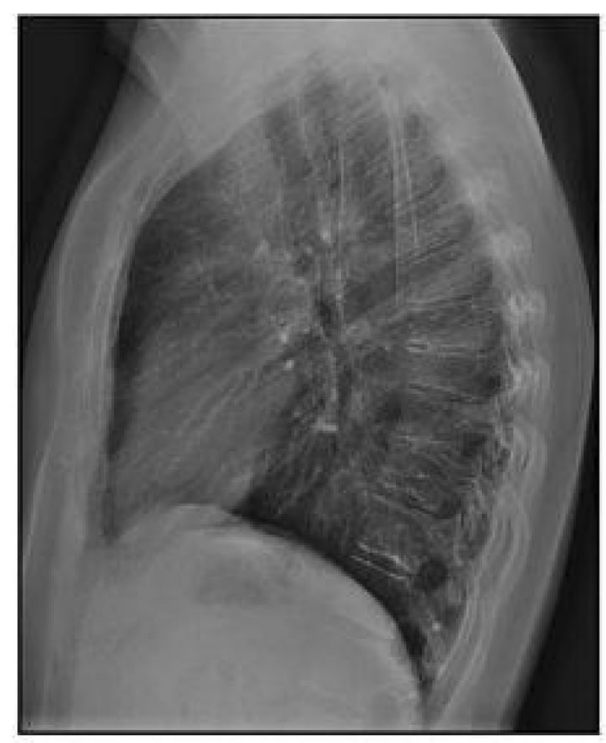

\section{$\mathrm{RX}$ flat dorsal vertebra}

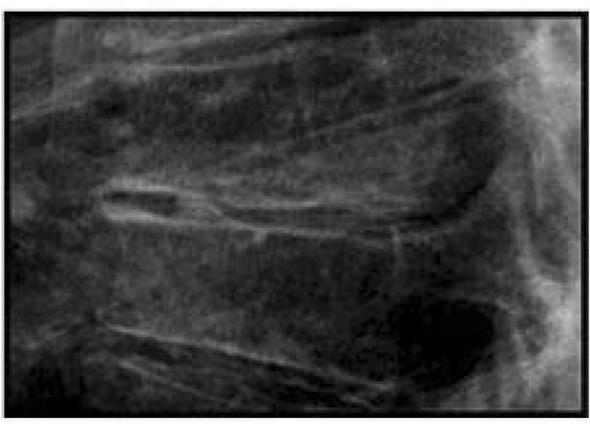

\section{$\mathrm{RX}$ flat dorsal vertebrae in detail}

\section{P282}

Effect of denosumab on vertebral bone microarchitecture in patients with rheumatoid arthritis, as assessed by clinical computed tomography in vivo

Taro Mawatari ${ }^{1,2}$, Gen Matsui ${ }^{1}$, Takahiro Iguchi ${ }^{1}$, Hiroaki Mitsuyasu $^{1}$, Shinya Kawahara ${ }^{1}$, Satoshi Ikemura ${ }^{2}$, Seiji Yoshizawa ${ }^{1}$, Yasuharu Nakashima ${ }^{2}$

${ }^{1}$ Hamanomachi Hospital, Fukuoka, Japan, ${ }^{2}$ Kyushu University, Fukuoka, Japan

Objective: Denosumab (DMAB) has been reported to increase bone mineral density (BMD) and reduce fracture risk for rheumatoid arthritis (RA) patients, while the effect on bone microarchitecture remains unclear. The purpose of this study was to clarify the effect of DMAB on the vertebral microarchitecture as assessed by clinical computed tomography in vivo.

Material and Methods: Treatment-naïve DMAB-treated RA patients ( $\mathrm{n}=11$, ave. 65.5 years old, 3 male and 8 female) and nontreated age-matched historical RA control $(\mathrm{n}=9$, ave. 65.4 years old, all female) were retrospectively evaluated at baseline and 1 year later. 8 patients from each group were receiving oral glucocorticoid therapy (2.5-7.5 mg/day). Areal BMD (aBMD) scanned by DXA, and 3D data of 3rd lumbar spine scanned by clinical computed tomography at a spatial resolution of $351 \times 351 \times 500 \mu \mathrm{m}$ were repeatedly evaluated. Bone volume fraction (BV/TV), trabecular thickness (Tb.Th), 
trabecular number (Tb.N), connectivity density (CD), and structure model index (SMI) were calculated by custom-made software. The study protocol was approved by the institutional review boards.

Results: After 1 year, non-treated control lost $-4.3 \%$ of aBMD, and the DMAB group gained $6.4 \%$, while BV/TV changed -26.4 and $+20.8 \%$, respectively. While DMAB could significantly improve several microarchitectural parameters including Tb.N $(-22.2$ vs. $+17.6 \%$ ), SMI (63.6 vs. $-40.5 \%)$, changes in Tb.Th (-5.8 vs. $2.9 \%)$ and $\mathrm{CD}(-19.8$ vs. $108.7 \%)$ varied widely and were not statistically significant.

Conclusion: DMAB increased bone volume and improved microarchitecture of lumber spine in patients with RA. Our results indicate that evaluation of aBMD by DXA may underestimate these changes. Despite the apparent increase in bone volume by DMAB, it might be difficult to reestablish trabecular connectivity. In order to prevent connectivity loss, earlier therapeutic intervention would be necessary before the connectivity has been lost.

\section{P283}

Study to evaluate the effect of radiation therapy on pelvic bones in patients of carcinoma cervix undergoing concurrent chemoradiation: an ongoing clinical trial interim analysis

\section{Divyesh Kumar ${ }^{1}$}

${ }^{1}$ Radiotherapy \& Oncology, Post Graduate Institute of Medical Education \& Research, Chandigarh, India

Introduction: Carcinoma cervix is the leading cause of morbidity in developing countries. Radiation therapy with or without chemotherapy forms an integral part of management for these patients. We in this interim analysis have tried to evaluate bone mineral density (BMD) in lumbar spine and femur in patients of carcinoma cervix undergoing concurrent chemoradiation therapy (CCRT).

Material and Method: DEXA scan is performed in patients of carcinoma cervix who meet the eligibility criteria, both before start of radiation therapy and after completion of 1 year of radiation therapy. Results analysed statistically.

Results: 20 patients have been analysed .T-scores of BMD at lumbar and pelvic bones 1 year after completion of CCRT is $89.2 \pm 4.2 \%$ ( $p<0.05$ in comparison to baseline). Conclusion-Pelvic radiation therapy given in patients of carcinoma cervix might increase the risk of bone resorption further leading to osteoporosis $><0.05$ in comparison to baseline).

Conclusion: Pelvic radiation therapy given in patients might increase the chances of bone resorption further leading to osteoporosis.

Keywords: Cancer cervix, osteoporosis, bone mineral density, radiation therapy, pelvic bones

\section{P284}

Obstructive sleep apnea syndrome in males: Bone mineral density and vitamin D3 levels in blood

Maria Del Pilar Ahijado Guzman ${ }^{1}$, Miguel Cantalejo Moreira ${ }^{2}$, Raul Maria Veiga Cabello ${ }^{3}$, Justo Ruiz Ruiz ${ }^{4}$, Antonio Zapatero Gaviria ${ }^{4}$

${ }^{1}$ Rheumatology, Htal Universitario de Fuenlabrada, Fuenlabrada, Spain, ${ }^{2}$ Rheumatology Unit, Htal Universitario de Fuenlabrada, Fuenlabrada, Spain, ${ }^{3}$ Rheumatology Service, Htal Central de la Defensa, Madrid, Spain, ${ }^{4}$ Internal Medicine Service, Htal Universitario de Fuenlabrada, Fuenlabrada, Spain

Introduction: It is considered that the prevalence of Obstructive Sleep Apnea Syndrome (OSAS) in the European population is $4 \%$. A significant reduction in bone mineral density has been described in patients with severe OSAS (apnea-hypopnea index $>30$ per hour).

Objective: To observe the variations in bone mineral density and vitamin D3 levels in a sample of male patients diagnosed with OSAS by the Pneumology Service of the Fuenlabrada's Hospital, comparing the data obtained with a control group of males of the same sociodemographic characteristics.

Patients and Method: Men diagnosed with OSAS were divided according to the severity of the apnea-hypopnea index. All patients were asked for vitamin D3 levels, including controls, obtaining the mean by groups, and DEXA Densitometry was performed exclusively on men with OSAS, calculating the average in the L2-L4 lumbar spine, and in the femoral neck.

Results: 48 patients were analyzed: 18 patients with severe OSAS, 12 patients with moderate OSAS and 18 patients with severe OSAS, and a control group with 22 male patients was collected. See Table 1 OSAS-OS..

Conclusion: This study is a small sample, and therefore a low power. However, if it seems that OSAS has a negative impact on bone metabolism. However, low levels of vitamin D3 are observed in all groups, including control. Given the prevalence of OSAS in the western population, which increases with age, it would be reasonable to influence the study of bone metabolism in these patients.

Table 1 Average Age (years), Vitamin D3 (ng/ml), T score Lumbar L2-4 and femoral neck

\begin{tabular}{lcccc}
\hline & Age & $\begin{array}{l}\text { Vitamin D3 } \\
(\mathrm{ng} / \mathrm{ml})\end{array}$ & $\begin{array}{l}\text { T score } \\
\text { lumbar }\end{array}$ & $\begin{array}{l}\text { T score } \\
\text { femoral } \\
\text { neck }\end{array}$ \\
\hline Mild OSAS & 55 & 14.8 & $<-1.2$ & $<-0.8$ \\
Moderate OSAS & 48 & 7.5 & $<-1.2$ & $<-0.7$ \\
Severe OSAS & 51 & 12.6 & $<-1.8$ & $<-1.2$ \\
Control group & 46 & 21.6 & & \\
\hline
\end{tabular}

Mild OSAS: between 10 and 20 episodes of night apnea per hour. Moderate SAOS: between 20 and 30 per hour. Severe SAOS: more than 30 episodes per hour. Normal values of vitamin D3: 30-80 ng/ $\mathrm{ml}$

\section{P285}

Bone mass improvement after liver transplant: case report

Iulia Simona Soare ${ }^{1}$, Anca Elena Sirbu ${ }^{2}$, Raluca Pascu ${ }^{3}$, Liliana Mazalu $^{3}$, Simona Fica ${ }^{2}$

${ }^{1}$ Endocrinology, Carol Davila University of Medicine and Pharmacology, Bucharest, Romania, ${ }^{2}$ Endocrinology, Diabetes and Metabolic Disease - Elias Hospital, Carol Davila University of Medicine and Pharmacology, Bucharest, Romania, ${ }^{3}$ Endocrinology, Diabetes and Metabolic Diseases, Elias Emergency Hospital, Bucharest, Romania

Background: Increased survival after organ transplantation has led to some long-term complications, among which osteoporosis and associated fractures affect the quality of life in patients. The pathogenesis of osteoporosis in transplant recipients is multifactorial and may be related to increased bone resorption and decreased bone formation; also it is involved the preexisting underlying metabolic bone disorders hepato dystrophy and the use of immunosuppressive agents and corticosteroids.

Case presentation: We report the case of a 49-year-old man, smoker, who received liver transplant for hepatocarcinoma 
(November 2014). At the evaluation pre-transplant, he was diagnosed with BMD (bone mass density) low for age and gender (L1-L4 BMD $0.817 \mathrm{~g} / \mathrm{cm}^{2}, \mathrm{Z}$ score $=-3.3 \mathrm{DS}$, left hip $0.885 \mathrm{~g} / \mathrm{cm}^{2}, \mathrm{Z}$ score $=-1$ DS). He did not receive treatment and he was lost for the follow-up. He received corticosteroid $16 \mathrm{mg} /$ day and cyclosporine for 6 months after the transplant, and then he was switched to tacrolimus $1.5 \mathrm{mg}$ /day. In august 2017, DXA showed BMD L1-L4 $1.049 \mathrm{~g} / \mathrm{cm}^{2}$, $\mathrm{Z}$ score $=-1.3 \mathrm{DS}$, left hip $0.903 \mathrm{~g} / \mathrm{cm}^{2}, \mathrm{Z}$ score $=-1.2 \mathrm{DS}$ and TBS score L1-L4 1.395. His blood tests showed normal liver function, no hypogonadism, 25 hydroxy vitamin D $15.7 \mathrm{ng} / \mathrm{ml}$ (insufficient level), normal resorption and formation markers $b$ CTx $=0.31 \mathrm{ng} / \mathrm{ml}(\leq 0.704)$, osteocalcin $28.7 \mathrm{ng} / \mathrm{ml}(14-46)$. He was prescribed vitamin D $6000 \mathrm{UI} /$ day, 6 weeks and then 1000 $\mathrm{UI} /$ day.

Conclusion: Increased risk of osteoporosis and fractures are described after receiving liver transplant, especially in the first year post-transplant. Using immunosuppressive instead of glucocorticoids and cyclosporine showed a favorable long-term effect on bone mass. Normalizing liver blood tests and adequate level of vitamin D has corrected the preexistent hepatic bone disease and decreased the risk of developing osteoporosis and a fragility fracture.

Keywords: osteoporosis, liver transplant, bone mass density

\section{P286}

PoCOsteo: Personalized fracture risk prediction via point-of-care device

Patricia Khashayar ${ }^{1}$, Ciara Kathleen O’Sullivan ${ }^{2}$, Ioanis Katakis ${ }^{2}$, Mayreli Ortiz ${ }^{2}$, Josep Lluis Acero ${ }^{2}$, Rainer Gransee ${ }^{3}$, Daniel Latta ${ }^{3}$, Richard Hoogenboom ${ }^{4}$, Frank Devlieghere ${ }^{5}$, Peter Ragaert ${ }^{5}$, An Vermeulen $^{5}$, Mieke Adriaens ${ }^{6}$, Frederik Leys ${ }^{1}$, Paula Lopes ${ }^{1}$, Gust Schols $^{7}$, Ian James Riley ${ }^{8}$, Phil Biggs ${ }^{8}$, Borja Barredo ${ }^{9}$, Afshin Ostovar ${ }^{10}$, Nahid Moradi ${ }^{10}$, Bagher Larijani ${ }^{11}$, Hans Peter Dimai ${ }^{12}$, Barbara Obermayer-Pietsch ${ }^{12}$, Jan Vanfleteren ${ }^{1}$

${ }^{1}$ CMST, Ghent University, Zwijnaarde-Gent, Belgium, ${ }^{2}$ Interfibio Research Group, Department of Chemical Engineering, Universitat Rovira i Virgili, Tarragona, Spain, ${ }^{3}$ Fraunhofer IMM, Mainz, Germany, ${ }^{4}$ Supramolecular Chemistry group, Centre of Macromolecular Chemistry (CMaC), Department of Organic and Macromolecular Chemistry, Ghent University, Gent, Belgium, ${ }^{5}$ Research Unit Food Microbiology and Food Preservation, Ghent University, Gent, Belgium, ${ }^{6}$ Department of Analytical Chemistry, Ghent University, Gent, Belgium, ${ }^{7}$ Fundico bvba, Zwijnaarde-Gent, Belgium, ${ }^{8}$ Labman Automation Ltd, Stokesely, Norther Yorkshire, United Kingdom, ${ }^{9}$ Microliquids, Arrasate, Spain, ${ }^{10}$ Osteoporosis Research Center, Endocrinology and Metabolism Clinical Sciences Institute, Tehran University of Medical Sciences, Tehran, Islamic Republic of Iran, ${ }^{11}$ Endocrinology and Metabolism Research Center, Endocrinology and Metabolism Clinical Sciences Institute, Tehran University of Medical Sciences, Tehran, Islamic Republic of Iran, ${ }^{12}$ Medical University of Graz, Department of Internal Medicine, Division of Endocrinology \& Diabetology, Graz, Austria

Osteoporosis and associated fractures are a major burden for individuals and society. Risk prediction is therefore highly warranted, as no simple, precise or sensitive tools for identification of individuals at risk of osteoporosis are currently available, mainly due to the multifactorial causes of osteoporosis including clinical, lifestyle and genetic factors.

Early stage and easily accessible identification of high-risk individuals who might best benefit from prophylaxis should therefore include various technologies such as molecular medicine, nanobiotechnology, microfluidics, and biochemistry.

The PoCOsteo project, funded by the European Commission in the framework of H2020-NMBP-X-KET-2017 (Grant Agreement number
767325), integrates proteomics and genomics technology into the development of a functional point of care ( $\mathrm{PoC})$ microfluidic device for osteoporosis risk detection. This device is aimed to be used by physicians to improve the prediction of potential fractures and to provide timely personalized care for affected individuals.

In addition, monitoring of the treatment processes via this in-office test should provide results in real-time during consultation, thus reducing the low-compliance-rate, commonly reported in osteoporotic patients more efficiently and at lower costs. The design of this PoC tool will limit the amount of both expensive reagents and complex procedures and blood volume needed for the tests and should therefore be suitable also in countries with lack of reimbursement and lower medical care standards.

The overall objective of the currently proposed PoCOsteo project therefore is the development, clinical validation and preparation for commercialization of a $\mathrm{PoC}$ tool for bone disease (i.e. osteoporosis) prevention, detection and treatment with academic/research partners from 5 countries (Gent University, University Rovira I Virgili, Fraunhofer IMM, Tehran University of Medical Sciences and Medical University of Graz) and 3 SME's (Labman, MicroLIQUID and Fundico) with a detailed project plan.

\section{P287}

Controlling hypoxia-inducible factor- $2 \alpha$ is critical to maintain bone homeostasis in mice

Je-Hwang Ryu ${ }^{1}$, Sun Young Lee ${ }^{1}$, Ka Hyon Park ${ }^{1}$, Jeong-Tae Koh ${ }^{1}$, Yun-Chan Hwang ${ }^{1}$, Yun Hyun Huh ${ }^{2}$

${ }^{1}$ School of Dentistry, Chonnam National University, Gwangju, Republic of Korea, ${ }^{2}$ School of Life Sciences, Gwangju Institute of Science and Technology, Gwangju, Republic of Korea

Objectives: To identify the function of HIF- $2 \alpha$ in the interplay between osteoblasts and osteoclasts using both constitutive HIF- $2 \alpha$ knockout (KO) mice and conditional mice with osteoblast- or osteoclast-specific depletion of HIF- $2 \alpha$.

Methods: The role of HIF-2 $\alpha$ was investigated by overexpression using adenovirus or knockdown analysis using primary cultured cells from mice. To determine the regulatory function of HIF- $2 \alpha$ in bone metabolism, we examined the bone microarchitecture in osteoblast or osteoclast conditional knockout mice using a micro-CT and analyzed bone mass using a bone histomorphometry.

Results: Overexpression of Epas1 decreased Osteocalcin mRNA level in osteoblasts through regulation of Twist $2(\mathrm{p}<0.05)$. Osteoblast overexpressing Epasl exerted an increased RANKL gene induction $(\mathrm{p}<0.01)$, which was resulted from improved binding to HRE site inside the RANKL promoter region. When osteoblast overexpressing Epas1 were co-cultured with osteoclast precursor cells, osteoclast differentiation was significantly induced even in absence of exogenous RANKL ( $\mathrm{p}<0.05)$. Osteoblast-specific HIF$2 \alpha \mathrm{KO}$ mice have augmented bone formation. The micro-CT images and quantitative parameters (BV/TV, Tb.Th, Tb.Sp and Tb. N) presented the increase of bone mineral density and trabeculae bone percentages in osteoblast-specific HIF-2 $\alpha$ KO mice compared with WT mice $(\mathrm{p}<0.05)$. H\&E staining images and bone histomorphometric analyses also showed an increased trabeculae percentage in osteoblast-specific HIF- $2 \alpha$ KO mice $(\mathrm{p}<0.05)$. MicroCT and quantitative analyses addressed that osteoclast-specific depletion of HIF$2 \alpha$ increased bone mineral density $(\mathrm{p}<0.05)$. Bone histomorphometric analyses with $\mathrm{H} \& \mathrm{E}$ and TRAP staining showed that N.Oc/BS and Oc.S/BS were significantly decreased in osteoclast-specific HIF$2 \alpha \mathrm{KO}$ mice $(\mathrm{p}<0.05)$ whereas N.Ob/BS and Ob.S/BS were not changed (not significant), which indicates that specific depletion of HIF- $2 \alpha$ in osteoclasts increased bone mineral density by preventing bone resorption. 
Conclusions: These results indicate that HIF- $2 \alpha$ may be a critical key in maintenance of bone homeostasis.

\section{P288}

The effect of anti-osteoporosis treatment on titanium particle-induced osteolysis in osteoporotic mouse calvarial model

\section{Yue Ding ${ }^{1}$, Guangtao $\mathrm{Fu}^{1}$, Junxiong Qiu ${ }^{1}$, Peng Peng ${ }^{1}$}

${ }^{1}$ Orthopedics, Sun Yat-sen memorial Hospital, Sun Yat-sen University, Guangzhou, China

Introduction: Postmenopausal osteoporosis is a common disorder that occurred in more than half of the patients who underwent total hip arthroplasty. Aseptic loosening due to wear particle-induced osteolysis is the main cause of arthroplasty failure, while the influence of postmenopausal osteoporosis on particle-induced osteolysis remains unclear.

Materials and Methods: 66 female C57BL/6J mice were randomly divided into osteoporosis group and Sham-ovariectomy (OVX) group. Several mice were randomly selected for micro-CT scanning to confirm the establishment of OVX-induced osteoporosis mice model. The modified Ti particle-induced mouse calvaria osteolysis model was established in the rest of the osteoporotic mice and Sham-OVX mice. Mice in control group underwent sham surgery only, while the mice in Titanium particle (Ti), Titanium particle + zoledronic acid $(\mathrm{Ti}+\mathrm{ZOL})$ and Titanium particle + teriparatide $(\mathrm{Ti}+\mathrm{PTH})$ groups received Ti particles, Ti particles with single dose zoledronic acid $(50 \mu \mathrm{g} / \mathrm{kg})$, and $\mathrm{Ti}$ particles with teriparatide $(40 \mu \mathrm{g} / \mathrm{kg} / \mathrm{d}$, subcutaneous injection*14d), respectively. Mice calvarias were collected for micro-CT scanning and histomorphometric analysis 2 weeks later.

Results: Osteoporotic mice had significantly decreased BMD, BV/ $\mathrm{TV}$, and increased total porosity when compared with Sham-OVX mice within the Ti group. Eroded surface area and osteoclast numbers were 34.9 and $59.7 \%$ (both $\mathrm{P}<0.05$ ) higher in osteoporotic mice. Single dose zoledronate treatment markedly inhibited Ti particle-induced osteolysis of mice with or without osteoporosis, with significantly increased $\mathrm{BMD}, \mathrm{BV} / \mathrm{TV}$, and decreased total porosity, eroded surface area and osteoclast numbers. There was not significant difference of the former-mentioned parameters between the Ti group and $\mathrm{Ti}+\mathrm{PTH}$ group in Sham-OVX mice or osteoporotic mice after 2 weeks teriparatide treatment.

Conclusion: Zoledronate could be developed into an effective therapy for wear particle-induced osteolysis, and patients with osteoporosis should consider close monitoring and early intervention after THA.

\section{P289}

The prevalence of newly diagnosed celiac disease in patients with a recent fracture at the FLS

Irma de Bruin $^{1,2}$, Lisanne Vranken ${ }^{1,2}$, Caroline Wyers ${ }^{1,2}$, Robert van de Velde ${ }^{1,2}$, Thera Trienekens ${ }^{3}$, Sjoerd Kaarsemaker ${ }^{4}$, Heinrich Janzing $^{5}$, Frank Wolters ${ }^{6}$, Siebe Wouda ${ }^{7}$, Piet Geusens, ${ }^{8,9}$, Joop van den Bergh ${ }^{1,2,9}$

${ }^{1}$ Department of Internal Medicine, VieCuri Medical Center, Venlo, Netherlands, ${ }^{2}$ NUTRIM, Department of Internal Medicine, Maastricht University Medical Center +, Maastricht, Netherlands, ${ }^{3}$ Department of Medical Microbiology, VieCuri Medical Center, Venlo, Netherlands, ${ }^{4}$ Department of Orthopedic Surgery, VieCuri Medical Center, Venlo, Netherlands, ${ }^{5}$ Department of Surgery, VieCuri Medical Center, Venlo, Netherlands, ${ }^{6}$ Department of Gastro-Enterology, VieCuri Medical Center, Venlo, Netherlands, ${ }^{7}$ Department of Pathology, VieCuri Medical Center, Venlo, Netherlands, ${ }^{8}$ CAPHRI, Department of Internal Medicine, Subdivision Rheumatology, Maastricht University Medical Center +, Maastricht, Netherlands, ${ }^{9}$ Biomedical Research Center, Hasselt University, Diepenbeek, Belgium

Introduction: Celiac disease (CD) is a known risk factor for osteoporosis and fractures. Accordingly, we expect to find a higher prevalence of CD in fracture liaison service (FLS) patients compared to the population prevalence. The prevalence of CD in an FLS population is currently unknown.

Objective: To investigate the prevalence of $\mathrm{CD}$ in the FLS population.

Methods: Patients with a recent fracture aged $\geq 50$ years were invited at the FLS. In FLS attendees, BMD and laboratory evaluation for metabolic bone disorders was performed. During a period of 18 months, serological screening for CD (Serum IgA and tissue transglutaminase antibodies (anti-tTG $\operatorname{Ig} \mathrm{A}$ )) was added to our evaluation. If serologic testing was positive, further evaluation was performed to confirm the diagnosis $\mathrm{CD}$.

Results: Included were 1042 FLS attendees, with a mean age of $66 \pm 9.4$ years, 719 were females $(69.0 \%)$. Fracture classification according to Center: $6.9 \%$ hip, $27.6 \%$ major, $54.4 \%$ minor and $11 \%$ finger and toe fractures. In $26.4 \%$ osteoporosis was diagnosed, $50.8 \%$ had osteopenia and $22.8 \%$ had a normal BMD. Four patients $(0.38 \%)$ had a positive serology for $\mathrm{CD}$. One patient refused further evaluation, one was already diagnosed with $\mathrm{CD}$, and in two patients $\mathrm{CD}$ was confirmed via duodenal biopsy. In addition, one patient with a negative serologic test was known with biopsy proven $\mathrm{CD}$. In two patients with $\operatorname{IgA}<0.2 \mathrm{~g} / \mathrm{l}$, also anti-tTG IgG was measured, and was negative. The prevalence of biopsy proven $\mathrm{CD}$ was therefore $0.38 \%$ $(n=4 / 1042)$ and $0.19 \%(n=2 / 1042)$ for newly diagnosed CD.

Conclusion: The prevalence of $\mathrm{CD}$ in patients with a recent fracture attending our FLS is low and comparable to the reported prevalence of $\mathrm{CD}$ in the Western-European population: $0.33-1.5 \%$. We conclude that systematic screening for $\mathrm{CD}$ in patients aged $\geq 50$ years, with a recent fracture at the FLS is not recommended.

\section{P290}

Porcupine inhibitors impair both trabecular and cortical bone subsequently altering bone strength in adult mice

Thomas Funck-Brentano ${ }^{1}$, Karin Nilsson ${ }^{1}$, Robert Brommage ${ }^{1}$, Petra Henning $^{1}$, Ulf Lerner ${ }^{1}$, Juha Tuukkanen ${ }^{2}$, Sofia Movérare-Skrtic ${ }^{1}$, Martine Cohen-Solal ${ }^{3}$, Claes Ohlsson ${ }^{1}$

${ }^{1}$ Center for Bone \& Arthritis Research, Gothenburg University, Gothenburg, Sweden, ${ }^{2}$ Unit of Cancer Research and Translational Medicine, University of Oulu, Oulu, Finland, ${ }^{3}$ INSERM UMR 1132, Université Paris Diderot, Paris, France

WNT signalling is involved in the tumorigenesis of various cancers and regulates bone homeostasis. Palmitoleoylation of WNTs by Porcupine is required for WNT activity. Porcupine inhibitors are under development for cancer therapy. As the possible side effects of Porcupine inhibitors on bone health are unknown, we determined their effects on bone mass and strength. Twelve-week-old C57BL/6N female mice were treated by the Porcupine inhibitors LGK974 (Low dose $=3 \mathrm{mg} / \mathrm{kg} / \mathrm{day} ;$ High dose $=6 \mathrm{mg} / \mathrm{kg} / \mathrm{day})$ or Wnt-C59 $(10 \mathrm{mg} /$ $\mathrm{kg} / \mathrm{day}$ ) or vehicle for 3 weeks. Bone parameters were assessed by serum biomarkers, DXA, $\mu \mathrm{CT}$, and histomorphometry. Bone strength was measured by the three-point bending test. The Porcupine inhibitors were well tolerated demonstrated by normal body weight. Both doses of LGK974 and Wnt-C59 reduced total body bone mineral density compared with vehicle treatment $(P<0.001)$. Cortical thickness of the femur shaft $(P<0.001)$ and trabecular bone volume fraction in the vertebral body $(P<0.001)$ were reduced by treatment with LGK974 or Wnt-C59. Porcupine inhibition reduced bone strength in the tibia $(P<0.05)$. The cortical bone loss was the result 
of impaired periosteal bone formation and increased endocortical bone resorption and the trabecular bone loss was caused by reduced trabecular bone formation and increased bone resorption. Porcupine inhibitors exert deleterious effects on bone mass and strength caused by a combination of reduced bone formation and increased bone resorption. We suggest that cancer targeted therapies using Porcupine inhibitors may increase the risk of fractures.

\section{P291}

Secular trends of hip fractures in France between 2002 and 2013: impact of the reference values

$\underline{\text { Romain Garofoli }^{1}}{ }^{\text {, Martine Cohen-Solal }}{ }^{1}$, Agnès Ostertag ${ }^{1}$

${ }^{1}$ Lariboisiere Hospital, Paris, France

Background: Hip fractures are a societal burden. With the aging of populations, worries grow about an increase of the incidence and incidence rate of hip fracture. An increased incidence whilst a decreased incidence rate of hip fracture has been reported in European countries and North America. Magnitude varies according to the reference population used. The aim of our study was to assess the impact of the choice of the reference population in the incidence rates.

Methods: From the French National Hospital Database, we extracted data of hospitalizations for hip fracture in patients over 59 years between 2002 and 2013. We calculated crude incidence rates and adjusted incidence rates of hip fracture using direct standardization with different reference populations (2002, 2013, mean population of 2002-2013).

Results: Between 2002 and 2013 in France, the incidence of hip fracture raised of $4.8 \%$ in women (from 49,287 to 51,661 ) and $21.8 \%$ in men (from 12,716 to 15,482 ), over 59 years. Meanwhile, the corresponding French population increased more: $+21.3 \%$ in women and $+28.7 \%$ in men, which explained a decrease in the crude incidence rates of $13.6 \%$ in women and $5.4 \%$ in men. This decrease was more obvious after direct standardization whatever population of reference was used: $25 \%$ in women and $19 \%$ in men. The incidence rates of hip fracture decreased in France between 2002 and 2013 in men and women aged 60 years and over. This decrease is larger after direct standardization whatever population used as a reference as a results of a difference in age-structure of the population.

Conclusion: The incidence of hip fractures continues to grow despite a reduced incidence rate throughout a 12 year-period. Sustained effort for the diagnosis and treatment should be maintained.

\section{P292}

Smoking is associated with trabecular structure and radiographic vertebral fractures in women independent of BMD

Fjorda Koromani ${ }^{1,2,3}$, Enisa Shevroja ${ }^{4}$, Ling Oei ${ }^{1}$, Carola Zillikens ${ }^{1}$, Arfan Ikram $^{2}$, Didier Hans ${ }^{4}$, Gabriel Krestin ${ }^{3}$, Andre Uitterlinden ${ }^{1,2}$, Edwin $\mathrm{Oei}^{3}$, Fernando Rivadeneira ${ }^{1,2}$

${ }^{1}$ Internal Medicine, Erasmus Medical Center, Rotterdam, Netherlands, ${ }^{2}$ Epidemiology, Erasmus Medical Center, Rotterdam, Netherlands, ${ }^{3}$ Radiology, Erasmus Medical Center, Rotterdam, Netherlands, ${ }^{4}$ Bone \& Joint, Lausanne University Hospital, Lausanne, Switzerland

Background: Smoking has been independently associated with osteoporosis and vertebral fracture (VFs) risk. We aimed to assess whether lower Trabecular Bone Score (TBS) levels among smokers might explain this association.

Methods: Analysis was conducted in subjects aged 55 years and over assessed for TBS, radiographic VFs and smoking status including 1609 (with TBS) and 1320 (with TBS and VFs) individuals
( 74\% women). TBS was measured on lumbar spine DXA scans (Medimaps TBS insight ${ }^{\circledR}$ ) and VFs scored using the Algorithm Based Qualitative ABQ method (and Quantitative Morphometry (QM) assisted by SpineAnalyzer $\left.{ }^{\circledR}\right)$ methods. Association models of smoking status were stratified by sex and adjusted for age, height, BMI and LS-BMD (for TBS using linear regression) and additionally for TBS (for VFs risk using logistic regression).

Results: Smokers had - 0.5 SD mean lower LS-TBS than smokers $(-0.55[95 \% \mathrm{CI}-0.72$ to -0.37$]$ in men) and $(-0.45[95 \% \mathrm{CI}$ -0.58 to -0.32$]$ in women); while no differences were identified with LS-BMD. Overall, $9.8 \%$ of smokers $(n=389)$ and $8.9 \%$ of nonsmokers $(n=931)$ sustained a fracture. Smoking increased the risk of VFs in women $(\mathrm{OR}=1.87 ; 95 \% \mathrm{CI} 1.12-3.14)$ and was reduced by $12 \%$ and not significant after additionally adjusting for LS-TBS $(\mathrm{OR}=1.64 ; 95 \%$ CI $0.96-2.79)$. There was no association observed among men $(\mathrm{OR}=0.73 ; 95 \%$ CI $0.27 ; 1.98)$ or in either sexes using the QM assessment.

Conclusions: Our results suggest that smoking affects trabecular structure and is associated with increased VF risk in women (at least partly through decreased TBS levels). TBS is superior to BMD for the assessment of bone health among smokers.

Keywords: vertebral fractures, smoking, trabecular bone score, BMD

\section{P293}

Effects of long-term use of unfractionated heparin or low-molecularweight heparin on bone mineral density in patients with nephrotic syndrome

\section{$\underline{\text { Simeon Monov }}^{1}$, Daniela Monova ${ }^{2}$}

${ }^{1}$ Department of Internal Medicine, Clinic of Rheumatology, Medical University - Sofia, Sofia, Bulgaria, ${ }^{2}$ Department of Internal Medicine, Medical University - Sofia, Medical Institute, Sofia, Bulgaria

Objective: The aim of this study is to determine the effects of unfractionated heparin (UFH) or low-molecular-weight heparin (LMWH) therapy of at least 1 year duration on bone mineral density (BMD) in patients with nephrotic syndrome (NS).

Material and Methods: 465 patients with NS received the prophylactic anticoagulation regimen were included. Patients received corticosteroids or with renal failure were excluded from the study. 312 patients received UFH and 153-LMWH at some point in the course of their disease. There was no difference in mean age, sex, or disease duration between both groups. 276 patients were switched from UFH or LMWH to acenocoumarol as a result of protracted hypoalbuminemia (serum albumin $<2.0 \mathrm{~g} / \mathrm{dl}$ ) at a median time of $24.3 \pm 9.3$ weeks' treatment. Bone mineral density (BMD) was measured at the lumbar spine and total hip region with dual X-ray absorptiometry.

Results: Poisson regression analysis showed that LMWH therapy was associated with a lower risk of osteoporosis compared with UFH (0.7 vs. 1.1 per 100 person-years). No statistically significant increase in the risk of fractures at 12 months was found for patients $(\mathrm{RR}=1$, 03; 95\% CI 0.27 - 3.34). UFH for 24 months decreased mean BMD by $2.6-3.5 \%$ (depending on the BMD site) compared to mean BMD decreases of $1.9-2.9 \%$ with LMWH. BMD of the spine and hip is significantly lower in patients with LMWH therapy than in subjects with UFH therapy, which is independent of age, sex, menopause, low BMI and altered body composition.

Conclusion: LMWH most likely have less effect on bone turnover when compared to UFH. LMWH for 6 months may not increase the risk of osteoporosis, but longer exposure for up to 24 months may adversely affect BMD. Clinicians should consider monitoring BMD in adults on long-term heparin therapy who are at increased risk of bone loss or fracture. 


\section{P294}

Effects of a selective phosphodiesterase 5 inhibitor (sildenafil) on the skeletal system of ovariectomized and non-ovariectomized rats Maria Pytlik $^{1}$, Leszek Śliwiński ${ }^{1}$, Małgorzata Zbrojkiewicz ${ }^{1}$, Joanna Folwarczna $^{1}$, Urszula Cegieła ${ }^{1}$, Aleksandra Janas ${ }^{1}$

${ }^{I}$ Department of Pharmacology, School of Pharmacy with the Division of Laboratory Medicine in Sosnowiec, Medical University of Silesia, Katowice, Sosnowiec, Poland

In the treatment of pulmonary arterial hypertension, selective phosphodiesterase 5 (PDE5) inhibitors are used. They induce the increase in cGMP cellular levels, and cGMP signal transduction takes part in regulation of bone remodeling. Sildenafil is the most commonly used selective inhibitor of PDE5; its effects on the skeletal system in conditions of estrogen deficiency have not been studied so far. The aim of the study was to investigate the effects of sildenafil on the skeletal system of ovariectomized (estrogen deficient) and nonovariectomized rats.

The experiments were carried out on 3-month-old female Wistar rats divided into 4 groups $(\mathrm{n}=10-11)$ : non-ovariectomized control rats, ovariectomized control rats, non-ovariectomized rats receiving sildenafil, and ovariectomized rats receiving sildenafil. Bilateral ovariectomy was performed 6 days before the start of sildenafil administration. Sildenafil was administered at a dose of $10 \mathrm{mg} / \mathrm{kg}$ by stomach gavage, 6 days a week, for a period of 30 days. Control rats were administered the vehicle, $0.9 \% \mathrm{NaCl}$. Bone mass and mineralization, macrometric and histomorphometric parameters, as well as mechanical properties of the tibial metaphysis, femoral diaphysis and femoral neck were evaluated.

Estrogen deficiency in ovariectomized rats induced osteopenia, with statistically significantly increased bone turnover and strong worsening of mechanical properties of the tibial metaphysis (maximum load and stress) and femoral neck (maximum load). In nonovariectomized rats, sildenafil significantly increased bone formation and the strength of cancellous bone of the tibial metaphysis (maximum load and energy for maximum load). In ovariectomized rats, sildenafil favorably affected the skeletal system, markedly increasing bone formation and counteracting some disorders of histomorphometric parameters induced by estrogen deficiency.

The results obtained in the animal model of osteoporosis suggest that sildenafil used in women with pulmonary arterial hypertension may exert beneficial effects on bone metabolism and reduce the risk of osteoporosis.

Keywords: PDE5 inhibitors, sildenafil, ovariectomized rats, bones

\section{P295}

Short term effects of ambient air pollution on osteoporotic hip fracture. An ecological study in a period of 16 years

Ramón Mazzucchelli ${ }^{1}$, Natalia Crespí Villarías ${ }^{2}$, Elia Perez Fernandez $^{3}$, Javier Quirós ${ }^{1}$, Olalla Guzón ${ }^{4}$, Alberto García-Vadillo ${ }^{5}$, Gil Rodriguez Caravaca ${ }^{6}$, Loreto Carmona ${ }^{7}$

${ }^{1}$ Rheumatology Unit, Hospital Universitario Fundación Alcorcón, Madrid, Spain, ${ }^{2}$ Centro de Salud de La Rivota, Alcorcón, Spain, ${ }^{3}$ Clinical Investigation, Hospital Universitario Fundación Alcorcón, Madrid, Spain, ${ }^{4}$ Rehabilitation, Hospital Universitario Fundación Alcorcón, Madrid, Spain, ${ }^{5}$ Rheumatology Unit, Hospital Universitario La Princesa, Madrid, Spain, ${ }^{6}$ Preventive Medicine and Public Health, Universidad Rey Juan Carlos, Alcorcón Madrid, Spain, ${ }^{7}$ Instituto de la Salud Musculoesquelética (INMUSC), Madrid, Spain

Objective: To examine the short-term effects of outdoor air pollution on the incidence of osteoporotic HFx in a southern European region.
Methods: This is a retrospective cohort study based on data from three databases. Daily HFx incidence was calculated using discharge data from the hospital of Alcorcon for the period 2000-2015. Daily weather conditions were compiled from records of the national meteorological station (AEMET). Daily air pollutant levels $\left(\mathrm{mcg} / \mathrm{m}^{3}\right)$ were calculated from data from the Ministry of Environment for the Madrid Community: sulfur dioxide $\left(\mathrm{SO}_{2}\right)$, nitrogen monoxide (NO), nitrogen dioxide $\left(\mathrm{NO}_{2}\right)$, ozone $\left(\mathrm{O}_{3}\right)$ and particulate matter in suspension $<2.5 \mu \mathrm{m}\left(\mathrm{PM}_{2.5}\right)$ and $<10 \mu \mathrm{m}\left(\mathrm{PM}_{10}\right)$, for the same period. Pollutant concentrations were categorized into quartiles (Q1 to Q4, lowest to highest). Associations between HFx incidence and air pollutant levels were examined through Generalized Additive Models (GAM) at a daily level with Poisson link function. Models were adjusted for a penalized spline function of time.

Main outcome measures: Daily hospital admissions for hip fracture.

Results: $\mathrm{HFx}$ incidence showed a direct association with $\mathrm{NO}, \mathrm{NO}_{2}$, $\mathrm{PM}_{2.5}$ and $\mathrm{PM}_{10}$ and inverse association with $\mathrm{O}_{3}$ levels. Incidence rate ratios for Q1vsQ2, Q1vsQ3 and Q1vsQ4 respectively were: 1.171 (1.103-1.244), 1.245 (1.173-1.322) and 1.331 (1.253-1.414) for NO; 1.057 (0.996-1.122), 1.185 (1.116-1.259) and $1.276(1.199-1.357)$ for $\mathrm{NO} 2 ; 1.028(0.943-1.12), 1.092(1.006-1.185)$ and 1.146 (1.049-1.253) for $\mathrm{PM}_{2.5} ; 1.083$ (1.016-1.155), 1.099 (1.034-1.168) and $1.213(1.136-1.294)$ for $\mathrm{PM}_{10}$; and $0.975(0.914-1.04), 0.868$ $(0.815-0.924)$ and $0.814(0.765-0.867)$ for $\mathrm{O}_{3}$. These associations persisted when the models were corrected for season, day of the week and weather conditions. When participants were stratified by age and sex, associations persisted only in women older than 75 years.

Conclusions: A short-term effect was observed of several indicators of air pollution on hip fracture incidence. This is the first study that finds this association.

\section{P296}

Senile rats: a clinically relevant osteoporotic animal model?

Deeksha Malhan ${ }^{1}$, Stefanie Kern ${ }^{1}$, Diaa Eldin S Daghma ${ }^{1}$, Sabine Stoetzel $^{1}$, Fathi Hassan ${ }^{1}$, Marian Kampschulte ${ }^{2,3}$, Jan Belikan ${ }^{2}$, Felix Schulze $^{4}$, Angela Rösen-Wolff ${ }^{4}$, Markus Rupp ${ }^{1,5}$, Christian Heiss ${ }^{1,5}$, Thaqif El Khassawna ${ }^{1}$

${ }^{1}$ Laboratory for Experimental Trauma Surgery, Faculty of Medicine, Justus Liebig University of Giessen, Giessen, Germany, ${ }^{2}$ Laboratory of Experimental Radiology, Faculty of Medicine, Justus Liebig University of Giessen, Giessen, Germany, ${ }^{3}$ Department of Diagnostic and Interventional Radiology, University Hospital of Giessen-Marburg, Giessen, Germany, ${ }^{4}$ Department of Pediatrics, University Hospital Carl Gustav Carus, TU Dresden, Dresden, Germany, ${ }^{5}$ Department of Trauma, Hand and Reconstructive Surgery, University Hospital of Giessen-Marburg, Giessen, Germany

Osteoporosis is characterized by lower bone mass and deteriorated bone microarchitecture resulting in higher fracture risks among elderly patients. This study used a senile rat animal to characterize and establish a clinically relevant osteoporotic model. Our study aimed to deepen the understanding of changes in extracellular matrix with progression of osteoporosis.

46 female Sprague-Dawley rats (age $=12$ months) were divided into five groups: (1) Control group $(\mathrm{M}=$ Months) $(0 \mathrm{M})$, (2) Sham operated (Sham, 3M), (3) bilaterally ovariectomized (OVX, 3M), (4) Sham operated combined with diet-deficiency (Diet, 3M), and (5) OVX combined with diet-deficiency (OVXD, 3M). After euthanasia, cryostatic micro-CT, histological analysis, and molecular analysis were performed. Furthermore, structural changes within groups were observed using Transmission Electron Microscopy (TEM) and Scanning Electron Microscopy (SEM). 
Micro-CT analysis showed significantly lower Bone Mineral Density (BMD) in OVX and OVXD (p-value $\leq 0.05$ ) compared to other groups after 3M. Von Kossa/Van Gieson stain depicted lower mineralized and non-mineralized bone matrix in OVX, Diet, and OVXD compared to Sham after 3M. Discrepancies in osteocytes morphology and canaliculi network were observed in OVXD group. Computational segmentation of collagen fibers obtained from TEM showed irregularities in fiber properties. Furthermore, SEM showed qualitative discrepancies in collagen fibers between groups. Higher relative gene expression of Col1a2 and Spp1 were observed in OVXD group.

In small animal models, osteoporosis is induced by combined OVX and multi-deficient diet. Previous studies utilizing pre-aged models with long treatment period showed osteomalatic status rather than osteoporotic. In accordance with clinical observation, our study showed deteriorated bone quality in OVX and OVXD group. Furthermore, the prominent effect of aging and estrogen deficiency was observed in OVX and OVXD group. Irregular fiber arrangement reflected on biomechanical properties of bone. Our study showed significant loss in bone matrix and bone density, thus mimicking the senile osteoporosis condition in patients.

Keywords: Osteoporosis, ECM

\section{P297}

Bone mineral density decline in subjects waiting for simultaneous pancreas kidney transplantation (retrospective analysis)

\section{Simona Kratochvílová ${ }^{1}$, Jana Brunová}

${ }^{I}$ Diabetes Centre, Institute for Clinical and Experimental Medicine, Prague, Czech Republic

Objective: Type 1 diabetes mellitus (T1DM) and chronic kidney disease (CKD) are both important risk factors for bone metabolism impairment. Data about bone mineral density (BMD) decline in terminal stages of diabetic nephropathy in T1DM patients are sparse. The aim of our study was to compare retrospectively BMD in subjects in waiting list (WL) for simultaneous pancreas kidney transplantation (SPK) in the period from the beginning of pre-transplant investigation till transplantation.

Design and Methods: Out of 182 subjects, who entered the WL for SPK in years 2011-2016 for the first time, 83 fulfilled criteria of having densitometry within 2 months after SPK and being on WL at least 12 months. Six subjects were excluded due to denosumab treatment. Final analysis comprised 77 subjects ( 55 men, 22 women), mean age $40.6 \pm 10.1$; diabetes duration $24.8 \pm 7.8$; glomerular filtration (MDRD) $0.27 \pm 0.14 \mathrm{ml} / \mathrm{s}$; median waiting time 20 months.

Results: Mean lumbal spine (LS) T-score did not differ $(-1.09 \pm 1.19$ vs $-1.18 \pm 1.18$; NS), proximal femur $(\mathrm{PF}) \mathrm{T}$-score declined $(-1.24 \pm 0.96$ vs $-1.38 \pm 1.04 ; \mathrm{p}<0.05)$. BMD decline calculated per 1 year of waiting was $-1.8 \pm 41.8 \mathrm{mg} / \mathrm{cm}^{2}$ $(-0.1 \pm 4.0 \%)$ in LS and $-10.2 \pm 28.6 \mathrm{mg} / \mathrm{cm}^{2}(-1.1 \pm 3.2 \%)$ in PF and was significantly accelerated in PF $(\mathrm{p}<0.05)$. The proportion of subjects having osteoporosis or osteopenia in either site slightly increased (osteoporosis $15.6 \%$ before and $19.5 \%$ after, osteopenia $53.2 \%$ before and $57.1 \%$ after). Significant decrease in vitamin D level was registered $(17.3 \pm 8.7$ vs. $11.6 \pm 4.7 \mu \mathrm{g} / \mathrm{l}$; $\mathrm{p}<0.001$; paired values in 45 subjects).

Conclusions: Though the proportion of subjects having pathological BMD values among patients with T1DM in terminal stages of diabetic nephropathy was high, the calculated annual BMD decline did not exceed the presumptive value in healthy subjects. Intensive correction of calcium-phosphate metabolism prevents profound bone loss in CKD 4-5 but adequate preventive measures should be introduced yet form very early stages of diabetic nephropathy.
Supported by the Ministry of Health of the Czech Republic (MZO 00023001)

\section{P298}

Development of a porcine model for bone effects of juvenile binge alcohol drinking

Peter Pietschmann $^{1}$, Ursula Föger-Samwald ${ }^{1}$, Christian Knecht ${ }^{2}$, Thomas Stimpfl ${ }^{3}$, Thomas Szekeres ${ }^{3}$, Katharina Kerschan-Schindl ${ }^{4}$, Peter Mikosch ${ }^{5}$, Wolfgang Sipos ${ }^{2}$

${ }^{1}$ Department of Pathophysiology and Allergy Research, Medical University of Vienna, Vienna, Austria, ${ }^{2}$ University Clinic for Swine, Department for Farm Animals and Veterinary Public Health, University of Veterinary Medicine Vienna, Vienna, Austria, ${ }^{3}$ Clinical Department for Medical and Chemical Laboratory Diagnostics, Medical University of Vienna, Vienna, Austria, ${ }^{4}$ Department of Physical Medicine and Rehabilitation, Medical University of Vienna, Vienna, Austria, ${ }^{5}$ Department of Medicine 2, Landesklinikum Mistelbach-Gänserndorf, Mistelbach, Austria

Background: Although chronic alcohol consumption in adults is an established risk factor of osteoporotic fractures, there is a huge gap in our knowledge about bone effects of binge drinking in adolescents. The aim of this pilot study was to develop a large animal model of skeletal effects of binge drinking in adolescents.

Methods: Piglets aged two months were offered alcohol orally as a mixture of hard liquour and apple juice. Those with the highest propensity to drink alcohol were included in the experiment and received $1.4 \mathrm{~g}$ alcohol $/ \mathrm{kg}$ body weight two times per week for two months (binge group); control piglets received apple juice in an identical manner. At the age of four months the animals were euthanized; bones harvested during autopsy were assessed by microcomputed tomography and dynamic histomorphometry. In addition, routine blood chemistry and blood alcohol determinations were performed.

Results: Blood alcohol levels assessed $1 \mathrm{~h}$ after alcohol administration were $1.08 \pm 0.18 \%$. In the binge group serum calcium $(2.1 \pm 0.7$ vs. $2.7 \pm 0.1 \mathrm{mmol} / \mathrm{l} ; \mathrm{p}<0.05)$ and phosphate $(2.4 \pm 0.2$ vs. $2.9 \pm 0.2 \mathrm{mmol} / \mathrm{l} ; \mathrm{p}<0.01$ ) levels were decreased. At the femur, trabecular number and connectivity density were lower in the binge than in the control group $(-17 \%, p<0.05$ and $-46 \%, p<0.05)$, at the humerus concerning trabecular number an opposite pattern was seen $(+39 \%, \mathrm{p}<0.05)$. At most bone sites investigated parameters of bone formation were lower in the binge than in the control group.

Conclusions: Pigs are a promising model for binge alcohol drinking during adolescence. Binge drinking has bone effects that are site specific. However, these data have to be confirmed in a larger study population.

Grant support: Austrian Science Foundation \# P28827-B30.

Protocol approval: Austrian Ministry of Health (BMWFW-68.205/ 0061-WF/V/3b/2015).

Keywords: alcohol, large animal model, pig, secondary osteoporosis

\section{P299}

Incidence and mortality of osteoporotic fracture in Rheumatoid arthritis over 50 years of age in South Korea using nationwide claims data

$\underline{\text { Deog-Yoon Kim }}^{1}$, Yong-Chan $\mathrm{Ha}^{2}$, Young-Kyun Lee ${ }^{3}$

${ }^{1}$ Department of Nuclear Medicine, Kyung Hee University, School of Medicine, Seoul, Republic of Korea, ${ }^{2}$ Department of Orthopaedic Surgery, Chung-Ang University, Seoul, Republic of Korea, ${ }^{3}$ Seoul National University Bundang Hospital, Seoul, Republic of Korea 
Purpose: The purpose of this study was to investigate incidence and mortality of osteoporotic fractures (including hip, spine, distal radius, and proximal humerus) in RA patients and compare them with those in the general population.

Methods: Data provided by NHIS were used to identify osteoporotic fractures in patients aged $>50$ years between 2010 and 2012. Patients with RA were identified by the diagnostic code for seropositive RA (V.223). Standardized mortality ratios (SMR; observed/expected deaths) of osteoporotic fractures were calculated based on age and gender-specific rates in the entire Korean population. Incidence, mortality, and SMR of osteoporotic fractures in RA patients and the general population were calculated and compared.

Results: Osteoporotic fractures in the general population and RA patients were increased by 11.6 and $17.4 \%$ over 3 years between 2010 and 2012 (195,271 and 1356 in 2010; 217,985 and 1592 in 2012, respectively). Mean age-specific incidence of osteoporotic fracture in RA patients increased from $932.1 / 100,000$ for those aged 50-59 year to $9377.1 / 100,000$ for those aged $\geq 80$ year in women and from $306.1 / 100,000$ for those aged $50-59$ year to $3700.9 / 100,000$ for those aged $\geq 80$ year in men. Cumulative mortality rate in the first year after osteoporotic fracture in patients with RA was higher than that in the general population $(7.8 \%$ in RA and $6.6 \%$ in the general population). SMR of osteoporotic fracture in RA patients was 1.4 times higher in men and 1.3 times higher in women than that for the general population.

Conclusions: This study demonstrated that incidence, 1-year mortality, and SMR of osteoporotic fracture in RA patients aged 50 years and older were higher than those in the general papulation. Therefore, antiosteoporotic management is needed for RA patients to prevent osteoporotic fracture.

\section{P300}

Iron overload in $\mathrm{Hfe}$-hemochromatosis mice does not cause osteoporosis

Maja Vujic Spasic ${ }^{1}$, Alessa Wagner ${ }^{1}$, Betül Alan ${ }^{1}$, Dilay Lai ${ }^{1}$, Naveen Tangudu $^{1}$, Mubashir Ahmad ${ }^{1}$, Jan Tuckermann ${ }^{1}$

${ }^{1}$ Institute of Comparative Molecular Endocrinology, University of Ulm, Ulm, Germany

Objectives: Clinical data and a several mouse studies have raised attention to the correlation between iron overload and the development of osteoporosis. In particularly, iron overload in a mouse model of $\mathrm{Hfe}$-hereditary hemochromatosis $(\mathrm{HH})$ has been associated with significant bone loss. Here we challenge these findings and show that under our experimental conditions, neither iron overload nor the lack of $\mathrm{Hfe}$ in bone cells affects bone status of $\mathrm{Hfe}-\mathrm{HH}$ mice.

Methods: To address the contribution of iron to bone loss, we performed an in-depth systematic analysis of major iron and bone parameters in 13, 29 and 53 weeks old males and females $\mathrm{Hfe-/}$ mice in regard to control littermates. We further used: liver-specific $H f e$-deficient mice since these mice present similar systemic iron overload as constitutive $\mathrm{He}-\mathrm{C}-\mathrm{mice}$, and a mouse model of transfusional siderosis where iron overload was induced by parenteral iron administration in wild-type mice. Finally, the consequences of the lack of $H f e$ in bone cells on iron and bone metabolism were evaluated by generating osteoblast- and osteoclasts-specific Hfe mutant mice.

Results: We show that iron overload present in constitutive $\mathrm{Hfe}-\mathrm{/}-$ and liver-specific Hfe mutant mice does not affect major bone parameters such as BV/TV, Trabecular number/separation/ thickness, bone formation rate, and the serum markers. Similarly, osteoblasts differentiation capacity was similar between control, constitutive and liver-specific Hfe mutant mice, as revealed by ALP staining. Likewise, parenteral iron injections had no effect on major bone markers. Finally, osteoblast- and osteoclast-specific Hfe-mutant mice showed normal iron and bone status.

Conclusions: Our data demonstrate that iron overload, present in $\mathrm{Hfe}$ - $\mathrm{HH}$ mouse models and in the experimentally induced transfusional siderosis, does not suffice to trigger bone loss contrasting previously published data. Our data raise critical question to the importance of iron overload on bone homeostasis in $\mathrm{Hfe}$ - $\mathrm{HH}$ mice and HFE-HH patients.

\section{P301}

Changes of periprosthetic BMD in proximal femur of patients with osteoporosis after cementless total hip arthroplasty

Yue Ding ${ }^{1}$, Guangtao $\mathrm{Fu}^{1}$, Changchuan $\mathrm{Li}^{1}$, Shixun $\mathrm{Li}^{1}$

${ }^{1}$ Orthopedics, Sun Yat-sen memorial Hospital, Sun Yat-sen University, Guangzhou, China

Introduction: The aim of the study was to investigate the effect of bisphosphonate treatment and osteoporosis on bone remodeling in proximal femur after cementless total hip arthroplasty.

Materials and Methods: 41 patients underwent unilateral cementless THA from January 2015 to May 2016 due to developmental dysplasia of the hip, femoral neck fracture and femoral head necrosis were included. Patients were divided into control group, osteopenia group and osteoporosis group on the basis of the BMD measurement in L1-L4 and femoral neck preoperative. Patients in osteoporosis group received a single dose of $5 \mathrm{mg}$ zoledronic acid with oral calcium and vitamin D administration 2 weeks post operation. Patients in the osteopenia group received only oral calcium and vitamin D. Patients in control group did not take any osteoactive drugs. After divided it into Gruen zone 1-Gruen zone 7, periprosthetic BMD were measured pre-operation, 1 week postoperation, 3 months postoperation and 1 year postoperation.

Results: 41 patients (16 male, 25 female) were included, with a mean age of 65.7 years. There were significant lower decreases of BMD percentage deviation from $3 \mathrm{~m}$ post-operation to $1 \mathrm{w}$ post-operation in Gruen zone $4(-0.14 \%, \mathrm{P}<0.05), 6(+1.42 \%, \mathrm{P}<0.01)$ and $7(+5.89 \%, \mathrm{P}<0.01)$ in osteoporosis group (treated with zoledronic acid) when compared with control group and osteopenia group. The decrease of periprosthetic BMD was retarded in normal group and there were a slight increase in osteopenia and osteoporosis group 1 year after total hip arthroplasty, but there was not significant difference between all the three groups.

Conclusions: Zoledronate therapy was effective in eliminating the decrease of periprosthetic BMD in proximal femur of osteoporosis patients after total hip arthroplasty.

\section{P302}

Proximal femoral geometry as fracture risk factor of osteoporotic hip fracture in Korean women

Myung Hoon Hahn ${ }^{1}$, Jun Han ${ }^{2}$

${ }^{1}$ Orthopedic Surgery, Cheil General Hospital and Women's Healthcare Center, Dankook University, Seoul, Republic of Korea, ${ }^{2}$ Orthopedic Surgery, Ajou University School of Medicine, Suwon, Republic of Korea

Background: Proximal femoral geometry may be a risk factor of osteoporotic hip fractures. However, there existed great differences among studies depending on race, sex and age of subjects. The purpose of the present study is to analyze proximal femoral geometry and bone mineral density (BMD) in the osteoporotic hip fracture patients. Furthermore, we investigated proximal femoral geometric parameters 
affecting fractures, and whether the geometric parameters could be an independent risk factor of fractures regardless of BMD.

Methods: This study was conducted on 197 women aged 65 years or more who were hospitalized with osteoporotic hip fracture. Control group included 551 women who visited to check osteoporosis. Femur BMD and proximal femoral geometry for all subjects were measured using dual energy X-ray absorptiometry (DXA), and compared between the control and fracture groups. Besides, proximal femoral geometric parameters associated with fractures were statistically analyzed.

Results: There were statistically significant differences in the age and weight, cross-sectional area (CSA)/length/width of the femoral neck and BMD of the proximal femur between fracture group and control group. BMD of the proximal femur in the control group was higher than in the fracture group. For the femoral neck fractures group, the odds ratio (OR) for fractures decrease in the CSA and neck length (NL) of the femur increased by 1.97 times and 1.73 times respectively, regardless of BMD. The OR for fractures increase in the femoral neck width increased by 1.53 times. In the intertrochanteric fracture group, the OR for fractures increase in the femoral neck width increased by 1.45 times regardless of BMD.

Conclusions: We found that an increase of the femoral neck width could be a proximal femoral geometric parameter which plays important roles as a risk factor for fracture independently of BMD.

Keywords: Hip fracture, Risk factors, Femoral neck width, Osteoporosis

\section{P303}

Trends of mortality after osteoporotic hip fracture in a period of 17 years

Olalla Guzón ${ }^{1}$, Ramón Mazzucchelli ${ }^{1}$, Elia Perez Fernandez ${ }^{1}$, Natalia Crespí Villarías ${ }^{2}$, Javier Quirós ${ }^{1}$, Marina Peña ${ }^{1}$, Alberto GarcíaVadillo $^{3}$

${ }^{1}$ Hospital U. Fundación Alcorcón, Madrid, Spain, ${ }^{2}$ C. S La Rivota, Alcorcón, Spain, ${ }^{3}$ Hospital Universitario La Princesa, Madrid, Spain

Background: It is known that the mortality after a hip fracture is increased with respect to the general population. However, the trend of mortality is a controversial issue.

Objective: The objective of this study is to analyze the incidence, trend and factors associated with mortality in patients with osteoporotic hip fracture.

Methods: This is a retrospective cohort study using the MBDS of our hospital that collects a minimum data set at hospital discharge. We identified patients older than 45 years who suffered an osteoporotic hip fracture during the period from 1999 to 2015. (3992 hip fractures). The identification of the deceased was obtained by consulting the MBDS and the INDEF (National Death Index facilitated by the Ministry of Health). A survival analysis was performed (regression of Cox and Kaplan-Meier). The incidence rate, standardized mortality index (SMI) was calculated with respect to the mortality of the general population of Madrid (mortality data of the general population obtained INE), trend (Poisson regression) and risk (Hazard Ratio) for the different clinical and demographic variables.

Results: The cumulative incidence of mortality was $72.69 \%$ in the study period. The crude mortality rate at 1, 3, 6 months and 1 and 3 years was $9.2,17.4,24.6,33$ and $56 \%$, respectively. In men it was $13.7,25,32.7,43.3$ and $65.6 \%$ and in women $7.9,15.7,22.3,30$, $53.2 \%$. The median overall survival was 886 days (95\% CI 836-951), with 576 for men and 998 for women. A statistically significant reduction in median survival was observed throughout the study period. The IME was 8.3 (95\% CI 7.98-8.59); (similar values in men and women).
Conclusion: We found an increase in mortality (or decrease in median survival) of patients with hip fracture during the last 17 years.

\section{P304}

12 weeks is the appropriate timing for ovariectomy to induce animal model for osteopenia in mature female C57/BL6J mice

Yue Ding ${ }^{1}$, Guangtao $\mathrm{Fu}^{1}$, Changchuan $\mathrm{Li}^{1}$, Guibin Fang ${ }^{1}$

${ }^{1}$ Orthopedics, Sun Yat-sen memorial Hospital, Sun Yat-sen University, Guangzhou, China

Introduction: The aim of the study was to investigate the influence of OVX on BMD and trabecular architecture in mature female C57/ BL6J mice, and to evaluate the appropriate timing for OVX to induce mice model of osteopenia.

Methods and Materials: 60 mature female C57/BL6J mice were randomly divided into $8 \mathrm{w}-\mathrm{OVX}$ group and $12 \mathrm{w}$-OVX group. The former underwent OVX at the age of 8 weeks while the latter was operated at the age of 12 weeks. DEXA was used for the assessment of BMD of total body, spine and lower extremity in vivo, and micro$\mathrm{CT}$ were used to measure trabecular architecture and reconstruct the 3D images of excised tibia at 0 day, 6 weeks, 8 weeks, 10 weeks and 12 weeks post-operation.

Results: There was no significant difference of BMD that measured by DEXA between all the time points in both groups. Profound improvement of the bone structure was observed at 12 weeks when comparing with 8 weeks (BV/TV: $+23.7 \%$, Tb.N: +29.4 , Tb.Sp: $-28.9 \%$, all $\mathrm{P}<0.05$ ). Changes of BV/TV, Tb.N and Tb.Sp of $8 \mathrm{w}-$ OVX group didn't reach statistical significance until 8 weeks (BV/ TV: $-46.1 \%$, Tb.N: -52.2 Tb.Sp: $+57.0 \%$, all $\mathrm{P}<0.05)$ after OVX and reached a plateau thereafter. The BV/TV, Tb.N and Tb.Sp of $12 \mathrm{w}-\mathrm{OVX}$ group changed markly after OVX and the difference first reached significance 6 weeks (BV/TV: $-26.6 \%$, Tb.N: $-37.1 \mathrm{~Tb} . \mathrm{Sp}:+59.6 \%$, all $\mathrm{P}<0.05)$ after OVX, followed by sustained decrease/increase in the next 6 weeks.

Conclusion: Micro-CT is more suitable for the evaluation of bone morphology and microarchitecture in mice models. 12 weeks, rather than 8 weeks, is the appropriate timing for OVX to induce mice model for osteopenia under the consideration of the success rate and the time it cost.

\section{P305}

Bisphosphonates use and risk of subtrochanteric and diaphyseal femur fractures in Korea: Results from the National Claim Registry

Young-Kyun Lee ${ }^{1}$, Yong-Chan $\mathrm{Ha}^{2}$, Tae-Young Kim ${ }^{3}$, Ha-Young $\mathrm{Kim}^{4}$, Deog-Yoon Kim ${ }^{5}$, Dong Won Byun ${ }^{6}$

${ }^{1}$ Seoul National University Bundang Hospital, Seongnam, Republic of Korea, ${ }^{2}$ Chung-Ang University, Seoul, Republic of Korea, ${ }^{3}$ Konkuk University, Seoul, Republic of Korea, ${ }^{4}$ Wonkwang University Sanbon Hospital, Gunpo, Republic of Korea, ${ }^{5}$ Kyung Hee University Medical Center, Seoul, Republic of Korea, ${ }^{6}$ Soonchunhyang University Hospital, Seoul, Republic of Korea

Background: Subtrochanteric and diaphyseal femur fractures after bisphosphonate use has been high-lightened. Although Asian with bisphosphonate has been considered to have higher risk of subtrochanteric and diaphyseal femur fractures, the occurrence of subtrochanteric and diaphyseal femur fractures has been still unclear in East Asian.

Methods: Using national health insurance claim database, we performed a propensity score-matched cohort study to determine the incidence rates and risk of subtrochanteric and diaphyseal femur fractures among bisphosphonate users compared with non-users. 
Results: A total of 682 subtrochanteric and diaphyseal femur fractures were observed among 348,311 bisphosphonate users. The estimated incidence rates of subtrochanteric and diaphyseal femur fractures per 100,000 person-years was 37.75 (95\% CI 35.02-40.70) among the bisphosphonate users and 24.41 (95\% CI 22.31-26.71) among non-users.

Significant association between bisphosphonate use and subtrochanteric and diaphyseal femur fractures was found (hazard ratio, $1.541 ; 95 \%$ CI $1.370-1.734)$.

Conclusion: With this large sized study, the occurrence of subtrochanteric or diaphyseal femur fracture was very rare in Korea. The raised concerns about bisphosphonate-associated subtrochanteric and diaphyseal femur fracture in Asia is too excess than the real situation, although the use of bisphosphonate might be associated with occurrence of $\mathrm{AFF}$.

\section{P306}

Impact of atrophic gastritis on bone mineral density in premenopausal women in their $40 \mathrm{~s}$

\section{A Sol Kim $^{1}$, Hae Jin $\mathrm{Ko}^{2}$}

${ }^{1}$ Department of Family Medicine, Kyungpook National University Chilgok Hospital, Daegu, Republic of Korea, ${ }^{2}$ Department of Family Medicine, Kyungpook National University School of Medicine, Daegu, Republic of Korea

Background: Atrophic gastritis can induce calcium malabsorption. Calcium malabsorption is a risk factor of osteoporosis. Thus, this study assessed the effects of atrophic gastritis on bone mineral density (BMD) in premenopausal females in their 1940s.

Methods: We performed a retrospective analysis of medical records on premenopausal female patients in their 1940s who visited hospital for health checkup and performed esophagogastroduodenoscopy and bone densitometry at least 3 times with 24-months increment between January 2006 and December 2016. Based on esophagogastroduodenoscopy results from their first visit, patients were divided into atrophic gastritis group and non-atrophic gastritis group and the changes in BMD over time were analyzed. Furthermore, patients were further divided into subgroups depending on the persistence of atrophic gastritis and differences and absolute changes in BMD were assessed.

Results: BMD between atrophic gastritis group and non-atrophic gastritis group exhibited group-by-time interaction $(\mathrm{P}<0.001)$. After adjusting for confounding factors, estimated mean BMD of the two groups were significantly different at 24-month follow-up with 1.109 and 1.101 and at 48-month follow-up with 1.103 and 1.066 $(\mathrm{P}=0.049, \mathrm{P}<0.001$ respectively). Subgroup analysis after adjusting for confounding factors showed estimated mean BMD of 1.128 and 1.104 at baseline, 1.110 and 1.100 at 24-month follow-up, and 1.106 and 1.065 at 48 -month follow-up for atrophic gastritis group and non-atrophic gastritis group respectively. The difference between mean BMD was not significant at baseline $(\mathrm{P}=0.171)$, but was significant at both 24-month and 48-month follow-up $(\mathrm{P}=0.044$ and $\mathrm{P}<0.001$ respectively). Absolute changes in BMD over 48 months was -0.010 and -0.051 for the two subgroups, which was significantly different $(\mathrm{P}<0.001)$.

Conclusion: Atrophic gastritis reduces BMD in premenopausal females in their 1940s. Patients with atrophic gastritis exhibited lower BMD than the patients without, and the patients with persistent atrophic gastritis exhibited greater decrease in BMD.

Keywords: Atrophic gastritis, Bone mineral density, Hypochlorhydria, Pre-menopause, Woman

\section{P307}

Weather conditions and their effect on seasonality of incident osteoporotic hip fracture in a Mediterranean area

Ramón Mazzucchelli ${ }^{1}$, Natalia Crespí Villarías ${ }^{2}$, Elia Perez Fernandez $^{3}$, Javier Quirós ${ }^{1}$, Olalla Guzón ${ }^{4}$, Alberto García-Vadillo ${ }^{5}$, Gil Rodriguez Caravaca ${ }^{6}$, Angel Gil de Miguel $^{6}$, Loreto Carmona ${ }^{7}$

${ }^{1}$ Rheumatology Unit, Hospital Universitario Fundación Alcorcón, Madrid, Spain, ${ }^{2}$ Centro de Salud de La Rivota, Alcorcón, Spain, ${ }^{3}$ Clinical Investigation, Hospital Universitario Fundación Alcorcón, Madrid, Spain, ${ }^{4}$ Rehabilitation Unit, Hospital Universitario Fundación Alcorcón, Madrid, Spain, ${ }^{5}$ Rheumatology Unit, Hospital Universitario La Princesa, Madrid, Spain, ${ }^{6}$ Preventive Medicine and Public Health, Universidad Rey Juan Carlos, Madrid, Spain, ${ }^{7}$ Instituto de la Salud Musculoesquelética (INMUSC), Madrid, Spain

Background: Most of the studies in which an association is established between the incidence of hip fracture (HFx) and seasonality-climatic variables, are carried out in geographical areas whose latitude is greater than $50^{\circ}$. There are few studies in Mediterranean countries.

Objective: To evaluate seasonality and the effect of weather conditions on the incidence of osteoporotic HFx in a Southern European region.

Methods: This retrospective cohort study included all patients admitted to Alcorcón (Spain) with a diagnosis of osteoporotic HFx between the years 1999-2015. In a time series analysis, we examined the association between HFx incidence and different weather conditions and seasonality using Poisson models. The incidence rate ratio (IRR) crude and adjusted by season was estimated for all parameters. HFx incidence was further analyzed by sex and age subgroups.

Results: 4.271 patients with an osteoporotic hip fracture were included. Season fracture rate were significantly higher in fall and winter (67.06 and 64.41 fractures/season) compared to summer and spring (59.71 and 60.06; $\mathrm{p}<0.001$ ). Hip fracture incidence was $15 \%$ greater in autumn and winter than in spring and summer. This seasonal pattern was observed in all subgroups, except in younger 75 years. Fog [IRR 1.15 (95\% CI 1.003-1.33)], Atmospheric pressure (per $100 \mathrm{mb}$ ) [IRR 1.05 (95\% CI 1.004-1.114)], and frost [IRR 1.15 (95\% CI 1.03-1.30)] were significantly associated with increased hip fracture. Daily average temperature (per $5{ }^{\circ} \mathrm{C}$ ) [IRR $0.98(95 \% \mathrm{CI}$ 0.957-0.996)], rain (per $10 \mathrm{ml}$ ) [IRR 0.99 (95\% CI 0.981-1.0)] and daily ultraviolet radiation (per 100 jules) [IRR 0.998 (95\% CI 0.996-1.0)] were negatively associated with fracture. After adjusting by season and trend all this associations disappear.

Conclusions: In this Southern region, hip fracture incidence exhibits a seasonal pattern different from those communicated in Northern regions. There is short-term association with different weather conditions that partly explain this seasonal pattern.

\section{P308}

Bone density and quality in long-term levothyroxine treated patients for thyroid cancer patients in premenopausal and postmenopausal state

Sonsoles Guadalix ${ }^{1}$, María Luisa De Mingo ${ }^{1}$, David Males Maldonado $^{1}$, Cristina Martín-Arriscado ${ }^{2}$, Guillermo Martinez DiazGuerra $^{1}$, Federico Hawkins ${ }^{1,3}$

${ }^{1}$ Servicio de Endocrinología, Hospital Universitario 12 de Octubre, Madrid, Spain, ${ }^{2}$ Clinical Research Institute, Hospital Universitario 12 de Octubre, Madrid, Spain, ${ }^{3}$ Instituto de Investigacion I+12, Hospital Universitario 12 de Octubre, Facultad de Medicina, Universidad Complutense de Madrid, Madrid, Spain 
The effect of suppressive doses of levothyroxine (SDLT) on bone mass of patients with Differentiated Thyroid Carcinoma (DTC) are controversial. Our aim was to analyse the effects on axial and appendicular Bone Mineral Density (BMD) and Trabecular Bone Score (TBS) in long-term SDLT suppressive therapy in a cross-sectional study to assess the potential influence of menopausal status.

Material and Method-Serum routine parameters, TSH, free thyroxine and PTH were assessed with standard procedures. BMD was measured by DXA Hologic QDR and TBS applying the iNsight2.0 software (Med-Imaps, Swiss) to the L-BMD DXA exams. Patients with postsurgical hypoparathyroidism, disease of bone metabolism, or taking drugs that interfere with bone metabolism were excluded.

Results: Three groups were evaluated: G-1: premenopausal women during the study ( $\mathrm{n}=14$, age $45.7 \pm 3.8$ years); G-2: postmenopausal women that were previous premenopausal during the study ( $\mathrm{n}=47$, age 58.5 years); G-3 postmenopausal women during the study ( $\mathrm{n}=84$, age $70 \pm 7.7$ years). Duration of SDT was similar: $10.57 \pm 6.7,14.47 \pm 5.9$ and $11.3 \pm 5.9$ years in G-1, G-2 and G-3. Densitometric osteoporosis was found in $47.6 \%$ of patients of $\mathrm{G} 3$ and $34 \%$ of G2. Incidental fractures during SDT therapy were 4, 8 and 5 in G1-3 respectively. BMD $\left(\mathrm{g} / \mathrm{cm}^{2}\right)$ values of Groups are shown in Table 1.

Table 1

\begin{tabular}{llllr}
\hline & G-1 & G-2 & G-3 & P value \\
\hline L-BMD & $0.86 \pm 0.12$ & $0.91 \pm 0.30$ & $1.00 \pm 0.11$ & 0.0005 \\
FN-BMD & $0.68 \pm 0.10$ & $0.71 \pm 0.11$ & $0.78 \pm 0.10$ & 0.0093 \\
TH-BMD & $0.84 \pm 0.13$ & $0.86 \pm 0.14$ & $0.95 \pm 0.10$ & 0.0097 \\
1/3DR-BMD & $0.59 \pm 0.06$ & $0.64 \pm 0.07$ & $0.71 \pm 0.04$ & $<0.001$ \\
TBS & $1.24 \pm 0.12$ & $1.28 \pm 0.10$ & $1.46 \pm 0.08$ & $<0.001$ \\
\hline
\end{tabular}

Significance differences: L-BMD (G-III and G-I \& G-II and G-I), FNBMD (G-III and G-I), TH (G-III and G-I \& G-II and G-I)

Conclusion: In our study postmenopausal patients with DTC with long-term SDT follow up we have not found lower trabecular and cortical BMD values.. We found a deterioration of TBS, whether the diagnosis was made before or after the menopausal status.

\section{P309}

Difference in bone markers and BMD in type (I) and (II) diabetic patients

Vaclav Vyskocil $^{1,2}$, Anna Planicova ${ }^{3}$

${ }^{1}$ Centre for Metabolic Bone Disease Department of Medicine II, Charles University Hospital, PLZEN, Czech Republic, ${ }^{2}$ Department of Orthopaedic Surgery, Charles University Hospital, PLZEN, Czech Republic, ${ }^{3}$ Centre for Metabolic Bone Disease Department of Medicine II., Charles University Hospital, PLZEN, Czech Republic

The authors observed 1887 patients with type 2 diabetes mellitus (T2DM), 1206 women and 681 men and 108 patients with type 1 diabetes mellitus (T1DM), 75 women and 33 men. 2197 non-diabetic patients with similar age were considered as a control group. Average age in control group was 69.7 years, average BMI was 29.7 in all groups. BMD, vitamin $\mathrm{D}$ and bone markers were measured in all patients.

DM2 patients had significantly lower osteocalcin level $(\mathrm{p}=0.03)$, higher CTX $(p=0.038)$, higher PINP $(p<0.01)$ than the control group. On the contrary DM1 patients had significantly higher osteocalcin $(p<0.01)$, higher CTX $(p=0.002)$, higher PINP $(\mathrm{p}<0.01)$ than the control group.

Statistically significant negative correlation was found between vitamin D levels and BMI $(\mathrm{p}<0.05)$, higher BMI was connected with lower vitamin D.

Statistically significant differences in vitamin D levels were found according to BMI category. There was no statistically significant correlation between BMI and T-score in DM1 patients. Statistically significant correlation between BMI and T-score in spine, total hip and femoral neck was found in DM2 patients. There was no statistically significant correlation between BMI and T-score in all measured areas in the control group.

1. Higher bone turnover in DM patients

2. Lower level of vit D particularly in DM2

3. Significant negative correlation between BMI and vit D level

4. No significant difference between BMI and T-score of spine and hip in DM1

5. Positive correlation between BMI and $\mathrm{T}$ score in the left hip and femoral neck in DM1 and all areas of DM2 patients

6. Higher effect of calcium and vit $\mathrm{D}$ supplementation than vit $\mathrm{D}$ only

7. There were no side differences in hip and femur neck BMD

8. Risk factors: lower vit D level and T-score, higher turnover, higher BMI, increased falling rate.

\section{P310}

Combining calcaneus QUS with OSTA in the application of osteoporosis screening

Yue Ding ${ }^{1}$, Wei Liu ${ }^{1}$, Changchuan $\mathrm{Li}^{1}$, Xumin $\mathrm{Hu}^{1}$

${ }^{1}$ Orthopedics, Sun Yat-sen Memorial Hospital, Sun Yat-sen University, Guangzhou, China

Purpose: To explore the regression equation for elder residents by combining osteoporosis self-assessment tool for Asians (OSTA) with calcaneus quantitative ultrasound (QUS), and assess the diagnostic value by calculating the area under the ROC curve (AUC).

Method: Calcaneus quantitative ultrasound of elderly residents in communities of Guangzhou were measured from FEB. 2017 to NOV. 2017, and other data, including age, height and weight, was also acquired at the same time. Only part of the residents accepted DXA scan because of some worrying about radiation. A total of 516 cases (including 413 postmenopausal women and 103 men aged $\geq 60$ years) met the inclusion criteria and exclusion criteria. The regression equations for each group were fitted by combining OSTA with calcaneus QUS, with receiver operating characteristic curve (ROC) drawed, AUC, cut-off value, standard error (SE) calculated, respectively.

Result: Regression equations of predicting osteoporosis are as follows: for postmenopausal women: $\mathrm{Y}=-1.084 * \mathrm{QUS}-0.280 *$ OSTA -2.071, AUC $=0.791$, cut-off value $=-0.543$, standard error $(\mathrm{SE})=0.022, \mathrm{P}=0.000$, for elder men (aged $\geq 60$ years): $\mathrm{Y}=-0.395^{*}$ OSTA $-0.545^{*} \mathrm{QUS}-0.956$, AUC $=0.752$, cut-off value $=-0.543$, standard error $(\mathrm{SE})=0.049, \mathrm{P}=0.001$.

Conclusion: This study is based on the previous result of our research group, and a total of 516 cases eligible (postmenopausal women \&elder men aged $\geq 60$ years in Guangzhou) are included, regression equations and AUCs are calculated for each group, meanwhile, the AUCs shows moderate diagnostic value. These regression equations together with this method, which are economic, convenient and radiation-free, could be generalized in the whole Asia, especially where lacks DXA. 


\section{P311}

Low peak bone mineral density of lumbar spine in Korean women of Korea National Health and Nutrition Examination Survey (2008-2010)

\section{Woong Choi ${ }^{1}$}

${ }^{1}$ Hanyang University Hospital, Seoul, Republic of Korea

Background: Increasing peak bone mineral density (BMD) in young people may help to reduce the incidence of osteoporosis.

Objective: We aimed to determine the age of the peak BMD and associated factors with peak BMD.

Design: We analyzed body composition and BMD data of 20-39 years old men $(\mathrm{n}=1768)$ and women $(\mathrm{n}=1959)$ from KNHANES 2008-2010 who had not liver, kidney, pulmonary, metabolic disease, cancer, pregnancy or breastfeeding. Body composition and bone mineral density (BMD) of femur neck were measured by DXA.

Results: Femur neck and spine BMD in men and femur neck BMD in women were negative correlated with age (all $\mathrm{p}<0.001)$ even after adjusting body weight, vitamin D level, exercise, smoking, and nutritional intake. Interestingly, spine BMD in women was positive correlated with age $(\mathrm{p}<0.001)$ even after adjusting. BMD of young adult also had positive association with exercise, vitamin D level, EQ$5 \mathrm{D}$, and body weight but negative correlated with recent weight change (all $\mathrm{p}<0.05$ ).

Conclusion: Korean young adult attained the peak BMD around early 20 years, but the lumbar BMD of women not. Low lumbar BMD in early 20 years old women might be associated bad life style (diets and no exercise) but further studies needed.

\section{P312}

Analysis in postmenopausal osteoporosis patients with fragility fracture during zoledronic acid treatment

Yue Ding ${ }^{1}$, Wei Liu ${ }^{1}$, Changchuan $\mathrm{Li}^{1}$, Chunhai $\mathrm{Li}^{1}$

${ }^{1}$ Orthopedics, Sun Yat-sen Memorial Hospital, Sun Yat-sen University, Guangzhou, China

Object: To analyze possible factors of fragility fracture during zoledronic acid (ZOL) treatment in postmenopausal osteoporosis patients (PMOP).

Methods: A retrospective study of 270 cases that received at least one ZOL treatment after confirmed PMOP from 2010.1.1 to 2017.1.1 in Memorial hospital of Sun Yat-Sen university were included. All patients were divided into two groups based on the presence of fragility fracture during ZOL treatment, fragility fracture group had 10 cases and the non-fragility fracture group had 260 cases. Previous fragility fracture history, age, BMI, bone mineral density (BMD), ALP, $25(\mathrm{OH})_{2}$ VitD at baseline were compared between two groups.

Results: 48 patients combined with previous fragility fracture history. 10 patients experienced fragility fracture during ZOL treatment. The proportion of the cases combined with previous fragility fracture history in fragility fracture group was significantly higher than that in the control group $\left(c^{2}=79.286, P<0.05\right)$. The baseline of BMD and T-score of the fragility fracture group was significantly lower than that of the control group $(t=-2.736, t=-2.170$, $t=-3.030, t=-2.271, P<0.05)$. While there were no significant difference of age, BMI, ALP, 25-hydroxy vitamin D at the baseline between two groups.

Conclusion: Under the condition of adequate intake of calcium, vitamin D and good compliance, The proportion of the cases combined with previous fragility fracture history in the fragility fracture group was significantly higher than that of the control group. The baseline of BMD and T-score of the fragility fracture group was significantly lower than that of the control group.

\section{P313}

Biochemical markers of osteoporosis in postmenopausal women

Raj Raghupathy ${ }^{1}$, Fawaz Azizieh $^{2}$, Olusegun Mojiminiyi ${ }^{3}$, Diaa Shehab $^{4}$, Khaled Al-Jarallah ${ }^{4}$, Renu Gupta ${ }^{5}$

${ }^{1}$ Department of Microbiology, Faculty of Medicine, Kuwait University, Kuwait, Kuwait, ${ }^{2}$ Department of Mathematics and Natural Sciences, Gulf University of Science and Technology, Kuwait, Kuwait, ${ }^{3}$ Department of Pathology, Faculty of Medicine, Kuwait University, Kuwait, Kuwait, ${ }^{4}$ Department of Medicine, Faculty of Medicine, Kuwait University, Kuwait, Kuwait, ${ }^{5}$ Department of Radiology, Faculty of Medicine, Kuwait University, Kuwait, Kuwait

Postmenopausal osteoporosis poses significant challenges to health; it is also significant research problem with much controversy related to factors contributing to its etiopathogenesis and diagnostic markers. This study was aimed at examining the possible relationships between selected markers and bone density in postmenopausal women with normal or low bone mineral density (BMD). We measured circulatory levels of serum procollagen type I N propeptide (P1NP), C-terminal cross-linking telopeptide of type I collagen (CTX), estradiol, Vitamin D, Vitamin D Binding Protein (VDBP) and routine bone profile parameters.

This study included 93 post-menopausal women, of whom 46 had normal BMD, 32 had osteopenia and 15 had osteoporosis. Serum P1NP and plasma CTX were measured by Roche assays; estradiol, Vitamin D, VDBP, corrected calcium, magnesium, phosphorus and alkaline phosphatase were measured by automated chemistry analyzers.

Postmenopausal women with normal BMD had significantly higher serum P1NP levels compared to subjects with low BMD $(p=0.03)$, osteopenia $(p=0.05)$ and osteoporosis $(p=0.04)$. While CTX levels were not different between groups, women with normal BMD had significantly higher P1NP/CTX ratios as compared to women with low BMD ( $\mathrm{p}=0.04)$. Women with normal BMD had significantly higher estradiol levels compared to women with low BMD $(\mathrm{p}=0.001), \quad$ osteopenia $(\mathrm{p}=0.001)$ and osteoporosis $(p=0.0001)$; osteopenic subjects had significantly higher levels of estradiol compared to osteoporotic women. All three groups had comparable levels of vitamin D and VDBP, but mean concentrations of magnesium were significantly lower in osteoporotic women compared to osteopenic subjects $(\mathrm{p}=0.01)$.

These data support the potential use of bone turnover markers in the management of postmenopausal osteoporosis. It would of great benefit to develop a multivariate panel of bone turnover markers that will help assess bone health, bone quality and bone remodeling balance and also assess the risk of fractures in postmenopausal osteoporosis.

Keywords: Postmenopausal osteoporosis; Bone turnover markers; Bone mineral density

\section{P314}

Positive effects of intermittent PTH on growing bone and dystrophic muscle in Mdx mouse model of Duchenne Muscular Dystrophy

$\underline{\text { Sung-Hee (Seanna) Yoon }}{ }^{1,2}$, Marc Grynpas ${ }^{2}$, Jane Mitchell ${ }^{1}$

${ }^{1}$ Pharmacology and Toxicology, University of Toronto, Toronto, Canada, ${ }^{2}$ Lunenfeld-Tanenbaum Research Institute, Toronto, Canada

Duchenne Muscular Dystrophy (DMD) is a progressive muscle disorder caused by genetic mutations on the dystrophin-encoding 
gene. Without functional dystrophin, children with DMD have progressive muscle wasting and inflammation, and compromised bone health with high risk of fracture. The use of high dose glucocorticoids as the standard of care also contributes to bone fragility. Our previous study showed that the prophylactic use of anti-resorptive bisphosphonates improved bone mineral density (BMD) but did not improve mechanical properties of bone in the Mdx mouse model of DMD and also dramatically decreased bone turnover. Thus, this study examined the effects of daily administered parathyroid hormone (PTH), the only available bone anabolic therapy, on growing bone and dystrophic muscle in the presence of continuous prednisone treatment in Mdx mice. Five-week prednisone treated $\mathrm{Mdx}$ mice showed decreased cortical bone thickness and area $(\mathrm{p}<0.001)$, which was significantly improved by PTH treatment $(\mathrm{p}<0.001)$. This bone increase by intermittent PTH was through suppression of osteoclasts on endocortical surfaces $(\mathrm{p}<0.001)$. While there was no significant effect of muscular dystrophy on cortical bone, Mdx mice showed significantly less volumetric BMD and bone mass of trabecular compartments in lumbar vertebrae. Prednisone or intermittent PTH by themselves did not have significant effect, but the combination of two treatments resulted in significant increase in volumetric BMD $(p<0.001)$ and percent bone volume $(\mathrm{p}<0.01)$. Moreover, prednisone treatment resulted in significantly improved grip strength and endurance in treadmill running, which were maintained and further improved, respectively, in co-treated Mdx mice. All together, our study demonstrated that intermittent PTH significantly improved glucocorticoid-induced bone loss and maintained or further enhanced dystrophic muscle function that is already improved by prednisone treatment. These findings could give insight into the use of teriparatide in patients with DMD, as well as other forms of glucocorticoid-induced pediatric osteoporosis.

\section{P315}

A novel triphasic calcium-based implant increases bone formation in ovariectomized rat femoral metaphyseal defects

Jonathan Shaul ${ }^{1}$, Peggy Lalor ${ }^{2}$, Shane Woods ${ }^{3}$, Scott Bruder ${ }^{4}$, Ronald Hill $^{1}$

${ }^{1}$ Research and Development, AgNovos Healthcare, Rockville, United States, ${ }^{2}$ Histion, Everett, United States, ${ }^{3}$ MPI Research, Mattawan, United States, ${ }^{4}$ Bruder Consulting and Venture Group, Franklin Lakes, United States

Objective: To evaluate implant resorption and bone formation facilitated by a novel calcium-based implant material (AGN1) in ovariectomized (OVX) rat metaphyseal defects.

Methods: Under IACUC-approved protocols, OVX was performed in 12-week old SD rats, followed by a 12-week bone loss induction period. Bilateral 2.5 by $4.0 \mathrm{~mm}$ metaphyseal defects were drilled in distal femurs at 24 weeks (T0). One was implanted with AGN1, the other left empty. Retrievals were at T0, 6 and $12(\mathrm{~N}=6 /$ group $)$ and 18 weeks $(n=8)$. Histology, histomorphometry and $\mu \mathrm{CT}$ scans were performed. $\mathrm{p}<0.05$ was considered significant.

Results: AGN1 rapidly resorbed with $2.4 \pm 1.9 \%$ remaining at 6 weeks and $1.3 \pm 1.0 \%$ at 18 weeks. Histology and $\mu \mathrm{CT}$ revealed residual AGN1 was present as discrete particles where new trabecular bone formed. More trabecular bone filled AGN1 compared to empty defects. At 18 weeks, trabecular architecture in AGN1 compared to empty defects demonstrated increased trabecular number and connectivity, and decreased trabecular spacing.
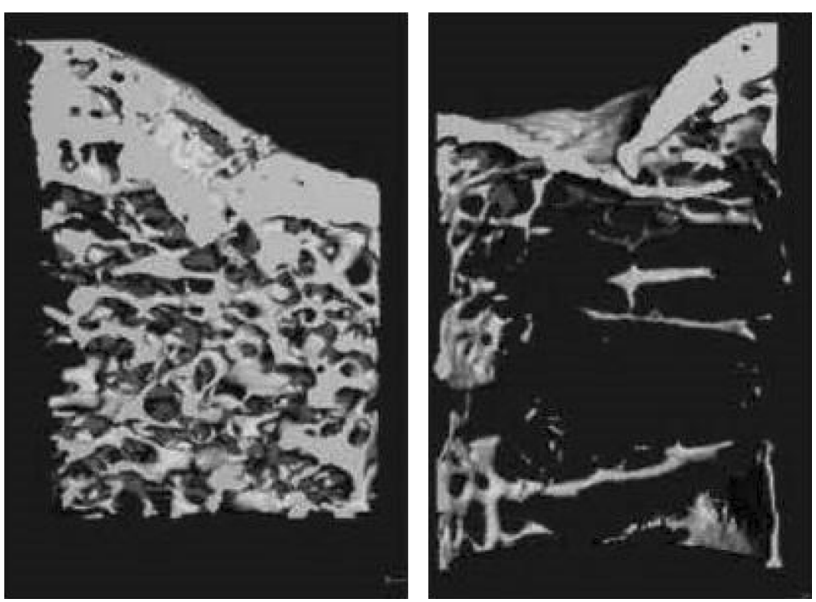

Figure AGN1 defects filled with trabecular bone (left) compared to empty defects (right)

Bone volume

\begin{tabular}{lll}
\hline Weeks & Empty $(\%$ BV/TV $)$ & AGN1 $(\% \mathrm{BV} / \mathrm{TV})$ \\
\hline 6 & $16.0 \pm 3.2$ & $28.4 \pm 5.1^{*}$ \\
12 & $21.6 \pm 12.3$ & $27.2 \pm 7.8$ \\
18 & $18.0 \pm 4.9$ & $24.6 \pm 5.3^{*}$ \\
\hline
\end{tabular}

Summary and Conclusions: A unique triphasic calcium-based implant (AGN1) was resorbed and replaced with new bone in metaphyseal defects in OVX rats. New bone architecture was consistent with normal trabecular bone structure, and suggests it may benefit patients suffering osteoporotic bone loss.

Keywords: Bone, Histomorphometry, Preclinical, Rat, $\mu \mathrm{CT}$

\section{P316}

Bone Union of spinal fusion surgery using local bone in long term bisphosphonates users

\section{$\underline{\text { SY Park }}^{1}$, TW Kang ${ }^{2}$, SW Suh ${ }^{2}$}

${ }^{1}$ Orthopaedicsurgery, Korea University, Seoul, Republic of Korea, ${ }^{2}$ Korea University, Seoul, Republic of Korea

Introduction: Bisphosphonates are the most popular drugs for treatment of postmenopausal osteoporosis. However, long-term use of bisphosphonates (BPs) may cause several complications such as atypical fracture of hip, osteonecrosis of jaw and delayed fracture healing. PLIF is a typical surgical technique for treatment of degenerative spinal disorders. Long-term use of BPs may also inhibit spinal fusion process after PLIF. We compared bony union rates of longterm BPs users and non-users after undergoing spinal fusion surgery.

Methods: The subject were 95 postmenopausal women whose course could be observed for at least 2 years after surgery. Single interbody PLIF was done using local bone graft from laminectomy. Participants were divided into two groups; there were 62 patients in long-term BPs users group and 33 in non-users group. Serum C-terminal cross linking telopeptide levels were checked for bone resorption marker which could be extremely decreased in long-term BPs users. Bone fusion rates were calculated, 2 years after the surgery 
using plain radiographs and computed tomographic scans. Clinical outcomes were measured using ODI and VAS.

Results: Serum CTX level was extremely decreased in long-term BPs user group $(\mathrm{p}<0.05)$. However, fusion rates turned out to be $82 \%$ in long-term BPs users group and $87 \%$ in non-users group ( $p>0.05$, not significant). There was no significant difference between two groups in ODI and VAS.

Discussion: At the 2-year postoperative follow-up, there was no significant difference in bone fusion rate between two groups. Longterm BPs users showed fusion rates greater than $80 \%$ and clinical outcome improvement that were compatible to those in non-users. No significant effect was found after long-term BPs use on fusion rate of PLIF

Keywords: fusion, bisphosphonates, spine, bone union, long term user

\section{P317}

Biodegradable screw containing bone morphogenetic protein-2 in an osteoporotic rat modell

Je Hoon Jeong ${ }^{1}$, Eun-Sun Jin ${ }^{2}$, Ji Yeon $\mathrm{Kim}^{3}$, Bora Lee ${ }^{4}$, Joong Kee Min $^{3}$, Sang Ryong Jeon ${ }^{5}$, Kyoung Hyo Choi ${ }^{6}$

${ }^{I}$ Department of Neurosurgery, Soon Chun Hyang Unviersity Bucheon Hospital, Bucheon-si, Republic of Korea, ${ }^{2}$ Department of Internal Medicine, College of Medicine, Kyung Hee University, Seoul, Republic of Korea, ${ }^{3}$ Laboratory of Stem Cell Therapy, College of Medicine, Asan Medical Center, University of Ulsan, Seoul, Republic of Korea, ${ }^{4}$ Dep. of Biostatistic Consulting, Soon Chun Hyang Medical Center, Bucehon-si, Kosovo, Republic of, ${ }^{5}$ Department of Neurological Surgery, College of Medicine, Asan Medical Center, University of Ulsan, Seoul, Republic of Korea, ${ }^{6}$ Department of Rehabilitation Medicine, College of Medicine, Asan Medical Center, University of Ulsan, Seoul, Republic of Korea

Introduction: The aim of this study was to evaluate the effect for biodegradable screws containing bone morphogenetic protein-2 (BMP-2) in an osteoporotic rat model.

Methods: Twenty-four female Wistar rat (250-300 g, 12 weeks of age) were randomized into four groups. Three groups underwent bilateral ovariectomy (OVX). Biodegradable screws with or without BMP-2 were inserted in the proximal tibia in two implantation groups. The extracted proximal metaphysis of the tibiae were scanned by exo-vivo micro-computed tomography. Evaluated parameters included bone mineral density (BMD), trabecular bone volume (BV), trabecular number (Tb.N), trabecular thickness (Tb.Th) and trabecular separation (Tb.Sp). The tibia samples were pathologically evaluated by staining with by Hematoxylin and Eosin, and trichrome.

Results: Trabecular formation near screw insertion site was evident only in rats receiving BMP-2 screws. BMD and BV/TV significantly differed between controls and the OVX and OVX with screw groups. However, there were no significant differences between control and OVX with screw BMP groups. Tb.Sp significantly differed between control and OVX and OVX with screw groups $(\mathrm{p}<0.05)$, and between the OVX and OVX with screw BMP group $(\mathrm{p}<0.05)$, with no statistically significant difference between control and OVX with screw BMP groups. Over the 12 weeks after surgery, bone lamellae in direct contact with the screw developed more extensive and thicker trabecular bone around the implant in the OVX with screw BMP group compared to the OVX with screw group.

Conclusion: Biodegradable screws containing BMP-2 improve nearby bone conditions and enhance ostoeintegration between the implant and the osteoporotic bone.

\section{P318}

Biological therapy or bisphosphonates in postmenopausal women with osteoporosis?

\section{Corina Galesanu ${ }^{1}$}

${ }^{1}$ University of Medicine and Pharmacy 'Grigore T. Popa', Iasi, Romania

Objective: The molecular mechanisms of bone formation and resorption led to promising therapeutic targets for osteoporosis. In the novel biological drugs, denosumab, has been clinically applied by positive effect on bone mineral density, preventive effect on fragility fractures and safety. Bisphosphonates inhibit bone resorption with relatively few side effects. We have proposed to compare the effect on the change of BMD during treatment with two powerful, parenteral antiresorptive therapies: Denosumab (DMAb) and Zoledronic Acid (ZOL).

Material and Methods: A randomized study, open-label in which 62 postmenopausal women with osteoporosis were randomized $1: 1$ to DMAb $60 \mathrm{mg}$ subcutaneously every 6 months or ZOL $5 \mathrm{mg}$ IV once yearly for 2 years. Thirty-two osteoporotic women received DMAb and thirty received ZOL. All patients have received supplement of $1000 \mathrm{mg}$ calcium and $1 \mathrm{mcg}$ alfacalcidol/day. BMD was measured by DXA at baseline and to 1- and 2-years. The diagnosis of osteoporosis was confirmed using BMD-WHO criteria. We analyzed also: changes in serum calcium, 25-OHD, PTH and bone alkaline phosphatase $(\mathrm{PAB})$ at baseline and at 1- and 2-years of treatment.

Results: At baseline mean BMD at LS was $0.727 \mathrm{~g} / \mathrm{cm}^{2}$ and increased at $0.811 \mathrm{~g} / \mathrm{cm}^{2}$ after 2 years, at women treated with DMAb, a total of $+5.4 \%$. For the women treated with $\mathrm{ZOL}$ the baseline was $0.673 \mathrm{~g} / \mathrm{cm}^{2}$ and increased at $0.689 \mathrm{~g} / \mathrm{cm}^{2}$ a total of $+2.4 \%$. At TH the increase of BMD was $+4.0 \%$ for DMAb and $1.8 \%$ for ZOL after 2 years. For FN the change in BMD was $+2.5 \%$ for DNAb and $+0.9 \%$ for ZOL. No adverse events or new fractures under the therapy. No significant change in serum calcium, 25-OHD3, PTH.

Conclusion: DMAb treatment increased more BMD at LS, TH and FN by comparing with ZOL in patients with postmenopausal osteoporosis. Both drugs were effective at 2 years of treatment.

\section{P319}

Effect of medications on secondary prevention of Osteoporotic Vertebral Compression Fracture: a meta-analysis of randomized controlled trials

Yuan-Zhe Jin $^{1}$, Jae Hyup Lee ${ }^{1}$

${ }^{1}$ Orthopedic Surgery, Seoul National University College of Medicine, Seoul, Republic of Korea

Osteoporotic vertebral compression fracture (OVCF) increases the risk of new fractures and deaths. Various medications have been prescribed to treat osteoporosis, but few study summarized their secondary prevention effects. Therefore, we investigated their effects on preventing subsequent OVCF via meta-analyses of randomized controlled trials. Electronic databases, including MEDLINE, EMBASE, CENTRAL, and Web of Science were searched for published randomized controlled trials until December 2017. The trials that recruited participants with at least one OVCF were included. We assessed the risk of bias of every study, estimated relative risk ratio of secondary OVCF, non-vertebral fracture, gastrointestinal complaints and discontinuation due to adverse events. Finally, we evaluated the quality of evidence.

Thirty-nine articles were included. Moderate to high quality evidence proved the effectiveness of alendronate (Relative Risk, RR: 0.54 ), risedronate (RR: 0.52), ibandronate (RR: 0.52), parathyroid hormone (RR: 0.30), and selective estrogen receptor modulators 
(Raloxifene, RR: 0.58; Bazedoxifene, RR: 0.66) in preventing secondary fractures. Moderate quality evidence proved romosozumab had better effect than alendronate (Romosozumab vs. alendronate, RR: 0.64) and high quality evidence proved that teriparatide had better effect than risedronate (risedronate vs. teriparatide, RR: 1.67).

High to moderate quality evidence proved zoledronic acid, alendronate, risedronate, etidronate, ibandronate, $\mathrm{PTH}$, denosumab and SERMs have significant effect on preventing secondary OVCF. Among them, zoledronic acid, risedronate and PTH also had significant effects on preventing non-vertebra fracture. Moderate quality evidence proved romosozumab had better effect than alendronate. High quality evidence proved that PTH had superior effect to risedronate, but that medication should be prescribed with caution because of its significantly higher risk of adverse events.

\section{P320}

Tromsoporosis: secondary fracture prevention program resulted in improved adherence to anti-osteoporotic drugs in Troms $\varnothing$, Norway Camilla Andreasen ${ }^{1,2}$, Veronica G. Rogli ${ }^{2}$, Jan Elvenes ${ }^{1,2}$, Ragnar M. Joakimsen $^{1,2}$, Tove T. Borgen ${ }^{3}$, Lene B. Solberg ${ }^{4}$, Frede Frihagen ${ }^{4,5}$, Lars Nordsletten $^{4,5}$, Erik F. Eriksen ${ }^{4,5}$, Trude Basso 6 , Cecilie Dahl ${ }^{5}$, Tone K. Omsland ${ }^{5}$, Åshild Bjørnerem ${ }^{1,2}$

${ }^{1}$ University Hospital of North-Norway, Troms $\phi$, Norway, ${ }^{2}$ The Arctic University of Norway, Troms $\phi$, Norway, ${ }^{3}$ Vestre Viken Hospital Trust, Hospital of Drammen, Drammen, Norway, ${ }^{4}$ Oslo University Hospital, Oslo, Norway, ${ }^{5}$ University of Oslo, Oslo, Norway, ${ }^{6}$ St. Olav University Hospital, Trondheim, Norway

Objective: Secondary fracture prevention is important, but often suboptimal. Adherence to anti-osteoporosis drugs (AOD) is crucial to achieve proper prevention of new fractures. We evaluated the adherence to AOD in fracture patients attending a secondary fracture prevention program.

Materials and Methods: Tromsoporosis is a quality assurance study, and part of Norwegian Capture the Fracture ${ }^{\circledR}$ Initiative (NoFRACT) at the Department of Orthopedic Surgery, University Hospital of North Norway. Patients $>50$ years with fragility fracture are offered evaluation and treatment. According to the NoFRACT protocol, AOD is recommended in patients with (i) hip fracture, vertebral fracture or $\geq 2$ low-energy fractures or (ii) one low-energy fracture and FRAX 10 year probability of major osteoporotic fracture $\geq 20 \%$ or BMD T-score $<-1.5$. Self-reported adherence was assessed by phone at 3 and 12 months. We present results on those who completed follow-up from October 2015 through August 2017.

Results: Of 260 patients: 195 were females, aged $69 \pm 11$ years (mean \pm SD). Of those 260 patients, 195 fulfilled the criteria for treatment with AOD, only 16 were already on treatment, 180 started taking AOD (128 alendronate, 6 zoledronic acid, 32 denosumab and 11 calcium and vitamin D only). Of 180 patients, 10 and 47 were lost to follow-up, while $116(64 \%)$ and $97(54 \%)$ patients reported adherence to AOD after 3 and 12 months, respectively. In fact, 15 (28\%) patients whom were non-adherent at 3 months were restarted on AOD and were adherent at 12 months follow-up. There was no significant difference in age, sex, fracture types, FRAX estimate or BMD T-score between those who were adherent vs. non-adherent to AOD at 12 months.

Conclusion: Of those recommended AOD, $90 \%$ were treatmentnaive at the time of fracture, the close follow-up resulted in high adherence rates, which seems promising for future fracture prevention in NOFRACT.

Keywords: Adherence

\section{P321}

Vitamin D status and refill compliance as determinants of BMD response to Oral Bisphosphonates in a real world setting

Martin T Ernst ${ }^{1,2}$, Katrine H Rubin ${ }^{1,2}$, Daniel Prieto-Alhambra ${ }^{3,4}$, Anne Pernille Hermann ${ }^{5}$, Bo Abrahamsen ${ }^{1,6}$

${ }^{1}$ Odense Patient Data Explorative Network, University of Southern Denmark, Odense, Denmark, ${ }^{2}$ Odense University Hospital, Odense, Denmark, ${ }^{3}$ NDORMS, University of Oxford, Oxford, United Kingdom, ${ }^{4}$ GREMPAL Research Group, Universitat Autonoma de Barcelona, Barcelona, Spain, ${ }^{5}$ Department of Endocrinology, Odense University Hospital, Odense, Denmark, ${ }^{6}$ Medicine, Holbak Hospital, Holbak, Denmark

Background: Most RCTs with oral bisphosphonates (oBPs) were conducted with (near) mandatory coadministration of calcium and vitamin $\mathrm{D}$ as is consistent with clinical practice. It is unclear if vitamin D deficiency is associated with a poorer BMD outcomes. Reid (JIM 2017) reported greater vitamin D effects on BMD with low baseline status.

Methods and study population: We identified 7533 treatment naïve oBP users with baseline BMD. The lab database for all hospitals and GP surgeries in the region was queried for $\mathrm{D}$ status in the 12 months before treatment $(\mathrm{N}=4359)$. Change in total hip BMD over 2 years was used as the outcome with adherence (MPR $>80$ in the National Prescription Register), gender and baseline D as the predictors.

Results: The increment in BMD was significantly associated with MPR $>80(+0.9,0.7-1.1 \% \mathrm{p}<0.001)$, and negatively with male gender $(-0.3 \%,-0.5$ to $-0.1 \mathrm{p}=0.015)$ and higher vitamin $\mathrm{D}$ level $(-0.003 \%$ per $n m o l / 1, p=0.018)$. Only a small proportion of patients with low values had a follow-up vitamin D measurement in the first 6 months of treatment. Adjusting for follow-up serum level did not add significantly to the prediction $(\mathrm{p}=0.10)$.

Limitations: Adherence to vitamin supplementation may be higher in persons who adhere closely to oBP.

\% Change/year in total hip BMD, mean and $95 \% \mathrm{CI}$

\begin{tabular}{|c|c|c|c|c|}
\hline $\begin{array}{l}\text { Baseline } \\
\text { serum } \\
25 \mathrm{OHD}\end{array}$ & $\mathrm{N}$ & $\begin{array}{l}\text { All } \quad \text { BP } \\
N=1961\end{array}$ & $\mathrm{~N}$ & $\begin{array}{l}\text { MPR } \geq 80 \% \\
N=1080\end{array}$ \\
\hline$\geq 50 \mathrm{nmol} / \mathrm{l}$ & 1650 & $0.90(0.80-1.00)$ & 921 & $1.26(1.14-1.38)$ \\
\hline $\begin{array}{c}25-49 \mathrm{nmol} / \\
1\end{array}$ & 239 & $1.19(0.91-1.46)$ & 120 & $1.78(1.42-2.14)$ \\
\hline$<25 \mathrm{nmol} / \mathrm{l}$ & 72 & $1.16(0.38-1.93)$ & 39 & $1.75(0.82-2.68)$ \\
\hline P-value & & 0.10 & & 0.01 \\
\hline
\end{tabular}

Discussion: Current treatment practices of $\mathrm{CaD}$ coadministration with oBPs appear sufficient to offset any detrimental effects of vitamin D deficiency in the first years of oBP treatment. Indeed the highest BMD increases were seen in patients with lower baseline values, consistent with what has been reported with vitamin D supplementation outside the context of oBP therapy.

\section{P322}

Teriparatide is superior to standard care in severe spinal osteoporosis: observational study in routine clinical practice

$\underline{\text { Philip Riches }}^{1}$, Ailsa Oswald ${ }^{1}$, Kathryn Berg ${ }^{1}$, Stuart Ralston ${ }^{1}$

${ }^{1}$ University of Edinburgh, Edinburgh, United Kingdom 
We have offered teriparatide (TPTD) to women with severe osteoporosis of the spine (T-score $<-4.0$ ) since 2005. We audited the outcome of 494 such patients in comparison with 230 eligible patients who received standard care (SC) with alendronic acid $(\mathrm{n}=159)$, zoledronic acid $(\mathrm{n}=30)$ risedronate $(\mathrm{n}=17)$ or other therapies $(n=24)$. The reasons that these treatments were given instead of TPTD included being unwilling or unable to self-inject $(n=120)$; being established on alternative treatment $(n=71)$; contraindication $(n=29)$ and other reasons $(n=10)$. The results are summarised in the table. Patients treated with TPTD were younger, had higher hip BMD and a lower 10-year fracture risk than those given standard care. The response of spine BMD was better with TPTD, but there was no difference at the hip. New vertebral fractures were significantly less common with TPTD (4.9 vs $10.0 \%)$, odds ratio 0.46 (95\% CI $0.25-0.83, \mathrm{p}=0.01$ ), and this remained significant even after adjusting for baseline characteristics by logistic regression (OR $0.49,0.26-0.89, p=0.02$ ). Non-vertebral fractures did not differ between the groups. This real-life study suggests that TPTD is the preferred treatment for patients with severe spinal osteoporosis.

Table 1 Characteristics of study population

\begin{tabular}{lllr}
\hline & $\begin{array}{l}\text { TPTD } \\
(\mathrm{n}=494)\end{array}$ & SC $(\mathrm{n}=230)$ & \multicolumn{1}{l}{$\begin{array}{l}\mathrm{p}- \\
\text { Value }\end{array}$} \\
\hline Age & $69.16 \pm 8.62$ & $73.97 \pm 9.22$ & $<0.01$ \\
10 year fracture risk & $25.5 \pm 12.5$ & $28.5 \pm 13.0$ & $<0.01$ \\
Lumbar spine T score & $-4.36 \pm 0.69$ & $-4.42 \pm 0.59$ & 0.29 \\
$\begin{array}{l}\text { Total hip T score } \\
\text { \% Change spine BMD after }\end{array}$ & $-2.72 \pm 0.85-3.12 \pm 0.89$ & $<0.01$ \\
$\quad \begin{array}{l}\text { 2 years } \\
\text { \% Change total hip BMD }\end{array}$ & $1.33 \pm 7.8 \%$ & $2.65 \pm 5.7 \%$ & 0.18 \\
$\quad$ after 2 years & & & \\
$\begin{array}{l}\text { New vertebral fractures } \\
\text { New non-vertebral }\end{array}$ & $24(4.9 \%)$ & $23(10.0 \%)$ & 0.01 \\
$\quad 101(20.4 \%)$ & $51(21.2 \%)$ & 0.59 \\
\hline
\end{tabular}

Keywords: Teriparatide, Severe osteoporosis, Vertebral fracture

\section{P323}

Periodontal infection affects the severity of the medication-related osteonecrosis of the jaw (MRONJ)

Jeong Keun Lee ${ }^{1,2}$

${ }^{1}$ Oral and Maxillofacial Surgery, Ajou University School of Medicine, Suwon, Republic of Korea, ${ }^{2}$ Oral and Maxillofacial Surgery, Ajou University Dental Hospital, Suwon, Republic of Korea

Periodontal infection is one of the most prevalent oral diseases. MRONJ is not frequent but once occurred, is one of the most tenacious diseases that severely affects the victims' quality of life. Although it is well known that periodontal disease is one of risk factors of the MRONJ, it is not clear if periodontal disease affects the severity of the MRONJ. Purpose of the study was to investigate the relationship of periodontal infection to the severity of the MRONJ.

Of all patients diagnosed as MRONJ since 2006 in our department, total number of the study object was 86 excluding one patient whose radiograph was unavailable and 6 patients who were totally edentulous. MRONJ stage was evaluated with usual manners recommended in AAOMS position paper and the patients were classified into 4 groups. Periodontal infection was evaluated by 2 indices, alveolar bone score and residual tooth rate, in each of the 4 groups. All the patients were included in this study according to recommendations of the institutional review board in our hospital. Statistical analysis was done by Kruskal-Willis test and $t$ test, according to classification criteria.

The number of patients in each groups were 10, 20, 47, and 9 in stage 0 , stage 1 , stage 2 , and stage 3 , respectively. Kruskal-Wallis test yielded statistical difference only in alveolar bone score between stage 1 and 2 groups in post-hoc test $(p=0.009)$. Those 4 groups were rearranged according to the criteria of infection to yield infection-free (stage 0 and 1) group and infection-related (stage 2 and 3) group. The result of $t$ test for mean comparison of these 2 groups in alveolar bone score was also statistically meaningful $(p=0.004)$. On the basis of these results we concluded that periodontal infection is related to stage of MRONJ and affects the severity of the MRONJ.

\section{P324}

A new approach for reversing osteoporosis bone loss with a conjugated drug (C3) in an Ovariectomized (OVX) rat model Marc Grynpas ${ }^{1}$, Zeeshan Sheikh ${ }^{2}$, Gang Chen ${ }^{3}$, Robert Young ${ }^{3}$, Michael Glogauer ${ }^{2}$

${ }^{1}$ Mount Sinai Hospital, Toronto, Canada, ${ }^{2}$ University of Toronto, Toronto, Canada, ${ }^{3}$ Simon Fraser University, Burnaby, Canada

Introduction: There is an unmet need to find new anabolic drugs to rebuild bone lost to osteoporosis. By linking a $\mathrm{PGE}_{2}$ receptor analog (EP4a) to a bisphosphonate (C1) we have shown that bone loss can be recovered. However this conjugated drug did not allow for bone remodeling. In this study we adopted a conjugate bone-targeting approach where a synthetic, stable EP4 agonist is covalently linked to an inactive alendronate (ALN) that still bind to bone and allow bone remodeling.

Methods: In this curative experiment, the following 7 groups were treated for 8 weeks (after losing bone for 12 weeks). (1) Sham, (2) OVX, (3) OVX + C3 low and high (4), 5) OVX + C1, 6) inactive ALN alone and 7) mixture of unconjugated ALN and EP4a (to assess the effect of conjugation). We use microCT to determine bone architecture; static and dynamic histomorphometry to determine bone turnover and biomechanics to measure bone mechanical properties.

Results: This experiment demonstrated that $\mathrm{C} 3$ treatment significantly increased vertebral bone mineral density and trabecular bone volume vs. OVX controls. Biomechanical testing showed that $\mathrm{C} 3$ treatment led to significant improvement in the load bearing abilities of the vertebrae compared to OVX controls; C3 stimulated bone formation and increased load bearing in femurs. We have shown that C3 led to significant anabolic effects on trabecular bone while anabolic effects associated with $\mathrm{C} 1$ were beyond physiological levels. Conjugation between the EP4a and ALN components was crucial to the drug's anabolic efficacy.

Conclusion: The $\mathrm{C} 3$ conjugate demonstrate, for the first time, that a combined therapy using an anabolic agent linked to an inactive ALN has significant anabolic effects, yet allow bone remodelling.

Keywords: Conjugated drug, osteoporosis, OVX rat model

\section{P325}

Why are patients with a recent fracture not attending the Fracture Liaison Service? A questionnaire-based study

Peter van den Berg ${ }^{1}$, Paul van Haard ${ }^{2}$, Piet Geusens ${ }^{3,4}$, Joop van den Bergh $^{3,5}$, Dave Schweitzer ${ }^{6}$

${ }^{I}$ FLS - orthopaedics, Reinier de Graaf Gasthuis, Delft, Netherlands,

${ }^{2}$ Medical Laboratories, Association of Clinical Chemistry, Reinier the 
Graaf Gasthuis, Delft, Netherlands, ${ }^{3}$ Subdivision Rheumatology, Maastricht University Medical Center, Maastricht, Netherlands, ${ }^{4}$ Hasselt University, Hasselt, Belgium, ${ }^{5}$ Internal Medicine, VieCuri Medical Center Noord-Limburg, Venlo, Netherlands, ${ }^{6}$ Internal Medicine and Endocrinology, Reinier de Graaf Gasthuis, Delft, Netherlands

Introduction: Fracture Liaison Service (FLS) is proposed as the most effective strategy for subsequent fracture prevention. The major shortcoming in FLS practice is a low response rate to attend the FLS. This study evaluated attendance gap reasons.

Patients and Methods: We analyzed patient registration, death, exclusion criteria and the results of a questionnaire examining reasons for not attending, based on demographics (age, gender and frailty), perception on aspects of bone strength (intrinsic factors) and effects of doctors ' and nurses " advices to attend the FLS (extrinsic factors).

Results: Out of 2006 consecutive fracture patients, 740 did not receive an invitation due to wrong fracture registration codes $(\mathrm{n}=278)$ and death $(\mathrm{n}=130)$ and excluded patients with chronic conditions already treated by other specialists with experience in bone diseases $(n=131)$, and with DXA prior to fracture $(n=179)$. Of the 1288 invited patients, [841 (64\%) attended the FLS], 971 were women (76\%) and 377 men (24\%). 745 (59\%) responded adequately. Of the FLS attendees, 537 (64\%) and of the non-attendees, 208 (47\%) responded.

Bivariate analysis concerning non-attendance was related to age (OR: $1.9>70$ year vs $<70$ year-old), gender (OR: 1.5 for man vs. women) and frailty (OR: Groningen Frailty Indicator: 1.8; Tilburg Frailty Indicator: 2.1) and absence of informing the patient (OR: 5.6). In multivariate logistic regression gender (OR: 1.7 for men), frailty (OR: 2.1 for the Tilburg Frailty Indicator) and adequate patient-information remained significant.

Conclusions: Our study shows that only $42 \%$ of all $50+$ patients with a recent fracture attended this FLS. Reasons were registration errors (14\%), death (7\%), exclusion (15\%) and $22 \%$ did neither attend nor respond to the invitation.

In multivariate analysis predictive variables for non-attendance at the FLS were men, frailty and lack of patient-information and motivation.

Keywords: Attendance; FLS; Prediction

\section{P326}

LC-MS/MS measurement of parathyroid hormone PTH (1-34): Use in studying oral PTH (1-34) administration and possible diagnostic application in pseudohypoparathyroidism

Sulaiman Al-Riyami ${ }^{1}$, Jonathan Tang ${ }^{1}$, Hillel Galitzer ${ }^{2}$, William Fraser $^{1}$

${ }^{1}$ Norwich Medical School, University of East Anglia, Norwich, United Kingdom, ${ }^{2}$ Hadassah Ein-Kerem, Jerusalem Bio Park, Entera Bio Ltd, Jerusalem, Israel

Background: Teriparatide PTH (1-34) is an osteoanabolic agent used in the treatment of osteoporosis. Measurement of PTH (1-34) can be useful in osteoporosis treatment and in the diagnosis of pseudohypoparathyroidism (PHP) by confirming administration of PTH (1-34).

Aims: (1) To assess PTH (1-34) profiles obtained using standard Forsteo treatment compared to using a novel oral administration. (2) To confirm the PTH (1-34) in a PHP patient receiving PTH (1-34) as part of an Ellsworth Howard Test (EHT). (3) To perform a method comparison of oxidised/non oxidised forms of PTH (1-34) detected by LC-MS/MS with immunoassay.
Methods: Using a LC-MS/MS method, PTH (1-34) was measured in Pharmacokinetic (PK) profiles from a human double blinded study. Participants were given teriparatide either by a single SC injection (Forsteo, $20 \mu \mathrm{g})(\mathrm{n}=6)$; or in an oral dose of $0.69 \mathrm{mg}(\mathrm{n}=4)$, or $2.07 \mathrm{mg}(\mathrm{n}=6)$ (EnteraBio). In an EHT, PTH (1-34), urinary PO4, and urine/plasma cyclic adenosine $3^{\prime} 5^{\prime}$-monophosphate (cAMP) were measured on samples before/after $20 \mu \mathrm{g}$ Forsteo injection. Oxidised/ non-oxidised forms of PTH $(1-34)(n=390)$ measured by LC-MS/ MS were compared against immunoassay (IDS; Boldon, UK).

Results: PK profiles showed rapid absorption of PTH (1-34) in plasma. The $2.07 \mathrm{mg}$ oral dose achieved $\mathrm{C}_{\max }$ of $271 \mathrm{pg} / \mathrm{mL}$ comparable to that of $20 \mu \mathrm{g}$ Forsteo, but the injection form showed slower rate of plasma clearance $\left(\mathrm{T}^{1} / 2_{\text {(injection })}=37.7 \mathrm{~min}, \mathrm{~T}^{1} / 2_{\text {(oral) }}=12.5\right.$ min). The EHT profile from a PHP patient showed a lack of cAMP response despite significant increase in plasma PTH (1-34) concentration. Method comparison showed LC-MS/MS results were correlated $\left(r^{2}=0.950\right)$, but biased $(-35.5 \%)$ against the immunoassay. The bias was caused partly by a matrix effect $(14.6 \pm 18.4 \%)$, cross reactivity of the immunoassay with PTH (1-84) $(7.1 \pm 0.45 \%)$ and to oxidised forms of PTH (1-34) $(23.9 \pm 6.1 \%)$.

Conclusion: Our LC-MS/MS method for PTH (1-34) can help validate the therapeutic use of osteoanabolic agents; confirm the lack of response to exogenous stimulation in EHT; and may explain the differences in responses to treatment due to oxidation of PTH (1-34).

\section{P327}

Health-related quality of life in postmenopausal osteoporosis connected to the adherence and persistence of Denosumab vs. weekly Bisphosphonate

Giorgio Carlino $^{1}$, Roberta Maggio ${ }^{2}$

${ }^{1}$ Rheumatology, ASL LECCE, Casarano, Italy, ${ }^{2}$ Internal Medicine, Scorrano Hospital, Scorrano, Italy

Background: Long term adherence and persistence in patients undergoing treatment for postmenopausal osteoporosis (PO) remains poor despite the proven efficacy of the therapy. We evaluated whether greater adherence and persistence in treatment can lead to an improvement in the quality of life.

Methods: 268 patients, all women, in PO divided into two groups were evaluated: "DEN Group" (DEN) in treatment with denosumab $(\mathrm{n}=131)$ and "BIS Group" (BIS) with bisphosphonates $(\mathrm{n}=137)$. Patients were followed for 3 years with baseline, 6-month, 18-month, and 36-month evaluation. The evaluation criteria were the persistence in therapy and the self-related treatment compliance, as well as the quality of life assessed with the QUALEFFO-41.

Results: At 6 months the percentage of patients who had left the therapy was $44.3 \%$ in the BIS and $5.3 \%$ in the DEN, at 36 months $54.9 \%$ in the BIS and $7.2 \%$ in the DEN. In the BIS the main reason for abandonment were adverse events, in the DEN drop-out. The majority of patients both BIS and DEN said they were satisfied with the treatment and wanted to continue. The QUALEFFO scores of patients from the baseline were significantly improved at 36 months (BIS $79.6 \pm 25.4$ vs. $65.4 \pm 14.6$; DEN $80.2 \pm 18.5$ vs. $55.6^{*} \pm 16.4$ ) $(* \mathrm{p}<0.001)$; the difference was not significant between BIS and DEN groups at both baseline and 36 months, but in the DEN group there was significance between baseline and 36 months.

Conclusions: In conclusion, the observation, although numerically limited, notes that the use of denosumab in patients with PO leads to a greater persistence in treatment and a statistically significant adherence to therapy, which allows to obtain the maximum therapeutic effect of the therapy, also determining in 36 months of treatment an improvement in the quality of life, which is not achieved in subjects treated with bisphosphonates. 
Table 1

\begin{tabular}{llll}
\hline Basal features & $\begin{array}{l}\text { BIS } \\
(\mathrm{N}=137)\end{array}$ & $\begin{array}{l}\text { group } \\
\text { DEN } \\
(\mathrm{N}=131)\end{array}$ & group \\
\hline Age (year) & $62 \pm 6$ & $63 \pm 10$ \\
BMI $\left(\mathrm{kg} / \mathrm{m}^{-2}\right)$ & $25.8 \pm 3.5$ & $25.1 \pm 4.2$ \\
GFR $\left(\mathrm{mL} / \mathrm{min} / 1.73 \mathrm{~m}^{2}\right)$ & $79 \pm 16$ & $77 \pm 25$ \\
$\begin{array}{l}\text { BMD femoral neck }(\mathrm{g} / \\
\left.\mathrm{cm}^{2}\right)\end{array}$ & $0.650 \pm 0.077$ & $0.700 \pm 0.070$ \\
$\begin{array}{l}\text { BMD lumbar spine (g/ } \\
\left.\mathrm{cm}^{2}\right)\end{array}$ & $0.746 \pm 0.061$ & $0.772 \pm 0.106$ \\
$\begin{array}{l}\text { History of fractures }(\# \mathrm{pts}) \\
\text { 25OHD (ng/ml) }\end{array}$ & 35 & 42 \\
PTH (pg/ml) & $39 \pm 12$ & $49 \pm 25$ \\
CTX (ng/ml) & $53 \pm 17$ & $44 \pm 13$ \\
\hline
\end{tabular}

\section{P328}

Abaloparatide: an effective osteoanabolic compared with PTH (1-34) and PTHrP (1-36) in mice

Carole Le Henaff ${ }^{1}$, Florante Ricarte ${ }^{2,3}$, Zhiming $\mathrm{He}^{1}$, Joshua Johnson $^{1}$, Nicola C. Partridge ${ }^{1}$

${ }^{1}$ Department of Basic Science and Craniofacial Biology, New York University Dental School, New York, United States, ${ }^{2}$ Molecular Pharmacology Training Program, Sackler Institute of Graduate Biomedical Sciences, New York University School of Medicine, New York, United States, ${ }^{3}$ Department of Basic Science and Craniofacial Biology, New York University College of Dentistry, New York, United States

Abaloparatide (ABL), a novel analog of parathyroid hormonerelated protein (PTHrP 1-36) had similar bone anabolic effects to teriparatide (PTH 1-34), but lesser bone resorption and became the second FDA-approved osteoanabolic therapy for the treatment of osteoporosis. This study aims to elucidate the effects of PTH (1-34), PTHrP (1-36), and ABL on bone remodeling in mice. Intermittent daily subcutaneous peptide injections of $80 \mu \mathrm{g} / \mathrm{kg} /$ day were administered to 4 month-old $\mathrm{C} 57 \mathrm{Bl} / 6$ male mice for 6 weeks ( $n=10$ /group). Dual Energy X-ray Absorptiometry was performed during the course of the treatment. Eighteen hours after the final injection, right femurs were harvested for $\mu \mathrm{CT}$ analyses, sera were assayed for P1NP and CTX, and tibiae were separated into cortical, trabecular, and bone marrow fractions for RT-qPCR analyses. We show that ABL resulted in a similar increase in whole body, femoral, and tibial bone mineral density (BMD) compared with PTH (1-34). $\mu \mathrm{CT}$ analyses revealed similar increases in cortical thickness with ABL and PTH (1-34). ABL was superior to PTH (1-34) and PTHrP (1-36) in stimulating greater levels of P1NP, and PTH (1-34) and ABL led to increases in CTX. Surprisingly, PTHrP (1-36) seemed to have no effect on P1NP and CTX levels, BMD or cortical thickness. RT-qPCR analyses of trabecular bone populations showed that PTH (1-34) and ABL led to a similar rise in osteoblastic gene expression. Surprisingly, PTH (1-34) and PTHrP (1-36) led to increases in osteoblastic mRNAs in bone marrow, while ABL did not. These data reveal that $\mathrm{ABL}$ is equivalent to $\mathrm{PTH}(1-34)$ in increasing BMD and bone osteoblastic gene expression. The relative serum P1NP and CTX levels showed that ABL is better able to stimulate bone formation. Taken together, this study provides greater insight into the effects of
PTH (1-34), PTHrP (1-36) and ABL in bone, and confirms that ABL is an effective osteoanabolic.

\section{P329}

$\mathrm{NaQuinate}$ treatment maintains bone architecture and improves bone quality in ovariectomized rats

Stephanie Gohin ${ }^{1}$, Robin Soper ${ }^{2}$, Behzad Javaheri ${ }^{1}$, Lars Marius Ytreb $\varnothing^{3}$, Mark Hopkinson ${ }^{1}$, Richard Meeson ${ }^{4}$, David Howat ${ }^{2}$, Andrew Pitsillides ${ }^{1}$, Stephen Hodges ${ }^{1,2,3}$

${ }^{1}$ Comparative Biomedical Sciences, Royal Veterinary College, London, United Kingdom, ${ }^{2}$ Haoma Medica, London, United Kingdom, ${ }^{3}$ Anaesthesiology and Intensive Care Medicine, Troms $\phi$ University Hospital, Troms $\phi$, Norway, ${ }^{4}$ Institute of Orthopaedics and Musculoskeletal Science, University College London, Stanmore, United Kingdom

Osteoporosis represents a major healthcare burden that is anticipated to increase. Emerging therapies are limited in efficacy and by their safety profile. NaQuinate, 2-methyl-1,4-naphthoquinone with a branched-chain aliphatic hydrocarbon carboxylic acid at the 3 position, has previously been demonstrated to preserve trabecular bone volume and architecture in mouse models of osteoporosis. Herein, we have expanded upon this original work to test whether $\mathrm{NaQuinate}$ also modifies bone mass and architecture in ovariectomized female rats. Female Sprague Dawley rats $(\mathrm{n}=10$ per group) were bilaterally ovariectomized and received either vehicle (OVX + Veh: $5 \%$ ethanol/PBS; i.p) or a daily NaQuinate dose (OVX $+\mathrm{NaQ}: 250 \mu \mathrm{g} / \mathrm{kg} / \mathrm{d}$; i.p) for 6 weeks; an additional group of rats underwent sham surgery and received the vehicle. Trabecular and cortical architecture in the tibiae was imaged with micro-CT ex vivo. Ovariectomy resulted in greater gain in body mass $(+25 \%, \mathrm{P}<0.0001)$ which was unaffected by NaQuinate treatment. Ovariectomized rats showed the expected diminution in trabecular bone mass and structure (BV/TV: $-80 \%, \mathrm{P}<0.0001)$. Interestingly, NaQuinate treatment for 6 weeks improved trabecular bone volume $(+58 \%, \mathrm{P}<0.05)$ compared with OVX + Veh, indicating a protection against the bone loss induced by ovariectomy. In the cortical compartment, NaQuinate treatment for 6 weeks increased cortical bone area, Imin, Imax and the predicted tibial resistance to torsion $(\mathrm{J})$ across a broad mid-diaphyseal region of the tibia compared to OVX + Veh. Direct measurements of bone strength will be determined to confirm these extrapolated data. To conclude, these results suggest that NaQuinate treatment effectively maintains bone integrity and improves bone strength in ovariectomized rats.

Keywords: Osteoporosis, NaQuinate, bone formation, microCT

\section{P330}

Comparison of the new bioactive Sclerostin ELISA with other commercially available Sclerostin immunoassays

Jacqueline Wallwitz ${ }^{1}$, Elisabeth Gadermaier ${ }^{1}$, Gabriela Berg ${ }^{1,2}$, Gottfried Himmler ${ }^{1}$

${ }^{1}$ The Antibody Lab GmbH, Vienna, Austria, ${ }^{2}$ Biomedica Medizinprodukte GmbH \& Co KG, Vienna, Austria

Objective: Sclerostin is a 190-amino acid glycoprotein of the DAN/Cerberus family and is mainly secreted by osteocytes. It is an inhibitor of the osteoanabolic Wnt signaling pathway achieved by binding with its second loop to the LRP5/6 complex. In a clinical setting, blocking active sclerostin by therapeutical antibodies is an important approach to decrease bone resorption. To our knowledge, the existing ELISAs on the market neither specifically detect bioactive sclerostin, nor specify the epitopes of their used antibodies and 
therefore it's not clear which sclerostin forms or fragments they detect. With this defined ELISA the measurement of bioactive sclerostin within human serum and plasma is possible.

Methods: We have developed an immunoassay for the detection of bioactive sclerostin in human samples. It contains a monoclonal and an affinity-purified polyclonal antibody. These antibodies were characterized and assay performance was validated to standard guidelines. Measured bioactive sclerostin concentrations in apparently healthy individuals as well as in diseased patients were compared with other commercially available ELISA (Biomedica, Teco and R\&D Systems).

Results: The recombinant monoclonal antibody recognizes an epitope within the bioactive site of the second loop of sclerostin, whereas the polyclonal detection antibody has five linear epitopes distributed throughout the whole molecule. Both antibodies have good binding kinetics of kdis of $<1.0 \mathrm{E}-07 \mathrm{~s}^{-1}$ for the monoclonal antibody and $1.19 \mathrm{E}-05 \mathrm{~s}^{-1}$ for the polyclonal antibody, respectively. All validation parameters were within the standard of acceptance demonstrating the robustness, accuracy and precision of the assay. The correlation between the assays was dependent on the sample type and disease state.

Summary and Conclusion: The presented ELISA is a tool for the defined detection of bioactive Sclerostin in human samples and may be helpful to further investigate sclerostin as a biomarker in the diagnosis of bone remodeling disorders and in the assessment of therapeutic effectiveness.

\section{P331}

Dose nail length effect on operative result of atypical subtrochanteric femur fracture?

\section{Kwangkyoun $\mathrm{Kim}^{1}$}

${ }^{\text {I}}$ Orthopedic Surgery, Konyang University, Daejeon, Republic of Korea

Objective: Severe anterior bowing of the femur can result in mismatch between the nail and the alignment of the femur. Such mismatch is a risk factor for anterior cortical perforation of the distal femur with subtrochanteric fractures, and leg length discrepancy with fractures of the femoral shaft. Moreover, there is more chance in radiation exposure during fixing distal interlocking screw in full length nail than partial length nail. The purpose of this retrospective study was to evaluate whether length of nail would influence on the radiologic and hemodynamic outcomes in the treatment of atypical insufficiency subtrochanteric fractures.

Materials and Methods: Seventy-two consecutive fractures with atypical insufficiency subtrochanteric fractures who had undergone intramedullary fixation using proximal femoral anti-rotation nail (PFNA, diameter, 10, 11, $12 \mathrm{~mm}$; length, 200, 240, 300, 340, $380 \mathrm{~mm}$, Synthes, Oberdorf, Switzerland) between March 2010 and March 2016 were followed-up for over 12 months. Patients were classified partial nail used group (200, $240 \mathrm{~mm})$ and full length nail used group $(300,340,380 \mathrm{~mm})$.

Results: Radiologically, there were no significant difference between partial length nail group and Full length nail group in union period, change of neck-shaft angle and leg length discrepancy (LLD) (In order, $p=0.429, p=0.273, p=0.359$ ). Clinically, There were no significant difference total blood loss but difference in operation time (In order, $p=0.249, p=0.034$ ). There were three operation related complications. One lateral cortical thinning around nail tip occurred in short nail used group. Four intraoperative iatrogenic fractures on ipsilateral side occurring nail insertion were occurred in long nail used group. There was one ipsilateral secondary fracture after operation in partial nail length groups.
Conclusion: Full nail length PFNA for atypical insufficiency subtrochanter fracture was not more efficient except ipsilateral femoral secondary fracture and has disadvantage in respect of long operation time.

Keywords: Osteoporosis, atypical insufficiency fracture, femur

\section{P332}

Bisphosphonate treatment in 6 patients with pregnancy- and lactationassociated osteoporosis

Kallipi Lampropoulou-Adamidou ${ }^{1}$, Ioannis K Triantafyllopoulos ${ }^{1}$, Alexia Balanika ${ }^{2}$, Konstantinos Makris ${ }^{3}$, Chrysoula G Liakou ${ }^{4}$, George Trovas ${ }^{1}$, Symeon Tournis ${ }^{1}$

${ }^{1}$ Laboratory for the Research of Musculoskeletal System "Th. Garofalidis", Medical School, University of Athens, KAT Hospital, Athens, Greece, ${ }^{2}$ Department of Computed Tomography, Asklepeion Voulas Hospital, Athens, Greece, ${ }^{3}$ Biochemistry Department, KAT Hospital, Athens, Greece, ${ }^{4}$ First Obstetrics and Gynaecology Department, 'Alexandra' Hospital, Athens, Greece

Pregnancy- and lactation-associated osteoporosis (PLO) is an uncommon disease. In some patients, the clinical symptoms of PLO resolve spontaneously, while others have severe, prolonged and disabling back pain caused by vertebral fractures (VFs). Because of the rarity of the disease and the lack of controlled trials, there is no consensus to date on how to treat PLO. We present our experience of 6 cases with PLO treated with bisphosphonates (BSP) combined with calcium and vitamin D supplementation for 2.4 years (range 6 months to 5 years). Patients sustained an average of 3.4 VFs (range $1-7)$. The mean patients' age at the time of fracture was 34 years (range 32-37). Five cases were diagnosed after the first pregnancy and one after the second. Secondary causes of osteoporosis were present in 2 patients. All women had a negative family history of osteoporosis. All patients complained of back pain during the postpartum period. The duration of lactation varied from 2 weeks to 3 months. The mean patients' follow-up was 7 years (range 4-17). They had a mean BMD increase at the lumbar spine $18.3 \%$ (range $7-28$ ) and at the total hip 3.3\% (range -2 to 11 ) at 12 months, $20 \%$ (range $8-31$ ) and $6.5 \%$ (range 2-11) at 2 years, 25\% (range 17-31) and $9.8 \%$ (range $5-15$ ) at 3 years, and $25 \%$ (range $17-40$ ) and $8.7 \%$ (range 5-12) at 5 years, respectively. One patient had other two gestations without presenting new fracture. New VF occurred in one case 17 years after diagnosis of PLO. BSP treatment along with weaning and calcium and vitamin D supplementation is associated with substantial increase in BMD and might prevent new VFs in women with PLO.

\section{P333}

Oxytocin did not affect the skeletal system in prednisolone-treated female rats

Aleksandra Janas ${ }^{1}$, Ewa Kruczek ${ }^{1}$, Dominika Gancarczyk ${ }^{1}$, Karolina Bałamucka $^{1}$, Adam Materla ${ }^{1}$, Joanna Folwarczna ${ }^{1}$

${ }^{I}$ Department of Pharmacology, School of Pharmacy with the Division of Laboratory Medicine in Sosnowiec, Medical University of Silesia, Katowice, Sosnowiec, Poland

Oxytocin, a hormone released from the posterior pituitary, takes part in the regulation of bone metabolism. Oxytocin has been reported to exert favorable effects on the skeletal system, especially in female organisms. It has been suggested that affecting oxytocin pathways may find application in the treatment of osteoporosis. Long-term glucocorticoid therapy is the most common cause of secondary osteoporosis. The aim of the present study was to investigate the 
effects of oxytocin on the skeletal system of prednisolone-treated rats with normal and decreased estrogen level (developing osteoporosis due to estrogen deficiency induced by bilateral ovariectomy).

The experiments were carried out on mature female Wistar rats, divided into 6 groups $(\mathrm{n}=9-10)$ : I-Non-ovariectomized (NOVX) control rats, II-Ovariectomized (OVX) control rats, III-Prednisolone-treated NOVX rats, IV-Prednisolone and oxytocin-treated NOVX rats, V-Prednisolone-treated OVX rats, VI-Prednisolone and oxytocin-treated OVX rats. Bilateral ovariectomy was performed 7 days before the start of drug administration. Prednisolone 21-hemisuccinate sodium salt $(7 \mathrm{mg} / \mathrm{kg})$ and oxytocin $(0.1 \mathrm{mg} / \mathrm{kg})$ were administered subcutaneously, once daily for 4 weeks. Serum bone turnover markers (CTX-I and osteocalcin), bone mass and mineralization, histomorphometric parameters (OsteoMeasure system) and mechanical properties (Instron apparatus) of cancellous and compact bone were determined. The results were statistically evaluated by ANOVA tests.

Estrogen deficiency induced typical osteoporotic (histomorphometric and mechanical) changes in OVX rats, with increased bone resorption and formation. Prednisolone administration induced only slight inhibition of bone resorption and formation in NOVX rats, and much stronger inhibition of bone turnover in OVX rats. Administration of oxytocin did not affect most of the investigated skeletal parameters in prednisolone-treated rats, independent of the estrogen status.

Results of this in vivo study do not support the hypothesis on the usefulness of oxytocin as a potential antiosteoporotic drug.

Keywords: oxytocin, glucocorticoids, osteoporosis, rats

\section{P334}

How much does bone mineral density increase after short-term teriparatide treatment ( $\leq 12$ months) in patients with severe osteoporosis?

Kyung Jung Kang ${ }^{1}$, Min Hyung Jung ${ }^{2}$

${ }^{1}$ Kyung Hee University Medical Center, Seoul, Republic of Korea, ${ }^{2}$ Kyung Hee University, Kyung Hee Medical, Seoul, Republic of Korea

Among various osteoporosis medications, to date, bone forming agent such as parathyroid hormone is considered as one of the most potent medication for the increase of bone mineral density (BMD) and fracture prevention in patients with osteoporosis. However, its application is limited due to its discomfort for use and high cost. Accordingly, that was applied during a short period in real clinical field with a lack of evidence. The aim of this study is to evaluated effects of short-term teriparatide on changes increase of BMD.

Total 116 patients (mean age: 74, female: 108) that underwent short-term teriparatide treatment (less than 12 months) in orthopedic department for severe osteoporosis were reviewed. Both spine and hip BMD were measured at baseline and 1 year after teriparatide treatment. Correlation between duration of teriparatide usage and BMD change was evaluated. According to duration of teriparatide treatment, changes of the BMD were also analyzed.

Mean duration of teriparatide treatment was 3.5 month (range 1-12). Mean spine and hip BMD were $0.638 \pm 0.111$ and $0.660 \pm 0.104 \mathrm{~g} / \mathrm{cm}^{2}$ at the baseline, respectively. After the teriparatide treatment, mean total increments of spine and hip BMD were $8.1 \pm 8.4$ and $0.6 \pm 5.2 \%$ at 1 year after the treatment, respectively. Although the increment of hip BMD was not correlated with duration of the teriparatide usage, the increments of spine BMD showed significant positive associations with the duration of teriparatide treatment $(r=0.329, p=0.002)$. Amount of the spine BMD increment were $8.79 \pm 8.07,8.63 \pm 8.48,10.76 \pm 9.09,12.8 \pm 9.4$, $13.4 \pm 9.84 \%$ when the teriparatide treatments were continued during more than 1, 2, 3, 4 and 5 months, respectively.

After short-term teriparatide treatment ( $\leq 12$ months), although hip BMD did not show any changes, significant increments were shown in spine BMD. The amounts of the BMD increments were proportional to longer duration of teriparatide usage.

\section{P335}

A hip fracture during the delivery because of transient migratory osteoporosis: the suspected diagnosis' importance

\section{Lorena Pena $^{1}$, Diego Bertrand ${ }^{1}$, Alejandro Braña ${ }^{1}$, Daniel Nuñez ${ }^{1}$}

${ }^{I}$ Traumatología y Cirugía Ortopédica, Hospital Universitario Central de Asturias, Oviedo, Spain

Introduction: Transient osteoporosis of the hip (TOH) is an uncommon condition. This painful regional osteoporosis affects previously healthy women in the third trimester of pregnancy. It is characterized by pain in the affected hip and pronounced osteopenia of the femoral head and neck. It has a relatively short clinical course (average 6 months) and a predictably benign prognosis.

Methods: We report a case of TOH in a 35-year-old woman who suffered a displaced femoral neck fracture during the delivery because an unknown TOH. She presented clinically with hip pain (during the last 3 months), limping gait, and limited ranges of hip motion. Radiographs are typically unremarkable but MR imaging studies yield findings consistent with bone marrow edema. TOH often resembles osteonecrosis but the two conditions must be differentiated due to different prognoses and management approaches. She was treated surgically with a total hip arthroplasty (THA) and also avoiding lactation, she increased intake of calcium and vitamin D and recovered without further medical treatment.

Results: A THA was the treatment for the displaced femoral neck fracture developed during the delivery because of a TOH and the patient could start weight bearing again and she has no pain since the surgery in the hip. Although the situation of the patient is good at the moment, it is very important to suspect this disease so as to give the patient the proper treatment.

Conclusions: The diagnosis is one of exclusion. The cause of the osteopenia is not known, although various aetiological factors have been implicated. We think that the suspected diagnosis is the most important thing for those patients who are able to benefit from a conservatively treatment and avoiding a hip fracture in so young patients.

Keywords: pregnancy, osteoporosis, hip fracture, prevention

\section{P336}

Teriparatide treatment in pregnancy-associated osteoporosis with multiple vertebra fractures

Vera Choida $^{1}$, Judith Bubbear ${ }^{1}$, Richard Keen ${ }^{1}$

${ }^{1}$ Royal National Orthopaedic Hospital, London, United Kingdom

Objectives: Fragility fractures secondary to pregnancy-associated osteoporosis can cause significant disability during pregnancy and post-partum. We report a case of successful treatment with Teriparatide.

Methods: A 36 year old lady treated with low molecular weight heparin for pregnancy-associated deep venous thrombosis developed acute back pain post-delivery. She was initially diagnosed with symphysis pubis dysfunction and 3 months later had an MRI scan that 
showed acute compression fractures of T12, L4 and L5 vertebrae. Her DXA scan was normal at the spine and hip sites. CT bone densitometry scan, however, revealed T-scores of -3.2 and -4.4 at the L3 and L4 vertebra respectively. Her bone profile, vitamin D, thyroid and parathyroid hormone levels were normal.

Results: She was initially treated in another hospital with subcutaneous calcitonin injections and balloon kyphoplasty of the T12, L4 and L5 vertebrae. Her pain worsened after the procedure and her mobility was significantly reduced despite high doses of opioid analgesia. A repeat MRI scan showed a new L2 fracture and a sacral insufficiency fracture. She was treated with Teriparatide injections $20 \mathrm{mcg}$ daily for 3 months. Her pain gradually improved from $6 / 10$ before Teriparatide treatment to $2 / 102$ months post treatment. She achieved a reduction of opioid doses by $75 \%$ in 2 months.

Summary and Conclusions: Teriparatide treatment improved pain from vertebral fractures in a lady with pregnancy-associated osteoporosis, who had also been exposed to heparin treatment. On the other hand, the initial balloon kyphoplasty was followed by another vertebral fracture and worsening pain.

\section{P337}

Rete Osteoporosi Sicilia (Sicilian Osteoporosis Network): an IT platform for the improvement of diagnosis and treatment of osteoporosis in Sicily

Agostino Gaudio $^{1}$, Rosario Rapisarda ${ }^{1}$, Anastasia Xourafa ${ }^{1}$, Anna Schillaci $^{1}$, Carmelo Erio Fiore ${ }^{1}$, Pietro Castellino ${ }^{1}$

${ }^{1}$ Department of Clinical and Experimental Medicine, University of Catania, Catania, Italy

The "Rete Osteoporosi Sicilia" (Sicilian Osteoporosis Network) is based on an IT platform that will connect family doctors with the Regional Reference Center for Osteoporosis (University Policlinic of Catania), providing "online counseling". Family doctors will have the capability to ask for opinions from a team of bone disease specialists, supplying information on a clinical case through the platform (e.g. laboratory analysis, radiographs, DXA exams, etc.) to facilitate diagnosis and treatment.

Sicily is among the three highest regions in Italy (after Sardinia and Campania) for bone fragility prevalence, with over $31 \%$ of patients over 65 years old receiving a diagnosis of osteoporosis.

Specifically, the territory in which the "pilot phase" of this project will take place, the Province of Catania, has over 1,100,000 inhabitants, of which over 200,000 are over 65 , and therefore, at risk of bone fragility.

The central idea of the network is for strong integration between territory and specialised centers, to minimise the need for hospital visits and displacement of elderly patients, because it will allow their doctors to directly address experts.

The "Rete Osteoporosi Sicilia" (Sicilian Osteoporosis Network) was developed within the PSN 2014, funded by the Health Department of the Sicily Region.

The IT platform was created by Agile srl, which registered the domain http://www.reteosteoporosisicilia.it, in the name of the University of Catania.

To the best of our knowledge, this is one of the very few IT platforms in Italy providing connection between family doctors and a center specialising in the treatment of osteoporosis.

We expect to soon be able to demonstrate how this tool is useful in improving diagnostic and therapeutic appropriateness in osteoporosis and other metabolic bone diseases.

Keywords: osteoporosis, IT platform, Sicily, appropriateness, online counseling

\section{P338}

Assessment of femoral cortical bone quality in individuals with type 2 diabetes mellitus reveals increased porosity accompanied by altered hard tissue characteristics in endocortical compartments

Eva Maria Wölfel ${ }^{1}$, Petar Milovanovic ${ }^{1,2}$, Katharina Jähn ${ }^{1}$, Herbert Mushumba $^{3}$, Birgit Wulff ${ }^{3}$, Michael Amling ${ }^{1}$, Klaus Püschel ${ }^{3}$, Graeme M. Campbell ${ }^{4}$, Björn Busse ${ }^{1}$

${ }^{1}$ Department of Osteology and Biomechanics, University Medical Center Hamburg-Eppendorf, Hamburg, Germany, ${ }^{2}$ Institute of Anatomy, Belgrade, Serbia, ${ }^{3}$ Department of Forensic Medicine, University Medical Center Hamburg-Eppendorf, Hamburg, Germany, ${ }^{4}$ Institute of Biomechanics, Hamburg University of Technology, Hamburg, Germany

Patients with type 2 diabetes mellitus (T2DM) experience an increased fracture risk despite presenting a normal or even higher bone mineral density. Since T2DM has an increasing prevalence it is needed to understand the underlying effects of T2DM on bone microstructure leading to an elevated fracture risk not detectable with the common clinical fracture risk assessment methods.

In this study the anterior part of the mid-diaphyseal femoral cortex of 12 T2DM-diagnosed individuals and age-matched controls were investigated following autopsy and local ethics approval. Cortical porosity was analyzed in 3D with micro-computed tomography $(\mu-$ $\mathrm{CT}$ ). Nanoindentation was performed to assess the osteonal Young's modulus and hardness in the periosteal and endocortical region. Furthermore, the endocortical region was analyzed performing histomorphometry on toluidine blue stained sections.

One-third of the analyzed diabetic individuals showed a highly elevated cortical porosity ranging between 20 and 60\%. Here, endocortical trabecularization was identified as a major contributor to the increased extent of cortical porosity in the T2DM group. Our preliminary results obtained by nanoindentation revealed a $10 \%$ lower Young's modulus and a $15 \%$ lower hardness in the endocortical region of the T2DM compared to the control group, while differences in the periosteal region of diabetic and age-matched controls were not evident. Furthermore, presence of osteoclasts was more pronounced in cases with T2DM reflecting processes of bone resorption at the endocortical region in the T2DM study group, while osteoclasts in aged-matched controls with a mean age of 77 years were essentially absent following static bone histomorphometry.

These results suggest an increased occurrence of cortical reorganization in the endocortical compartment due to T2DM affecting alterations of the mechanical characteristics. Higher porosity and changes of endocortical mechanical properties may play a role in fracture susceptibility in the femoral cortex of T2DM patients.

Keywords: $\mu$-CT, type 2 diabetes mellitus, nanoindentation, histomorphometry

\section{P339}

All-cause mortality with use of antidepressants and benzodiazepines after major osteoporotic fracture

Irma de Bruin $^{1,2}$, Corinne Klop ${ }^{3}$, Caroline Wyers ${ }^{1,2}$, Jetty Overbeek ${ }^{4}$, Joop van den Bergh ${ }^{1,2,5}$, Piet Geusens ${ }^{5,6}$, Johanna Driessen ${ }^{3,7,8}$, Frank de Vries ${ }^{3,8,9}$

${ }^{I}$ Department of Internal Medicine, VieCuri Medical Center, Venlo, Netherlands, ${ }^{2}$ NUTRIM, Department of Internal Medicine, Maastricht University Medical Center +, Maastricht, Netherlands, ${ }^{3}$ Division of Pharmacoepidemiology \& Clinical Pharmacology, Utrecht Institute for Pharmaceutical Sciences, Utrecht University, Utrecht, Netherlands, ${ }^{4}$ PHARMO institute, Utrecht, Netherlands, ${ }^{5}$ Biomedical Research Center, Hasselt University, Diepenbeek, Belgium, 
${ }^{6}$ CAPHRI, Department of Internal Medicine, Subdivision Rheumatology, Maastricht University Medical Center +, Maastricht, Netherlands, ${ }^{7}$ NUTRIM/CAPHRI, Maastricht University Medical Center +, Maastricht, Netherlands, ${ }^{8}$ Department of Clinical Pharmacy and Toxicology, Maastricht University Medical Center +, Maastricht, Netherlands, ${ }^{9}$ CAPHRI, Maastricht University Medical Center +, Maastricht, Netherlands

Objectives: to study the association between use of antidepressants and benzodiazepines and the risk of mortality after major osteoporotic fracture (MOF). Fractures of hip, humerus, forearm and spine were considered as a MOF.

Methods: A cohort study was performed within the Dutch PHARMO Database. All patients aged 65 years or older with a first record of a MOF between 2002 and 2011 were included. Oral use of antidepressants and benzodiazepines was determined using pharmacy dispensing data. Cumulative incidences and adjusted hazard ratios were estimated with Kaplan Meier analyses and Cox-proportional hazards models, respectively. Time-dependent adjustments were made for age, sex, comorbidity and concomitant drug use.

Results: A total of 4854 patients sustained a MOF. The cumulative incidence of use of antidepressants following the first fracture increased significantly from $10.6 \%(95 \%$ CI $9.8-11.6 \%)$ at 3 months to $14.7 \%$ ( $95 \%$ CI $13.7-15.7 \%$ ) at 12 months; for benzodiazepines it increased significantly from $24.0 \%$ (95\% CI $22.8-25.3 \%$ ) at 3 months to $31.4 \%$ (95\% CI $30.1-32.8 \%)$ at 12 months.

After a major osteoporotic fracture, current use of antidepressants or benzodiazepines was not associated with all-cause mortality compared to no use of antidepressants and benzodiazepines (adjusted HR 0.96 (95\% CI 0.76-1.21) and 1.16 (95\% CI 0.96-1.41) respectively). In contrast, all-cause mortality was significantly increased after a MOF among patients who previously used but stopped taking antidepressants (defined as the most recent dispensing more than 3 months ago) [adjusted HR 1.39 (95\% CI 1.15-1.66)] or benzodiazepines [adjusted HR 1.20 (95\% CI 1.02-1.42)].

Conclusion: This study did not show an association between mortality risk and current use of antidepressants or benzodiazepines after a MOF. However, risk of mortality increased after cessation of antidepressants or benzodiazepines. Patients with a MOF and previous antidepressant or benzodiazepine therapy should be monitored for reasons that increase mortality in order to elucidate these findings.

\section{P340}

SQSTM1 mutations in a Hungarian cohort of Paget's disease of bone: associations with disease severity

Judit Donáth ${ }^{1}$, Márton Pálinkás ${ }^{1}$, Rita Rásonyi ${ }^{1}$, Gyula Vastag ${ }^{2,3}$, Nerea Alonso $^{4}$, Beatriz Larraz Prieto ${ }^{4}$, Mahéva Vallet ${ }^{4}$, Stuart Ralston ${ }^{4}$, Gyula Poór ${ }^{1,5}$

${ }^{1}$ Rheumatology, National Institute of Rheumatology and Physiotherapy, Budapest, Hungary, ${ }^{2}$ Széchenyi University, Györ, Hungary, ${ }^{3}$ National University of Public Service, Budapest, Hungary, ${ }^{4}$ Edinburgh Clinical Trials Unit, University of Edinburgh, Western General Hospital, Edinburgh, United Kingdom, ${ }^{5}$ Semmelweis University, Budapest, Hungary

Objective: This study provides information about the frequency and severity of SQSTM1 mutations in a Hungarian cohort of patients with Paget's disease of bone (PDB).

Material and Methods: We performed mutation analysis in 82 sporadic cases of PDB. Skeletal affection, disease onset, bone fractures and need for orthopedic surgery were assessed. All Pagetic cases had radionuclide bone scan. 67 patients were examined before and after bisphosphonate treatment. Serum alkaline phosphatase (SAP) levels and bone pain on visual analogue scale (VAS) were measured.
Results: Mutations of SQSTM1 were detected in 12/82 patients $(14.6 \%)$. Mutation carriers had slightly younger age at diagnosis $(60.6 \pm 9.8$ vs. $61.6 \pm 10.0$ years) with shorter range (from 46 to 74 years vs. 39 and 86 years). Greater number of bones were affected $(1.67 \pm 0.19 \quad$ vs. $1.21 \pm 0.08, \quad \mathrm{p}=0.035)$ and deformed $(0.41767 \pm 0.669$ vs. $0.157 \pm 0.366 ; p=0.052)$ in the group of mutation carriers. Orthopedic surgery was also needed in higher percentage in carriers (16.67 versus $2.86 \%$ ). The average of SAP at diagnosis was not significantly different in the two groups (983.90 U/ $\mathrm{L}$ carriers vs. $771.59 \mathrm{U} / \mathrm{L}$ non-carriers). The need for hearing aid was higher in patients with SQSTM1 mutations (16.67 versus $2.86 \%$ ). There was no significant difference between the two groups in comorbidity number $(0.9167 \pm 0.900$ vs. $1.114 \pm 0.986)$. BP treatment responses on SAP levels showed significant differences between SQSTM1 mutation carriers and non-carriers but no significant differences were detected in the pain scale.

Conclusions: Our results confirmed that SQSTM1 mutations represent about $14.6 \%$ in sporadic Paget cases in Hungary. The study demonstrates that SQSTM1 mutations are strongly associated with bone affection, deformity and response to BP treatment, and may have influence on clinical complications such as the need for hearing aid or orthopedic surgery.

Keywords: Paget's disease of bone, SQSTM1 mutations, Hungarian data

\section{P341}

Reduction of arrhythmias in primary hyperparathyroidism by parathyroidectomy, evaluated with $24 \mathrm{~h}$ ecg monitoring Jessica Pepe ${ }^{1}$, Cristiana Cipriani ${ }^{1}$, Mario Curione ${ }^{1}$, Federica Biamonte $^{1}$, Luciano Colangelo ${ }^{1}$, Vittoria Danese ${ }^{1}$, Veronica Cecchetti $^{1}$, Chiara Sonato ${ }^{1}$, Federica Ferrone ${ }^{1}$, Mirella Cilli ${ }^{1}$, Salvatore Minisola ${ }^{1}$

${ }^{I}$ Department of Internal Medicine and Medical Disciplines, Sapienza University of Rome, Rome, Italy

Objective: Hypercalcemia may induce arrhythmias. There are no data on the prevalence of arrhythmias in primary hyperparathyroidism (PHPT) in daily life. Aim of this study was to investigate both the prevalence of arrhythmias in patients with PHPT compared to controls and the impact of parathyroidectomy, evaluated by $24 \mathrm{~h}$ electrocardiogram (ECG) monitoring.

Methods: 26 postmenopausal women with PHPT and 26 controls were enrolled (age range $45-80$ years). PHPT patients were randomized into two groups: 13 underwent parathyroidectomy (Group A) and 13 were followed-up conservatively (Group B). After 6 months, patients were examined again. Each patient underwent mineral metabolism biochemical evaluation, bone mineral density measurements, standard ECG and $24 \mathrm{~h}$ ECG monitoring.

Results: We found no differences in anthropometric parameters and cardiovascular risk factors between patients and controls. PHPT patients showed higher calcium and parathyroid hormone compared to controls. A significantly higher prevalence of supraventricular premature beats (SVPBs) and ventricular premature beats (VPBs) were found in Group A and B compared to controls: almost $70 \%$ of patients in both Groups had VPBs compared to $27 \%$ of the subjects in the control group (all $\mathrm{p}<0.05$ ). Group A and B showed no differences in mean baseline biochemical values and ECG parameters. Mean value of QTc was in the normal range at baseline (Group A: $400.6 \pm 14.9$; Group B: $399.0 \pm 14.7 \mathrm{msec}, \mathrm{p}=0.78$ ), but significantly shorter than controls $(411.9 \pm 17.2 \mathrm{msec}, \mathrm{p}<0.05)$. A negative correlation was found between QTc and ionized calcium levels $(\mathrm{r}=-0.48, \mathrm{p}<0.05)$. Six months following parathyroidectomy, a significant reduction of SVPBs $(p=0.01)$ and of VPBs 
$(\mathrm{p}=0.04)$ was noted in Group A. Group B showed no significant changes after a six month period.

Conclusions: The increased prevalence of SVPBs and VPBs is significantly reduced by parathyroidectomy, and it is mainly related to the short QTc caused by hypercalcemia.

Keywords: parathyroid hormone, ecg, qt interval

\section{P342}

High bone mineral density on routine bone density scanning: Frequency and causes

Aurore Nottez ${ }^{1}$, Sami Kolta ${ }^{2}$, Isabelle Legroux-Gérot ${ }^{1}$, Georges Lion $^{1}$, Bernard Cortet ${ }^{1}$, Julien Paccou ${ }^{1}$

${ }^{1}$ Lille University Hospital, Lille, France, ${ }^{2} \mathrm{CHU}$ de Cochin, Paris, France

A finding of high BMD on routine DXA scanning is not infrequent. This study was performed to assess the frequency and causes of high BMD within the general population referred for DXA scanning in a tertiary referral hospital.

DXA databases were initially searched for individuals with a BMD $\mathrm{T}$ - or Z-score $\geq+3$ at any site. Frequency of high BMD was evaluated at the lumbar spine and hip, as were causes associated with high BMD.

In total, 27,247 historical DXA hip scans (9857 in men and 17,390 in women) and 28,438 lumbar spine scans $(10,209$ in men and 18,229) were screened over a period of 12 years (between 2005 and 2017). The frequency of high BMD was $0.44,0.55$ and $2.93 \%$ at total hip, femoral neck and lumbar spine respectively. A total of 16,695 individuals with at least one scan were identified among all scanners performed.

In total, DXA scans and medical records of 728 individuals (237 men and 491 women) with high BMD were screened. Then, the frequency of high BMD was $4.36 \%$. High BMD at the hip (total hip and/or femoral neck) without high BMD at the lumbar spine was only found in 90 individuals $(0.54 \%, 19$ men). "Generalized" increase in bone mass (BMD T- or Z-score $\geq+3$ at both total hip and lumbar spine) was only found in 62 individuals $(0.37 \%, 16$ men). DXA scans were inspected by two reviewers; approximately $60 \%$ were considered to have artefactually raised BMD due to degenerative changes. Of the remaining cases, rare hereditary diseases (e.g. osteopetrosis...), acquired-high BMD (e.g. renal osteodystrophy, myelofibrosis, sclerotic bone metastases...) and unexplained high BMD were found.

The frequency of high BMD (T- or Z-score $\geq+3$ at any site) was higher than expected. This study indicates that the causes of high BMD were mainly due to osteoarthritis.

\section{P343}

Osteoporosis in Crohn's Disease is dependent on Serum factors which change protein expression in a linear relationship to patients' disease stage and bone mineral density

Martina Blaschke ${ }^{1,2}$, Regine Köpp ${ }^{1}$, Christoph Lenz ${ }^{3,4}$, Jochen Kruppa $^{5,6}$, Klaus Jung ${ }^{5,6}$, Heide Siggelkow ${ }^{1,2}$

${ }^{1}$ Gastroenterology and gastrointestinal Oncology, University of Göttingen, Göttingen, Germany, ${ }^{2}$ MVZ Endokrinologikum Göttingen, Göttingen, Germany, ${ }^{3}$ Bioanalytical Mass Spectrometry, Max Planck Institute for Biophysical Chemistry, Göttingen, Germany, ${ }^{4}$ Institute of Clinical Chemistry, University Medical Center, Göttingen, Germany, ${ }^{5}$ Department of Medical Statistics, University Medical Center, Göttingen, Germany, ${ }^{6}$ Genomics and Bioinformatics of Infectious Diseases, University of Veterinary Medicine Hannover, Hannover, Germany

Background: Crohn's disease (CD) is associated with a higher prevalence of osteoporosis, a complication that is recognized as a significant cause of morbidity. Its pathogenesis is controversial, but the activity of $\mathrm{CD}$ is one contributing factor.

Methods: We stimulated SCP-1 cells (mesenchymal stem cell line) under osteogenic conditions with serum from adult patients with $\mathrm{CD}$ and compared the effect of sera collected in the symptomatic phase (SP) versus remission (R). Concentrations of IL-6, IL-1 beta, and TNF alpha in the sera were measured. Patients were classified as normal or osteopenic/osteoporotic based on bone mineral density (BMD) T-score measurements. After 14 days in culture, protein expression and gene ontology (GO) annotation analysis was performed.

Results: Cytokine concentrations (IL-6, IL-1 beta, TNF alpha) varied within sera groups. None of the cytokines were significantly increased in the symptomatic phase compared to remission. Protein analysis revealed 17 proteins regulated by the SP vs. $\mathrm{R}$ phase sera of disease. A linear relationship between CDAI (Crohn's disease activity index) and normalized protein expression of APOA1/2, TTR, CDKAL1 and TUBB6 could be determined. Eleven proteins were found to be differentially regulated comparing osteoporosis-positive and osteoporosis-negative sera. Gene annotation and further analysis identified these genes as part of hem and erythrocyte metabolism as well as involved in hypoxia. A significant linear relationship between bone mineral density and normalized protein expression could be determined for proteins FABP3 and TTR.

Conclusion: Our explorative results confirm our hypothesis that factors in serum from patients with $\mathrm{CD}$ change the protein expression pattern of human immortalized osteoblast like cells. The correlation to BMD suggests, that these shorttime changes indeed influence factors of bone metabolism.

Keywords: Crohn's disease; osteoporosis; proteome analysis; cytokines; Gene ontology

\section{P344}

Patterns of spinal ossifications in 500 chronic spinal injury patients Shamsa Shariatpanahi ${ }^{1}$

\section{${ }^{1}$ Shahed Univercity, Tehran, Islamic Republic of Iran}

Introduction: Chronic spinal cord injury has an important role in sensorimotor disorders which is associated with many complications and the rate of these complications should be certainly considered. The most important complications are the changes in bones and joints which come with pain and lead to increase in their motor disability. In this paper, the radiographic images were studied and the ossifications of spinal cord were described and also the type of changes and their prevalence was evaluated.

Materials and Methods: In this study, 500 patients and veterans with spinal cord injuries were examined. The spinal X-ray of the patients have been studied for spinal cord ossification, this changes classified to psoriasis like ossifications, syndesmophytes, bone spores (osteophytes), DISH like ossifications.

Results: Among the patients, 485 cases were males and 15 cases were females, the average age was 50.26 and the mean duration of injury was 26 years. Four hundred and forty six patients were paraplegic and 54 cases were quadriplegic. Psoriasis like ossifications were seen in $25.1 \%$ of paraplegics and $13 \%$ of quadriplegics, osteophytes were seen in $16.7 \%$ of paraplegics and $7.4 \%$ of quadriplegics, syndesmophytes were seen in $6.7 \%$ of paraplegics and $1.9 \%$ of quadriplegics, DISH like ossifications were seen in $5.6 \%$ of paraplegics and $5.4 \%$ of quadriplegics respectively. There was a significant relationship between age and the number of involved vertebrae $(P=0.000)$, psoriasis like ossifications $(P=0.048)$ and large osteophytes $(\mathrm{P}=0.037)$.

Conclusions: In lumbar spine radiography of the patients, psoriasis like ossifications and osteophytes were mostly seen. These changes 
must differentiate from spondyloarthropaties and degenerative disease of spine.

\section{P345}

The relationship between the renin-angiotensin-aldosterone system and bone metabolism in patients with secondary aldosteronism caused by anorexia nervosa or laxative abuse

Gen Yasuda $^{1}$, Akira Fujiwara ${ }^{1}$, Sanae Saka ${ }^{1}$, Nobuhito Hirawa ${ }^{1}$

${ }^{1}$ Yokohama City University, Yokohama, Japan

Objectives: Angiotensin II is one of the strongest vasoconstrictive substances, but it has been known that angiotensin II is a potent stimulator of osteoclastic bone resorption. Patients with anorexia nervosa or long-term laxative abuse show clinical and laboratory signs of secondary aldosteronism, stimulating the renin-angiotensinaldosterone (RAA) system to maintain blood pressure. However, there are few reports regarding how the RAA system relates with bone metabolism in patients with secondary aldosteronism. We evaluated the relationship between the RAA system and bone metabolism parameters in these patients.

Methods: Eighteen patients with anorexia nervosa or laxative abuse (mean age, $32 \pm 6$ years old; men: women, $2: 16$ ) and 18 ageand sex-matched healthy subjects (mean age, $33 \pm 5$ years old; men: women, $2: 16)$ as the control group participated in the study. Serum concentrations of type I collagen C-terminal telopeptide (ICTP) and bone alkaline phosphatase (BAP), and urinary concentrations of type I collagen cross-linked N-terminal telopeptide (NTx) were measured as the bone metabolism parameters. Plasma renin activity (PRA), angiotensin II and aldosterone concentrations were measured.

Results: ICTP, BAP, and NTx in the secondary aldosteronism group were $8.8 \pm 2.0 \mathrm{ng} / \mathrm{mL}, 58 \pm 16 \mathrm{U} / \mathrm{L}$ and $133 \pm 30 \mathrm{nmol}$ $\mathrm{BCE} / \mathrm{mmol}$, which were significantly $(\mathrm{P}<0.05)$ higher than $3.6 \pm 1.4 \mathrm{ng} / \mathrm{mL}, \quad 28 \pm 11 \mathrm{U} / \mathrm{L}$, and $40 \pm 18 \mathrm{nmol} \mathrm{BCE} / \mathrm{mmol}$, respectively, in the control group. PRA, plasma angiotensin II and aldosterone concentrations in the secondary aldosteronism group were $5.1 \pm 1.3 \mathrm{ng} / \mathrm{mL} / \mathrm{h}, \quad 27 \pm 8 \mathrm{pg} / \mathrm{mL}$, and $132 \pm 28 \mathrm{pg} / \mathrm{mL}$, which were significantly higher $(\mathrm{P}<0.05)$ than $1.2 \pm 0.6 \mathrm{ng} / \mathrm{mL} / \mathrm{h}$, $12 \pm 4 \mathrm{pg} / \mathrm{mL}$, and $78 \pm 22 \mathrm{pg} / \mathrm{mL}$, respectively, in the control group. Plasma angiotensin II concentrations significantly $(\mathrm{P}<0.01)$ correlated to ICTP $(r=0.40)$, BAP $(r=0.44)$, and NTx $(r=0.29)$, respectively.

Conclusions: The results suggest that, in patients with secondary aldosteronism, osteolytic bone metabolism increased in line with the elevated RAA system. This infers that the RAA system stimulates osteoclastic bone resorption, resulting in osteoporosis.

\section{P346}

Obesity and vitamin D deficiency and insufficiency in postmenopausal women

Vladyslav Povoroznyuk $^{1}$, Anna Musiienko ${ }^{1}$, Nataliia Dzerovych ${ }^{1}$, Roksolana Povoroznyuk $^{1}$, Oksana Ivanyk ${ }^{1}$

${ }^{1}$ Department of Clinical Physiology and Pathology of Locomotor Apparatus, D. F. Chebotarev Institute of Gerontology AMS Ukraine, Kyiv, Ukraine

Introduction: Deficiency and insufficiency of Vitamin D is a pandemic of the twenty-first century. Obesity patients have a lower level of vitamin $\mathrm{D}$, but the literature data are contradictory.

The aim of this study is to examine vitamin D deficiency and insufficiency prevalence in postmenopausal women suffering from obesity.
Materials and Methods: We examined 1007 women aged 50-89 years (age $-65.74 \pm 8.61$ years; height $-1.61 \pm 0.07 \mathrm{~m}$; weight $-70.65 \pm 13.50 \mathrm{~kg}$; body mass index $-27.27 \pm 4.86 \mathrm{~kg} / \mathrm{m}^{2}$ ) and mean $25(\mathrm{OH}) \mathrm{D}$ levels in serum-26.00 $\pm 12.00 \mathrm{nmol} / \mathrm{l}$. The women were divided into the following 6 groups depending on body mass index: I group-338 women with normal body weight, II group - 16 women with insufficient body weight, III group-382 women with excessive body weight, IV group-199 women with obesity of class I, V group- 60 women with obesity of class II, and VI group-12 women with obesity of class III. Level of 25(OH)D in serum was measured by means of an electrochemiluminescent method-Elecsys 2010 analyzer (Roche Diagnostics, Germany).

Results: In the Ukrainian women with obesity, BMI significantly influences vitamin D level, and this influence does not depend on the season. Vitamin D deficiency was found in $34.4 \%$ postmenopausal women, deficiency in $31.4 \%$ and normal levels in $34.4 \%$. $25(\mathrm{OH}) \mathrm{D}$ levels were significantly lower in women with obesity of class I $(23.60 \pm 10.24 \mathrm{ng} / \mathrm{ml})$ and obesity of class II $(22.38 \pm 10.34 \mathrm{ng} / \mathrm{ml})$, compared with women who had normal body mass $(28.24 \pm 12.99 \mathrm{ng} / \mathrm{ml}), \mathrm{p}=0.00003$. The study results reveal a weak correlation between 25(OH)D level and BMI $(r=0.15)$.

Conclusion: In patients with obesity, significant influence of BMI was found on the level of vitamin D. The presented results should be taken into account for prevention and treatment of vitamin D deficiency in obese women.

\section{P347}

Association of dorsal flat vertebra/platyspondylia as a form of vertebral dysplasia within type II collagen disorders

Maria Del Pilar Ahijado Guzman ${ }^{1}$, Raúl Veiga Cabello ${ }^{2}$, Miguel Cantalejo Moreira $^{1}$, Justo Ruiz Ruiz ${ }^{3}$, Antonio Zapatero Gaviria ${ }^{3}$

${ }^{1}$ Rheumatology, Hospital de Fuenlabrada, Fuenlabrada, Spain, ${ }^{2}$ Rheumatology, Hospital Central de la Defensa, Madrid, Spain, ${ }^{3}$ Internal Medicine, Hospital de Fuenlabrada, Fuenlabrada, Spain

Background/Purpose: Defects in the type II collagen gene are described. These predispose humans to various phenotypic combinations in families with skeletal dysplasia, and/or disease by causing deposits of microcrystals and/or early osteoarthritis and/or synovial osteochondromatosis. Flat vertebra is defined as the flattening or wedging of one or more vertebral bodies with irregular surface or with nodules of Schmorl. Calvé in 1925 firstly described it as isolated location ("The affection (...) is, I believe, to the spinal column what coxa plana is to the hip, and what Koehler's disease is to the foot"). But later varied etiologies and various pathological mechanisms have been implicated, broadening its horizon, also including the dysplastic vertebrae suggested by Rimoin in 1990 .

The purpose of this study is to demonstrate the possibility of "dorsal flat vertebra" in relation to pathologies associated with type II collagen disorders.

Methods: Medical history was taken for patients attending physician since 1994, in whom Type II collagen disease or vertebral dysplasia was suspected. It analyzes a series of 84 patients, (Mean Age 47.1 years, 43 woman and 41 man), with two adjacent flattened vertebrae according to the mentioned definition. Scheuermann's Disease according to the criteria of Sorensen was excluded, since this diagnosis is made after a minimum of three contiguous vertebrae affected. Inclusion criteria: Suspicion of flat vertebra (according to the defined criteria)-X-rays assessed by triple-observer (two rheumatologists and one radiologist) through available radiographs, including dorsal lateral spine. DEXA osteoporosis screening Exclusion criteria: Densitometric osteoporosis-Chest trauma.

Results: 
Table 1 Radiological findings

\begin{tabular}{lr}
\hline Atypical arthrosis & $64.3 \%$ \\
Synovial osteochondromatosis & $15.5 \%$ \\
Chondrocalcinosis & $7.1 \%$ \\
Calcifying enthesopathies & $4.8 \%$ \\
Exostoses & $4.8 \%$ \\
Other calcifications & $20.2 \%$ \\
Scoliosis & $33.3 \%$ \\
Family history of flat vertebrae & $3.6 \%$ \\
Dysplastic peripheral traits & $27.4 \%$ \\
Personal history of microcrystalline arthritis & $4.8 \%$ \\
\hline
\end{tabular}

Conclusions: The association of dorsal flat vertebra (according to the defined criteria) is seen as a form of vertebral dysplasia within type II collagen disorders. All patients or their parents were born in small towns, leading to suspicion in endogamy.

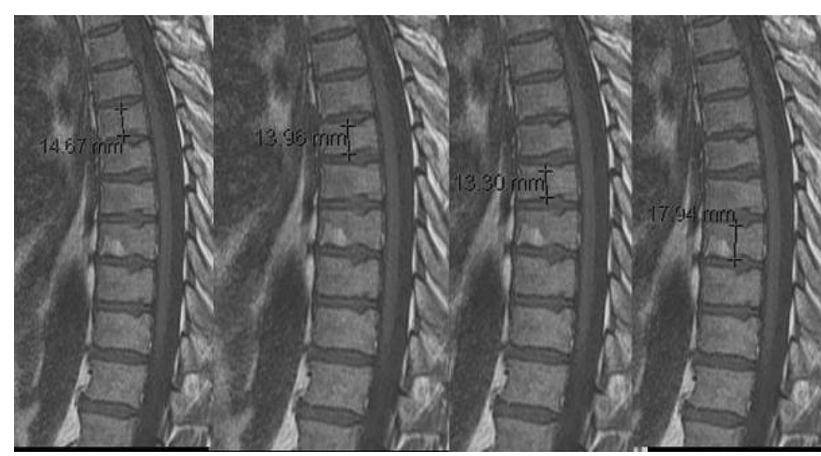

MRI of the spine with two dorsal flat vertebrae (7th and 8th)

\section{P348}

Bone parameters in experimental colitis following the corticosteroid therapy in rats

Ivana Maric $^{1}$, Ivana Smoljan ${ }^{2}$, Igor Erjavec ${ }^{3}$, Andrica Lekic ${ }^{4}$, Tamara $\overline{\text { Soic Vranic }}^{1}$, Dragica Bobinac ${ }^{1}$, Sanja Zoricic Cvek ${ }^{1}$

${ }^{1}$ Department of Anatomy, Faculty of Medicine, University of Rijeka, Rijeka, Croatia, ${ }^{2}$ Department of Cardiovascular Disease, University Hospital Rijeka, Rijeka, Croatia, ${ }^{3}$ Laboratory for Mineralized Tissues, University of Zagreb School of Medicine, Rijeka, Croatia, ${ }^{4}$ Department of Basic Medical Sciences, Faculty of Health Studies, University of Rijeka, Rijeka, Croatia

Background: Bone loss is a long-term complication of inflammatory bowel disease (IBD). Our aim was to analyze the effect of experimentally induced chronic colitis and corticosteroid therapy on bone mass and quality.

Materials and Methods: Chronic colitis was induced by onceweekly administration of increased doses of trinitrobenzenesulfonic acid (TNBS) for 4 weeks. After the last dose of TNBS, the rats were treated with a daily dose of $2 \mathrm{mg} / \mathrm{kg}$ dexamethasone for 5 days. The animals were divided into three groups: colitis, dexa, and control groups. After 5-weeks, the animals were sacrificed. The samples of femur and tibia trabecular and cortical bone were subjected to $\mathrm{mCT}$.
Data were analyzed by the one-way ANOVA. Ethical approval was obtained from the Ministry of Agriculture of the Republic of Croatia, Veterinary and Food Safety Directorate.

Results: Trabecular BV/TV values in the femur were higher in the control group $(2.6 \pm 0.82 \%)$ compared to colitis $(2.4 \pm 0.56 \%)$ and dexa groups $(2.4 \pm 0.45 \%)$. In the colitis group, the trabeculae tended to be thinner $(0.092 \pm 0.002 \mathrm{~mm})$ with higher separation $(1.9 \pm 0.08 \mathrm{~mm})$ compared to control $(0.93 \pm 0.003 \mathrm{~mm}$ $1.8 \pm 0.16 \mathrm{~mm}$ ) whereas the trabecular number was higher in the control group $(0.28 \pm 0.07)$ vs. colitis and dexa groups $(0.26 \pm 0.06$, $0.26 \pm 0.04$, respectively). Tb.Th in the tibia was similar in all groups. A decrease in Tb.N $(0.17 \pm 0.08)$ with larger spacing $(1.94 \pm 0.19 \mathrm{~mm})$ was observed in the control group compared to colitis and dexa groups $(0.19 \pm 0.04$ and $0.18 \pm 0.04$, $1.80 \pm 0.17 \mathrm{~mm}$ and $1.89 \pm 0.18 \mathrm{~mm}$, respectively).

Conclusion: The results showed no significant evidence of experimental IBD model impact on bone microstructure. Therefore, further studies are required to determine the disease severity, duration, and therapy which influence bone $\mu \mathrm{CT}$ parameters.

Supported by the University of Rijeka under the project number 13.06.1.3.46.

\section{P349}

Mechanisms of bone morphogenetic protein-7 protective effect against cold ischemia induced renal injury

Tanja Celic ${ }^{1}$, Hrvoje Omrcen ${ }^{1}$, Olga Cvijanovic-Peloza ${ }^{1}$, Ivana $\overline{\text { Maric }}^{1}$, Dragica Bobinac ${ }^{1}$

${ }^{1}$ University of Rijeka, Medical faculty, Rijeka, Croatia

Deceased donor kidneys are exposed to cold ischemic insult which makes them particularly susceptible to the effects of cold ischemic injury during hypothermic preservation resulting in high rates of delayed graft function. BMP-7 is a valuable reagent in the field of tissue regeneration and preservation under ischemic conditions. Following these insights, we investigated the effect of rhBMP-7 on graft preservation during cold ischemia.

The study was conducted on an experimental model of kidney cold ischemia in rats. Kidneys were perfused with: saline, University of Wisconsin (UW), rhBMP-7 or rhBMP-7 + UW and exposed to cold ischemia during 6, 12 and $24 \mathrm{~h}$.

In tubular epithelial cells of kidneys perfused with rhBMP-7 and rhBMP-7 + UW solution the expression of BMP-7 and E-cadherin was observed after $24 \mathrm{~h}$ of cold ischemia. In kidneys which were not perfused with rhBMP-7 high expression of TGF- $\beta 1$ and $\alpha$-SMA was found. Also, in kidneys perfused with rhBMP-7 solution statistically higher levels of Smad1, Smad5 and Smad8 mRNA expression were proved.

BMP-7 maintains the morphology of kidney tissue better than UW solution during $24 \mathrm{~h}$ of cold ischemia. BMP-7 prevents epithelial to mesenchymal transformation and consequently maintains epithelial phenotype of tubular cells.

Keywords: cold ischemia, bone morphogenetic protein 7, acute renal injury, kidney transplantation

\section{P350}

Bone density in duchenne muscular dystrophy: longitudinal changes Silvia Vai ${ }^{1}$, Barbara Pasanisi ${ }^{2}$, Giovanni Baranello ${ }^{2}$, Francesca Broggi $^{1}$, Maria Luisa Bianchi ${ }^{1}$

${ }^{1}$ Istituto Auxologico Italiano IRCCS, Milano, Italy, ${ }^{2}$ Fondazione Istituto Neurologico C. Besta IRCCS, Milano, Italy 
Duchenne muscular dystrophy (DMD) is a severe X-linked recessive disorder due to mutations in the dystrophin gene. There is no cure, but glucocorticosteroids (GCs) improve muscle strength, prolong ambulation, and significantly extend life.

We studied the yearly evolution of bone mineral density (BMD) in 64 boys (aged $11.2 \pm 2.8$ years) with genetic diagnosis of DMD, on GC therapy (starting age $6.8 \pm 2.4$ years).

BMD was measured at spine (LS) and total body less head (TBLH), always with the same scanning and analyzing protocol, on a Hologic Discovery DXA instrument, at baseline (T0) until up to 5 years later (T5y; in 37 boys).

All DMD children had reduced LS and TBHL BMD at T0, and the mean Z-score (calculated with respect to healthy age-matched boys) progressively decreased, from -1.6 at $\mathrm{T} 0$ to -2.7 at T5y.

An inverse correlation was observed between $\mathrm{Z}$-scores and length of GC therapy $(\mathrm{r}=-0.533, \mathrm{p}=0.005)$. In 29 non-ambulant boys, the loss-of-ambulation age (11.8 \pm 2 years) was correlated to TBLH $(\mathrm{r}=0.409, \mathrm{p}=0.02)$ and lower limb BMD $(\mathrm{r}=0.378, \mathrm{p}=0.04)$ at T0.

Separately studying two age groups, we observed an increasing BMD (from 450 to $530 \mathrm{mg} / \mathrm{cm}^{2}$ ) in boys up to 7 years (group A, 33 boys, age $5.2 \pm 1.1$ years) and stable or decreasing BMD in boys over 7 years (group B, 31 boys, age $9.3 \pm 2.4$ years). Group A showed a negative correlation between length of GC therapy and change of TBLH $(r=-0.532, p=0.013)$ and lower limb BMD $(\mathrm{r}=0.562, \mathrm{p}=0.01)$ over 4 years.

In conclusion:

- DMD boys have lower BMD than healthy age-matched boys

- BMD only increases in younger DMD children

- the observed correlations confirm the negative influence of GC therapy on BMD and suggest a negative influence of BMD decrease on a relevant hallmark of disease progression (loss of ambulation).

Keywords: Duchenne muscular dystrophy, bone mineral density, glucocorticosteroids

\section{P351}

Osteosarcoma cells show laminopathic nuclear phenotypes: study on the nuclear envelope composition

\section{Enrica Urciuoli ${ }^{1}$, Stefania Petrini ${ }^{2}$, Valentina D'Oria ${ }^{2}$, Barbara} Peruzzi $^{1}$

${ }^{1}$ Multifactorial Diseases Unit - Research Laboratories, Bambino Gesù Children's Hospital, IRCCS, Rome, Italy, ${ }^{2}$ Confocal Microscopy Core Facility, Bambino Gesù Children Hospital, IRCCS, Rome, Italy

The nuclear lamins are essential for the maintenance of nuclear shape and mechanics, gene regulation, chromatin organization, chromosome positioning, DNA replication and repair. Mutations in lamin genes have been identified in a heterogeneous spectrum of rare human diseases commonly known as "laminopathies" associated with nuclear envelope defects and deregulation of cellular functions. Alterations in the expression of lamins and nuclear lamina-associated proteins are recently explored in cancer development and tumor progression. Given that osteosarcoma is the only neoplasm described in the literature in association with laminopathies, the objective of this study was to investigate the expression of nuclear envelope proteins in osteosarcoma. To this aim, we used human primary osteoblasts, as control, and osteosarcoma cell lines with increasing aggressiveness to assess A-type, B-type lamins and emerin expression by confocal microscopy analysis. We observed a higher percentage of dysmorphic nuclei in osteosarcoma cells in comparison to normal osteoblasts, finding all the hallmarks of laminopathic features as folds and blebs, honeycombs, donut nuclei and micronuclei. As regarding nuclear envelope proteins, A-type lamin and emerin expression was significantly higher in normal osteoblasts and decreased along with osteosarcoma aggressiveness, starting from low aggressive SaOS2 cells to high metastatic 143B cells. B-type lamin was significantly more expressed in all osteosarcoma cell lines in comparison to normal osteoblasts and correlated with tumor aggressiveness, being highly expressed in SaOS2 cells and low expressed in 143B cells. An altered functionality of nuclear envelope proteins in osteosarcoma cells was also confirmed by the assessment of MKL1 subcellular localization and actin polymerization, resulted delayed in high aggressive, laminA low 143B cells. In conclusion, we described for the first time laminopathic nuclear phenotypes in osteosarcoma cells, providing evidence for a deregulated nucleoskeleton architecture likely involved in the cytoskeletal rearrangement, the transcriptional activity and the mechanics of this tumor.

\section{P352}

Bone densitometric analysis in two children with Hadju-Cheney syndrome

Jean De Schepper ${ }^{1}$, Armand Laumen ${ }^{2}$, Caroline Ernst ${ }^{3}$, Kathlijne Keymolen $^{4}$, Marjan De Rademaeker ${ }^{4}$, Olivia Louis ${ }^{3}$

${ }^{1}$ Pediatrics, UZ Brussel, Brussels, Belgium, ${ }^{2}$ Orthopedics, UZ Brussel, Brussels, Belgium, ${ }^{3}$ Radiology, UZ Brussel, Brussels, Belgium, ${ }^{4}$ Genetics, UZ Brussel, Brussels, Belgium

Hadju-Cheney syndrome (CHS) is beside osteoporosis, skeletally characterized by acro-osteolysis. The experience with intravenous bisphosphonates during childhood is limited to 4 CHS patients.

The aim is to report the result of bone densitometric assessments in two young CHS children and the effect of 2 years of pamidronate treatment in the oldest patient.

Patient 1 was born at 35 weeks (length $49 \mathrm{~cm}$ ). Prenatally bilateral renal cysts were diagnosed. Mild facial dysmorphism and a bicuspid aortic valve were present at birth. The diagnosis was genetically confirmed at the age of 3 years (c.6792C $>$ G (p.Tyr2264X) mutation of the NOTCH2 gene), when presenting with painful fingers, related to osteolysis of the distal phalanx of the 2nd and 3rd fingers. In addition, wormian bones at the skull and bowing of the fibula were observed. Given a lumbar spine (LS) BMD loss of $7 \%$ in the preceding year, pamidronate infusions ( $1 \mathrm{mg} / \mathrm{kg}$ every 3 months) were initiated at the age of 7 years. LS BMD remained at -2.3 SDS, whereas radial trabecular BMD (by pQCT) increased from +2.8 to +4.0 SDS. Height decreased to -1.73 SDS. Patient 2 was born at 38 weeks (length $46 \mathrm{~cm}$ ). She was treated for congenital hip luxation and operated for umbilical hernia during infancy. Diagnosis of CHS was made at the age of 7.5 years (c.6403_6404delCT (pLeu2135fs) mutation) at referral for unexplained proportionate short stature (standing height -2.5 SDS), dysmorphism, renal cysts and osteolysis of the distal phalanx (1st and 2th finger) and scoliosis. Lumbar spine BMD z-score was -2.3 .

In conclusion, the finding of increased trabecular BMD at the distal radius is in accordance with cortical bone trabecularization. The used pamidronate treatment schedule could not increase the spinal BMD in the boy with several skeletal anomalies.

Keywords: densitometry osteoporosis

\section{P353}

The effect of growth hormone treatment in a child with a novel TRPS1 gene mutation

Yael Levy-Shraga ${ }^{1,2}$, Shlomo Wientroub ${ }^{2,3}$, Leonid Zeitlin ${ }^{2,3}$ 
${ }^{1}$ Pediatric Endocrine and Diabetes Unit, Safra Children's Hospital, Ramat Gan, Israel, ${ }^{2}$ Sackler Faculty of Medicine, Tel Aviv University, Tel Aviv, Israel, ${ }^{3}$ Pediatric Orthopaedics, Dana Children's Hospital, Tel Aviv, Israel

Background: Tricho-rhino-phalangeal syndrome (TRPS) is an autosomal dominant disorder characterized by craniofacial and skeletal malformations including short stature, cone shaped phalangeal epiphyses of the hand and Perthes-like changes of the hip. We report a case of a boy with TRPS and severe growth hormone deficiency.

Case presentation: The patient presented at age 4 years for evaluation of short stature $(87 \mathrm{~cm},-3.7 \mathrm{SD})$. On physical examination characteristic phenotype of TRPS was found: sparse thin hair, lateral rarefaction of the eyebrows, a pear-shaped nose, a long philtrum and a thin upper lip. Radiographs of the left hand at a chronological age of 4.8 years revealed a bone age of 2.8 years. Furthermore, cone-shaped epiphyses were identified. Two growth hormone stimulation tests revealed severe growth hormone $(\mathrm{GH})$ deficiency (pick $\mathrm{GH}$ after stimulation of 3.3 and $1.7 \mathrm{mcg} / \mathrm{l})$ with low IGF1 $(30.1 \mathrm{ng} / \mathrm{ml}$, normal 35-217). Further work up revealed bilateral Perthes-like changes of the hips on pelvis radiographs. GH treatment was initiated $(0.03 \mathrm{mg} /$ $\mathrm{kg} /$ day).

Genetic analysis: Sanger sequencing of the TRPS1 gene was performed identified a heterozygous sequence variant, c. $3698 \mathrm{G}>\mathrm{A}$, in exon 7. This missense mutation leads to an amino acid change at codon 1233, (p.Cys1233Tyr). To the best of our knowledge, this mutation was not described previously. Two prediction programs (PolyPhen, SIFT) rat this mutation as disease causing. Both his parents did not carry this mutation.

Follow up: Growth velocity improved under GH treatment $(8-9 \mathrm{~cm} /$ year). His current height at the age of 7.5 years is $116.4 \mathrm{~cm}$ (-1.6 SD). Furthermore, marked improvement of the femoral epiphysis was shown on sequential pelvis radiographs.

Conclusion: Our observations may suggest the benefits of growth hormone treatment on both height and epiphyses status in TRPS. Further studies are needed to support this observation.

\section{$\mathbf{P 3 5 4}$}

Dysplasia epiphysealis hemimelica of the proximal femur

Leanid Hlazkin ${ }^{1}$, Iryna Maliovanaya ${ }^{1}$, Mikhail Mikhovich ${ }^{1}$, Aleh Sakalouski ${ }^{2}$

${ }^{1}$ Orthopaedics and Traumatology, Mogilev Regional Children's Hospital, Mogilev, Belarus, ${ }^{2}$ Belarus Republican Scientific and Practical Centre for Traumatology and Orthopaedics, Minsk, Belarus

Introduction: Dysplasia epiphysealis hemimelica or Trevor disease is a rare developmental disorder of epiphyseal osteocartilaginous growth in children, usually in the lower limbs. The lesion consists of osteocartilaginous tissue arising from the epiphysis and usually is hemimelic. The most common sites of involvement are the distal femur, proximal tibia, talus, and tarsal navicular.

Objective was to describe the case of this pathology in unusual localization.

Materials and Methods: Child P., 5 years old, had complaints about range of motion limitation in the right hip. Maximal passive hip flexion was 118 degrees. After radiographic evaluation additional bone tissue at the lateral part of the proximal femur physis was founded. Computed tomography helped in more exact localization of neoplasm - anterolateral part of the physis, and in establishing of the diagnosis.

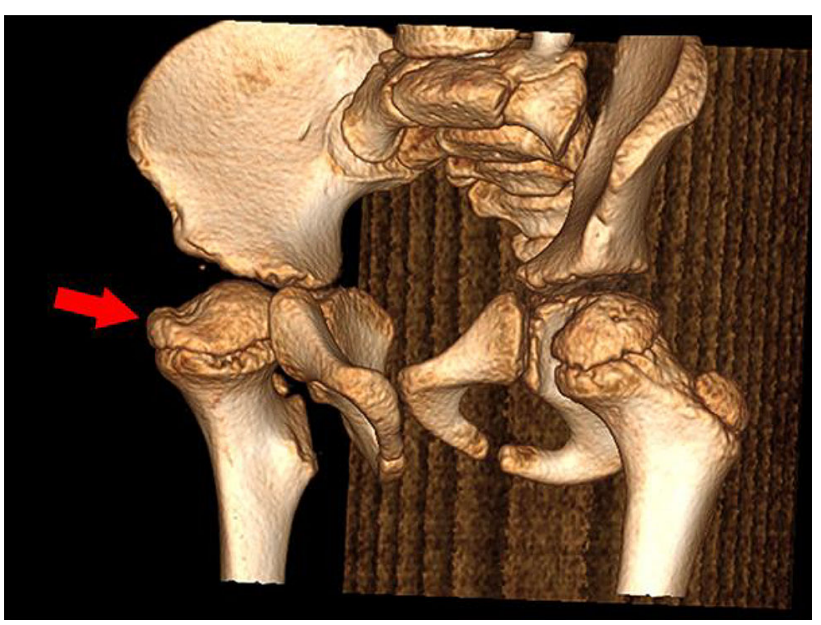

CT-image

Arthrotomy of the right hip and excision of the neoplasm was performed. The size of it was $3 * 3 * 2.5 \mathrm{~cm}$. Immobilization was not needed. Child was outpatient 8 days after surgery with recommendations not to load lower limb for 3 weeks.

Results and Conclusion: After 2 months patient had no complaints and walked without limping. The ROM was full and motions in right hip was painless.

Discussion: Although the incidence of this disease has been reported as 1 per million, physician must take it into account when he observing a child with limb deformity, limited range of motion in the joint, limp, muscle wasting and even limb length discrepancy. Radiographic evaluation may help in differential diagnosis.

Keywords: Dysplasia Epiphysealis Hemimelica, Unusual Localization, ROM Limitation

\section{P355}

Quantification of four mouse periostin isoforms with an in depth characterized sandwich ELISA

Elisabeth Gadermaier $^{1}$, Jacqueline Wallwitz ${ }^{1}$, Gabriela Berg ${ }^{1,2}$, Gottfried Himmler $^{1}$

${ }^{1}$ The Antibody Lab GmbH, Vienna, Austria, ${ }^{2}$ Biomedica Medizinprodukte $\mathrm{GmbH} \&$ Co KG, Vienna, Austria

Objective: Periostin (osteoblast-specific factor OSF-2) is an extracellular matrix protein which belongs to the FAS1 superfamily. It consists of a conserved $\mathrm{N}$-terminus and a $\mathrm{C}$-terminal region which is affected by alternative splicing leading to different isoforms. In bone, periostin is mainly expressed in the periosteum of long bones and in osteocytes. It has a general function in bone homeostasis and is regulated by factors like mechanical stimulation, $\mathrm{PTH}$, growth factors and cytokines. It is upregulated during bone development and remodeling, and it acts on bone formation by increasing osteoblast function. To further study periostin in preclinical mouse models, there is the need for a high-quality assay for periostin quantification.

Methods: We developed a sandwich ELISA employing polyclonal and monoclonal anti-periostin antibodies. Antibodies were characterized by epitope mapping with microarray technology. Assay parameters like specificity, dilution linearity and spike recovery were determined, and different sample matrices were tested (serum, plasma, urine). The binding pattern to periostin isoforms was analyzed.

Results: The novel assay is calibrated with mouse periostin isoform 1 and detects periostin in mouse serum and plasma, but not in mouse urine. It employs only minimal amounts $(<2 \mu \mathrm{l})$ of mouse 
sample for the quantification of periostin. Linear epitopes are located in the fourth FAS1 domain for the monoclonal coating antibody, or distributed over the whole periostin sequence for the polyclonal detection antibody. All assay characteristics (specificity, dilution linearity, spike recovery) meet the standards of acceptance. From five currently known mouse periostin isoforms, four are recognized in the assay, binding to isoform 4 is not known yet.

Conclusion: This novel mouse periostin ELISA provides a reliable and accurate tool for the quantification of mouse periostin in minute amounts of serum and plasma samples.

Keywords: periostin, ELISA, mouse

\section{P356}

Low sound wave propagation in cortical bone as a novel, noninvasive imaging biomarker in children and adolescents with hypophosphatemic rickets

Johannes Schneider ${ }^{1}$, Adalbert Raimann ${ }^{2}$, Andrea Boni-Mikats ${ }^{3}$, Martin Krssak ${ }^{4}$, Radka Klepochovás, Gabriele Haeusler ${ }^{2}$, Janina Patsch $^{3}$, Kay Raum ${ }^{1}$

${ }^{1}$ Berlin-Brandenburg Center for Regenerative Therapies, Charité Universitätsmedizin Berlin, Berlin, Germany, ${ }^{2}$ Department of Pediatrics and Adolescent Medicine, Medical University of Vienna, Vienna, Austria, ${ }^{3}$ Department of Biomedical Imaging and ImagedGuided Therapy, Division of General Radiology and Pediatric Radiology, Medical University of Vienna, Vienna, Austria, ${ }^{4}$ Division of Endocrinology and Metabolism, Department of Internal Medicine III, Medical University of Vienna, Vienna, Austria, ${ }^{5}$ Department of Biomedical Imaging and Imaged-Guided Therapy, High Field MR Centre, Medical University of Vienna, Vienna, Austria

Purpose: The assessment of ultrasound wave propagation in cortical bone is a novel, non-invasive quantitative imaging tool in bone research. The purpose of this study was to determine wave propagation properties in the distal extremities in pediatric patients with $\mathrm{X}$-linked hypophosphatemic rickets (XLH) and healthy controls.

Methods and Materials: Following study approval by institutional review and written informed consent, we performed quantitative bone ultrasound in children, adolescents and young adults with XLH and healthy controls. The majority of patients and controls also underwent high-resolution peripheral quantitative computed tomography (HR$\mathrm{pQCT}$ ) of the radius and tibia as a standard of reference. Bone ultrasound was performed at the distal radius and the distal tibia according to a structured examination protocol, using a dedicated linear $1 \mathrm{MHz}$ transducer with one receiver array and two transmitter arrays.. Based on bidirectional axial transmission and propagation of ultrasound waves, the 'first arriving signal velocity' (vFAS) was measured and post-processed. HR-pQCT images were reviewed by a board-certified radiologist, data were evaluated by the manufacturer's quantification software.

Results: Quantitative bone ultrasound of wave propagation was feasible in all study participants. At the distal radius, we found vFAS to be significantly lower in XLH patients than in healthy controls $(-6.8 \%$; ANOVA: $p=0.0013)$. A similar reduction of vFAS was observed in the distal tibia $(-6.5 \%$; ANOVA: $p=0.0009)$. HRpQCT scans revealed heterogenous microstructural deficits in both, cortical and trabecular bone.

Conclusion: Quantitative bone ultrasound of wave propagation is a promising imaging biomarker in children and adolescents with hypophosphatemic rickets.

\section{P357}

Somatic activating mutations in $M A P 2 K 1$ cause melorheostosis

Heeseog Kang ${ }^{1}$, Smita $\mathrm{Jha}^{2,3}$, Zuoming Deng ${ }^{4}$, Nadja Fratzl-Zelman ${ }^{5}$, $\overline{\text { Wayne Cabral }}^{1}$, Aleksandra Ivovic ${ }^{6}$, Françoise Meylan ${ }^{6}$, Eric
Hanson $^{7}$, Eileen Lange ${ }^{8}$, James Katz ${ }^{8}$, Paul Roschger ${ }^{5}$, Klaus Klaushofer ${ }^{5}$, Edward Cowen ${ }^{9}$, Richard Siegel ${ }^{6}$, Timothy Bhattacharyya $^{2}$, Joan Marini ${ }^{1}$

${ }^{1}$ Section on Heritable Disorders of Bone and Extracellular Matrix, National Institute of Child Health and Human Development, National Institutes of Health, Bethesda, United States, ${ }^{2}$ Clinical and Investigative Orthopedics Surgery Unit, National Institute of Arthritis and Musculoskeletal and Skin Diseases, National Institutes of Health, Bethesda, United States, ${ }^{3}$ Program in Reproductive and Adult Endocrinology, Eunice Kennedy Shriver National Institute of Child Health and Human Development, National Institutes of Health, Bethesda, United States, ${ }^{4}$ Biodata Mining and Discovery Section, Office of Science and Technology, National Institute of Arthritis and Musculoskeletal and Skin Diseases, National Institutes of Health, Bethesda, United States, ${ }^{5}$ Ludwig Boltzmann Institute of Osteology at the Hanusch Hospital of WGKK, and AUVA Trauma Center Meidling, 1st Medical Department Hanusch Hospital, Vienna, Austria, ${ }^{6}$ Immunoregulation Section, Autoimmunity Branch, National Institute of Arthritis and Musculoskeletal and Skin Diseases, National Institutes of Health, Bethesda, United States, ${ }^{7}$ Immunodeficiency and Inflammation Unit, Autoimmunity Branch, National Institute of Arthritis and Musculoskeletal and Skin Diseases, National Institutes of Health, Bethesda, United States, ${ }^{8}$ National Institute of Arthritis and Musculoskeletal and Skin Diseases, National Institutes of Health, Bethesda, United States, ${ }^{9}$ Dermatology Branch, National Institute of Arthritis and Musculoskeletal and Skin Diseases, National Institutes of Health, Bethesda, United States

Melorheostosis is a rare dysostosis of unknown etiology, with characteristic radiographs of "dripping candle wax". Because of its sporadic occurrence, somatic mutations have been hypothesized as causative, but bone tissue was not previously investigated. We recruited 15 unrelated melorheostosis patients, who underwent paired biopsies of affected and contralateral unaffected bone. Using exome sequencing, we identified somatic mosaic mutations in $M A P 2 K 1$, encoding the Mitogen-Activated Protein Kinase Kinase 1 (MEK1), in gDNA from affected, but not unaffected bone, of 8 of 15 patients, and confirmed these mutations by ddPCR. Mosaicism for MAP2K1 mutations was also detected in skin overlying bone lesions in some patients, but not in blood samples. Located within the negative regulatory domain of MEK1, these activating mutations (p.Q56P, p.K57E, p.K57N) were previously identified in various malignancies. Immunohistochemical analysis in affected bone displayed a mosaic pattern of activation of ERK1/2, the substrates of MEK1. In addition, flow cytometry of osteoblasts cultured from affected bone demonstrated two cell populations with distinct ph-ERK1/2 levels. Histology of melorheostotic bone was distinctive, with exuberant initial deposition of distinctive parallel layers of primary lamellar bone, followed by intense bone remodeling with increased cellularity. The elevated RANKL/OPG ratio in affected osteoblasts correlates with increased osteoclast number and bone remodelling. In cultured osteoblasts, the $M A P 2 K 1$ mutations enhanced cell proliferation with increased cyclin D expression. Interestingly, MAP2K1 mutations inhibited BMP2-mediated osteoblast differentiation and mineralization in vitro, underlying the significant elevation of unmineralized osteoid in melorheostotic bone. Melorheostosis, a benign bone overgrowth condition, is caused by somatic mosaicism for $M A P 2 K 1$ activating mutations previously identified as cancer-associated. Increased MEK1-ERK1/2 signaling enhanced osteoblast growth and bone remodeling, while inhibiting osteoblast differentiation and bone. This is the first demonstration that the $M A P 2 K 1$ oncogene is important to human bone formation. MEK1 inhibition, already in trials for cancer, may be a treatment for melorheostosis.

\section{P358}

Lentiviral-mediated gene transfer of TCIRG1 to correct osteoclast function in Autosomal Recessive Osteopetrosis 
Sara Penna ${ }^{1,2}$, Valentina Capo $^{1}$, Elena Draghici ${ }^{1}$, Lucia Sergi Sergi ${ }^{1}$, Eleonora Palagano ${ }^{3,4}$, Erika Zonari ${ }^{1}$, Francesca Ficara ${ }^{3,5}$, Cristina Sobacchi $^{3,5}$, Bernhard Gentner ${ }^{1}$, Anna Villa ${ }^{1,5}$

${ }^{1}$ San Raffaele Telethon Institute for Gene Therapy, Milan, Italy, ${ }^{2}$ School of Medicine and Surgery, University of Milano-Bicocca, Monza, Italy, ${ }^{3}$ Humanitas Clinical and Research Center, Rozzano, Italy, ${ }^{4}$ Department of Medical Biotechnologies and Translational Medicine, University of Milan, Milan, Italy, ${ }^{5}$ Milan Unit, CNR, Milan, Italy

Autosomal recessive osteopetrosis (ARO) is a group of rare genetic disease characterized by dysfunctional osteoclasts. The majority of ARO patients (55\%) have mutations in TCIRG1 gene, encoding the a3 subunit of V-ATPase, necessary for bone resorption. ARO patients present a limited bone marrow cavity, resulting in extramedullary hematopoiesis and increased number of circulating CD34 + cells. Bone marrow transplantation is the only therapeutic option for ARO patients, but HLA-matched donors are rare and condition regimens are toxic. Therefore, gene therapy (GT) represents an alternative cure for these patients.

We developed two lentiviral vectors, driving TCIRG1 expression under the control of the PGK promoter, with or without the $d N G F R$ marker gene. We transduced Lin- isolated from splenocytes of 12-daysold Tcirg 1-mutated mice, the ocloc spontaneous model. Differentiation and resorption assays were performed in vitro on Tcirg1-corrected cells showing rescue of osteoclasts resorptive function. Tcirgl-corrected linwere injected intra-liver into irradiated $o c-o c$ newborn mice at 2 days of life. Untreated $o c-o c$ mice have a severe osteopetrotic phenotype with a life expectancy of 2-3 weeks. After GT, mice show an improved lifespan and absence of circling behavior. However they have a smaller body weight compared to wild-type littermates. In parallel, we transduced and expanded CD34+ cells isolated from the peripheral blood of five ARO patients. Transduced cells were differentiated in vitro towards myeloid lineage and forced to osteoclasts differentiation. Transduced cells plated on bone slice showed restoration of bone resorption capacity. Moreover, in vitro expanded ARO CD34+ transduced cells were transplanted into immunodeficient NSG mice to evaluate engraftment capacity and differentiation potential. We observed up to $30 \%$ of human CD45 + cells in NSG peripheral blood 13 weeks after transplantation.

Our results suggest that gene therapy could represent a feasible alternative treatment for ARO patients when HLA-matched donor is not available.

Keywords: osteopetrosis, gene therapy, osteoclasts

\section{P359}

Impaired proteoglycan glycosylation, elevated TGF- $\beta$ signaling, and abnormal osteoblast differentiation as the basis for bone fragility in a mouse model for gerodermia osteodysplastica

Uwe Kornak $^{1}$, Wing-Lee Chan ${ }^{1}$, Magdalena Steiner ${ }^{1}$, Tomasz M. Witkos $^{2}$, Bettina Willie ${ }^{3}$, Thorsten Schinke ${ }^{4}$, Antonio Rossi ${ }^{5}$, Michael Amling ${ }^{4}$, Martin Lowe ${ }^{2}$, Danny Chan ${ }^{6}$, Stefan Mundlos ${ }^{1}$

${ }^{1}$ Institut für Medizinische Genetik, Charité-Universitätsmedizin Berlin, Berlin, Germany, ${ }^{2}$ Faculty of Biology, Medicine and Health, University of Manchester, Manchester, United Kingdom, ${ }^{3}$ Department of Pediatric Surgery, McGill University, Montreal, Canada, ${ }^{4}$ Department of Osteology and Biomechanics, University Medical Center Hamburg-Eppendorf, Hamburg, Germany, ${ }^{5}$ Department of Molecular Medicine, University of Pavia, Pavia, Italy, ${ }^{6}$ LKS Faculty of Medicine, The University of Hong Kong, Hong Kong, China

Gerodermia osteodysplastica (GO) is characterized by skin laxity and early-onset osteoporosis. GORAB, the responsible disease gene, encodes a small Golgi protein of poorly characterized function. To circumvent neonatal lethality of the Gorab ${ }^{\text {Null }}$ full knockout, Gorab was conditionally inactivated in mesenchymal progenitor cells (Prx1cre), pre-osteoblasts (Runx2-cre), and late osteoblasts/osteocytes (Dmp1-cre), respectively. While in all three lines a reduction in trabecular bone density was evident, only Gorab $^{\operatorname{Prx} 1}$ and Gorab ${ }^{\text {Runx2 }}$ mutants showed dramatically thinned, porous cortical bone and spontaneous fractures. Collagen fibrils in the skin of Gorab ${ }^{\text {Null }}$ mutants and in bone of Gorab ${ }^{\operatorname{Prx} 1}$ mutants were disorganized, which was also seen in a bone biopsy from a GO patient. Measurement of glycosaminoglycan contents revealed a reduction of dermatan sulfate levels in skin and cartilage from Gorab ${ }^{\text {Null }}$ mutants. In bone from Gorab $^{\operatorname{Prx} 1}$ mutants total glycosaminoglycan levels and the relative percentage of dermatan sulfate were both strongly diminished. Accordingly, the proteoglycans biglycan and decorin showed reduced glycanation. Also in cultured GORAB-deficient fibroblasts reduced decorin glycanation was evident. The Golgi compartment of these cells showed an accumulation of decorin, but reduced signals for dermatan sulfate. Moreover, we found elevated activation of TGF-b in $G o r a b^{\text {Prx1 }}$ bone tissue leading to enhanced downstream signalling, which was reproduced in GORAB-deficient fibroblasts. Our data suggest that the loss of Gorab primarily perturbs pre-osteoblasts. GO may be regarded as a congenital disorder of glycosylation affecting proteoglycan synthesis due to delayed transport and impaired posttranslational modification in the Golgi compartment.

\section{P360}

MSC-seeded biomimetic scaffolds as factory of human soluble RANKL in Rankl-deficient osteopetrosis

Ciro Menale $^{1,2}$, Eleonora Palagano ${ }^{2,3}$, Stefano Mantero ${ }^{1,2}$, Elisabetta Campodoni $^{4}$, Marco Erreni ${ }^{2}$, Francesca Schena ${ }^{5}$, Antonio Inforzato ${ }^{6}$, Paolo Vezzoni ${ }^{1,2}$, Anna Tampieri ${ }^{4}$, Monica Sandri ${ }^{4}$, Anna Villa ${ }^{1,2}$, Cristina Sobacchi ${ }^{1,2}$

${ }^{1}$ IRGB-CNR UOS Milan, Rozzano-Milan, Italy, ${ }^{2}$ Humanitas Clinical and Research Center, Rozzano, Italy, ${ }^{3}$ Department of Medical Biotechnologies and Translational Medicine, University of Milan, Milan, Italy, ${ }^{4}$ ISTEC-CNR, Faenza, Italy, ${ }^{5}$ Laboratory of Immunology and Rheumatic Diseases, Pediatrics II, Gaslini Institute, Genova, Italy, ${ }^{6}$ Humanitas University, Rozzano, Italy

Biomimetic scaffolds have been hitting the scene since some decades for their versatility in terms of chemical composition and physical properties, which can be ad hoc defined based on the intended specific application. Among different applications, the exploitation of the biomaterial itself or of hybrid cell-seeded constructs as a source of bioactive soluble factors can be envisaged. We reasoned pursuing this strategy might be interesting in the setup of a cell-based therapy for RANKL-deficient Autosomal Recessive Osteopetrosis, a very rare skeletal genetic disease in which lack of the essential osteoclastogenic factor RANKL impedes osteoclast formation. The exogenously provided RANKL cytokine is effective in achieving osteoclast formation and function in vitro and in vivo, thus we produced murine Rankl-/- MSCs overexpressing human soluble RANKL (hsRL) because of lentiviral transduction (LvhsRL). In this work, we developed a 3D culture system on a biocompatible scaffold closely reproducing the physicochemical properties of bone. Murine MSCs seeded on it showed improved properties, as compared to 2D culture, in terms of proliferation and hsRL production, with respect to LvhsRL-transduced cells. Once implanted subcutaneously in Rankl-/- mice, these cell constructs were well tolerated, colonized also by cells of the host and intensely vascularized. Of note, specifically in the bone of Rankl-/- mice that carried scaffolds with either WT or LvhsRL-transduced Rankl-/- MSCs, we observed the formation of TRAP + cells, likely due to sRL released from the scaffolds into the circulation. Our strategy has the potential to elicit an effect on bone, but 
further work is needed to maximize the possible benefit achieved and to improve the skeletal pathology in treated $\mathrm{Rankl}^{-1-}$ mice.

Keywords: Osteopetrosis, MSC, Rankl, Scaffold

\section{P361}

Disruption of a PTHrP-SIK3 mediated pathway alters mTOR signaling and causes a spectrum of skeletal dysplasias

Fabiana Csukasi ${ }^{1}$, Ivan Duran ${ }^{1}$, Maya Barad ${ }^{1}$, Jorge Martin ${ }^{1}$, Daniel H Cohn $^{1}$, Pavel Krejci ${ }^{2}$, Deborah Krakow ${ }^{1}$

${ }^{1}$ University of California Los Angeles, Los Angeles, United States, ${ }^{2}$ Masaryk University, Brno, Czech Republic

Mammalian target of rapamycin (mTOR) is an evolutionarily conserved pathway that functions in integrating numerous inputs to regulate cellular metabolism, growth, proliferation and survival. We studied a family with a previously undescribed skeletal disorder with similarities to Jansen Type Methaphyseal Chondrodysplasia, a disorder caused by constitutive activation of the PTH/PTHrP receptor. Exome analysis identified homozygosity for a missense mutation (p. Arg129Cys) altering the catalytic domain, and thereby the Ser/Thr kinase activity, of Salt-Inducible Kinase 3 (SIK3). Biochemical analysis of cells from the affected individuals showed decreased activity of mTOR complexes mTORC1 and mTORC2. SIK3 directly interacted with DEPTOR, a negative regulator common to both mTOR complexes, and as a result of SIK3 deficiency DEPTOR accumulated to high levels in mutant cells due to its impaired interaction with the proteasome machinery. Similarly to SIK3 deficient cells, cells from a patient with Jansen's showed elevated levels of DEPTOR besides reduced levels of phosphorylation of SIK3, indicating a common mechanism of disease. Furthermore, PTHrP treatment of wild-type chondrocytes also induced the accumulation of DEPTOR and the reduction of phospho-SIK3 supporting that PTHrP signals through SIK3 to control DEPTOR degradation. Our results reveal a role for SIK3 in the regulation of mTOR activity in response to PTHrP signaling and establish that SIK3 activity during development is essential for skeletogenesis.

Keywords: skeletal dysplasias, rare diseases, mTOR, SIK3, DEPTOR

\section{P362}

The high bone mass phenotype of Lrp5-mutant mice is not affected by megakaryocyte depletion

Timur Yorgan ${ }^{1}$, Nele Vollersen ${ }^{1}$, Jean-Pierre David ${ }^{1}$, Michael Amling $^{1}$, Thosten Schinke ${ }^{1}$

${ }^{1}$ Department of Osteology and Biomechanics, University Medical Center Hamburg-Eppendorf, Hamburg, Germany

Objective: The putative Wnt co-receptor Lrp5 is a key regulator of bone remodeling and activating mutations cause elevated bone formation in mice and humans. We have previously reported increased numbers of bone marrow megakaryocytes in mice carrying a high bone mass mutation in $\operatorname{Lrp} 5\left(\operatorname{Lrp} 5^{\mathrm{G} 170 \mathrm{~V} / \mathrm{G} 170 \mathrm{~V}}\right)$. Since megakaryocytes were reported to increase bone formation, and since we detected expression of the osteoanabolic molecule Wnt1 in this cell type, we addressed the question, if the bone remodeling phenotype of $L r$ $p 5^{\mathrm{G} 170 \mathrm{~V} / \mathrm{G} 170 \mathrm{~V}}$ mice is dependent on megakaryocyte-derived signals.

Methods: $M p l^{\mathrm{hlb} 219 / 219} / \operatorname{Lrp}^{\mathrm{G} 170 \mathrm{~V} / \mathrm{G} 170 \mathrm{~V}}$ mice with a high bone mass and lacking megakaryocytes as well as $\mathrm{Wnt}^{\text {flox/flox}} / \mathrm{Pf4}-\mathrm{Cr} \mathrm{e}^{+}$ mice with a megakaryocyte-specific Wnt1-deficiency were characterized by $\mu \mathrm{CT}$ and histomorphometric analysis.

Results: $M p l^{\mathrm{hlb} 219 / 219} / L r p 5^{\mathrm{G} 170 \mathrm{~V} / \mathrm{G} 170 \mathrm{~V}}$ mice displayed a high bone mass comparable to $M p l^{\mathrm{wt} / \mathrm{wt}} / \operatorname{Lrp}^{\mathrm{G} 170 \mathrm{~V} / \mathrm{G} 170 \mathrm{~V}}$ mice (BV/TV: $37.0 \pm 3.7$ vs. $34.7 \pm 3.8 \%$, p-value: 0.34 ). Indeed, despite a nearly complete depletion of megakaryocytes, no structural or cellular difference in the femur and vertebral body was observed compared to
$M p l^{\mathrm{wt} / \mathrm{wt}} / L r p 5^{\mathrm{G} 170 \mathrm{~V} / \mathrm{G} 170 \mathrm{~V}}$ mice. Furthermore, $M p l^{\mathrm{hlb} 219 / 219} / L r p 5^{\mathrm{wt} / \mathrm{wt}}$ mice did not display a different bone phenotype in comparison to littermate wild-type animals (BV/TV: $10.7 \pm 1.0$ vs. $10.4 \pm 1.7 \%$, p-value: 0.80). Finally, the megakaryocyte-specific inactivation of Wntl did not affect the skeletal microarchitecture (BV/TV: Wnt $1^{\text {flox } /}$ flox $/$ Pf4-Cre ${ }^{+}: 15.2 \pm 2.9 \%$, Wnt $1^{\text {flox/flox }} /$ Pf4-Cre $^{-}: \quad 15.5 \pm 0.9 \%$, p-value: 0.88).

Conclusion: Despite the increased number of megakaryocytes in the bone marrow of mice carrying a high bone mass-causing mutation of Lrp5 and the observed expression of Wnt1 in megakaryocytes, the depletion of this cell type did not affect the high bone mass phenotype of the $\operatorname{Lrp} 5^{\mathrm{G} 170 \mathrm{~V} / \mathrm{G} 170 \mathrm{~V}}$ mice, nor did it influence the skeletal structure under normal conditions. Likewise, inactivation of Wnt1 in megakaryocytes had no impact on bone structure. These results clearly demonstrate that megakaryocyte-derived factors and especially megakaryocyte-derived Wnt 1 are not required for the proper formation and turnover of skeletal tissue.

Keywords: Mouse models, Wnt signaling, High bone mass, Megakaryocyte

\section{P364}

Material properties in bone tissue from patients with Melorheostosis caused by somatic activation mutations in $M A P 2 K 1$

Nadja Fratzl-Zelman $^{1}$, Paul Roschger ${ }^{1}$, Heeseog Kang ${ }^{2}$, Smita Jha ${ }^{3,4}$, Andreas Roschger $^{5}$, Stephane Blouin ${ }^{1}$, Zuoming Deng ${ }^{6}$, Wayne A. $\mathrm{Cabral}^{2,7}$, Aleksandra Ivovic ${ }^{8}$, Francoise Meylan ${ }^{8}$, Eric P. Hanson ${ }^{9}$, Eileen Lange ${ }^{10}$, James Katz ${ }^{10}$, Edward W. Cowen ${ }^{11}$, Klaus Klaushofer ${ }^{1}$, Richard Siegel ${ }^{8}$, Peter Fratzl ${ }^{5}$, Timothy Bhattacharyya ${ }^{3}$, Joan C. Marini ${ }^{2}$

${ }^{1}$ Ludwig Boltzmann Institute of Osteology at the Hanusch Hospital of WGKK, and AUVA Trauma Center Meidling, 1st Medical Department Hanusch Hospital, Vienna, Austria, ${ }^{2}$ Section on Heritable Disorders of Bone and Extracellular Matrix, National Institute of Child Health and Human Development, National Institutes of Health, Bethesda, United States, ${ }^{3}$ Clinical and Investigative Orthopedics Surgery Unit, National Institute of Arthritis and Musculoskeletal and Skin Diseases, National Institutes of Health, Bethesda, United States, ${ }^{4}$ Program in Reproductive and Adult Endocrinology, Eunice Kennedy Shriver National Institute of Child Health and Human Development, National Institutes of Health, Bethesda, United States, ${ }^{5}$ Max Planck Institute of Colloids and Interfaces, Department of Biomaterials, Potsdam, Germany, ${ }^{6}$ Biodata Mining and Discovery Section, Office of Science and Technology, National Institute of Arthritis and Musculoskeletal and Skin Diseases, National Institutes of Health, Bethesda, United States, ${ }^{7}$ Current Address: Molecular Genetics Section, National Human Genome Research Institute, National Institutes of Health, Bethesda, United States, ${ }^{8}$ Immunoregulation Section, Autoimmunity Branch, National Institute of Arthritis and Musculoskeletal and Skin Diseases, National Institutes of Health, Bethesda, United States, ${ }^{9}$ Immunodeficiency and Inflammation Unit, Autoimmunity Branch, National Institute of Arthritis and Musculoskeletal and Skin Diseases, National Institutes of Health, Bethesda, United States, ${ }^{10}$ Office of the Clinical Director, National Institute of Arthritis and Musculoskeletal and Skin Diseases, National Institutes of Health, Bethesda, United States, ${ }^{11}$ Dermatology Branch, National Institute of Arthritis and Musculoskeletal and Skin Diseases, National Institutes of Health, Bethesda, United States

Somatic activating mutations in $M A P 2 K 1$ have been recently identified as a cause of melorheostosis, a rare skeletal dysostosis characterized by dense hyperostotic lesions. These consist of osteonal-like remodeled bone covered by a shell formed through apposition of compact multi-layered lamellae oriented parallel to the periosteal surface. Here we characterize bone tissue properties in affected and 
unaffected bone of six patients using quantitative back-scattered electron imaging and nanoindentation.

Melorheostotic bone lesions have lower average mineralization density (CaMean, $\mathrm{P}=0.03)$ and increased amount of matrix undergoing primary mineralization (CaLow, $\mathrm{P}=0.008$ ) compared to unaffected cortical tissue (paired $t$ test). The parallel lamellar portion of the lesion, was even less mineralized, possibly because the newly deposited material consists of younger tissue. Overall, there is a positive correlation between CaLow and osteoid thickness, osteoid surface (both $\mathrm{P}=0.005, \mathrm{r}=0.68)$ and osteoblast surface $(\mathrm{P}=0.5$, $r=0.51$ ) supporting dependence of mineral content on bone turnover. Indentation modulus characterizing material stiffness is dependent on tissue mineralization (positive correlation with CaPeak: $\mathrm{P}=0.0007$, $r=0.98$ ) in the same manner in affected and unaffected bone. Finally, we find a higher 2D-porosity in sections of affected bone due to increased osteocyte lacunar number $(+39 \%, \mathrm{p}=0.03)$ and mean area $(+7.5 \%, \mathrm{p}=0.03)$. There was also a higher density ( $2-3$ fold $)$ of osteon-like voids in affected bone.

In conclusion, the mechanical properties of bone tissue in melorheostotic lesions reflect its structural heterogeneity, and they correlate with local variations in tissue-age dependent mineral content and with remodeling rates in the same way as normal bone.

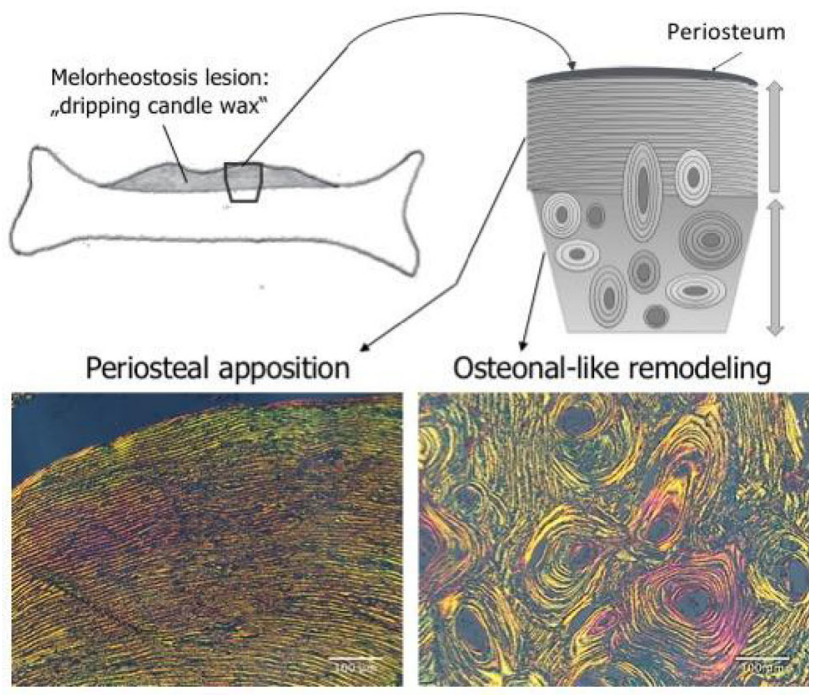

Bone Mineralization Density Distribution

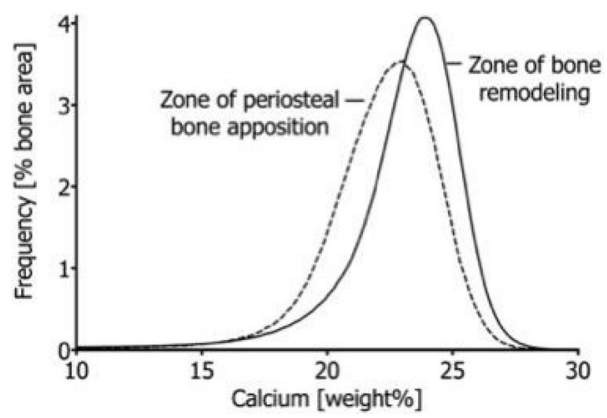

Melorheostotic bone lesion

Keywords: Histology, Periosteum, Matrix Mineralization, Nanoindentation

\section{P365}

A homozygous CREB $3 L 1$ missense mutation expands the mutational spectrum of CREB3L1-related osteogenesis imperfecta

B Guillemyn $^{1}$, D Syx ${ }^{1}$, L Demuynck $^{1}, \mathrm{H} \mathrm{Kayserili}^{2}$, A De Paepe ${ }^{1}, \mathrm{P}$ Coucke $^{1}$, F Malfait ${ }^{1}$, S Symoens ${ }^{1}$

${ }^{1}$ Center for Medical Genetics Ghent $(C M G G)$ - Medical Research Building (MRB), Ghent University, Ghent, Belgium, ${ }^{2}$ Medical Genetics, University of Istanbul, Istanbul, Turkey

Objectives: Previously, we identified CREB3L1 (encoding the ERstress transducer OASIS) as a novel autosomal recessive (AR) lethal/severe osteogenesis imperfecta (OI) gene in a fetus in whom this gene was homozygously deleted. Hitherto, only two additional mutations have been reported. One mutation concerns an in-frame deletion c.934_936delAAG (p.(Lys312del)), affecting both heterozygous carriers (mild) and homozygous patients (severe). The other mutation is a homozygous frameshift mutation c.1365del (p.(Pro458Argfs*25)) in a family with severe AR OI. We now report the first homozygous CREB3L1 missense mutation associated with lethal AR OI.

Methods: Fetal DNA, parental blood samples and signed informed consent were obtained. Genetic testing was performed for all known OI genes. The pathogenic nature of the variant was investigated by evaluating population/disease/sequence-specific databases and in silico predictive algorithms.

Results: The proband is a fetus which was aborted at 19 weeks of gestation because of multiple fractures and short/bowed extremities. Genetic testing revealed a homozygous CREB3L1 missense mutation c. $911 \mathrm{C}>\mathrm{T}$, p.(Ala304Val). Both parents are heterozygous carriers. The variant is located within the highly conserved basic leucine zipper domain and is positioned only four amino acids upstream of the DNA binding domain. In silico tools predict this variant to be pathogenic (unknown in gnomAD; PROVEAN $=-3.722$ (Deleterious); $\quad$ MutationTaster $=$ disease $\quad$ causing; $\quad$ PolyPhen $2=5.735$; PhastCons $=1$ ).

Conclusion: We highlight the first CREB3L1 missense mutation associated with lethal AR OI and herewith expand the mutational spectrum of this poorly understood OI type. The variant is located in the same protein domain as the earlier reported in-frame deletion. As such, we hypothesize that this missense mutation-similar to the reported in-frame deletion-might affect OASIS transcriptional activity; thereby influencing the formation of the secretory coat protein II complex, which is in turn postulated to disturb the OASISmediated secretory pathways, necessary for normal bone development.

\section{P366}

The cardiovascular profile among patients with Non-Surgical Hypoparathyroidism and Pseudohypoparathyroidism: a cohort study $\underline{\text { Line Underbjerg }}^{1}$, Tanja Sikjaer ${ }^{1}$, Lars Rejnmark ${ }^{1}$

${ }^{I}$ Department of Endocrinology and Internal Medicine, Aarhus University Hospital, Aarhus C, Denmark

Background: Both Non-Surgical Hypoparathyroidism (NS-HypoPT) and Pseudohypoparathyroidism (PHP) are rare diseases. PTH has both a direct and indirect effect on the cardiovascular system. Previously studies have found a significantly increased risk of cardiovascular diseases, especially ischemic heart diseases, stroke and arrhythmia, among patients with NS-HypoPT compared to age- and gender matched controls, whereas no difference between PHP and matched controls were seen.

We aimed to investigate the cardiovascular profile among Danish patients with either NS-HypoPT or PHP and compare the two groups. 
We hypothesize no difference in the cardiovascular profile between groups.

Methods: 56 patients with Ns-HypoPT and 30 with PHP were included and underwent a clinical examination including blood sampling and measurements of blood pressure, arterial stiffness and pulse wave velocity. Arterial stiffness and pulse wave velocity was measured using AtCor SpygmoCor-XCEL (Atcor Medical Pty Ltd, Sydney, Australia).

Results: Patients with Ns-HypoPT had an average age of $47 \pm 17$ years (68\% females) and PHP patients $36 \pm 13$ years $(80 \%$ females). Over $70 \%$ in both groups were been genetically verified. No difference between groups were seen in history of cardiovascular disease, smoker status, use of calcium and vitamin D intake nor cholesterol lowering drugs or antihypertensive treatment. Compared with Ns-HypoPT, PHP patients had a significantly lower HDL cholesterol and average glucose from $\mathrm{HbAlc}\left(\mathrm{p}_{\mathrm{both}}=0.01\right)$. Office blood pressure did not differ between groups $\left(\mathrm{BP}_{\mathrm{Ns}-\mathrm{HypoPT}} 129 / 77\right.$, pulse 68 ; $\mathrm{BP}_{\mathrm{PHP}} 127 / 78$, pulse 68 ), neither were differences seen after stratification on gender. Pulse wave velocity was significantly higher among patients with Ns-HypoPT ( $\mathrm{p}_{\text {crude }}$ 0.02), even after adjustment for middle arterial pressure, BMI, age and gender $\left(p_{\text {adjusted }}<0.01\right)$.

Conclusion: The cardiovascular profile between groups differ. Compared with PHP, Ns-HypoPT have a higher pulse wave velocity, associated with an increased risk of cardiovascular diseases.

Keywords: Non-surgical Hypoparathyroidism, pseudohypoparathyroidism, cardiovascular profile

\section{P367}

[18F]NaF PET: A good modality for identifying progression of HO in Fibrodysplasia ossificans progressiva

Esmée Botman ${ }^{1}$, Pieter G.H.M. Raijmakers ${ }^{2}$, Maqsood M. Yaqub ${ }^{2}$, Bernd Teunissen $^{3}$, J. Coen Netelenbos ${ }^{1}$, Wouter Lubbers ${ }^{4}$, Lothar Schwarte $^{4}$, Adriaan A. Lammertsma ${ }^{2}$, Marelise (Elisabeth) Eekhoff ${ }^{1}$, on behalf of the FOP research group

${ }^{I}$ Endocrinology, $V U$ University Medical Center, Amsterdam, Netherlands, ${ }^{2}$ Nuclear Medicine, VU University Medical Center, Amsterdam, Netherlands, ${ }^{3}$ Radiology, VU University Medical Center, Amsterdam, Netherlands, ${ }^{4}$ Anesthesiology, VU University Medical Center, Amsterdam, Netherlands

Background: Fibrodysplasia ossificans progressiva (FOP) is a rare, autosomal dominant disorder characterized by heterotopic ossification (HO) in muscles, ligaments and tendons. Flare-ups often precede the formation of $\mathrm{HO}$. Whether $\mathrm{HO}$ progresses in the absence of a flare-up has not been confirmed yet. A recent study showed that $\left[{ }^{18} \mathrm{~F}\right] \mathrm{NaF}$ PET is a promising modality for early identification of ossifying flare-ups. To examine whether existing $\mathrm{HO}$ progresses asymptomatically and whether the $\left[{ }^{18} \mathrm{~F}\right] \mathrm{NaF}$ PET can identify these chronic asymptomatic lesions, consecutive $\left[{ }^{18} \mathrm{~F}\right] \mathrm{NaF}$ PET/CT-scans were analyzed retrospectively.

Method: A total of thirteen $\left[{ }^{18} \mathrm{~F}\right] \mathrm{NaF}$ PET/CT-scans from five FOP patients were analyzed. The scans were acquired over $0.5-2$ years. An in-house tool was used for identifying and analyzing the heterotopic bone structures. Amongst others, volume of $\mathrm{HO}$ and standardized volume uptake (SUV) values were obtained.

Results: Two out of five patients experienced $\geq 1$ active clinical flare-ups at the time of the $\left[{ }^{18} \mathrm{~F}\right] \mathrm{NaF}$ PET scan. Apart from the clinical flare-ups, in four out of five patients the serial scans showed progression of asymptomatic $\mathrm{HO}$ varying from 3 to $7 \mathrm{~mL}$. This volumetric increase was present for one out of ten of the 52 identified HO structures, which were all characterized by increased sodium fluoride uptake. The degree of progression of $\mathrm{HO}$ had a moderate correlation with $\mathrm{SUV}_{\text {peak }}$ values (Spearman: 0.570)
Conclusion: In FOP, HO volume may progress chronically without a clinical flare-ups. $\left[{ }^{18} \mathrm{~F}\right] \mathrm{NaF}$ PET enables to identify all asymptomatic chronic progressive disease lesions. Future drug trials should not only target new $\mathrm{HO}$ formation but also chronic $\mathrm{HO}$ progression, for which $\left[{ }^{18} \mathrm{~F}\right] \mathrm{NaF}$ PET/CT appears to be an adequate imaging technique for detection and follow-up.

Keywords: Fibrodysplasia Ossificans Progressiva, Positron Emission Tomography, Heterotopic ossification, flare-up

\section{P368}

Implementation of an in-house designed skeletal dysplasia gene panel as a first screening step to diagnose unsolved osteogenesis imperfecta (-like) patients

B Guillemyn $^{1}$, D Syx $^{1}$, T Rosseel ${ }^{1}$, H Kayserili $^{2}$, AM CuetoGonzález $^{3}$, L Van Maldergem ${ }^{4}$, B Menten ${ }^{1}$, A De Paepe ${ }^{1}$, P Coucke $^{1}$, $\mathrm{F}_{\text {Malfait }}{ }^{1}, \mathrm{~S}$ Symoens ${ }^{1}$

${ }^{1}$ Center for Medical Genetics Ghent $(C M G G)$ - Medical Research Building (MRB), Ghent University, Ghent, Belgium, ${ }^{2}$ Medical Genetics, University of Istanbul, Istanbul, Turkey, ${ }^{3}$ Clinical Genetics, Vall D'Hebron Hospital Materno Infantil, Barcelona, Spain, ${ }^{4}$ Centre Hospitalier Universitaire de Besançon - Génétique, Besancon Cedex, France

Objectives: The pace of disease-related gene discovery has accelerated phenomenally in recent years due to advances of next generation sequencing (NGS) technologies, such as whole exome sequencing (WES). In the field of skeletal dysplasias, particularly for osteogenesis imperfecta (OI), NGS added a remarkable number of disease loci, thereby expanding the brittle bone disease to a predominantly collagenrelated disorder. However, for a small subset of OI patients the genetic cause remains elusive. We designed a skeletal dysplasia gene panel and evaluated the detection rate in twenty-one unsolved OI patients.

Methods: Twenty-one OI-like patients, in who no type I collagen defect was detected, were selected based on clinical severity and positive familial anamnesis. Signed informed consents were obtained. Known loci for heritable skeletal disorders were combined with candidate genes for bone fragility, resulting in a skeletal dysplasia gene panel including 566 genes. WES data were mapped and analysed by using the in-house developed pipeline SeqPlorer, which integrates (population) database information/ quality scores/coverage data and in silico prediction algorithms.

Results: Hitherto, causal mutations were identified in three cases. The first proband carries a homozygous missense mutation in the known OI-gene LEPRE1 (c.446T > G, p.(Leu149Arg)), encoding prolyl-3-hydroxylase-1, confirming the diagnosis of autosomal recessive (AR) OI. The second proband harbours a homozygous missense mutation (c.1444C > A, p.(His482Asn)) in the ALPL gene, linked to AR hypophosphatasia. In a third consanguineous family a homozygous nonsense mutation was detected in the osteopetrosisassociated gene CTSK (c.721C > T, p.(Arg241*)). For the remaining patients, suggestive variants were selected for confirmational Sanger sequencing and segregation analysis.

Conclusion: Our findings show that the implementation of this skeletal dysplasia gene panel is a powerful first screening step in molecularly diagnosing OI (-like) patients, especially for those patients whom are difficult to diagnose due to phenotypically overlapping clinical symptoms.

\section{P369}

Trabecular bone score in osteogenesis imperfecta. Is it useful?

Helena Florez ${ }^{1}$, Africa Muxi ${ }^{2}$, Eva Gonzalez ${ }^{3}$, Ana Monegal ${ }^{1}$, Núria Guañabens ${ }^{1}$, Pilar Peris ${ }^{1}$ 
${ }^{1}$ Metabolic Bone Diseases Unit, Department of Rheumatology. Hospital Clinic. University of Barcelona, Barcelona, Spain, ${ }^{2}$ Department of Nuclear Medicine. Hospital Clinic, University of Barcelona, Barcelona, Spain, ${ }^{3}$ Department of Immunology. Hospital Clinic, University of Barcelona, Barcelona, Spain

Background: Osteogenesis imperfecta (OI) is a congenital bone disease characterised by a low bone mineral density (BMD) and poor bone quality and strength. The usefulness of Trabecular Bone Score (TBS) in OI has been scarcely evaluated.

Objectives: To analyse the clinical usefulness of TBS determination in patients with $\mathrm{OI}$ and its relation with anthropometric and clinical features (especially skeletal fractures and BMD results).

Methods: Twenty-four patients (18F:6M) with OI, aged $38 \pm 15$ years (19-63) were included. The clinical reports were reviewed, with especial attention to anthropometric parameters, previous fractures, disease severity, associated mutations and treatments received. Lumbar spine (LS), total hip (TH), and femoral neck (FN) BMD were measured using DXA equipment (Lunar). TBS was analysed in LS, and the results were classified in three categories ${ }^{1}$ : TBS $>1.310$ (normal), 1.230-1.310 (partially degraded microarchitecture), $<1.230$ (degraded microarchitecture). TBS values were compared with a healthy control group.

Results: $5 / 24$ patients $(21 \%)$ had a degraded microarchitecture, 4 $(17 \%)$ a partially degraded microarchitecture and $15(63 \%)$ normal TBS. All patients with TBS $<1230$ were over 40 years. 21/24 patients had multiple fractures. Regarding BMD, $54 \%$ of the patients had osteoporosis, $42 \%$ osteopenia and one had normal values. A correlation was observed between TBS and age $(r=-0.5$, $\mathrm{p}=0.006)$ and $\operatorname{LS}$ BMD $(\mathrm{r}=0.5, \mathrm{p}=0.014)$, showing a trend to significance with BMI $(r=-0.4, p=0.058)$. No significant differences were observed on comparing TBS in patients and controls (1.321 vs. $1.391, \mathrm{p}=$ N.S.).

Conclusions: TBS measurement does not seem to be useful for evaluating bone strength in patients with OI. Despite most patients presenting a history of multiple fractures, only $21 \%$ showed degraded microarchitecture with TBS.

References: ${ }^{1}$ McCloskey EV, et al. J Bone Miner Res. 2016

Keywords: Osteogenesis imperfecta, TBS, DXA, bone microstructure

\section{P370}

European reference network on bone rare diseases (BOND ERN)

Luca Sangiorgi ${ }^{1}$, Matias de la Calle ${ }^{2}$

${ }^{1}$ SSD Genetica Medica e Malattie Rare Ortopediche, Istituto Ortopedico Rizzoli, Bologna, Italy, ${ }^{2}$ SSG Genetica Medica e Malattie Rare Ortopediche, Istituto Ortopedico Rizzoli, Bologna, Italy

ERN BOND 38 HCPs from 10 EU countries.

The ambition of the ERN BOND is to implement measures that facilitate multidisciplinary, holistic, continuous, patient-centred and participative care provision to people living with rare bone diseases (RBD), supporting them in the full realisation of their fundamental human rights. To meet this goal, BOND gathers EU professionals highly specialized in the field of RBD for both scientific research and multidisciplinary care to increase knowledge on RDs, to improve healthcare quality and patient safety, to increase access to ultra specialized medical expertise and accessible information beyond national borders.

ERN BOND aspiration is to support patients affected by RBD and their families, to increase their capacity to undertake a participative role in care provision, to set priorities and to participate in decisions regarding their care plan and their life project.

BOND will bring rapid interchange of information, skills and practice to shorten time to diagnosis, and treatment, in collaboration with Patients Representatives (ePAGs).

Efforts to target less developed affiliate partners, will be carried, meeting the target of improving healthcare in ALL Members States. EU Parliamentary Meeting "ERNs accelerating and improving diagnosis for rare diseases patients: An ERN BOND White Paper on Diagnosis"

The White Paper provides a picture on the current situation in several EU countries in regard to diagnosis and to identify the main challenges and potential solutions that could help reduce diagnosis delays. The survey identifies existing care gaps within and among ERN BOND HCPs, collects patients information on their experience leading to the disease diagnosis. The results of the survey sheds light on the unmet needs of patients in this specific area and serve as a basis for a dialogue with policy-makers on the challenges related to diagnosis of rare bone diseases.

\section{P371}

Do femoral fractures in adult osteogenesis Imperfecta resemble atypical femoral fractures?

Jane Andersen $^{1,2}$, Mathias Hauge Bünger $^{3}$, Ole Rahbek ${ }^{3}$, Jannie Dahl Hald $^{1}$, Torben Harsløf ${ }^{1}$, Bente Langdahl ${ }^{1}$

${ }^{I}$ Department of Endocrinology and Internal Medicine, Aarhus University Hospital, Århus, Denmark, ${ }^{2}$ Department of Internal Medicine, Horsens Sygehus, Horsens, Denmark, ${ }^{3}$ Department of Paediatric Orthopaedics, Aarhus University Hospital, Århus, Denmark

Objectives: Atypical femoral fractures (AFF) are low energy femoral fractures with characteristic radiological features and a suspected relation to bisphosphonate (BP) treatment (Shane et al. JBMR, 2013). In osteogenesis imperfecta (OI) BPs are currently the drug of choice when medical treatment is necessary. OI is characterized by skeletal fragility and pronounced bone deformity. Due to bone deformities, the radiologic appearance of femoral fractures may be different in patients with OI. Thus, we speculated whether femoral fractures in adult OI resemble AFF.

Methods: We investigated the prevalence of femoral fractures in adult patients with confirmed OI, in a cohort of adult patients who attended the Day Care Clinic at Aarhus University Hospital. We collected information on severity of OI and length of BP treatment. The fractures were compared to major and minor criteria of AFF.

Results: In a cohort of 55 patients, eight patients (15\%) had suffered a femoral fracture in adult years $(20+$ years). One patient had OI type I, two had OI type III, four had OI type IV and one had OI type V. All fractures were associated with no or minimal trauma, were complete and non-comminuted. Six patients had fractures with a transverse configuration, located distal to the small trochanter and fulfilled the criteria of AFF. Two patients had fractures of the femoral neck in combination with coxa vara. Three patients had not received BP treatment prior to the fracture. Four patients had severe bone deformity in the femoral region due to OI.

Conclusion: Femoral fractures in OI resemble AFF. This suggests that bone deformity and collagen deficiencies rather than BPs cause fractures resembling AFF.

Continuous dull or aching pain in the groin or thigh requires a radiographic examination.

Keywords: Osteogenesis Imperfecta, Atypical Femoral Fractures, Case studies, Femoral Fractures, Bisphosphonate treatment 


\section{P372}

New mechanisms and therapies for Osteogenesis Imperfecta caused by mutations in type I procollagen chaperones

Ivan Duran ${ }^{1}$, Fabiana Csukasi ${ }^{1}$, Jorge Martin ${ }^{1}$, Pavel Krejci ${ }^{2}$, Dan Cohn $^{1}$, Deborah Krakow ${ }^{1}$

${ }^{1}$ University of California Los Angeles, Los Angeles, United States, ${ }^{2}$ Masaryk University, Brno, Czech Republic

Over the last decade we have learned that Osteogenesis Imperfecta (OI) is not solely caused by mutations in COL1A1 and COL1A2, the genes that encode type I collagen. Many of the newly identified OI genes have been shown to affect functions in the endoplasmic reticulum (ER), the organelle that is critical for the delivery of properly synthesized type I procollagen to the extracellular matrix. In the lumen of the ER, type I procollagen is post-translationally modified and processed by a number of enzymes and chaperones. Recently, our laboratory and others have shown that mutations in the genes encoding ER chaperones can produce autosomal recessive forms of OI. We have shown that FKBP65, HSP47, BiP and LH2 form a chaperone complex, which participates in the modification of type I procollagen. Loss of function of these key type I procollagen chaperones cause ER dysfunction and abnormal procollagen production. Interestingly, these ER defects are also observed in more typical OI cases with mutations in type I procollagen genes, suggesting that a defective ER is commonly associated with the disease and could be a therapeutic target. To test this hypothesis, we are using chemical chaperones to evaluate the effects of ER stress reduction in OI mouse models. Preliminary results show that the treatment is effective in reducing the ER stress and it ameliorates the OI phenotype.

Keywords: Osteogenesis Imperfecta; Type I procollagen; ER stress; chaperone

\section{P373}

Retrospective study of hypophosphatasia prevalence in Granada (Spain)

Cristina García Fontana ${ }^{1}$, Juan Miguel Villa Suárez ${ }^{2}$, Maria Teresa Márquez Hernández ${ }^{3}$, Francisco Andújar Vera ${ }^{1}$, José María Gómez Vida $^{4}$, Tomás de $\mathrm{Haro}^{2}$, Beatriz García Fontana ${ }^{5}$, Manuel Muñoz Torres $^{6}$

${ }^{1}$ Instituto de Investigación Biosanitaria de Granada (Ibs.GRANADA), Granada, Spain, ${ }^{2}$ Clinical Analyses Unit, Hospital Universitario Campus de la Salud, Granada, Spain, ${ }^{3}$ Instituto de Investigación Biosanitaria de Granada (Ibs.GRANADA); Department of Medicine. University of Granada, Granada, Spain, ${ }^{4}$ Pediatric Unit, Hospital Universitario Campus de la Salud, Granada, Spain, ${ }^{5}$ Endocrinology and Nutrition Unit, Hospital Universitario Campus de la Salud. Instituto de Investigación Biosanitaria de Granada (Ibs.GRANADA); CIBERFES. Instituto de Salud Carlos III, Granada, Spain, ${ }^{6}$ Endocrinology and Nutrition Unit, Hospital Universitario Campus de la Salud. Instituto de Investigación Biosanitaria de Granada (Ibs.GRANADA); CIBERFES. Instituto de Salud Carlos III; Department of Medicine. University of Granada., Granada, Spain

Introduction: Hypophosphatasia (HPP) is a rare, serious and potentially mortal genetic disease caused by one or several mutations in the gene coding for the alkaline phosphatase without tissue specificity (TNSALP). A low serum total alkaline phosphatase (ALP) level is the hallmark for the diagnosis of HPP.

Objective: To assess the recognition of persistent low ALP levels in a tertiary care hospital in Granada to identify the patients affected with HPP, to provide an appropriate management avoiding potentially harmful drugs.
Methods: An assessment of ALP levels was performed in 78,590 patients between the 1st of January and the 31st of December 2016 in the Biochemistry Unit of our hospital. The database was divided into adult population and pediatric population. Ninety-eight patients (66 adults and 32 children) had several serum ALP values persistently below the reference interval. Twenty-two patients were discarded because of potential causes of secondary HPP. Twenty-four potential HPP patients were contacted to fulfill a questionnaire about clinical manifestations HPP-related. Pyridoxal-5'-phosphate (PLP) was determined by high-performance liquid chromatography (HPLC) and sequencing TNSALP gene was performed. This project was approved by Ethical Committee from Granada.

Results: $0.12 \%$ of the studied patients had persistently low value of ALP (65\% females). Twenty-four were contacted. Ten of them had fractures, 4 had symptomatic chondrocalcinosis and 4 had dental abnormalities. From them, 11 patients ( 9 adults and 2 children) presented decreased ALP and increased PLP levels, of which, 7 adults had TNSALP mutations (4 corresponding to new variants not described previously).

Conclusions: Our study shows that there are several omitted diagnostics of HPP in a tertiary care hospital. From 78,590 patients analyzed, 7 of them presented TNSALP mutation obtaining 4 new genetic variants. These data indicate an estimated prevalence of $9 / 100.000$ in our area.

Keywords: hypophosphatasia, new genetic variants, prevalence

\section{P374}

Establishing an Osteogenesis Imperfecta pediatric cellular collection to study TGFbeta pathway activation

Arantza Infante $^{1}$, Andrea Goitia ${ }^{1}$, Ana Bueno $^{2}$, Belén Sagastizabal $^{3}$, Blanca Gener ${ }^{4}$, Clara I Rodríguez

${ }^{1}$ Stem Cell and Cell Therapy Lab, BioCruces Health Research Institute, Barakaldo, Spain, ${ }^{2}$ Department of Orthopedic Surgery, Getafe University Hospital, Madrid, Spain, ${ }^{3}$ Endocrinology Department, Getafe University Hospital, Madrid, Spain, ${ }^{4}$ Genetics Service, Cruces University Hospital, Barakaldo, Spain

Osteogenesis Imperfecta (OI) is a genetic disorder of connective tissue (1/15,000-20,000 births) characterized by brittle bones, fractures and extraskeletal manifestations with broad heterogeneity at the molecular and clinical levels. Most cases of OI are caused by autosomal dominant mutations in the genes encoding type I collagen (COL1A1 and COL1A2). In these situations the osteoblasts (which are derived from mesenchymal stem cells (MSCs)) are unable to synthesize and transfer outside the cells the appropriate collagen amount and/or quality, leading to poor bone matrix. Mutations in other genes responsible for recessive OI types are associated with defects in the collagen processing or impairment of osteoblastic differentiation. The molecular mechanisms of OI are incompletely understood, however, an interesting study carried out in murine models reported that excessive TGFbeta pathway activation is a common mechanism between different types of OI. Modulation of this pathway reverted the skeletal alterations in those murine models of OI.

The objectives of this work are: (1) To establish a collection of human mesenchymal stem cells (hMSCs) from pediatric OI patients (after obtaining the required ethical authorizations) in order to have an in vitro human model of OI disease. (2) To study TGFbeta pathway activation in the OI-hMSCs during the osteoblastic differentiation process.

Results: Currently, we have isolated OI-hMSCs from 17 pediatric patients clinically and genetically characterized which meet the minimum criteria for defining MSCs by the International Society of Cellular Therapy. Until now, we have studied the mRNA expression of three TGFbeta target genes (IGFBP7, PAI-1 and JunB) in three OI- 
hMSCs lines and in two control-hMSCs lines. The obtained data show an altered expression of TGFbeta target genes during the osteogenic differentiation process in OI-hMSCs.

We pretend to extend these analysis to the complete OI cellular collection.

\section{P375}

Osteitis condensans ilii: Differential diagnosis and management.

A case of back pain with combined OCI and syringomyelia findings

Peter Peev $^{1}$, Francesca Mellor ${ }^{1}$, Daniela Dyankova ${ }^{2}$

${ }^{1}$ London Northwest Hospitals NHS Trust, Ealing Hospital, London, United Kingdom, ${ }^{2}$ Royal National Orthopaedic Hospital, London, United Kingdom

Background: Osteitis condensans ilii refers to an increase in bone density (sclerosis) located on the inferomedial aspect of the ilium adjacent to the sacroiliac joint. It is often bilateral, symmetric, and triangular. It is a rare condition of benign cause of axial low back pain. The reason remains unknown since it is first described in 1926. Typically affects females following pregnancy, but males and nulliparous females have also been reported.

Objectives: Describe diagnostic process and management of OCI as a rare condition of low back pain. Compare the findings with the available data from literature.

Method: 29 years old woman presents with lower back pain and polyarthralgia without a history of trauma or injuries. She is an office worker and the condition affects her daily life activities and disturbs her night sleep. Finds it particularly difficult to lie flat and get in and out of bed. No other comorbidities recorded.

Clinically: Paraspinal and midline tenderness at L4/L5 levels, Good spinal flexion, neurovascular intact. Hip examination unremarkable. Distraction, Compression FABER and Gaenslen's test for sacroiliac joints were positive.

Radiology findings demonstrate sclerotic area, adjacent to Left SIJ with a triangular shape. MRI scan of the whole spine and pelvis demonstrated finding of syrinx from $\mathrm{T} 5$ to the termination of spinal cord and OCI lesion.

Laboratory studies demonstrated: Vit D and B12 deficiency, Subclinical hyperthyroidism, Normal CRP and ESR and Rh factor.

Further testing for HLA antigen is performed.

Discussion: OCI is a rare cause of lower back pain. History, examination, XR and MRI are diagnostic standards. Other spine pathology must be ruled out. Further laboratory tests are also required to distinguish this idiopathic condition from ankylosing spondylitis, seronegative spondyloarthropathy, metastatic disease or sacroiliitis. The condition could represent a diagnostic challenge in patients with polyarthralgia.

Keywords: Osteitis Condensans Ilii, Syringomyelia, Lumbalgia

\section{P376}

Bilateral multiple insufficiency femoral fractures in a patient with Paget disease treated 7 years with calcitonin and 20 years with etidronate

\section{George Trovas $^{1}$, Evagelia Kaskani ${ }^{2}$, Chistos Kosmidis ${ }^{3}$}

${ }^{1}$ Laboratory for Research of the Musculoskeletal System 'Th. Garofalidis', KAT Hospital, National and Kapodistrian University of Athens, School of Medicine, Athens, Greece, ${ }^{2}$ Health Center Chalandri, 1st Health District Attica, Athens, Greece, ${ }^{3}$ Health Center Alexandras, 1st Health District Attica, Athens, Greece

The current consensus of the Second report of the Task Force of the American Society for Bone and Mineral Research is that Atypical
Femoral Fractures are stress or insufficiency fractures that develop over time (JBMR 2014).

We present a 77 years old man who was diagnosed with Paget disease of bone in 1990. At the beginning received nasal calcitonin for 7 years 200UI and later he was started on etidronate $400 \mathrm{mg}$ daily which he has been taken ever since.

The patient presented earlier this year with a gradually increasing right hip/groin pain and a frontal radiograph showed a linear lucency in trochanteric region and lateral shaft of the right femur through pagetic bone (Fig. 1) and an oblique lucency in the diaphysis of the otherwise normal left femur (Fig. 2).

A bone scintigraphy demonstrates increased radionuclide uptake in the right femur right anonumous $* * * *$ bone and L 3 vertebra and the laboratory investigation concerning bone metabolism was as follows: $\mathrm{ALP}=257 \mathrm{UI}(25-129) \mathrm{Ca}=10.2 \mathrm{mh} / \mathrm{dl}, \mathrm{P}=4.3 \mathrm{mg} / \mathrm{dl}, \mathrm{PTH}=19$ $\mathrm{pg} / \mathrm{ml}(15-65), 26 \mathrm{OHD} 3=26.3 \mathrm{ng} / \mathrm{ml}$ Osteocalcin $=32.4(14-46)$.

We report a case of insufficiency fractures with features of atypical femoral fracture in a patient with Paget's disease associated with long term use of antiresorptive drugs calcitonin and non-nitrogen containing bisphosphonate (etidronate).

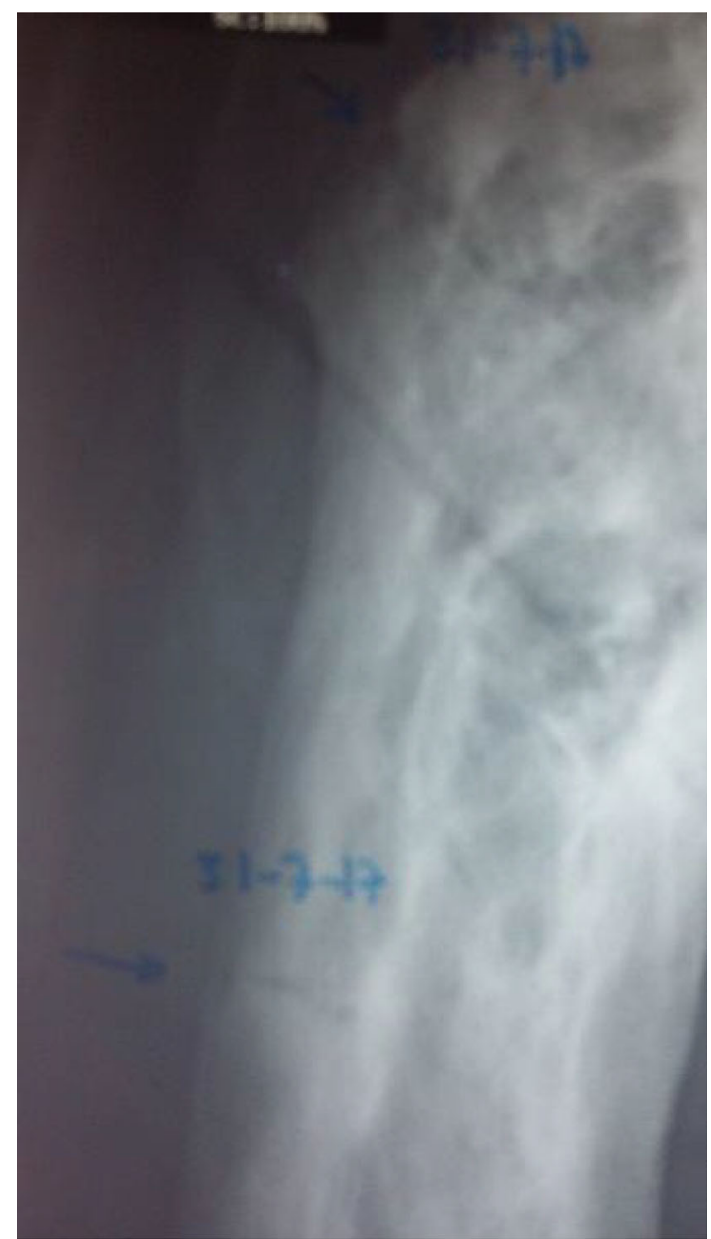

Figure 1

Paget disease insufficiency fractures-bisphosphonates 


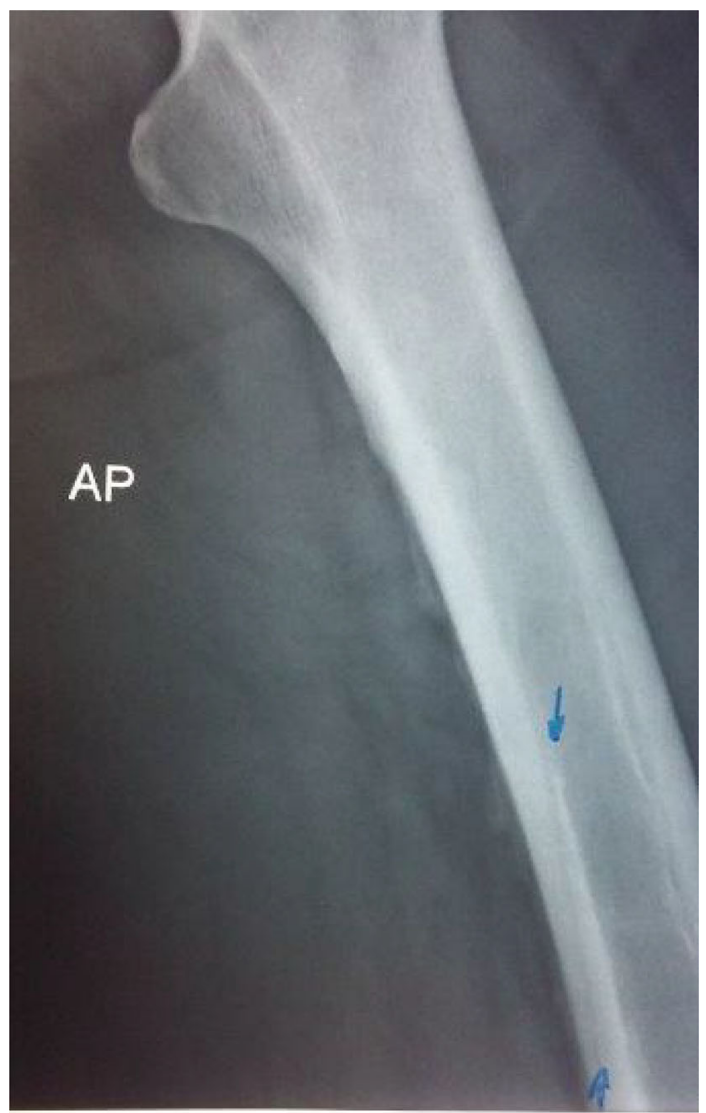

Figure2

\section{P377}

The Dual Roles of ENPP1, an Enzyme Suppressing Ectopic Mineralization and Supporting Bone Mineral Density

Dillon Kavanagh $^{1}$, Kristin Zimmerman ${ }^{1}$, Paul Stabach ${ }^{1}$, Thomas Carpenter $^{2}$, Mark Horowitz ${ }^{3}$

${ }^{1}$ Department of Pathology, Yale University, New Haven, United States, ${ }^{2}$ Department of Pediatrics, Yale University, New Haven, United States, ${ }^{3}$ Department of Orthopaedics and Rehabilitation, Yale University, New Haven, United States

Paradoxic Mineralization is a pathologic state characterized by over-mineralization in the peripheral tissues and under-mineralization of the skeleton. A satisfying physiologic mechanism accounting for the mineralization imbalance and therapeutics capable of addressing the morbidity of these diseases is lacking. Humans with ENPP1 loss of function develop a paradoxic mineralization disorder resulting in lethal vascular calcifications leading to death in $60-70 \%$ of patients by 6 months. This disease, called Generalized Calcification of Infancy (GACI), is then followed by a bone mineralization disorder in the surviving patients called Autosomal Hypophosphatemic Rickets type
2 (ARHR2). We recently demonstrated the efficacy of enzyme replacement therapy with an ENPP1-Fc fusion protein. Here, we demonstrate that the same strategy also rescues bone mineral deficits in the ENPP $1^{\text {asj/asj }}$ animal model. Our findings support a dual role for ENPP1 in suppressing ectopic vascular calcifications and maintaining bone mineral density.
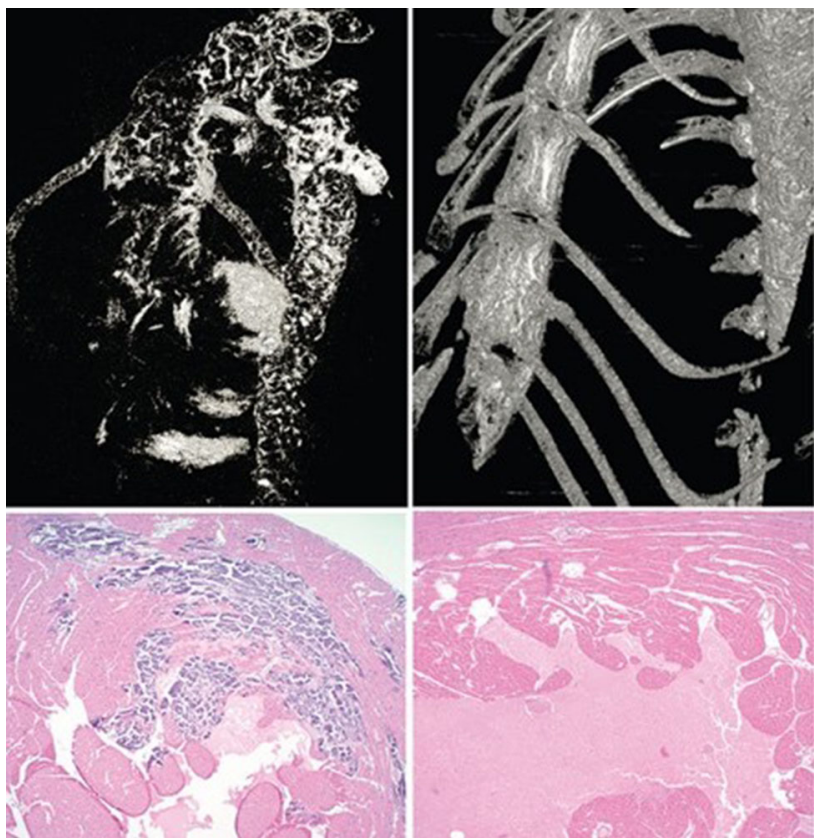

Top: MicroCT of untreated ENPP1 $1^{\text {asj/asj }}$ mice (left), and mice treated with ENPP1-Fc (right).

Bottom: Heart Histology of untreated ENPP1 ${ }^{\text {asj/asj }}$ mice (left) and mice treated with ENPP1-Fc (right).

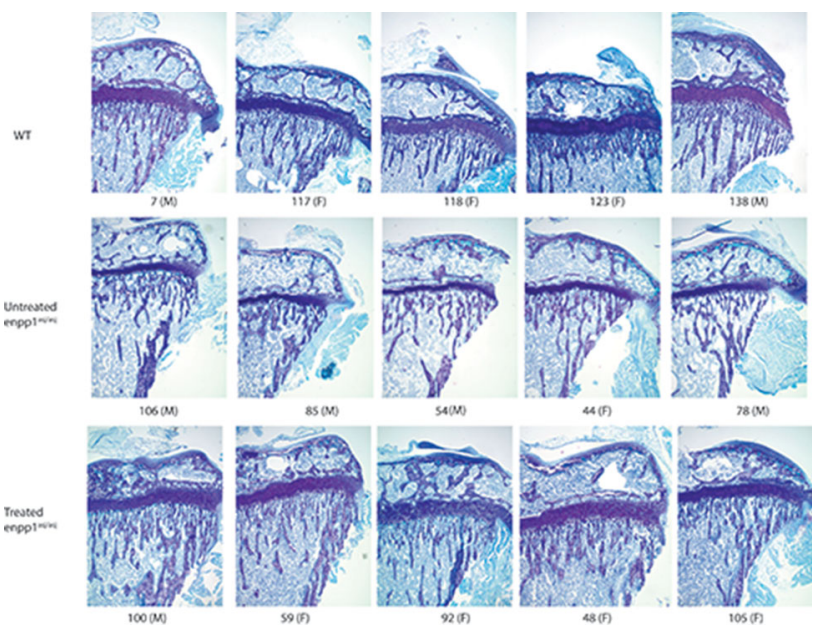

Tibia Epiphysis from WT (top) and ENPP1asj/asj mice treated with either vehicle (PBS - middle) or ENPP1-Fc (bottom), demonstrating the effect of ENPP1 on bone mineral density. 